\title{
IntechOpen
}

\section{Current Issues \\ and Recent Advances in \\ Pacemaker Therapy}

Edited by Attila Roka

R 



\section{CURRENT ISSUES AND RECENT ADVANCES IN PACEMAKER THERAPY}

Edited by Attila Roka 


\section{Current Issues and Recent Advances in Pacemaker Therapy}

http://dx.doi.org/10.5772/2618

Edited by Attila Roka

\section{Contributors}

Spyridon Koulouris, Sofia Metaxa, Baris Bugan, Ching-Sung Wang, Teng-Wei Wang, John Kassotis, Majid Haghjoo, Michele Rossi, Attilio Renzulli, Giuseppe Musolino, Giuseppe Filiberto Serraino, Jeffrey Williams, Dirk Bastian, Klaus Fessele, Béla Merkely, Levente Molnár, Attila Roka, Charles Webber, Jr., Alessandro Capucci, Federico Guerra, Michela Brambatti, Maria Vittoria Matassini, Salim Surani

\section{(c) The Editor(s) and the Author(s) 2012}

The moral rights of the and the author(s) have been asserted.

All rights to the book as a whole are reserved by INTECH. The book as a whole (compilation) cannot be reproduced, distributed or used for commercial or non-commercial purposes without INTECH's written permission.

Enquiries concerning the use of the book should be directed to INTECH rights and permissions department (permissions@intechopen.com).

Violations are liable to prosecution under the governing Copyright Law.

\section{(cc) BY}

Individual chapters of this publication are distributed under the terms of the Creative Commons Attribution 3.0 Unported License which permits commercial use, distribution and reproduction of the individual chapters, provided the original author(s) and source publication are appropriately acknowledged. If so indicated, certain images may not be included under the Creative Commons license. In such cases users will need to obtain permission from the license holder to reproduce the material. More details and guidelines concerning content reuse and adaptation can be foundat http://www.intechopen.com/copyright-policy.html.

\section{Notice}

Statements and opinions expressed in the chapters are these of the individual contributors and not necessarily those of the editors or publisher. No responsibility is accepted for the accuracy of information contained in the published chapters. The publisher assumes no responsibility for any damage or injury to persons or property arising out of the use of any materials, instructions, methods or ideas contained in the book.

First published in Croatia, 2012 by INTECH d.o.o.

eBook (PDF) Published by IN TECH d.o.o.

Place and year of publication of eBook (PDF): Rijeka, 2019.

IntechOpen is the global imprint of IN TECH d.o.o.

Printed in Croatia

Legal deposit, Croatia: National and University Library in Zagreb

Additional hard and PDF copies can be obtained from orders@intechopen.com

Current Issues and Recent Advances in Pacemaker Therapy

Edited by Attila Roka

p. cm.

ISBN 978-953-51-0703-3

eBook (PDF) ISBN 978-953-51-7023-5 


\section{We are IntechOpen, \\ the world's leading publisher of Open Access books}

Built by scientists, for scientists

\section{$4,100+$}

Open access books available

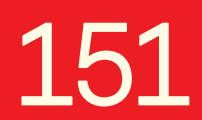

Countries delivered to
$116,000+$

International authors and editors
$120 \mathrm{M}+$

Downloads

Our authors are among the

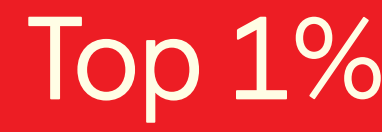

most cited scientists

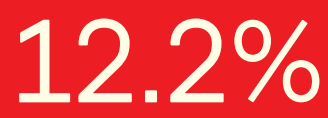

Contributors from top 500 universities

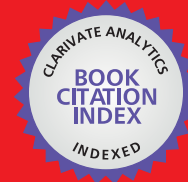

WEB OF SCIENCE ${ }^{\mathrm{TM}}$

Selection of our books indexed in the Book Citation Index in Web of Science ${ }^{\mathrm{TM}}$ Core Collection (BKCI)

Interested in publishing with us?

Contact book.department@intechopen.com

Numbers displayed above are based on latest data collected.

For more information visit www.intechopen.com

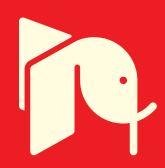





\section{Meet the editor}

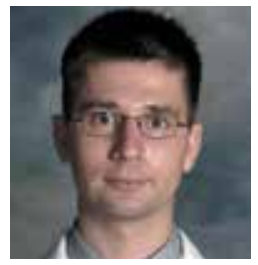

Dr Attila Roka graduated from Semmelweis University (Budapest, Hungary) as an MD in 2002, where he also earned a PhD in 2008. His current research interests are in vivo/in silico cardiac electrophysiology and optimization of device-based arrhythmia therapy. His collaborations include projects such as research on atrioventricular conduction in atrial fibrillation and pacemaker-based non-pharmacological rate control, avoidance of phrenic nerve stimulation during cardiac resynchronization therapy, use of remote monitoring to predict adverse events in patients with implanted defibrillators, and an international effort to investigate factors affecting utilization of cardiac resynchronization therapy in Europe. Currently he is a cardiovascular diseases fellow at the Hospital of St. Raphael (New Haven, CT, USA). He was awarded the Steve Kunos prize by the Arrhythmia Research and Treatment Foundation in 2008. 



\section{Contents}

\section{Preface XI}

Section 1 Novel Implantation Techniques 1

Chapter 1 Techniques of Permanent Pacemaker Implantation 3 Majid Haghjoo

Chapter 2 Interventional and Minimally Invasive Surgical Techniques Facilitating Cardiac Resynchronization Therapy 13

Bela Merkely, Levente Molnar and Attila Roka

Section 2 Follow Up and Optimization of Device Function 33

Chapter 3 Electrocardiographic Troubleshooting of Implanted Cardiac Electronic Devices 35

Attila Roka

Chapter 4 Strategies and Pacemaker Algorithms for Avoidance of Unnecessary Right Ventricular Stimulation 73

D. Bastian and K. Fessele

Chapter 5 Implanted Devices and Atrial Fibrillation 105

Federico Guerra, Michela Brambatti,

Maria Vittoria Matassini and Alessandro Capucci

Section 3 Management of Complications 129

Chapter 6 Complications of Pacemaker Implantation 131

Jeffrey L. Williams and Robert T. Stevenson

Chapter 7 Device-Related Endocarditis and Infected Leads Extraction: The Dark Side of The Moon 159

Michele Rossi, Giuseppe Musolino,

Giuseppe Filiberto Serraino and Attilio Renzulli 
Chapter 8 Mechanism and Management of Pacing Lead Related Cardiac Perforation 173

Sanjay Kumar, Haidar Yassin, Opesanmi Esan,

Adam S. Budzikowski and John T. Kassotis

Chapter 9 Intravascular Lead Extractions: Tips and Tricks 189

Spyridon Koulouris and Sofia Metaxa

Chapter 10 Emergencies of Implantable Cardioverter Defibrillator 217 Baris Bugan

Section 4 Frontiers of Pacemaker Technology 231

Chapter 11 Nonlinear Cardiac Dynamics 233

Charles L. Webber, Jr.

Chapter 12 Mobile and Wireless Technologies on Sphygmomanometers and Pulsimeters for Patients with Pacemakers and Those with Other Cardiovascular Diseases 247

Ching-Sung Wang and Teng-Wei Wang

Chapter 13 Phrenic Nerve Pacing: Current Concepts 269

Jorge F. Velazco, Shekhar Ghamande and Salim Surani 


\section{Preface}

Implantable cardiac pacemakers have been utilized for more than 50 years now. Continuous development transformed them from rudimentary, asynchronous pulse generators to sophisticated devices with advanced diagnostic and therapeutic capabilities. Implantable defibrillators and biventricular devices, treating malignant tachyarrhythmias and dyssynchrony, use a similar technology. As initial issues of bradycardia-related pacing got resolved, new issues have been recognized, such as the deleterious effects of chronic right ventricular pacing or high risk of complications due to utilization in a frail patient population.

Evidence-based practice guidelines were initially developed with a focus on indications and contraindications. Safe utilization of implantable antiarrhythmia devices begins with proper patient selection; implantation method and technique, device programming and follow up are also important aspects that determine the outcome. Lack of high quality data, which could guide these decisions, leads to large individual and geographical variations. Recently, expert consensus documents addressed several important topics, such as follow-up or end-of-life considerations, however, further research is still needed to provide a basis for a more uniform, safe therapy.

This book reviews several current issues and recent advances in pacemaker therapy. Improving safety of implantation is of utmost importance to decrease complications. Transvenous route became the standard approach for antiarrhythmic cardiac device implantation as it poses only a minimal burden to the patient. Cardiac resynchronization therapy can still be challenging from this approach due to highly variable individual anatomy - recently, interventional and minimally invasive surgical methods have been developed and studied in detail, with great success. Although the risk of severe periprocedural complications is low for pacemaker or defibrillator implants, there are still dreaded consequences, such as endocarditis or lead perforation, which may be lethal if not addressed in a timely and appropriate manner. Transvenous lead extraction revolutionized management of these complications. Other common issues encountered during follow up include unnecessary right ventricular pacing and atrial fibrillation, with its potential to interact with device function. Several new algorithms have been introduced lately to address these issues - familiarity with these may prevent misinterpreting appropriate device function as malfunction. 
Patients are frequently seen by multiple healthcare providers in various settings, and questions may arise whether the implanted device contributes to, or is responsible for the clinical presentation. As device-specific programmers are not always immediately available, routine diagnostic modalities, such as a 12-lead ECG should be evaluated first as these may often identify or rule out a potential issue.

Multiple ongoing research projects address potential applications related to implantable cardiac arrhythmia devices: studies on nonlinear cardiac dynamics, mobile telemonitoring and phrenic nerve stimulation in patients with diaphragmatic paralysis will be reviewed, displaying the potential of the technology beyond antibradycardia pacing.

I am extremely grateful to the international group of authors who contributed this book - their expertise clearly shows the worldwide abundance of pacemaker technology and thriving research activity in this field. I would like to also appreciate the help of the wonderful InTech editorial staff - Ms. Tanja Skorupan and Mr. Hrvoje Pecotic, who expertly guided us through the publication process. A special thanks to my family - wife, Erika, and children, Fruzsina, Alice and Adam, who provided a supportive environment while working on this project.

Attila Roka MD, PhD

Hospital of St. Raphael, New Haven, CT, 


Section 1

Novel Implantation Techniques 



\title{
Techniques of Permanent Pacemaker Implantation
}

\author{
Majid Haghjoo
}

Additional information is available at the end of the chapter

http://dx.doi.org/10.5772/48639

\section{Introduction}

Currently available permanent pacemakers contain a pulse generator and one or more pacing leads. Early in the era of pacemaker implantation, this procedure was only performed by the cardiac surgeons because of the initial mandate for epicardial lead implantation. Further advancements in the pacing hardware and percutaneous venous catheterization simplified the implantation technique and made it feasible to implant the transvenous leads. Simultaneously, further innovations in the pulse generator and its circuitry extended the utility of the percutaneous technique even in the very young patients.

All device trainees will require basic skills in pacemaker implantation. However, first step is to identify whether a patient needs a permanent pacemaker. This chapter will summarize the necessary equipments, patient preparation, and implantation techniques. Like any practical skill it is only possible to give a flavor of the methodology in writing, and nothing can replace the practical tuition of an experienced implanter in the pacing theatre during a number of pacemaker implants.

\section{Equipments}

The pacemaker implantation can be performed in electrophysiology (EP) laboratory, catheterization laboratory, or operating room [1]. Pacemaker implantation by interventional electrophysiologist in EP lab or catheterization laboratory resulted in a significant reduction in medical cost and hospital stay [2].

Minimum required personnel for pacemaker implantation consist of implanting physician, scrub nurse, and circulating nurse or technician. Scrub nurse is required to help the implanter throughout the procedure. The circulating nurse or technician is required to prepare and administer medications, and to operate pacing system analyzer. 
Fluoroscopy and electrocardiography (ECG) are necessary equipments in every device implant. Single-plane fluoroscopy via anteroposterior, $30^{\circ}$ right anterior oblique, and $45^{\circ}$ left anterior oblique views is usually adequate for transvenous implantation from either the right or the left pectoral approach. Currently, initial lead sensing and capture measurements are obtained by pacing system analyzers (Figure 1), which may be stand-alone or built into the pacer programmer.

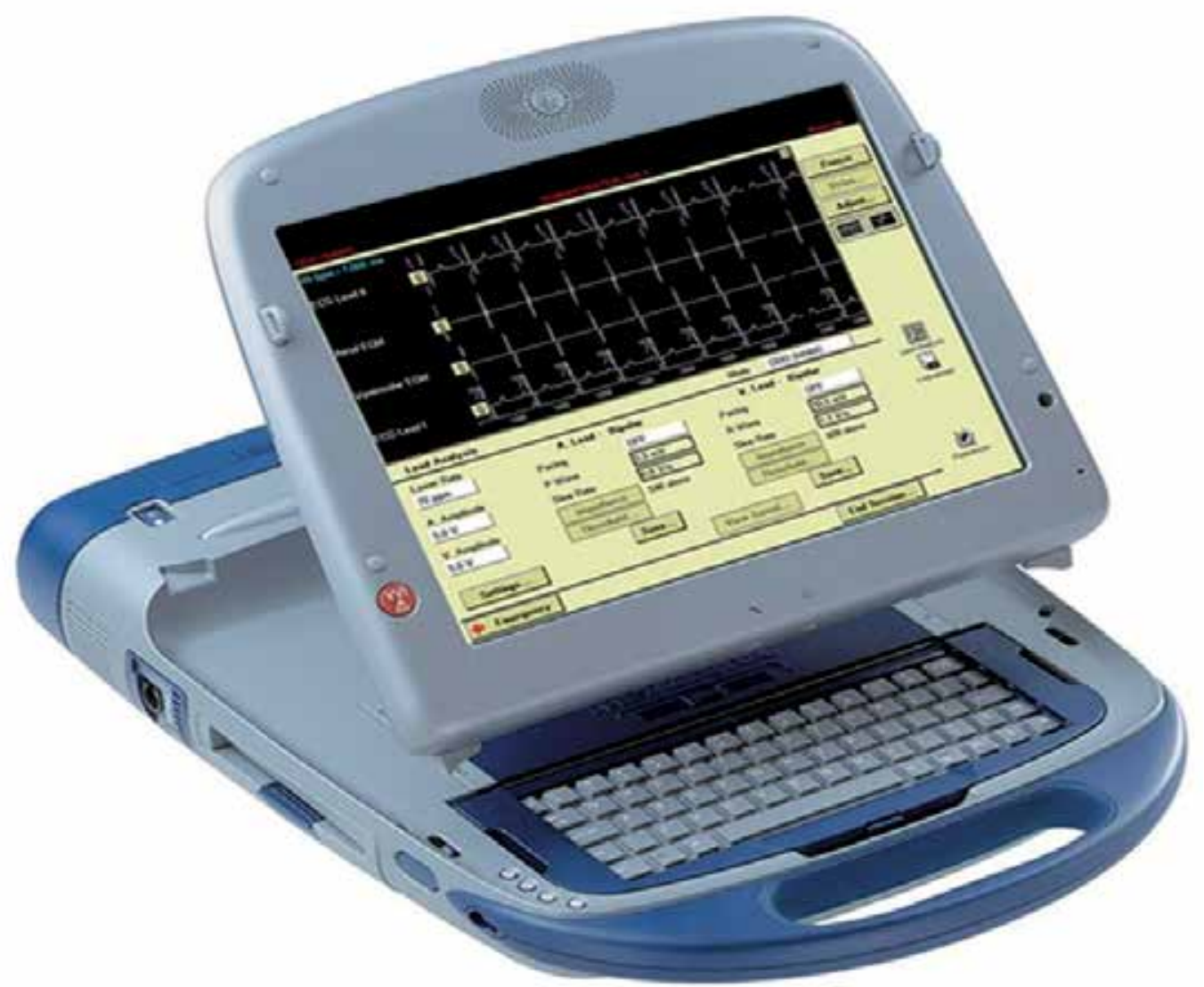

Figure 1. Built-in pacing system analyzer

Apart from the fluoroscopy equipment and vital observation monitors, there are a number of sterile surgical instruments and equipment that are needed (Figure 2). Suture materials include both nonabsorbable material for lead and device anchoring and absorbable material for pocket closure. Antimicrobial flush and saline for pocket irrigation should be available. If venography is to be performed, an appropriate intravenous contrast agent must be available. 


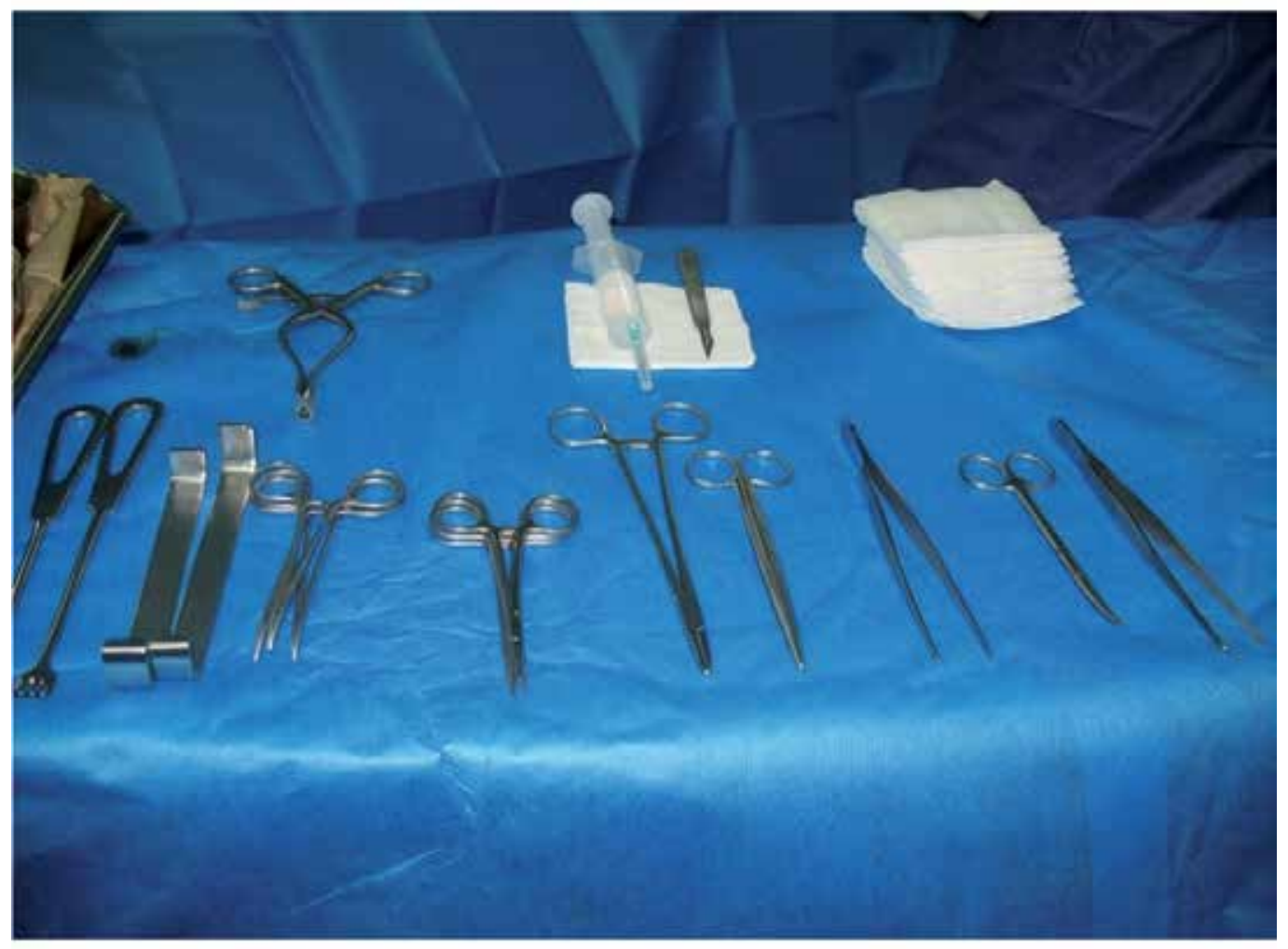

Figure 2. Minor surgical tray for permanent pacemaker implantation

\section{Patient preparation}

Before pacemaker implantation, an informed consent should be obtained. Any anticipated risks and benefits should be honestly discussed with patient or the patient's family. The indication for pacing should be thoroughly described to the patient. The need for lifelong follow-up should be emphasized and patient should be informed about the generator change and possible lead replacement in the future. Any physical or occupational restrictions related to the pacemaker implantation including rules regarding the driving should be discussed in detail with the patient.

Routine pre-implant lab tests are 12-lead ECG, chest x-ray, complete blood count, prothombin and partial thromboplastin times, serum electrolytes, blood urea nitrogen, and serum creatinine. Many of the patients requiring a pacemaker may be on oral anticoagulant [3]. Perioperative management of these patients is often challenging and needs special experience. In the past, standard practice was to discontinue warfarin 48 hours before the procedure, bridge with intravenous heparin, and then reinitiate warfarin the day of the procedure or even the night before. This practice has been associated with higher risk of 
hematoma formation compared with that encountered in unanticoagulated patients (up to $20 \%$ ) [4]. Recently, there has been an increasing interest in performing the pacemaker implantation without reversal of the anticoagulant. This practice was associated with lower risk of pocket bleeding and shorter hospital stay [3, 5-7].

Antibiotic prophylaxis is a controversial issue, but most implanters prefer to give oral or intravenous (IV) antibiotics to decrease the incidence of local or systemic infections based on limited data available [8]. Although there is a distinct lack of either national or international guidance in this area, meta-analysis of the randomized trials suggests a benefit from pre-procedure intravenous antibiotics [9]. Our routine practice is to give 1 gram of cefazolin or vancomycin (in penicillin-allergic patients) one hour before the procedure.

Implantation of pacemaker usually involves a combination of local anesthesia and conscious sedation. Infiltration of skin and subcutaneous tissue at the implant site with 1-2\% lidocaine provides sufficient local anesthesia for the majority of implant procedures. However, to obtain optimal anesthesia, conscious sedation in the form of carefully titrated IV midazolam and fentanyl is recommended. On rare occasions, general anesthesia may be required in an extremely uncooperative patient.

Implant area from the angle of jaw to the nipple line bilaterally should be completely cleansed and shaved. Placement of an IV line ipsilateral to the intended implant site is routine for administration of prophylactic antibiotics, administration of IV analgesia/sedation, and potentially to perform venography.

On entering the procedure room, the patient is usually placed on his or her back with the arms tucked and physiologic monitoring (ECG, pulse oximetry, and noninvasive blood pressure) should be quickly established to detect any arrhythmia or hemodynamic abnormality. Preparing the procedure field is also crucial to minimizing complications. Sterility is obviously of paramount importance; the chest is prepared with an antiseptic solution, and the area is covered with sterile drapes to keep the incision area as clean as possible.

\section{Implant techniques}

\subsection{Central venous access techniques}

A central vein (ie, the subclavian, internal jugular or axillary vein) is accessed via a percutaneous approach. Alternatively, target vein is accessed via direct visualization by a cut down technique (most commonly, cephalic vein). Figure 3 shows a standard prepackaged introducer set for implantation. In patients in whom this is technically difficult because skeletal landmarks are deviated, an initial brief fluoroscopic examination will greatly reduce the time and complications associated with obtaining the access (Figure 4). 


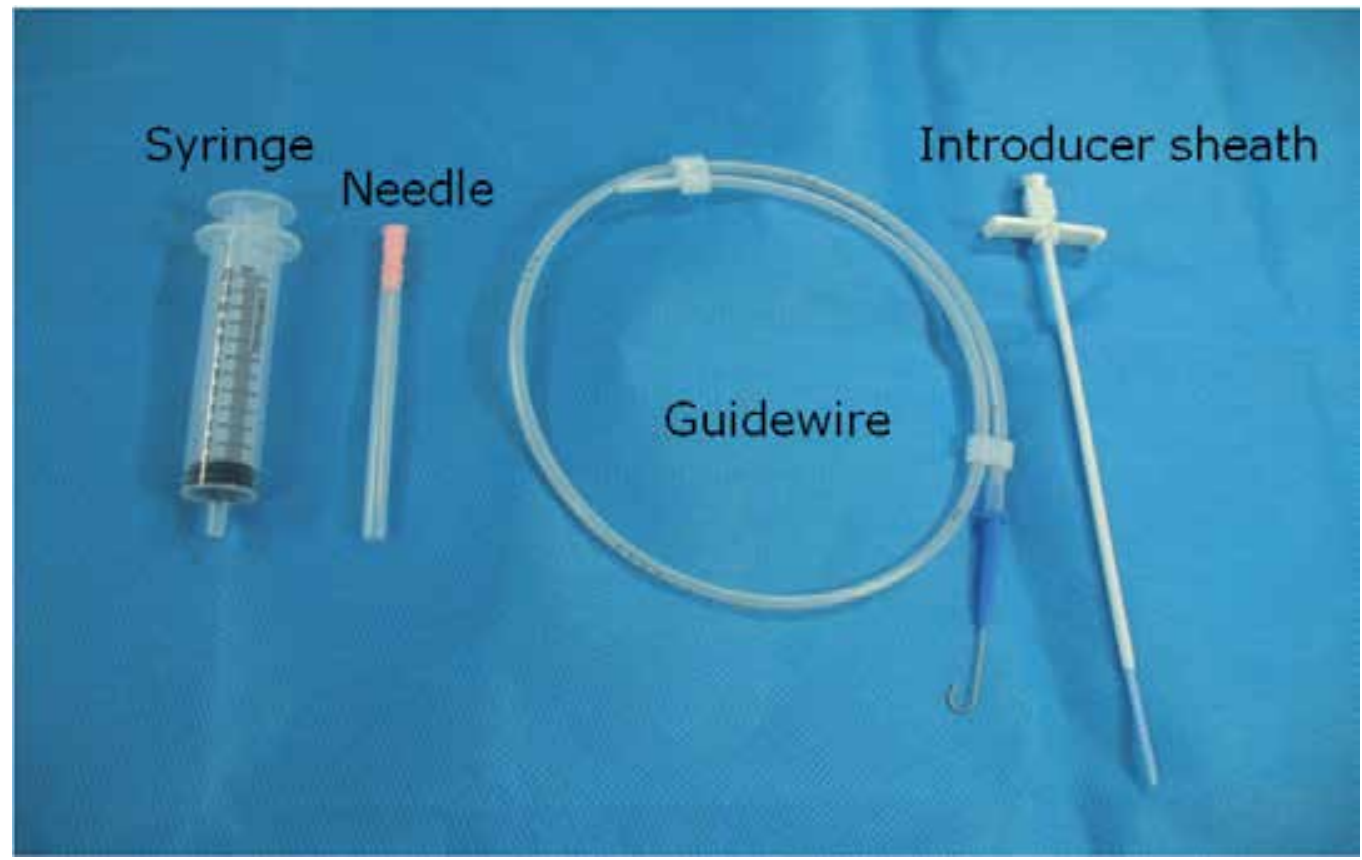

Figure 3. Introducer set used for percutaneous access to central veins

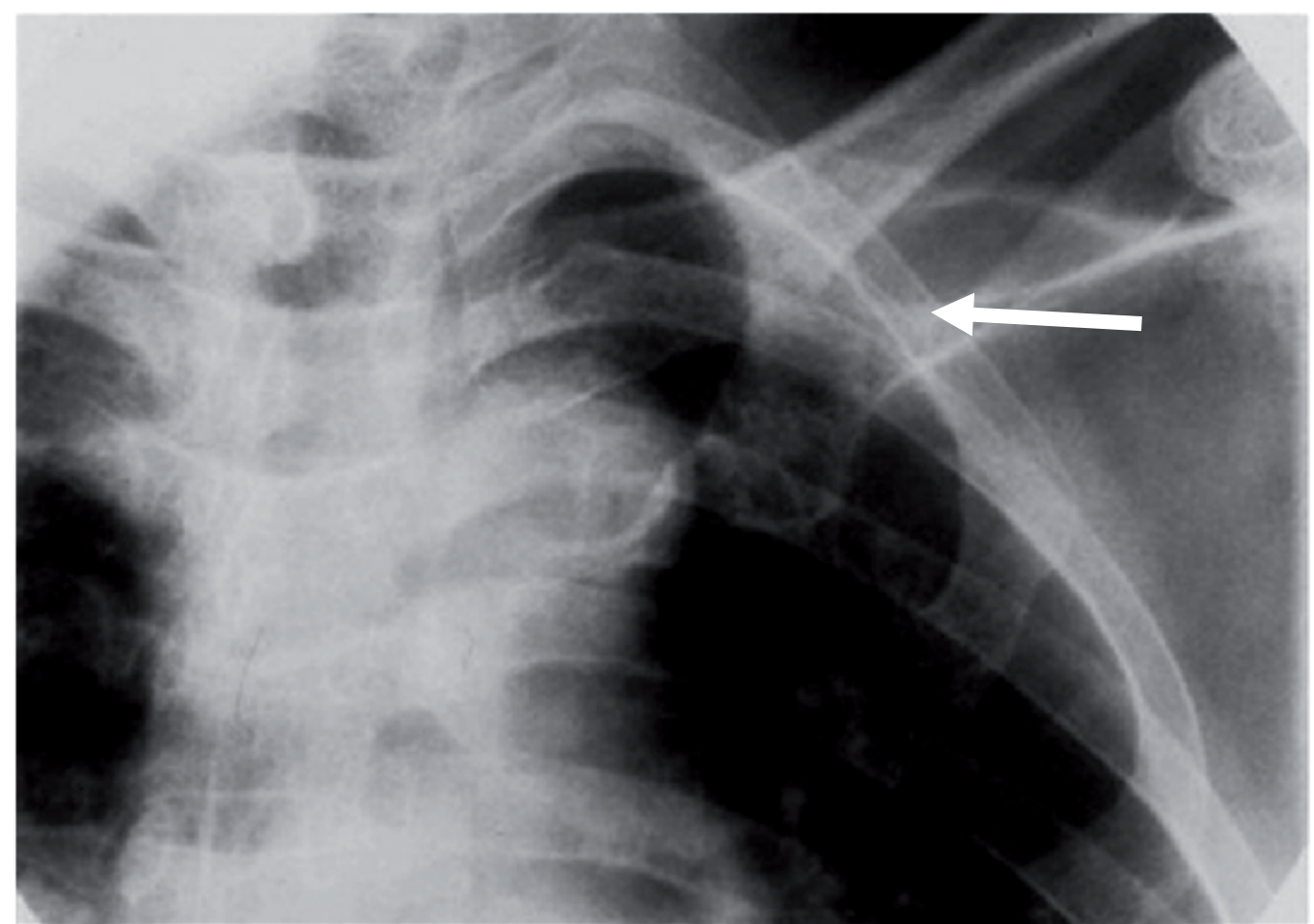

Figure 4. Target area for subclavian vein puncture is marked by white arrow. 
Subclavian vein puncture is the first choice technique for most operators. The needle is advanced (gently aspirating on an attached syringe as with any other indirect puncture), aiming for the space below the clavicle and over the first rib until either the vein is cannulated or the rib is struck. The subclavian vein is typically accessed at the junction of the first rib and the clavicle. On occasion, venography may be required to visualize the vein adequately or to confirm its patency (Figure 5). This approach is associated with minimal incidence of pneumothorax.

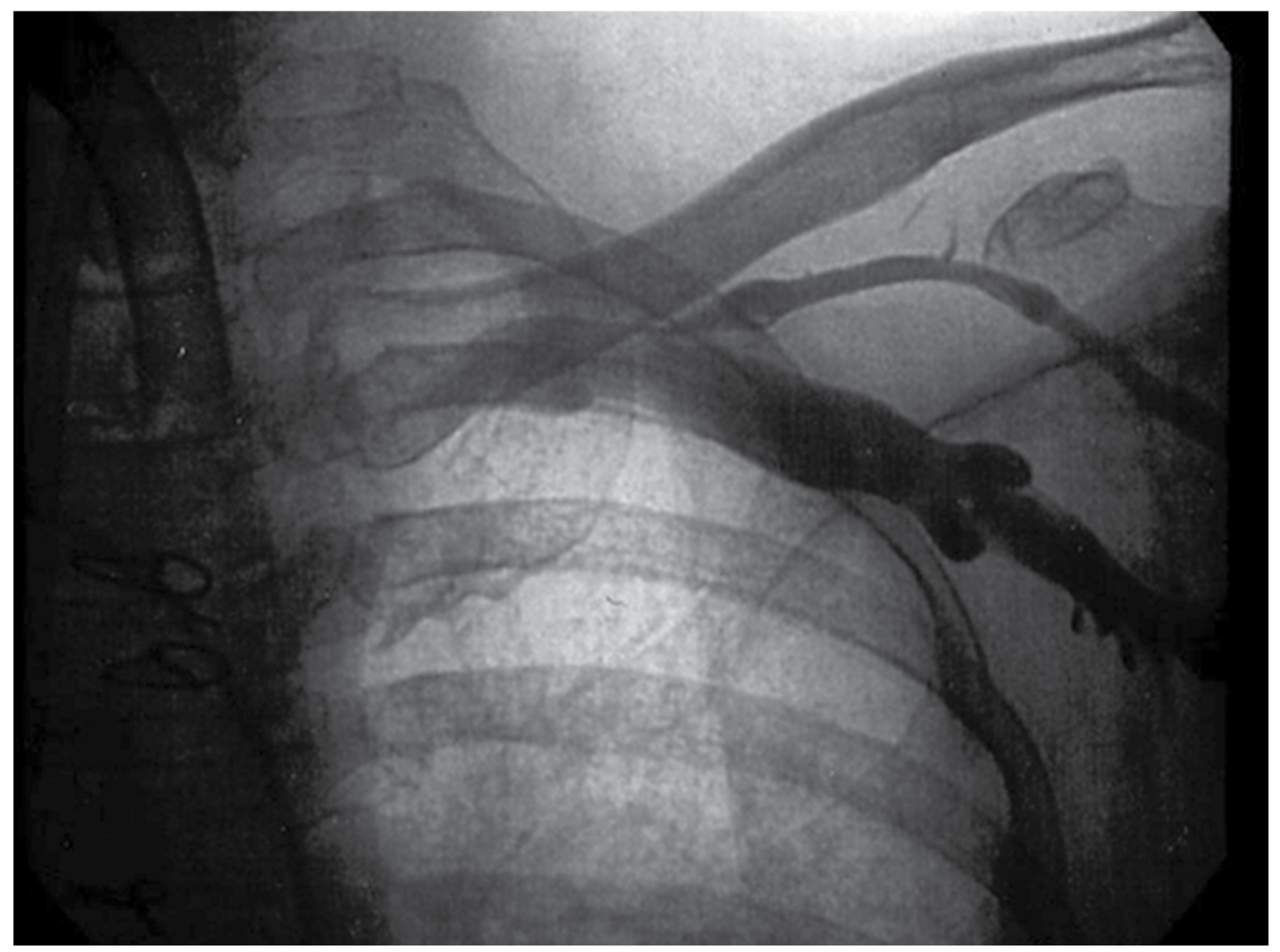

Figure 5. Contrast venography performed from left brachial vein. The figure clearly shows axillary vein, cephalic vein, subclavian vein

Other important alternative central vein techniques for lead implantation are cephalic vein cut down and axillary vein puncture. The cephalic vein resides in the sulcus between the deltoid and pectoral muscles. This area is easily identified by palpation and is occupied by loose connective tissue and fat, which can be dissected to identify the cephalic vein. Occasionally, the vein is deep or consists of a plexus of tiny veins. In these circumstances, other routes should be used for lead insertion. After vein isolation for 1 to $2 \mathrm{~cm}$ within the groove, it is ligated distally. A ligator is placed around the proximal part of the vein for hemostasis. The vein can be entered using venotomy or with 16- or 18-guage peripheral IV catheter. The axillary vein can be accessed by blind percutaneous puncture by entering the pectoral muscle just medial to the acromion process on anteroposterior fluoroscopy. The 
needle then is directed to the point at which the lateral border of the first rib appears to cross the inferior margin of the clavicle. Alternatively, the axillary vein can be accessed using contrast venography.

After venous access is obtained, a guide wire is advanced through the access needle, and the tip of the guide wire is positioned in the right atrium or the venacaval area under fluoroscopy. The needle is then withdrawn, leaving the guide wire in place. If indicated, a second access will be obtained in a similar fashion for positioning of a second guide wire.

Sometimes, a double-wire technique is used, whereby 2 guide wires are inserted through the first sheath and the sheath then withdrawn, so that 2 separate sheaths can be advanced over the 2 guide wires. This technique can cause some resistance or friction during sheath or lead advancement.

\subsection{Pocket formation}

Although the pocket may be formed in the axilla or in the abdomen (for epicardial or femoral systems), the most common site is the pectoral region. In the latter approach, a 1.5to 2-inch incision is made in the infraclavicular area parallel to the middle third of the clavicle, and a subcutaneous pocket is created with sharp and blunt dissection where the pacemaker generator will be implanted. Some physicians prefer to make the pocket first and obtain access later through the pocket or via venous cutdown; once access is obtained, they position the guide wires as described above.

\subsection{Positioning of atrial and ventricular leads}

Over the guide wire, a special peel-away sheath and dilator are advanced. The guide wire and dilator are withdrawn, leaving the sheath in place. A stylet (a thin wire) is inserted inside the center channel of the pacemaker lead to make it more rigid, and the lead-stylet combination is then inserted into the sheath and advanced under fluoroscopy to the appropriate heart chamber. Usually, the ventricular lead is positioned before the atrial lead to prevent its dislodgment.

Making a small curve at the tip of the stylet renders the ventricular lead tip more maneuverable, so that it can more easily be placed across the tricuspid valve and positioned at the right ventricular apex.

Once correct lead positioning is confirmed, the lead is affixed to the endocardium either passively with tines (like a grappling hook) or actively via a helical screw located at the tip. The screw at the tip of the pacemaker is extended or retracted by turning the outer end of the lead with the help of a torque device. Adequate extension of the screw is confirmed with fluoroscopy. Each manufacturer has its own proprietary identification marks for confirming adequate extension of the screw.

Once the lead is secured in position, the introducing sheath is carefully peeled away, leaving the lead in place. After the pacing lead stylet is removed, pacing and sensing thresholds and 
lead impedances are measured with a pacing system analyzer, and pacing is performed at $10 \mathrm{~V}$ to make sure that it is not causing diaphragmatic stimulation. After confirmation of lead position and thresholds, the proximal end of the lead is secured to the underlying tissue (ie, pectoralis) with a nonabsorbable suture that is sewn to a sleeve located on the lead.

If a second lead is indicated, it is positioned in the right atrium via a second sheath, with the lead tip typically positioned in the right atrial appendage with the help of a preformed Jshaped stylet.

In a patient who is without an atrial appendage as a result of previous cardiac surgery, the lead can be positioned medially or in the lateral free wall of the right atrium. As with the ventricular lead, the atrial lead position is confirmed, impedance is assessed, the stylet is withdrawn, and the lead is secured to the underlying pectoralis with a nonabsorbable suture.

\subsection{Lead connection and pulse generator insertion in the pocket}

When the leads have been properly positioned and tested and sutured to the underlying tissue, the pacemaker pocket is irrigated with antimicrobial solution, and the pulse generator is connected securely to the leads. Many physicians secure the pulse generator to underlying tissue with a nonabsorbable suture to prevent migration or twiddler syndrome.

Typically, the pacemaker is positioned superficial to the pectoralis, but occasionally, a subpectoral or inframammary position is required. After hemostasis is confirmed, a final look under fluoroscopy before closure of the incision is recommended to confirm appropriate lead positioning.

\subsection{Pocket closure}

The incision is closed in layers with absorbable sutures and adhesive strips. Sterile dressing is applied to the incision surface. An arm restraint or immobilizer is applied to the unilateral arm for 12-24 hours to limit movement.

\subsection{Post-procedural care}

Pain levels are typically low after the procedure, and the patient can be given pain medication to manage breakthrough pain associated with the incision site. There is controversy over the routine use of IV or oral antibiotics after the procedure. A postoperative chest radiograph is usually obtained to confirm lead position and rule out pneumothorax. Before discharge on the following day, posteroanterior and lateral chest radiographs will be ordered again to confirm lead positions and exclude delayed pneumothorax. Pacemaker interrogation is also recommended to ensure proper pacing function before patient leaving the hospital. 


\section{Author details}

Majid Haghjoo,

Cardiovascular Medicine, Cardiac Electrophysiology Research Center,

Department of Cardiac pacing and Electrophysiology, Rajaie Cardiovascular Medical Center,

Tehran University of Medical Sciences, Tehran, Iran

\section{References}

[1] Garcia-Bolao I, Alegria E [1999] Implantation of 500 consecutive cardiac pacemakers in the electrophysiology laboratory. Acta. Cardiol. 54: 339-343.

[2] Yamamura KH, Kloosterman EM, Alba J, Garcia F, Williams PL, Mitran RD, Interian A Jr [1999] Analysis of charges and complications of permanent pacemaker implantation in the cardiac catheterization laboratory versus the operating room. Pacing. Clin. Electrophysiol. 22: 1820-1824.

[3] Guidici MC, Barold SS, Paul DL, Bontu P [2004] Pacemaker and implantable cardioverter defibrillator implantation without reversal of warfarin. Pacing. Clin. Electrophysiol. 27:358-360.

[4] Chow V, Ranasinghe I, Lau J, Stowe H, Bannon P, Hendel N, Kritharides L [2010] Peri-procedural anticoagulation and the incidence of haematoma formation after permanent pacemaker implantation in the elderly. Heart. Lung. Circ. 19:706712.

[5] Li HK, Chen FC, Rea RF, Asirvatham SJ, Powell BD, Friedman PA, Shen WK, Brady PA, Bradley DJ, Lee HC, Hodge DO, Slusser JP, Hayes DL, Cha YM [2011] No increased bleeding events with continuation of oral anticoagulation therapy for patients undergoing cardiac device procedure. Pacing. Clin. Electrophysiol. 34: 868874.

[6] Cheng A, Nazarian S, Brinker JA, Tompkins C, Spragg DD, Leng CT, Halperin H, Tandri H, Sinha SK, Marine JE, Calkins H, Tomaselli GF, Berger RD, Henrikson CA [2011] Continuation of warfarin during pacemaker or implantable cardioverter-defibrillator implantation: a randomized clinical trial. Heart. Rhythm. 8: 536-540.

[7] Ahmed I, Gertner E, Nelson WB, House CM, Dahiya R, Anderson CP, Benditt DG, Zhu DW [2010] Continuing warfarin therapy is superior to interrupting warfarin with or without bridging anticoagulation therapy in patients undergoing pacemaker and defibrillator implantation. Heart. Rhythm. 7: 745-749.

[8] Mounsey JP, Griffith MJ, Tynan M, Gould FK, MacDermott AF, Gold RG, Bexton RS [1994] Antibiotic prophylaxis in permanent pacemaker implantation: a prospective randomized trial. Br. Heart. J. 72: 339-343. 
[9] Da Costa A, Kirkorian G, Cucherat M, Delahaye F, Chevalier P, Cerisier A, Isaaz K, Touboul P [1998] Antibiotic prophylaxis for permanent pacemaker implantation: a meta-analysis. Circulation. 97:1796-1801. 


\title{
Interventional and Minimally Invasive Surgical Techniques Facilitating Cardiac Resynchronization Therapy
}

\author{
Bela Merkely, Levente Molnar and Attila Roka
}

Additional information is available at the end of the chapter

http://dx.doi.org/10.5772/50291

\section{Introduction}

Cardiac resynchronization therapy (CRT) with atriobiventricular pacing has been utilized for more than a decade for severe congestive heart failure associated with intraventricular dyssynchrony. The primary objective of CRT is to coordinate myocardial contraction with stimulation via right atrial (RA), right ventricular (RV) and left ventricular (LV) leads, using a biventricular pacemaker (CRT-P) or defibrillator (CRT-D). Although it provides both morbidity and mortality benefits in addition to medical therapy and implantable cardioverter-defibrillator, several issues remain unresolved, most importantly the high number of non-responder patients (up to 30\%) and the technically challenging implantation. These are linked, as optimal lead position is essential for successful resynchronization.

Atriobiventricular pacing is most commonly accomplished with transvenous placement of the system. Endocardial right atrial and right ventricular leads are conventional. The left ventricle is stimulated via a branch of the coronary sinus, performing epicardial stimulation. Unfavorable coronary sinus or vein anatomy, such as valves, tortuosity or focal stenosis may render left ventricular lead implantation very difficult. Even in the absence of these, there may be no appropriate vein in the area which would provide effective resynchronization. The average implantation time of CRT in high volume centers is under 2 hours, with a procedural success rate of $87 \%-96 \%$ (Alonso, 2009). Perioperative complications are seen in $10 \%$ and the rate of late complications is $5.5 \%$ (Khan, 2009).

Recent evidence suggests that LV lead position is crucial to ensure effective CRT. This chapter will review the indications for CRT, use of imaging modalities to facilitate targeted lead placement, perioperative issues and techniques shown to be useful in challenging cases. 


\section{Indications for CRT}

Most clinical CRT trials were performed in patients with advanced heart failure: LVEF $\leq 35 \%$, wide QRS, sinus rhythm, NYHA III-IV functional stage despite optimal medical treatment (COMPANION, CARE-HF, MIRACLE, MUSTIC SR etc.). Few studies included patients with less severe symptoms (MADIT CRT, REVERSE, RAFT) or who were in atrial fibrillation (MUSTIC-AF and partially RAFT). The guidelines for implantation were recently updated by the HFA and ESC (McMurray, 2012). (Figure 1)

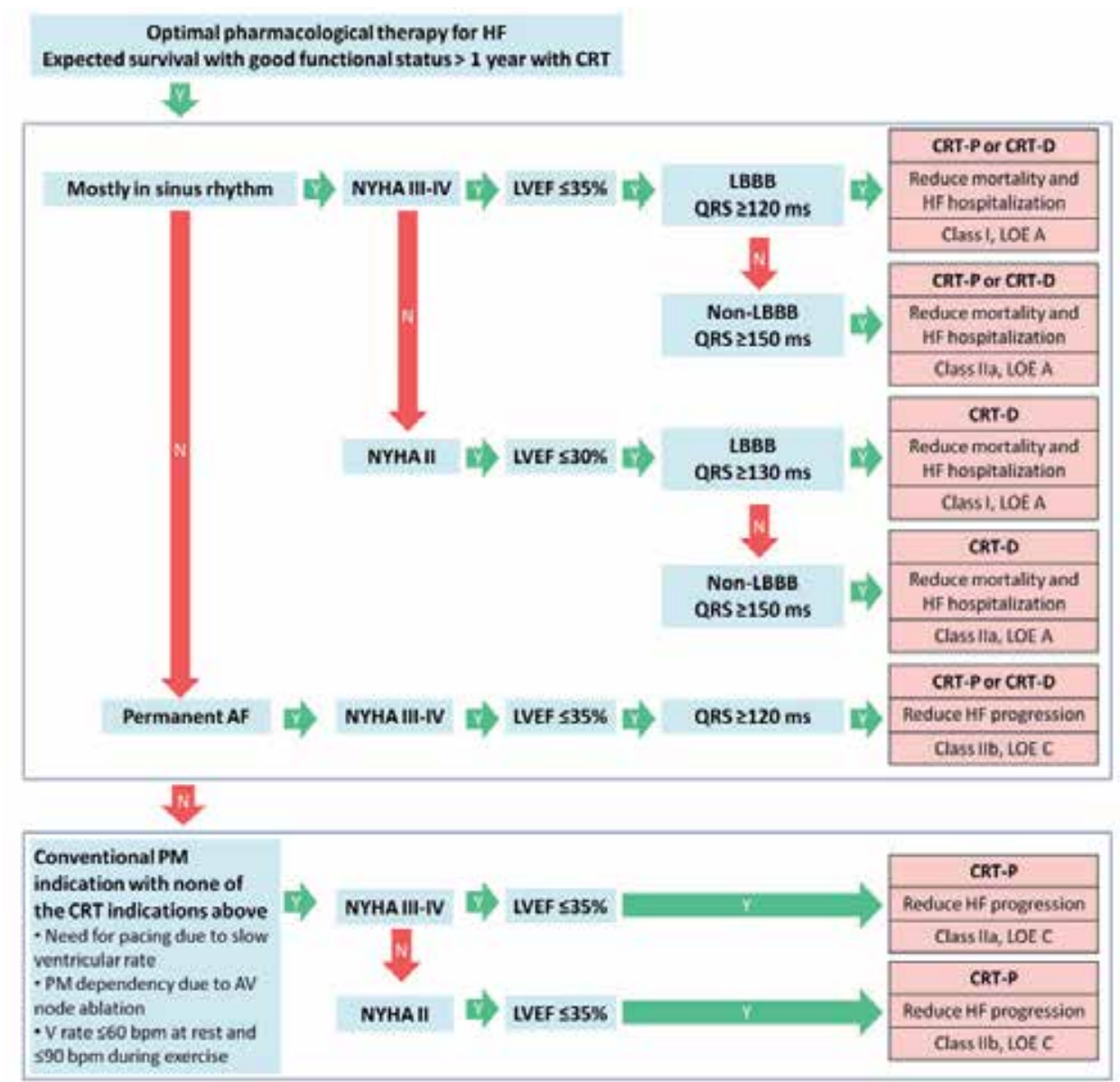

Figure 1. Indications for cardiac resynchronization therapy, Heart Failure Association and European Society of Cardiology, 2012 (McMurray, 2012). Stage IV heart failure patients should be "ambulatory" pressor-dependent or acutely decompensated patients are generally not good candidates for CRT. HF: heart failure. CRT: cardiac resynchronization therapy. NYHA: New York Heart Association functional class. LVEF: left ventricular ejection fraction. LBBB: left bundle branch block. CRT-P: cardiac resynchronization with biventricular pacemaker. CRT-D: cardiac resynchronization with biventricular implantable cardioverter defibrillator. PM: pacemaker. AV: atrioventricular. Class: strength of indication (class I-implantation recommended, class IIa - implantation should be considered, class IIb - implantation may be considered). LOE: level of evidence (A: multiple randomized trials or meta-analysis, C: expert opinion) 


\section{Pre-implantation evaluation}

Patient evaluation prior to implantation should include assessment of functional stage, left ventricular ejection fraction and 12-lead ECG to determine whether the patient is a candidate for CRT. Potentially reversible factors contributing to heart failure and cardiomyopathy should be looked for and corrected (ischemia, hypertension, suboptimal medical management etc.). Co-morbidities limiting life expectancy or decreasing possible benefits of CRT should be identified (cardiac cachexia, advanced renal disease, frailty etc.) (Theuns, 2011). Advanced age alone is not a contraindication to CRT. History of arrhythmias and eligibility for ICD should be also assessed to guide device and lead selection. The patient should not be in a decompensated condition at the time of implantation. Heart failure medications must be utilized at maximally tolerated doses for at least 3 months prior to considering the patient as a candidate for CRT implantation. In the immediate preimplantation period, basic laboratory parameters should be checked and corrected as necessary to minimize surgical risks (complete blood cell count, electrolytes and kidney function, coagulation tests) (Epstein, 2008). A standardized evaluation of heart failure may help to assess response to CRT (serum BNP, 6 minute walking test).

Echocardiography is the most common test performed to assess severity of left ventricular dysfunction. Right ventricular dysfunction may affect response to CRT and should also be evaluated (Burri, 2010). Although multiple echocardiographic measurements exist to evaluate intraventricular or interventricular mechanical dyssynchrony, so far these were not proven to be helpful to guide patient selection for CRT. Absence of echocardiographic dyssynchrony should not defer utilization of CRT if the patient would otherwise be a candidate, however, the risk of deterioration is higher in these cases (Mullens, 2009).

Cardiac MRI with delayed enhancement imaging may be considered when the presence and location of myocardial scar is relevant and echocardiography is non-diagnostic (Bleeker, 2006).

\section{Assessment of coronary vein anatomy}

Transvenous endovascular left ventricular lead placement is limited by the anatomic constraints of the coronary veins. Patients with history of thoracic irradiation or cardiac surgeries may be at high risk for unsuccessful left ventricular lead implantation. Imaging studies prior to implantation may help to determine if the patient is suitable for transvenous implantation.

Coronary angiography is routinely performed during the workup of heart failure. Delayed images after contrast injection into the coronary arteries may outline the anatomy of the coronary sinus and its main branches. A major advantage of this method is that it does not provide any additional burden to the patient. The position of the orifice, the angle of the proximal CS, tortuousity and diameter of the vein are valuable clues for the selection of target vessel and the implantation approach. Most useful projections are anteroposterior and left anterior oblique, as these are common working views during CRT implantation. 
Although the image quality is rarely good enough to assess terminal branches of the CS, the data obtained during coronary angiography may be used to identify fluoroscopic markers for CS cannulation as the ostium is almost always visualized. Another limitation is that this approach provides only two dimensional data (Kovacs, 2004) (Figure 2).
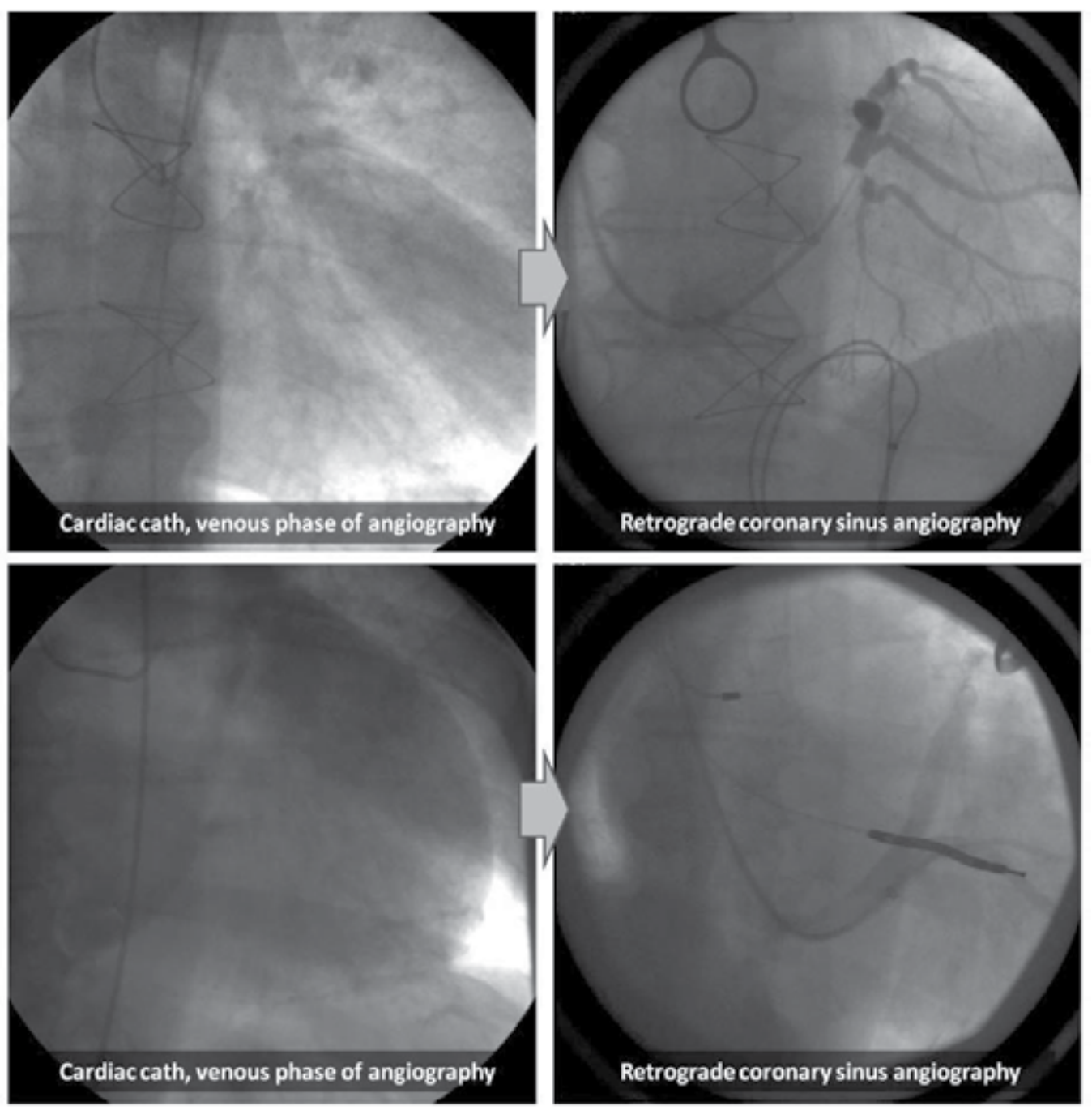

Figure 2. Evaluation of coronary vein anatomy during cardiac catheterization. The venous phase after contrast injection into the left coronary artery shows the coronary sinus and its major branches.

Although the smaller branches are not visualized, the images are helpful to localize the CS ostium, the angle of the CS and origin of its major branches. The images correlate with those obtained during retrograde CS angiography.

Cardiac CT angiography provides detailed assessment of coronary arterial and venous anatomy, but requires x-ray radiation and contrast. Pre-implantation evaluation of the coronary venous anatomy can facilitate CRT, leading to decreased procedure time (Girsky, 
2010 and Doganay, 2011). Area of latest mechanical activation may be correlated with echocardiographic images and veins in this region should be targeted for LV lead implantation - this approach correlates with improved acute response to CRT, however, no long term data available yet (Van de Veire, 2008). The phrenic nerve may also be visualized to identify high risk regions for inadvertent diaphragmatic stimulation (Matsumoto, 2007). Fusion of CT and fluoroscopic images may be of even greater benefit (Auricchio, 2009). Contrast allergy and advanced renal disease are contraindications for cardiac CT.

Cardiac MRI can also be used to assess coronary veins. These images may be overlayed with other MRI data, such as scar distribution or area of latest activation (Duckett, 2011a; White, 2010 and Kronborg, 2012). Imaging of scar burden improves prediction of response to CRT: in ischemic cardiomyopathy, less than $15 \%$ of total myocardium infarcted and absence of significant posterolateral scar is associated with better response (Bilchick, 2008 and Chalil, 2007). Real time MRI-guided intubation of the coronary sinus is being investigated (Neizel, 2010). Cardiac MRI requires gadolinium-based contrast and is contraindicated in patients with advanced renal dysfunction. Previously implanted non-MRI compatible hardware may also limit its utilization. The scanning times are longer and image acquisition requires some patient cooperation, which may be poorly tolerated in severe heart failure.

Although these advanced imaging modalities have improved both implantation success rate and responder rate in small studies, currently it is not known whether routine application in all patients undergoing CRT would be cost-efficient. In patients undergoing CRT, review of all existing relevant imaging data is recommended for implant strategy planning. Advanced modalities should be reserved for cases where difficult anatomy is anticipated or if the patient had an unsuccessful implantation attempt. If cardiac CT and MRI will be shown to predict and facilitate CRT in a cost-efficient manner, these modalities may potentially be implemented on a more routine basis in the future.

\section{Perioperative period}

The patient should be on a stable heart failure medication regimen for at least 3 months before CRT implantation. Vasopressor-dependent or significantly fluid overloaded/congested patients are not good candidates for the procedure. Following implantation, careful monitoring is required as occasionally significant diuresis is observed with initiation of CRT, leading to electrolyte imbalances. The dose of heart failure medications should be titrated as tolerated after recovery from the implantation procedure. Bradycardia may not be a limiting factor after CRT implantation when considering utilization of higher dose of beta-blockers. Hypotensive side effects of ACE inhibitors and other vasodilators may be less pronounced, however, individual responses vary significantly. The dose of diuretics should be decreased if significant improvement in volume status is observed with CRT, to avoid prerenal azotemia and hypotension.

Almost all patients undergoing CRT implantation are on antiplatelet or anticoagulation therapy, which increases bleeding risks. Compared to untreated patients, the likelihood of bleeding complications is doubled in patients on aspirin and quadrupled with dual 
antiplatelet therapy $(1.6 \%, 3.9 \%$ and $7.2 \%$, respectively) (Jamula, 2008). Antiplatelet medications for primary prevention can be safely discontinued for a period of 5-7 days prior to the implantation. Dual antiplatelet therapy following PCI should not be discontinued in patients high risk for subacute stent thrombosis (such as early after coronary stent implantation, with timing dependent on stent type) (Tompkins, 2011). Bridging anticoagulation with heparin is associated with higher bleeding risk (up to 20\%), where short-term discontinuation of anticoagulation is not an option - in these cases, continuation of coumadin is recommended (Tompkins, 2010). Continuation of coumadin has a risk of postoperative pocket bleeding of 1.9-6.6\% (Wiegand, 2004).

After decades of debate, now there is evidence that perioperative antibiotic prophylaxis decreases risk of infectious complications. Although these complications are rare (generally $<1 \%$ ), their consequences are devastating, leading to significant morbidity, mortality and costs (Klug, 2007 and Da Costa, 1998). Iv. cephazolin administered immediately before the procedure reduced the risk of infection from $3.28 \%$ to $0.63 \%$ in a large randomized trial (de Oliveira, 2009). Although some implant centers continue antibiotic utilization after the implantation, there is no proven benefit for this approach. Data regarding antibiotic prophylaxis for non-transvenous CRT implantation is scarce - generally, cardiothoracic surgical guidelines should apply as the optimal duration and selection of antibiotics may be affected by the given surgical approach (Mertz, 2011; Edwards, 2006 and Engelman, 2006).

Most interventional techniques may be safely utilized in the pacemaker laboratory. It is preferable that the physician attempting a complex implantation or a device upgrade is well trained and current in the appropriate interventional and surgical techniques, or a physician with this training is immediately available. In case lead extraction is planned, a hybrid operating room or prompt access to surgical backup is mandatory. The incidence of unsuccessful implantations is declining, which is partly due to the advances in lead technology and implanting tools, however, interventional cardiology techniques have also been increasingly utilized with excellent efficacy and safety records. Minimally invasive surgical options are rapidly evolving and should be considered in case transvenous implantation is not feasible.

Most common perioperative complications are failure to implant the LV lead, pocket hematoma, hemothorax or pneumothorax, CS dissection, cardiac perforation or tamponade, extracardiac stimulation, complete heart block, LV lead dislodgement, exacerbation of HF, acute renal failure, and death. Overall perioperative complication rates range from $4 \%$ in more recent trials to $28 \%$ in earlier CRT trials (Leon, 2005 and Linde, 2008).

\section{Targeting LV lead placement}

There is considerable variability in the ventricular activation pattern and distribution of mechanical dyssynchrony even in the LBBB population, and consequently inter-individual variability in the most optimal pacing site (Auricchio, 2004 and Derval, 2010). In addition, a significant number of patients don't even have typical LBBB. Lead placement via endocardial and surgical epicardial approach may have more potential for individualized 
targeted pacing. Targeting methods include those assessing electrical, mechanical and anatomic parameters, but there is no consensus yet regarding the best method to improve long term outcomes (Ansalone, 2002; Merchant, 2010; Gold, 2011 and Ypenburg, 2008).

Apically positioned LV lead location is associated with a worse clinical outcome in the REVERSE and MADIT-CRT trials (Thebault, 2012 and Singh, 2011). The COMPANION and MADIT-CRT studies showed a comparable response between lateral, anterior or posterior LV lead locations, while patients in REVERSE benefited from a lateral lead location (Saxon, 2009). Imaging may help to select specific sites for left ventricular pacing based on anticipated optimization of electromechanical effects (Toumoux, 2010). Echocardiography (tissue Doppler or tissue synchronization imaging) may identify LV sites with marked mechanical delay (Cannesson, 2006 and Murphy, 2006). Pacing these sites may result in greater ventricular remodeling and improved clinical outcomes. Currently there is not enough data supporting the role of acute hemodynamic measurements during implantation to target lead implantation (Duckett, 2011b).

Multisite ventricular stimulation (more than one LV lead) may provide even greater benefits with more homogenous ventricular activation. More clinical data will be needed to evaluate whether it is superior to biventricular stimulation.

\section{Transvenous CRT implantation and reinterventions}

The standard approach to CRT is to implant three endovascular leads for cardiac stimulation: endocardial RV and RA leads, and an epicardial LV lead into a coronary vein through the CS. Transvenous implantation should be the preferred way for CRT as most evidence is with this approach. The standard transvenous approach has significant drawbacks as it is dependent on the highly variable venous anatomy. Main reasons of failed LV lead implantations are inability to cannulate the CS due to RA dilatation or prominent Thebesian valve, diminutive CS, severe kinking of the vein or venous valve in the CS. Even with successful lead placement, unstable position, high pacing threshold or phrenic nerve stimulation may hinder effective delivery of LV pacing. Implantation success rate is above $90 \%$ in experienced centers, most failures are due to unsuccessful placement of the LV lead. In case of an unsuccessful procedure, repeat transvenous procedure with a more experienced operator or with interventional backup is recommended, if the venous anatomy seems to be suitable for implantation. In unsuitable cases, alternative approaches (surgical epicardial, transseptal endocardial) should be considered.

The EHRA and HRS has recently published guidelines for recommended approach for transvenous CRT implantation (Daubert, 2012). Implantation should be performed from the left subclavian vein system, unless preexisting pathology (venous occlusion, infection) makes this approach unfeasible. The right ventricular lead should be placed first as it is less likely to dislodge during manipulation of other leads and provides information about the position of the tricuspid valve and right atrial size. 
New guidelines emphasize the role of cardiac resynchronization therapy in subgroups of patients who already have conventional pacemakers, to avoid pacing-induced dyssynchrony and remodeling. Upgrading an existing device may pose difficulties due to the necessity to operate in a previously scarred area and the presence of previously implanted leads in the venous system. The perioperative risks in these patients are significantly higher: the 6-month major complication rate was $18.7 \%$ in the REPLACE registry in patients undergoing upgrade to a CRT device with addition of a new endocardial LV lead to the existing leads (Poole, 2010). The risk of subclavian vein thrombosis is related to the number of leads implanted, among recipients of CRT devices severe obstruction or occlusion can be observed in 30\% (Bulur, 2010). Subclavian venography with injection through the upper extremity veins is a simple and effective technique to evaluate venous anatomy prior to an upgrade or lead revision. Venoplasty may be performed if ipsilateral implantation is favored (Worley, 2011).

Extraction of non-used leads during an upgrade or revision has to be considered as the risk of long-term complications from abandoned leads is not negligible and correlates with the number of leads implanted and the number of prior procedures performed (Diemberger, 2011). Implantation via the jugular or contralateral subclavian vein, with subcutaneous tunneling is required if the anatomy does not permit ipsilateral addition of a new lead. Although primary transvenous device implantations are routinely performed using conscious sedation without much patient discomfort, deep sedation or general anesthesia may be required for lead tunneling (Fox, 2007). In case lead extraction has to be performed prior to the upgrade, general anesthesia, invasive monitoring and availability of immediate surgical backup is recommended (Kratz, 2010).

For CS cannulation, a wide array of sheaths are available. The ostium may be probed with a standard angiographic soft-tip wire, an angiographic catheter (such as Amplatz) may be inserted into the sheath to adjust its distal curve. Alternatively, a steerable CS EP catheter may also be used for mapping, individual practices vary significantly. Mapping with stiff catheters should be performed very cautiously as after overcoming any resistance from the Thebesian valve, the catheter may advance to the CS at an oblique angle, dissecting it, rendering continuation of the implantation procedure extremely difficult. This is most commonly encountered during insertion or advancement of the occlusion balloon catheter, as it has a relatively stiff distal portion. Transesophageal echocardiography may facilitate CS cannulation when traditional methods have proven ineffective (Bashir, 2003). In difficult cases intracardiac echocardiography may be more tolerable in patients under conscious sedation (Shalaby, 2005).

Cannulation of the CS is followed by coronary sinus angiography using an occluder balloon. Fluoroscopic acquisition should allow time to image late filling terminal branches, due to collateral flow. Some centers perform a single venography image, however, two orthogonal views (RAO and LAO) are preferred for better visualization of 3 dimensional anatomy. A minority of centers perform rotational venography, which may provide more detailed information (Blendea, 2007). The location and takeoff of the ostium may vary considerably, can be distorted by right atrial enlargement or prior surgery. Distally in the CS, the valve of 
Vieussens (typically 3-5 cm from the ostium) may hinder cannulation of the distal vessel (Ho, 2004).

LV pacing leads may be implanted over a guidewire or directly. The guidewire may help to achieve more distal position by providing a rail when advancing the lead in a tortuous vein. Care should be taken to place the preformed fixation mechanism (curves, spiral or tags) with adequate distal penetration, to have a large area of contact with the vein wall (Hansky, 2002). Most current leads are at least bipolar with size and flexibility similar to previous unipolar leads. The advantage of multiple electrodes is the possibility of electrical "repositioning" when high pacing threshold or phrenic nerve stimulation is encountered (Gurevitz, 2005 and Forleo, 2011). (Figure 3)

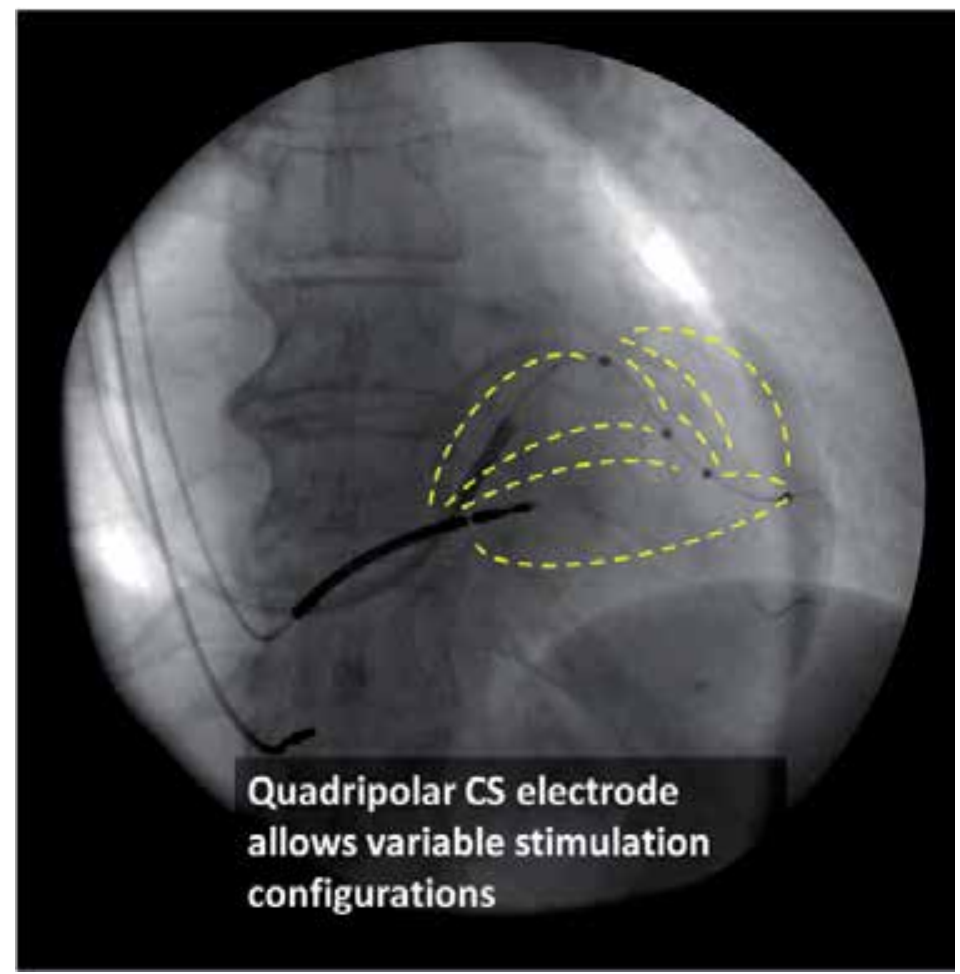

Figure 3. A quadripolar CS lead allows multiple pacing configurations, providing options to avoid phrenic nerve stimulation or sites with high threshold. It also enables a more stable distal lead position, while still allowing pacing on the proximal electrodes if desired.

If the vein is tortuous, a stiffer wire or buddy wire may be used with caution, as the wall thickness and tear resistance of coronary veins is much less compared to coronary arteries. In case severe tortuosity or kinking of the vein does not allow adequate force transition to advance the lead, use of telescopic guides may be considered (Russo, 2009). Inflated venogram (occluder) and coronary balloons can also be used as anchors to facilitate CS cannulation and left ventricular lead placement and help to recover lost CS and target vein access (Worley, 2009). 
In case the target vein is stenotic, balloon angioplasty may be attempted. Although the pathophysiological basis for coronary artery stenoses and coronary sinus/vein abnormalities are different, in most cases these obstacles can be overcome with the use of conventional interventional cardiology techniques. The required instrumentation is the same as for coronary artery angioplasties. In the majority of cases with a focal stenosis or valve, balloon angioplasty is a safe method to facilitate passage of the lead. In selected cases coronary atherectomy or stent implantation may be required (Soga, 2007). Angioplasty may also be used as a rescue when dissection of the coronary sinus or the target vein is observed during implantation, which would otherwise prohibit further attempts for lead placement (Bosa, 2008 and Gutleben, 2008). In case of unfavorable coronary vein anatomy in the target area, dilatation and use of collateral veins may be considered (Abben, 2010). Complications from venoplasty are rare, however, venous rupture has been reported (Worley, 2008). With access to these techniques, implantation success rate may be as high as $99 \%$ : a retrospective single center analysis showed that $3.5 \%$ of patients required venoplasty for LV implantation (of these, $77 \%$ coronary vein, $13 \%$ subclavian vein, $10 \%$ valvular structures within the CS or a Marshall vein). The required inflation pressures for ring-like structures were high (16 \pm 3 atm), complications were rare. Mostly short balloons with $3 \mathrm{~mm}$ diameter were used (Luedorff, 2008). The presence of a persistent left superior vena cava may prohibit successful CRT implantation due to severely dilated CS and distorted anatomy, however, successful use of angioplasty in this case was reported (Cagin, 2010).

High pacing threshold or phrenic nerve stimulation may require repositioning of the lead from a stable to a less optimal position, where its fixation mechanism may not be as effective. In these cases, a coronary stent implanted near the distal end of the lead may stabilize the new position. A large single center study of 312 patients treated with stent implantation showed that the method was safe and effective in long term to prevent lead dislocation. $95 \%$ of patients had stenting due to intraoperative lead instability or phrenic nerve stimulation, while $5 \%$ required it due to previous lead disclocation. The bare metal stents were implanted $5-35 \mathrm{~mm}$ proximal to the most proximal electrode (unipolar and bipolar LV leads). There was no evidence of mechanical damage to the lead or CS perforation. During the follow-up of average 28.4 months, a significant increase in the left ventricular pacing threshold was found in four cases and reoperation was necessary in two patients $(0.6 \%)$. Phrenic nerve stimulation was observed in 18 instances, and closed repositioning with an ablation catheter was performed in seven cases. In three cases the leads were extracted without complication after 3-49 months (infection, heart transplant) (Geller, 2011). (Figure 4)

Coronary sinus dissection is a rare complication of LV lead placement. It may be recognized as inability to pass instruments though a previously accessible area, and pooling of contrast after angiography. Although complete perforation is even less common and the pressure in the CS is low, cardiac tamponade may occasionally develop. If the dissection is small, it may be possible to finish the implantation, although balloon angioplasty or stenting of the dissected segment may be necessary. In these cases, patients need to be monitored after the implantation, however, the exact duration that is required before a safe discharge is unknown (Figure 5). 


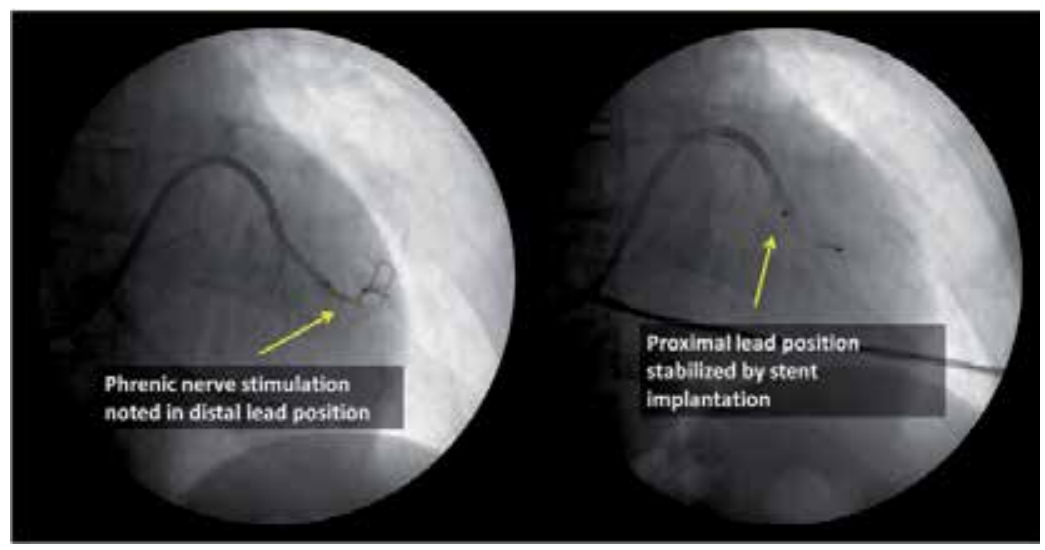

Figure 4. Phrenic nerve stimulation was encountered in the distal lateral vein. In a more proximal position, the pacing threshold was acceptable with no diaphragmatic stimulation, however, the lead position was unstable. A bare metal coronary stent was implanted just proximal to the proximal electrode, after which the lead position stabilized.

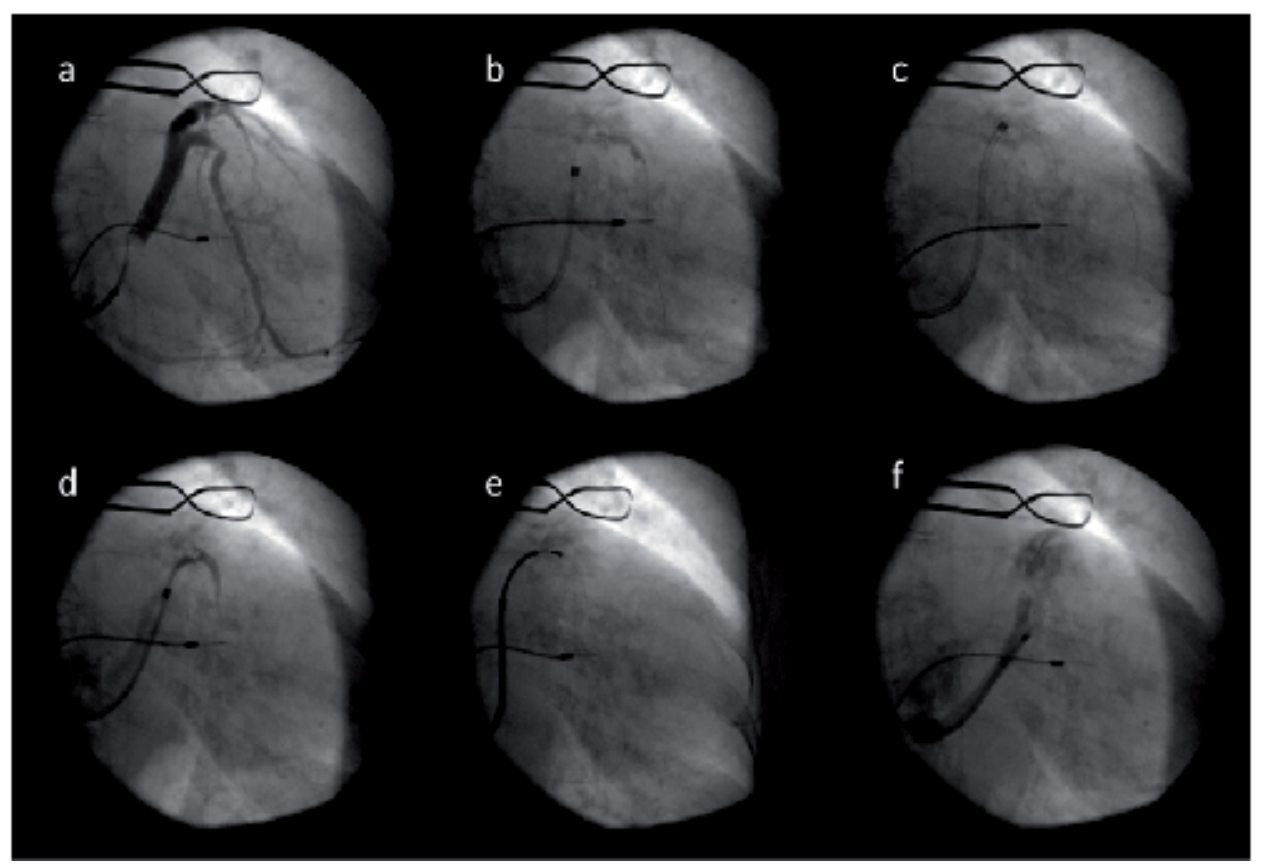

Figure 5. Complicated CS anatomy may prohibit transvenous lead implantation even with extensive use of interventional techniques. The targeted lateral vein has a narrowed proximal segment with a kink, which did not allow lead advancement (a). After passing a coronary guidewire, a bare metal stent was deposited into this region (b). Although the guidewire easily passed through this area, lead advancement was still not feasible, the stent is clearly visible (c). Selective venography shows dissection proximal to the stent (d). Even after multiple attempts with balloon angioplasty in this area, lead advancement was still not possible (e). After multiple attempts with a selective guide and buddy wire, repeat venography shows dissection of CS with distal occlusion and staining, at this point the procedure was aborted (f). No significant pericardial effusion or further sequelae were noted. 
Epicardial left ventricular stimulation poses a unique challenge as the electrode position has to be stable to provide effective cardiac resynchronization with low energy output, while avoiding phrenic nerve stimulation. The coronary veins lack trabeculation and the thin wall prohibits the use of conventional screw-in active fixation leads. The majority of coronary sinus leads are passive fixation, which try to maintain stability with pre-shaped tips. Recently, built-in active lead fixation mechanisms became available. Up to $7 \%$ of transvenously placed LV leads may require revision (Borleffs, 2008) over long-term follow up (2 years). One third of the interventions are required more than 6 months after the implantation. A dislodged coronary sinus lead can be the source of multiple complications, such as inadvertent stimulation of extracardiac structures, arrhythmia or perforation, and loss of effective cardiac resynchronization. Repositioning most of the time requires access to the lead via a device pocket revision. As open revision carries a higher risk of system infection, minimally invasive procedures should be considered when appropriate. In some cases, the dislocation may be resolved by catheter-based techniques. In each case, an attempt has to be made to provide a more stable final lead position. A transfemoral approach was effective for the retraction of the LV lead from distal dislocation in a single center case series, where distal dislocation of the electrode lead to intolerable phrenic nerve stimulation (Szilagyi, 2008). The CS was cannulated with an Amplatz catheter, then a coronary stent was introduced over a guidewire besides the lead into the side branch in 7 patients or in 2 patients, into the CS. A steerable ablation catheter was looped around the LV lead in the atrium with bent tip and was drawn backwards together. The stent was then inflated to stabilize the lead tip in a new position. During follow-up of median 7.7 months, stable pacing thresholds and impedances were measured; transient and avoidable phrenic nerve stimulation was present in only one patient. There were no procedural complications or infections.

Stabilizing the lead position with retained stylets is not recommended due to high risk of late lead failure (Nagele, 2007). With active fixation of the CS leads, lead extraction may be an issue if the device has to be explanted. In the above mentioned case series, the few leads that had to be explanted and were previously stabilized with stent implantation, came out with manual traction most of the time, however, more data will be needed to assess if active anchoring of LV leads into the coronary veins poses any long-term risk.

\section{Transseptal endocardial left ventricular pacing}

Transseptal approach through the interatrial septum has been used for multiple interventional and electrophysiological procedures for decades. It is a relatively safe procedure in experienced hands, however, the facility should be prepared to address potential complications (mainly pericardiocentesis and emergent periocardiotomy). Using the transseptal approach, a conventional endocardial PM lead may be implanted into the LV cavity for endocardial stimulation. The thromboembolic risks are not negligible and these patient require long term anticoagulation. Mitral valve damage and endocarditis are rare, but serious compliations. 
The transseptal implantation begins with a standard transseptal puncture via the femoral vein (van Gelder, 2007). After the successful puncture, full anticoagulation with iv. heparin is initiated. A 0.035 -inch guidewire is inserted into one of the pulmonary veins and then the dilator and the sheath is removed. The puncture site is dilated with a 6-8 mm balloon. A steerable EP catheter is advanced from the subclavian area towards the interatrial septum, then into the left ventricle after deflating the balloon. A sheath is then advanced into the LA over the EP catheter. The endocardial surface of the LV may be mapped with the EP catheter to localize a site most suitable for lead implantation, then a standard endocardial bipolar pacemaker lead is implanted. Using a modified technique, electroanatomical mapping may be used to precisely identify the area of latest activation in the LV (Kutyifa, 2012).

The main disadvantage of this technique is the unknown long term thromboembolic risk, which may be similar to a mechanical valve, with INR goals in the higher range (3-4). Over an average of 85 month follow up of 6 patients in a case series, one patient had LV lead dislodgment at 3 months requiring reintervention. One patient had a transient ischemic attack, when anticoagulation was accidentally interrupted (Pasquie, 2007). The need to start full dose anticoagulation immediately after the implantation increases the risk of periprocedural bleeding complications.

This technique is a feasible and safe second option with a benefit of endocardial pacing site and implantation procedure with no more burden to the patient than conventional transvenous CRT implantation. Main disadvantage is the need for long term anticoagulation. Patients who are ineligible for surgical epicardial implantation and have no contraindications for lifelong oral anticoagulation can be selected for this approach. Biventricular pacing with endocardial stimulation may provide more homogenous intraventricular resynchronization than with epicardial stimulation, and is associated with better LV filling and systolic performance (Garrigue, 2001).

\section{Surgical epicardial LV lead placement}

In early cases of CRT, the LV lead was surgically placed via thoracotomy. This approach was associated with considerable morbidity, requiring general anesthesia and longer recovery time. Long term electrical stability of surgically placed electrodes is inferior when compared to transvenous leads (Lau, 2009). Major advantage of the surgical implantation is that it is not constrained by the venous anatomy and the latest contracting segment may be visually identified. Phrenic nerve stimulation may also be more easily avoided and there is no need for fluoroscopy. As transvenous implantation has a high success rate with excellent long-term stability, surgical implantation should not be considered are first line therapy. The risk of failed transvenous implant is higher in patients with prior cardiac surgeries (however, this also increases the risk of consecutive sternotomy/thoracotomy). In patients eligible for CRT, undergoing cardiothoracic surgical procedure, implantation of epicardial LV lead may be considered, which may be tunneled to the planned PM/ICD generator site. Surgical implantation may also be preferred in patients with complex congential heart disease, where the venous anatomy may interfere with the transvenous approach. 
Usually, there is no need for full left thoracotomy for epicardial LV lead implantation as minimal thoracotomy provides adequate window for the procedure. The surgery is performed using single-lung ventilation on a beating heart. Transeosophageal echocardiography (TEE) control is needed throughout the procedure. A 3 to $5 \mathrm{~cm}$ incision is made over the 4 th or 5 th intercostal space anterior to the midaxillary line. The lung is pushed back and the pericardium is opened anterior to the phrenic nerve. The left ventricle is mapped for optimal pacing site and an epicardial lead placement device is used to attach the electrode (unipolar or bipolar) (Mair, 2005). The leads are tunneled to the PM/ICD generator site, usually in the left infraclavicular region. Short-term chest drainage is required postoperatively. A recent investigation described this technique as a safe and acceptable option, with benefits comparable to transvenous CRT: in 33 patients, functional and echocardiographic parameters showed similar improvement, however, with a delayed onset of peak VO2 improvement (Patwala, 2009).

Instead of minimal thoracotomy, video assisted thoracoscopy may be used for lead placement. It uses two incisions for the ports in the 4th or 5th intercostal space along the anterior and midaxillary line. General anaesthesia and single lung ventilation enables the deflation of the left lung. The camera and the manipulating instruments are inserted through the ports, the pericardium is opened laterally to the phrenic nerve under visual control, then the epicardial lead is screwed into the lateral wall of the LV. The lead is passed through the medial incision and then tunneled subcutaneously to the generator site. The procedure is well tolerated, it has minimal postoperative recovery and very good cosmetic results. In a series of 15 patients, who previously failed transvenous implantation, mean skin-to-skin operating time was $55 \pm 16 \mathrm{~min}$, no conversion to thoracotomy was necessary. All patients were extubated in the operating room and remained in the intensive care unit for less than $24 \mathrm{~h}$. Chest tubes were removed after a mean of $1.6 \pm 0.5$ days and the patients were discharged after a mean of $4 \pm 1.3$ days. Intraoperative and postoperative pacing thresholds at 1 and 7 months were satisfactory in all cases and there was no lead dislocation. All but two patients had an improvement of their NYHA function class. There was neither surgical morbidity nor mortality (Gabor, 2005).

Endoscopic robotic surgery is rapidly evolving and has been investigated for CRT. This technique also needs general anesthesia, single lung intubation and TEE. The left and right arms for the DaVinci system are placed in the 5th and 9th intercostal space. The pericardium is opened posterior to the phrenic nerve and the region of the obtuse marginal is identified to find the latest activating area with a temporary pacing electrode, the procedure is similar to the endoscopic approach. The patient is extubated immediately after the procedure and there is no need for a chest tube. Patients are usually discharged on the first postoperative day. Follow-up results of 42 patients showed a procedure time on the plateau of the learning curve of $45 \pm 13$ minutes, with a responder rate of $81 \%$ and $70 \%$ at 3 and 6 month follow up. Three patients experienced loss of LV capture at 1, 9, and 14 months (a second lead, which was simultaneously implanted with the first one and also tunneled to the generator site had to be activated in these patients) (Joshi, 2005). With growing experience and availability of surgical robotic system, this method may gain more widespread utilization. 


\section{Transapical endocardial lead implantation}

This technique combines the minimally invasive surgical approach with the advantages of endocardial pacing. This may be the only option for patients with extensive epicardial adhesions prohibiting access to the pericardial space (Kassai, 2008). After induction of general anaesthesia and selective bronchial intubation, the LV apex is localized with transthoracic echocardiography, then a small left thoracotomy is performed. The apex of the left ventricle is punctured and an active fixation lead is inserted into the cavity, using Seldinger technique. The bleeding is controlled with purse-string sutures. The lead is guided into its final position with a guide, using fluoroscopy. As transapical endocardial lead implantation does not involve the mitral valve, the risk of mitral valve endocarditis is reduced. The lead is subcutaneously tunneled to the infraclavicular pocket to be connected to the generator. Long term anticoagulation is required, similar to patients with transseptal endocardial leads. Advantages of this technique are the accessibility of the endocardial segments without limitations of the CS anatomy and absence of phrenic nerve stimulation (Kassai, 2009).

\section{Conclusion}

Transvenous implantation of CRT systems is the preferred way as it poses the least risk for the patient, has excellent long term results and is supported by large amount of evidence from clinical trials. Familiarity with interventional angioplasty methods may facilitate the implant procedure. Although newer left ventricular leads have better maneuverability and improved fixation mechanisms, interventional techniques are useful adjuncts if difficult anatomy is encountered. In case of unsuitable anatomy of failed implantation, minimally invasive surgical procedures should be considered for LV lead placement.

\section{Author details}

Bela Merkely and Levente Molnar

Heart Center, Semmelweis University, Hungary

Attila Roka

Hospital of St. Raphael, New Haven, CT, USA

\section{References}

Abben, R.P., et al., V.Traversing and dilating venous collaterals: a useful adjunct in left ventricular electrode placement. J Invasive Cardiol, 2010. 22(6): p. E93-6.

Alonso, C., In the field of cardiac resynchronization therapy is left ventricular pacing via the coronary sinus a mature technique. Europace. 2009. 11(5): p. 544-5.

Ansalone, G., et al., Doppler myocardial imaging to evaluate the effectiveness of pacing sites in patients receiving biventricular pacing. J Am Coll Cardiol, 2002. 39(3): p. 489-99 
Auricchio, A., et al., Characterization of left ventricular activation in patients with heart failure and left bundle-branch block. Circulation, 2004. 109(9): p. 1133-9.

Auricchio, A., et al., Accuracy and usefulness of fusion imaging between three-dimensional coronary sinus and coronary veins computed tomographic images with projection images obtained using fluoroscopy. Europace, 2009. 11(11): p. 1483-90.

Bashir, J.G., et al., Combined use of transesophageal ECHO and fluoroscopy for the placement of left ventricular pacing leads via the coronary sinus. Pacing Clin Electrophysiol, 2003. 26(10): p. 1951-4.

Bilchick, K.C., et al., Cardiac magnetic resonance assessment of dyssynchrony and myocardial scar predicts function class improvement following cardiac resynchronization therapy. JACC Cardiovasc Imaging, 2008. 1(5): p. 561-8.

Bleeker, G.B., et al., Effect of postero-lateral scar tissue on clinical and echocardiographic improvement following cardiac resynchronization therapy. Circulation, 2006. 113 p. 969-976.

Blendea, D., et al., Variability of coronary venous anatomy in patients undergoing cardiac resynchronization therapy: a high-speed rotational venography study. Heart Rhythm, 2007. 4(9): p. 1155-62.

Bosa, F., et al., Prolonged inflation of coronary angioplasty balloon as treatment for subocclusive dissection of the coronary sinus during implantation of a coronary sinus pacing lead. J Interv Card Electrophysiol, 2008. 23(2): p. 139-41.

Borleffs, C.J., et al., Requirement for coronary sinus lead interventions and effectiveness of endovascular replacement during long-term follow-up after implantation of a resynchronization device. Europace, 2008. 11(5): p. 607-11.

Bulur, S., et al., Incidence and predictors of subclavian vein obstruction following biventricular device implantation. J Interv Card Electrophysiol, 2010. 29(3): p. 199-202.

Burri, H., et al., Right ventricular systolic function and cardiac resynchronization therapy. Europace, 2010. 12(3): p. 389-94.

Cagin C., et al.,, Coronary venoplasty-assisted implantation of cardiac resynchronization device in a patient with persistent left superior vena cava. Heart Rhythm. 2010. 7(1): p.141-2.

Cannesson, M., et al., Velocity vector imaging to quantify ventricular dyssynchrony and predict response to cardiac resynchronization therapy. Am J Cardiol, 2006. 98(7): p. 949-53.

Chalil, S., et al., Effect of posterolateral left ventricular scar on mortality and morbidity following cardiac resynchronization therapy. Pacing Clin Electrophysiol, 2007. 30(10): p. 1201-9.

Da Costa, A., et al., Antibiotic prophylaxis for permanent pacemaker implantation: a metaanalysis. Circulation, 1998. 97(18): p. 1796-801.

Daubert, J.C., et al., 2012 EHRA/HRS Expert Consensus Statement on Cardiac Resynchronization Therapy Implant and Follow-up Considerations. Europace 2012, in press

de Oliveira, J.C., et al., Efficacy of antibiotic prophylaxis before the implantation of pacemakers and cardioverter-defibrillators: results of a large, prospective, randomized, double-blinded, placebo-controlled trial. Circ Arrhythm Electrophysiol, 2009. 2(1): p. 29-34. 
Derval, N., et al., Optimizing hemodynamics in heart failure patients by systematic screening of left ventricular pacing sites: the lateral left ventricular wall and the coronary sinus are rarely the best sites. J Am Coll Cardiol, 2010. 55(6): p. 566-75.

Diemberger, I., et al., From lead management to implanted patient management: indications to lead extraction in pacemaker and cardioverter-defibrillator systems. Expert Rev Med Devices, 2011. 8(2): p. 235-55.

Doganay, S., et al., Usefulness of multidetector computed tomography coronary venous angiography examination before cardiac resynchronization therapy. Jpn J Radiol, 2011. 29(5): p. 342-7.

Duckett, S.G., et al., Cardiac MRI to investigate myocardial scar and coronary venous anatomy using a slow infusion of dimeglumine gadobenate in patients undergoing assessment for cardiac resynchronization therapy. J Magn Reson Imaging, 2011. 33(1): p. 87-95.

Duckett, S.G., et al., Invasive acute hemodynamic response to guide left ventricular lead implantation predicts chronic remodeling in patients undergoing cardiac resynchronization therapy. J Am Coll Cardiol, 2011. 58(11): p. 1128-36.

Edwards, F.H., et al., Society of Thoracic Surgeons. The Society of Thoracic Surgeons Practice Guideline Series: Antibiotic Prophylaxis in Cardiac Surgery, Part I: Duration. Ann Thorac Surg, 2006. 81(1): p. 397-404.

Engelman, R., et al.. Workforce on Evidence-Based Medicine, Society of Thoracic Surgeons. The Society of Thoracic Surgeons practice guideline series: Antibiotic prophylaxis in cardiac surgery, part II: Antibiotic choice. Ann Thorac Surg, 2007. 83(4): 1569-76.

Epstein, A.E., et al., ACC/AHA/HRS 2008 Guidelines for Device-Based Therapy of Cardiac Rhythm Abnormalities: a report of the American College of Cardiology/American Heart Association Task Force on Practice Guidelines (Writing Committee to Revise the ACC/AHA/NASPE 2002 Guideline Update for Implantation of Cardiac Pacemakers and Antiarrhythmia Devices) developed in collaboration with the American Association for Thoracic Surgery and Society of Thoracic Surgeons. J Am Coll Cardiol, 2008. 51(21): p. e1-62.

Forleo, G.B., et al., Left ventricular pacing with a new quadripolar transvenous lead for CRT: early results of a prospective comparison with conventional implant outcomes. Heart Rhythm, 2011. 8(1): p. 31-7.

Fox, D.J., et al., Safety and acceptability of implantation of internal cardioverterdefibrillators under local anesthetic and conscious sedation. Pacing Clin Electrophysiol, 2007. 30(8): p. 992-7.

Gabor, S., et al., A simplified technique for implantation of left ventricular epicardial leads for biventricular re-synchronization using video-assisted thoracoscopy (VATS). European Journal of Cardio-thoracic Surgery, 2005. 28(6), p. 797-800.

Garrigue, S., et al. Comparison of chronic biventricular pacing between epicardial and endocardial left ventricular stimulation using Doppler tissue imaging in patients with heart failure American Journal of Cardiology, 2001. 88(8), p. 858-862.

Geller L., et al., Long-term experience with coronary sinus side branch stenting to stabilize left ventricular electrode position. Heart Rhythm. 2011. 8(6): p. 845-50. 
Girsky, M.J., et al., Prospective randomized trial of venous cardiac computed tomographic angiography for facilitation of cardiac resynchronization therapy. Pacing Clin Electrophysiol, 2010. 33(10): p. 1182-7. 49.

Gold, M.R., et al., The relationship between ventricular electrical delay and left ventricular remodelling with cardiac resynchronization therapy. Eur Heart J, 2011. 32(20): p. 2516-24.

Gurevitz, O., et al., Programmable multiple pacing configurations help to overcome high left ventricular pacing thresholds and avoid phrenic nerve stimulation. Pacing and clinical electrophysiology : PACE, 2005. 28(12): p. 1255-9.

Gutleben, K.J., et al., Rescue-stenting of an occluded lateral coronary sinus branch for recanalization after dissection during cardiac resynchronization device implantation. Europace, 2008. 10(12): p. 1442-4.

Hansky, B., et al., Left heart pacing--experience with several types of coronary vein leads. J Interv Card Electrophysiol, 2002. 6(1): p. 71-5.

Ho, S.Y., et al., A review of the coronary venous system: a road less travelled. Heart Rhythm, 2004. 1(1): p. 107-12.

Jamula, E., et al., Perioperative anticoagulation in patients having implantation of a cardiac pacemaker or defibrillator: a systematic review and practical management guide. J Thromb Haemost, 2008. 6(10): p. 1615-21.

Joshi, S., et al., Follow-up of robotically assisted left ventricular epicardial leads for cardiac resynchronization therapy. Journal of the American College of Cardiology. 2005. 46(12): p. 2358-2359.

Kassai, I., et al. New method for cardiac resynchronization therapy: transapical endocardial lead implantation for left ventrical free wall pacing. Europace, 2008. 10(7): p. 882-3.

Kassai, I., et al., A Novel Approach for Endocardial Resynchronization Therapy: Initial Experience with Transapical Implantation of the Left Ventricular Lead. Heart Surg Forum, 2009. 12(3): p. 137-40.

Khan, F.Z., et al., Left ventricular lead placement in cardiac resyncrnisation therapy: where and how? Europace, 2009. 11(5), p. 554-61.

Klug, D., et al., Risk factors related to infections of implanted pacemakers and cardioverterdefibrillators: results of a large prospective study. Circulation, 2007. 116(12): p. 1349-55. 93

Kovacs, B., et al., Anatomy of the coronary veins can be assessed with left coronary angiography (abstract). European Journal of Heart Failure, 2004. 5(Suppl 1): 820.

Kratz, J.M., et al., Pacemaker and internal cardioverter defibrillator lead extraction: a safe and effective surgical approach. Ann Thorac Surg, 2010. 90(5): p. 1411-7.

Kronborg, M.B., et al., Non-contrast magnetic resonance imaging for guiding left ventricular lead position in cardiac resynchronization therapy. J Interv Card Electrophysiol, 2012. 33(1): p. 27-35.

Kutyifa, V., et al., Usefulness of electroanatomical mapping during transseptal endocardial left ventricular lead implantation. Europace, 2012. 14(4): p. 599-604.

Lau, W.E. Achieving permanent left ventricular pacing - options and choice. Pacing Clinical Electrophysiology, 2009. 32(11), p. 1466-77.

Leon, A.R., et al., Safety of transvenous cardiac resynchronization system implantation in patients with chronic heart failure: combined results of over 2,000 patients from a multicenter study program. J Am Coll Cardiol, 2005. 46(12): p. 2348-56. 
Linde, C., et al., Randomized trial of cardiac resynchronization in mildly symptomatic heart failure patients and in asymptomatic patients with left ventricular dysfunction and previous heart failure symptoms. J Am Coll Cardiol, 2008. 52(23): p. 1834-43.

Luedorff, G., et al. Different venous angioplasty manoeuvres for successful implantation of CRT devices. Clin Res Cardiol, 2009. 98(3): p. 159-64.

Mair, H., et al. Surgical epicardial left ventricular lead versus coronary sinus lead placement in biventricular pacing. European Journal of Cardiothoracic Surgery, 2005. 27(2), p. 235-42.

McMurray, J.J.V., et al. ESC Guidelines for the diagnosis and treatment of acute and chronic heart failure 2012. Eur Heart J, 2012. doi:10.1093/eurheartj/ehs104 (in press)

Merchant, F.M., et al., Interlead distance and left ventricular lead electrical delay predict reverse remodeling during cardiac resynchronization therapy. Pacing Clin Electrophysiol, 2010. 33(5): p. 575-82.

Mertz, D., et al. Does duration of perioperative antibiotic prophylaxis matter in cardiac surgery? A systematic review and meta-analysis. Ann Surg, 2011. 254(1): p. 48-54.

Matsumoto, Y., et al., Detection of phrenic nerves and their relation to cardiac anatomy using 64-slice multidetector computed tomography. Am J Cardiol, 2007. 100(1): p. 133-7.

Mullens, W., et al., Insights from a cardiac resynchronization optimization clinic as part of a heart failure disease management program. J Am Coll Cardiol, 2009. 53(9): p. 765-73. 42. Knappe, D., et al., Dyssynchrony, contractile function, and response to cardiac resynchronization therapy. Circ Heart Fail, 2011. 4(4): p. 433-40.

Murphy, R.T., et al., Tissue synchronization imaging and optimal left ventricular pacing site in cardiac resynchronization therapy. Am J Cardiol, 2006. 97(11): p. 1615-21.

Nagele, H., et al., Coronary sinus lead fragmentation 2 years after implantation with a retained guidewire. Pacing Clin Electrophysiol, 2007. 30(3): p. 438-9.

Neizel, M., et al., Magnetic resonance imaging of the cardiac venous system and magnetic resonance-guided intubation of the coronary sinus in swine: a feasibility study. Invest Radiol, 2010. 45(8): p. 502-6.

Pasquie, J.L., et al. Long-Term Follow-Up of Biventricular Pacing Using a Totally Endocardial Approach in Patients with End-Stage Cardiac Failure. Pacing Clinical Electrophysiology, 2007. 30(1), p. S31-33.

Patwala, A., et al. (2009) A prospective longitudinal evaluation of the benefits of epicardial lead placement for cardiac resynchronization therapy. Europace, 2009. 11(10): p. 1323-9.

Poole, J.E., et al., Complication rates associated with pacemaker or implantable cardioverterdefibrillator generator replacements and upgrade procedures: results from the REPLACE registry. Circulation, 2010. 122(16): p. 1553-61.

Russo, V., et al. Superselective cannulation of coronary sinus branch with telescopic system during left ventricular lead placement. Acta Biomed, 2009. 80(2):p. 153-5.

Saxon, L.A., et al., Influence of left ventricular lead location on outcomes in the COMPANION study. J Cardiovasc Electrophysiol, 2009. 20(7): p. 764-8.

Shalaby, A.A., Utilization of intracardiac echocardiography to access the coronary sinus for left ventricular lead placement. Pacing Clin Electrophysiol, 2005. 28(6): p. 493-7.

Singh, J.P., et al., Left ventricular lead position and clinical outcome in the multicenter automatic defibrillator implantation trial-cardiac resynchronization therapy (MADITCRT) trial. Circulation, 2011. 123(11): p. 1159-66. 
Soga, Y., et al., Efficacy of coronary venoplasty for left ventricular lead implantation. Circ J, 2007. 71(9): p. 1442-5.

Szilagyi, S., et al. Minimal invasive coronary sinus lead reposition technique for the treatment of phrenic nerve stimulation. Europace, 2008. 10(10): p. 1157-60.

Thebault, C., et al., Sites of left and right ventricular lead implantation and response to cardiac resynchronization therapy observations from the REVERSE trial. Eur Heart J, 2012, in press.

Theuns, D.A., et al. The prognosis of implantable defibrillator patients treated with cardiac resynchronization therapy: comorbidity burden as predictor of mortality. Europace, 2011. 13(1): p. 62-9.

Tompkins, C., et al., Dual antiplatelet therapy and heparin "bridging" significantly increase the risk of bleeding complications after pacemaker or implantable cardioverterdefibrillator device implantation. J Am Coll Cardiol, 2010. 55(21): p. 2376-82.

Tompkins, C., et al. Optimal strategies for the management of antiplatelet and anticoagulation medications prior to cardiac device implantation. Cardiol J, 2011. 18(1): p. 103-9.

Tournoux, F., et al., Integrating functional and anatomical information to guide cardiac resynchronization therapy. Eur J Heart Fail, 2010. 12(1): p. 52-7.

Van de Veire, N.R., et al., Noninvasive imaging of cardiac venous anatomy with 64-slice multi-slice computed tomography and noninvasive assessment of left ventricular dyssynchrony by 3-dimensional tissue synchronization imaging in patients with heart failure scheduled for cardiac resynchronization therapy. Am J Cardiol, 2008. 101(7): p. 1023-9.

Van Gelder, B.M., et al. Transseptal endocardial left ventricular pacing: An alternative technique for coronary sinus lead placement in cardiac resynchronization therapy. Heart Rhythm, 2007. 4(4), p. 454-60.

White, J.A., et al., Fused whole-heart coronary and myocardial scar imaging using 3-T CMR. Implications for planning of cardiac resynchronization therapy and coronary revascularization. JACC Cardiovasc Imaging, 2010. 3(9): p. 921-30.

Wiegand, U.K., et al., Pocket hematoma after pacemaker or implantable cardioverter defibrillator surgery: influence of patient morbidity, operation strategy, and perioperative antiplatelet/anticoagulation therapy. Chest, 2004. 126(4): p. 1177-86.

Worley, S.J., Implant venoplasty: dilation of subclavian and coronary veins to facilitate device implantation: indications, frequency, methods, and complications. J Cardiovasc Electrophysiol, 2008. 19(9): p. 1004-7.

Worley, S.J. How to use balloons as anchors to facilitate cannulation of the coronary sinus left ventricular lead placement and to regain lost coronary sinus or target vein access. Heart Rhythm, 2009. 6(8): p. 1242-6.

Worley, S.J., et al., Subclavian venoplasty by the implanting physicians in 373 patients over 11 years. Heart Rhythm, 2011. 8(4): p. 526-33.

Ypenburg, C., et al., Optimal left ventricular lead position predicts reverse remodeling and survival after cardiac resynchronization therapy. J Am Coll Cardiol, 2008. 52(17): p. 1402-9. 
Section 2

\section{Follow Up and Optimization of Device Function}



Chapter 3

\title{
Electrocardiographic Troubleshooting of Implanted Cardiac Electronic Devices
}

\author{
Attila Roka \\ Additional information is available at the end of the chapter
}

http://dx.doi.org/10.5772/50983

\section{Introduction}

\subsection{Evaluation of CIED function}

Pacemakers, ICDs and cardiac resynchronization devices are implanted and followed mostly by cardiac electrophysiologists. Detailed diagnostic data (pacing statistics, lead function, arrhythmia episode intracardiac electrograms etc.) are available using manufacturer-specific programmer devices or remote follow-up (Figure 1). However, patients may present with suspected cardiac or arrhythmia-related symptoms when these measures are not immediately available. Using conventional diagnostic methods basic device function can be evaluated and correlation with the clinical presentation may be assessed (McPherson, 2004). In certain cases, such as with transient events, these may be the only diagnostic clues available as current CIEDs do not have full Holter capability - only episodes of significance, as determined by the device, are stored.

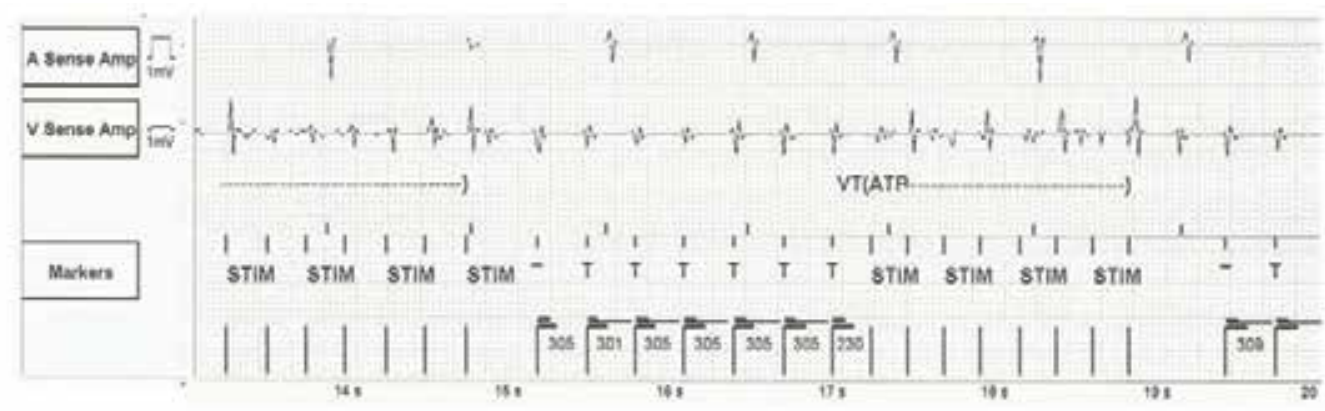

Figure 1. Device interrogation provides detailed information about intracardiac signals, their interpretation and device response. The tracing depicts an episode of ventricular tachycardia, where the implanted cardioverter-defibrillator attempted burst antitachycardia stimulation. 
Basic evaluation of CIED function requires a 12-lead ECG and review of past medical records to identify device type and settings. If prior records are not available, a simple chest X-ray may provide important clues (pacemaker, ICD, or CRT; lead locations) (Jacob, 2011). In case intermittent or transient malfunction is detected and device interrogation does not provide clear answer, Holter monitoring or an event recorder may be required. If a programmer is available, diagnostic tests should be performed according to the guidelines (Wilkoff, 2008). Patient symptoms, if any, should be assessed, whether they can be signs of a possible device malfunction.

\section{Electrocardiographic evaluation of device function}

A 12-lead ECG may raise the suspicion of device malfunction. Careful evaluation of patientrelated factors is required as these interact with device function (Table 1). Occasionally, very advanced forms of electrophysiological abnormalities may be identified as the devices generally do not prevent natural progression of underlying pathophysiology. In case an arrhythmia or device malfunction is suspected on a telemetry recording, a full 12-lead ECG is recommended to avoid misinterpretation (Figures 2-6). Artifacts may severely impact interpretation and tracings with good technical quality should be obtained (Figures 7-10). Atrial rhythm and characteristics of atrioventricular/ventriculoatrial conduction should also be assessed (Figures 11-17).

\begin{tabular}{|c|c|}
\hline ECG feature & $\begin{array}{l}\text { Importance - diagnostic clues and possible interaction with } \\
\text { devices }\end{array}$ \\
\hline \multicolumn{2}{|l|}{ Atrial rhythm } \\
\hline Bradycardia & $\begin{array}{l}\text { Should be paced unless there is oversensing or no atrial lead } \\
\text { present }\end{array}$ \\
\hline Premature beats & $\begin{array}{l}\text { Blocked PACs should elicit different response than sinoatrial } \\
\text { block if atrial sensing is present }\end{array}$ \\
\hline Atrial flutter & May be tracked with high ventricular rate \\
\hline Atrial fibrillation & May be undersensed, leading to ineffective atrial pacing \\
\hline \multicolumn{2}{|c|}{ Atrioventricular conduction } \\
\hline $\begin{array}{l}\text { Variable AV conduction } \\
\text { interval }\end{array}$ & May lead to fusion and pseudofusion beats \\
\hline Complete heart block & May be intermittent or unidirectional \\
\hline Retrograde conduction & May lead to pacemaker-tachycardia or pacemaker syndrome \\
\hline \multicolumn{2}{|l|}{ Ventricles } \\
\hline $\begin{array}{l}\text { Native QRS } \\
\text { morphology }\end{array}$ & $\begin{array}{l}\text { Assess biventricular capture during cardiac resynchronization } \\
\text { If atrial pacing only, may be used to identify ischemia, etc. }\end{array}$ \\
\hline Premature beats & $\begin{array}{l}\text { May trigger safety or sense response pacing or activate rate } \\
\text { smoothing algorithms }\end{array}$ \\
\hline
\end{tabular}

Table 1. Important ECG features that should be assessed when evaluating CIED function. 
Certain conditions, such as acute heart failure may require adjustment of device settings, even without device malfunction - pacemaker algorithms do not provide optimal hemodynamics for all situations. Unfortunately, evidence-based approach is limited due to scarcity of data (Lahiri, 2011).

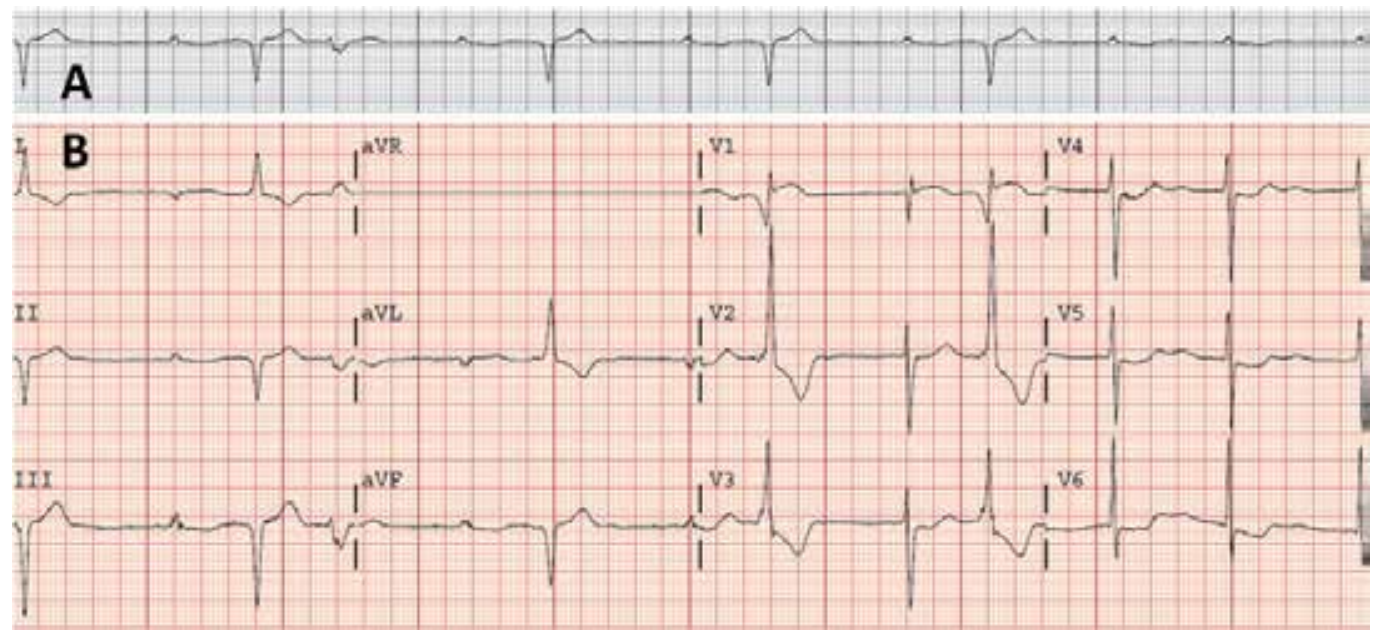

Figure 2. Rhythm strip suggestive of high degree AV block (A). 12 lead ECG obtained at the same time actually shows that the low amplitude signals are QRS complexes and the higher amplitude ones are PVCs (B).

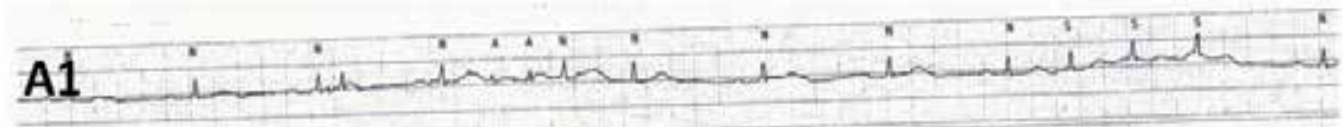

A2

B1

B2

Figure 3. Artifacts masking AV block. High frequency artifacts mimic fast, irregular ventricular rate, resembling atrial fibrillation (A1). However, these artifacts are not present on the simultaneous tracing in a different lead (A2). Once the artifacts disappear, $\mathrm{P}$ waves are easily recognizable with high degree AV block (B1, B2). 


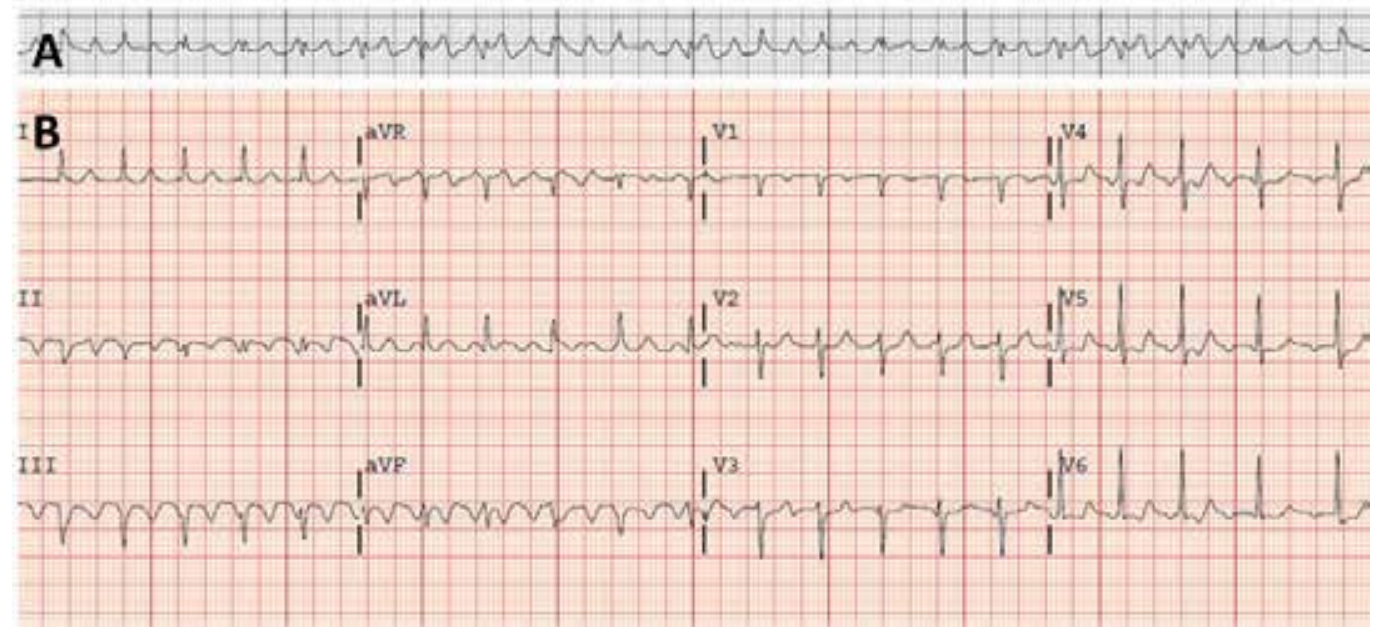

Figure 4. Atrial flutter may mimic ventricular tachycardia in a rhythm strip (A), however, 12-lead ECG clearly identifies the flutter with dominantly 2:1 conduction (B).
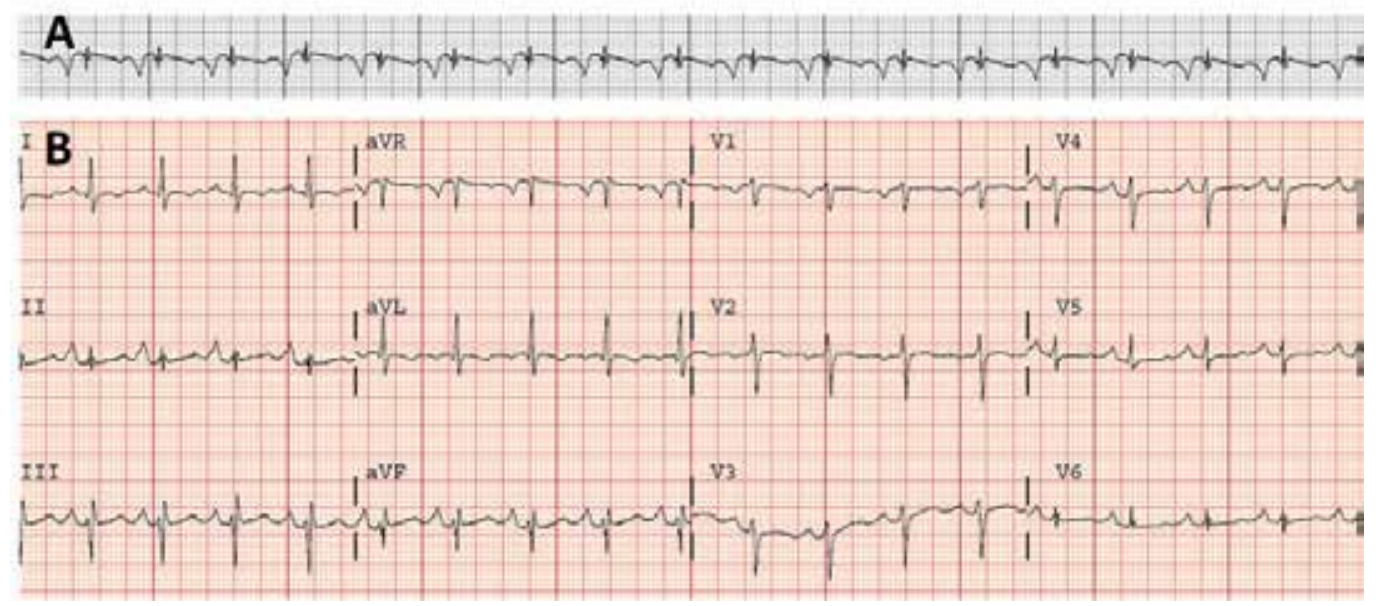

Figure 5. Rhythm strip suggestive of atrial pacing with prolonged AV interval (A). 12 lead ECG shows no evidence of pacing, however, $\mathrm{P}$ pulmonale is present and the QRS is low amplitude in II (B). 


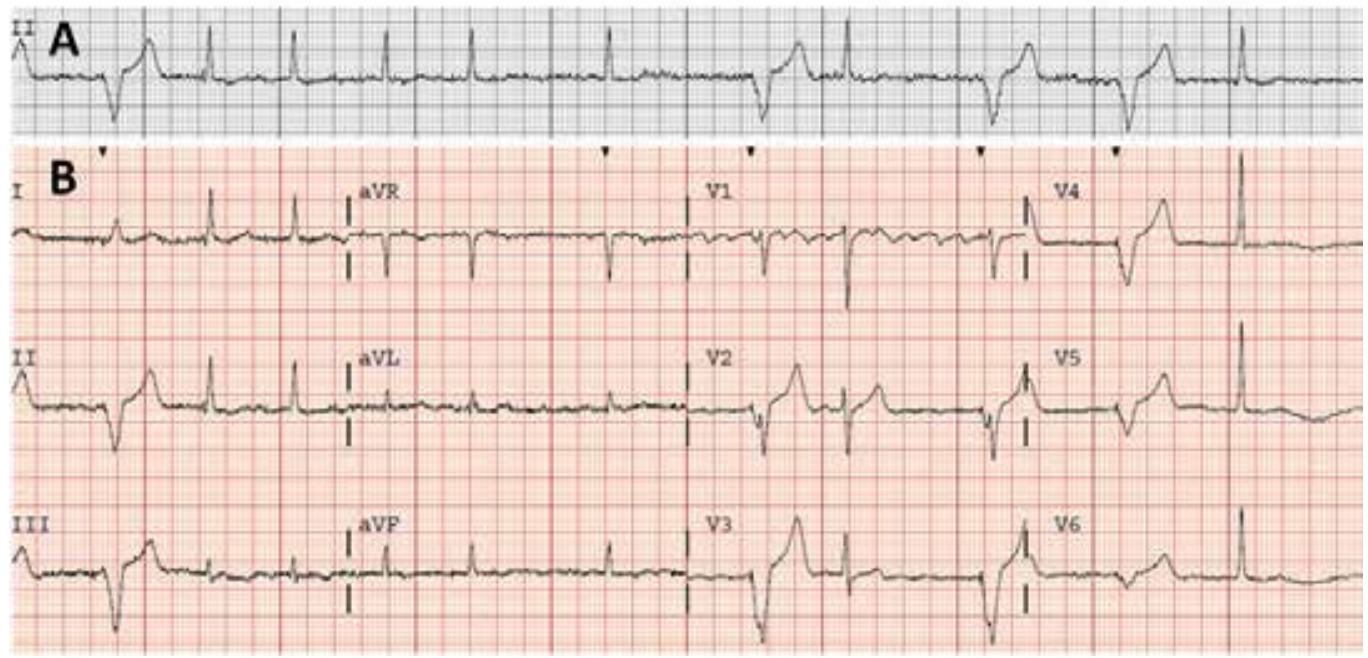

Figure 6. The rhythm strip suggests atrial fibrillation with PVCs or escape beats (A). Simultaneous 12 lead ECG shows evidence of VVI pacing at 60/minute (B). Even when pacing spikes are not visible, wide QRS beats with constant coupling interval, and no R-R cycle longer than this interval should suggest ventricular demand pacing.

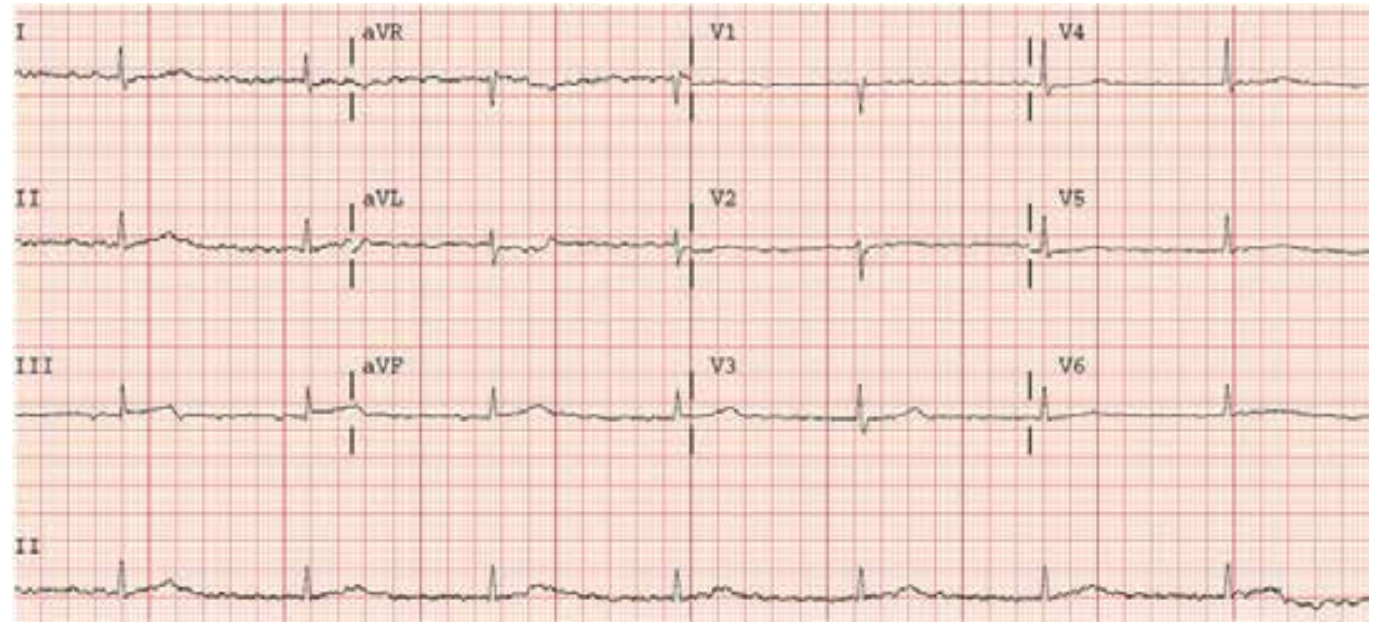

Figure 7. Low amplitude, high frequency artifact masking sinus or ectopic atrial bradycardia. The rhythm may be confused with atrial fibrillation and junctional rhythm, however, P waves can be identified in III and aVF. 


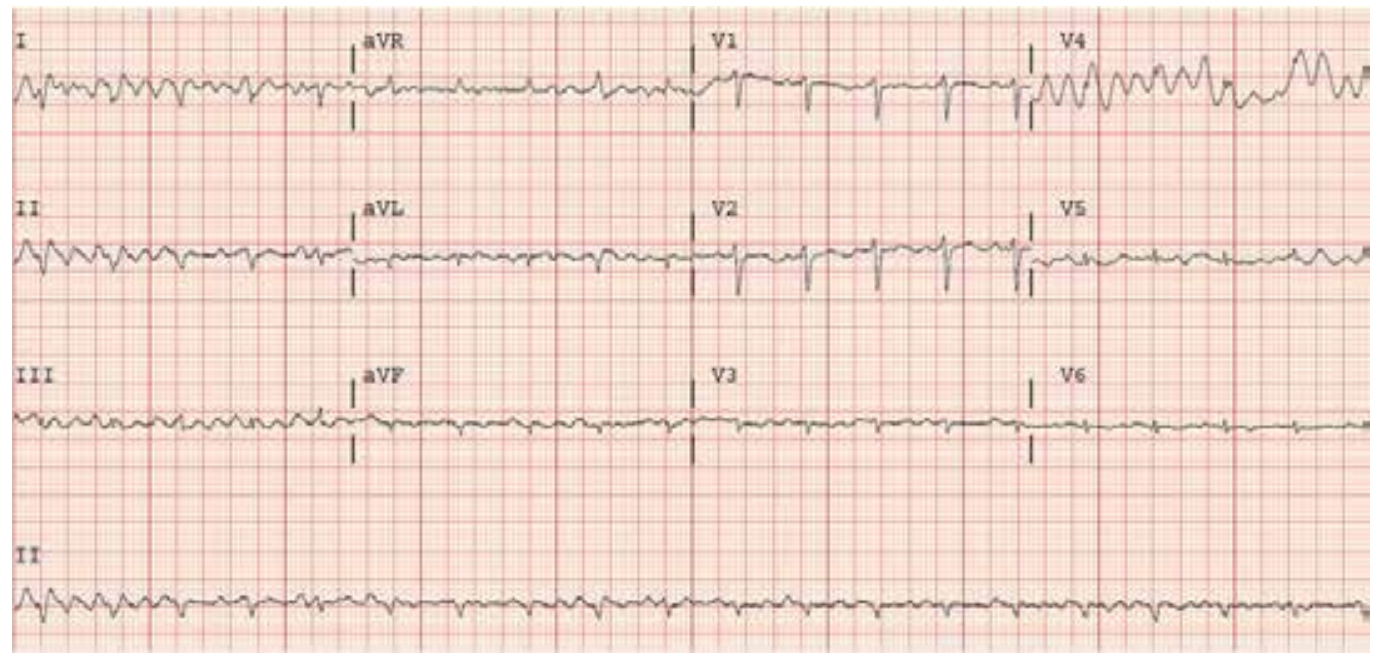

Figure 8. High amplitude artifacts with low ECG voltage may be misinterpreted as atrial flutter of fibrillation. However, sinus tachycardia is easy to recognize in V1 and V2.
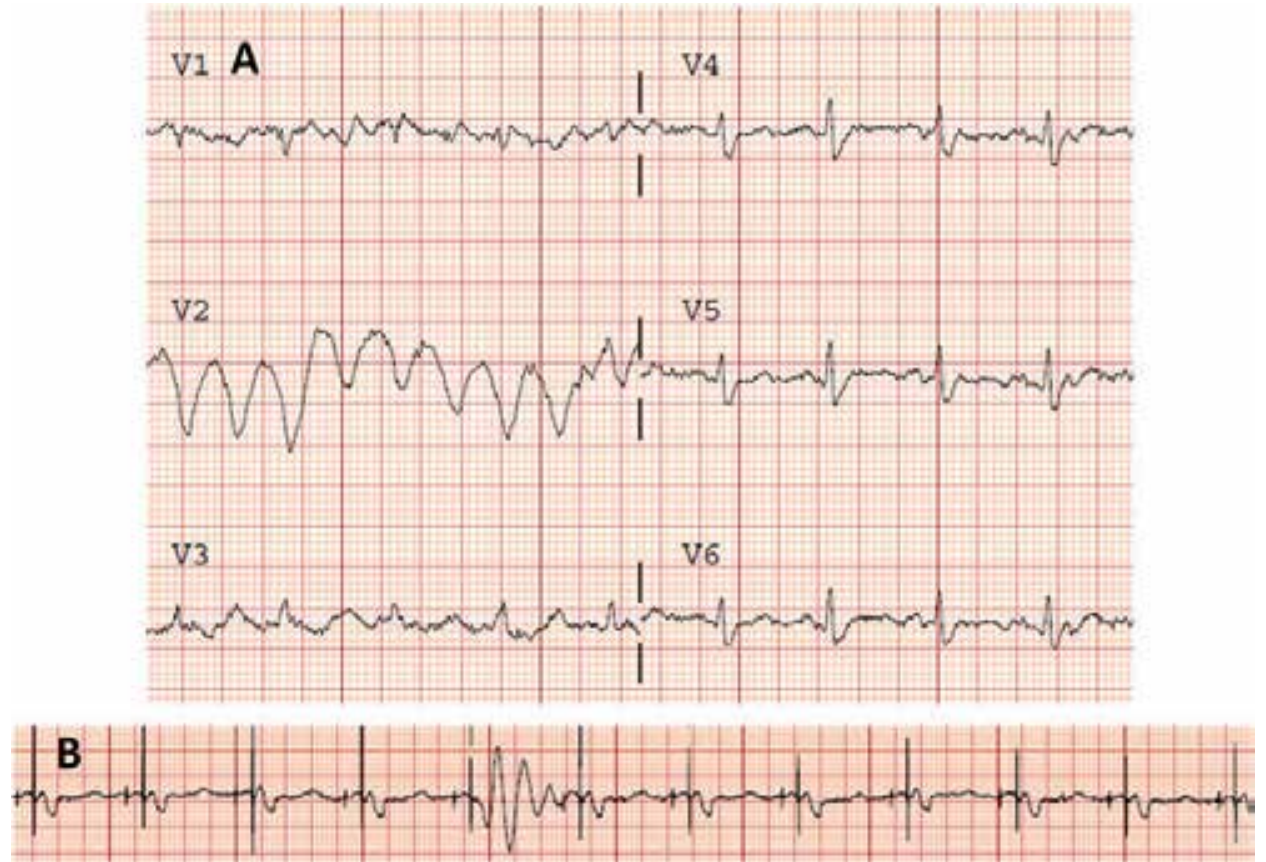

Figure 9. Artifacts suggestive of NSVT. However, the simultaneous V1 and V3, and the following V4-6 leads show that the underlying rhythm is sinus (A). Biventricular pacing is not affected by the artifact this would be unlikely with any true ventricular arrhythmia (B). 


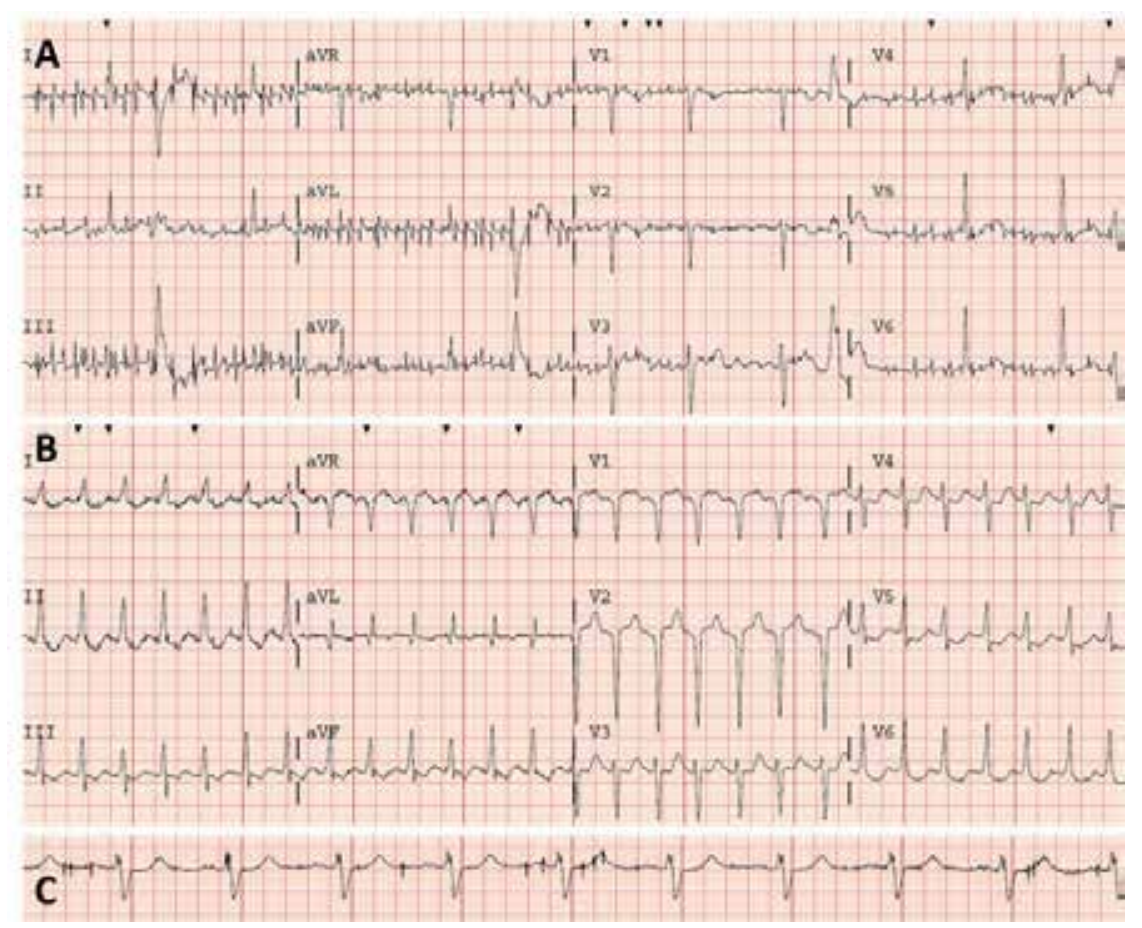

Figure 10. High frequency artifacts causing false detection of pacing in automated ECG device. Although the pacemaker-spike gain and marker functions of the ECG systems may be very helpful to identify small pacing spikes, these systems may be overcalling artifacts. This patient does not have a pacemaker, the ECG improperly identifies some artifacts as pacing (black triangles on top (A). In some cases, the artifacts may be less obvious (B), or may closely resemble pacing spikes (C).

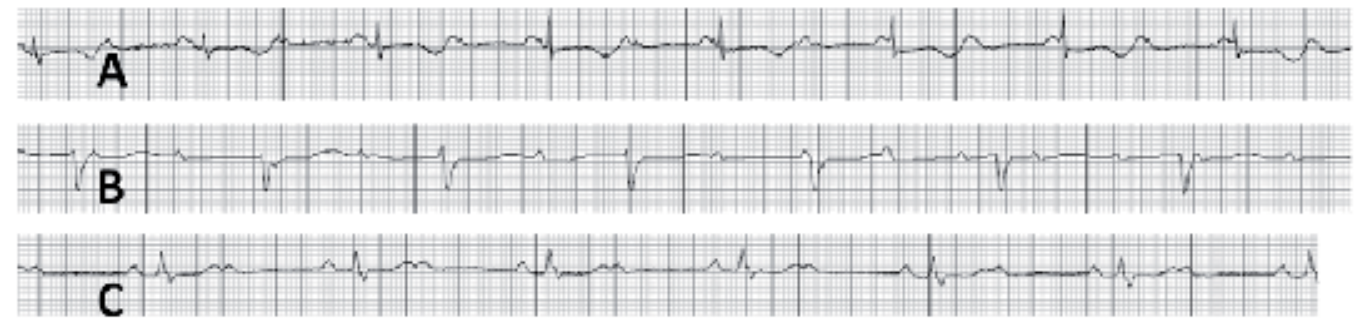

Figure 11. Rhythm strip suggestive of 2:1 AV block (A). However, the QRS complexes are "creeping in" on the preceding $\mathrm{P}$ waves - there is complete AV dissociation, more typical for complete heart block with junctional escape rhythm. A similarly difficult tracing (B), suggestive of first degree AV block. As the atrial rate accelerates, complete AV dissociation becomes apparent. (C) True 2:1 AV block - the PR interval following the conducted $\mathrm{P}$ waves is constant. Note that the P-P interval slightly irregular (shortlong), which may represent ventriculophasic sinus arrhythmia or atrial bigeminy. 


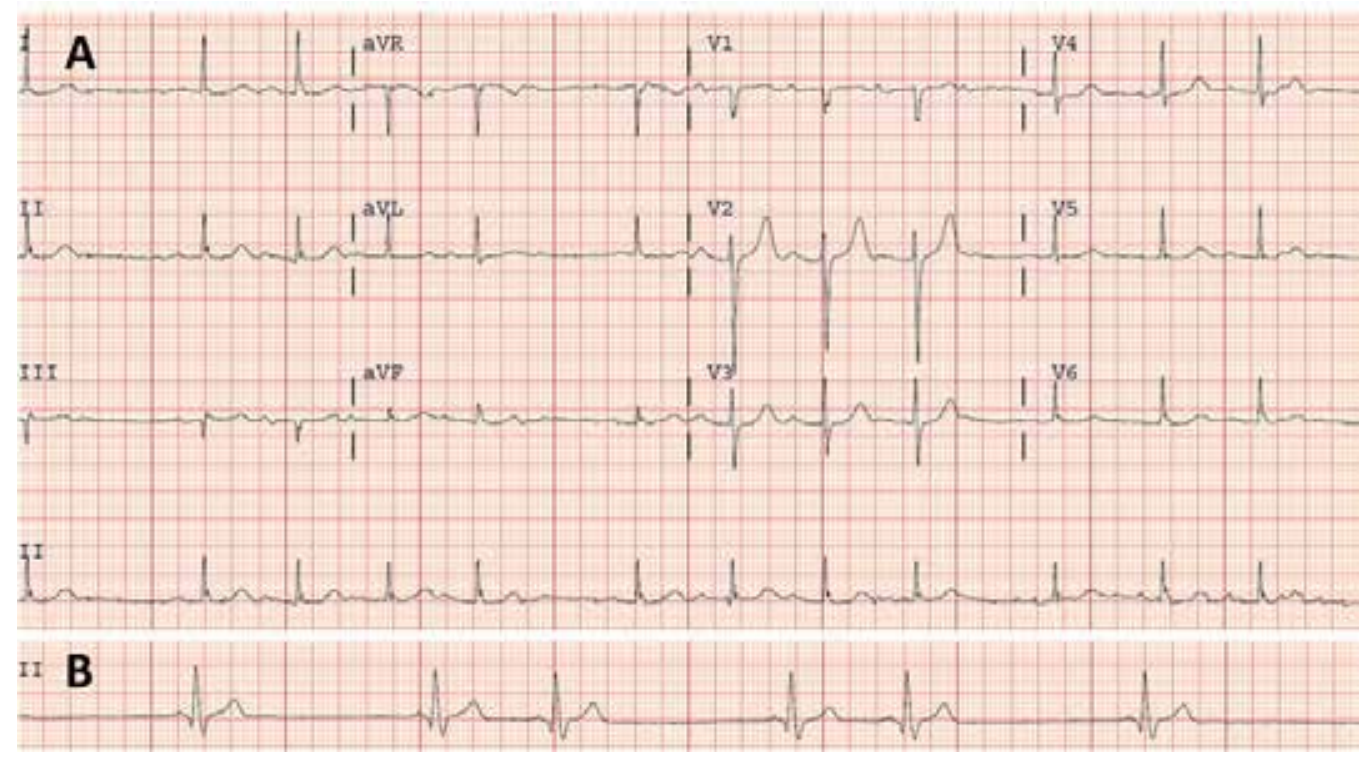

Figure 12. Blocked premature atrial beats (PACs) should be identified as they elicit a different response during atrial pacing (inhibition) than sinus arrest (pacing). In (A), V1 gives the clue for the arrhythmia mechanism - blocked PACs. In (B), there are no visible early P waves - this is 3:2 sinoatrial block.

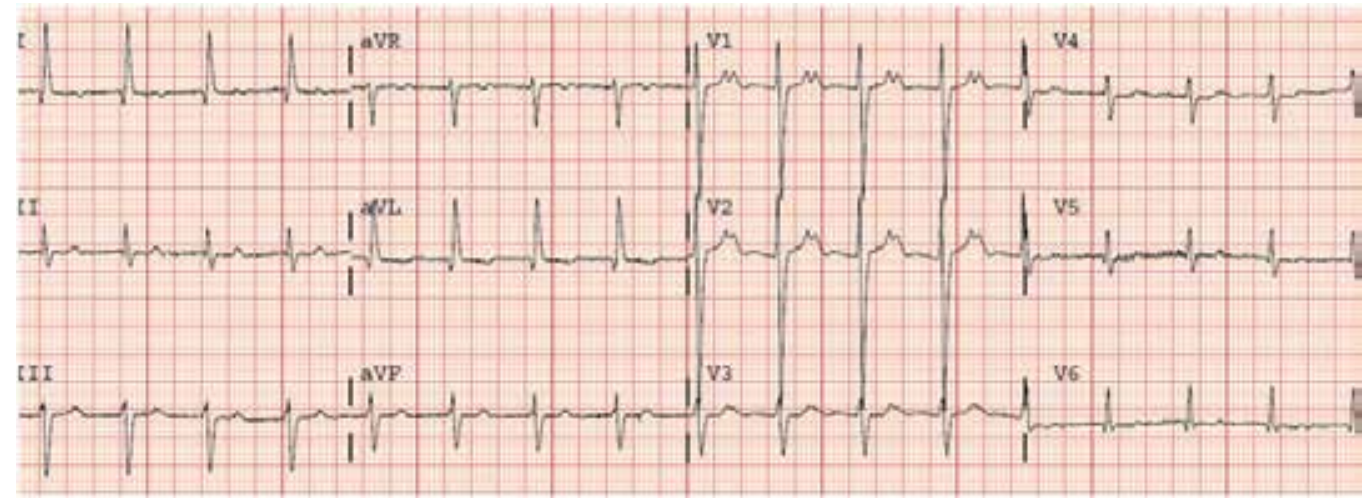

Figure 13. Sinus tachycardia with 1st degree AV block resembling junctional tachycardia. $P$ waves with constant PR interval can be seen in V1-2. 

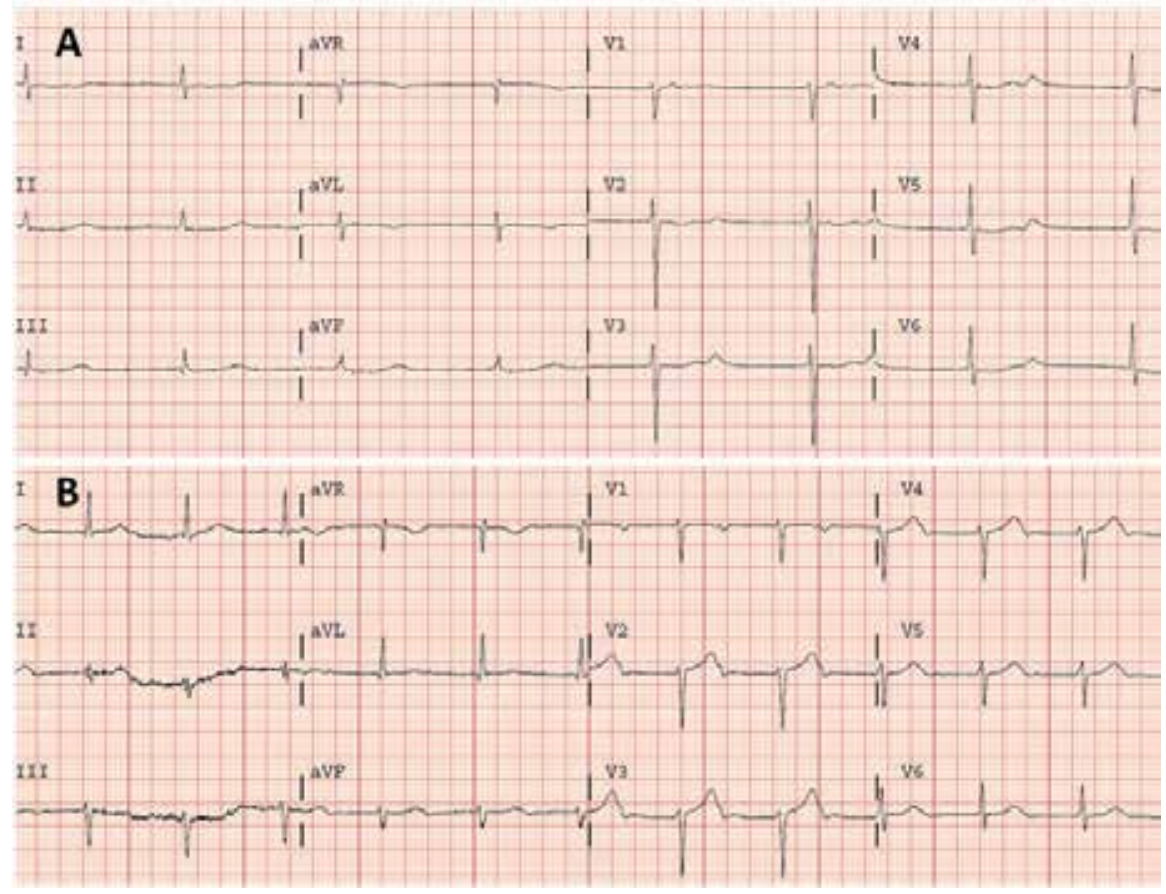

Figure 14. Junctional rhythm with 1:1 VA conduction. Retrograde $P$ waves are visible in V1, which may be misinterpreted as T waves. Note, however, the prolonged QT in all other leads (A). Very long (640 $\mathrm{ms}$ ) first degree AV block may be confused with junctional rhythm, however, P waves are seen in V1 (B).

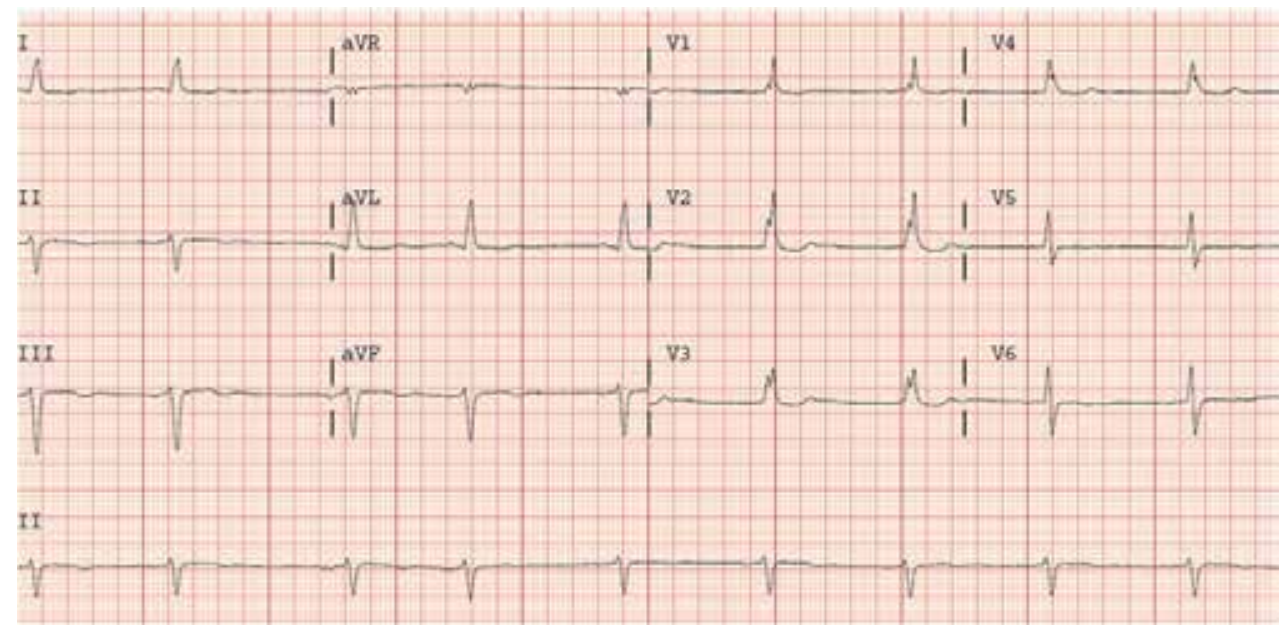

Figure 15. Irregular atrial rhythm resembling atrial fibrillation. The actual rhythm is most likely sinus with PACs, The P waves are of low amplitude, however, they can be identified in III and aVL with 1:1 relationship to QRS and with constant AV delay. 


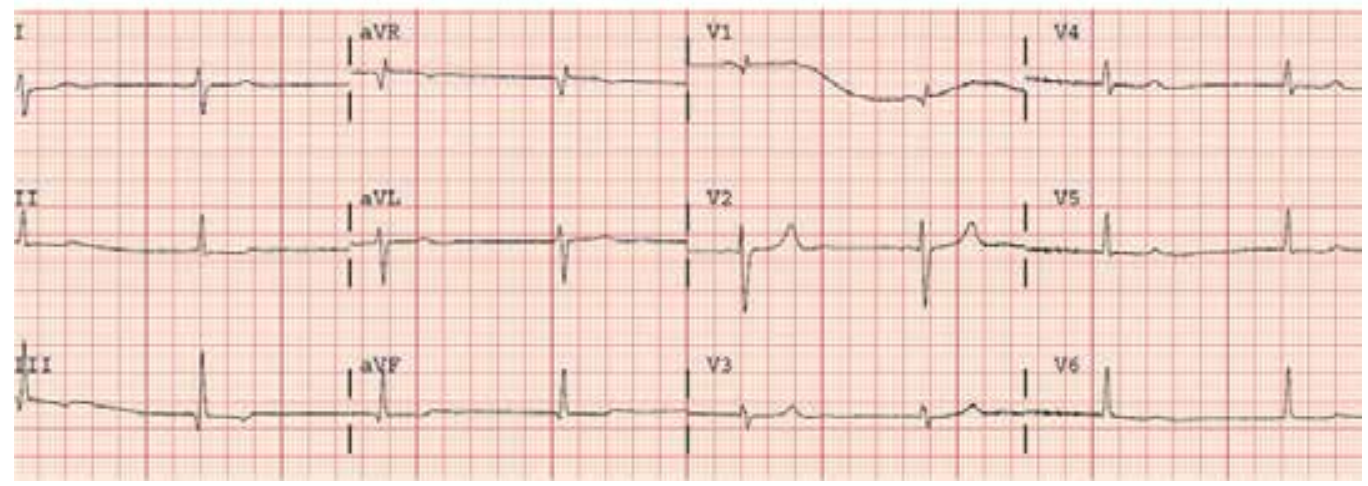

Figure 16. Regular bradycardia with narrow QRS would suggest junctional rhythm, however, $\mathrm{P}$ waves are seen before each QRS in V1 - the driving focus is atrial. Advanced atrial conduction disease is not uncommon in pacemaker recipients, leading to low amplitude, fragmented $\mathrm{P}$ waves.

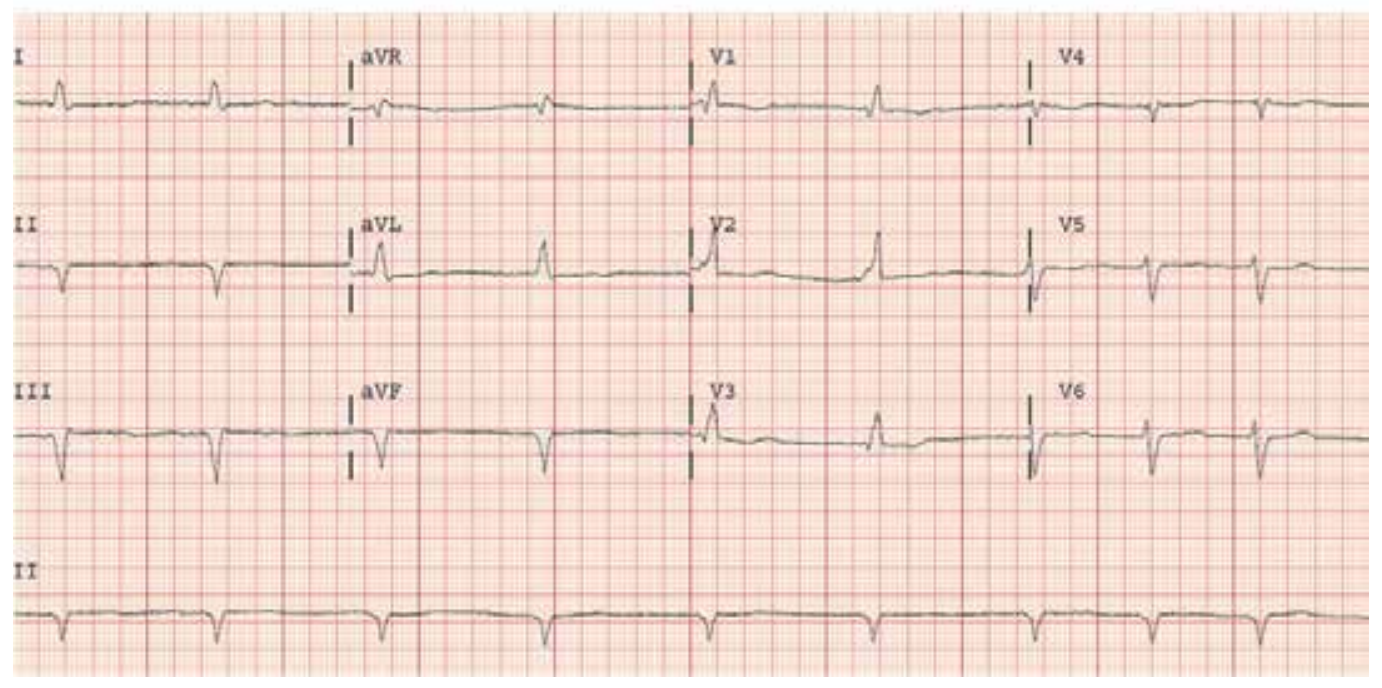

Figure 17. Atrial fibrillation with junctional escape. The regular rhythm may be misinterpreted as pure junctional rhythm, however, the ventricular rate changes when atrioventricular conduction improves and conducted activity overtakes junctional escape. Proper identification of atrial rhythm is important when evaluating pacemaker function - atrial fibrillation should suppress atrial pacing, while atrial pacing should take place with pure junctional rhythm, if an atrial lead is present. 


\section{Unexpected findings with normal device function}

Certain artifacts or interaction of pacemaker algorithms with underlying rhythm may lead to electrocardiographic findings, which may be difficult to distinguish from abnormal function (Balachander, 2011). P/QRS morphology, timing and response to pacing spikes should be addressed, when analyzing the ECG. With ubiquity of bipolar systems, spikes may be difficult to identify (Figure 18). In addition, myocardial depolarization has a vector, which may be isoelectric in certain leads, or may be delayed by intraatrial or intraventricular conduction delay, suggesting ineffective stimulation (Figure 19). Spike morphology may be affected be automatic signal gain function of the ECG system or issues with digital sampling (Figure 20). „Anticipated" spikes may be missing due to very small variations in heart rate, inhibiting demand pacing (Figure 21).

Variable signal morphology may be caused by fusion beats (when the resulting signal morphology is the sum of activation from the pacemaker and spontaneous/conducted activation) or pseudofusion beats (pacing occurs when the myocardium is already refractory from spontaneous/conducted activation, Figures 22-24). Identification of the pacing site is crucial to prove appropriate device function (Figures 25-28).

Occasionally, pacing mode may be difficult to identify based solely on the ECG (Figure 29). It may change due to algorithms trying to minimize right ventricular stimulation (Figures 30-32), rate smoothing function (Figure 33), or arrhythmia - mainly, atrial fibrillation (Israel, 2002).

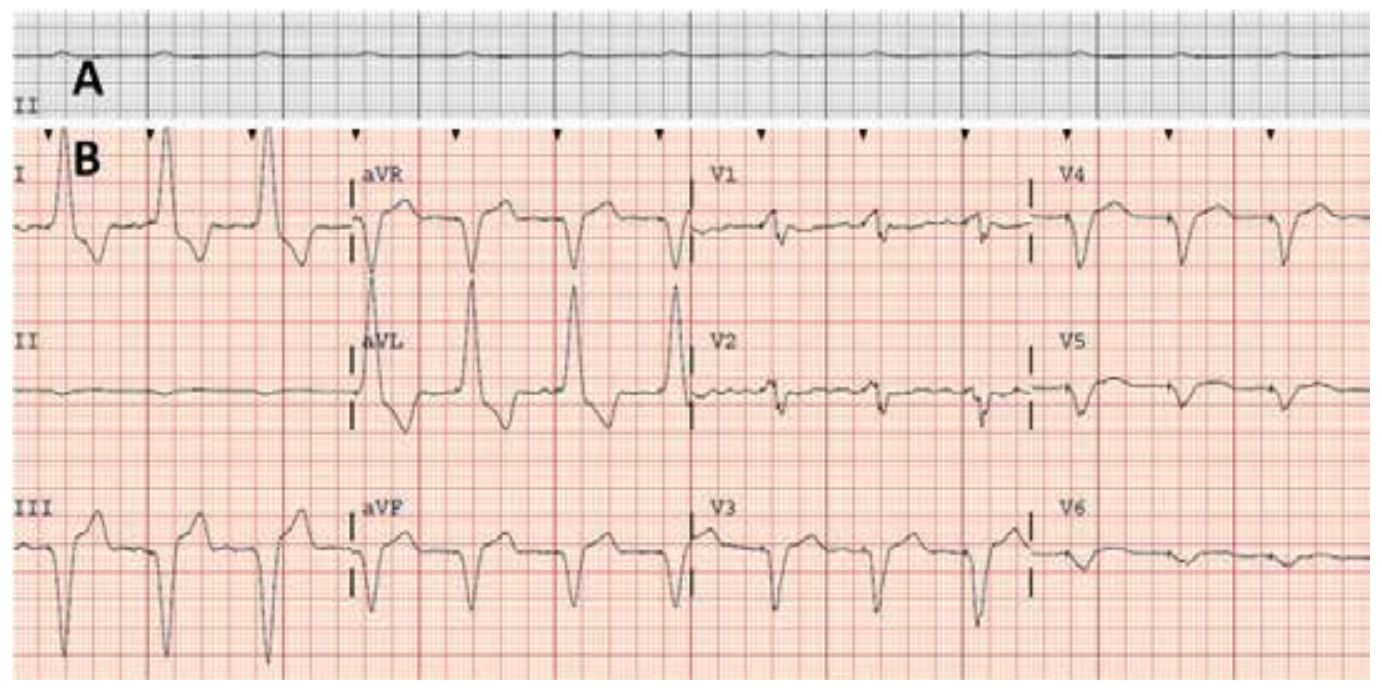

Figure 18. Rhythm strip suggestive of complete heart block and absence of pacing (A). Simultaneous 12 lead ECG shows appropriate ventricular stimulation - the vector of the myocardial activation is close to isoelectric in II. 


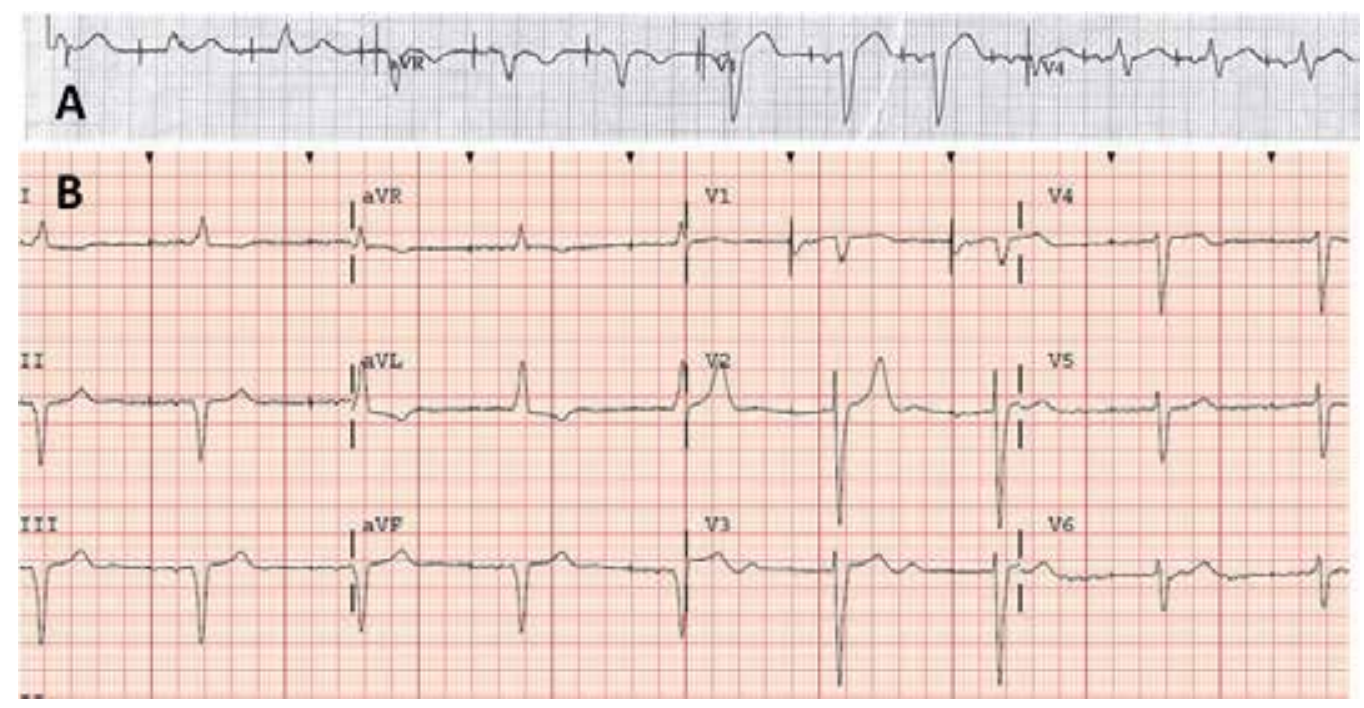

Figure 19. $\mathrm{P}$ waves are not seen in I despite effective atrial pacing - the atrial depolarization vector may be isoelectric in certain leads, depending on the atrial pacing site and pathologic conditions. Note that there is a delay in each lead from the atrial spike to the $P$ wave, suggestive of conduction delay (A). Intra-atrial conduction delay may present even in regions far from the pacing electrode - note instant capture in V1, however, significantly prolonged, fragmented P wave in the frontal leads (200 ms) (B).

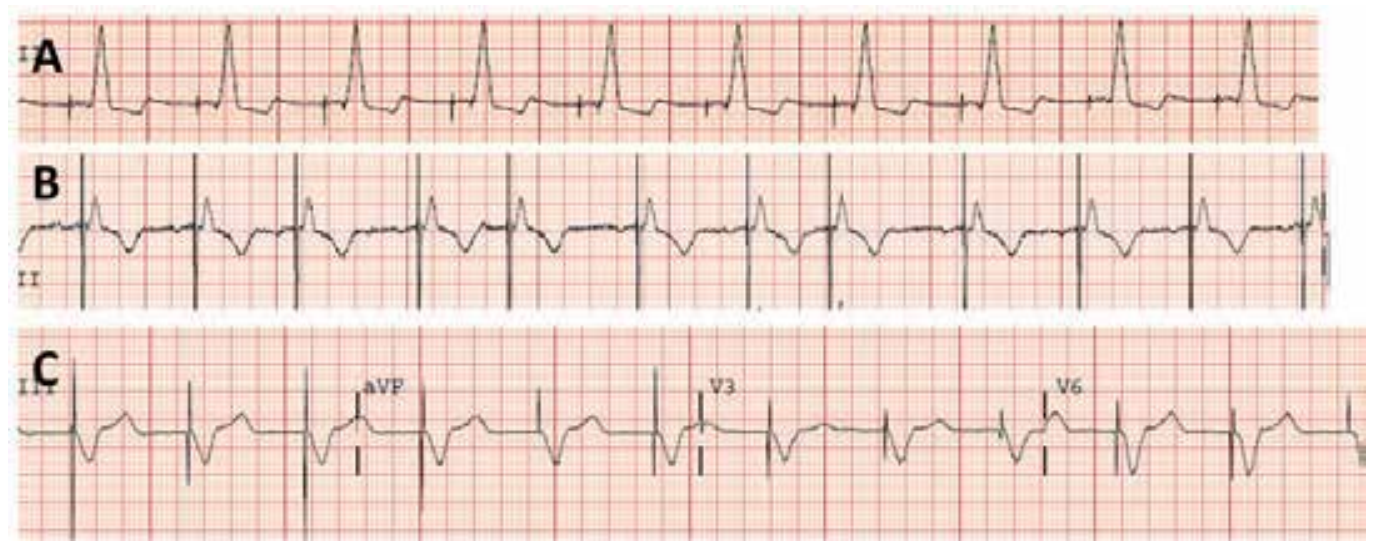

Figure 20. Variable spike morphology (A). This is a normal finding as the spike morphology is affected by the digital sampling of the ECG system and whether it uses pacemaker signal identification/amplification. There is no clinically useful correlation between the spike height and pacing energy. Generally, unipolar pacing (B) leads to much higher amplitude signals than bipolar (A). Spike height may vary not just between different ECG leads, but even with each beat (C). 


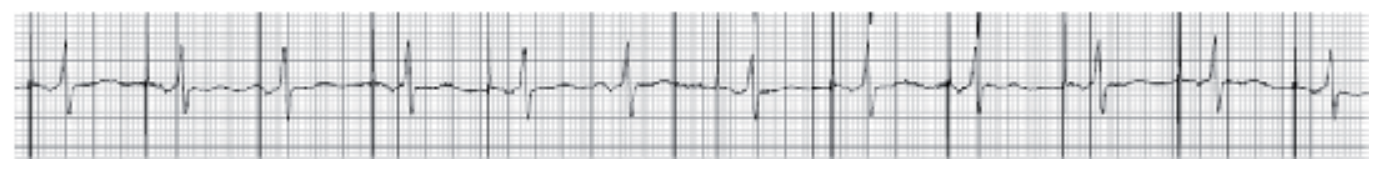

Figure 21. Sinus rhythm competing with AAI pacemaker - there is appropriate inhibition of atrial pacing when the P-P interval is shorter than the basic pacing cycle length.

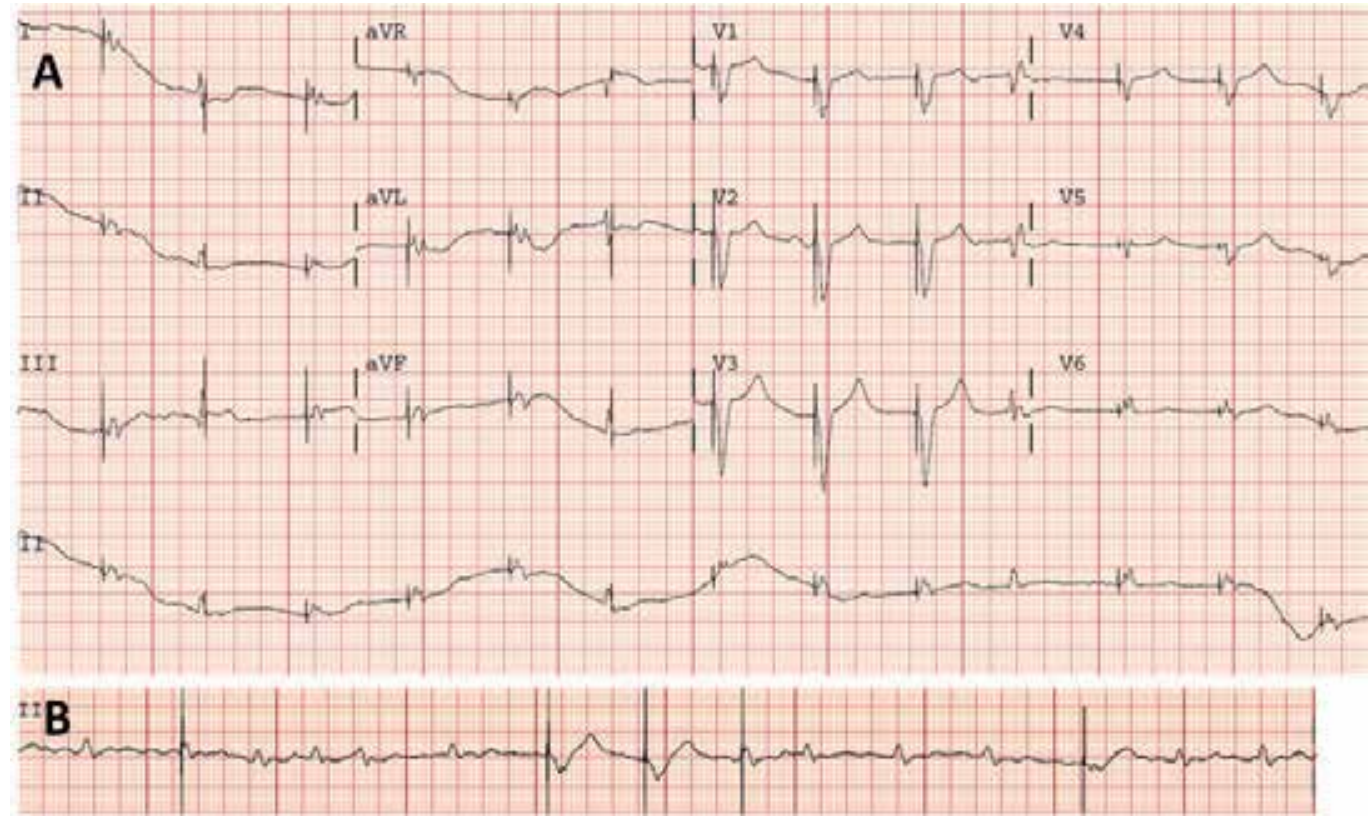

Figure 22. Pseudofusion beats during ventricular stimulation - this is a normal phenomenon as detection of ventricular activation is delayed due to lead tip position (usually right ventricular apex the ventricles may be partially depolarized, when signal is sensed in this region). Beats 2 and 6 show pseudofusion, ventricular pacing is delivered after the ventricles are depolarized and refractory to further stimuli. Beat 10 is sensed appropriately and pacing is inhibited, as the coupling interval is somewhat shorter - this makes ventricular undersensing very unlikely as the cause for pseudofusion (A). Fusion and pseudofusion beats are very common during atrial fibrillation due to the wide range of coupling intervals (B). 


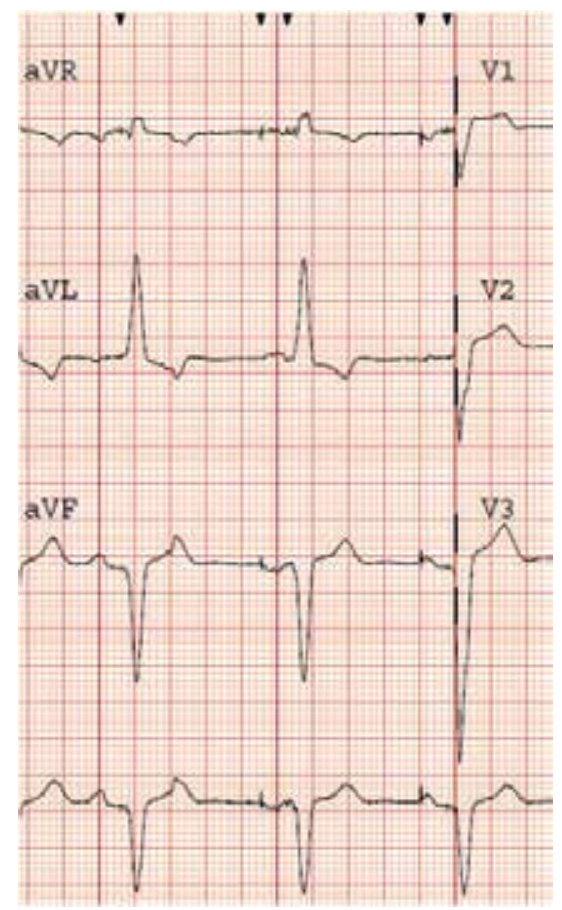

Figure 23. Fusion can be also encountered in the atria, although may be more difficult to identify due to lower signal amplitudes. Undersensing of PACs should be excluded and may require longer tracings or device interrogation.

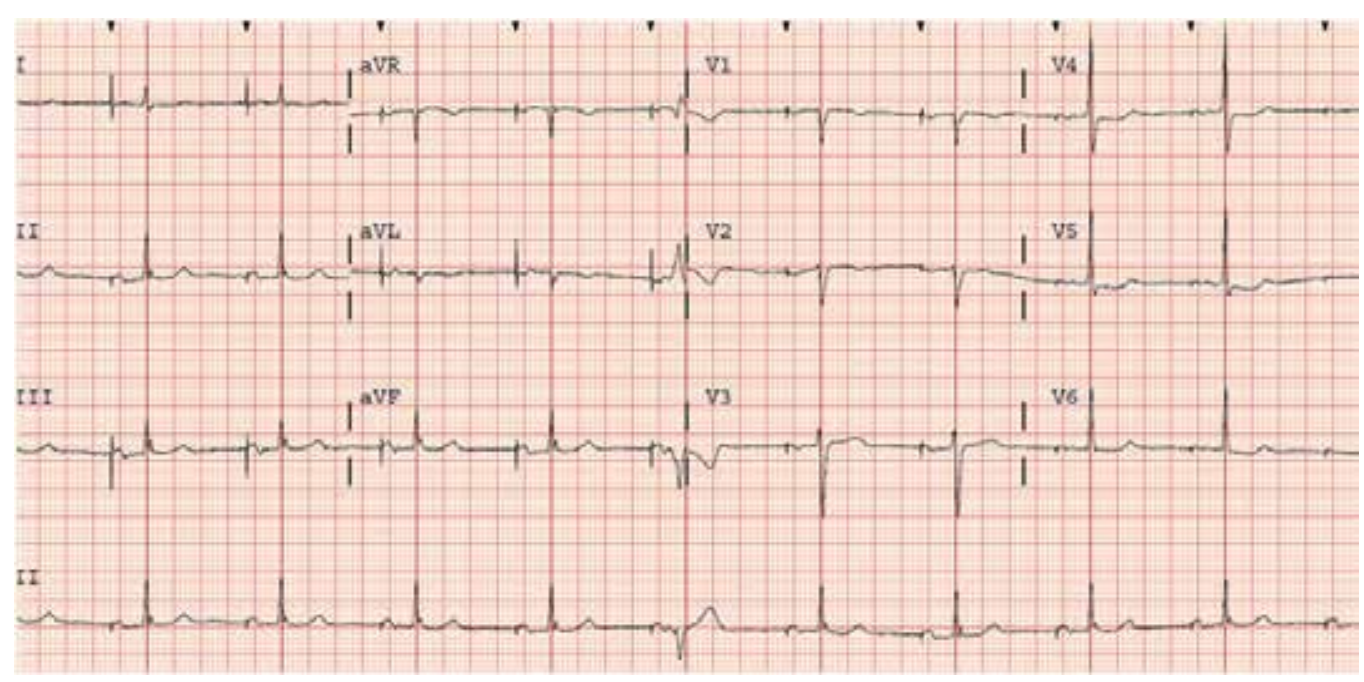

Figure 24. Wide QRS beat encountered during regular atrial pacing with short PR interval. This is most likely a premature ventricular contraction (PVC), fusing with the atrial paced, spontaneously conducted beat. 


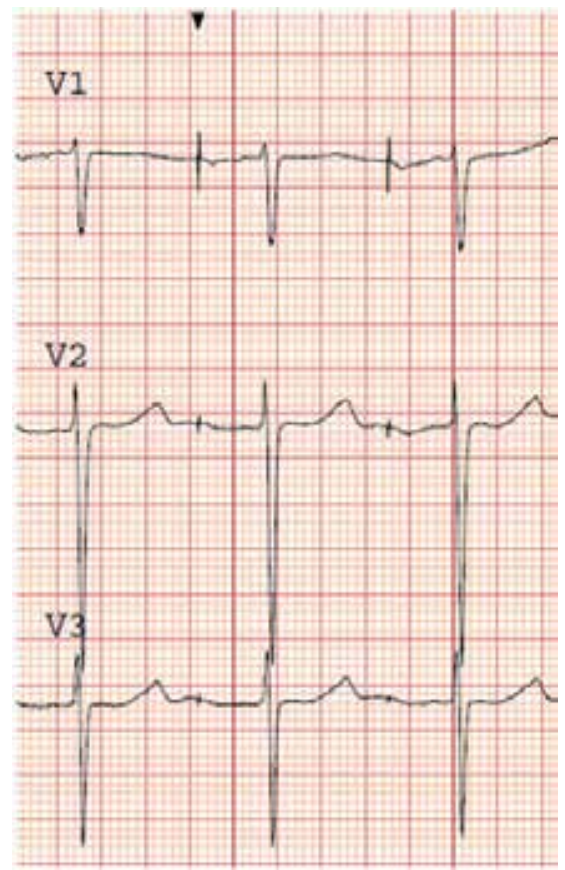

Figure 25. Pacing spikes fall into the $\mathrm{U}$ waves in V2 and V3 and are not followed by apparent capture. However, in V1 atrial capture is clear.

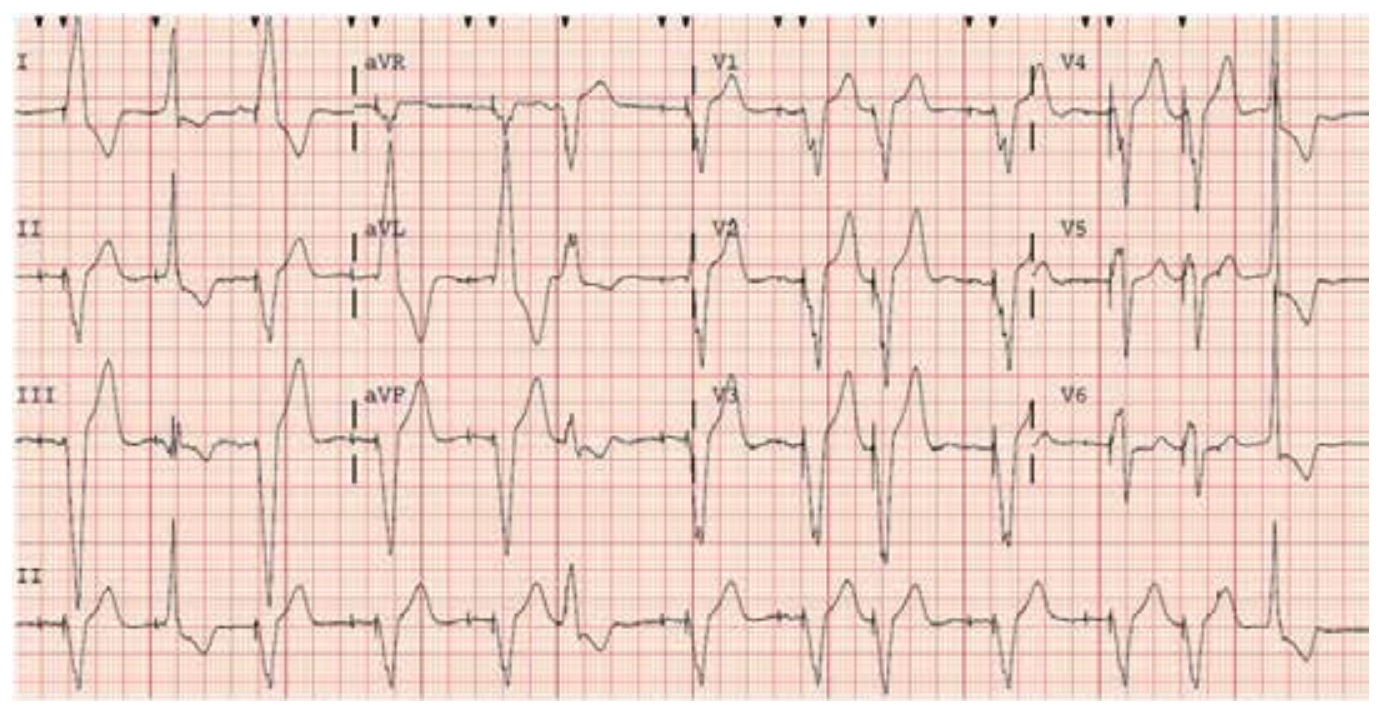

Figure 26. Pseudo pseudofusion - a spike appears immediately before (3rd spike) or within a QRS (9th spike). However, these are atrial spikes and the tracing represents appropriate DDD pacemaker response to frequent premature ventricular and atrial beats. Origin of pacing spikes should be identified based on their timing and sequence to avoid misinterpretation as undersensing or ineffective capture. 


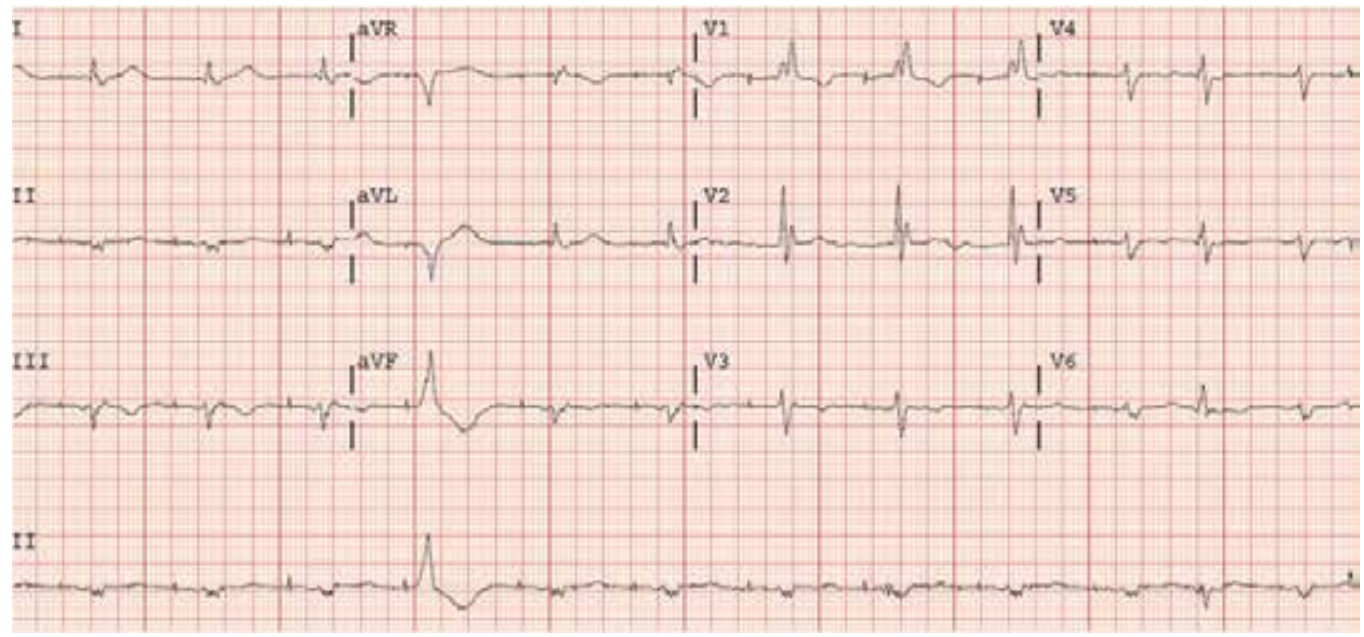

Figure 27. Identification of pacing site is important to avoid misinterpretation. The $4^{\text {th }}$ spike seems to be non-capturing and is followed by a wide QRS beat with an $80 \mathrm{~ms}$ delay. However, this is an atrial stimulus as it is apparent by reviewing the consecutive beats. The wide QRS beat is a PVC, which does not have any correlation with atrial pacing. The $\mathrm{P}$ waves are of low amplitude and difficult to identify, however, regular atrial pacing followed by regular ventricular activation with the same atrioventricular delay suggests consistent atrial capture.

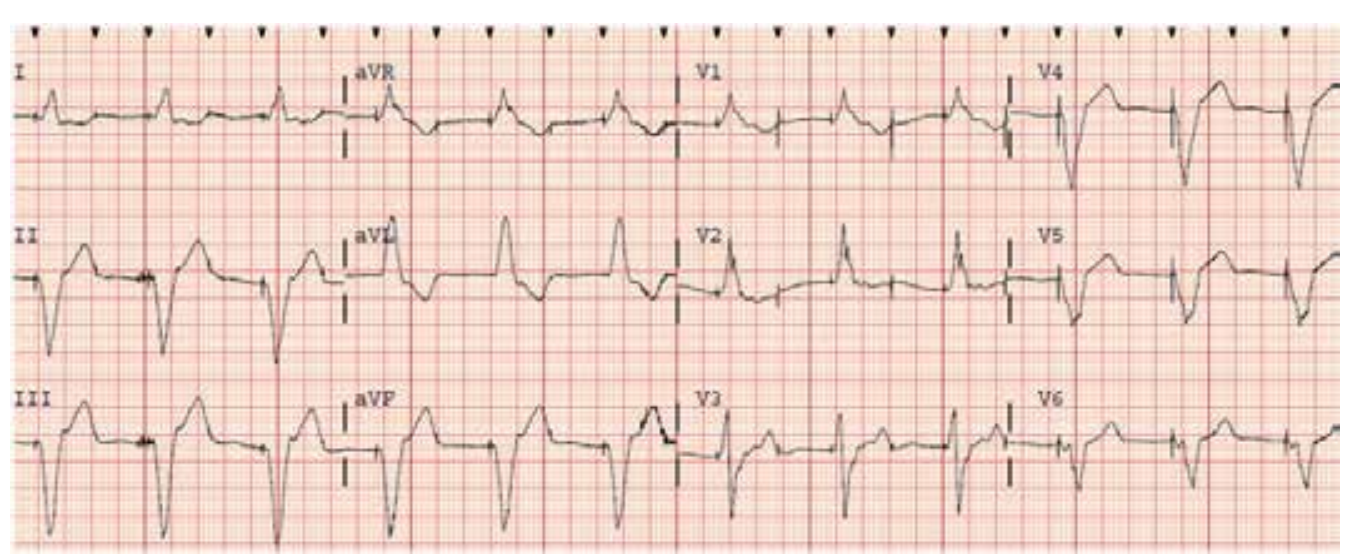

Figure 28. High frequency pacing may occasionally be a sign of serious pacemaker malfunction (runaway pacemaker) or appropriate response to an arrhythmia (tracked sinus/atrial tachycardia). In dual chamber systems, proper identification of pacing spikes is necessary for troubleshooting. In this tracing, 150/minute pacing seems to be capturing 2:1. Note, however, that the pacing is regularly irregular (short-long) and the spike morphology is alternating in V1 - every other one is an atrial spike. The patient is in atrial fibrillation, which is undersensed and the DDD pacemaker is delivering dual chamber stimulation at 70/minute with an AV delay of $400 \mathrm{~ms}$. 


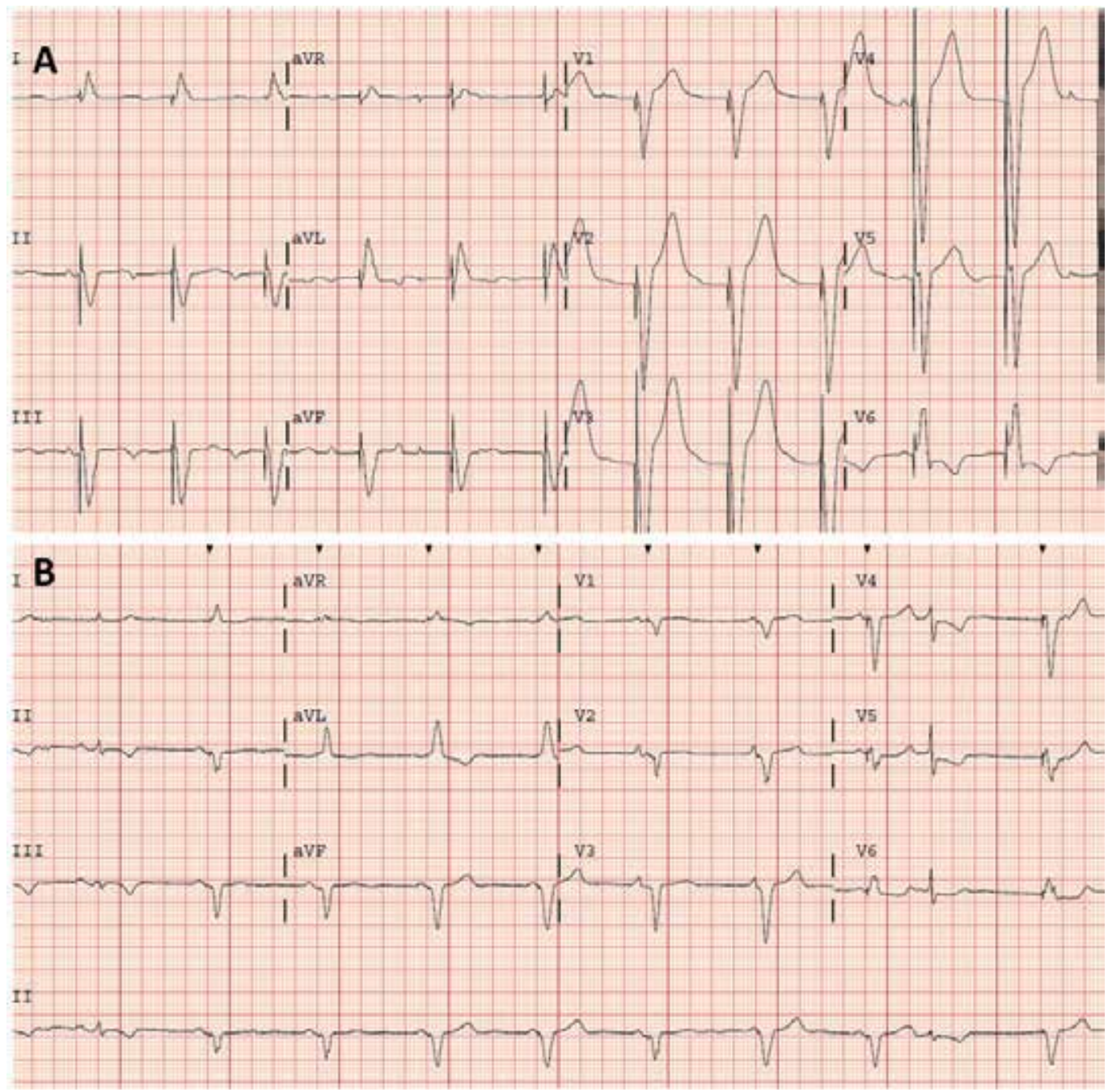

Figure 29. Pacing mode may be difficult to identify from surface ECG. (A) In III and aVF it may appear that the atrial activity is tracked to the ventricles. However, the ventricular rate is completely regular despite variable P-P intervals. AV dissociation is seen in V4 and V5. This device is a VVI pacemaker in a patient with complete heart block. (B) Isorhythmic dissociation between sinus rhythm and VVI pacing close inspection of the PR intervals reveals that the atrial activity is not tracked

If pacing spikes are seen during tachycardia, most common causes are atrial tachyarrhythmia tracked by the pacemaker, or true PM mediated tachycardia (caused by retrograde conduction or atrial oversensing of ventricular events, leading to endless loop tachycardia). Rate response function may also cause transient increase in pacing rate. The differential is usually difficult based on surface ECG alone, unless initiation and termination can be clearly identified. Device interrogation is strongly recommended (Ip, 2011). Transient changes in rhythm may elucidate the mechanism of a suspected malfunction (Figure 34). 
Both atrial and ventricular tachyarrhythmias may raise the concern of device malfunction. If no spikes are seen, the rhythm is likely not related to pacing and patient-related issues should be suspected (Figures 35-38). Underlying rhythm should be identified: atrial fibrillation/flutter may be difficult to recognize with asynchronous pacing, but still pose a thromboembolic risk (Figures 39-40).

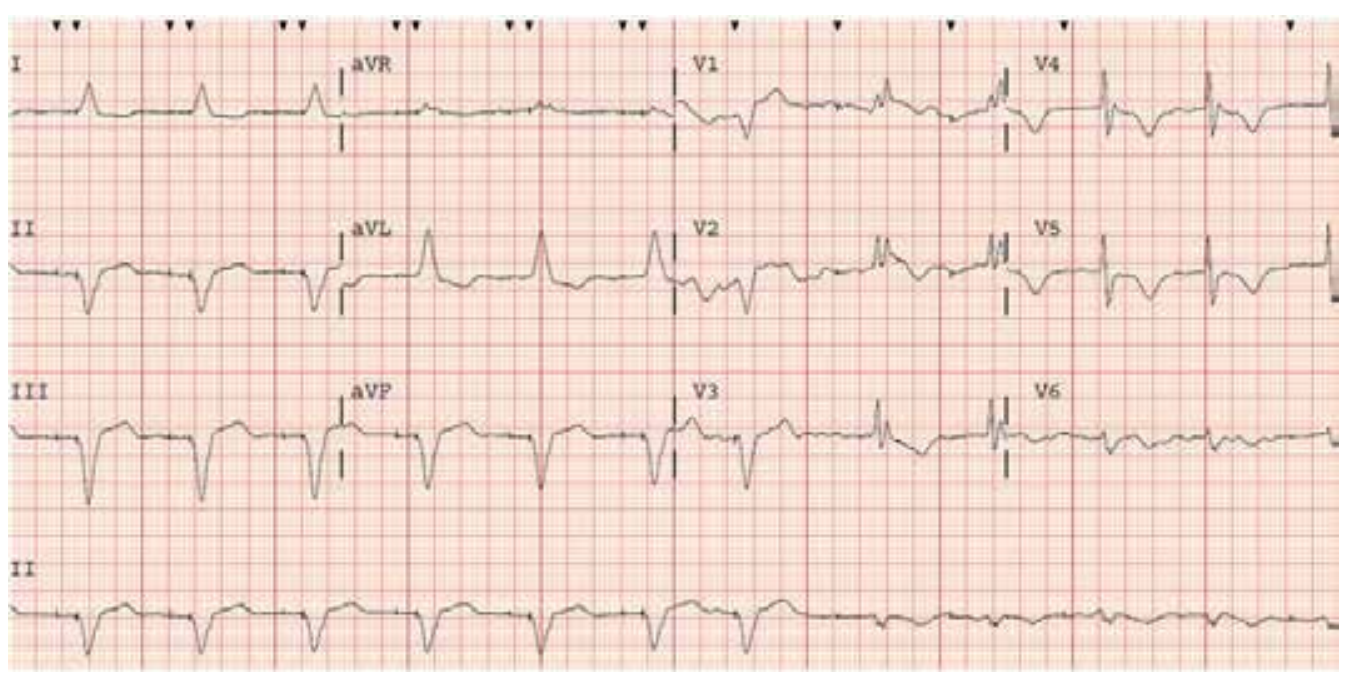

Figure 30. Response to a premature atrial beat with managed ventricular pacing algorithm (Medtronic, Inc). The DDD pacemaker is delivering atrioventricular stimulation, then an atrial-sensed ventricular pace following a premature atrial beat. Following this beat, an atrial stimulus is delivered with mode switch to ADI. As atrioventricular conduction is detected, the device continues with atrial stimulation and allows spontaneous conduction with prolonged AV delay.

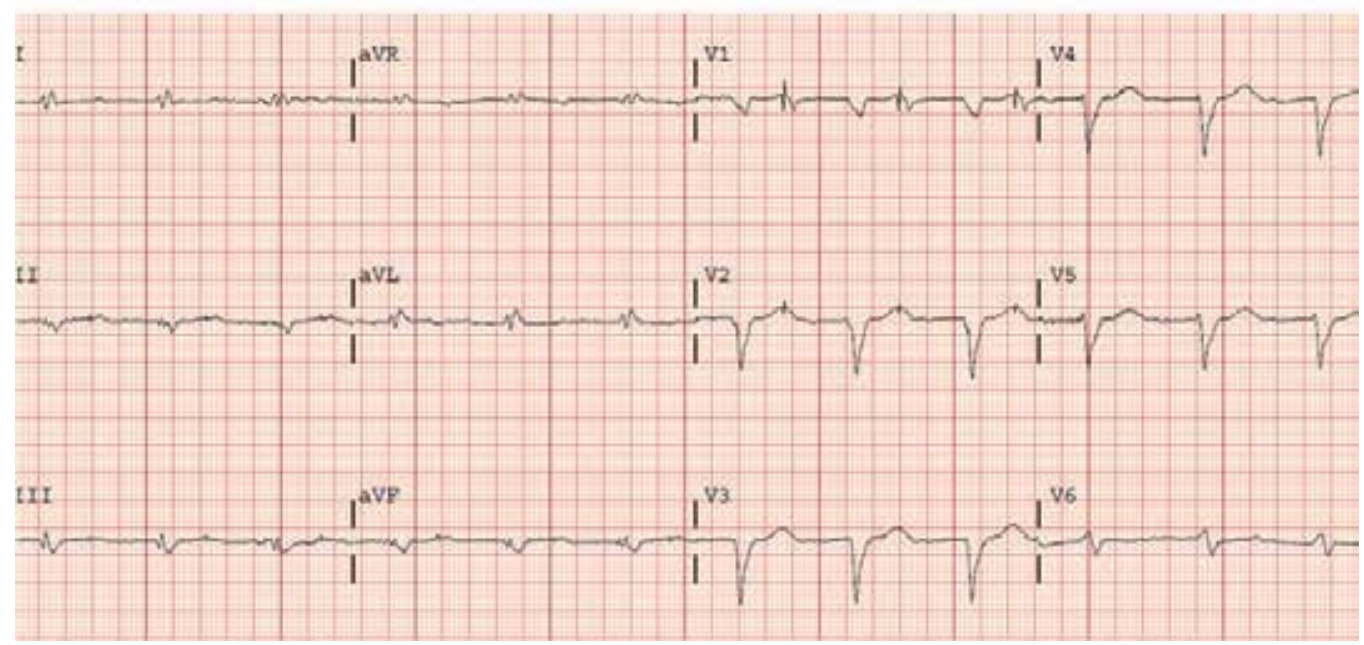

Figure 31. Managed ventricular pacing may maintain very long AV interval, if the 1:1 atrial:ventricular ratio is maintained. In this case, atrial pacing spikes occur after the QRS, in the T waves. V1 shows atrial capture with an AV delay of $460 \mathrm{~ms}$. 


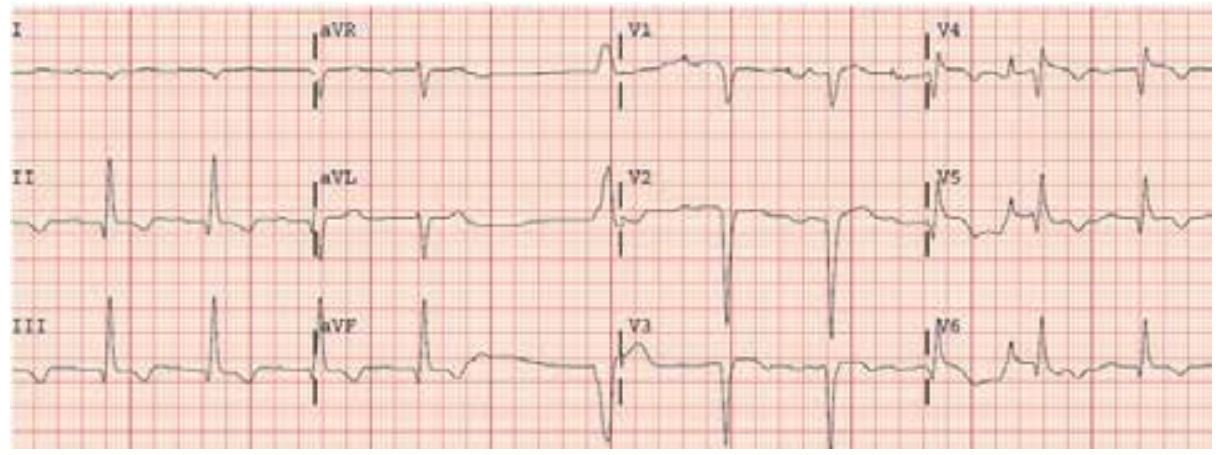

Figure 32. Heart rate may drop down to $\approx 50 \%$ of the basic rate for one cycle with managed ventricular pacing - in this case a late blocked PAC is followed by an atrial stimulus, followed by a ventricular stimulus with very short AV delay (wide QRS beat, the spikes are not visualized in these leads). V1 gives the clue that the rhythm is paced at 70/minute. The artifact in V4-6 is not related to pacing.

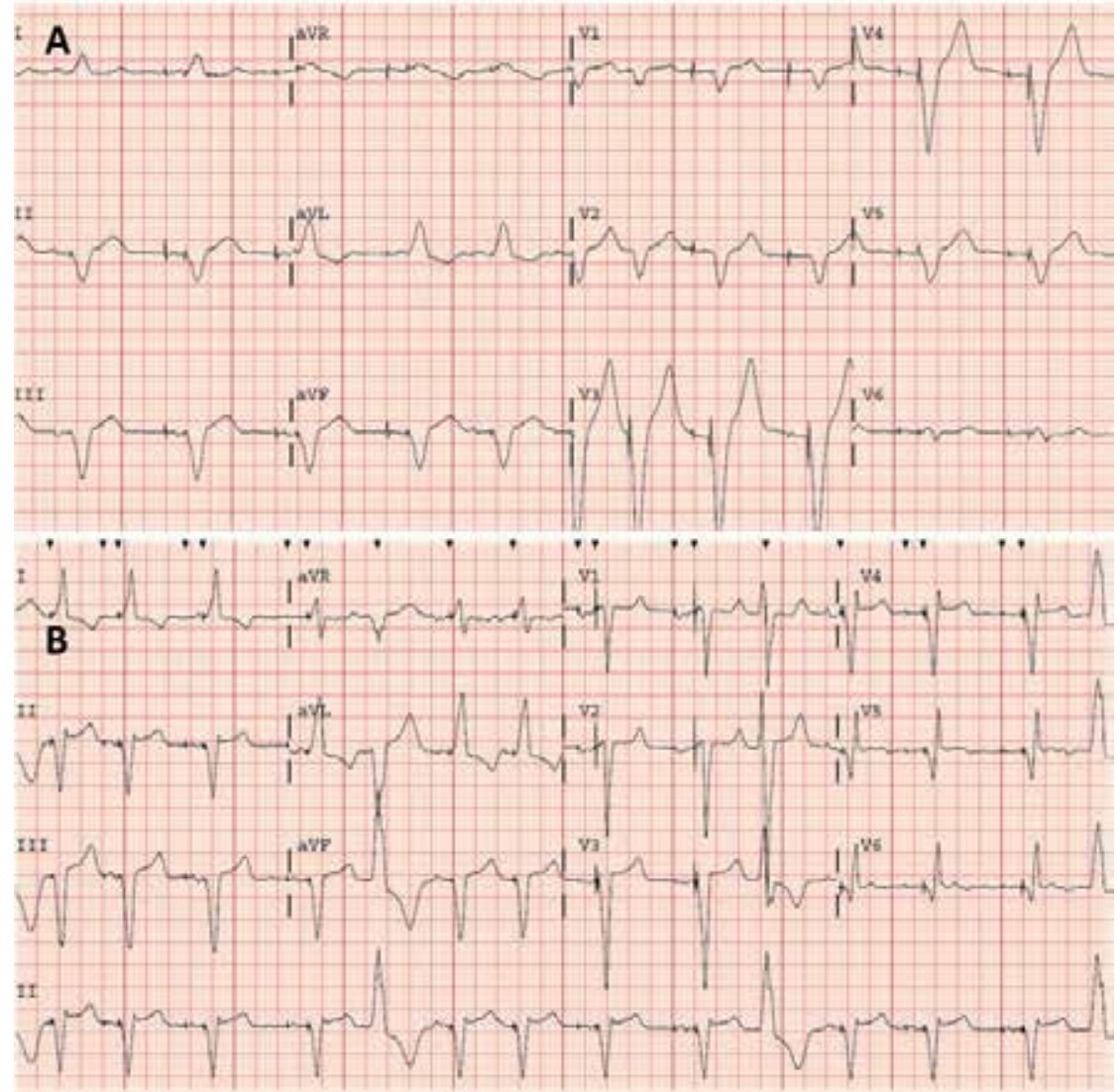

Figure 33. Rate smoothing with a DDD pacemaker (A). Following the premature beats, the atrial pacing rate is gradually decreased to the basic pacing rate. Irregular pacing caused by rate smoothing in a biventricular system (B). All premature beats are sensed (ventricular and atrial), and either a sense response pace or an tracked biventricular pace is delivered. The basic pacing rate is gradually decreased after these over a few cycles, the lowest pacing rate on this tracing is $65 /$ minute. 


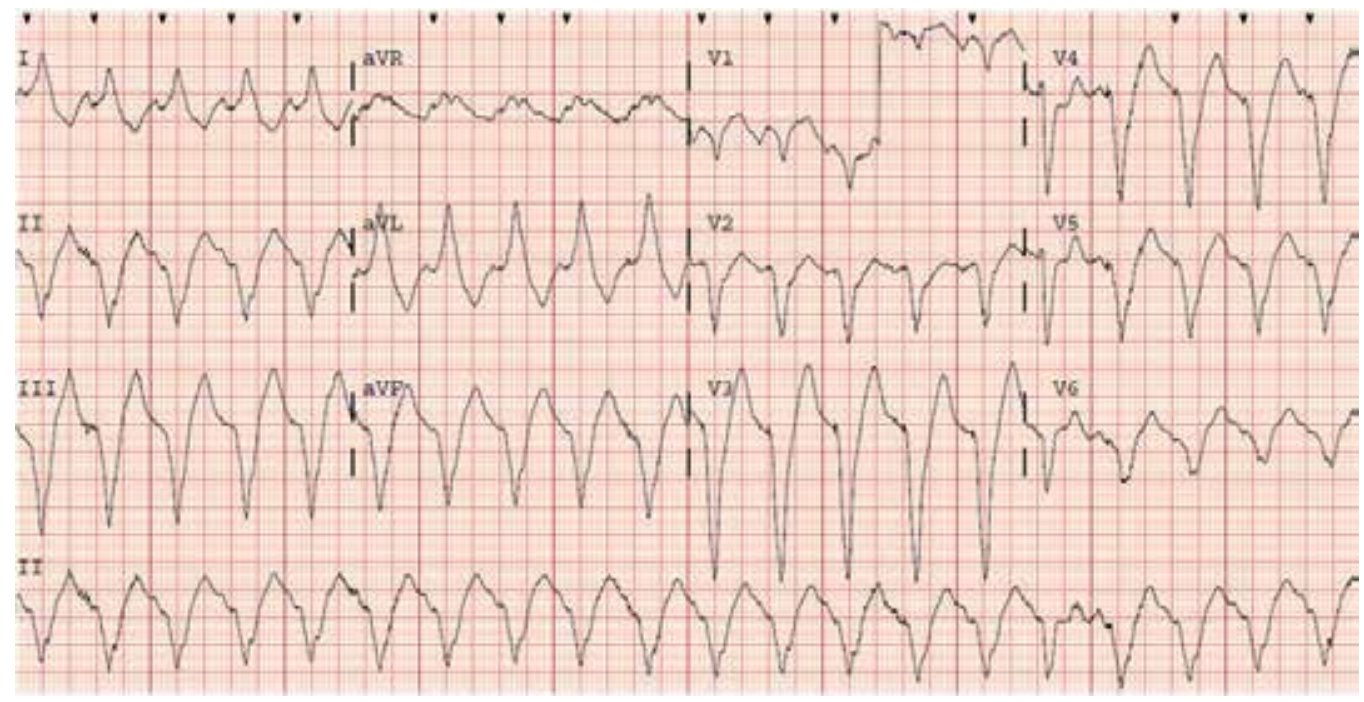

Figure 34. Sudden changes in regular tachycardia may elucidate the mechanism. The premature beat unmasks a $P$ wave, followed by a ventricular paced beat - this is a supraventricular tachycardia tracked by the dual chamber pacemaker.

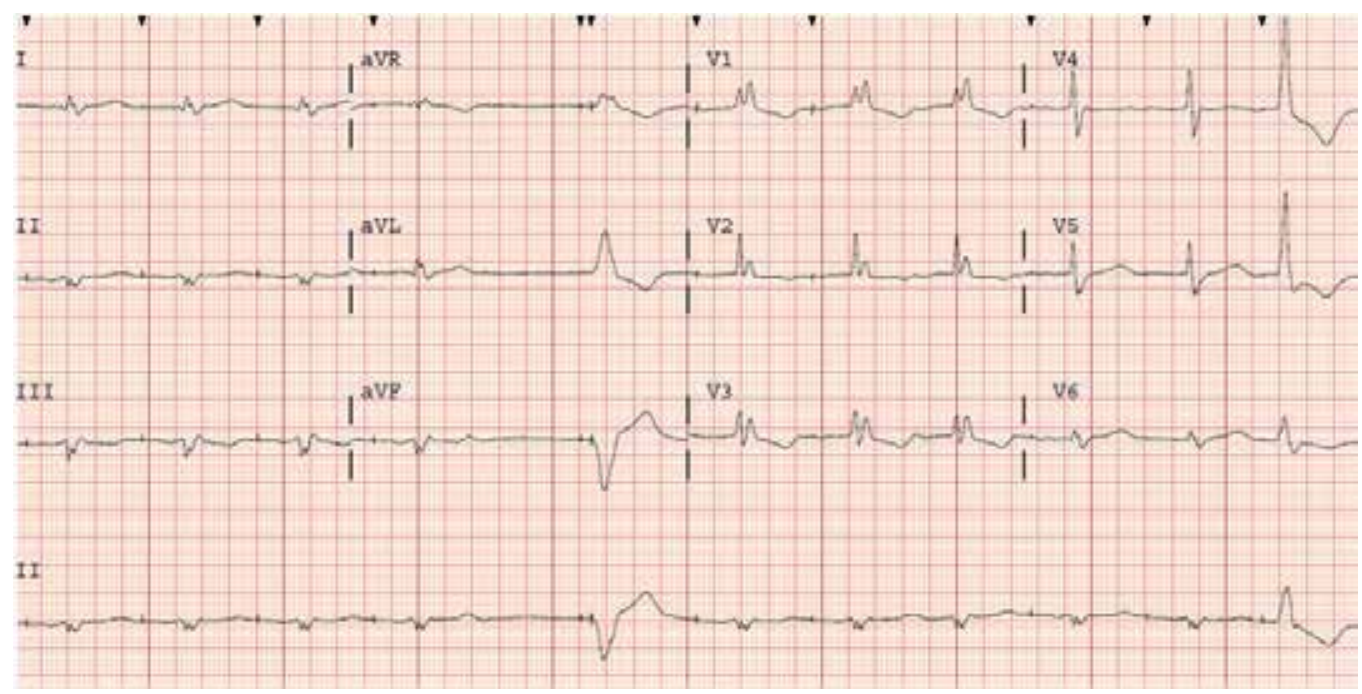

Figure 35. Hidden premature atrial beat mimics pacemaker malfunction. The $5^{\text {th }}$ atrial spike is delayed, suggestive of oversensing, however, the $4^{\text {th }} \mathrm{T}$ wave in the rhythm strip is different from the previous three, suggestive of a buried premature atrial beat, with AV block. The $5^{\text {th }}$ atrial spike actually comes right on time as the pacing cycle was reset by the premature atrial beat. Additionally, a ventricular pace is delivered by the managed ventricular pacing algorithm. 


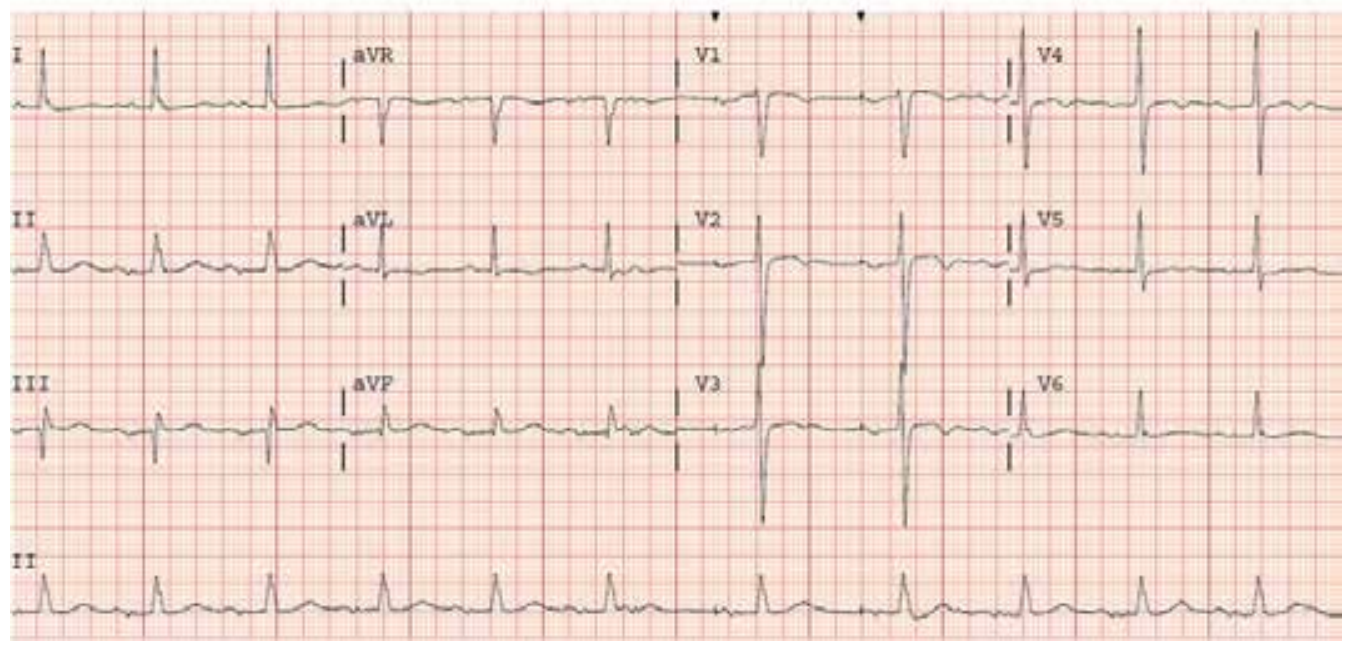

Figure 36. Blocked PAC without tracking with a DDD pacemaker. This is normal function, as the blocked PAC (after the $6^{\text {th }}$ QRS) comes very early and was sensed in the post-ventricular atrial refractory period. Instead, an atrial stimulus is delivered later to maintain the basic rate. As there is spontaneous AV conduction, ventricular pacing is inhibited. After 2 atrial paced beats, sinus rhythm takes over again.
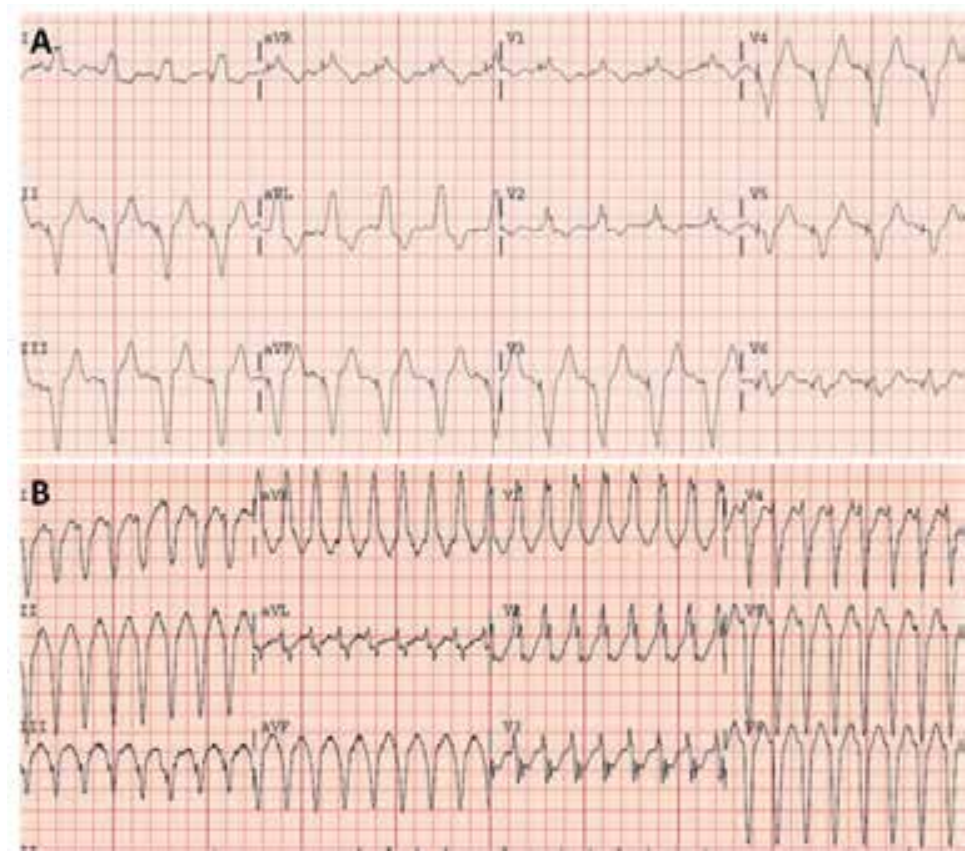

Figure 37. Wide QRS tachycardia in pacemaker recipients. Sinus tachycardia with appropriate sensing and ventricular pacing (A). With bipolar pacing the spikes may not be visible in all leads and the rhythm may be misinterpreted as ventricular tachycardia - especially in telemetry tracings. When ventricular rate is higher than the upper tracking rate (if known) or the QRS morphology is not compatible with usual pacing sites, VT (B) or SVT with aberrancy should be suspected. 


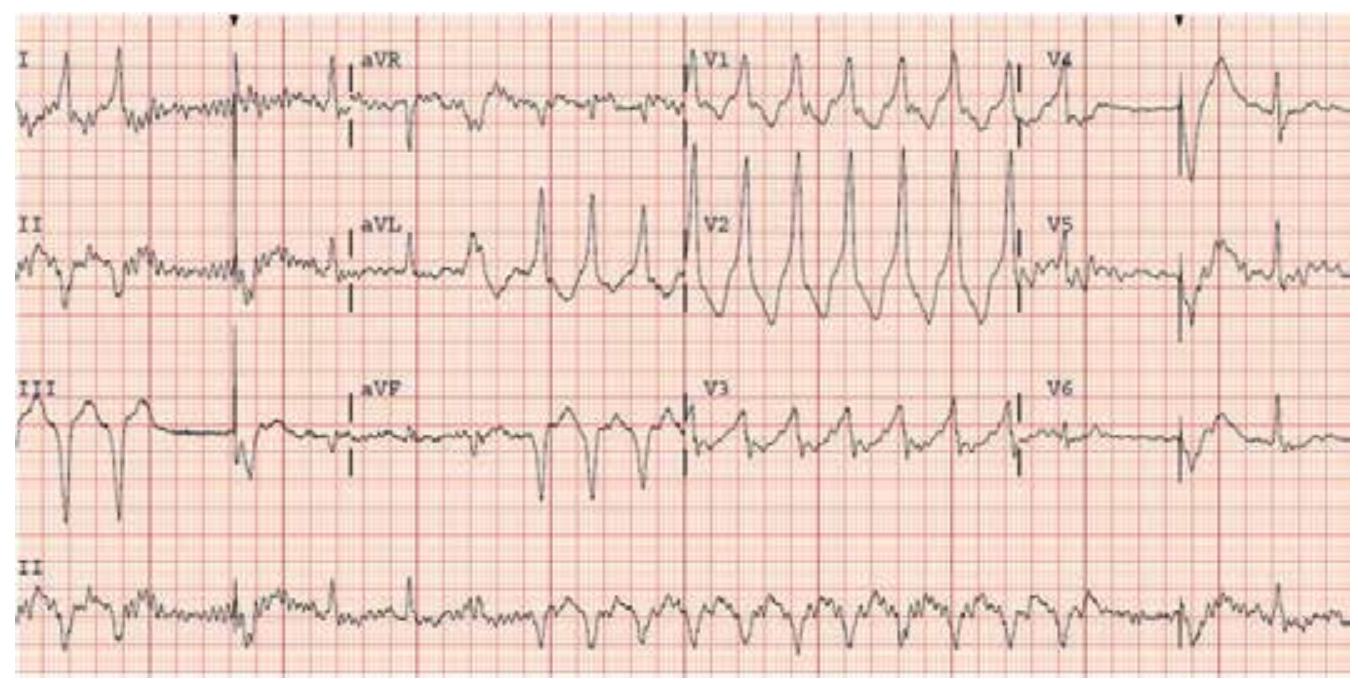

Figure 38. Non-sustained wide QRS tachycardia in a patient with a VVI pacemaker. Note appropriate demand pacing and the absence of spikes during the tachycardia - this is not pacing-related, but a true non-sustained ventricular tachycardia.

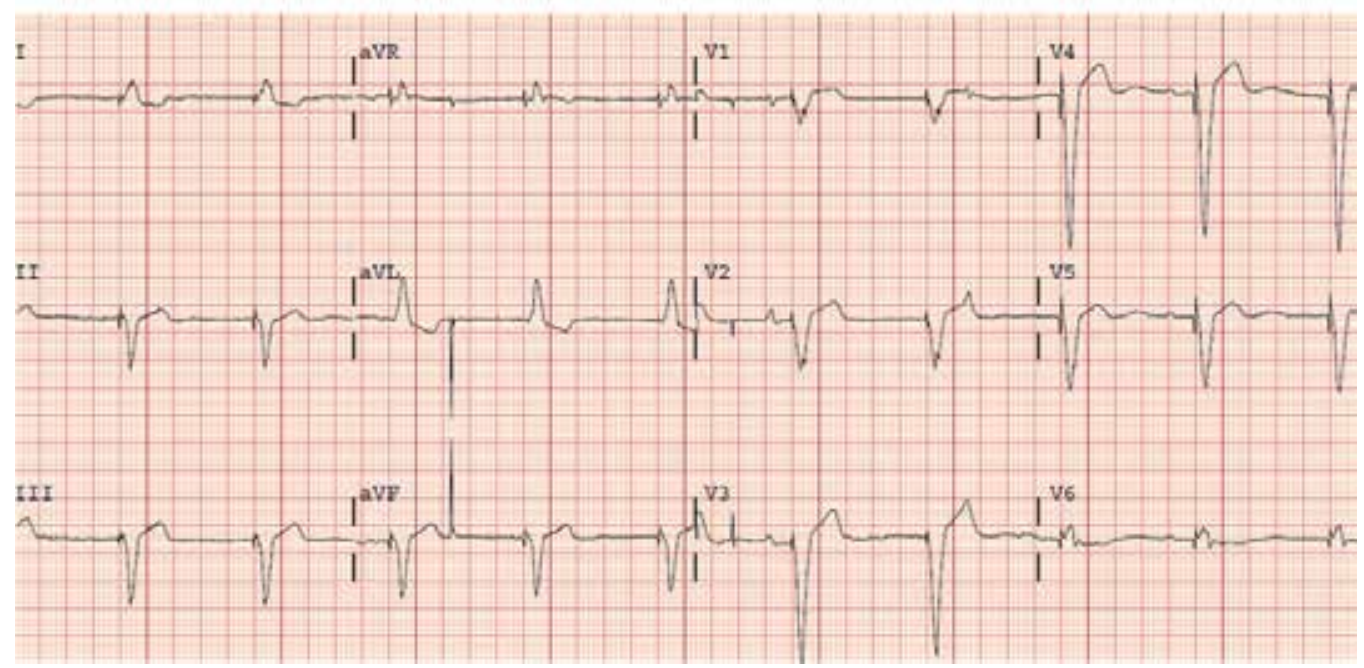

Figure 39. VVI pacing without retrograde conduction. An underlying, slow regular atrial rhythm is seen in V2 and V5. The spikes after the 3rd and 5th beats are artifacts.

ECG analysis should always include assessment of QRS and ST-T, even in patients with paced rhythms. Atrial pacing preserves normal ventricular activation, so it may be interpreted without interference from the device. Underlying conduction blocks may mimic paced beats (Figures 41-42). If artifacts limit interpretation, comparing multiple simultaneous ECG leads may be helpful (Figure 43).

In patients presenting with symptoms suspicious for pacemaker syndrome (hypotension, shortness of breath, dizziness, most commonly in an intermittent pattern), atrioventricular 
activation sequence and presence of ventriculoatrial conduction should be assessed. If these are compatible with PM syndrome, device settings should be adjusted (or the device should be upgraded), to restore AV synchrony and avoid atrial contraction during ventricular systole (Figures 44-45).

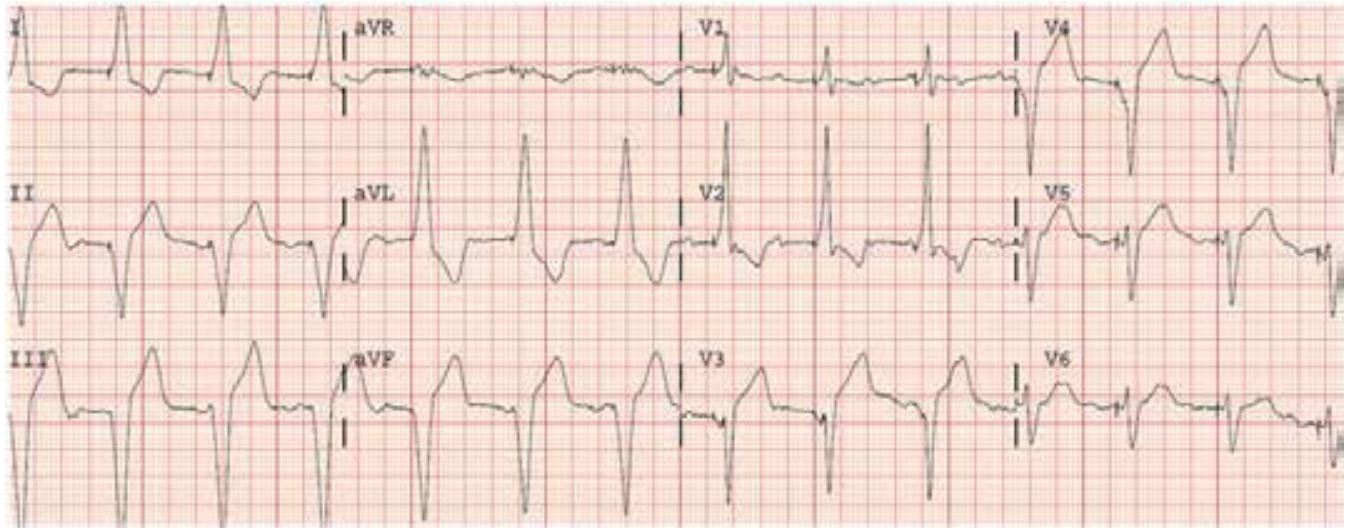

Figure 40. Underlying rhythm is atrial tachycardia or slow atrial flutter with a cycle length around 320 $\mathrm{ms}$. This is neither spontaneously conducted, nor tracked by the pacemaker to the ventricles, $80 /$ minute ventricular stimulation is seen.
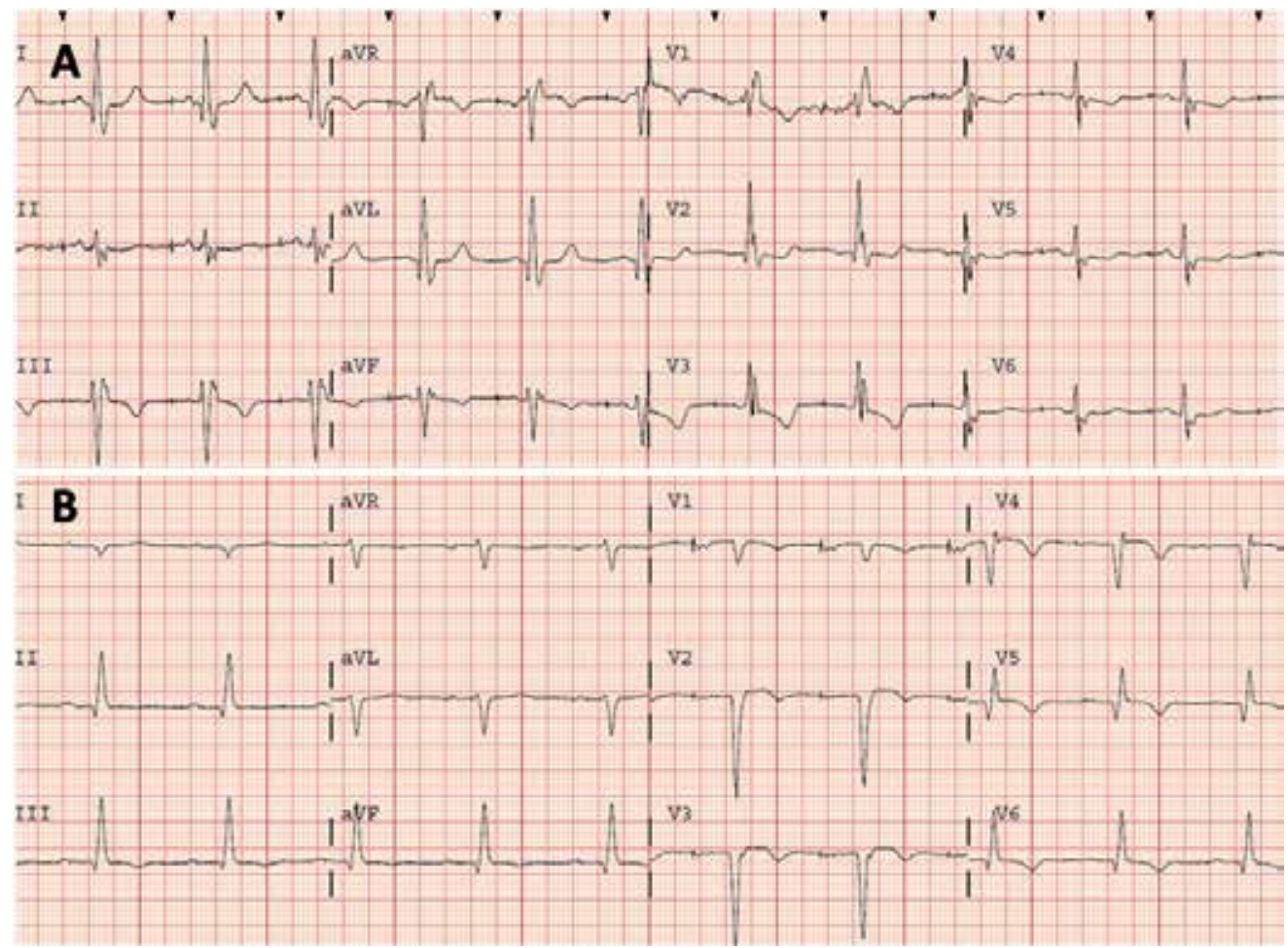

Figure 41. Atrial pacing preserves normal ventricular activation sequence, conventional ECG criteria may be used to identify ischemia, blocks, hypertrophy. RBBB (A), remote anterior MI (B). 


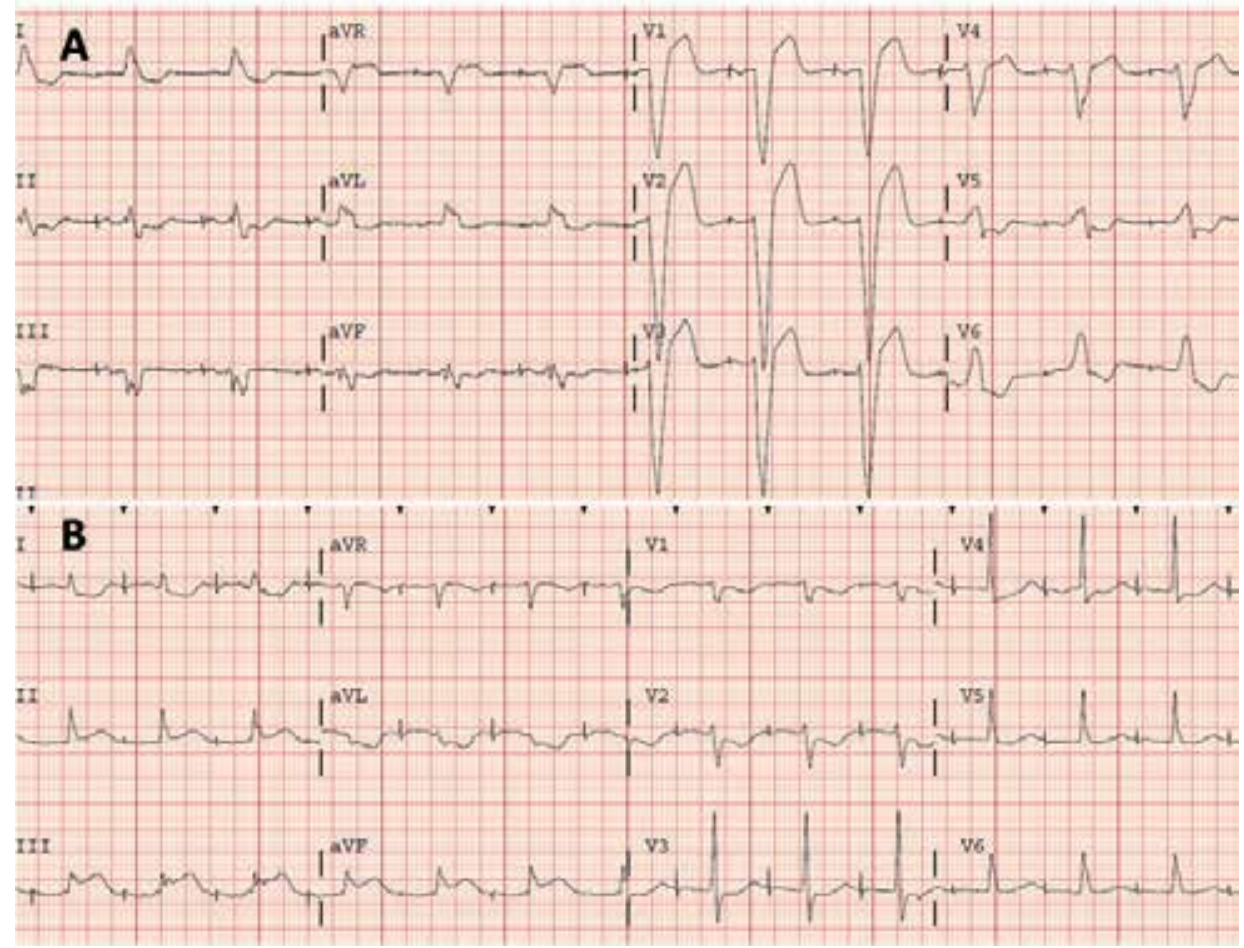

Figure 42. Preexisting LBBB may be confused with dual chamber pacing. Close inspection of the QRS complexes reveals atrial pacing and typical LBBB (A). Acute inferior MI with atrial pacing - typical ST elevation with reciprocal changes (B).

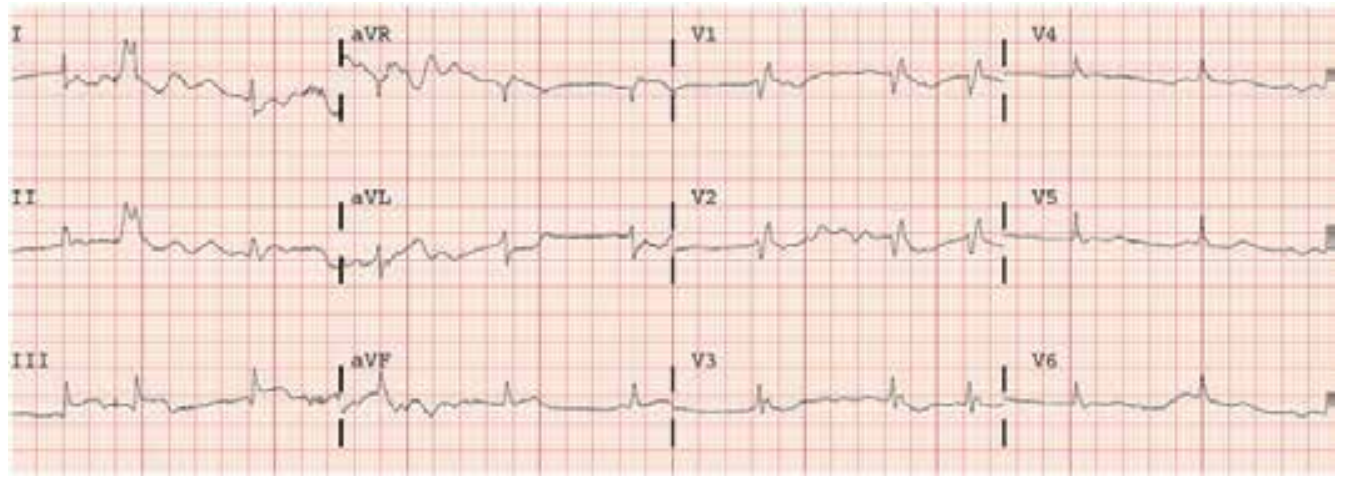

Figure 43. Artifact suggestive of ventricular undersensing with a recently placed temporary right ventricular lead. There appears to be a pacemaker spike shortly after the first QRS with a captured beat, suspicious for undersensing. However, the morphology of this "paced" beat is not typical and note that in III there is a 120 ms delay between the "spike" and the QRS and no change in depolarization/repolarization compared to non-paced beats - this would be impossible with a ventricular paced beat. This phenomenon was caused by an artifact causing a high amplitude, positive deflection in I and II, imitating a LBBB pattern, and there was actually no pacing - the artifact was gained as a spike by the ECG. There are multiple artifacts in I, II and aVR, suggestive of noise coming from the right upper extremity ECG electrode. 

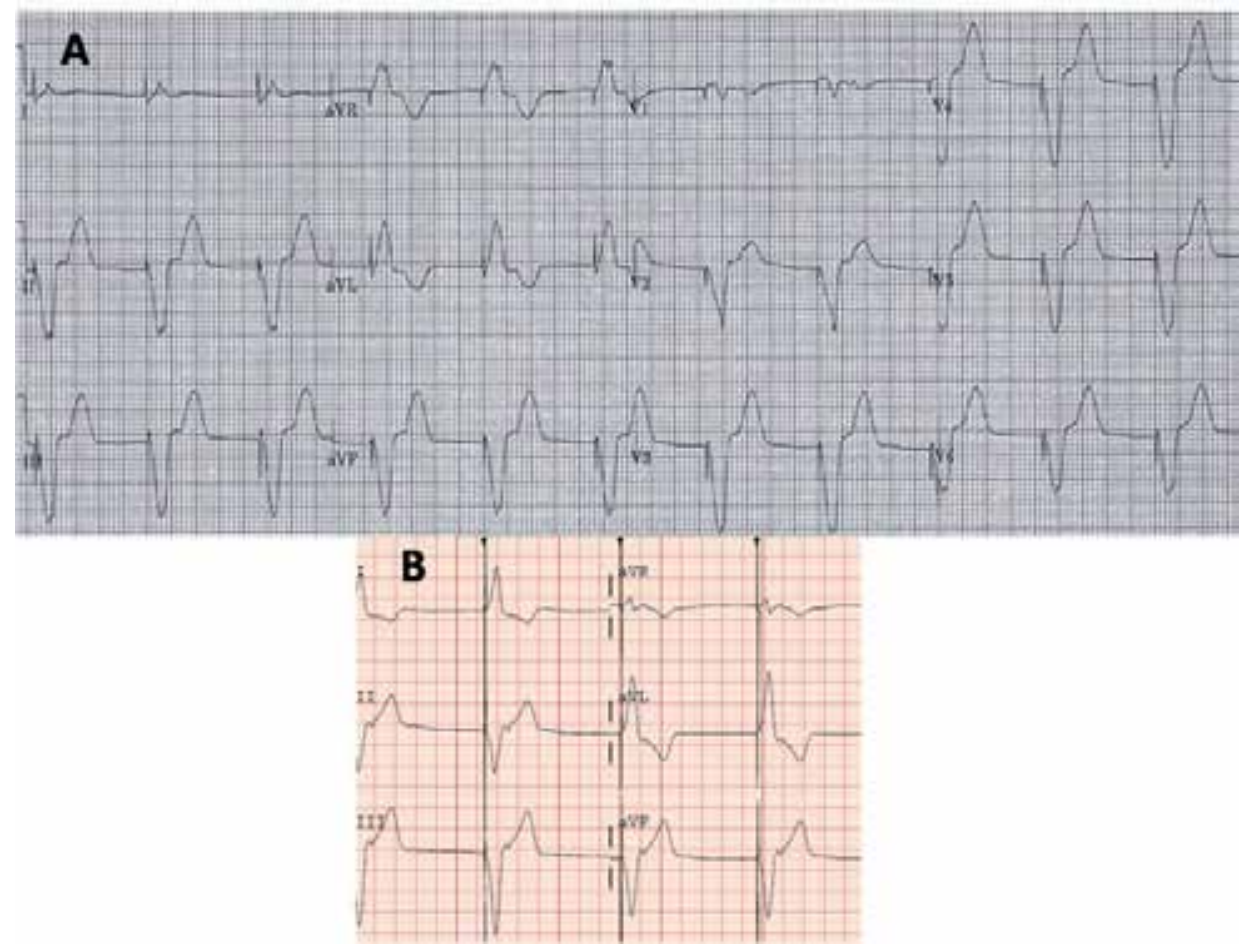

Figure 44. Mode switch due to battery depletion (A). The patient with a DDD PM presented with sudden onset complaints typical for pacemaker syndrome. ECG shows 65/minute ventricular stimulation with 1:1 VA conduction (best seen in V1) - this pacemaker converted to VVI 65/min backup mode when battery condition reached end-of-service. (B) is a more typical presentation of VVI stimulation with 1:1 retrograde conduction - without significant atrial conductive system disease, retrograde $P$ waves are easily recognized

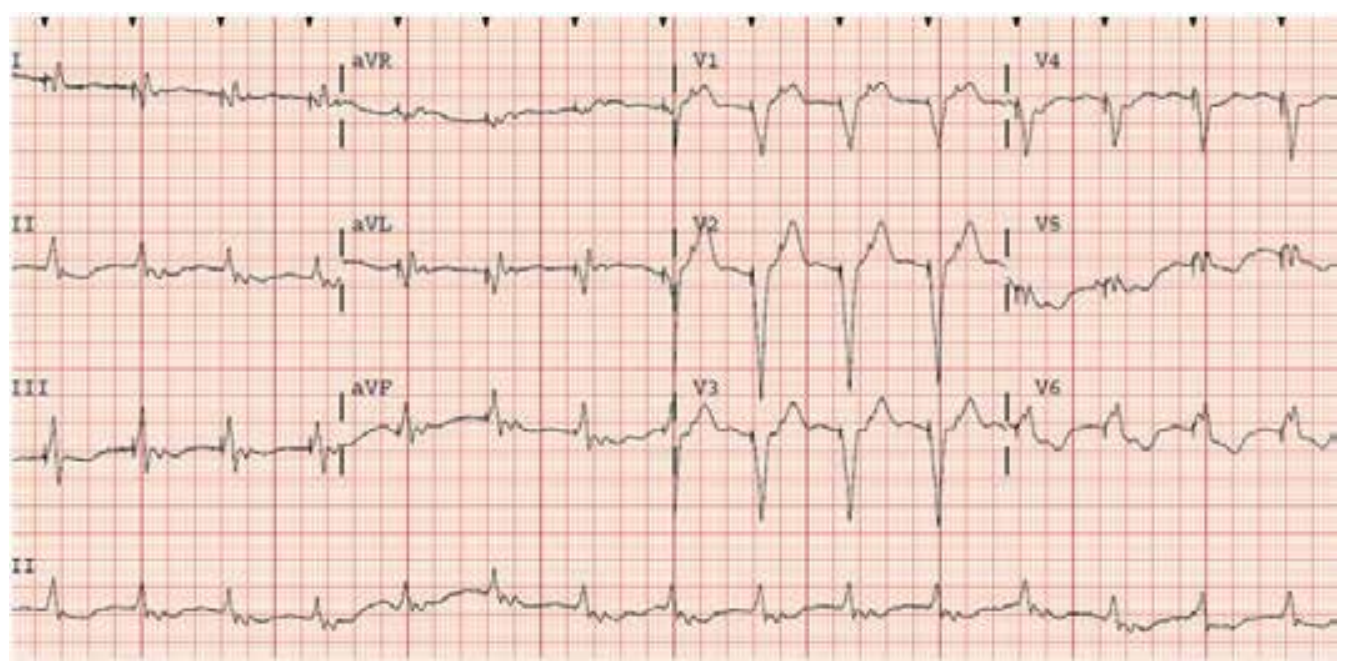

Figure 45. Retrograde conduction may be intermittent even during VVI pacing at constant rate - in this case, it starts after the 2 nd beat and ends 3 beats before the recording ends. 


\section{Pacemaker or lead malfunction}

Most common pacemaker and lead related malfunctions, that should be promptly identified and corrected, include oversensing, undersensing and ineffective stimulation. These may be related to inappropriate settings that may be easily corrected with a programmer, however, pacemaker lead related issues (dislocation, fracture, insulation failure) may present similarly and require hardware revision. If true pacemaker dysfunction cannot be ruled out with certainty based on ECG, device interrogation should be performed - this is especially important, if the patient was exposed to factors with potential device interaction, such as MRI, therapeutic irradiation, trauma, or drugs with known effect on pacing threshold (Goldschlager, 2001).

Pure undersensing may be identified by a pacing spike that comes early compared to the anticipated timing, with appropriate capture, if the paced chamber is not refractory. Transient arrhythmias, such as premature ventricular beats, may lead to intermittent undersensing, as their intracardiac signal amplitude may be low (Figure 46). Atrial fibrillation is often undersensed and elicits different behavior in AAI and DDD systems (Figures 47-48).

Loss of capture is easily recognized, however, post-pacing artifacts should not be misinterpreted as capture (Figure 49). Complete lead fracture usually leads to exit block with no visible spikes, while lead dislocation or insulation failure may manifest in various ways (Figures 50-54).

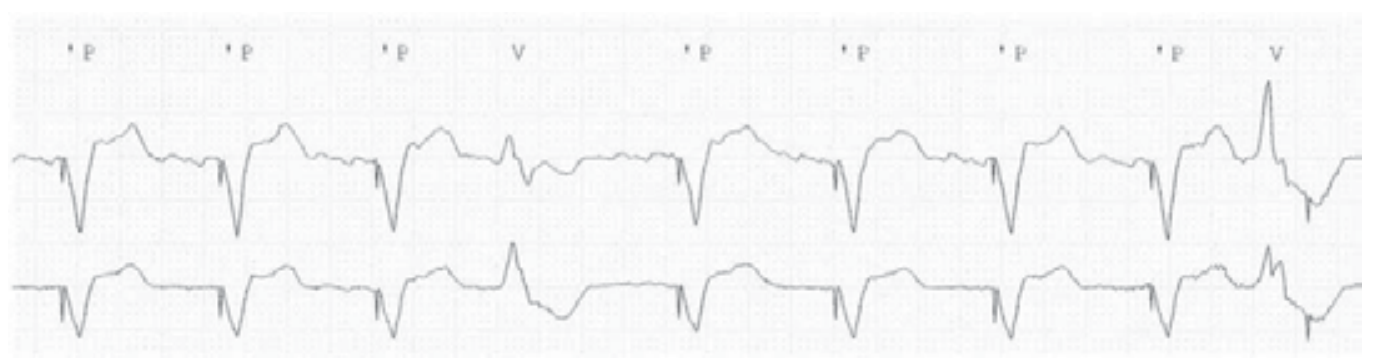

Figure 46. Ventricular undersensing in a patient with VVI pacemaker after AV node ablation for AF. The first PVC was detected and the pacing cycle was reset, however, the second PVC with a different morphology was not detected and inappropriate ventricular pacing occurred in the refractory period of the ventricles. 


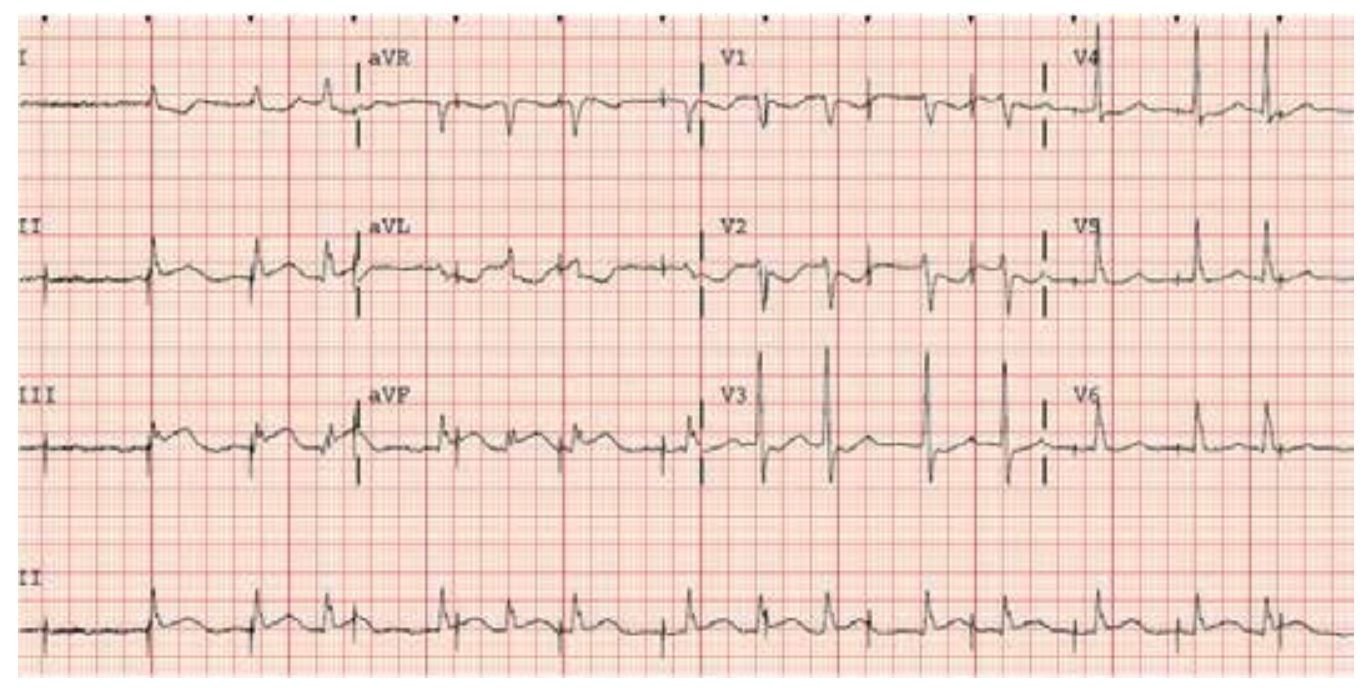

Figure 47. Undersensing of atrial fibrillation with an AAI pacemaker - there is asynchronous atrial pacing without capture. Pseudo pseudofusion beats are seen (2nd, $3 \mathrm{rd}$ ). VVI pacing would give a similar picture in case of complete sensing failure and loss of capture.

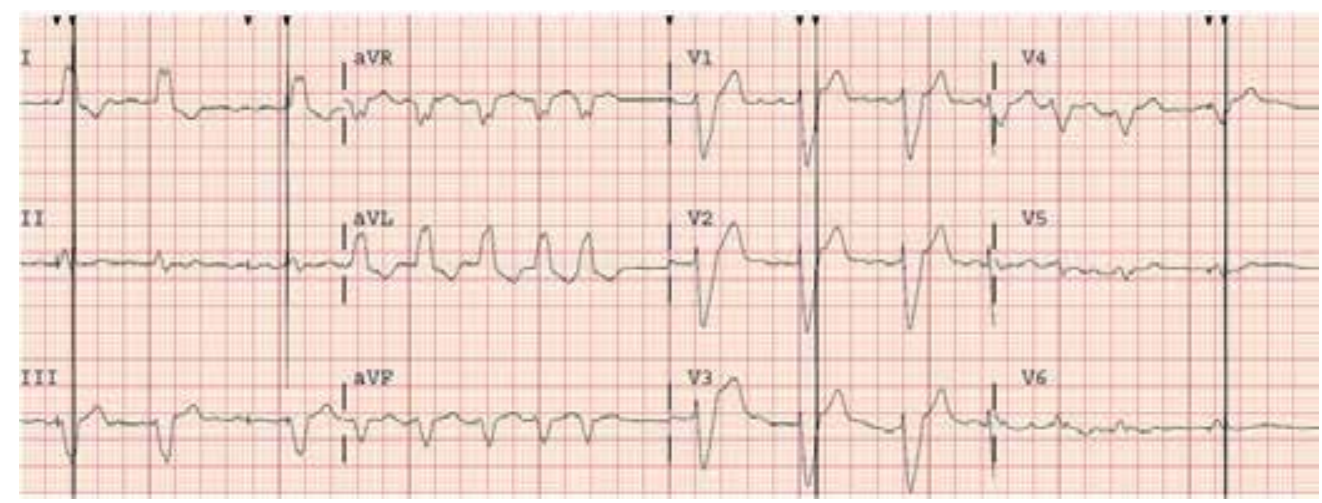

Figure 48. Undersensing of atrial fibrillation with a DDD pacemaker. When the ventricular rate during $\mathrm{AF}$ falls below the basic pacing rate, the PM delivers an atrial stimulus, which is not capturing as the patient is in AF. If conduction does not occur after the preset AV delay, a ventricular stimulus is delivered. If conduction occurs after the atrial spike within the ventricular safety period, a ventricular safety pace is delivered, which is not capturing as the ventricles are refractory. 


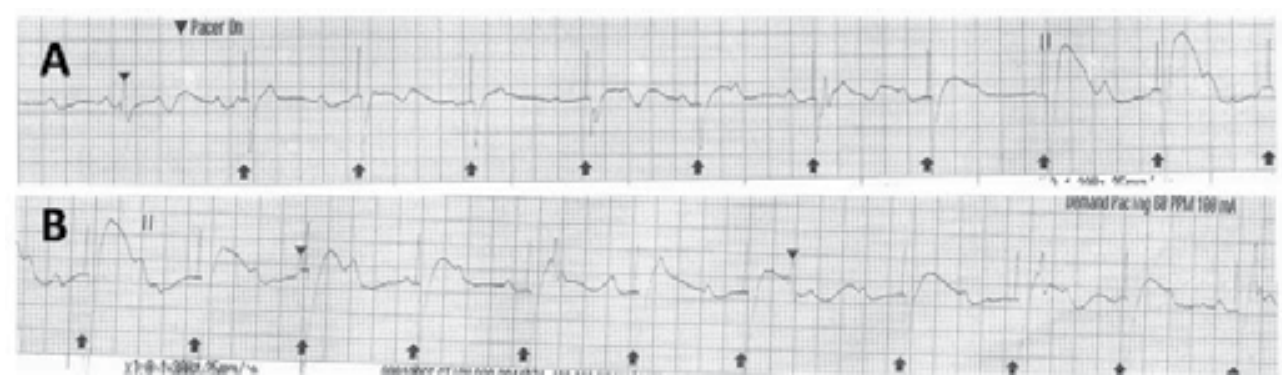

Figure 49. Pseudocapture during temporary external pacing. Transcutaneous pacing was initiated due to complete heart block (underlying rhythm is sinus tachycardia). An escape beat is seen (marked with a black triangle), then pacing is initiated and pacing energy is increased rapidly, causing progressively increasing post-pacing artifacts, which may be misinterpreted as capture (A). However, the slow escape rhythm is still visible between the spikes (best seen after the 6th spike). Later, dissociation between pacing and ventricular rhythm is even more evident despite marked post-pacing artifacts (B).

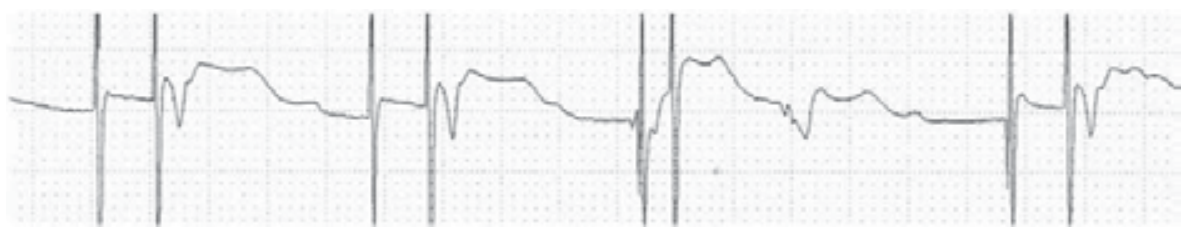

Figure 50. Atrial lead dislocation of a DDD pacemaker. The atrial activity is not sensed, which leads to asynchronous pacing without atrial capture, followed by ventricular pacing with capture. The 3rd beat is a sinus beat conducted with prolonged AV delay, which is sensed in the ventricular safety pacing interval, so a ventricular safety pace is delivered without capture - the ventricle is refractory at this time.

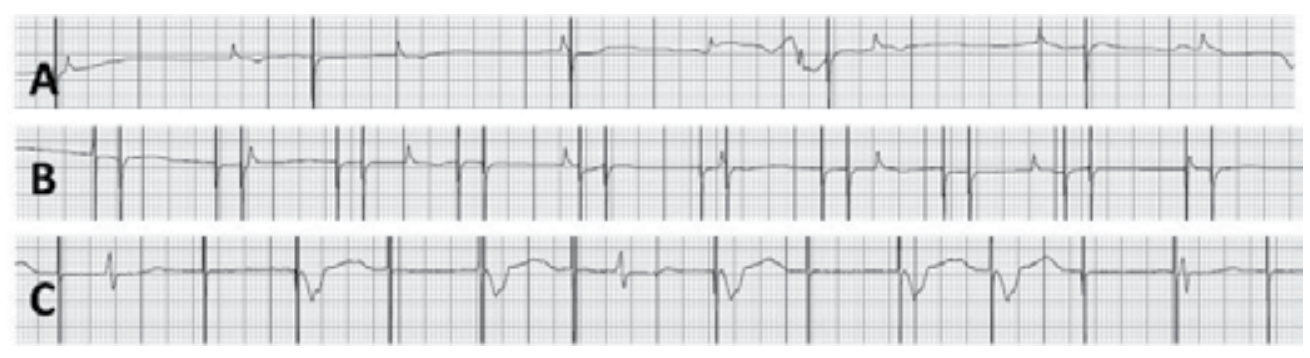

Figure 51. (A) Undersensing and ineffective pacing with a unipolar pacemaker. Both single chamber atrial and ventricular pacemakers would present similarly in case of lead dislocation. (B) Complete failure of sensing and pacing in a dual chamber pacemaker. There is asynchronous dual chamber pacing, without capture in either chamber. Unipolar pacing causes notable post-spike artifacts, which should not be confused with cardiac electrical activity. (C) Intermittent loss of capture with a ventricular pacemaker. Sensing appears to be normal as each spontaneous QRS resets the pacing cycle. The last spontaneous beat comes very early after the pacing stimulus and is likely not detected due to sensing in the blanking period. 


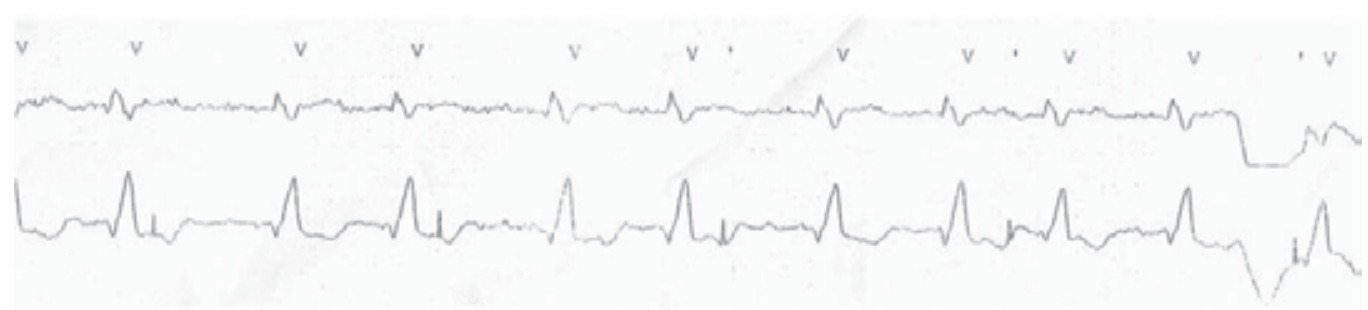

Figure 52. Lead dislocation in a recently implanted single chamber ICD. There is no ventricular sensing, so the pacing is at $40 / \mathrm{min}$, asynchronous to the intrinsic rhythm. There is also lack of capture attention should be paid when assessing capture as spikes 1-3 come very early when the ventricles may still be refractory. However, spike 5 should have lead to capture.

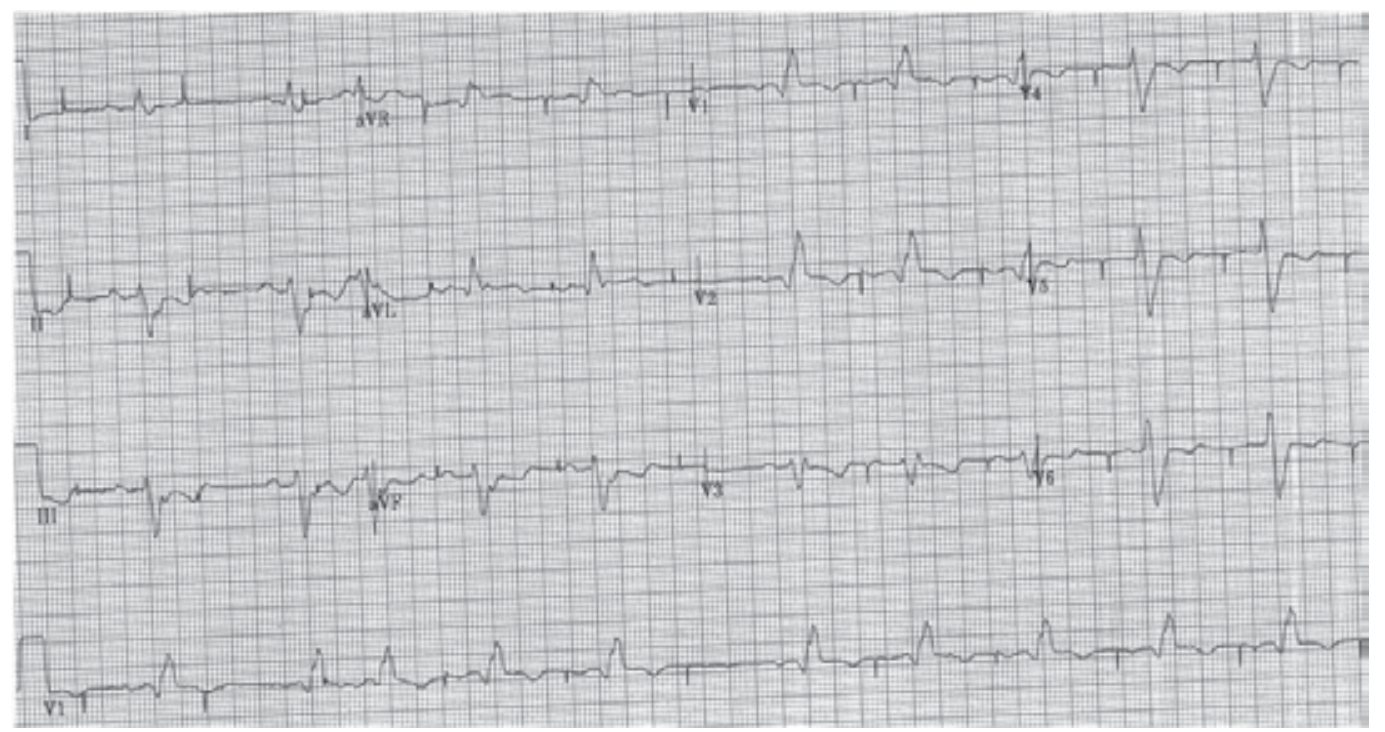

Figure 53. Intermittent loss of atrial capture during AAI stimulation. There is also intermittent undesensing - the atrial activation before the 3rd spike was not detected by the device. This scenario is suspicious for lead disclocation. 


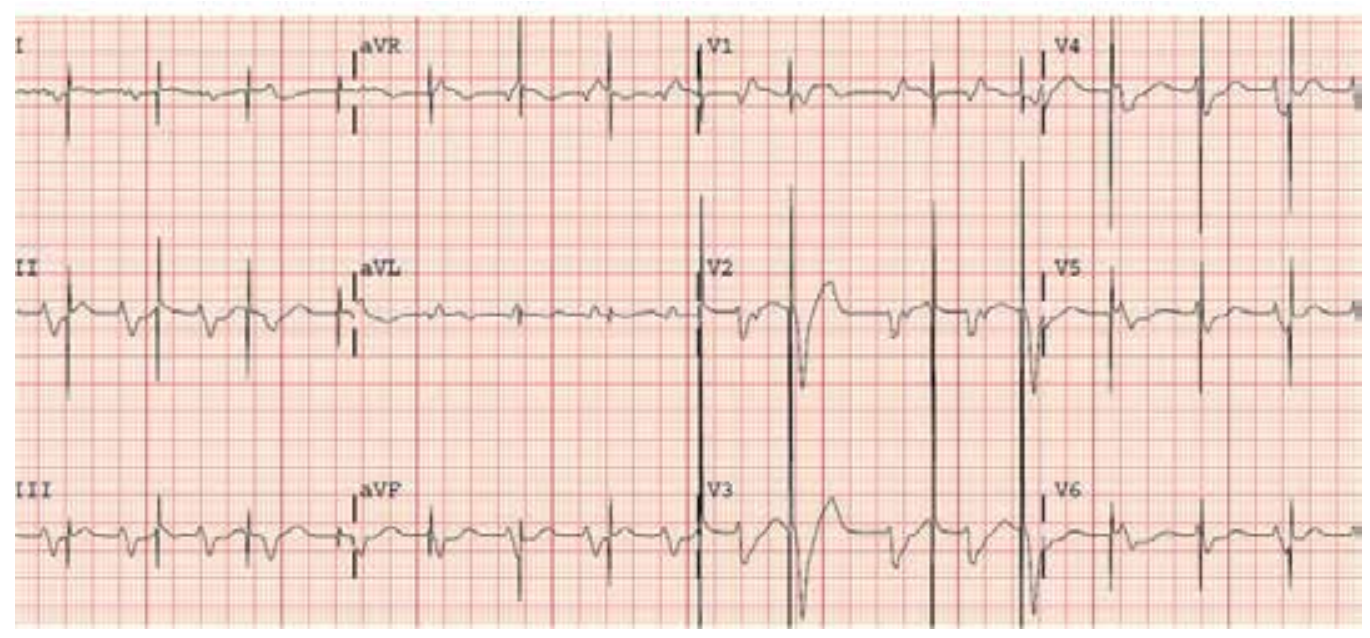

Figure 54. Loss of sensing with oversensing in a ventricular pacemaker. The first few beats may be misinterpreted as atrial pacing, however, the spike to QRS interval is not constant. Fusion and paced beats are seen when pacing occurs during an excitable period. Transient oversensing caused delayed pacing (3rd spike in V3). This scenario is suggestive of lead dislocation or failure.

\section{Evaluation of ICD function}

Implantable cardioverter defibrillators have multiple therapeutic zones (bradycardia, "physiological”, ventricular tachycardia and fibrillation - VT, VF), that should be taken into account when interpreting ECG changes. While issues due to undersensing or ineffective capture usually manifest similarly to a pacemaker, oversensing may lead to inappropriate therapy due to false VT/VF detection.

As ICD therapies may cause severe patient distress or proarrhythmia, prompt device interrogation and expert consultation is required after such events, unless appropriate device behavior is evident (Figures 55-56). Even when appropriate therapies have been delivered, the patient has to be fully evaluated and appropriate measures should be taken to reduce the risk of arrhythmia recurrence (Mishkin, 2009). In cases when inappropriate therapy is suspected and the risk of recurrence is high (atrial fibrillation with rapid ventricular rate, oversensing), a magnet may be applied to temporarily inhibit tachyarrhythmia therapies, until the device may be interrogated and appropriately reprogrammed (Figure 57). Continuous monitoring is required in the meantime as the patient will not be protected from malignant tachyarrhythmias while in magnet effect. 


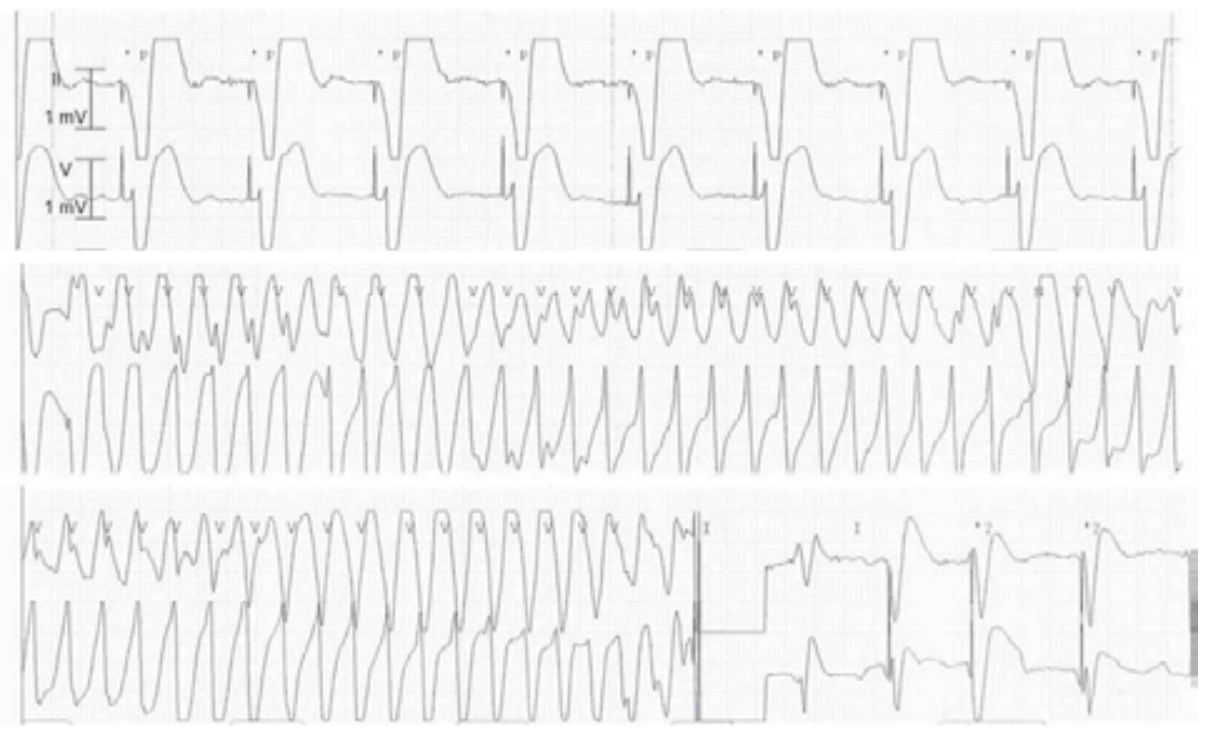

Figure 55. Appropriate ICD function recorded on telemetry. Following ventricular paced rhythm, rapid polymorphic ventricular tachycardia develops, which is terminated by a single endocardial shock after appropriate detection.

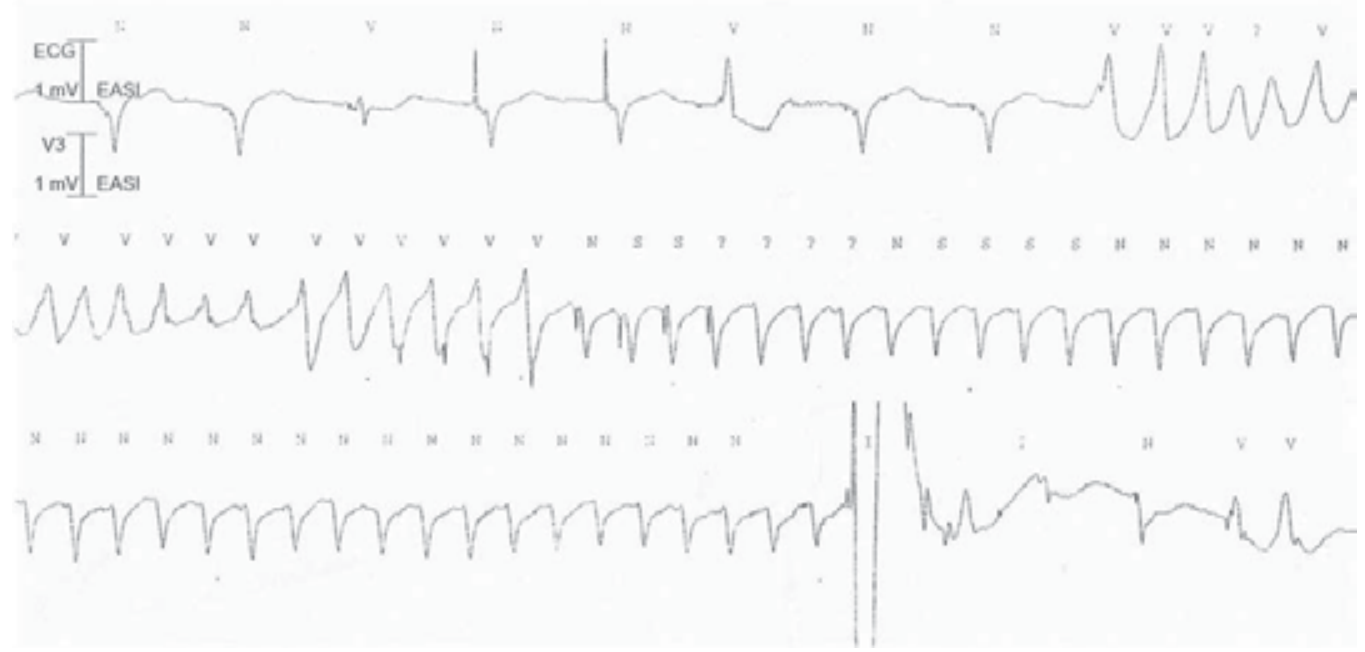

Figure 56. Appropriate ICD function recorded on telemetry. Following ventricular paced rhythm, rapid polymorphic ventricular tachycardia develops, then burst antitachycardia stimulation is attempted, however, fails to terminate the arrhythmia, although changes it to monomorphic VT. The tachyarrhythmia is terminated by an endocardial shock. 

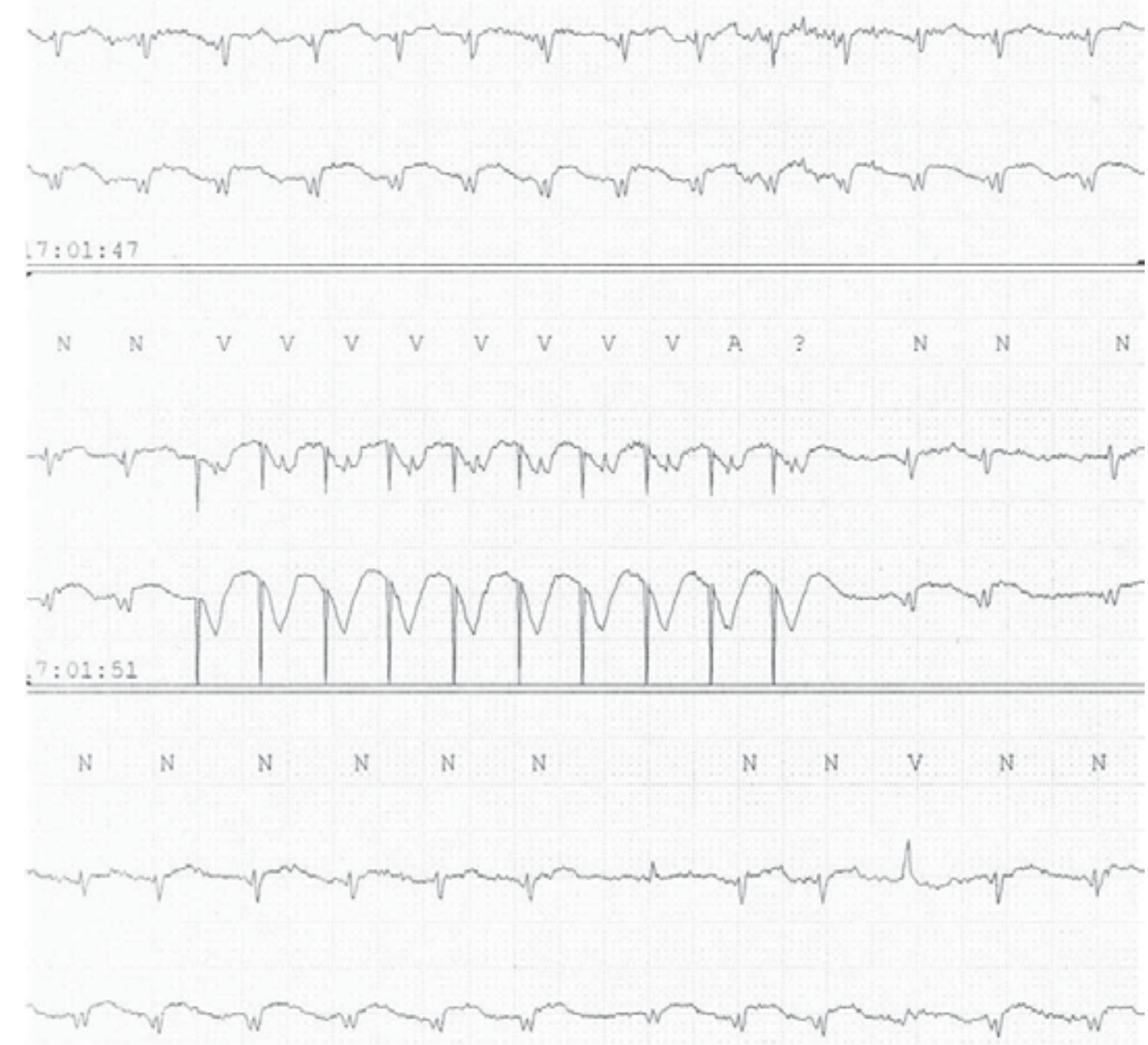

Figure 57. Atrial fibrillation with rapid ventricular rate sensed as ventricular tachycardia inappropriate burst antitachycardia pacing burst was delivered. The patient is at risk of further inappropriate therapies as the underlying rhythm did not change.

\section{Evaluation of cardiac resynchronization devices}

Consistent biventricular capture is required to maintain cardiac resynchronization. Paced QRS morphology may vary based on underlying conduction abnormalities, lead location, interventricular delay and the amount of myocardium captured by each lead, relative to each other. Typically, right axis deviation and atypical RBBB pattern is present. If interventricular delay is set greater than $0 \mathrm{~ms}$, usually two pacing spikes can be seen prior to the QRS (Figure 58). In rare cases, conventional RV pacing may mimic biventricular paced QRS morphology (Figure 59). QRS morphology may change due to variable fusion with conducted beats either from variable AV delay or atrial fibrillation (Figures 60-61). 


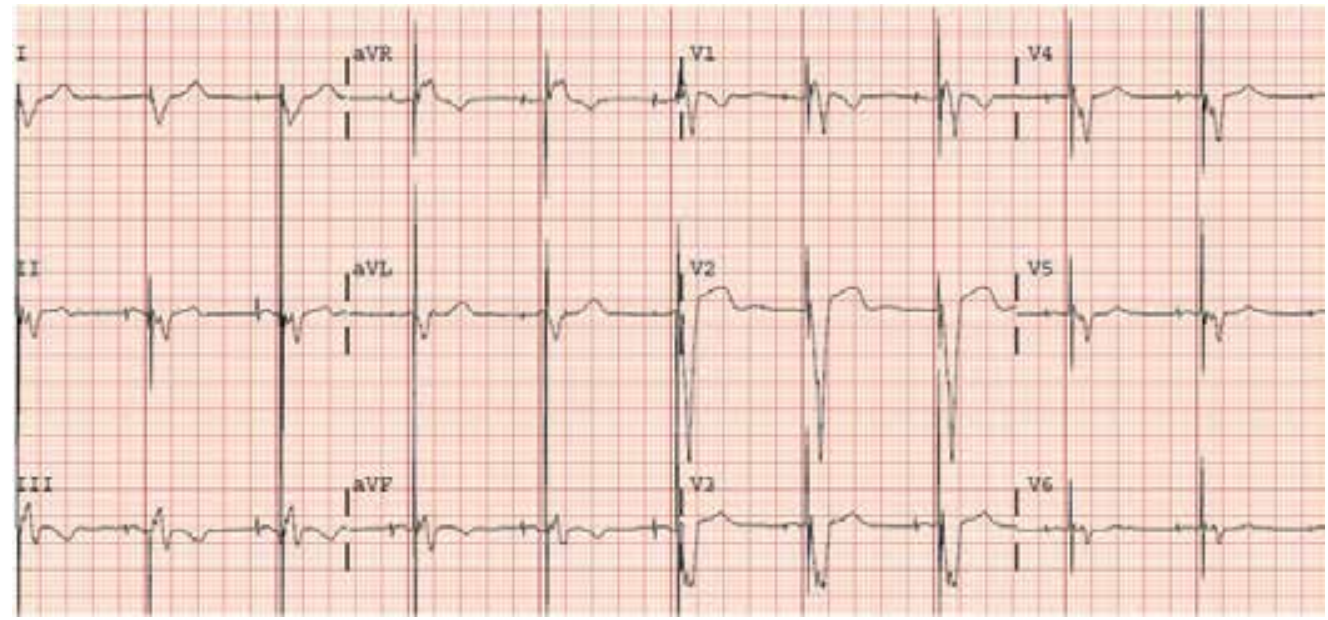

Figure 58. Typical atriobiventricular pacing. The paced QRS usually shows right axis deviation and an atypical RBBB pattern in V1. Two distinct pacing spikes, representing right and left ventricular stimulation with a delay around $20 \mathrm{~ms}$, can be best seen in II and V3 on this tracing.

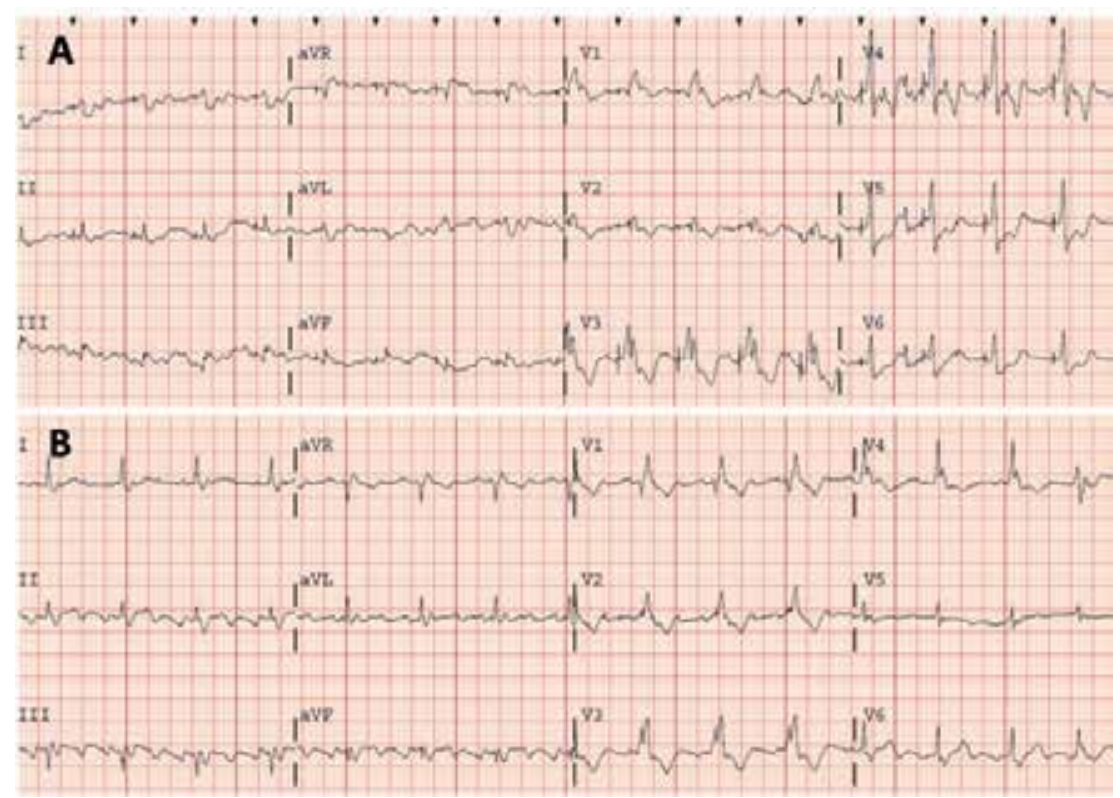

Figure 59. Although biventricular pacing may be recognized in most cases, underlying RBBB may mimic this QRS morphology during right ventricular pacing, especially, if fusion is present - this patient has a DDD pacemaker (A). His previous ECG showed atrial flutter with RBBB (B).

Sense response pacing is an algorithm that was designed to maintain the benefits of biventricular stimulation with premature beats or fast AV conduction - in case a ventricular event is sensed, a pacing stimulus is delivered simultaneously to decrease ventricular activation time. The resulting QRS morphology is affected by the origin of the premature beat and the amount of fusion (Figures 62-64). 
Loss of left ventricular lead capture changes QRS morphology, so it becomes similar to RV pacing. A full 12-lead ECG should always be obtained during follow-up (Barold, 2011a and Barold, 2011b). Comparison with previous tracings is recommended as biventricular paced QRS morphology varies individually (Figure 65). Undersensing or oversensing may be more difficult to identify with resynchronization devices, than with conventional pacemakers, due to the algorithms designed to maintain biventricular pacing (Figure 6667). In uncertain cases, device interrogation should be performed to prevent loss of resynchronization.

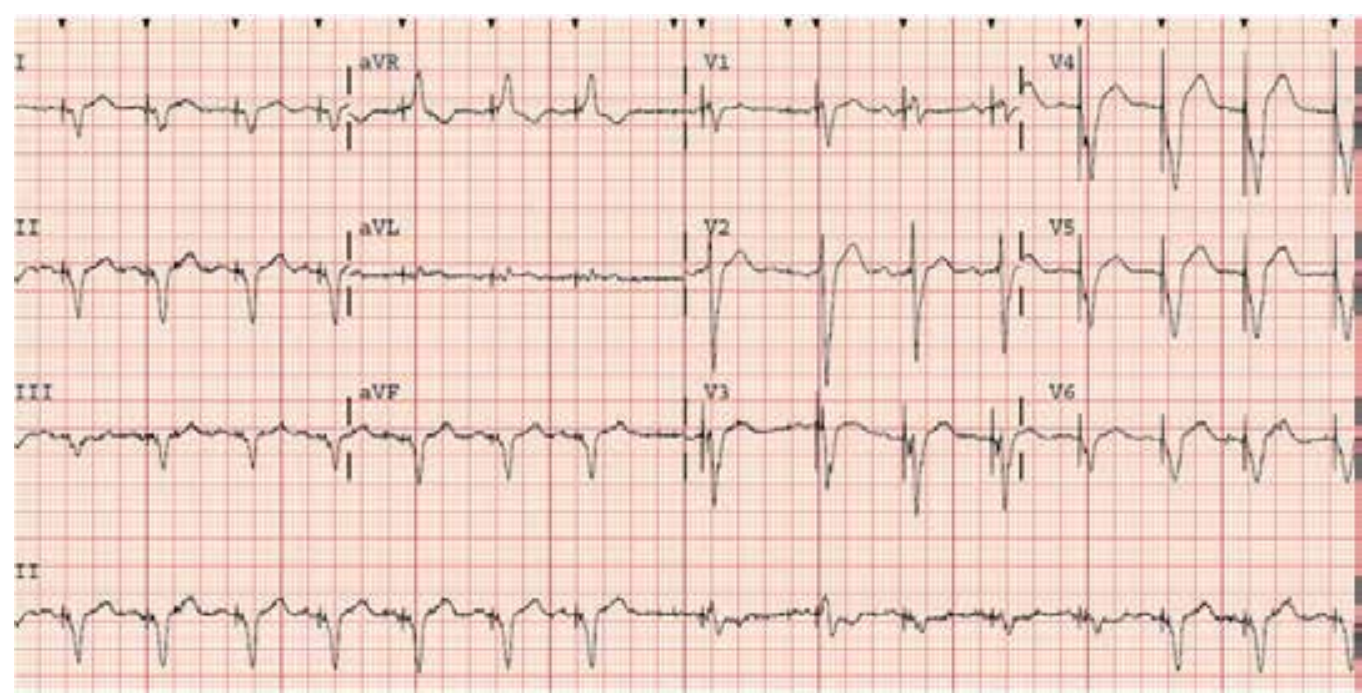

Figure 60. Variable fusion during biventricular pacing due changes in the atrial rate - the degree of ventricular fusion is different for atrial sensed and atrial paced beats, due to different atrioventricular delay, affecting how much of the ventricular myocardium can be activated through the native conduction system during biventricular pacing.

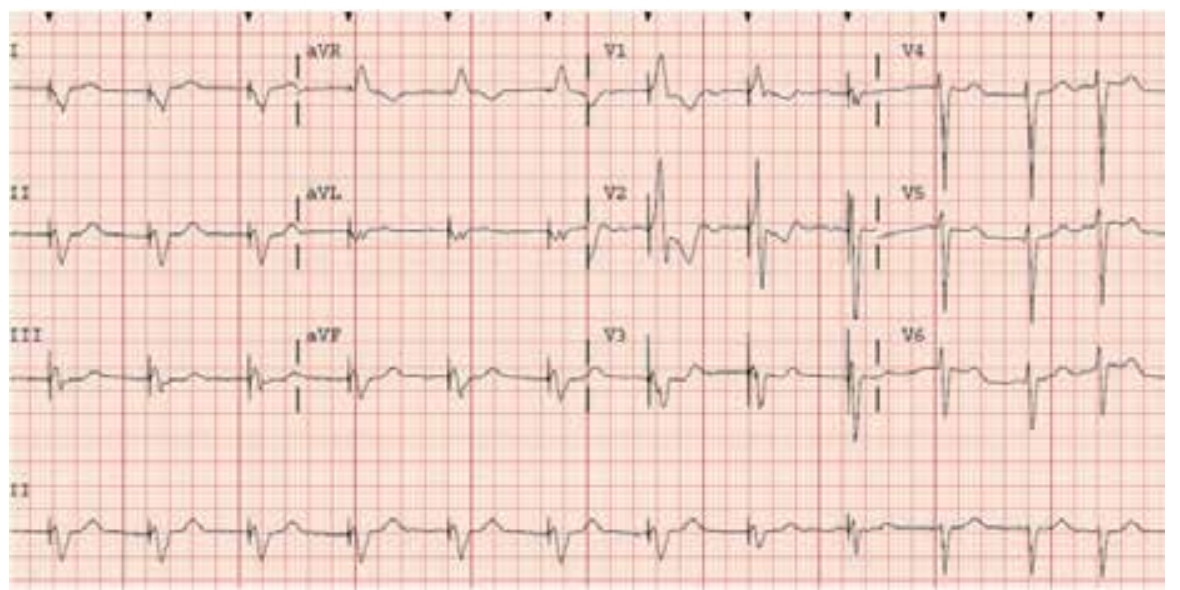

Figure 61. AF with biventricular pacing. When the ventricular rate increases, first it leads to more fusion, then to sense response pacing - appropriate response of the system. 


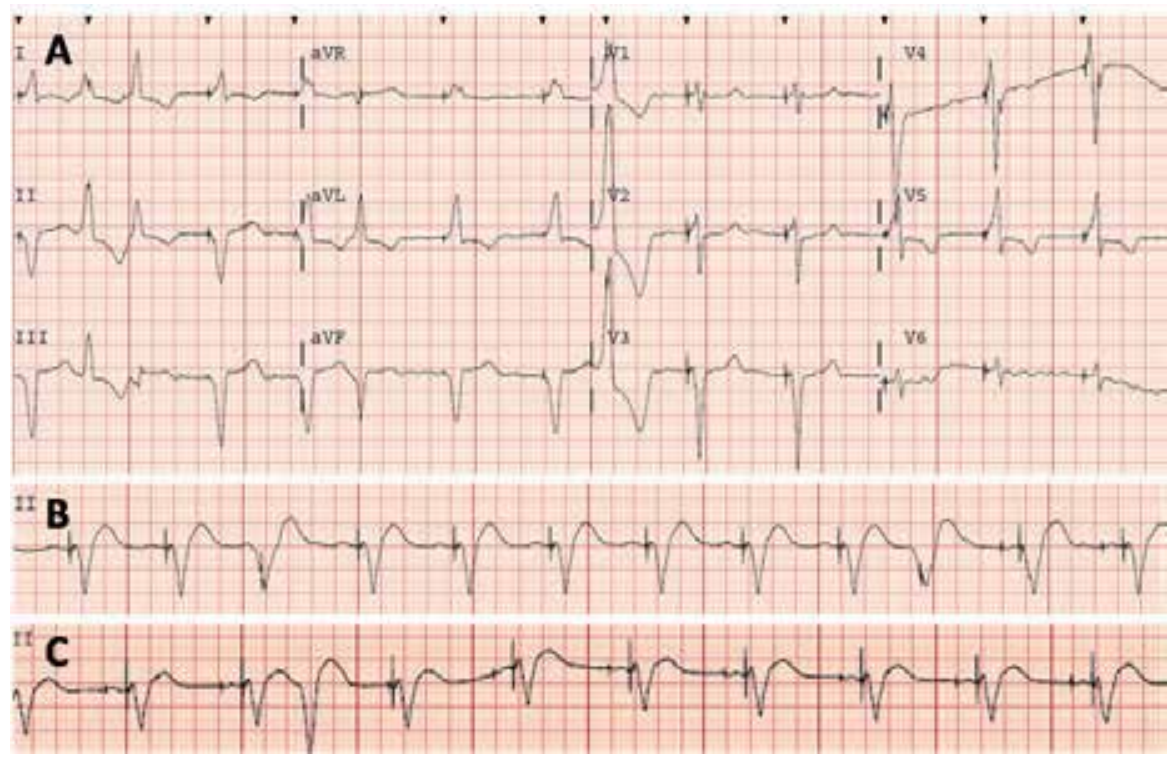

Figure 62. Sense response pacing during biventricular stimulation - each ventricular sensed event (PVC, rapidly conducted AF) leads to simultaneous pacing, aiming to maintain optimal hemodynamics of biventricular pacing. Due to the various origin of these early beats, the result can be fusion of even pseudofusion. Despite irregular rate and variable QRS morphology, this tracing shows appropriate biventricular pacemaker function (A). This function may be easier to evaluate when the underlying rhythm is regular, such as in sinus rhythm (B). There is an appropriate sense response pace for each premature beat. Depending on the coupling interval of the premature beat and the atrial rate, this may lead to post-event atrial pacing, if the compensatory pause exceeds the basic pacing rate. Very early PVCs do not trigger sense response pacing if that would exceed a maximal tracking rate (C).

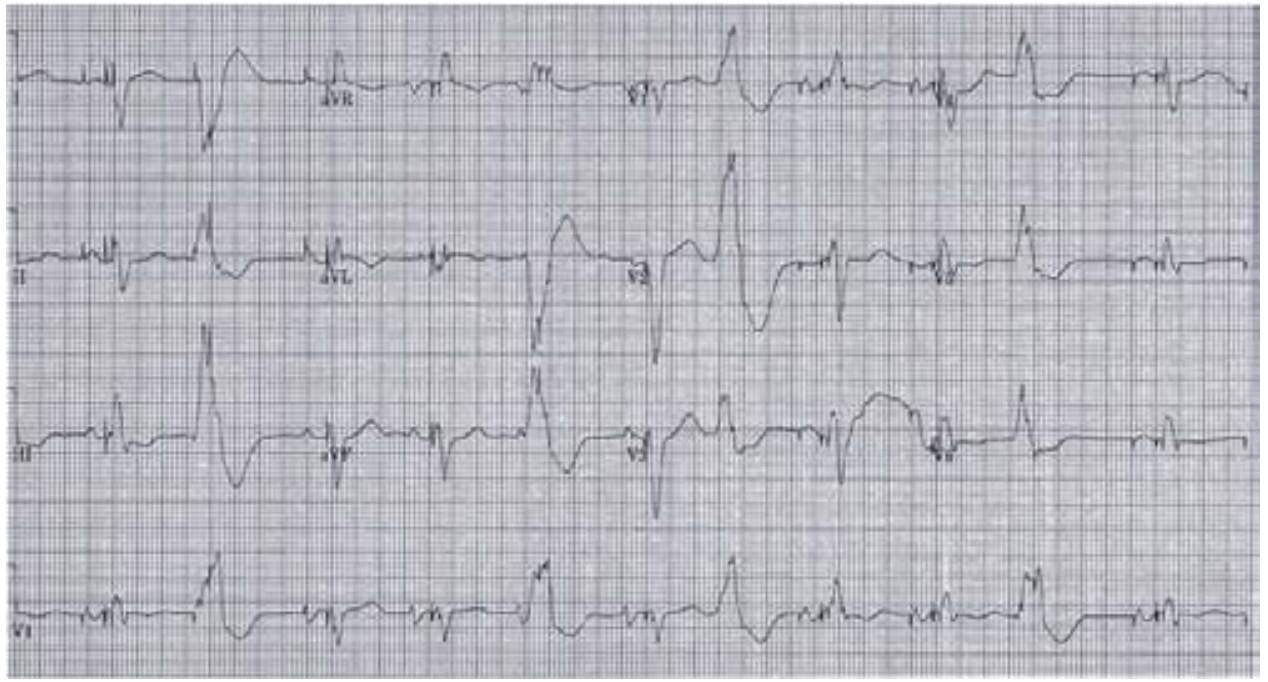

Figure 63. Atriobiventricular pacing with irregular ventricular rhythm due to frequent PVCs.

Appropriate device behavior with sense response pacing during PVCs (best seen during the 1st PVC). There is notable interventricular delay between the right and left ventricular stimulation (60 ms). 


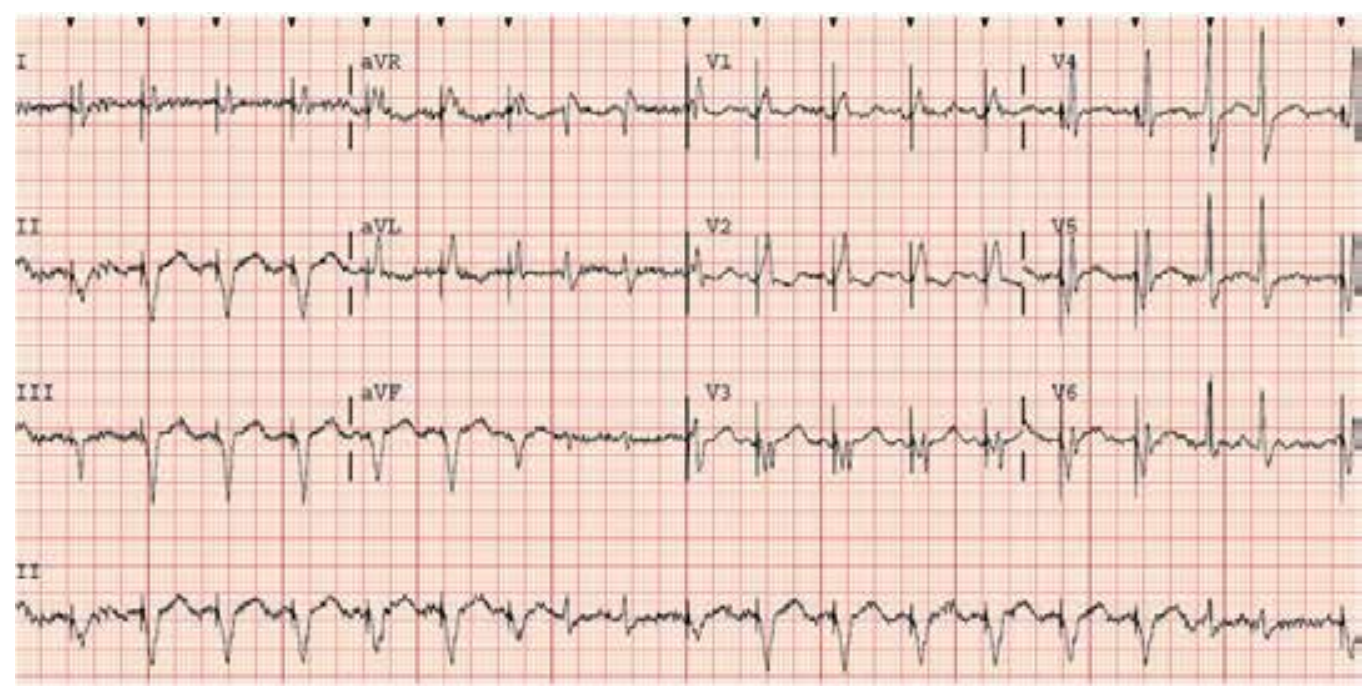

Figure 64. Biventricular (sense response) pacing ceases above the upper biventricular tracking rate this is appropriate pacemaker function, however, may lead to rapid deterioration if the tachycardia persists.

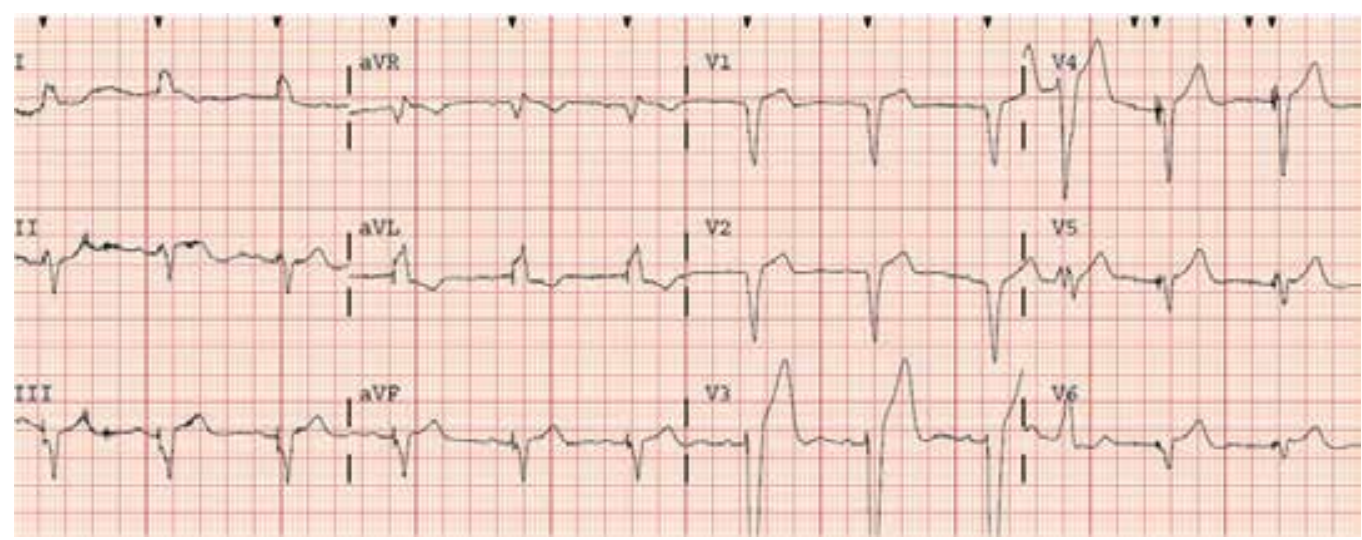

Figure 65. LBBB QRS morphology in a patient with a biventricular system should raise the suspicion of left ventricular non-capture. Other causes include suboptimal LV lead placement or too long RV-LV delay - these may diminish the amount of myocardium activated by the LV lead during biventricular pacing. In this case, biventricular pacing with $40 \mathrm{~mm} \mathrm{VV}$ delay is apparent in V4. 


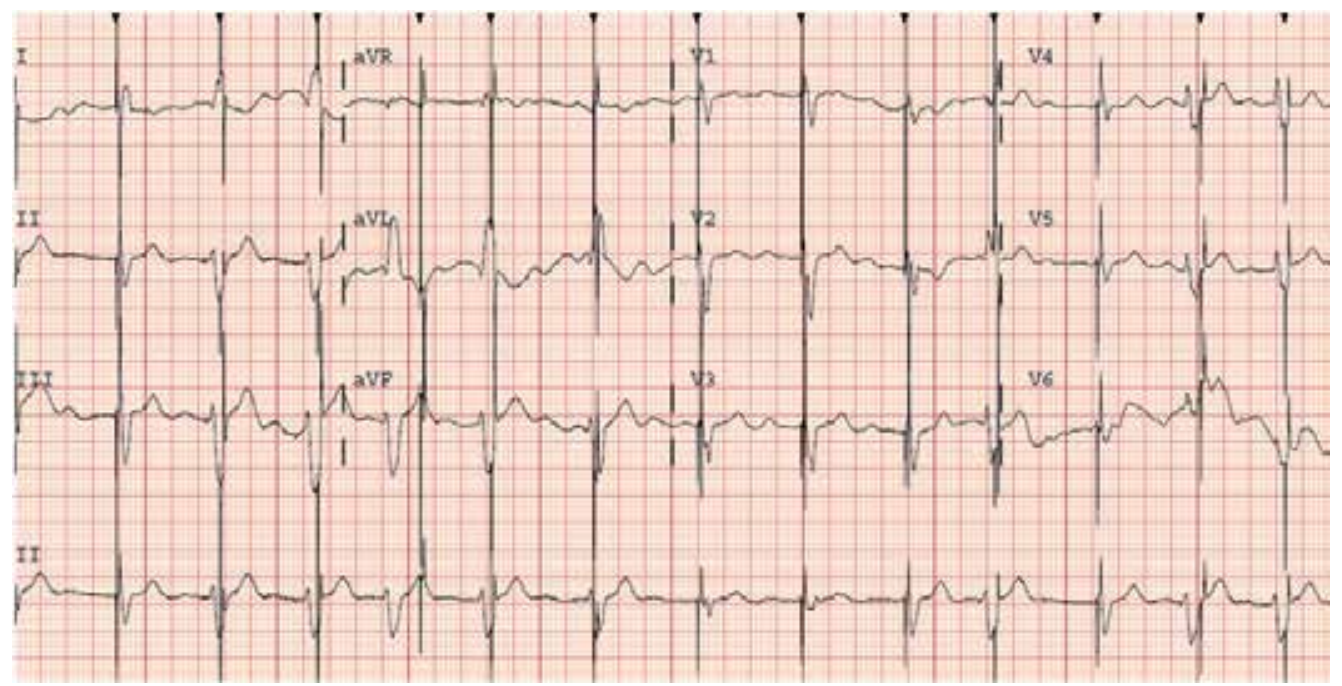

Figure 66. Intermittent ventricular undersensing in a biventricular system. Most ventricular beats are biventricular paced at 75/minute or sense response paced (occurring faster that 75/min). However, there are few spikes coming late (instead of a sense response pace), at 75/minute - the ventricular activation was not detected by the device. The undersensing is intermittent, as the sense response paced beats present on this tracing require a sensed event.

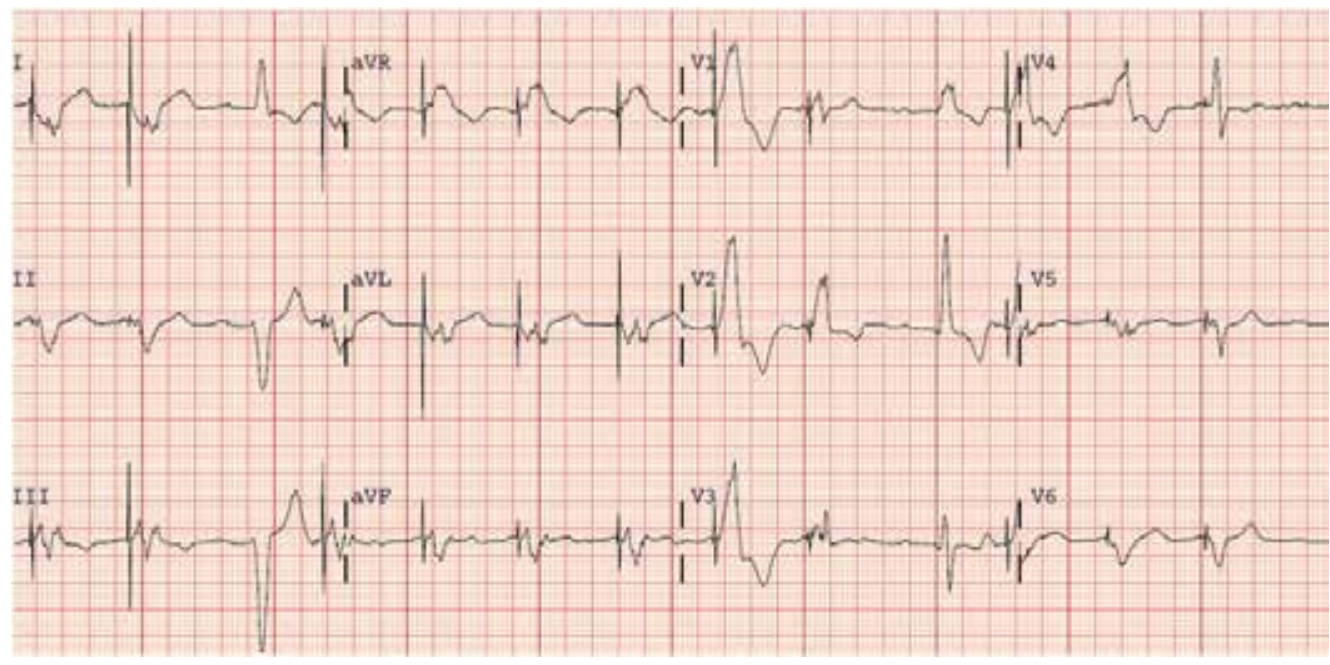

Figure 67. Pacing below the basic rate in an atriobiventricular system is always abnormal - algorithms are designed to maintain the ventricular rate, track premature beats and provide sense response pacing. This patient with a biventricular defibrillator had a fracture of the right ventricular sensing/pacing/shock ICD lead, leading to intermittent ventricular oversensing and multiple inappropriate shocks. 


\section{Conclusion}

Conventional 12-lead ECG is an important tool to evaluate CIED function. A systematic approach is required to identify appropriate device function and to decide whether further investigation is necessary. As advanced devices, such as implantable cardioverterdefibrillators and cardiac resynchronization systems become more abundant, even common malfunctions and pseudo-malfunctions may be more difficult to identify, due to the presence of special pacing algorithms. In uncertain cases, review of prior patient data, device interrogation and expert consultation is required.

\section{Author details}

Attila Roka

Hospital of St. Raphael, New Haven, CT, USA

\section{References}

Balachander, J., et al., Pacemaker trouble shooting and follow up. Indian Heart J, 2011. 63(4): p. 356-370.

Barold, S.S., et al., Usefulness of the 12-lead electrocardiogram in the follow-up of patients with cardiac resynchronization devices. Part I. Cardiol J, 2011. 18(5): p. 476-486.

Barold, S.S., et al., Usefulness of the 12-lead electrocardiogram in the follow-up of patients with cardiac resynchronization devices. Part II. Cardiol J, 2011. 18(6): p. 610-624.

Goldschlager, N., et al., Environmental and drug effects on patients with pacemakers and implantable cardioverter/defibrillators: a practical guide to patient treatment. Arch Intern Med, 2001. 161(5): p. 649-655.

Ip, J.E., et al., Differentiating pacemaker-mediated tachycardia from tachycardia due to atrial tracking: Utility of $\mathrm{V}-\mathrm{A}-\mathrm{A}-\mathrm{V}$ versus $\mathrm{V}-\mathrm{A}-\mathrm{V}$ response after postventricular atrial refractory period extension. Heart Rhythm, 2011. 8(8): p. 1185-1191.

Israel, C.W., Analysis of mode switching algorithms in dual chamber pacemakers. Pacing Clin Electrophysiol, 2002. 25(3): p. 380-393.

Jacob, S., et al., Cardiac rhythm device identification algorithm using X-Rays: CaRDIA-X. Heart Rhythm, 2012. 8(6): p. 915-922.

Lahiri, M.K., et al., Strategies for pacemaker programming in acute heart failure. Heart Fail Rev, 2011. 16(5): p. 441-448.

McPherson, C.A., et al., Permanent pacemakers and implantable defibrillators: considerations for intensivists. Am J Respir Crit Care Med, 2004. 170(9): p. 933-940.

Mishkin, J.D., et al., Appropriate evaluation and treatment of heart failure patients after implantable cardioverter-defibrillator discharge: time to go beyond the initial shock. J Am Coll Cardiol, 2009. 54(22): p. 1993-2000.

Wilkoff, B.L., et al., HRS/EHRA expert consensus on the monitoring of cardiovascular implantable electronic devices (CIEDs): description of techniques, indications, personnel, frequency and ethical considerations. Heart Rhythm, 2008. 5(6): p. 907-925. 


\title{
Strategies and Pacemaker Algorithms for Avoidance of Unnecessary Right Ventricular Stimulation
}

\author{
D. Bastian and K. Fessele \\ Additional information is available at the end of the chapter
}

http://dx.doi.org/10.5772/48747

\section{Introduction}

Since the first implantation of a pacemaker (PM) was performed in 1958, this effective form of antibradycardia therapy has evolved in an amazing way. Besides ensuring the survival of patients with asystole or complete AV block who lack a sufficient intrinsic escape rhythm, today there is a wide range of further indications including advanced therapy strategies for pacing therapy. After it was shown that frequent right ventricular (RV) stimulation (especially in the RV apex (RVA)) can be associated with clinical deterioration in patients with implanted cardioverter-defibrillators (ICD) the avoidance of unnecessary RV pacing (RVP) has become one of the cornerstones of modern ICD- and PM-therapy.

\section{Pathophysiology of right ventricular stimulation}

With intact cardiac conduction, the physiological excitation of the ventricles occurs with high velocity $(3-4 \mathrm{~m} / \mathrm{s})$ via the His-Purkinje-system (HPS) nearly synchronously. This is the basis for an optimum hemodynamic contraction sequence in all heart chambers.

Similar to the excitation in a premature ventricular contraction (PVC) or following conduction through an antegrade conducting bypass tract, ectopic ventricular stimulation will result in a more or less non-physiologic activation and therefore non-physiologic contraction sequence of the ventricles. For example, the conduction after stimulation in the RV apex (RVA) will occur mostly in the working myocardium with a significantly lower velocity $\leq 1 \mathrm{~m} / \mathrm{s}$ in an apico-basal direction from the RV via the interventricular septum to the left ventricle (LV). The exact individual sequence of excitation and contraction will be influenced by stimulus location, activation of parts of the specific conduction system and electroanatomical characteristics of the myocardium (fibrosis, scars). The dyssychronous 
contraction resulting from atypical excitation due to pacing can be compared to the situation in patients with conduction delay or block in the left Tawara-bundle (left bundle branch block (LBBB)).

At the start of systole (isovolumetric phase) the myocardial fibers near the RVA-pacing site will be the first ones to shorten, whereas the more remote left lateral ventricular areas will go into passive stretching, as they are lacking electrical excitation at this point. In the ejection phase the early activated areas will be able to contract only a little further, whereas the later activated areas will contract 1) delayed and 2) increased due to the previous stretching (Fig. 1 and 4).

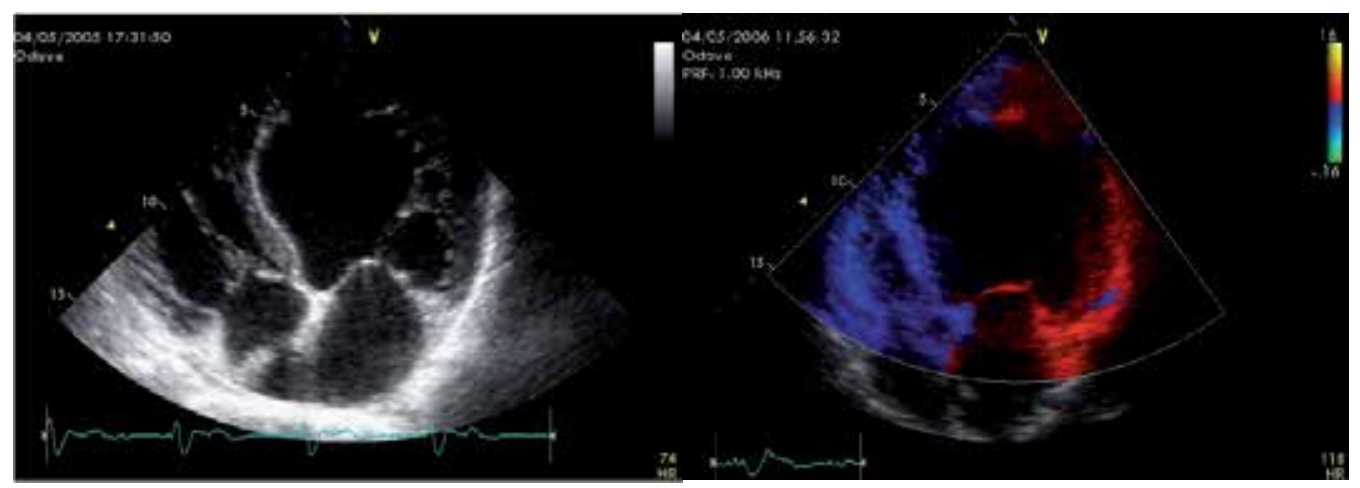

Figure 1. a. Transthoracic echocardiogram (TTE, apical 4-chamber view (A4CH)); $2 \mathrm{~b}$. Tissue Doppler. There is preserved global systolic LV-function but longstanding left ventricular dyssynchrony (here resulting from LBBB) causes a typical hypertrophy of the left lateral wall compared to the interventricular septum. This is due to the delayed systolic LV lateral wall activation and the increased contraction after the preceding passive stretching. The different colors in the Tissue Doppler demonstrate the dyssynchronous wall motion.

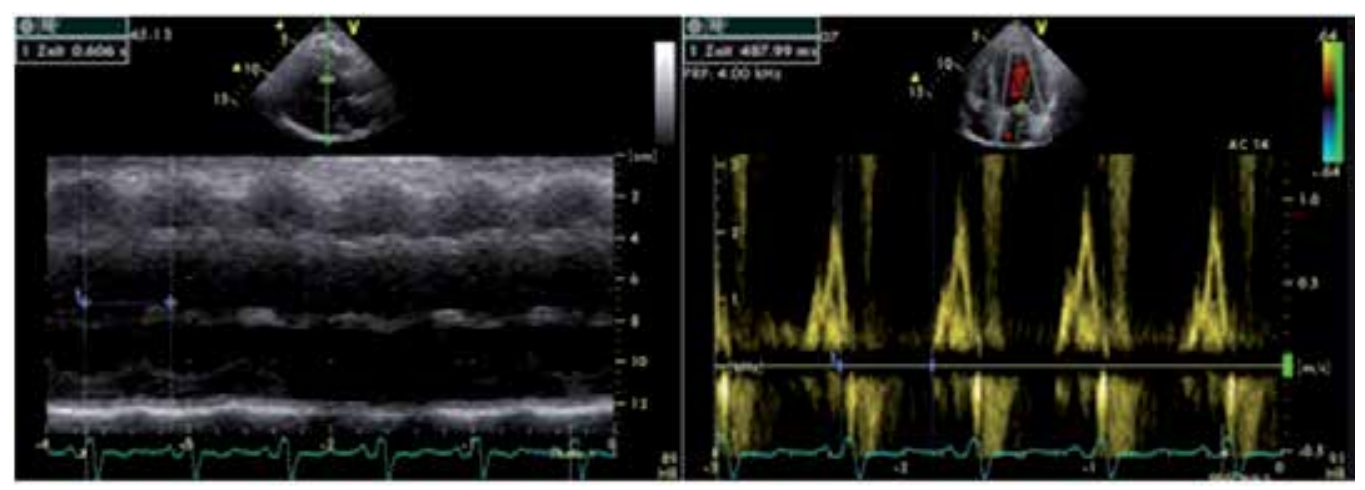

Figure 2. a. TTE, m-mode, parasternal long axis (PLAX); 2b. Doppler at mitral inflow, $\mathrm{A} 4 \mathrm{CH}$.

Demonstration of systolic contraction of the left posterolateral ventricular wall (LV-PLW) while there is already left ventricular filling. The time interval $\Delta t 1$ from the beginning of the QRS complex to the end of anterior movement of the LV-PLW (fig. 2a) is longer than $\Delta t 2$ : from the beginning of the QRS complex to the start of transmitral filling (fig. 2b). Transmitral Doppler shows fusion of E- and Awave, representing an overlap of the passive and active LV filling, which is therefore shortened and reduced. 
The electromechanical dyssynchrony influences the diastole as well. Whereas the first activated areas of myocardium already enter the relaxation cycle, the delayed activated ones can still be in systolic contraction. This late systolic contraction delays the passive ventricular filling and thereby shortens the effective duration of diastole (Fig. 2) [1].

The delayed dyssynchronous activation of the ventricles with RV stimulation is visible in the ECG by a more or less deformed and widened QRS complex (Fig. 3).

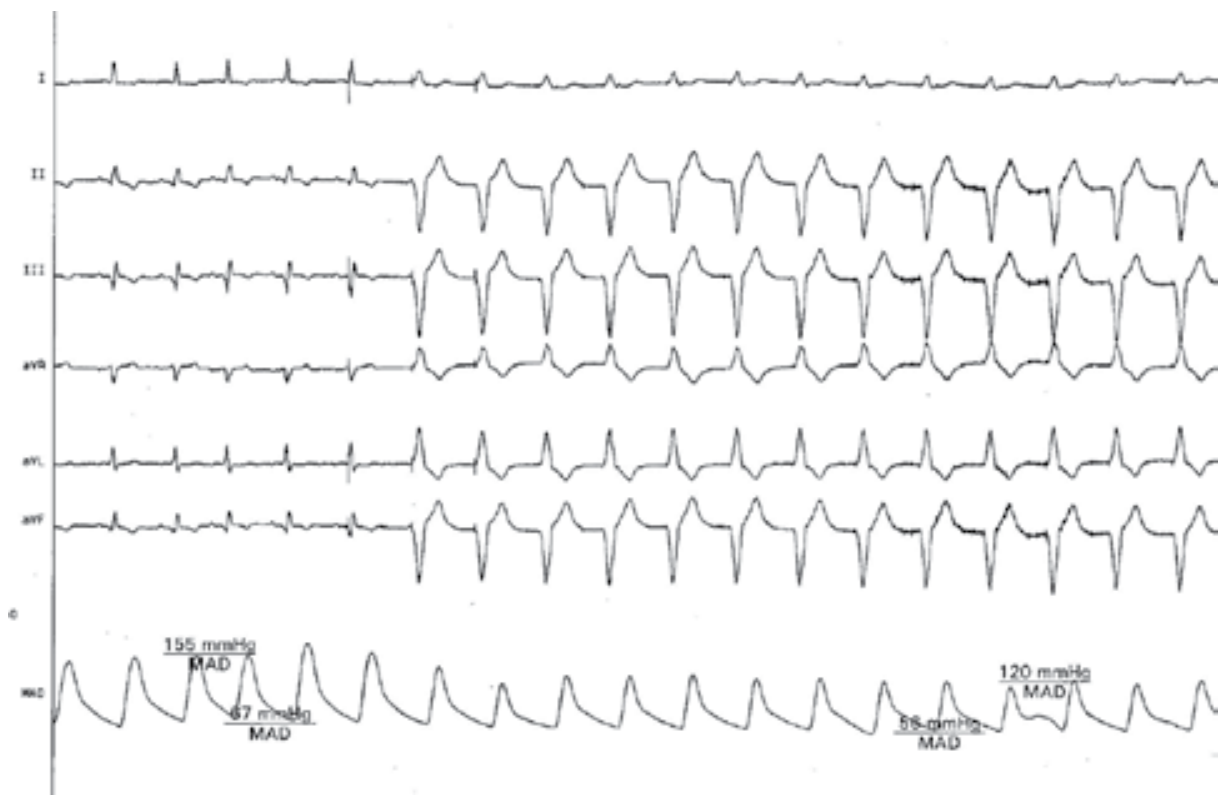

Figure 3. 12 Lead-ECG of VVI pacemaker stimulation in the right ventricular apex. The stimulated QRS complex is visibly widened, shows a negative deflection in the inferior leads (II, III, aVF) and LBBB-like deformation. Note the fall of aortic pressure with VVI stimulation here in a patient with sinus rhythm.

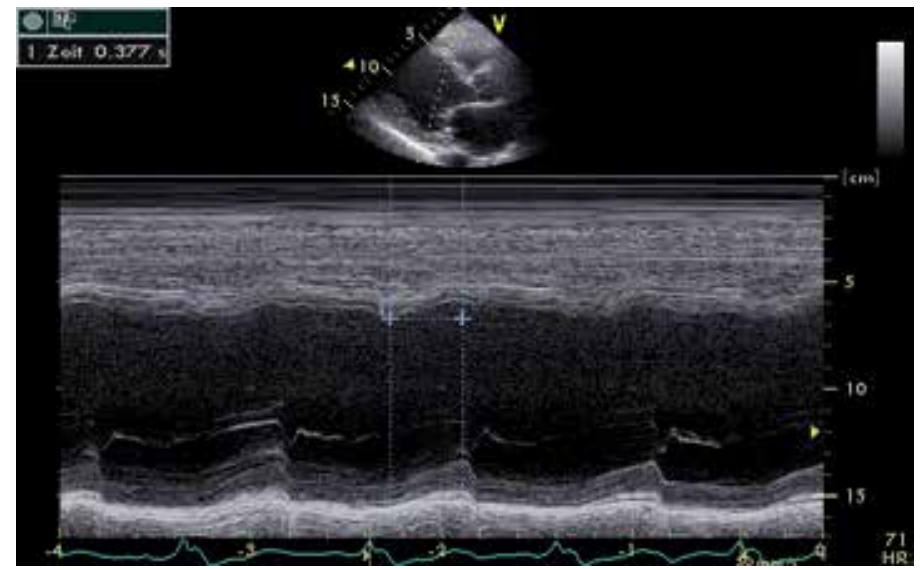

Figure 4. TTE, m-mode, PLAX. Demonstration of the different times for maximum systolic contraction of the interventricular septum and the LV posterolateral wall. The "septal to posterior wall motion delay" (SPWMD) is measured as $377 \mathrm{~ms}$ in this case. Values > 130 (140) ms are considered pathological. 
Echocardiography allows excellent noninvasive estimation of global and regional electromechanical dyssynchrony and hemodynamic consequences. Examples are shown in Fig. 1, 2 as well as 4 and 5. For more detailed information further specialized reading is recommended [2-5].

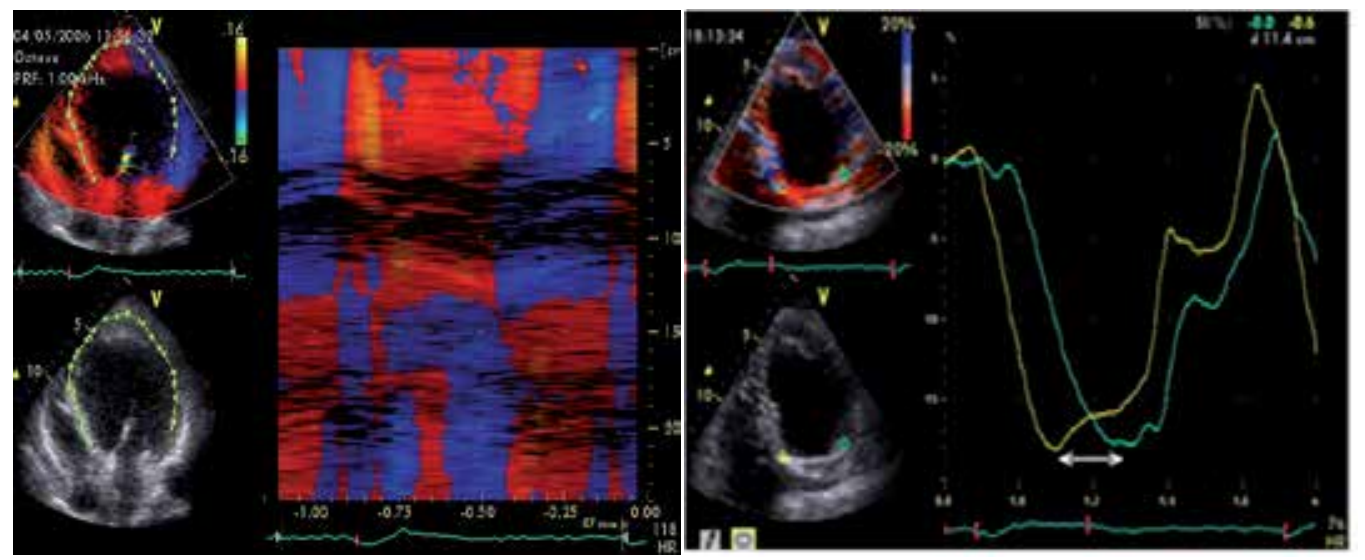

Figure 5. Visible shift of the contraction phases of interventricular septum and lateral LV wall, color-mmode (5a) and Tissue Doppler/ strain (5b).

\section{Clinical consequences of unnecessary right ventricular pacing}

The first evidence for clinically relevant negative effects of a high percentage of RV pacing was found interestingly in studies that were originally intended to show benefits of "physiologic" AV sequential DDD stimulation compared to VVI stimulation.

The original intention of the DAVID trial (Dual Chamber and VVI Implantable Defibrillator) was to show a survival benefit of dual chamber ICD systems compared to single chamber ICDs. Patients with an ejection fraction $<40 \%$ and chronic heart failure were enrolled, who didn't have an indication for antibradycardia stimulation. The study was stopped early after enrollment of 506 patients: the patients with the supposedly "physiological" AV sequential DDD pacing (at least 70/min) had a 1.61 increased risk for mortality or hospitalization because of new onset or deteriorated heart failure compared to patients with VVI backup stimulation $(40 / \mathrm{min})$ only $[1,6,7]$. The proportion of ventricular stimulation was $55.7 \%$ in the DDD group compared to $2.9 \%$ in the VVI patients.

In a sub-study of the MOST trial (Mode selection Trial) it was shown that patients with $\operatorname{DDD}(\mathrm{R})$ stimulation for sick sinus syndrome / sinus node dysfunction (SSS / SND) and normal QRS duration ( $<120 \mathrm{~ms}$ ) had a significant increase of their risk for heart failure hospitalization (HFH) associated with an increase of cumulative percentage RVP (cum\%RVP) up to $40 \%[1,8]$.

Interestingly the correlation between the percentage of RV stimulation and the risk for $\mathrm{HFH}$ was different for patients with DDDR versus VVIR stimulation. 
After detailed review it seemed that the risk for HFH stayed nearly constant for percentages of VP $>40 \%$ in the DDDR mode. It was therefore speculated that the risk of HFH in the DDDR mode would not increase with further increases in cum $\%$ RVP above $40 \%$, but a further risk reduction to about $2 \%$ could be achieved with minimization of unnecessary $\mathrm{RV}$ pacing ( $\mathrm{VP}<10 \%)$.

Overall the relative risk for hospitalization due to heart failure was always higher for VVIR patients compared to DDDR patients who had a comparable percentage of cumulative right ventricular stimulation.

Furthermore a linear relationship, between RV pacing and the incidence of atrial fibrillation $(\mathrm{AF})$, was found up to a right ventricular stimulation percentage of $85 \%$ [8].

This observation was confirmed in a prospective randomized study including 177 patients with SSS, by demonstrating that AAIR stimulation was associated with a significantly lower incidence of AF compared to DDD stimulation with a short ( $\leq 150 \mathrm{~ms})$ or a long (300 ms) AV interval [9]. Furthermore no significant changes were observed for left atrial (LA) or left ventricular diameters in the $\mathrm{AAI}(\mathrm{R})$ population, whereas in both $\mathrm{DDD}(\mathrm{R})$ groups the $\mathrm{LA}$ diameter increased significantly.

A sub-analysis of the MADIT II study (Multicenter automatic defibrillator trial II) was also able to show a clear correlation between more frequent RV stimulation and increasing morbidity and mortality for the included collective of ICD patients [10]. With increased RV stimulation the percentage of VT episodes was higher. If there was a cum $\%$ RVP of $\geq 50 \%$ a significant increase of the risk for HFH was observed $(\mathrm{p}<0.001)$.

Gardiwal et al. showed that apart from an LV ejection fraction (EF) $<40 \%$ a cum $\%$ RVP $>2 \%$ is an independent predictor for the occurrence of ventricular tachyarrhythmias, mortality and episodes of heart failure in ICD patients (who had predominantly secondary prophylactic ICD indications) [11].

Since the implementation of specific algorithms for avoidance of unnecessary RV stimulation in modern PM and ICD systems several studies have shown the clinical relevance and necessity of this approach. The details of these studies will be shown in the following sections respectively.

\section{Strategies and algorithms for avoiding unnecessary right ventricular stimulation}

\subsection{Single-chamber AAI(R) pacing}

In patients with single SND and completely intact AV conduction, the implantation of an $\mathrm{AAI}(\mathrm{R})$ system theoretically appears to be the best form of pacemaker therapy especially considering the - here $100 \%$ - avoidance of RV stimulation [12]. It has to be emphasized, that this pacing modality is so far the only one with a prognostic benefit (overall and cardiovascular mortality of 225 PM patients with SND) that was proven in a study: in the 
DANISH trial the AAI(R) stimulated patients had a higher survival rate, less deaths due to heart failure (HF) and other cardiac causes, less AF and fewer thromboembolic complications compared to the group with $\operatorname{VVI}(\mathrm{R})$ stimulation [7,12-14].

Indication:

In principle all AAI systems can be indicated in single SND without evidence of impaired $\mathrm{AV}$ conduction or concern over development of AV block in the future.

The following conditions have to be present:

- $\quad$ No AV block of any degree (including ${ }^{\circ} 1$ )

- Narrow QRS complex

- $\quad$ Antegrade 1:1 conduction / Wenckebach point >120 (130)/min

- $\quad$ No need for any medication causing conduction delay

- No carotid sinus syndrome

- No loss of consciousness as primary indication for pacing therapy.

Patients with carotid sinus syndrome or vasovagal syncope are not suitable for AAI systems, because apart from the inhibition of the sinus node intermittent AV block is encountered. [14].

Whereas the overall percentage of $\mathrm{AAI}(\mathrm{R})$ pacemaker systems is about $9-10 \%$ in Scandinavian countries [1,7], atrial single-chamber pacemakers are only implanted in selected cases in other countries. The German PM register lists for the year 2009 a nearly constant low implantation rate of $0.5 \%$ AAI systems [15]. The reasoning behind it is, that relevant AV conduction disturbances are often not detectable or foreseeable at the time of implantation, however a later manifestation cannot be predicted or excluded for the individual patient. The incidence of new onset AV block is overall low and is reported to be approximately between $0.65 \%$ and $1.8 \%$ per year $[1,12,16]$. In studies on atrial pacing for SND a median annual incidence of third-degree AV block of $0.6 \%(0 \%-4.5 \%)$ with a total prevalence of $2.1 \%(0-11.9 \%)$ was revealed. Potential clinical manifestations include

- Clinical symptoms due to AV block associated bradycardia, pauses or asystole, if there is no sufficient intrinsic escape rhythm. The incidence of syncope or near-syncope is high within the group of patients with onset of higher degree AV block.

- AAI pacemaker syndrome with non-physiologic long intrinsic, hemodynamically unfavorable AV delay (Fig. 6)

The fixation of the atrial lead in the appendage usually provides a stable position and good results for sensing and pacing threshold. Relevant ventricular far field signals should be ruled out carefully. Alternatively septal atrial (active) lead placement in the area of Bachmann's bundle (Fig. 7) can provide a more synchronous atrial activation resulting in a shorter $\mathrm{P}$ wave duration. There is some evidence that septal atrial pacing might have preventive effects on the incidence and progression of atrial fibrillation [51,52]. 


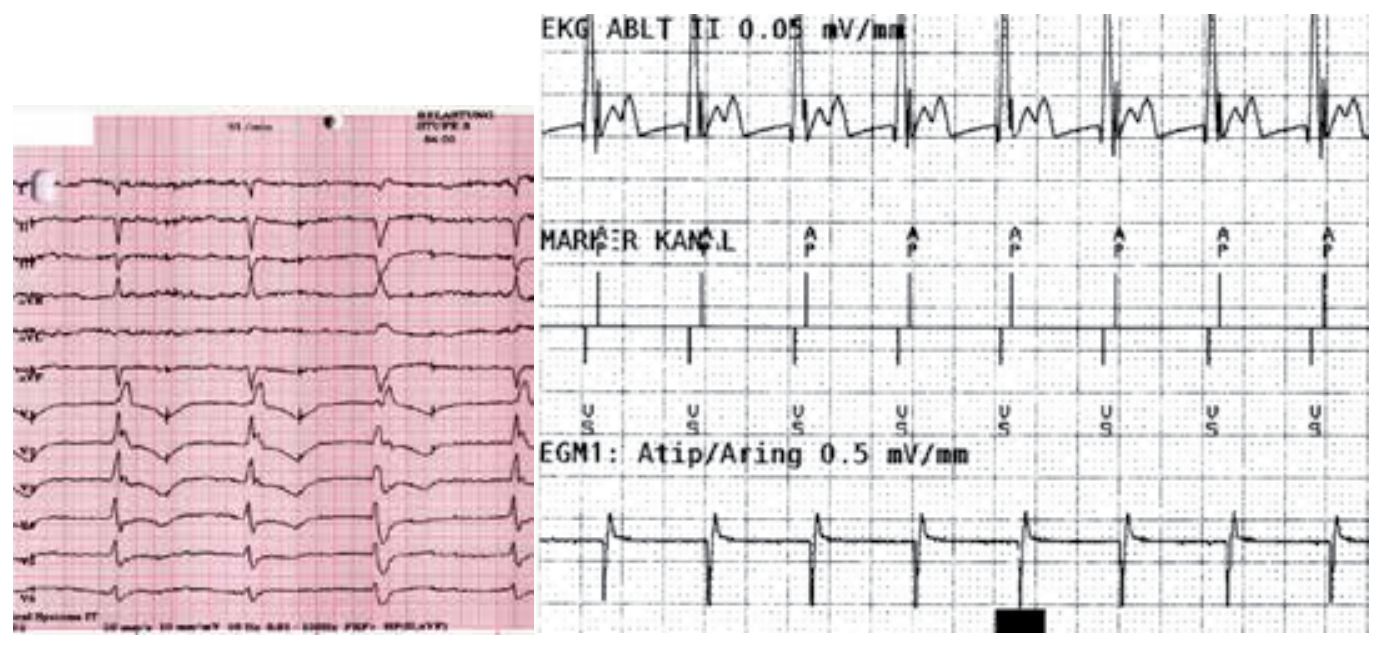

Figure 6. a. 12-lead stress test ECG: Unlike the normal physiologic response there can be a lack of shortening or even an increase of the intrinsic AV conduction delay during AAI stimulation with exercising in some patients with SND: the atrial stimulation can appear in extreme cases within the preceding systole (atrial stimulus shortly after or even within the QRS complex), the (nearly) simultaneous contraction of atria and ventricles can be the consequence, resulting in an AAI PM syndrome (6b).

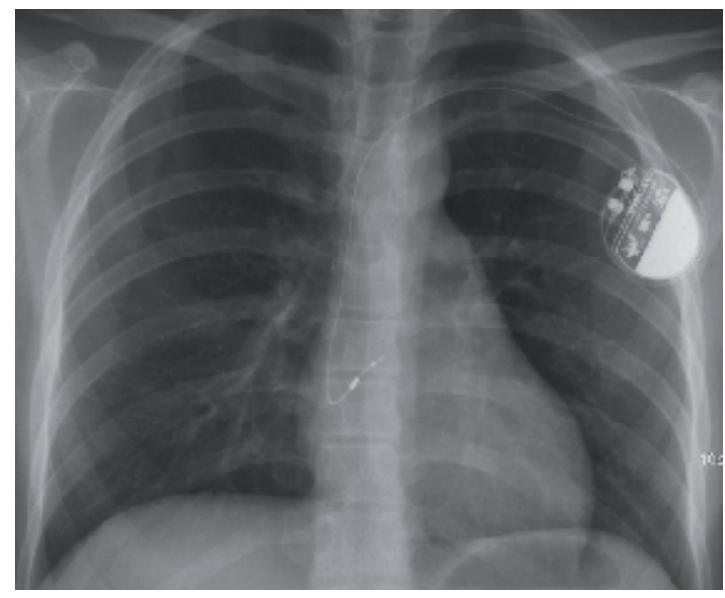

Figure 7. Chest $x$ ray. Single-chamber AAI PM implanted on the right side in a young female patient with symptomatic idiopathic sinus bradycardia. The active atrial electrode is placed at the septum, resulting in a narrow $\mathrm{P}$ wave indicating better synchronized activation of the atria.

If there is impaired AV conduction, a dual- or triple-chamber pacemaker system should be implanted. This should be considered in patients with advanced cardiac disease as well [14].

The indication for implantation of single-chamber AAI PM in patients with SND is currently questioned even more after the results of the DANPACE trial (The Danish multicenter randomised trial on AAIR versus DDDR pacing in sick sinus syndrome) [Nielsen, ESC Congress 2010]. The aim of this study was to compare AAIR with DDDR stimulation (lower 
rate $60 / \mathrm{min}$, upper rate $130 / \mathrm{min}$, paced/sensed AV-interval $\leq 220$ / $\leq 200 \mathrm{~ms}$ ). As main finding there was no survival difference for the 1415 patients in the two groups after follow up over $5.4 \pm 2.6$ years. The mortality of all causes was $29.6 \%$ in the AAIR group versus $27.3 \%$ in the DDDR group $(p=0.53)$. There was a doubling of reoperation risk with AAIR pacing. After correction for baseline variables, the patients in the AAIR group had a $27 \%$ risk increase for the development of atrial fibrillation. This contradicts the results of previous studies and therefore was not expected. However there was no monitoring regarding atrial fibrillation before enrollment, which means that a preexisting difference between the groups in AF prevalence already at baseline cannot be excluded. Additionally, monitoring in the follow up was not very sensitive for recognizing of AF episodes. The conclusion of the DANPACE authors was, that single-chamber (AAIR) pacing should be avoided in patients with SND and that DDDR stimulation using an AV interval $\leq 220 \mathrm{~ms}$ should be the pacing modality of choice for SND.

In his guest editorial for PACE in $2001 \mathrm{~S}$. Barold concluded that permanent single-chamber atrial pacing is obsolete: "Proponents of $\mathrm{AAI}(\mathrm{R})$ pacing claim it is safe but dual-chamber pacing is safer than $\mathrm{AAI}(\mathrm{R})$ pacing and more suitable for the overall care of SSS patients." [17].

\subsection{VVI stimulation with low intervention rate (,VVI backup $\left.{ }^{\prime \prime}\right)$}

The guidelines of the German society of cardiology state that VVI stimulation with a low intervention rate (e.g. $<45 / \mathrm{min}$ ) can be indicated, if there is rare disturbance of $\mathrm{AV}$ conduction (occurrence $<5 \%$ ) [indication class I B]. [14,18,19].

Fröhlig justifies the indication for implantation of a simple „VVI backup“-PM system in patients with recurrent syncope due to paroxysmal AV block, BBB and normal HV interval [1]. These patients have a high risk for further syncopal episodes. If there is no need for antibradycardia pacing apart from the short phases of paroxysmal AV block, this subgroup can be fitted adequately with a sole VVI „backup“ stimulation of 40/min.

Compared to pacemaker patients the situation looks different in patients with an ICD indication only, without need for antibradycardia pacing. The above mentioned DAVID study showed superiority of single-chamber ICD systems with a programming of VVI $40 /$ min to a dual-chamber mode [1,6,7].

In 2007 the INTRINSIC RV trial enrolling 988 ICD patients showed that the group with DDD pacing (60-130/min) with AV search hysteresis (AVSH, Boston Scientific) was not inferior to the VVI backup pacing group (40/min) in terms of all-cause mortality and heart failure hospitalization after 10 months follow up [20]. In the DDDR AVSH group the mean cum $\%$ RVP was $10 \%$ compared to $3 \%$ in the VVI pacing group. It has to be emphasized however, that prior to randomization only patients that had $<20 \%$ ventricular stimulation in the first week after implantation in DDDR AV search hysteresis mode were selected and therefore would be regarded as likely "responders" to AVSH. 
The MVP trial was a prospective, multicenter, randomized, single-blind, parallel, controlled clinical trial which didn't succeed in showing that atrial-based dual-chamber managed ventricular pacing mode (MVP $\left.{ }^{\mathrm{TM}}\right)$ is equivalent or superior to backup only ventricular pacing (VVI 40/min) with regard to time to death, heart failure hospitalization and heart failure-related urgent care in patients with standard indication for ICD therapy and no indication for antibradycardia pacing. The overall HF event rate was found to be slightly higher during AAI pacing and was mainly seen in patients with a PR interval $\geq 230 \mathrm{~ms}$ in the MVP-60 group compared to VVI-40. There were no differences between the two compared ICD pacing modes for atrial fibrillation, ventricular tachyarrhythmias, quality of life, or echocardiographic measurements. [21].

There is an ongoing discussion with regard to a possible improvement of discrimination between supraventricular and ventricular tachyarrhythmias due to additional atrial information in dual-chamber ICD compared to VVI systems as this could avoid inadequate therapy deliveries by the ICD.

The DATAS trial found a reduction of clinically significant adverse events (CSAE) in dualchamber ICD versus single-chamber devices or simulated single-chamber mode in implanted dual-chamber systems [22]. It was possible to reduce the occurrence of inadequate ICD shocks for atrial fibrillation in dual-chamber ICDs. Procedure related complications were more frequent with dual-chamber devices.

A meta-analysis from 2008 (748 patients) found less inappropriate treated episodes with dual-chamber discrimination but the number of patients experiencing inadequate therapies was not reduced [23]. It has to be known that the programmed criteria for differentiation of supraventricular and ventricular tachyarrythmias in the VVI ICD in this study were most commonly "onset" and "stability". Modern single-chamber ICDs offer markedly improved discrimination algorithms as standard today.

In summary the majority of patients with ICD indication only, i.e. lacking foreseeable demand for antibradycardia pacing or indication for CRT at the time of implantation, can be fitted adequately with a modern VVI ICD system and a backup-rate of 30-40/min. Advantages include avoidance of unnecessary RV stimulation, less expensive and less complex systems, less complications at implantation and in the long term course by using just a single lead. Most of the time in current single-chamber ICDs using modern algorithms there isn't worse SVT/VT discrimination compared to dual-chamber ICDs.

The disadvantage of this strategy is: If the need for regular antibradycardia pacing arises in the clinical course of patients with single-chamber ICDs the upgrade to a dual-chamber or CRT system is often a more complex procedure.

Therefore, dual-chamber ICD systems should be preferred in the following situations (of course unnecessary RVP should still be avoided when possible):

- Conventional PM indication (especially SND; with permanent AV block II ำ or III ${ }^{\circ}$ consider CRT-system)

- Long-QT-Syndrome 
- History of (frequent) atrial tachyarrhythmias.

\subsection{DDD stimulation with fixed long AV delay}

Programming of a fixed long atrioventricular delay (AVD) supports intrinsic conduction in patients with largely intact or only mildly impaired atrial and atrioventricular conduction and thereby avoids unnecessary RV stimulation.

However, even in patients with isolated disease of the sinus node and a programmed fixed AVD of $300 \mathrm{~ms}$, a percentage of $>10 \%$ RV stimulation is found in about every third patient $[1,24]$. It has to be kept in mind that the IEGM determined AVD of the PM is not identical with the PQ interval measured in the surface ECG. The relevant AV interval for the timing of the PM in atrial stimulation consists of: conduction time from the stimulus to the atrium, intra- and interatrial conduction times, AV conduction and the time to the expected actual detection of ventricular activation, which sometimes can be markedly delayed up to the $S$ wave of the chamber complex [25].

In clinical practice the following problems have to be considered with fixed long AVD programming [25]:

- $\quad$ A prolonged AVD results in an extension of the total atrial refractory period (TARP, fig. 8). Depending on the programmed postventricular atrial refractory period (PVARP) a limitation of the upper rate behavior can be the consequence, i.e. respectively lower limitation of the upper 1:1 AV conduction rate (upper tracking rate, 2:1-block rate), which can lead to problems with higher degree AV block on exercising.

Example: with an AVD of $300 \mathrm{~ms}$ plus a PVARP of $300 \mathrm{~ms}$, the PM is not able to detect atrial rates above 100/min 1:1 anymore and if there is a higher degree AV block to track AV sequentially 1:1.

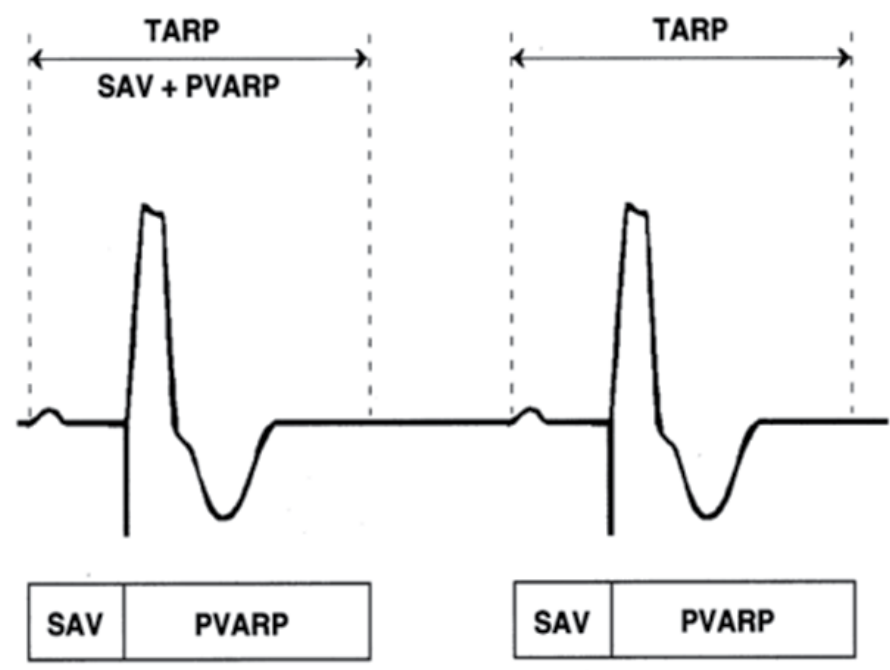

Figure 8. TARP $=$ AV Delay + PVARP 
- If as compensation the PVARP is programmed shorter in patients with preserved VA conduction, the occurrence of pacemaker mediated tachycardias (PMT) is facilitated.

- With an extremely long AVD the frame for detection of intrinsic atrial activities is limited, which sometimes - depending on the postventricular atrial blanking period (PVAB) - can possibly lead to an impairment of the mode switch reaction in atrial tachyarryhthmias [26].

- In case of higher degree AV block with resulting need of ventricular stimulation the (ultra-) long fixed AVD may result in a less favorable hemodynamic situation.

- If there is intermittent atrial undersensing (typically in atrial fibrillation) a very long AVD may favor proarryhthmogenic pacemaker-induced R-on T-stimulation. To avoid this a short postatrial ventricular blanking period should be programmed and the ventricular safety stimulation (safety window pacing) should be activated [25].

With SND and preserved AV conduction DDIR mode is recommended if a long AV delay is programmed [25,27]. Unfortunately this is not really an option in patients with intermittent AV block, as intrinsic P waves (AS-events) can't trigger AV sequential response then.

In summary the programming of fixed long AVD is associated with numerous problems. Nielsen et al. entitled a publication in 1999: "Programming a fixed long atrioventricular delay is not effective in preventing ventricular pacing in patients with sick sinus syndrome" [24]. For the effective avoidance of unnecessary RV stimulation the following modern algorithms should be preferred, if the implanted DDD PM offers these options.

\subsection{AV hysteresis}

To escape the problems associated with long fixed AVD the AV hysteresis was developed. The term "hysteresis" originates from the Greek hysteros = thereafter, later.

The algorithm distinguishes intact versus impaired / non-physiologically prolonged intrinsic $\mathrm{AV}$ conduction. A longer intrinsic AV conduction time is permitted ensuring stimulation with an optimized AV interval in case of a higher degree AV block.

Basically there are 2 sets of sensed or paced (AV / PV) atrioventricular intervals:

- $\quad$ The shorter AV-/PV delay becomes active, if the conducted intrinsic ventricular sensed (VS) event is missing.

- The longer (hysteresis) AVD will be switched to after VS events or when there is a search for intrinsic conduction [28].

The AV sequential cycle is mandatory for every beat.

- If $A V$ hysteresis is activated the stimulated or sensed (short base) AV time will be extended by a programmable amount of time after a spontaneous VS event. This now long AV interval remains unchanged, as long as there is intrinsic AV conduction within this interval (VS). After ventricular stimulation (VP) the shorter AV interval becomes active. (fig. 9). 


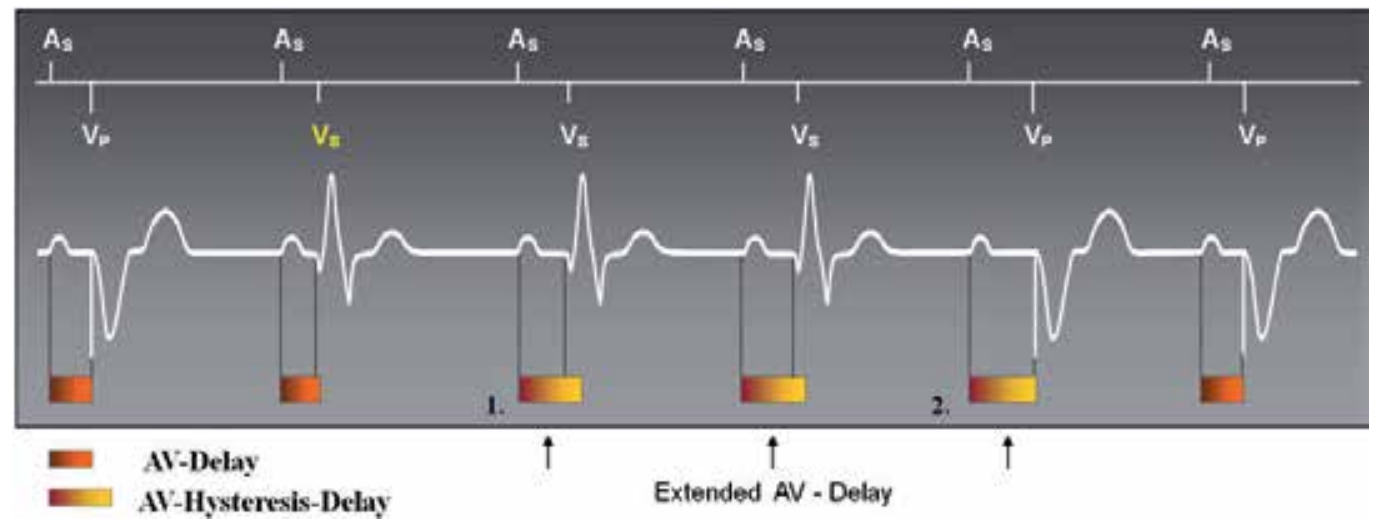

Figure 9. AV hysteresis: 1. After ventricular sensing (VS) the short AVD is extended by the programmed hysteresis interval. 2. A ventricular stimulation (VP) after the active long AVD deactivates the hysteresis and stimulation continues with the programmed short AV/PV delay.

- If an $A V$ repetitive hysteresis is activated, a single VP event will not immediately result in switching to the short base AV interval, but the long AV hysteresis interval will remain for a programmed number of cycles. The set back to the short AVD will come if there is no intrinsic conduction during these cycles.

- $\quad$ The $A V$ search hysteresis looks actively for preserved intrinsic conduction: In determined intervals the short base AV interval will be prolonged actively by the hysteresis duration for a set number of cycles (fig. 10).

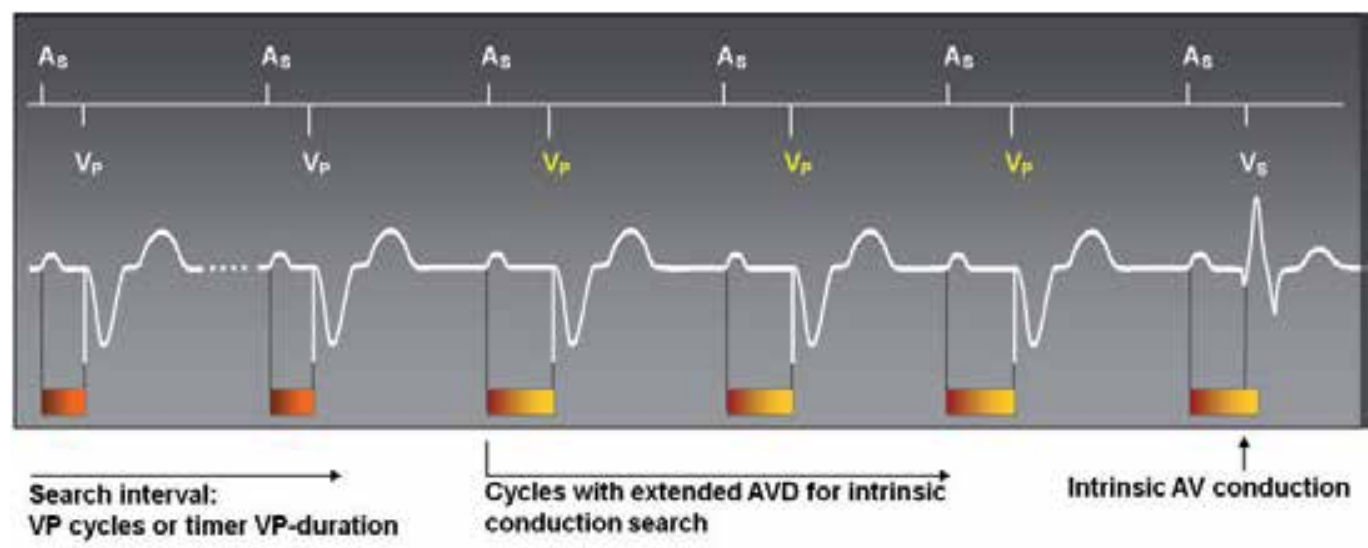

Figure 10. AV search and repetitive hysteresis.

Generally in modern devices these 3 algorithms can be activated combined in 1 function. The exact criteria that can be programmed (maximum AV time extension, search intervals) vary between the device manufacturers and models.

As examples available algorithms of 4 manufacturers are explained:

a. AV search hysteresis (AVSH (+)), Boston Scientific 
- $\quad$ Example devices: INSIGNIA $^{\circledR}$, ALTRUA $^{\circledR}$, ADVANTIO $^{\mathrm{TM}}$, INGENIO ${ }^{\mathrm{TM}}$

- Programming options (depending on the model):

- $\quad$ AV delay 10 (30) up to 300 respectively $400 \mathrm{~ms}$ in $10 \mathrm{~ms}$ steps (device dependent)

- Dynamic AVD

- Minimum (10 to $290 \mathrm{~ms}$ ) and maximum AVD (20 to 300 respectively $400 \mathrm{~ms}$; device dependent)

- AV search interval (off, 32, 64, 128, 256, 512, 1024 cycles)

- AV increase (proportional increase of AVD extension during one search cycle; $10 \%$ to $100 \%)$

- The AV delay will be extended periodically fixed or dynamical for up to 8 cycles to look for intrinsic conduction.

- If the search was successful (ventricular sensing: VS), the extension will be continued, as long as there is intrinsic conduction (fig. 11). A switch back to the programmed AV / PVD is done after the first ventricular stimulus with long hysteresis AVD.

- If the search is not successful, the stimulation continues with the programmed short AVD and a new AV search interval starts.

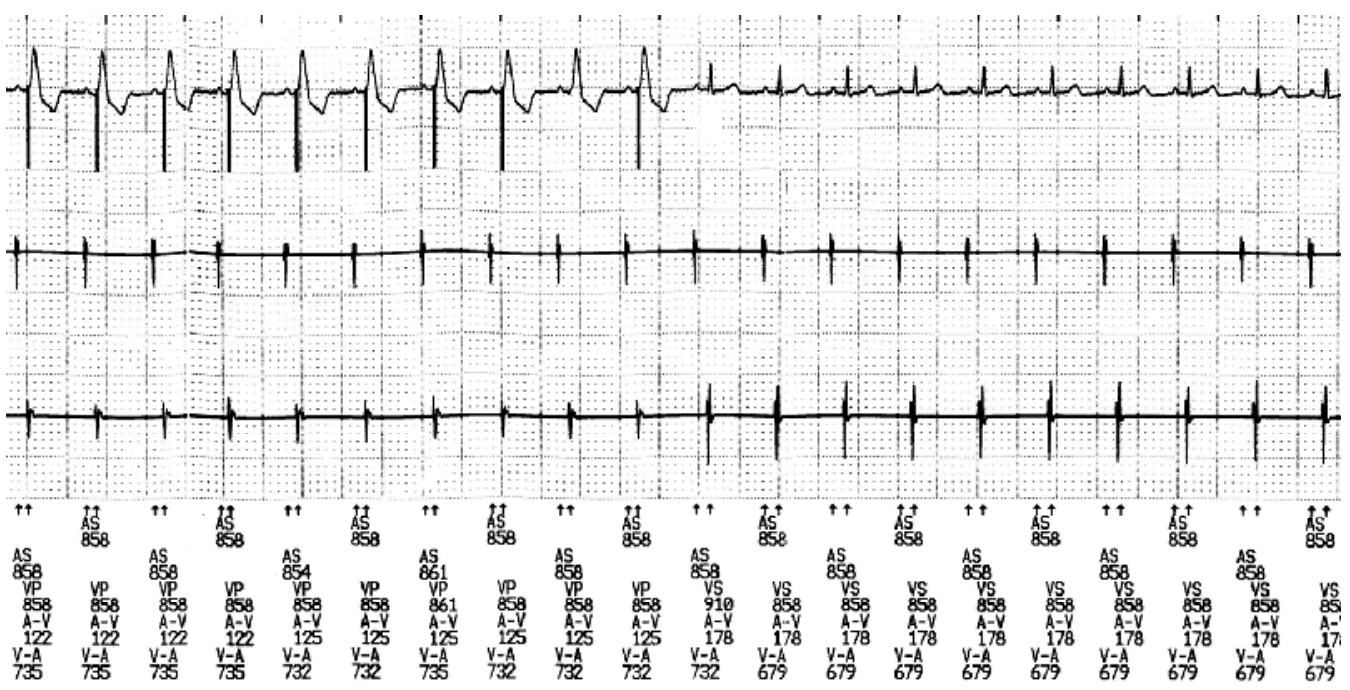

Figure 11. AVSH, BSCI. The tracing begins with AV sequential ventricular stimulation (AS/VP) with a programmed AVD of $125 \mathrm{~ms}$. The extension of the AVD by the AV search hysteresis results in intrinsic conduction: AS/VS with an intrinsic AV interval of $178 \mathrm{~ms}$.

b. AutoIntrinsic Conduction Search ${ }^{\mathrm{TM}}$ (AICS), St. Jude Medical

- $\quad$ Example device: INTEGRITY ${ }^{\mathrm{TM}}[53]$

- With ventricular stimulation the function extends the AV / PVD every 5 minutes with a programmable hysteresis time (in $\mathrm{ms}$ ) to search for intrinsic conduction.

- On ventricular sensing the extension of the AV / PVD is set, a switch back is done after the first ventricular stimulus.

- The maximum AVD is $350 \mathrm{~ms}$. 
- The function becomes inactive in the following situations:

- $\operatorname{DDD}(\mathrm{R})$ or $\operatorname{VDD}(\mathrm{R})$ mode + base rate $\geq 90 / \mathrm{min}$ + active rate dependent AVD

- Intrinsic atrial rate or sensor rate $\geq 90 / \mathrm{min}$

- During rate search hysteresis.

c. Intrinsic Rhythm Support (IRSplus), Biotronik

- Example device: Philos II DR.

- When the IRSplus is activated, the following features are set:

- $\quad$ AV hysteresis is at a fixed length of $300 \mathrm{~ms}$. The long AV interval stays active if an intrinsic ventricular signal is sensed (VS).

- In AV repetitive hysteresis there are five cycles with the prolonged AV / PV interval after a VS event has occurred. The AV hysteresis remains active, if intrinsic ventricular activity is sensed during one of these five cycles. However after five repetitive cycles without spontaneous $\mathrm{AV}$ conduction the device changes back to the short AV / PV interval.

- In AV scan hysteresis there is extension of the AV delay for five cycles after 180 consecutive ventricular paced cycles. If in these five cycles a spontaneous AV conduction is detected, the AV hysteresis stays active. If no ventricular event has been detected within these five cycles the device switches back to the short AV delay interval and the cycles end with ventricular stimulation. The cycle counter is reset and commences counting the consecutive paced cycles.

d. Search $A V^{\mathrm{TM}} /$ Search $\mathrm{AV}^{\mathrm{TM}}+$, Medtronic

- $\quad$ Example devices: Kappa 700 DR, EnPulse ${ }^{\mathrm{TM}}$

- The PM will try to detect intrinsic conducted events in an "AV delay window" that precedes scheduled VP events by -55 to $-15 \mathrm{~ms}$.

- If the device classifies 8 out of 16 AV conduction sequences as too long /"late" ( $\leq 15 \mathrm{~ms}$ before scheduled VP), it prolongs the operating SAV and PAV intervals by 31 / $62 \mathrm{~ms}$ for the next 16 pacing cycles to facilitate intrinsic conduction until the maximum AVD. If the previous $8 / 16 \mathrm{AV}$ intervals are defined as too short ( $>55 \mathrm{~ms}$ before scheduled VP), the device will shorten the operating SAV and PAV intervals by $8 \mathrm{~ms}$ for the next 16 pacing cycles.

- In the case of inadequate AV conduction (8/16 VP with maximum AVD) the search will be repeated after 15 and 30 minutes and then after $1,2 \ldots 16$ hours. The algorithm is deactivated after 10 unsuccessful searching attempts / 16 hours until the next device interrogation.

- With the help of this algorithm intrinsic conduction is promoted even in cases with slightly changing AV conduction times and unnecessary long AV delay intervals are avoided.

- $\quad$ The maximum AVD is 350 (Search $A V^{\mathrm{TM}}$ ) respectively $600 \mathrm{~ms}$ (Search $A V^{\mathrm{TM}+}$ ).

Melzer et al. compared the above mentioned algorithms Search AV TM (max. AVD sensed 230 / paced $260 \mathrm{~ms}$ ) versus Search AV+ ${ }^{\mathrm{TM}}$ (max. AVD $300 / 360 \mathrm{~ms}$ ) in a randomized study with 30 PM patients [29]. They showed that prolonging the AV interval above $300 \mathrm{~ms}$ results in an additional significant reduction of the percentage of ventricular stimulation $(19 \pm 28 \%$ versus $70 \pm 40 \%$, $\mathrm{p}<0.001)$. 
A larger prospective non randomized multi-center study enrolling 197 patients with a dualchamber PM (EnPulse) demonstrated a reduction of cum\%RVP from $97.2 \%$ without AV interval extension to $23.1 \%$ with Search $\mathrm{AV}+{ }^{\mathrm{TM}}$ [30]. There were no adverse events reported under Search $\mathrm{AV}{ }^{\mathrm{TM}}$.

\section{5. $\mathrm{AAI}(\mathrm{R}) \Leftrightarrow \mathrm{DDD}(\mathrm{R})$ mode switch}

This strategy is currently considered the most effective form of reducing unnecessary RV stimulation. A dual-chamber system (PM or ICD) is implanted in the usual way with conventional atrial and ventricular leads. The programming follows a special AAI(R) mode, by which the device controls AV conduction with every beat. If intrinsic conduction is preserved, the stimulation will be in a functional AAI(R) mode. However, as the algorithm still maintains ventricular sensing to assess AV conduction, it acts technically like ADI(R) mode. In contrast to conventional dual-chamber systems with e.g. AV hysteresis these devices are allowed to accept even single non conducted $\mathrm{p}$ waves, e.g. as in second-degree AV block type Wenckebach (fig. 12).

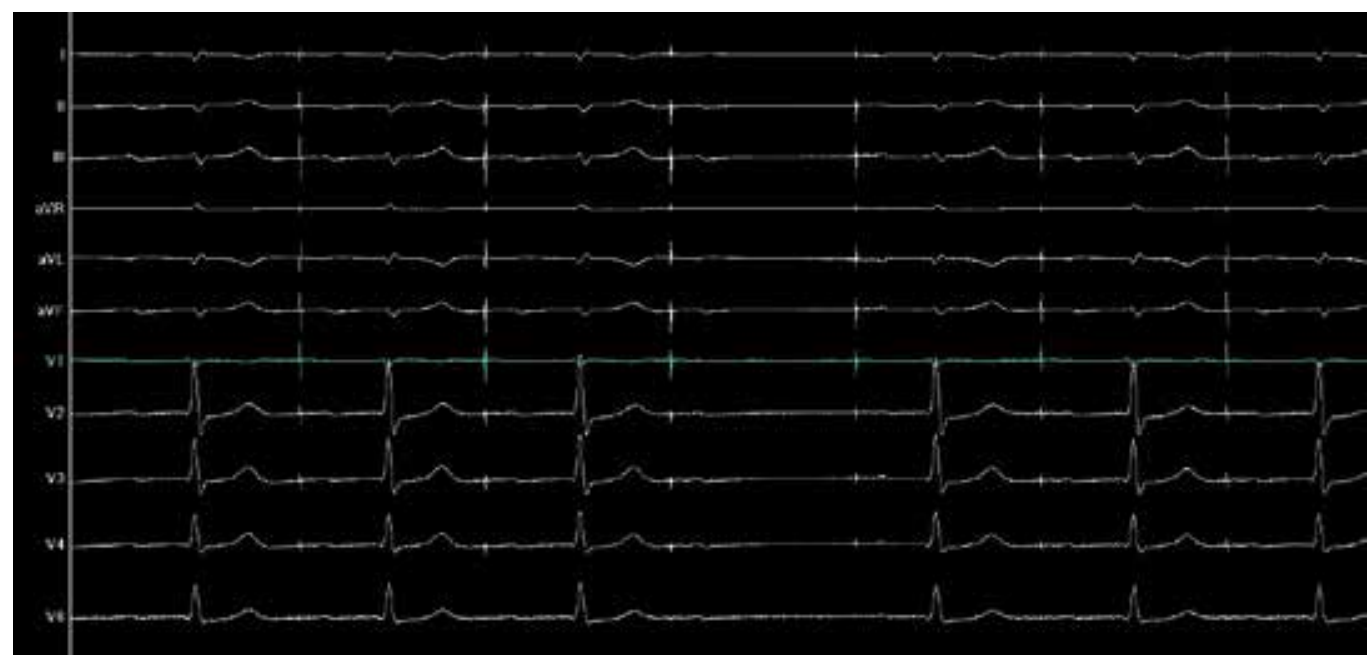

Figure 12. A dual-chamber PM with $\mathrm{AAI} \Leftrightarrow \mathrm{DDD}$ mode switch (Reply DR, AAISafeR2 ${ }^{\mathrm{TM}}$ ) accepts a single non conducted $\mathrm{p}$ wave in second-degree AV block type Wenckebach.

If a higher degree AV block occurs the device switches automatically to a dual-chamber mode according to defined criteria and keeps this up until improvement of intrinsic conduction.

This strategy thereby is thought to combine the advantages of the AAI(R) mode in avoiding unnecessary RV stimulation with the safety of $\mathrm{DDD}(\mathrm{R})$ backup.

To show examples 4 currently available systems will be explained.

a. AAISafeR ${ }^{\text {TM }}$, AAISafeR2 $^{\text {TM }}$, ELA Medical, Sorin Group

- $\quad$ Example devices: Symphony ${ }^{\mathrm{TM}} \mathrm{DR}, \mathrm{Reply}^{\mathrm{TM}} \mathrm{DR}$. 
- $\quad$ AAISafeR ${ }^{\mathrm{TM}}$ : loss of sufficient intrinsic AV conduction:

The switch to dual-chamber mode occurs following a defined pattern, by which the AV conduction is classified:

- 7 consecutive AV / PV intervals, that are too long (programmable for rest and exercise "first-degree AV block" criterion, fig. 13)

- 3 AS / AP events without VS within the last 12 atrial cycles ("second-degree AV block" criterion, fig. 14,15)

- 2 consecutive AS / AP without VS (“high degree AV block” criterion, fig. 16)

- Ventricular pause $>2$ up to 4 sec. (length of pause programmable, fig. 20)

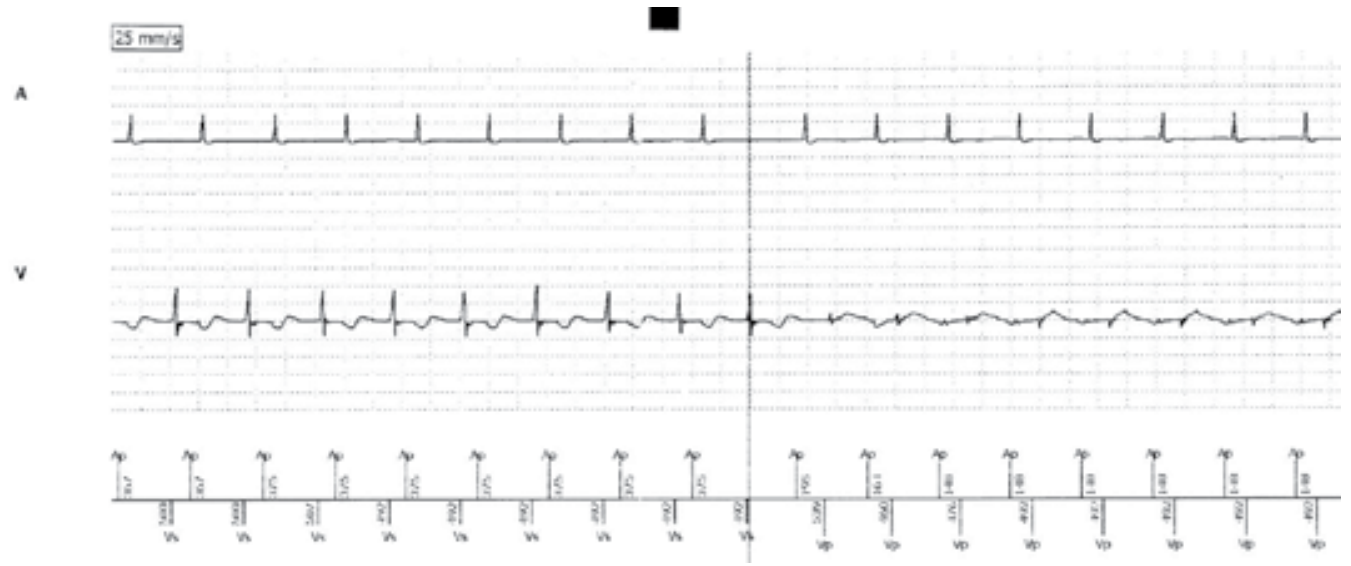

Figure 13. AAISafeR2 $2^{\text {Tw }}$, Reply ${ }^{\text {Tw }}$ DR: pacing mode switch with consecutive long stimulated AVD.

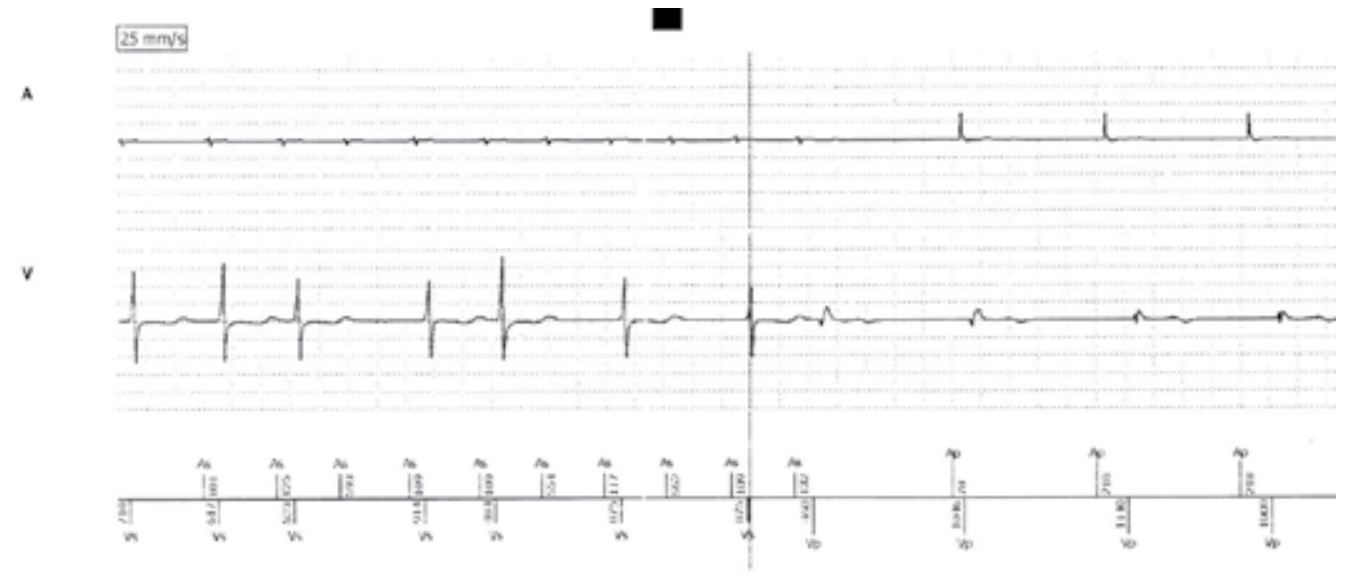

Figure 14. AAISafeR $2^{\text {Tw }}$, Reply ${ }^{\text {Tu }}$ DR: mode switch after 3 of 12 consecutive sensed or stimulated atrial events without VS. (In this particular case the pacing mode switch is triggered by a frequencydependent AV block caused by a short run of an atrial tachycardia, atrial CL about $450 \mathrm{~ms}$ ). 


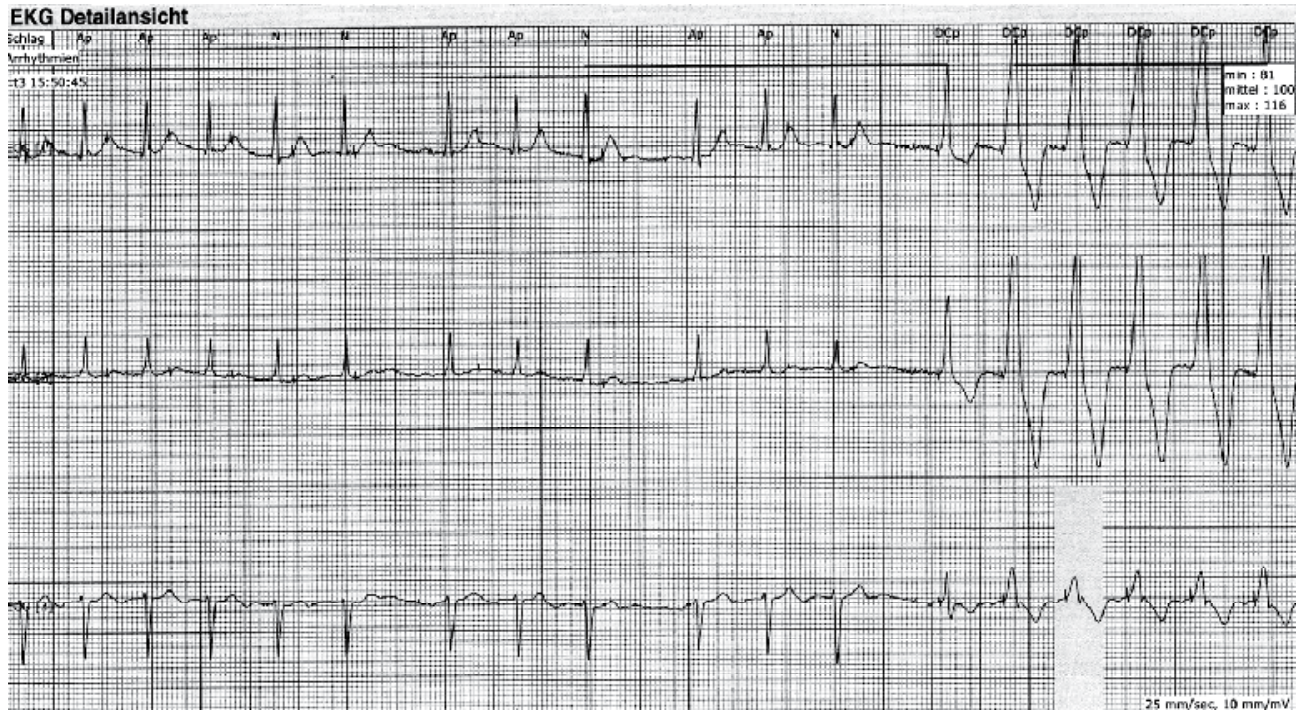

Figure 15. AAISafeR2 ${ }^{\mathrm{TM}}$, Reply DR, Holter-monitoring: switch from AAI(R) to DDD(R) after 3 (in this case stimulated) atrial events without intrinsic conduction within the last 12 AA intervals.

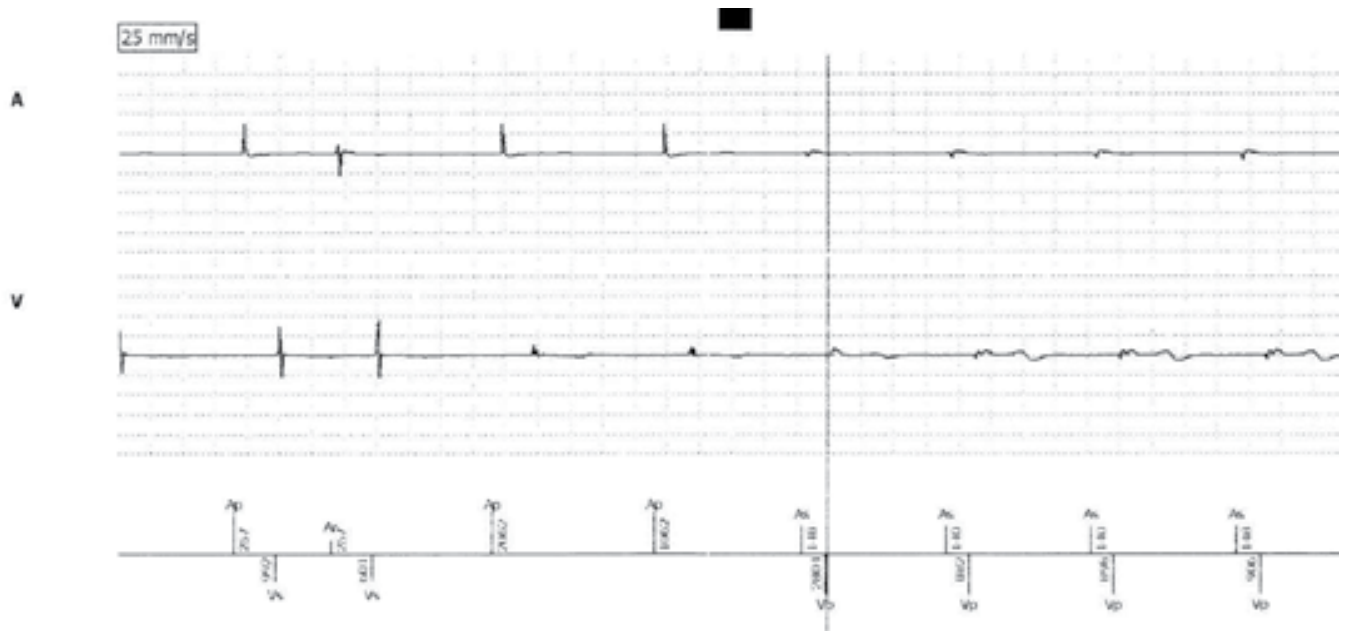

Figure 16. AAISafeR $2^{\mathrm{TM}}$, Reply $^{\mathrm{TM}} \mathrm{DR}$ : paroxysmal AV block, possibly phase 4 block caused by critical prolongation of the PP interval after a conducted atrial premature beat ("Ar"). Switch from AAI to DDD after 2 consecutive atrial stimulated events (AP) without intrinsic conduction.

- $\quad$ AAISafeR ${ }^{\mathrm{T} M}:$ Recurrence of sufficient intrinsic AV conduction

After 100 ventricular stimulations (VP) the device checks intrinsic AV conduction (fig. 17, $18,20)$. A switch back from $\operatorname{DDD}(\mathrm{R})$ to $\mathrm{AAI}(\mathrm{R})$ takes place after 12 cycles of spontaneous conduction. 


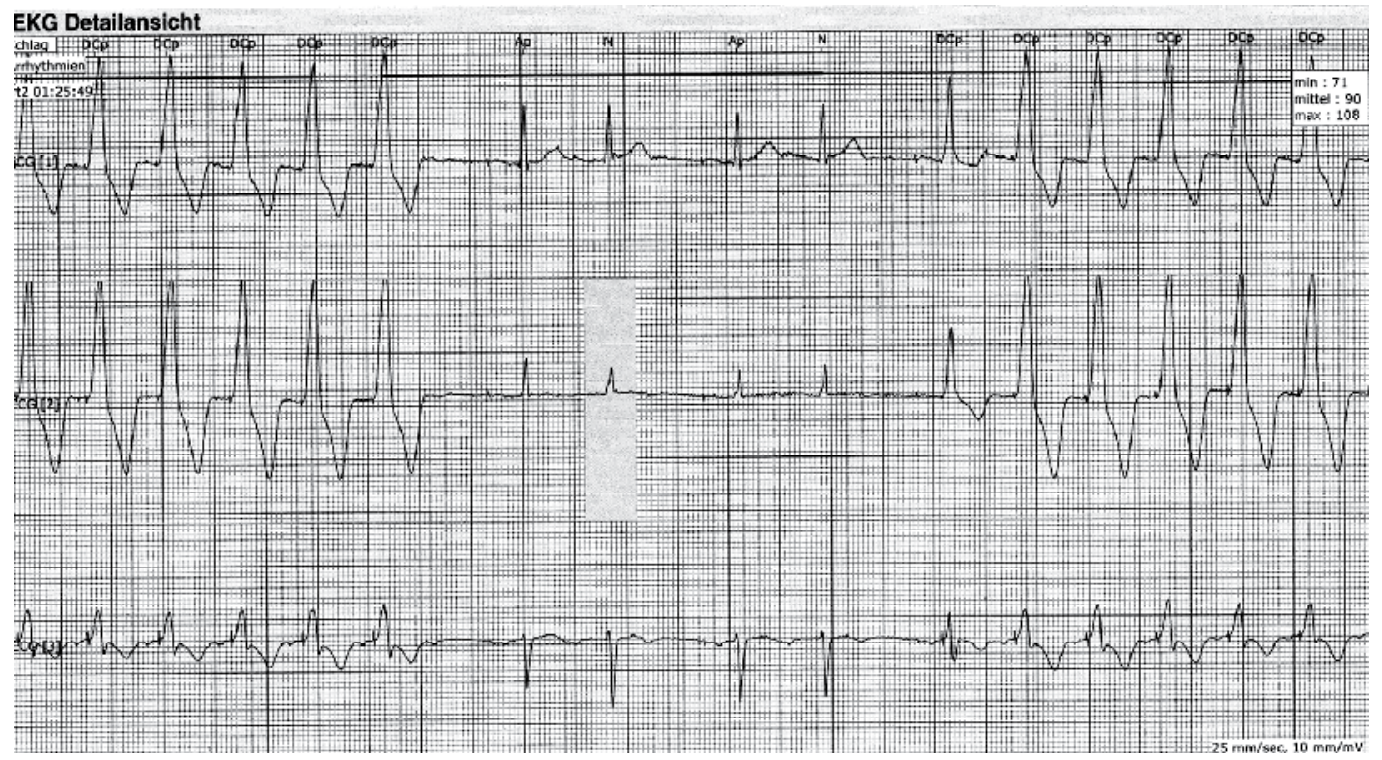

Figure 17. AAISafeR2 $2^{\text {Tw}}$ : After unsuccessful search for sufficient intrinsic conduction DDD(R) stimulation is continued.

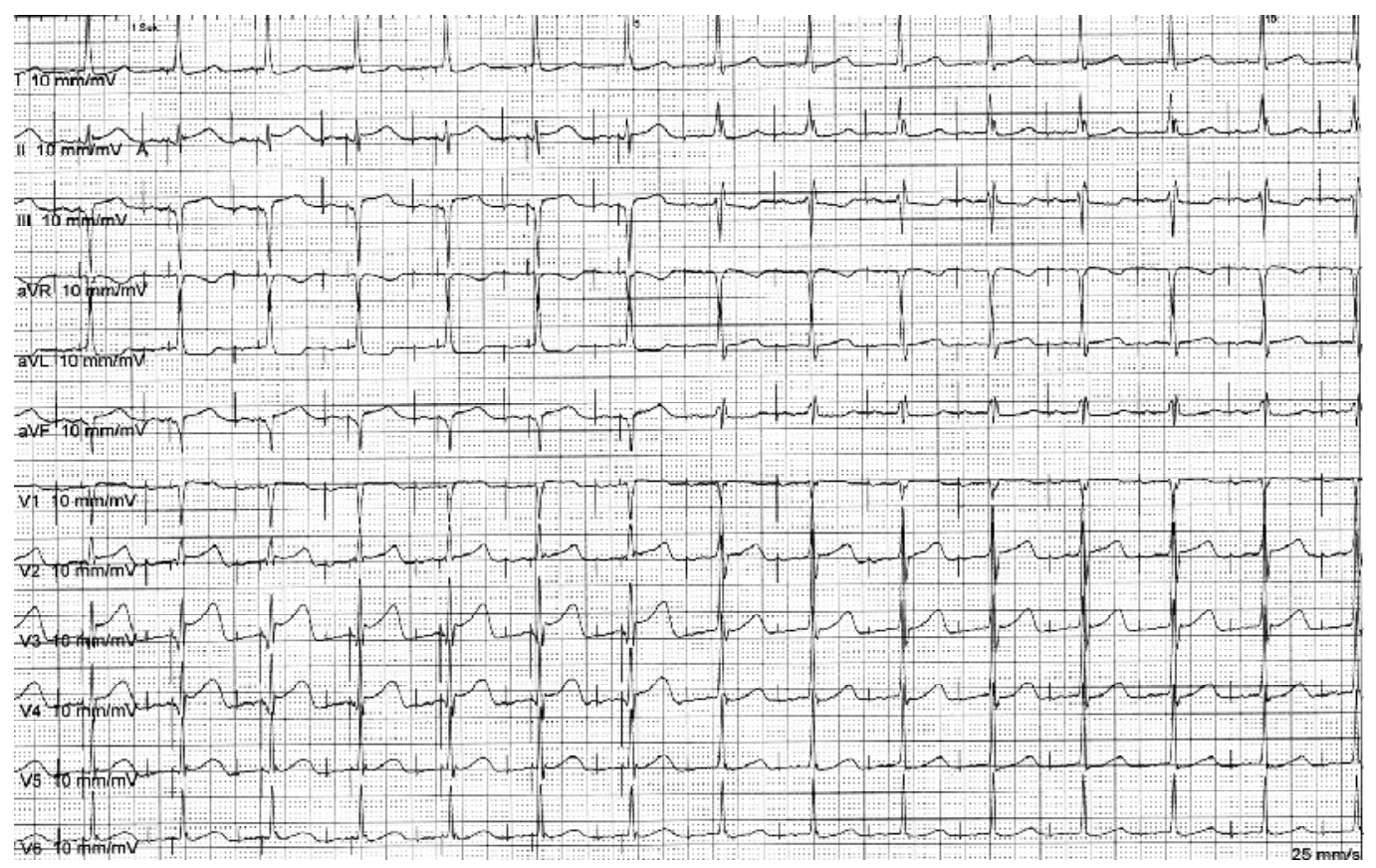

Figure 18. AAISafeR2 ${ }^{\text {TM }}$, Symphony ${ }^{\text {TM }}$ DR: Successful mode switch from AV sequential DDD pacing with ventricular fusion to AAI mode resulting in intrinsic AV conduction (APace/VSense).

In contrast to $\mathrm{MVP}^{\circledR}$ the $\mathrm{AAISafe \textrm {R } ^ { \mathrm { TM } }}$ is programmed for a permanent switch to $\mathrm{DDD}(\mathrm{R})$ mode in case of persisting AV conduction disturbance: 
- If there are $\geq 15$ mode switches within 24 hours

- If there are $>5$ mode switches per day on 3 consecutive days

A specific feature is the pacing mode switch when there is sensing of a ventricular event within the committed interval (ventricle, $94 \mathrm{~ms}$ after atrial stimulation: $\mathrm{Vr}$ ). Whereas in the DDD mode after the Vr event a ventricular safety stimulation occurs, the SafeR mode is not counting a sensed $\mathrm{Vr}$ as conducted in this committed interval. This can lead to a switch from $\mathrm{AAI}(\mathrm{R})$ to $\mathrm{DDD}(\mathrm{R})$ (fig. 19)

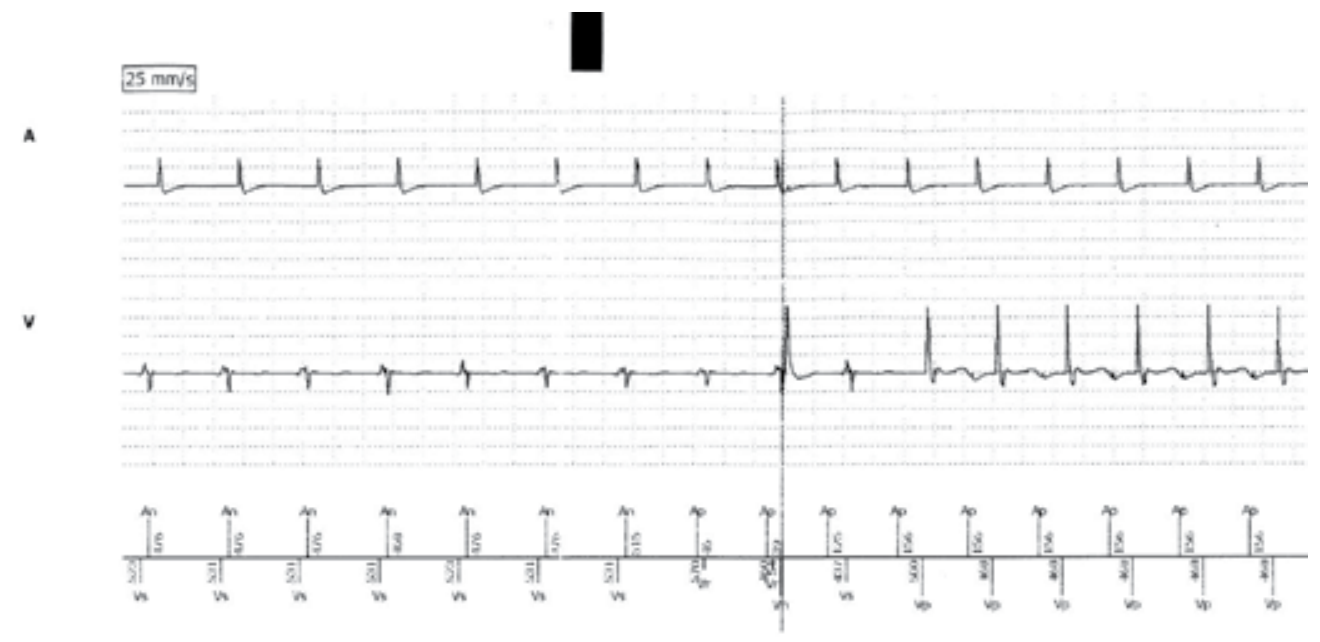

Figure 19. AAISafeR $2^{T M}$, Reply ${ }^{\mathrm{TM}}$ DR: With AAISafeR mode the occurrence of 2 consecutive ventricular events within the committed interval (Vr) results in mode switch to DDD. Once in DDD mode a safety stimulus is delivered here $(\mathrm{Vn})$.

The efficiency of the AAISafeR ${ }^{\mathrm{TM}}$ algorithm was shown in an approval study enrolling 43 patients with SSS and intermittent AV block: after 1 month $65 \%$ of the patients remained in $\mathrm{AAI}(\mathrm{R})$ mode with a ventricular stimulation percentage of only $0.2 \pm 0.4 \%$, in $35 \%$ of the patients the device automatically changed to permanent $\operatorname{DDD}(\mathrm{R})$ mode due to frequent mode switches $(73 \pm 23 \% \mathrm{VP})[1,7,31,32]$.

$\mathrm{AAISafeR}^{\mathrm{TM}}$ offers the following modifications [33]:

- The amount of time with stimulation in dual-chamber mode $(>50 \%)$ is used as a criterion for a persisting impairment of AV conduction.

- If there is AV block with an exercise induced heart rate $>100$ this is not used as criterion for "persisting AV conduction impairment".

- The switch criterion "too long consecutive AV intervals" can be inactivated for resting.

- Even after switch to $\operatorname{DDD}(\mathrm{R})$ with persisting AV block the device runs a search for intrinsic conduction every morning (fig. 20).

Fröhlig et al. investigated the algorithm in 123 PM patients with SND, paroxysmal AV block or Bradycardia Tachycardia Syndrome (BTS). In 97/123 patients an adequate switch to DDD was seen, with 69 patients (56\%) this wasn't persisting, average \%VP was $0.2 \pm 0.5 \%$ [33]. 


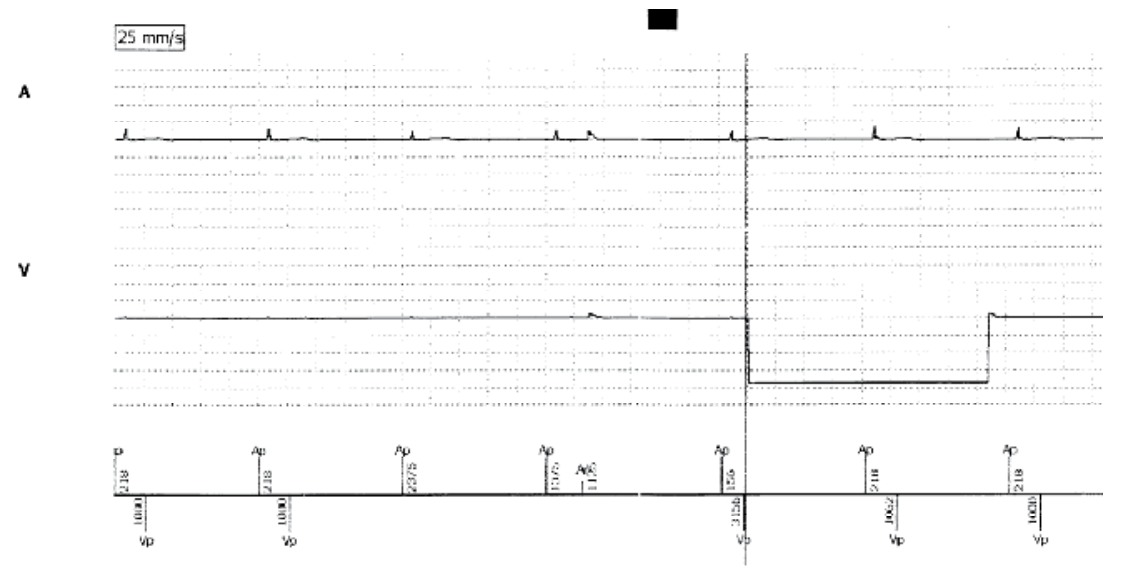

Figure 20. AAISafeR2 ${ }^{\text {Tw }}$, Reply: Unsuccessful search for intrinsic conduction in a patient with thirddegree AV block, resulting in a long pause $>3$ sec. DDD mode is maintained.

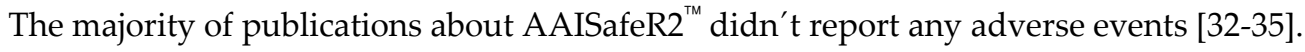

Thibault et al. observed 2 SafeR related adverse events among 208 PM patients:

1 patient with SND and second-degree AV block complained of dizziness. 24-hour electrocardiogram revealed ventricular pauses as cause, another patient with SND and firstdegree AV block presented with unexplained syncope. In both of these cases the device was reprogrammed to DDD [36].

b. Ventricular pace suppression (Vp Suppression $\left.{ }^{\circledR}\right)$, Biotronik.

- Example devices: Evia / Entovis, Estella series

Depending on evaluation of intrinsic AV conduction the device works either in $\operatorname{DDD}(R)$ or $\mathrm{ADI}(\mathrm{R})$ mode. Independent of that the system offers a mode switch to DDI(R) for atrial tachyarrhythmias (fig. 21).

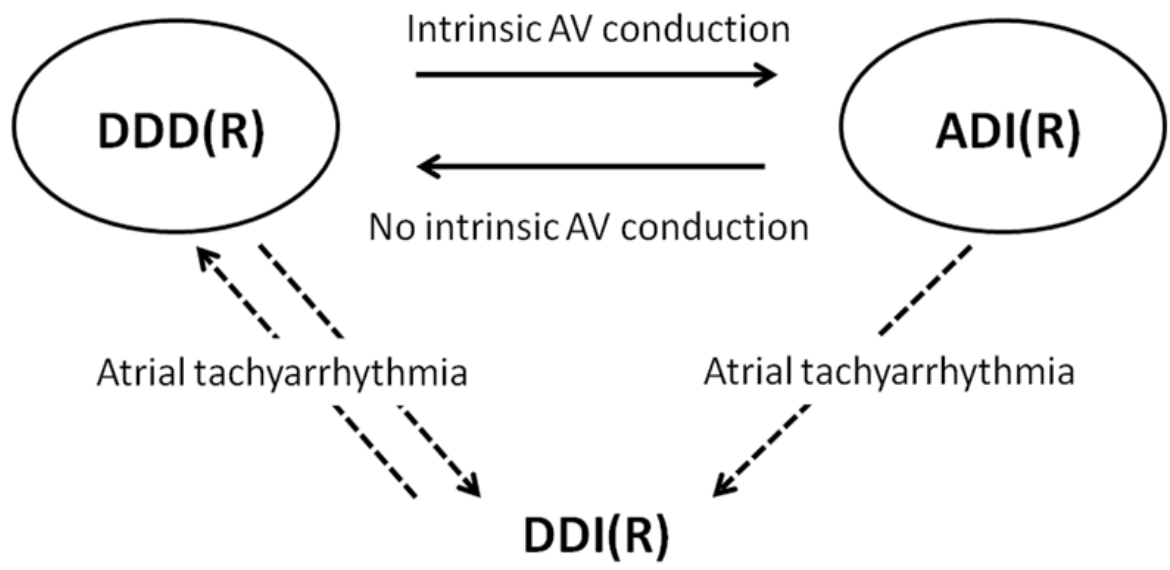

Figure 21. Stimulation forms of the Vp Suppression ${ }^{\circledR}$ algorithm, Biotronik 
- $\quad$ Vp Suppression ${ }^{\circledR}: \operatorname{DDD}(\mathrm{R})$ mode

With Vp Suppression ${ }^{\circledR}$ activated the system stimulates first in the $\operatorname{DDD}(\mathrm{R})$-mode with a programmed AVD. A VS search is started after a VS event or if there is no intrinsic conduction within $0.5 \mathrm{~min}$. AVD is then extended to $450 \mathrm{~ms}$ to search over 8 cycles for intrinsic conduction. The device switches to $\mathrm{ADI}(\mathrm{R})$, if the programmable criterion " $x$ consecutive $\mathrm{VS}^{\prime \prime}$ is met. It is possible to program $1,2 \ldots 8$ consecutive VS events in individual steps, default 6 . If the criterion isn't met within the VS search period, the device continues to work in the $\operatorname{DDD}(\mathrm{R})$ mode with the programmed AVD and the searching interval is doubled up to maximum of $128 \mathrm{~min}$. Thereafter the next search is initiated every $20 \mathrm{~h}$, as long as Vp Suppression ${ }^{\circledR}$ is activated.

- $\quad$ Vp Suppression ${ }^{\circledR}: \operatorname{ADI}(\mathrm{R})$ mode

A cycle without intrinsic conduction / VS within $450 \mathrm{~ms}$ triggers an observation period of 8 repetitive cycles, to make the decision about switching back to $\operatorname{DDD}(\mathrm{R})$ mode according to the following criteria:

- $\quad$ x/8 cycles without VS (programmable)

- 2 consecutive cycles without VS

- $\quad$ No VS for at least 2 sec.

c. RYTHMIQ ${ }^{\mathrm{TM}}$, Boston Scientific

- $\quad$ Example devices: ADVANTIO ${ }^{\mathrm{TM}}, \mathrm{INGENIO}^{\mathrm{TM}}, \mathrm{ENERGEN}^{\mathrm{TM}}, \mathrm{INCEPTA}^{\mathrm{TM}}$

The algorithm is automatically activated, if the indication-based programming (IBP) is used.

- $\quad$ RYTHMIQ ${ }^{\text {TM }}$ : Intact intrinsic AV conduction

If there is preserved intrinsic $\mathrm{AV}$ conduction, the system works in the $\mathrm{AAI}(\mathrm{R})$ mode at the lower rate limit (LRL) or sensor rate (SIR) with backup VVI stimulation at a rate which is 15/min below the programmed LRL [37]. The VVI backup can be provided between a rate not slower than 30/min but not faster than 60/min. During AAI(R) mode the device continuously checks AV synchrony.

- $\quad$ RYTHMIQ ${ }^{\text {TM }}$ : Loss of sufficient intrinsic AV conduction

The device automatically switches to $\operatorname{DDD}(\mathrm{R})$ mode, if three blocked or "slow ventricular beats" are documented within a rolling detection window of 11 beats. RYTHMIQ ${ }^{\text {TM }}$ defines a "slow ventricular beat" as a ventricular event (VS or VP) occurring at least $150 \mathrm{~ms}$ slower than the atrial pacing rate (LRL or SIR) [37].

- $\quad$ RYTHMIQ ${ }^{\text {TM }}$ : Reoccurrence of sufficient intrinsic AV conduction

From $\mathrm{DDD}(\mathrm{R})$ mode a regular search for intrinsic conduction is carried out by using the AV Search+ algorithm. The pacing mode is switched back to AAI(R) with VVI backup, if AV Search+1) can remain in AV hysteresis for a minimum of 25 intervals and 2) less than two out of the last ten cycles are VP events [37]. 
- $\quad$ RYTHMIQ ${ }^{\mathrm{TM}}:$ Mode switch for atrial tachyarrhythmias

The algorithm is able to detect atrial tachyarrhythmias from either AAI(R) with VVI backup or $\operatorname{DDD}(\mathrm{R})$. In the case of detection of atrial tachyarrhythmias the system immediately changes to the ATR mode switch.

d. $\quad$ Managed Ventricular Pacing ${ }^{\circledR}\left(\mathrm{MVP}^{\circledR}\right)$, Medtronic

- $\quad$ Example devices: Adapta L ADDRL, Ensura DR MRI'T ${ }^{T M}$ Protecta DR.

- $\quad$ MVP ${ }^{\circledR}$ : Intact intrinsic AV conduction [38]

The device works in the $\mathrm{AAI}(\mathrm{R})$ mode with programming and timing for atrial singlechamber stimulation. At the same time there is active surveillance of AV conduction.

The atrial refractory period (ARP) cannot be programmed. It is set to $600 \mathrm{~ms}$ for rates $<75 /$ min respectively $75 \%$ of ventricular cycle length (CL) for rates $\geq 75 / \mathrm{min}$. This dynamic ARP is intended to stop unnecessary switch episodes with singular non conducted atrial extra beats (PAC) or R wave far-field sensing.

If there are fast intrinsic ventricular events (e.g. PVC, VT) the atrial stimulation is inhibited. This is done to avoid unnecessary atrial stimulation, if the intrinsic ventricular rate is higher than the stimulation rate. Additionally the recognition of tachyarrhythmias is facilitated, if there is no interference by the blanking periods after atrial stimulation.

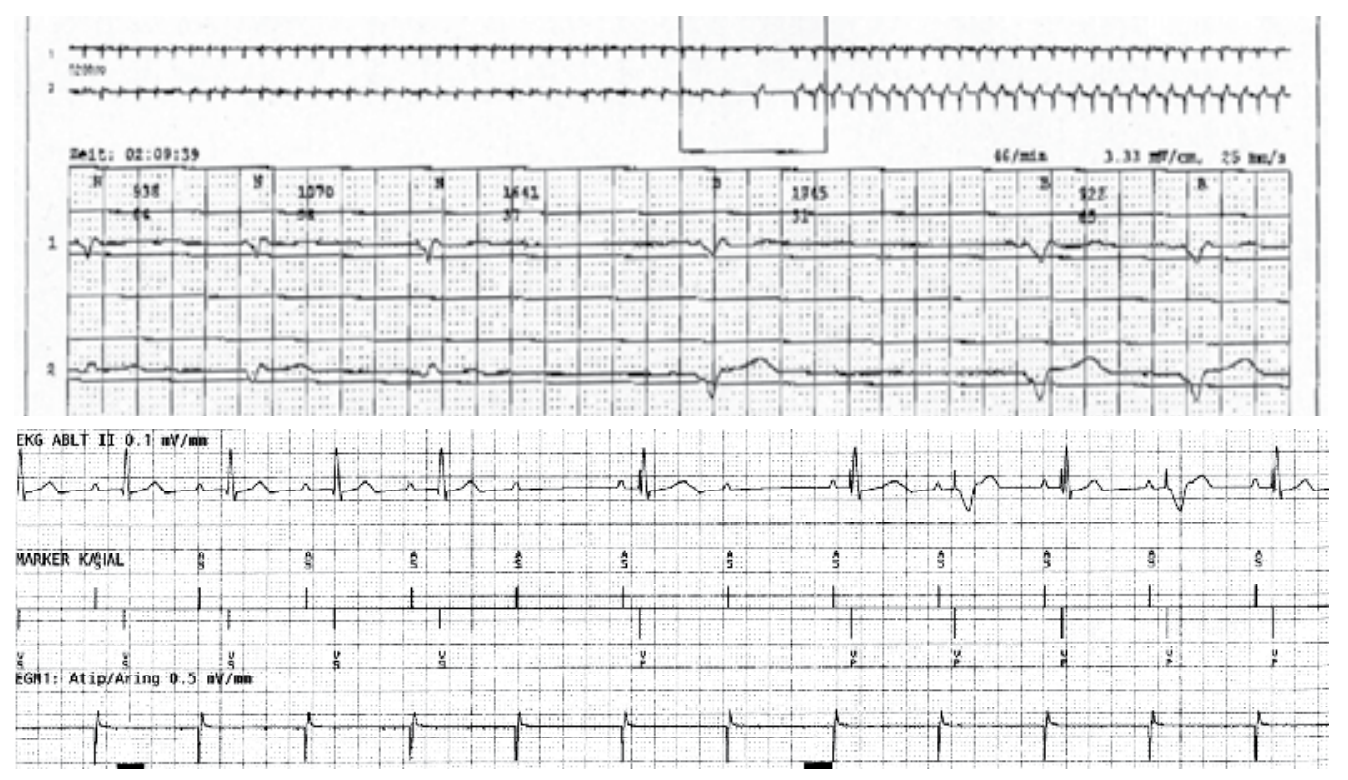

Figure 22.a. $\mathrm{MVP}^{\circledR}$ (EnTrust): Switch from $\mathrm{AAI}(\mathrm{R})$ to $\mathrm{DDD}(\mathrm{R})$ after intrinsic $\mathrm{AV}$ conduction was missing during 2 of the last 4 AA intervals. Special attention has to be paid to the fact, that the ventricular backup stimulation fires $80 \mathrm{~ms}$ after the intended (actually given or inhibited!) atrial stimulus after each AA interval without ventricular sensing (VS). (This ECG was sent to us per emergency fax as suspected ICD dysfunction: the specifics of AAI $\Leftrightarrow$ DDD mode switch can lead to substantial uncertainties).

b. $\mathrm{MVP}^{\circledR}$ (Protecta DR): Switch from AAI $(\mathrm{R})$ to $\mathrm{DDD}(\mathrm{R})$ due to sudden second-degree AV block with 2:1 AV conduction. 


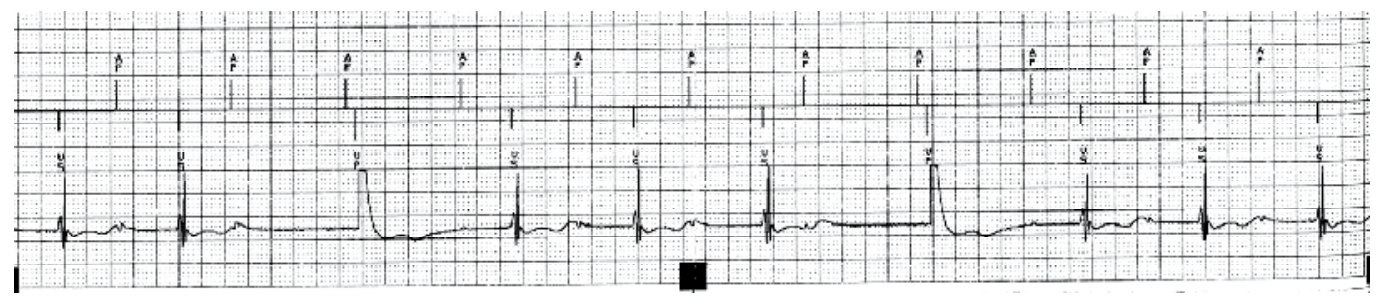

Figure 23. MVP® (Adapta DR): Action of the algorithm in a patient with second-degree AV block type Wenckebach. After there is a consecutive prolongation of the intrinsic AV conduction time (AP/VS) a singular AV conduction is missing and after the next stimulated atrial beat a ventricular stimulus is given.

The $\mathrm{AAI}(\mathrm{R})$ mode is maintained as long as intrinsic AV conduction is present. The criterion of intact intrinsic AV conduction is considered to be met, if there is a sensed ventricular event detected before the next atrial sensed event (AS) or atrial stimulation (AP).

The programmed AVD (PAV / SAV) is not relevant in this mode and will be only active after switch to $\mathrm{DDD}(\mathrm{R})$ mode.

- $\quad$ MVP ${ }^{\circledR}:$ loss of sufficient intrinsic AV conduction

The device switches automatically to a temporary $\operatorname{DDD}(\mathrm{R})$ mode, if there was no intrinsic ventricular event (VS) during 2 of the last 4 atrial intervals. After a missing VS event, there will be a ventricular backup stimulation following the next atrial action (fig. 22). If the following atrial event is not conducted either, the device switches to $\operatorname{DDD}(\mathrm{R})$ mode. Thereby singular missing VS events are tolerated (fig. 23). Two consecutive missing ventricular events however are not permitted. This behavior can cause pauses with duration of twice the cycle length of the intervention rate before switch to $\operatorname{DDD}(\mathrm{R})$, if there is a sudden loss of intrinsic AV conduction (fig. 24).
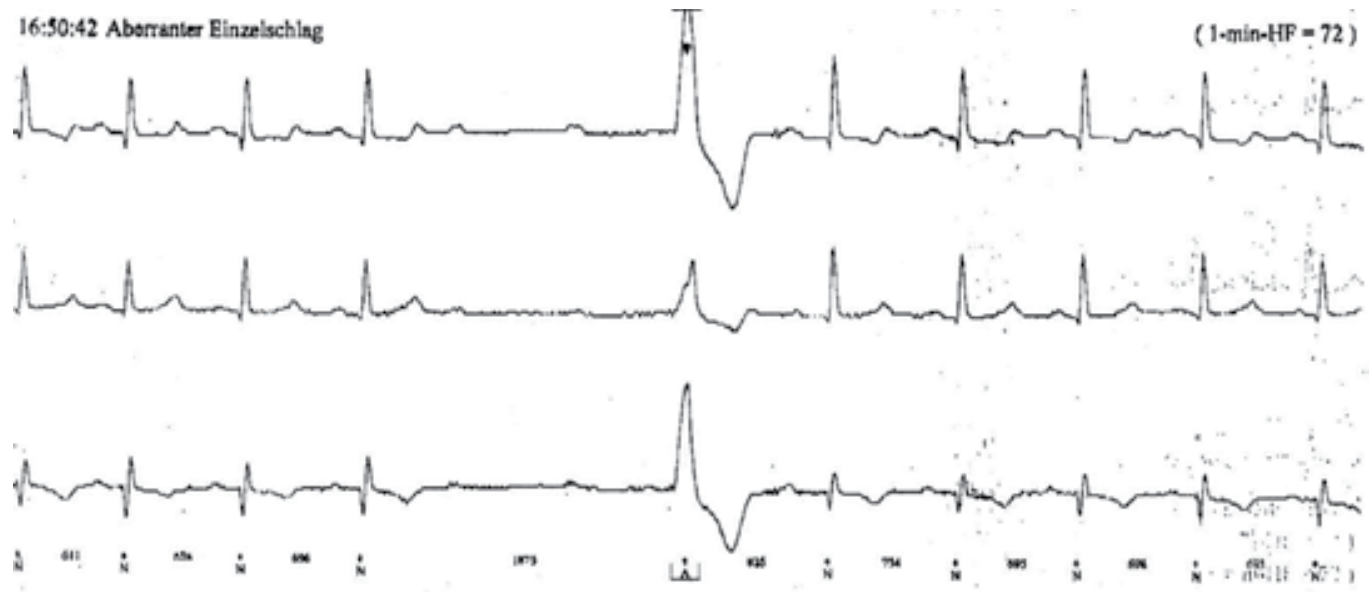

Figure 24. $\mathrm{MVP}^{\circledR}$ (Adapta DR): basic rate 50/min (1200 ms). When AV conduction is lost, a ventricular backup stimulus is given, but not with the normal AV time after the $2^{\text {nd }}$ blocked $\mathrm{P}$ wave, but $80 \mathrm{~ms}$ after the next intended (here inhibited!) atrial stimulus. The "pause" between the first blocked P wave and 
the ventricular backup-stimulus is here $1280 \mathrm{~ms}$. Because the following intrinsic AV conduction was intact again, the device stayed in the $\mathrm{AAI}(\mathrm{R})$ mode.

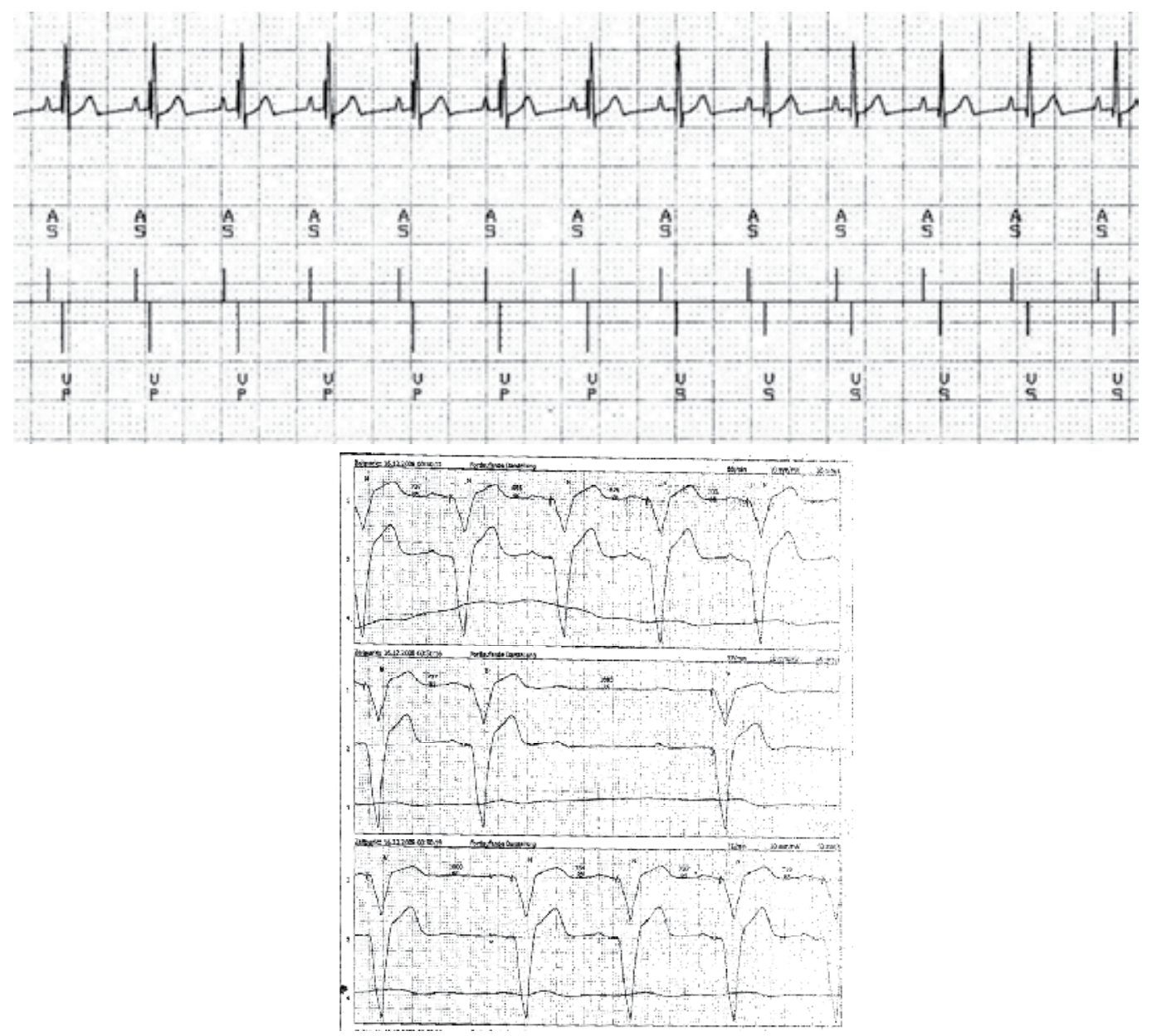

Figure 25. a. MVP® (Protecta DR): Successful test for intrinsic AV conduction, switch from DDD (AS/VP, ventricular fusion) to AAI (AS/VS).

b. MVP ${ }^{\circledR}$ : Negative test for intrinsic AV conduction in a patient with third-degree AV block.

There is no automatic switch over to a permanent $\operatorname{DDD}(\mathrm{R})$ mode.

- $\quad$ MVP $^{\circledR}$ : Reoccurrence of sufficient intrinsic AV conduction

After switching to $\operatorname{DDD}(\mathrm{R})$, the device checks the intrinsic conduction in regular intervals and thereby checks the possibility for return to $\mathrm{AAI}(\mathrm{R})$. This starts already one minute after change to $\operatorname{DDD}(R)$ with a switch to $\mathrm{AAI}(\mathrm{R})$ for one cycle.

- If there is a VS event following the next AA interval, the device remains in AAI(R) (fig 25a).

- If there is no VS event following the next AA interval, the conduction test was negative and the device remains in $\operatorname{DDD}(\mathrm{R})$ (fig. 25b). 
After each negative test the time interval doubles to the next control $(1 \Rightarrow 2 \Rightarrow 4 \Rightarrow 8$ $=>$...min). The maximum time interval is 16 hours.

As a consequence of this periodic check, patients with permanent complete AV block will have a single missing ventricular beat every 16 hours.

- $\quad$ MVP $^{\circledR}$ : Mode switch for atrial tachyarrhythmias

The device switches to DDIR both from $\operatorname{AAI}(\mathrm{R})$ and $\operatorname{DDD}(\mathrm{R})$ on the onset of an atrial tachyarrhythmia to avoid fast atrial 1:1 triggering. The mode switch to DDIR for atrial tachyarrhythmias is given a higher priority than $\mathrm{MVP}^{\circledR}$. Once the termination of the atrial tachyarrhythmia is recognized, there is a change to $\operatorname{DDD}(\mathrm{R})$ no matter which mode was active before that episode. Then an AV conduction test (1 beat) is performed and the device returns to functional $\mathrm{AAI}(\mathrm{R})$, if $\mathrm{AV}$ conduction is verified. If not, the $\mathrm{DDD}(\mathrm{R})$ mode is maintained and regular conduction tests are carried out $(1,2,4,8 \mathrm{~min} \ldots 16 \mathrm{~h})$, as described above.

The MVP ${ }^{\circledR}$ algorithm is highly effective in avoiding unnecessary RV stimulation.

In a randomized pilot study including 30 patients with dual-chamber ICDs without history of AV block MVP ${ }^{\circledR}$ "dramatically" reduced cum\%RVP from $80.6 \pm 33.8 \%$ to $3.79 \pm 16.3 \%$ $(\mathrm{p}<0.0001)$ [39]. $15 \%$ of $\mathrm{AV}$ intervals under $\mathrm{MVP}^{\circledR}$ were longer than $300 \mathrm{~ms}$. There were no relevant symptoms or adverse effects with $\mathrm{MVP}^{\circledR}$.

In 181 ICD patients, that were randomized in a prospective manner, a $99 \%$ relative reduction of the cumulative ventricular pacing percentage (cum\%VP $4.1 \pm 16.3$ vs. $73.8 \pm 32.5$, $\mathrm{p}<0.0001$ ) by application of the $\mathrm{MVP}^{\circledR}$-algorithm versus $\operatorname{DDD}(\mathrm{R})$ was found, again without adverse events [40].

In principle it is possible to reduce cum\%RVP in all patients with SND and intermittent impairment of AV conduction. According to an investigation by Gillis et al. the reduction of ventricular stimulation percentage is higher in PM patients with SND than in patients with AV block (median relative reduction $99.1 \%$ vs. 60.1\%) [41]. In a mixed PM population it was possible to reduce cum $\% \mathrm{VP}$ to $\leq 40 \%$ with the $\mathrm{MVP}^{\circledR}$ algorithm in $72 \%$ of patients [42]. Compared to AV search hysteresis Search $\mathrm{AV}+{ }^{\mathrm{TM}}$ there was a significantly lower median percent VP by application of MVP ${ }^{\circledR}$ with the exception of the patients with persisting thirddegree AV block [43]. Of the $322 \mathrm{PM}$ patients in this study the best VP reduction was found in mildly impaired AV conduction.

The safe and effective use of MVP ${ }^{\circledR}$ was shown for pediatric patients and grown up patients with congenital heart disease as well [44]. In this study it was necessary to change the programming to DDD in one case of symptomatic intermittent AV block.

The (hemodynamically) optimal form of stimulation for patients with a long AV conduction time ("long AV-block ${ }^{\circ} 1^{\prime}$ ) remains still unclear and has to be tested in the individual patient. Especially for these patients one has to consider that MVP ${ }^{\circledR}$ depending on the intervention rate will tolerate any length of $\mathrm{AV}$ conduction time, as long as there is a ventricular sensed event (VS) before the next atrial sensing (AS) or atrial stimulation (AP). 
This can lead to hemodynamically unfavorable (ultra-) long intrinsic AV times (Fig. 6). In this clinical scenario permanent $\operatorname{DDD}(\mathrm{R})$ stimulation with a fixed AV delay optimized e.g. by echocardiography may be more favorable [45].

As already mentioned before, the effective ventricular rate can drop to half of the intervention rate before switch to $\operatorname{DDD}(\mathrm{R})$, if there is a sudden loss of intrinsic conduction. Therefore it is recommended to program the basic rate of patients with sinus bradycardia or frequent $\mathrm{AV}$ block to a minimum of $50 / \mathrm{min}$.

When the available literature is reviewed, only few cases concerning clinical problems with $\mathrm{MVP}^{\circledR}$ are to be found. Mostly the algorithm worked as specified; the most common findings were:

- Atrial events, in which functional undersensing occurred due to the long atrial refractory period (Fig. 26)

- Ventricular events, in which functional undersensing occurred during the ventricular blanking time following the next atrial stimulation

- Major variations in AV delays

- Ultra long AV delays being accepted with the result of short VA intervals

- Long atrial pauses

- Occurrence of unnecessary RV pacing because of "linking", i.e. repetitive retrograde invasion of ventricular depolarization into the AV junction resulting in non-conducted P waves

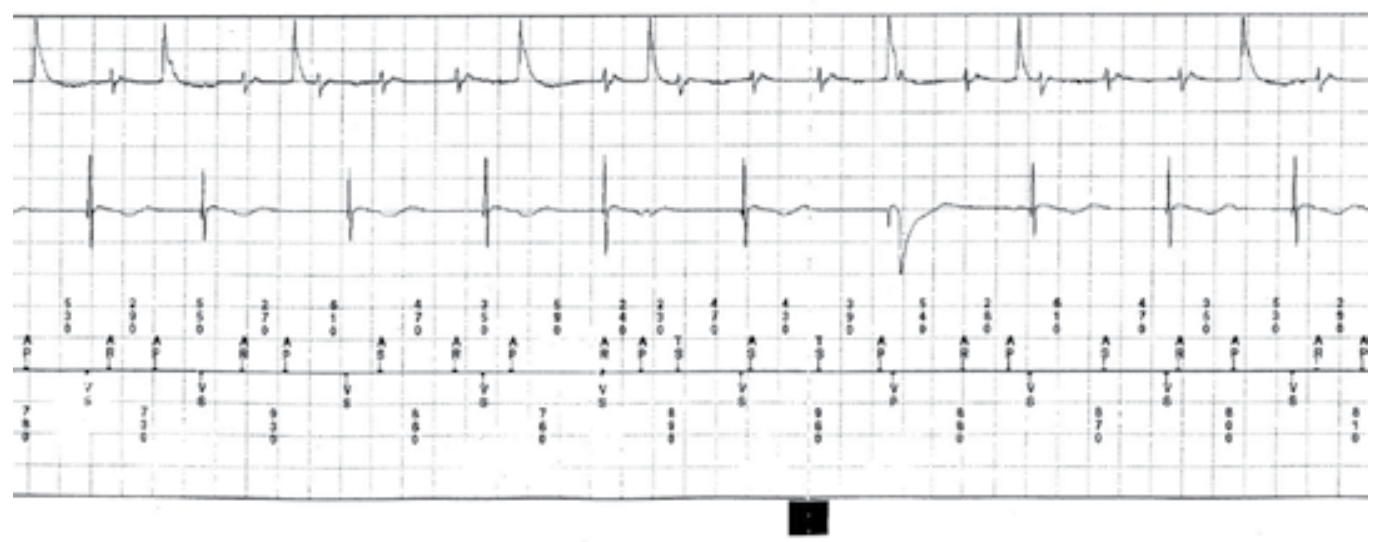

Figure 26. $\mathrm{MVP}^{\circledR}$, EnRhythm DR: Functional undersensing of atrial events due to the long atrial refractory period in a patient with a fast atrial rhythm (sinus or atrial tachycardia), CL about $480 \mathrm{~ms}$.

Murakami et al. reported 2 out of 127 Patients suffering from chest discomfort and one case with mild dizziness due to $2^{\text {nd }}$ or $3^{\text {rd }}$ degree AV block with frequent non-conducted atrial events in MVP mode [46]

A serious potentially proarrhythmogenic effect of MVP was observed by van Mechelen and Schoonderwoerd. In a patient with implanted PM for complete AV block a polymorphic VT 
degenerated to ventricular fibrillation which was successfully terminated by external defibrillation. Pacemaker interrogation showed correct device function in AAI mode (MVP) before the VT episode with irregular ventricular events (slow escape rhythm with frequent PVCs) and no AV synchronicity. The slow ventricular escape rhythm together with short coupled PVCs constituted proarrhythmogenic short-long-short cycles. Combined with documented hypokalemia, this caused the VT. As a consequence the MVP algorithm was switched off [47].

Clinical Benefit of Ventricular Pacing Reduction by $\mathrm{AAI}(\mathrm{R}) \Leftrightarrow \mathrm{DDD}(\mathrm{R})$ mode switch

Meanwhile, first study results became available showing the clinical benefit of reducing unnecessary $\mathrm{RV}$-stimulation by $\mathrm{AAI}(\mathrm{R}) \Leftrightarrow \mathrm{DDD}(\mathrm{R})$ mode switch algorithms.

In the SAVE PACe trial patients with symptomatic bradycardia due to sinus node disease were evaluated for the primary endpoint "time to persistent atrial fibrillation" [48]. Excluded were patients with persistent atrial fibrillation or cardioversion for atrial fibrillation in the preceding 6 months, second- or third-degree AV block and wide QRS complex. The 1065 enrolled pacemaker patients were randomized to either $\operatorname{DDD}(\mathrm{R})$ pacing mode (AVD 120 to $180 \mathrm{~ms}$ ) or to a dual-chamber pacing mode with a minimal ventricular pacing algorithm (MVP or SAV+). The study was stopped when in the interims analysis the pre-specified efficacy boundary $(\mathrm{P}=0.007)$ for difference in persistent atrial fibrillation between the two groups was reached (after $1.7 \pm 1.0$ years). The difference in the median cum\%RVP was substantial: $99 \%$ in the DDD group vs. $9.1 \%$ in the MVP group ( $<<0.001)$. The reduction of the risk for development of persistent atrial fibrillation was significant: $40 \%$ relative risk reduction $(\mathrm{P}=0.009)$ and $4.8 \%$ absolute risk reduction in the $\mathrm{SAV}+/ \mathrm{MVP}$ group. As clinical outcome there was a trend towards more strokes in patients who developed persistent atrial fibrillation, compared to those who did not (n.s., $\mathrm{P}=0.18$ ). There was no significant difference for mortality between the two groups. The conclusion of the authors was that in their examined group of patients with SND dual-chamber pacing with the use of a minimal ventricular pacing feature (MVP or SAV+) prevents ventricular desynchronization and is of advantage in reducing risk of persistent atrial fibrillation.

A single center randomized clinical trial done by Xue-Jun et al. compared follow up results after 3 months of pacing in DDD mode (AV delay $250 \mathrm{~ms}$ with $30 \mathrm{~ms}$ extension) with AAISafeR mode. 30 patients with sick sinus syndrome were randomized to one of the two modes for 3 months and then switched over to the other mode for another 3 months. After 3 months in DDD mode echocardiographic analysis showed that left atrial diameter, left ventricular end-diastolic diameter and left ventricular end-systolic diameter had increased significantly and left ventricular ejection fraction had decreased. However after 3 months of pacing in the AAISafeR mode no obvious changes were noted. In the AAISafeR mode cum\%RVP was significantly reduced compared to the DDD mode. The authors of this randomized trial using echocardiographic follow up concluded that AAISafeR mode is not only effective in reducing the amount of unnecessary RV pacing in sick sinus syndrome substantially, but also prevents harmful effects on cardiac performance [49]. 


\section{Conclusion}

Recently the importance of reducing unnecessary RV stimulation has been recognized widely. This is reflected in the ACC/AHA/HRS 2008 Guidelines for Device-Based Therapy of Cardiac Rhythm Abnormalities, which for the first time includes a separate chapter regarding this topic ("Importance of Minimizing Unnecessary Ventricular Pacing") [50].

Today we have several options available for this task. Which one to use has to be considered individually for each patient with an indication for a device - at the time of implantation and during follow-up.

The use of atrial single-chamber systems will stay limited to singular cases even in patients with SND, because of the missing ventricular backup if impairment of AV conduction occurs. Apart from the indication for bradycardia in permanent atrial fibrillation, singlechamber VVI PM systems as "backup" can be used as an option for patients with rare paroxysmal AV block. The other major application of single-chamber VVI backup devices with low intervention rate is in ICD therapy, if there is no need for concomitant antibradycardia pacing.

In dual-chamber systems the programming of a fixed long AVD offers a "makeshiftprogramming", if there is no other specific algorithm available. AV search hysteresis permits markedly longer intrinsic AV conduction times with stimulation with optimized AV interval in the event of higher degree AV block. An effective reduction of unnecessary RV stimulation is possible with the new $\mathrm{AAI} \Leftrightarrow \mathrm{DDD}$ mode switch algorithms. The clinical effects of wider use of these new functions need to be further evaluated in ongoing trials.

\section{Author details}

D. Bastian and K. Fessele

Klinikum Nürnberg Süd, Nuremberg, Germany

\section{References}

[1] Fröhlig G. [Why, when and how should right ventricular pacing be avoided?] Herzschr Elektrophys 2004; 15:165-176.

[2] Pitzalis MV, Iacoviello M, Romito R et al. Cardiac Resynchronisation Therapy Tailored by Echocardiographic Evaluation of Ventricular Asynchrony. J Am Coll Cardiol 2002; 40:1615-1622.

[3] Bax JJ, Ansalone G, Breithardt OA et al. Echocardiographic evaluation of cardiac resynchronisation therapy: ready for routine clinical use? J Am Coll Cardiol 2004; 44: 19.

[4] Søgaard P, Egeblad H, Kim WY et al. Tissue Doppler Imaging Predicts Improved Systolic Performance and Reversed Left Ventricular Remodeling During Long-Term Cardiac Resynchronisation Therapy. J Am Coll Cardiol 2002; 40: 723-730. 
[5] Breithardt OA, Sinha AM. Improved identification of suitable patients for cardiac resynchronisation therapy by transthoracic echocardiography. Herzschr Elektrophys $2005 ; 16: 10-19$.

[6] Wilkoff BL, Cook JR, Epstein AE et al. Dual-Chamber Pacing or Ventricular Backup Pacing in Patients With an Implantable Defibrillator: The Dual Chamber and VVI Implantable Defibrillator (DAVID) Trial. JAMA 2002; 288:3115-3123.

[7] Anelli-Monti M, Mächler H, Anelli-Monti B et al. [Avoiding Unnecessary Ventricular Stimulation in Sinus Node Disease. New strategies in bradycardia pacing.] J Kardiol 2005; 12:238-242.

[8] Sweeney M, Hellkamp A, Ellenbogen K, et al, for the MOde Selection Trial (MOST) Investigators. Adverse effect of ventricular pacing on heart failure and atrial fibrillation among patients with normal baseline QRS duration in a clinical trial of pacemaker therapy for sinus node dysfunction. Circulation 2003; 107:2932-2937.

[9] Nielsen JC, Kristensen L, Andersen HR et al. A randomized comparison of atrial and dual-chamber pacing in 177 consecutive patients with sick sinus syndrome: echocardiographic and clinical outcome. J Am Coll Cardiol 2003; 42(4): 614-623.

[10] Steinberg JS et al. The clinical implications of cumulative right ventricular pacing in the multicenter automatic defibrillator trial II. J Cardiovasc Electrophysiol 2005; 16(4):35965.

[11] Gardiwal A, Yu H, Oswald $\mathrm{H}$ et al. Right ventricular pacing is an independent predictor for ventricular tachycardia/ventricular fibrillation occurrence and heart failure events in patients with an implantable cardioverter-defibrillator. Europace 2008; 10:358363.

[12] Andersen HR, Thuesen L, Bagger JP, Vesterlund T, Thomsen PEB. Prospective randomised trial of atrial versus ventricular pacing in sick-sinus syndrome. Lancet 1994; 344:1523-1528.

[13] Andersen HR, Nielsen JC, Thomsen PEB, et al. Long-term follow-up of patients from a randomised trial of atrial versus ventricular pacing for sick-sinus syndrome. Lancet 1997;350:1210-1216.

[14] Deutsche Gesellschaft für Kardiologie (DGK) [German Cardiac Society]. Lemke B, Nowak B, Pfeiffer D (Hrsg). Leitlinien zur Herzschrittmacher-Therapie [Guidelines for pacemaker therapy]. Z Kardiol 2005; 94:704-720.

[15] Markewitz A. Jahresbericht 2009 des Deutschen Herzschrittmacher-Registers. Herzschr Elektrophys 2011; 22:259-280.

[16] Rosenqvist M, Brandt J, Schüller H. Long-term pacing in sinus node disease: effects of stimulation mode on cardiovascular morbidity and mortality. Am Heart J 1988; 116:1622.

[17] Barold SS. Permanent Single Chamber Atrial Pacing Is Obsolete. Pacing Clin Electrophysiol 2001; 24:271-275.

[18] Connolly SJ, Kerr CR, Gent M, et al. Effects of physiologic pacing versus ventricular pacing on the risk of stroke and death due to cardiovascular causes. N Engl J Med 2000; 342:1385-1391. 
[19] Tang ASL, Roberts RS, Kerr C, et al. Relationship between pacemaker dependency and the effect of pacing mode on cardiovascular outcomes. Circulation 2001; 103:3081-3085.

[20] Olshansky B, Day JD, Moore S et al. Is dual-chamber programming inferior to singlechamber programming in an implantable cardioverter-defibrillator? Results of the INTRINSIC RV (Inhibition of Unnecessary RV Pacing With AVSH in ICDs) study. Circulation 2007; 115(1):9-16.

[21] Sweeney MO, Ellenbogen KA, Tang AS et al. Atrial pacing or ventricular backup-only pacing in implantable cardioverter-defibrillator patients. Heart Rhythm 2010; 7:15521560.

[22] Almendral J, Arribas F, Wolpert Ch et al. Dual-chamber defibrillators reduce clinically significan adverse events compared with single-chamber devices: results from the DATAS (Dual chamber and Atrial Tachyarrhythmias Adverse events Study) trial. Europace 2008; 10:528-535.

[23] Theuns DA, Rivero-Ayerza M, Boersma E, Jordaens L. Prevention of inappropriate therapy in implantable defibrillators: A meta-analysis of clinical trials comparing singlechamber and dual-chamber arrhythmia discrimination algorithms. Int J Cardiol 2008; 125(3): 352-357.

[24] Nielsen JC, Pedersen AK, Mortensen PT et al. Programming a fixed long atrioventricular delay is not effective in preventing ventricular pacing in patients with sick sinus syndrome. Europace 1999; 1:113-120.

[25] Wiegand UKH. Avoidance of ventricular pacing in patients with sinus node disease or intermittend AV block. Herzschr Elektrophys 2008; 19:3-10.

[26] Israel CW. Analysis of mode switching algorithms in dual chamber pacemakers. Pacing Clin Electrophysiol 2002; 25:380-393.

[27] Nitardy A, Langreck H, Dietz R, Stockburger M. Reduction of right ventricular pacing in patients with sinus node dysfunction through programming a long atrioventricular delay along with the DDIR mode. Clin Res Cardiol 2009; 98(1):25-32.

[28] Fröhlig G, Koglek W. Schrittmacherfunktion: Algorithmen zum Erhalt der intrinsischen AV-Überleitung. In: Herzschrittmacher- und Defibrillator-Therapie. Thieme 2006: 200204.

[29] Melzer C, Sowelam S, Sheldon T, et al. Reduction of right ventricular pacing in patients with sinus node dysfunction using an enhanced search AV algorithm. Pacing Clin Electrophysiol. 2005; 28(6):521-527

[30] Milasinovic G, Sperzel J, Smith TW et al. Reduction of RV Pacing by Continuous Optimization of the AV Interval. Pacing Clin Electrophysiol 2006; 29:406-412.

[31] Savoure A, Anselme F, Galley D et al. A new dual-chamber pacing mode to prevent ventricular pacing. Europace 2003; 4(Suppl B):B175.

[32] Savoure A, Fröhlig G, Galley D, Defaye P, Reuter S, Mabo P, Sadoul N, Amblard A, Limousin $\mathrm{M}$, Anselme F. A new dual-chamber pacing mode to minimize ventricular pacing. Pacing Clin Electrophysiol 2005; 28 (1):43-46.

[33] Fröhlig G, Gras D, Victor J, Mabo P, Galley D, Savoure A, Jauvert G, Defaye P, Ducloux $\mathrm{P}$, Amblard A. Use of a new cardiac pacing mode designed to eliminate unnecessary ventricular pacing. Europace 2006; 8 (2):96-10. 
[34] Pioger G, Leny G, Nitzsche R, Ripart A. AAIsafeR limits ventricular pacing in unselected patients. Pacing Clin Electrophysiol 2007; 30 (1):66-70.

[35] Stockburger M, Trautmann F, Nitardy A, Teetzmann MJ, Schade S, Celebi O, Krebs A, Dietz R. Pacemaker-Based Analysis of Atrioventricular Conduction and Atrial Tachyarrhythmias in Patients with Primary Sinus Node Dysfunction. Pacing Clin Electrophysiol 2009; 32:604-613.

[36] Thibault B, Simpson C, Gagné CE. Blier L, Senaratne M, McNicoll S, Stuglin C, Williams R, Pinter A, Khaykin Y, Nitzsche R. Impact of AV Conduction Disorders on SafeR Mode Performance Pacing Clin Electrophysiol 2009; 32:S231-S235.

[37] INCEPTA ICD Manual, Boston Scientific, 358431-001 EN Europe 05/10, E 0086, 4-27.

[38] Medtronic, Inc. ENSURA DR MRI ${ }^{\mathrm{TM}}$ SURESCAN ${ }^{\mathrm{TM}}$ ENDR01. [Handbuch für Ärzte und Klinikpersonal]. Medtronic, Inc. Minneapolis, MN; 2010.

[39] Sweeney MO, Shea JB, Fox V et al. Randomized pilot study of a new atrial-based minimal ventricular pacing mode in dual-chamber implantable cardioverterdefibrillators. Heart Rhythm 2004; 1:160-167.

[40] Sweeney MO, Ellenbogen KA, Kasavant D et al. Multicenter, Prospective, Randomized Safety and Efficacy Study of a New Atrial-Based Managed Ventricular Pacing Mode (MVP) in Dual Chamber ICDs. J Cardiovasc Electrophysiol 2005; 16:811-817.

[41] Gillis AM, Pürerfellner H, Israel CW et al. Reducing Unnecessary Right Ventricular Pacing with the Managed Ventricular Pacing Mode in Patients with Sinus Node Disease and AV Block. Pacing Clin Electrophysiol 2006; 29:697-705.

[42] Milasinovic G, Tscheliessnigg K, Boehmer A et al. Percent ventricular pacing with managed ventricular pacing mode in standard pacemaker population. Europace 2008; 10:151-155.

[43] Pürerfellner H, Brandt J, Israel CW et al. Comparison of Two Strategies to Reduce Ventricular Pacing in Pacemaker Patients. Pacing Clin Electrophysiol 2008; 31:167-176.

[44] Kaltman JR, Ro PS, Zimmerman F, Moak JP, Epstein M, Zeltser IJ, Shah MJ, Buck K, Vetter VL, Tanel RE. Managed Ventricular Pacing in Pediatric Patients and Patients With Congenital Heart Disease. Am J Cardiol 2008; 102:875-878.

[45] Bastian D. Präsynkope unter physiologischer Schrittmacherstimlation [Pre-syncope under physiologic pacemaker stimulation]. 31st Autumn Meeting German Cardiac Society, Cologne 2007. Abstract L174. Oral Presentation. [German]

[46] Murakami Y, Tsuboi N, Inden Y, Yoshida Y, Murohara T, Ihara Z, Takami M. Difference in percentage of ventricular pacing between two algorithms for minimizing ventricular pacing: results of the IDEAL RVP (Identify the Best Algorithm for Reducing Unnecessary Right Ventricular Pacing) study. Europace 2010; 12:96-102.

[47] van Mechelen MR, Schoonderwoerd R. Risk of managed ventricular pacing in a patient with heart block. Heart Rhythm 2006; 3 (11):1384-1385.

[48] Sweeney MO, Bank AJ, Nash E, et al, for the Search AV Extension and Managed Ventricular Pacing for Promoting Atrioventricular Conduction (SAVE PACe) Trial. N Engl J Med. 2007; 357(10):1000-1008.

[49] Xue-Jun R, Zhihong $H$, Ye $W$ et al. A clinical comparison between a new dual-chamber pacing mode-AAI safeR and DDD mode. Am J Med Sci 2010; 339(2):145-147. 
[50] ACC/AHA/HRS 2008 Guidelines for Device-Based Therapy of Cardiac Rhythm Abnormalities.

[51] Epstein AE, DiMarco JP, Ellenbogen KA, et al [published correction appears in JACC. 2009;53:147]. JACC 2008;51(21):e1-62.

[52] Bailin SJ, Adler S, Giudici M. Prevention of chronic atrial fibrillation by pacing in the region of Bachmann's bundle: Results of a multicenter randomized trial. J Cardiovasc Electrophysiol 2001; 12:912-917.

[53] Israel CW. [Alternative Stimulationsorte zur Prävention von Vorhofflimmern.] Herzschr Elektrophys 2002; 13:30-43.

[54] INTEGRITY ${ }^{T M}$ AFx DR Modell 5346. User's Manual. St. Jude Medical, Inc. 2000:28-29. 


\title{
Implanted Devices and Atrial Fibrillation
}

\author{
Federico Guerra, Michela Brambatti, \\ Maria Vittoria Matassini and Alessandro Capucci
}

Additional information is available at the end of the chapter

http://dx.doi.org/10.5772/47449

\section{Introduction}

Atrial fibrillation (AF) is a supraventricular tachyarrhythmia characterized by uncoordinated atrial activation with consequent deterioration of atrial mechanical function. On the electrocardiogram (ECG), AF is characterized by the replacement of consistent $\mathrm{P}$ waves with rapid oscillations or fibrillatory waves associated with an irregular ventricular response. AF is the most common arrhythmia in clinical practice: its prevalence varies from $0.4 \%$ to $1 \%$ in the general population and increases with age, reaching $8 \%$ in patients older than 80 years [1]. AF may occur in a temporary causing condition setting, such as acute myocardial infarction, cardiac surgery, pericarditis, myocarditis, hyperthyroidism and pulmonary embolism, or in association with underlying cardiac disease such as valvular disease, coronary artery disease, hypertensive cardiomyopathy and others cardiomyopathies, especially those associated with left ventricular dysfunction and heart failure (HF). AF may also occur in younger patients without underlying cardiovascular disease and it is often referred to as "lone AF". AF may develop in isolation or in association with other tachyarrhythmias, most commonly atrial flutter or atrial tachycardia, or bradyarrhtythmias especially due to sinus node dysfunction [1].

Atrial fibrillation is very common in pacemaker recipients because of the wide range of conditions that could require device implantation and promote AF development.

\subsection{Patients implanted with dual chamber pacemaker}

Paced patients could develop AF for several reasons. On average, half of patients with dual chamber pacemaker have sinus node disease (SND) that is in turn associated with the development of $\mathrm{AF}$ in $20-50 \%$ of patients, defining the clinical picture of bradycardiatachycardia (brady-tachy) syndrome [2,3]. Moreover, nearly one third of all patients with complete atrioventricular (AV) block also shows tachy-brady syndrome. Right ventricular (RV) pacing has been demonstrated to increase the risk of developing AF in patients with 
permanent pacemaker implantation for both SND or AV block [4,5], because of its association with a number of pathophysiological changes which reduce left ventricular function and may promote AF. These changes include: an abnormal activation sequence (intraventricular and interventricular dyssynchrony), depressed left ventricular ejection fraction (EF), diastolic abnormalities and reduced myocardial perfusion [6]. As in general population, in paced patients aging is associated with a higher prevalence of AF, irrespectively of the pacing mode.

\subsection{Patients implanted with Implantable Cardioverter Defibrillator (ICDs)}

$\mathrm{AF}$ is particularly common in patients with left ventricular dysfunction and its prevalence depends on the severity of the underlying pathology. Prevalence is usually $10 \%-20 \%$ in mild to moderate $\mathrm{HF}$, and up to $50 \%$ in patients with more advanced disease [7]. Therefore, $\mathrm{AF}$ is frequent in ICD recipients, the vast majority of whom have structural heart disease. Approximately $25 \%$ of patients who receive an ICD have documented atrial tachyarrhythmias before implantation. Furthermore, a large proportion of patients without prior history of atrial tachyarrhythmias will develop AF after ICD implantation. AF occurs in about $25 \%$ of the patients with secondary prevention indication while its prevalence in patients with primary prevention indication is more difficult to define. Prevalence seems to be higher in patients with left ventricular dysfunction due to non-ischemic etiology (ranging from $15 \%$ to $25 \%$ ), and lower in patients with ischemic cardiomyopathy (about 5-10\%). Management of atrial tachyarrhythmias in patients with ICDs is important because of the increased morbidity and mortality and the increased cost of medical care.

\subsection{Patients implanted with Cardiac Resynchronization Therapy}

Cardiac resynchronization therapy (CRT) has emerged as an important and established therapy for patients with end-stage drug refractory HF due to systolic dysfunction and cardiac dyssynchrony. Several clinical trials have demonstrated the efficacy of CRT; however, the vast majority of patients included in all major trials were in sinus rhythm (SR) $[8,93]$. The low prevalence of AF patients in these trials is justified for several reasons. First, patients with AF are usually patients with more comorbidities and therefore less likely to be included in a clinical trial. On the other hand, in the absence of atrioventricular node ablation, AF reduces the likelihood of obtaining adequate pacing percentage and introduces a reasonable element of confusion when interpreting study results. Daily clinical practice is however quite different: approximately one-fifth of all patients receiving CRT in Europe has permanent AF, as reported in a recent ESC survey [9]. Patients suffering from AF are typically older, more likely to receive a CRT with pacemaker function (CRT-P), and have higher morbidity and mortality rates than patients in SR.

\section{International recommendations for pacemaker and defibrillator implants in AF patients}

Implantable devices are being commonly used for patients with AF. First of all, AF can occur in patients with SND. In SND, a wide range of cardiac arrhythmias such as sinus 
bradycardia, sinus arrest, sinoatrial block and junctional rhythm, often coexist with concomitant episodes of supraventricular tachyarrhythmias, generally AF. This peculiar clinical entity is also called brady-tachy syndrome. AF is often triggered by sudden deceleration in heart rate, sinus arrest and, in other cases, by long-short cycle sequence induced by extrasystoles. Sometimes a prolonged sinus arrest or severe bradycardia may follow the end of AF and this phenomenon is probably due to the prolonged suppression of sinus node induced by tachyarrhythmia. Patients with SND may be symptomatic for both bradyarrhythmias (fatigue, exercise intolerance, dizziness, syncope or pre-syncope) and tachyarrhythmia (palpitations, dyspnea, angina, heart failure). In these clinical situations, the implantation of a pacemaker may be necessary in order to prevent AF recurrence and control the symptoms related to bradyarrhythmia. Cardiac pacing can also facilitate the use of optimal dosages of antiarrhythmic drugs, thus preventing the bradycardia induced by the drugs themselves. Moreover, the activation of rate-responsive and dedicated algorithms can increase the benefits of antiarrhythmic atrial stimulation especially during exercise.

Permanent AF with low ventricular rate is another important clinical condition requiring pacemaker implantation. The degenerating process culminating into long-standing AF may be associated to a spontaneous disturbance of the atrio-ventricular conduction or it could be due to a scarce adherence to therapy. In this instance, the implantation of a single-chamber rate-responsive $\mathrm{PM}$ is indicated in presence of symptoms, such as fatigue, dizziness, presyncope, syncope or heart failure.

In patients with permanent or paroxysmal $\mathrm{AF}$, in which adequate control of ventricular rate cannot be achieved with drug therapy, device implantation may be also beneficial. Patients with symptoms related to rapid ventricular rates during AF require prompt medical management, and cardioversion should be considered if symptomatic hypotension, angina, or HF occurs. A sustained, uncontrolled tachycardia may lead to deterioration of ventricular function, the so-called tachycardia-induced cardiomyopathy, which tends to resolve within 6 months of rate or rhythm control. When tachycardia recurs, LV ejection fraction declines faster and HF develops over a shorter period. In this case, when pharmacologic antiarrhythmic treatment fails, atrioventricular junction ablation and pace-maker implantation and substrate catheter ablation are reasonable non-pharmacologic alternatives. A meta-analysis of 21 studies published between 1989 and 1998 that included a total of 1181 patients concluded that AV nodal ablation and permanent pacemaker implantation significantly improved cardiac symptoms, quality of life, and healthcare utilization for patients with symptomatic AF refractory to medical treatment [10].

The last issue concerns AF patients eligible to CRT. The European Society of Cardiology guidelines were the first to include patients with AF between the candidates for CRT in 2007 [11], and were recently revised and updated [12]. Nowadays, it is reasonable to implant a CRT-P in patients with LVEF $\leq 35 \%$, QRS duration $>130 \mathrm{~ms}$ and NYHA III-IV despite optimal pharmacological treatment with level of evidence $B$ for patients dependent on ventricular pacing and $C$ for those with slow ventricular response who are expected to achieve an adequate percentage of biventricular pacing. 


\section{Pacemakers and ICDs in detecting AF}

\subsection{Definition, prevalence and prognosis of asymptomatic AF}

AF can manifest itself through symptoms or it can be silent and consequently subclinical. The true prevalence of AF is dependent upon the method used for the diagnosis of this arrhythmia [13]: in general, current data showed an increased AF detection rate with longer monitoring durations. Twelve leads electrocardiogram controls are not very sensitive, detecting less than $30 \%$ of all AF episodes [14], whereas more AF paroxysms can be unmasked by using Holter recordings, telemedicine techinques, and loop recorders. Implanted devices, whether pacemakers or ICDs, further enhance the diagnostic yield of asymptomatic atrial arrhythmias by providing continuous rhythm monitoring.

In a study of patients with paroxysmal $\mathrm{AF}$ and conventional indication for permanent pacemakers, Israel et al. found that more than one third of all device documented AF of at least 48 hours duration were asymptomatic [15]. A multicenter study on pacemaker diagnostics also reported a high incidence of atrial arrhythmia in the pacemaker population, with atrial high rate episodes (AHRE) documented in $89 \%$ and $46 \%$ of patients with and without prior history of atrial tachyarrhythmia by 24 months of follow-up, respectively [16]. This study demonstrated that patients with prior history of atrial arrhythmia had higher arrhythmia burden and, again, most device-detected AHRE were asymptomatic.

However, silent AF and symptomatic AF share exactly the same risk for cardiovascular death and cardiovascular events [1]. A major concern with the onset of AF that progresses without any symptoms is the risk of cerebral embolism, with acute stroke its first manifestation. In about $25 \%$ of patients who have ischemic strokes, no etiologic factor is identified but subclinical AF is often suspected to be the cause. In the AFFIRM trial, which tested rate control versus rhythm control, stroke occurred in both groups, particularly in patients among whom anticoagulants were discontinued on the assumption that sinus rhythm was successfully maintained [17]. Recently, the ASSERT study found that pacemaker patients who have no history of atrial tachycardia AT or AF, but do have devicedetected arrhythmias, are approximately 2.5 times more likely to have a stroke than patients who do not have device-detected arrhythmias [18]. Nevertheless, it is currently unknown if treatment of asymptomatic episodes detected by the device should be the similar to what already recommended for symptomatic AF.

Another relevant aspect related to the absence of symptoms is the development of atrial remodeling. Intermittent asymptomatic AF episodes can cause changes in the electrophysiological and histological matrix of the atria, thereby facilitating the creation of appropriate conditions for degenerating AF into permanent type.

\subsection{Usefulness of implanted device in AF detection - New diagnosis, relapse monitoring, event recording, fibrillation burden and rate-control}

All implanted devices (pacemakers, ICDs and CRTs) including loop recorders are capable of identification, recording and transmission of electrocardiographic data. Among all the data 
available, a modern device can store total number of episodes, time of onset and duration of each episode, the overall burden of arrhythmia and the ventricular electrocardiogram morphology (VEGM) associated with each detected episode. These continuous monitoring modalities have improved knowledge into the characteristics of AF.

As previously underlined, implanted devices are useful to detect new episodes of AF. Ricci et al. detected AF in 42 of 166 patients using implanted pacemakers or ICD over a period of 18 months. Interestingly enough, AF was not known before device implantation in more than half of these patients [19].

In patients without a definitive device implant indication implantable leadless loop recorders may be useful in order to detect $\mathrm{AF}$ and quantify its burden. The XPECT trial tested an AF detection algorithm incorporated into an implantable leadless loop recorder against traditional monitoring through 48-h ECG Holter [20]. The results of this study indicated a higher sensitivity of loop recorder for detecting AF. However, specificity was limited by falsely stored AF episodes in $15 \%$ of the patients. In addition, continuous monitoring can contribute to better therapy management, through better rate control therapy or monitoring of rhythm control especially after catheter ablation. In the latter case, continuous assessment of freedom from tachyarrhythmia recurrences may lead to discontinuation of oral anticoagulation after a successful procedure in selected patients.

\subsection{Supraventricular tachycardia or ventricular tachycardia? Discriminating algorithms in modern devices}

The MADIT-II, SCD-HeFT, and MUSTT trials indicate that ICD therapy improves survival with a significant reduction in mortality [21-23]. However, nearly half of all shocks experienced by ICD patients are inappropriate, an outcome resulting in poorer quality of life, pain, psychological distress, shorter battery life and device-induced arrhythmias. To reduce inappropriate therapy of supraventricular tachycardia (SVT), ICDs include algorithms engineered to discriminate ventricular tachycardia (VT) from SVT. Programming of discrimination algorithms varies from single chamber to dual chamber ICDs, and should be tailored according to the patient's specific needs, comorbidity and previous history of SVT such as paroxysmal AF.

\subsubsection{Single-Chamber SVT-VT discriminators}

Most single chamber devices currently include four elements into detection algorithms: RR onset, RR stability, VEGM and sustained rate duration. RR onset is based on the evidence of abrupt onset present in most VT, in contrast to the gradual onset of sinus tachycardia. It has high specificity for rejecting sinus tachycardia but may prevent detection of VT that originates during SVT and VT that starts abruptly with an initial rate below the VT detection rate. 


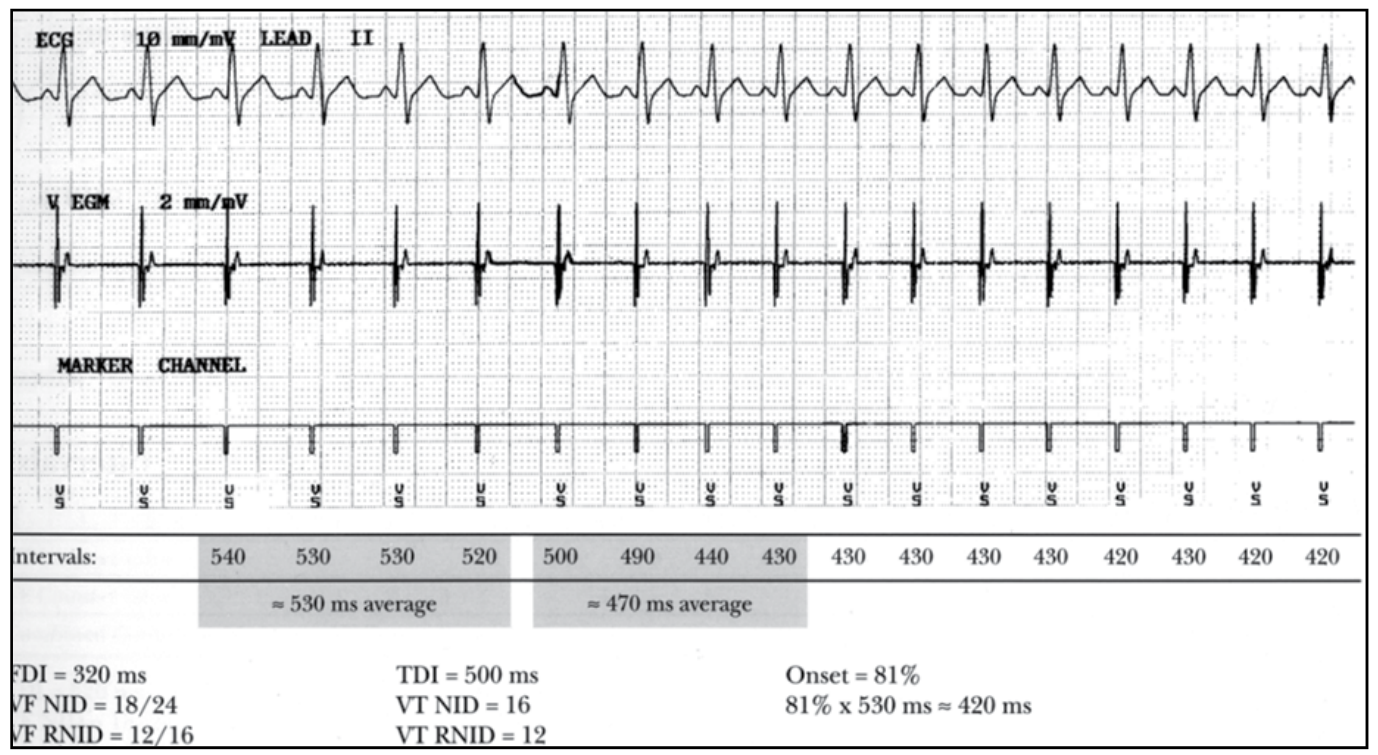

Figure 1. This particular RR onset algorithm analyses eight beats and compares mean ventricular rate of last four beats to mean ventricular rate of first four beats. If the ratio is over a pre-specified cut-off then the device recognises the tachycardia as VT.

RR stability discriminates monomorphic VT from AF based on regularity of the RR interval. The criterion depends on the analysis of cycle length variations and is continuously active during an episode; hence, when programmed "on", the stability algorithm withholds therapy despite ventricular rates in the tachycardia zone if the cycle length intervals are irregular. For most VT, the measured stability or the variation between RR intervals is $<21$ $\mathrm{ms}$, as opposed to AF, where typically varies by $35-50 \mathrm{~ms}$. Unlike onset, which is determined only once, stability algorithm continuously reevaluates the rhythm diagnosis during tachycardia. Nevertheless, it may misclassify a supraventricular arrhythmia with 2:1

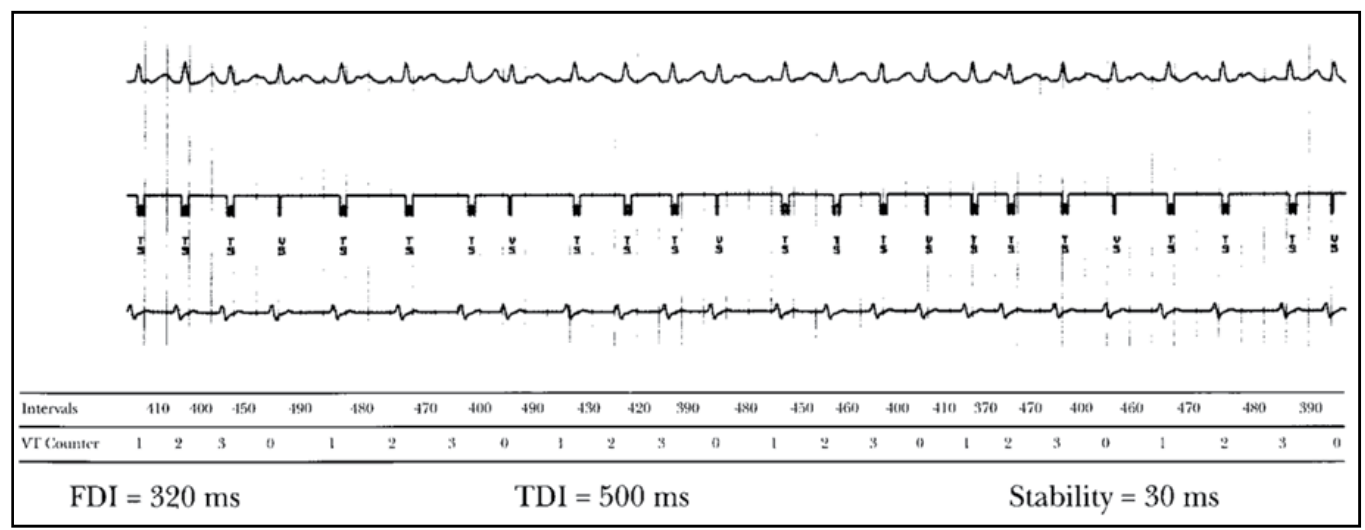

Figure 2. RR stability. Each RR interval is compared to the previous three. If the differences between the RR interval and each of the previous three are under a pre-specified stability cut-off then the arrhythmia is considered as VT. 
conduction block, such as atrial flutter or a rapidly conducted AF with a pseudoregular ventricular pattern. Antiarrhythmic drugs may also affect the algorithm's performance. Use of amiodarone or Class IC antiarrhythmic drugs (e.g., flecainide or propafenone) may cause monomorphic VT to become irregular or polymorphic VT to slow down, leading to rhythm misclassification. For this reason, stability should not be programmed "on" unless AF with a rapid ventricular response has been documented.

Morphology discriminates VT from SVT on the basis of morphology-based algorithms that compare the differences between ventricular EGM in a previously stored template of a normally conducted beat with VT EGMs during a tachycardia episode. Common elements present in all morphology algorithms include: creation of a template by mathematically extracting electrocardiogram features and storing them; recording of electrocardiograms during an unknown tachycardia; time aligning templates and tachycardia electrocardiograms; classifying each tachycardia electrocardiogram as a match or non-match based on its comparison with the template; classifying the tachycardia as SVT or VT based on the number of electrocardiograms that match the template. Morphology analysis goes on until insufficient normal beats are recognized (and VT detection occurs) or until the heart rate slows out of the detection zone. Various morphology algorithms differ in their electrocardiogram source, methods of quantitative representation and alignment. In single-chamber ICDs, morphology algorithms are the only discriminators that distinguish regular SVT, such as atrial flutter or atrioventricular nodal reentrant tachycardia (AVNRT), from VT.

Nevertheless, morphology may detect VT inappropriately because of patient-related or algorithm-related factors. Patient-related factors include rate-dependent bundle branch block during SVT and exercise-induced myopotentials in sinus tachycardia [24]. Algorithmrelated factors include misalignment of the sinus template with the tachycardia electrocardiogram, clipping of high-amplitude electrocardiograms, electrocardiogram changes during lead maturation, and shock-induced electrocardiogram changes [25]. In case of a possible sustained VT, a useful discriminator is the sustained-duration which overrides inhibition and starts therapy if the fast rate is persistent. It delivers therapy if a tachycardia

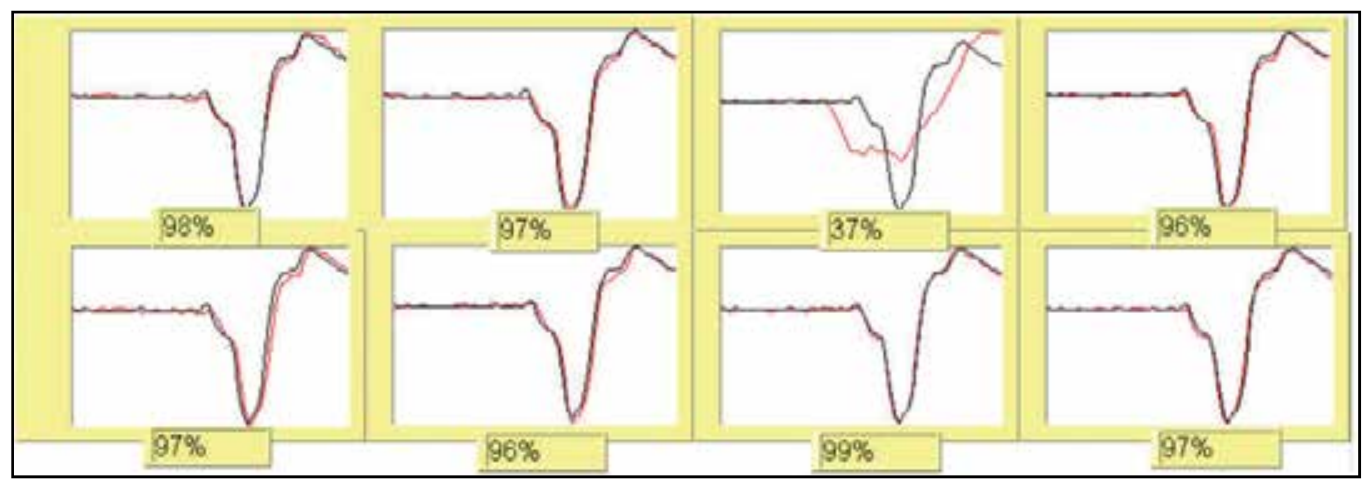

Figure 3. In this case morphology algorithm successfully discriminates a sinus tachycardia by matching tachycardia ventricular morphology with another morphology previously stored during sinus rhythm. 


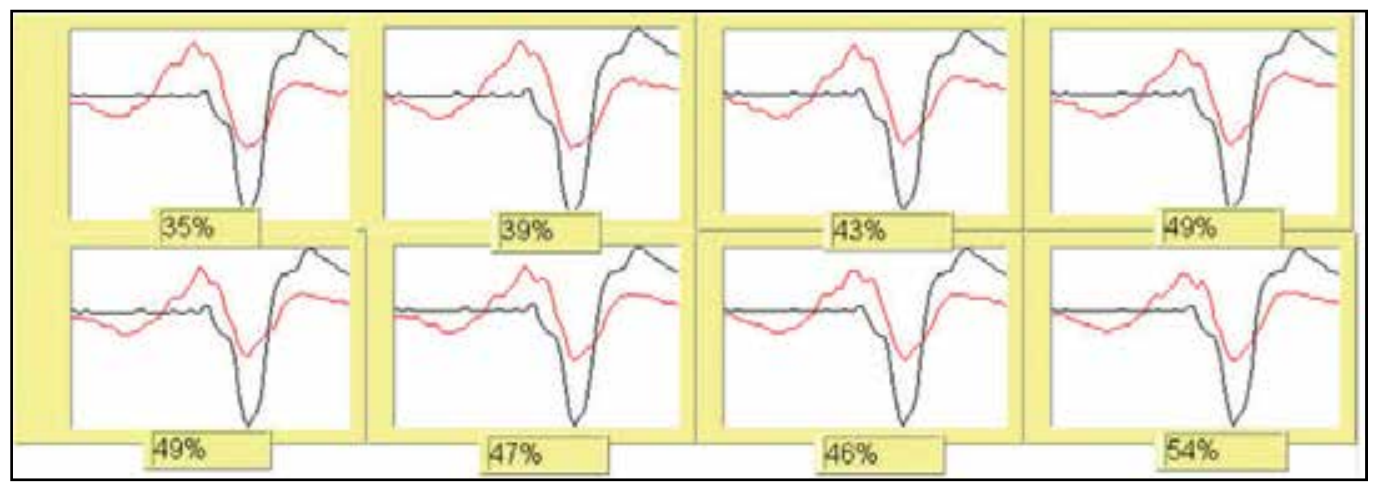

Figure 4. Ventricular morphology recorded during a tachycardia episode is recognized as different by morphology algohirthm, which in turn starts VT counter.

satisfies the VT rate criterion for a programmed duration, even if the discriminator indicates SVT. Since many cardiac patients are unable to maintain prolonged sinus tachycardia, the presumption is that sustained regular tachycardia lasting more than 3 min reflects VT. Thus, this feature ensures appropriate therapy of sustained VT at the price of decreased specificity for rejecting SVT. Indeed, inappropriate therapy is delivered for those SVT that remain above the rate boundary at the end of the programmed period.

\subsubsection{Dual-Chamber SVT-VT Discriminators}

Dual-chamber discriminators include analysis of atrial and ventricular rates and AV relationship, since dual chamber defibrillators acquire information simultaneously from the atrial and the ventricular leads. VT therapy is delivered immediately if ventricular rate is greater than atrial rate for a set number of beats. This occurs in more than $90 \%$ of detected arrhythmias which fall in the VT zone of dual-chamber ICD and in this case, no other data are necessary for therapy delivery. Therefore additional criteria for device diagnosis may be required only in less than $10 \%$ of VT. For instance, patients with AF may develop a slow VT, an atrial/ventricular counting algorithm would classify the situation as SVT because the atrial rate exceeds the ventricular rate, and other SVT discriminators may be necessary for a proper diagnosis. The best additional discriminator is morphology or stability.

Measures of AV association analyze the stability of the PR/RP interval and provide an implicit comparison of atrial and ventricular rates. Stable 2:1 AV association is what distinguishes atrial flutter from VT. Although 1:1 AV association does not discriminate between SVT and VT with 1:1 VA conduction, VT with 1:1 VA conduction begins often with transient AV dissociation.

P:R pattern identifies consistent timing relationships between atrial and ventricular electrocardiograms that occur during specific SVT, such as sinus tachycardia and atrial flutter. It identifies complex timing relationships, such as those present during variable AV block, and is insensitive to many transient perturbations. Yet, it is highly sensitive to sustained VT during SVT. The last discriminator, chamber of origin, is applied only to tachycardias with 1:1 AV 
association. It discriminates sinus or atrial tachycardia with 1:1 AV conduction from VT with 1:1 VA conduction. Atrial tachycardia begins with a short PP interval, whereas VT begins with a short RR interval. Chamber of origin depends on accurate sensing of all atrial and ventricular events at the onset of tachycardia. Thus, it is susceptible to errors based on a single oversensed or undersensed event. In summary, data have demonstrated that dual-chamber discriminators are able to diagnose $60-95 \%$ of SVT episodes correctly without significantly missing true VTs. Above all, they may be useful in selected patients, as those with a VT zone with rates $<200$ bpm and patients with no permanent AF and no AV block.

\subsection{Remote monitoring of AF through implanted devices}

Remote monitoring, especially wireless monitoring, offers multiple advantages over traditional office-based device follow-up. For instance it is useful for diagnosing technical issues such as lead fracture, device malfunction, early detection of tachyarrhythmias and heart failure progression, permitting a prompt clinical reaction [26]. The main potential advantage of remote control application in AF management is represented by early detection and early reaction to the arrhythmia occurrences. The current device diagnostics are very sophisticated and may give the physician full information about arrhythmia episodes, their number and duration, date and time of occurrence, onset mechanism, arrhythmia burden, effects of antitachycardia therapies and heart rate during the arrhythmia. The remote transmission of all these data allows a quicker identification of asymptomatic episodes, anticoagulation treatment and cardioversion decisions in a timely manner while the risk of thrombus formation is limited. Currently, the exact impact of the remote control as a clinical tool is still unknown. Moreover, it is still a matter of debate if device detection of AF with remote monitoring could be useful in selecting anticoagulation strategies, as well as the critical threshold of AF burden warranting anticoagulant therapy [27]. Despite the fact that anticoagulation has been shown to significantly improve the outcome in patients with AF, it is also associated with potentially life-threatening side effects and is therefore not well tolerated by patients. Automatic AF detection could make anticoagulation approach easier, allowing a swift therapeutic intervention. This technology seems particularly promising in patients with ICDs. Despite improvements in discrimination algorithms, SVTs continue to be the most common cause of inappropriate shocks. In these cases, the quick identification of the arrhythmia underlying an inappropriate shock may help physician in order to avoid further episodes. In table 1, advantages of remote monitoring in supraventricular tachycardia management are resumed.

- Early detection of symptomatic and asymptomatic supraventricular tachycardia

- Electrical cardioversion within 48 hours of the onset of AF

- Evaluation of the effectiveness of antiarrhythmic therapy

- Modification of the previous antiarrhythmic therapy or anticoagulation

- Rapid reprogramming of ICD in case of inappropriate shocks

- Decrease of number of to traditional office-based device follow-up

Table 1. Main advantages of remote monitoring in SVT detection. 


\section{Cardioversion in patients with implanted pacemakers and defibrillators}

Large-scale randomized trials have questioned the benefits of restoring normal sinus rhythm in patients with AF $[17,28]$.However, the presence of AF in patients with implanted devices could lead to increased morbidity and mortality through adverse device-related complications, such as inappropriate shock deliveries or pacemaker mediated tachycardia with associated tachycardia induced cardiomyopathy. Therefore, AF termination could become necessary and, when appropriate precautions are taken, cardioversion becomes safe and effective in patients with implanted devices.

\subsection{Internal cardioversion}

Internal cardioversion (ICV) is a defibrillation technique performed through intracardiac electrodes which allows successful CV with very low energy levels. Low-energy ICV does not interfere with pacemaker function in patients with electrodes positioned in the RA, coronary sinus, or left pulmonary artery. ICV has been shown to be superior to conventional external cardioversion (ECV) in term of primary success rate, energy requirements and need of sedation instead of general anaesthesia [29]. However, there are some disadvantages related to the procedure such as the need for an electrophysiology laboratory and of specific technical competence for lead positioning that greatly hamper widespread use in clinical practice. Oesophageal cardioversion is another method to perform CV. This technique is quite simple, very fast and provides several advantages. The most important is a low energy requirement thanks to proximity of the oesophagus to the left atrium which warrants a lower energy dispersion and a lower defibrillation impedance. For this reason its safety has been demonstrated in patients with implantable devices [30]. In patients with ICDs, a programmed ventricular ICD discharge could be another option for a quick and safe cardioversion.

\subsection{External cardioversion: how-to and precautions}

ECV has long been a cause of concern regarding the potential adverse effects on the generator and on the leads induced by the electrical shocks. First of all, electricity conducted along an implanted electrode may cause endocardial injury and bring to a temporary or permanent increase in stimulation threshold, resulting in acute or chronic loss of ventricular capture. Moreover, other ECV related problems include physical dysfunction of the device, spurious programming or electrical reset induced by the shock and changes in sensing function or pacing impedance [31,32].

\begin{tabular}{|l|l|l|l|}
\hline & Monophasic & Biphasic & p-value \\
\hline AF duration before CV & $18(1-394)$ days & $27(1-1359)$ days & 0.65 \\
\hline CV success & $20 / 21(95 \%)$ & $22 / 23(96 \%)$ & 1.0 \\
\hline Success with initial shock & $15 / 21(71 \%)$ & $17 / 23(74 \%)$ & 1.0 \\
\hline Cumulative shock energy & $200(200-1220) \mathrm{J}$ & $100(100-650) \mathrm{J}$ & 0.001 \\
\hline
\end{tabular}

Table 2. Comparison between AF cardioversion in patients with implanted rhythm devices treated with monophasic or biphasic shocks. For AF duration and cumulative shock energy, the median values and range are provided. Adapted from [31]. 
Devices assembled in last decades are considerably more sophisticated and better protected against sudden external discharges, even if data loss due to current surges is still possible. Voltage regulators protect the pacemaker circuitry by shunting the current away from the device through the lead to the electrode tip. However, this may result in concentration of the energy at the lead tip, causing burns and electrical trauma at the electrode-myocardial interface. This is the pathophysiological basis of the aforementioned pacing threshold and sensing changes.

Some precautions when performing ECV could be useful to ensure appropriate device function. First of all, the implanted device should be interrogated and, if necessary, reprogrammed before and after cardioversion. Prior to ECV, it is recommended to check pacemaker and leads function. Further precautions are necessary in pacemaker dependent patients such as programming higher voltage output in order to avoid loss of capture and switching to asynchronous mode (VOO or AOO). Current guidelines [33] do not set any interrogation timetable to follow after ECV. However, after the current discharge, it is safe to reassess device function immediately, at hospital discharge and 4-6 weeks later. Additional tests are needed in case of device malfunctioning.

Devices are typically implanted just under the collarbone, so the paddles used for ECV should be positioned as distantly as possible from the device. The positioning of paddles at least $8 \mathrm{~cm}$ away from pulse generator [31] did not produce changes in pacing thresholds or sensing measurements, preferably in the anterior-posterior configuration, as specified in AHA Guidelines [33]. The best paddles configuration is the anterior-posterior one. The antero-lateral orientation of cardioversion electrodes creates an electrical field parallel to the course of the leads thus maximizing current shunting through leads. The use of an anteroposterior orientation aims to prevent this 'antenna effect' since it creates an electrical field typically perpendicular to the main lead orientation. Furthermore, the anterior-posterior configuration needs, on average, lower energy requirements for termination of AF [31-33]. Different studies have demonstrated that biphasic shocks are superior to monophasic shocks for cardioversion because of the higher percentage of SR restoration, the fewer shocks needed and the lower energy delivered. However, few studies have focused the attention on patients with implanted devices. From the comparison of monophasic and biphasic shock energy application, it seems that there are no significant differences in terms of safety and efficacy. However, energy requirements are significantly lower for biphasic shocks when compared with monophasic shock waveforms.American and European guidelines do not specify the time between two consecutive shocks in patients with implanted devices. However, safe common practice might be a waiting time of at least 1 minute between shocks, as recommended for patients with AF without intracardiac devices [33].

\section{Atrial pacing for AF prevention}

Sustained AF depends on a complex electrophysiological substrate which has not been fully elucidated yet. Nonetheless, some animal models demonstrated that specific triggers, such as premature atrial beats, are needed for AF initiation and multiple atrial refractoriness 
patterns facilitate AF persistency. Some device strategies have been developed in order to prevent AF onset.

\subsection{Trigger suppression algorithms}

Suppression of AF triggers strategy is mainly based on suppression of premature atrial beats, either by elevating the lower rate or by dynamic overdrive. Both these options, although cheap and easy to perform in an out-of-hospital setting, are not very effective. Reprogramming a lower rate $10 \mathrm{bpm}$ faster than 24 hours mean heart rate prevented AF recurrence in approximately $60 \%$ of patients over a 30-day follow up [34]. In another study, while atrial pacing vastly reduced total premature atrial beats from 3.8 per hour to 0.5 per hour, no difference was found in AF recurrence prevalence or time to first recurrence between paced and not paced patients [35]. At one year, 43\% of all patients developed permanent $\mathrm{AF}$, suggesting that atrial pacing does not prevent the natural progression of $\mathrm{AF}$.

This strategy has also the drawback or requiring specific programming whenever mean heart rate changes, a quite common occurrence in AF patients. To address these issues, dynamic overdrive algorithms has been introduced. These algorithms work by increasing the paced rate upon detection of a premature atrial complex. The increased lower rate is then decreased again in steps until spontaneous atrial rate appears or a new premature atrial beat is detected. Premature beats can also increase dispersion of atrial refractoriness through the long-short cycle which is caused by the extrasystolic pause. In this setting, overdrive suppression algorithms are optimized to deliver either a series of paced beats or a higher pacing rate for a prespecified duration.

The advantage of these algorithms is to reduce atrial pauses following a premature beat while avoiding a fixed high heart rate, possibly leading to palpitations and tachycardiomyopathy. In general, mean heart rate is not significantly increased when these algorithms are working, and are well tolerated by the patients [36].

Efficacy of these algorithms in preventing AF is still a matter of debate. In one of the first prospective studies, AF suppression algorithm (St. Jude Medical, Sylmar, CA) was associated with a $35 \%$ reduction of AF burden in patients already implanted with a DDDR device [37]. Moreover, some effects on atrial remodeling, although little in magnitude, have been demonstrated. Other algorithms, such as the Atrial Preference Pacing algorithm (Medtronic, Minneapolis, MN) failed to show any reduction in the number of modeswitching episodes and symptomatic episodes despite a reduction of premature atrial beats [38].

Multicenter trials specifically designed to test efficacy and safety of these algorithms failed to demonstrate a net benefit over traditional pacing. The ATTEST trial evaluated three different algorithms for AF prevention (Atrial Preference Pacing, Atrial Rate Stabilization and Post Mode Switching Overdrive), and showed no difference in total number of AF episodes or median AF burden between patients with either the algorithms switched "on" or "off" [39]. The PIPAF trial had similar results, showing no difference in number of AF 
paroxysms per week in a crossover population during a 3 months follow up [40]. Post Mode Switching Overdrive algorithm (Medtronic, Minneapolis, MN) provided no reduction of AF episodes at $120 \mathrm{bpm}$ and was actually arrhythmogenic when programmed at $80 \mathrm{bpm}$.

\subsection{Alternative site and multi-site pacing for AF prevention}

Paroxysmal AF is usually related to an important inter-atrial and intra-atrial delay, which further increases atrial refractoriness dispersion and helps sustain AF. Unconventional atrial pacing techniques have been proposed in order to synchronize atrial activation and reduce dispersion of refractoriness. Alternative site pacing and dual-site pacing have been the most extensively studied strategies.

Alternative site atrial pacing attempts to reduce atrial activation time by selective pacing right atria at preferential conduction sites. The most common of these sites is probably the Bachmann's bundle, a band of fibrous tissue that travels from the superior part of the right atrium near the ostium of the vena cava superior to the upper part of the left atria appendage. Pacing at the Bachmann's bundle has been shown to reduce AF recurrence [41]. The incidence of AF recurrences at 1 year has been lowered with Bachmann's bundle pacing compared to traditional right atrial appendage pacing, and in those patients $\mathrm{P}$-wave duration was actually shorter, providing an indirect evidence of a better inter-atrial and intra-atrial activation [42]. Another alternative site for atrial pacing is the low interatrial septum near the triangle of Koch, where most of the connections between right and left atria have been discovered. Pacing at the low interatrial septum has been associated with a reduced AF burden and again, with shorter P-wave duration [43]. These alternative pacing sites obviously require active-fixation leads, but appear to be as stable and safe as conventional, passive-fixation, atrial appendage leads.

Multi-site pacing is another promising strategy which has been quite successful in reducing atrial refractoriness dispersion. This approach is characterized by the use of two atrial leads. The first one is placed in the atrial appendage or actively fixated into the interatrial septum. The second one can be either placed into the distal coronary sinus or near its ostium. In the former case, specifically designed leads are necessary in order to avoid dislodgement whereas in the latter case an active-fixation lead is required.

Only a single multicenter trial has currently been performed in order to test feasibility and efficacy of multi-site pacing in AF prevention [44]. This study randomized 118 patients to multi-site pacing, overdrive atrial pacing or simple support pacing at a lower rate of $50 \mathrm{bpm}$. The study showed a reduction in AF recurrences and mean dose of antiarrhythmic drugs taken in both multi-site pacing and overdrive pacing, with no significant difference between these two groups.

To conclude, patients with paroxysmal AF and a P duration $\geq 90 \mathrm{~ms}$ may benefit from dualsite pacing as well as alternative site pacing. Hybrid therapy, using both alternative site pacing and suppression algorithms, could represent a future approach to AF prevention, though multicenter trials are still needed in order to gather enough evidence. 


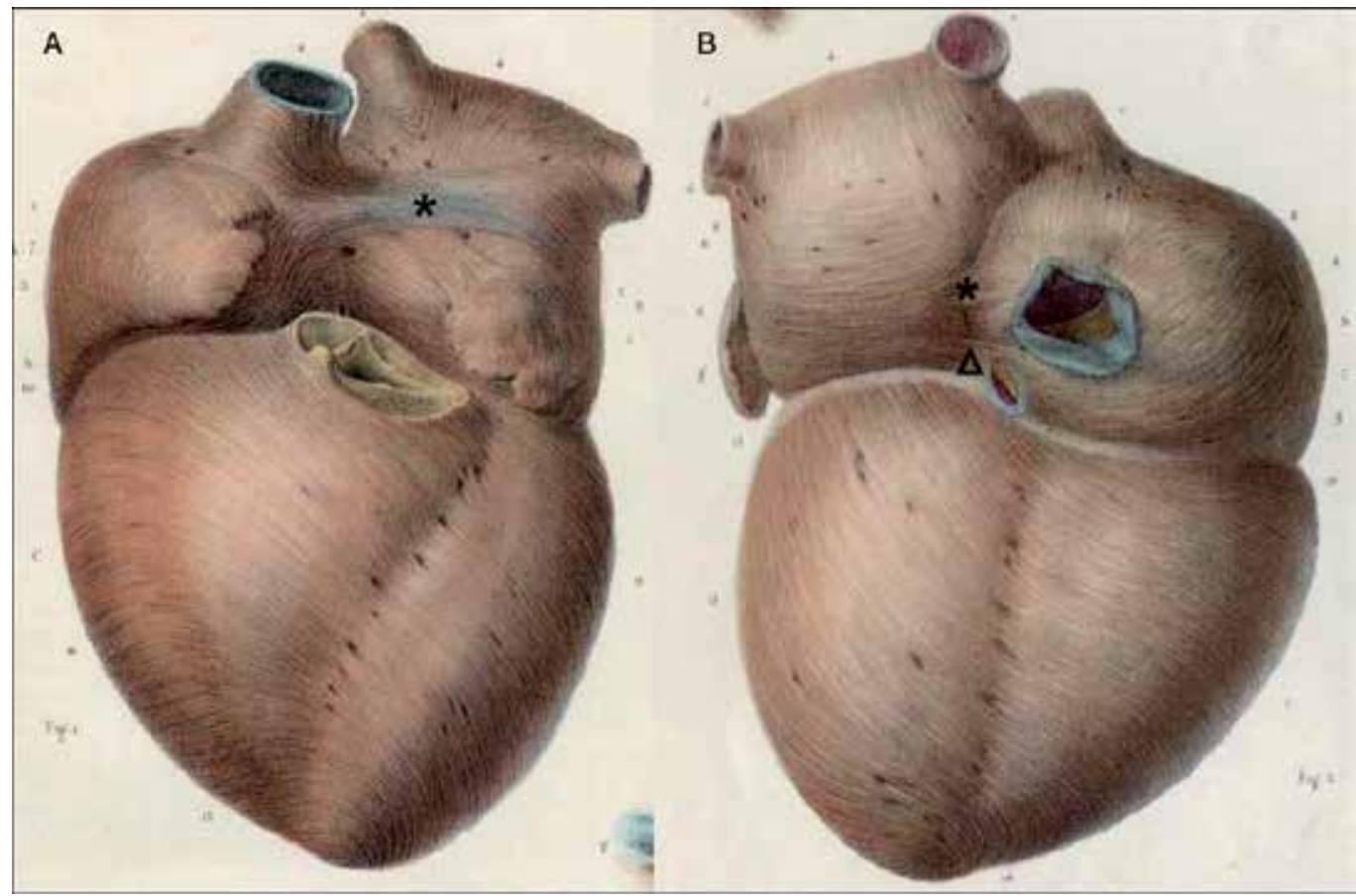

Figure 5. Superior (A) and inferior (B) view of the human heart. (A) Bachmann's bundle travels from the superior part of the right atrium near the ostium of the vena cava superior to the upper part of the left atrium $\left(^{*}\right)$. (B) components of the inferior interatrial route: asterisk denotes muscular bundles between the right atrium near the orifice of the vena cava inferior and the inferior surface of the left atrium; Arrowhead denotes interatrial bundles in the vicinity of the coronary sinus orifice (coronary sinus is removed). Reproduced from [41].

\section{Atrial pacing for AF termination}

Antitachycardia pacing (ATP) is a reliable method of suppressing ventricular arrhythmias, and is currently widespread as a standard ICDs therapy. More recently, its usefulness has been demonstrated also in terminating atrial tachyarrhythmias with a wide excitable gap such as atrial tachycardia or atrial flutter. Unfortunately, AF lacks a wide excitable gap, and its multiple reentrant wavelets do account for its resistance to ATP termination. Atrial ATP is now available in all modern implantable devices. Many different types of atrial ATP are available, as they differ from each producing company. The most common are: atrial burst (steady rate pacing), atrial ramp (auto-decremental pacing), atrial burst plus (steady rate pacing followed by two extrastimuli) and $50-\mathrm{Hz}$ burst pacing for 3 seconds, none of which proved to be more effective than the others in terminating AF.

Efficacy of atrial ATP is low, mainly because of the aforementioned mechanisms, and it varies from 30 to 54\% [39]. However, several variables must be taken into account. First of all, ATP success in terminating AF greatly depends on the cycle length of the arrhythmia: 
being as low as $29 \%$ if the cycle length is $\leq 190 \mathrm{~ms}$ and as high as $65 \%$ when the cycle length is $>320 \mathrm{~ms}$ [45]. Also, atrial ATP is more effective if delivered in recent-onset AF paroxysms. Another possible use of atrial ATP depends on the common pathophysiology between AF and atrial flutter. In patients in whom those two arrhythmias coexist, ATP delivery while the patient is in atrial flutter may prevent further degeneration of atrial flutter into AF.

\section{Ablate and pace}

\subsection{Rationale and indications for ablate and pace}

Atrioventricular junction (AVJ) ablation and subsequent pacing, also called "ablate and pace" may be considered whenever it is not possible to achieve adequate rate control despite optimal pharmacological therapy or after unsuccessful AF ablation. Complete AV block is achieved by selective catheter-mediated destruction of the AV node or His bundle, with radiofrequency current serving as the predominant source energy. AVJ ablation is usually performed by a right-sided approach, which offers lower prevalence of complications and is related to shorter hospitalizations. When the right-sided approach fails, left-sided ablation can be performed during the same session or at another session. Leftsided ablation is usually faster, as requires fewer radiofrequency applications, but is associated with a significantly higher risk of bleeding [46,47]. An expert electrophysiologist can usually produce an effective AV block in more than $98 \%$ of cases. Regression of AV block late after ablation is rare, and occurs in less than $5 \%$ of total procedures [48].

Current European [1] and American [33] guidelines list ablate and pace as a palliative treatment. Indeed, in contrast to other ablative procedures in which the ablation erases the electrophysiologic substrate of the disease, ablate and pace only works indirectly, changing an irregular and fast ventricular rate into a regular, pacemaker-dependent, normofrequent rate. It could also be said that ablate and pace replaces one disease (uncontrolled AF) with another (iatrogenic complete AV block) [49]. Moreover, the procedure is completed by a pacemaker implant, with all the short- and long-term complications associated with a device implant procedure.

Nonetheless, ablate and pace improves symptoms and quality of life compared to medical therapy, and improves survival making it similar to survival rates in the general population $[1,33]$. Usually, patients who benefit the most of ablate and pace strategy are those patients with tachycardia-mediated cardiomyopathy, as symptoms are usually related to a fast ventricular response.

A meta-analysis, including less than 1200 patients affected by symptomatic permanent AF, demonstrated that AVJ ablation and pacemaker implantation significantly improved quality of life, reducing symptoms and hospitalizations [10]. Similar results have been shown for patients with paroxysmal or persistent AF refractory to any form of pharmacological rate control [50]. Ablate and pace seems also beneficial in restoring an adequate cardiac function: in the Ablate and Pace Trial (APT) 156 patients undergoing AV node ablation and pacemaker implant experienced an improvement of cardiac systolic function along with 
clinical status and quality of life [51]. Moreover, in a small study recruiting 56 patients with EF less than $40 \%$, ablate and pace strategy was associated with an improvement of mean EF from 0.26 to 0.34 , with one fourth of the patients actually achieving the goal of a normal $(\geq 55 \%)$ EF.

The outcome of an ablate and pace strategy could be further improved by alternative sites pacing, such as the right ventricular outflow tract, the interventricular septum and the His bundle, although no clear evidence is currently available.

(A) AF Patients: Overall Survival

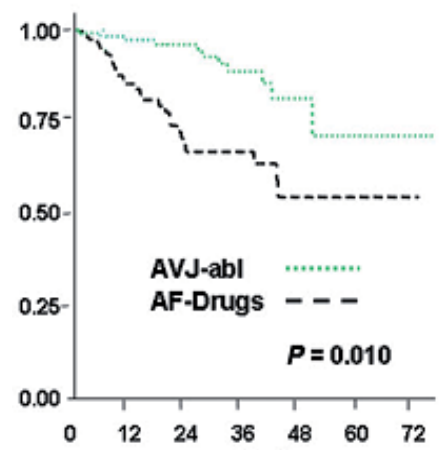

(B) AF Patients: Cardiac Survival

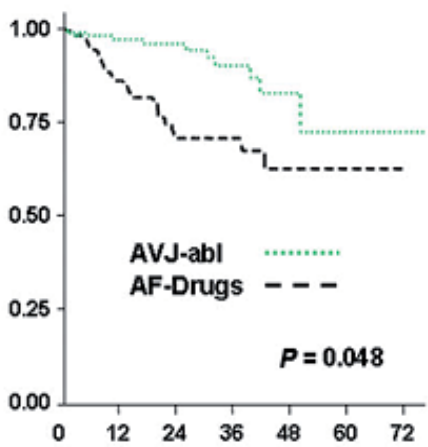

(C) AF Patients: HF Survival

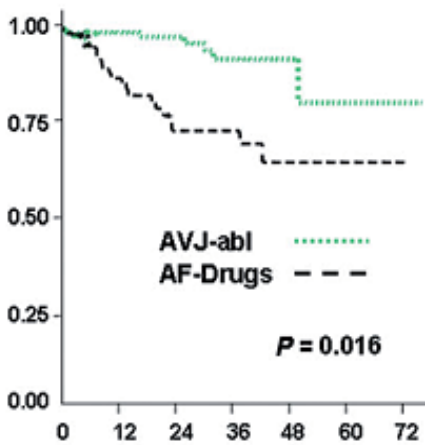

Figure 6. Comparison of Kaplan-Meier estimates of overall (A), cardiac (B), and heart failure (C) survival between AF patients who underwent AVJ ablation and AF patients treated only with antiarrhythmic drugs. The p-values presented derive from the adjusted hazards ratio analysis stratified according to the corresponding cause of death. Reproduced from [51].

\subsection{Which device in which patient? Recommendations for different types of devices in "ablate-and-pace" strategy}

Selection of the appropriate device (pacemaker or ICD) and the optimal stimulation modality (VVI, DDD, or CRT) must be based on a number of clinical variables. First of all, type of $\mathrm{AF}$ (paroxysmal, persistent or permanent) is of the utmost importance to determine the correct strategy. In patients with paroxysmal AF, dual-chamber pacemakers are obviously preferred in an effort to maintain AV synchrony during sinus rhythm following ablation. Automatic mode switching capability of modern dual-chamber devices prevents rapid ventricular rate when AF occurs and, as already said, provides a major diagnostic tool in detecting AF episodes. The device can be easily reprogrammed to VVIR modality when AF becomes persistent or permanent.

Associated ischemic or valvular heart disease, systolic and diastolic function and the presence and severity of heart failure symptoms should also be taken into account. Current recommendations for cardiac resynchronization are still valid in this subtype of patients. In fact, it has been demonstrated that AF patients undergoing AVJ ablation before CRT implant have a better survival rate when compared with AF patients under a pharmacological rate control strategy [52]. Upgrading to a biventricular device should be considered for patients with HF and a right ventricular pacing system when AV node 
ablation is performed. It is not currently established whether biventricular pacing could be useful in patients without left ventricular dysfunction, although some recent evidences are in favor of a possible role in reducing AF-related symptoms [53]. In severely symptomatic patients with permanent $\mathrm{AF}, \mathrm{CRT}$ significantly prevents hospitalization and worsening of clinical conditions during a 2-years follow-up [53].

\subsection{Safety of ablate and pace therapy}

As said before, ablation of the AV node produces a new illness (the iatrogenic AV complete block) out of an old one. Complications related to ablate and pace strategy include those commonly associated with device implant, thromboembolism associated with anticoagulation withhold and progression from paroxysmal to permanent AF. AVJ ablation procedure creates a temporary proarrhythmic state, which in turn can rarely lead to polymorphic VT and cardiac arrest. In fact, the 1-year mortality rate after ablate and pace is approximately $6.3 \%$, which include a $2 \%$ risk of sudden cardiac death [54]. Although this issue has raised some criticisms, programming a higher lower rate of about $80-90$ beats per minute for the first 1-2 months seems a viable option for reducing the risk of sudden death [55]. Nonetheless, mortality associated with ablate and pace is still low, reaching $10.5 \%$ in 5 years [56], with no significant difference in mortality between ablation and pharmacological control rate.

\section{Two birds with one stone: Usefulness of implanted devices in AF associated with other cardiac diseases}

\subsection{Bradycardia and syncope: Recommended type of devices and optimal settings in low-response AF}

VVIR is the mode of choice for patients with permanent AF and a slow ventricular response, in whom pacing is necessary. On the contrary, for most patients with brady-tachy syndrome, a dual chamber pacemaker is indicated. As most patients with symptomatic bradycardia often have associated chronotropic incompetence or at least the potential for this to occur, a dual-chamber pacemaker with rate-adaptive capabilities (DDDR) is recommended. In patients with frequent AF relapses associated with slow ventricular rates, DDDR becomes essential. Rate responsiveness in patients with chronic AF depends on autonomic regulation of the AV node, which is usually poorly regulated in this population. the DDDR pacemaker could be reprogrammed to VVIR afterwards if AF degenerates over time into permanent type. A DDD pacemaker, which has no rate-adaptive capability, could be reprogrammed only to VVI, which could not be optimal for the patient.

\subsection{Heart failure: Cardiac resynchronization therapy in AF}

Latest ESC [12] and AHA/ACC/HRS Guidelines [57] have considered atrial fibrillation patients, who constitute an important subgroup of HF patients, as eligible to receive CRT. 
Both ventricular conduction delay and AF are associated with poor prognosis in HF and CRT may be therefore indicated in these patients. Few randomized trials have been done in order to assess the efficacy of CRT in AF patients [58-60]. The MUSTIC trial showed good one-year results of biventricular pacing in patients with severe $\mathrm{HF}$ and major intraventricular conduction disturbances with either SR or AF [58]. Because of the high drop-out rate, the impact of these results were limited even though the results underlined the importance of an high percentage of biventricular pacing to achieve real CRT-related benefits in patients with AF. The PAVE study demonstrated that in patients undergoing AV node ablation for AF, biventricular pacing provided a significant improvement in the 6-minute walk test and ejection fraction compared to right ventricular pacing [59]. These beneficial effects of CRT were greater in patients with impaired systolic function or symptomatic HF [59]. An intrinsic, intermediate-to-high, irregularly spontaneous $\mathrm{AF}$ rhythm reduces the percentage of effectively biventricular paced captured beats. Even in a patient who has normofrequent AF, phases of effective biventricular capture alternate with phases of competing AF rhythm which translates into spontaneous, fusion, or pseudo-fusion beats. The presence of fusion and pseudo-fusion leads to inaccuracy and overestimation of the effective pacing capture. Furthermore, the global effective 'CRT-dose' may be markedly reduced compared with atrial synchronous rhythm with a short AV interval achieved during SR, since the number of effective biventricular captured beats are reduced. Moreover, in AF patients, spontaneous ventricular rate tends to overdrive biventricular pacing rates during exertion, determining a further reduction of paced beats precisely when patients are most in need of biventricular capture, and thus greatly limiting functional capacity [60,61]. Another problem is the possible negative impact on prognosis of negative chronotropic therapy in achieving adequate rate control. In fact, the SCD-HeFT study has shown that amiodarone therapy provided no benefit in patients in NYHA class II and decreased survival among patients in NYHA class III, as compared with those who received placebo [62].

To achieve a ventricular rate control delivery, devices-derived features have been developed in order to maximize ventricular pacing during potentially disruptive events. These features are ventricular rate regularization and in ventricular sense response pacing. The former consist in performing biventricular pacing, which 'overrides' intrinsic rhythm, through faster ventricular-paced depolarization, allowing to reduce short cycles through retrograde concealed penetration of the AV node. The other feature is characterized by the activation of LV pacing soon after a premature RV sensed event is detected. Another mechanism in the management of AF is the combination between CRT and atrial tachyarrhythmia prevention pacing algorithm. The ADOPT trial [37] has demonstrated the efficacy and safety of the AF suppression algorithm in reducing symptomatic AF burden in patients with permanent pacemaker with prior history of AF and with normal EF. A subsequent study, the MASCOT trial, has been designed to determine whether the addition of atrial overdrive pacing to CRT could reduce the incidence of permanent AF. The overall incidence of permanent AF was low and was similar for the two treatment groups underlying that probably the advanced atrial remodelling in the setting of HF and AF may preclude benefit from atrial algorithms in this altered milieu [63]. 
AVJ ablation and placement of a permanent pacemaker has been used in patients with fast, symptomatic and drug-refractory AF to confer symptomatic relief and improve exercise tolerance. In the setting of CRT and AF, the AVJ ablation is emerging as a useful tool to optimize CRT delivery because it allows a complete heart rate control through a constant biventricular pacing.

From the current evidences it emerges that the real benefits of CRT in AF population seem to be confined only to AF patients treated with AVJ ablation. The magnitude of reverse remodelling, of improving of the systolic function and of the decrease in NYHA functional class, the percentage of responders seems to be higher in AF patients treated with CRT and AVJ ablation [51,52]. AVJ ablation in addition to CRT appears also to improve long-term overall mortality and hospitalization compared with CRT alone, primarily by reducing HF death [53].

The outcomes of AF with AVJ ablation patients are similar to the outcomes of patients in SR. Several trials have concluded that AF patients display similar survival as sinus rhythm patients provided that AVJ ablation is performed. However, data from larger randomized clinical trials will be needed before utilizing this as a standard practice since this would create a large number of pacemaker- dependent HF patients with all stimulation dependency-related problems.

In patients with permanent $\mathrm{AF}$ who undergo CRT without AVJ ablation, a few studies have suggested that cardioversion and aggressive rhythm control result in better clinical outcomes [64]. Limited data showed that in patients with severely depressed left ventricular $\mathrm{EF}$, left bundle branch block and permanent AF a more vigorous approach to restoring SR is justified prior to implantation of a CRT device or after, in case of recurrences conferring clinical and instrumental improvement. However, currently available antiarrhythmic drugs are only partially effective in maintaining sinus rhythm, and this is achieved at the cost of potential risk [17]. In contrast, catheter ablation may offer another approach for achieving sinus rhythm in these patients. Several clinical trials have demonstrated catheter ablation as a promising alternative compared to pharmacologic therapy, that significantly improved LV function, NYHA class and exercise capacity in patients with AF and symptomatic LV dysfunction [65-68]. The results of these nonrandomized series provide a potent rationale for a randomized clinical trial comparing ablation to pharmacologic therapy.

\section{Author details}

Federico Guerra*, Michela Brambatti, Maria Vittoria Matassini and Alessandro Capucci Cardiology Clinic, Marche Polytechnic University, Ancona, Italy

\section{References}

[1] Camm AJ, Kirchhof P, Lip GY, Schotten U, Savelieva I, Ernst S, et al. (2010) Guidelines for the management of atrial fibrillation: the Task Force for the Management of Atrial Fibrillation of the European Society of Cardiology (ESC). European Heart Journal. 31:2369-2422.

" Corresponding Author 
[2] De Sisti A, Attuel P, Manot S, Fiorello P, Halimi F, Leclercq JF. (2000) Electrophysiological determinants of atrial fibrillation in sinus node dysfunction despite atrial pacing. Europace. 2:304-311.

[3] Lamas GA, Lee KL, Sweeney MO, Silverman R, Leon A, Yee R, et al. (2002) Ventricular pacing or dual-chamber pacing for sinus node dysfunction. New England Journal of Medicine. 346:1854-1862.

[4] Veasey RA, Arya A, Silberbauer J, Sharma V, Lloyd GW, Patel NR, Sulke AN. (2011) The relationship between right ventricular pacing and atrial fibrillation burden and disease progression in patients with paroxysmal atrial fibrillation: the long-MinVPACE study. Europace. 13:815-820.

[5] Sweeney MO, Bank AJ, Nsah E, Koullick M, Zeng QC, Hettrick D, et al. (2007) Minimizing Ventricular Pacing to Reduce Atrial Fibrillation in Sinus-Node Disease. New England Journal of Medicine. 357:1000-1008.

[6] Silberbauer J, Veasey RA, Freemantle N, Arya A, Boodhoo L, Sulke N. (2009) The relationship between high-frequency right ventricular pacing and paroxysmal atrial fibrillation burden. Europace. 11:1456-1461.

[7] Savelieva I, Camm J. (2003) Atrial fibrillation and heart failure: natural history and pharmacological treatment. Europace. 5: S5-S19.

[8] Cleland JG, Daubert JC, Erdmann E, Freemantle N, Gras D, Kappenberger L, Tavazzi L, for the Cardiac Resynchronization-Heart Failure (CARE-HF) Study Investigators. (2005) The effect of cardiac resynchronization on morbidity and mortality in heart failure. New England Journal of Medicine. 352:1539-1549.

[9] Dickstein K, Bogale N, Priori S, Auricchio A, Cleland JG, Gitt A, et al. (2009) The European cardiac resynchronization therapy survey. European Heart Journal. 30:2450 -2460.

[10] Wood MA, Brown-Mahoney C, Kay GN, Ellenbogen KA. (2000) Clinical outcomes after ablation and pacing therapy for atrial fibrillation: a meta-analysis. Circulation. 101:1138-1144.

[11] Vardas PE, Auricchio A, Blanc JJ, Daubert JC, Drexler H, Ector H, et al. (2007) Guidelines for cardiac pacing and cardiac resynchronization therapy. European Heart Journal. 28:2256-2295.

[12] Dickstein K, Vardas PE, Auricchio A, Daubert JC, Linde C, McMurray J, et al. (2010) Focused update of the European Society of Cardiology guidelines on device therapy in heart failure. An update of the 2008 European Society of Cardiology guidelines for the diagnosis and treatment of acute and chronic heart failure and the 2007 European Society of Cardiology guidelines for cardiac and resynchronization therapy. European Heart Journal. 31:2677-2687.

[13] Brignole M, Vardas P, Hoffman E, Huikuri H, Moya A, Ricci R, et al. (2009) Indications for the use of diagnostic implantable and external ECG loop recorders. Europace. 11:671-687.

[14] Nieuwlaat R, Capucci A, Camm AJ, Olsson SB, Andresen D, Davies DW, et al. (2005) Atrial fibrillation management: a prospective survey in ESC member countries: the Euro Heart Survey on Atrial Fibrillation. European Heart Journal. 26:2422-2434.

[15] Israel CW, Grönefeld G, Ehrlich JR, Li YG, Hohnloser SH. (2004) Long-term risk of recurrent atrial fibrillation as documented by an implantable monitoring device: implications for optimal patient care. Journal of the American College of Cardiology. 43:47-52. 
[16] Orlov MV, Ghali JK, Araghi-Niknam M, Sherfesee L, Sahr D, Hettrick DA. (2007) Asymptomatic atrial fibrillation in pacemaker recipients: incidence, progression, and determinants based on the atrial high rate trial. Pacing and Clinical Electrophysiology. 30:404-411.

[17] Wyse DG, Waldo AL, DiMarco JP, Domanski MJ, Rosenberg Y, Schron EB, et al. (2002). A comparison of rate control and rhythm control in patients with AF. New England Journal of Medicine. 347:1825-1833.

[18] Healey JS, Connolly SJ, Gold MR, Israel CW, Van Gelder IC, Capucci A, et al. (2012) Subclinical AF and the Risk of Stroke. New England Journal of Medicine. 366: 120-129.

[19] Ricci RP, Morichelli L, Santini M. (2009) Remote control of implanted devices through Home Monitoring ${ }^{\mathrm{TM}}$ technology improves detection and clinical management of AF. Europace. 11:54-61.

[20] Hindricks G, Pokushalov E, Urban L, Taborsky M, Kuck KH, Lebedev D, et al. (2010) Performance of a new leadless implantable cardiac monitor in detecting and quantifying AF: Results of the XPECT trial. Circulation: Arrhythmias and Electrophysiology 3:141-147.

[21] Buxton AE, Lee KL, Fisher JD, Josephson ME, Prystowsky EN, Hafley G. (1999) A randomized study of the prevention of sudden death in patients with coronary artery disease. New England Journal of Medicine. 341:1882-1890.

[22] Bardy GH, Lee KL, Mark DB, Poole JE, Packer DL, Boineau R, et al. (2005) Amiodarone or an implantable cardioverter-defibrillator for congestive heart failure. New England Journal of Medicine. 352:225-237.

[23] Daubert JP, Zareba W, Cannom DS, McNitt S, Rosero SZ, Wang P, et al. (2008) Inappropriate ICD Shocks in MADIT II. Journal of the American College of Cardiology. 51:1357-1365.

[24] Swerdlow CD, Ahern T, Chen PS, Hwang C, Gang E, Mandel W, et al. (1994) Underdetection of ventricular tachycardia by algorithms to enhance specificity in a tiered therapy cardioverter-defi brillator. Journal of the American College of Cardiology. 24:416-424.

[25] Duru F, Bauersfeld U, Rahn-Schonbeck M, Candinas R. (2000) Morphology discriminator feature for enhanced ventricular tachycardia discrimination in implantable cardioverter defibrillators. Pacing and Clinical Electrophysiology. 23:1365-1374.

[26] Brugada P (2006) What evidence do we have to replace in-hospital implantable cardioverter defibrillator follow-up? Clinical Research in Cardiology. 95(Supplement 3):iii3-iii9.

[27] Ip J, Waldo AL, Lip GY, Rothwell PM, Martin DT, Bersohn MM, et al. (2009) Multicenter randomized study of anticoagulation guided by remote rhythm monitoring in patients with implantable cardioverter-defibrillator and CRT-D devices: Rationale, design, and clinical characteristics of the initially enrolled cohort. The IMPACT study. American Heart Journal. 158:364-370.

[28] Carlsson J, Miketic S, Windeler J, Cuneo A, Haun S, Micus S, et al. (2003) Randomized trial of rate-control versus rhythm-control in persistent atrial fibrillation: the Strategies of Treatment of Atrial Fibrillation (STAF) study. Journal of American College of Cardiology. 41(10):1690-6. 
[29] Lévy S. (2005) Internal defibrillation: where we have been and where we should be going? Journal of Interventional Cardiac Electrophysiology. 13:61-66.

[30] Santini L, Forleo GB, Romeo F. (2011) Esophageal electrical cardioversion of atrial fibrillation: when esophagus gives a help to cardiologists. Cardiology Research and Practice. 2011:983937.

[31] Manegold JC, Israel CW, Ehrlich JR, Duray G, Pajitnev D, Wegener FT, Hohnloser SH. (2007) External cardioversion of atrial fibrillation in patients with implanted pacemaker or cardioverter-defibrillator systems: a randomized comparison of monophasic and biphasic shock energy application. European Heart Journal. 28:1731-1738.

[32] Gammage MD. (2007) External cardioversion in patients with implanted cardiac devices: is there a problem? European Heart Journal $28: 1668-1669$

[33] Fuster V, Rydén LE, Cannom DS, Crijns HJ, Curtis AB, Ellenbogen KA, et al. (2011) 2011 ACCF/AHA/HRS focused updates incorporated into the ACC/AHA/ESC 2006 Guidelines for the management of patients with atrial fibrillation: a report of the American College of Cardiology Foundation/American Heart Association Task Force on Practice Guidelines developed in partnership with the European Society of Cardiology and in collaboration with the European Heart Rhythm Association and the Heart Rhythm Society. Journal of American College of Cardiology. 57:101-198.

[34] Garrigue S, Barold SS, Cazequ S, Gencel L, Jaïs P, Haissaguerre M, Clémenty J. (1998) Prevention of atrial arrhythmias during DDD pacing by atrial overdrive. Pacing and Clinical Electrophysiology. 21:250-255.

[35] Gillis AM, Wyse G, Connolly SJ, Dubuc M, Philippon F, Yee R, et al. (1999) Atrial pacing periablation for prevention of paroxysmal atrial fibrillation. Circulation. 99:2553-2558.

[36] Lam CTF, Lau CP, Leung SK, Tse HF, Lee KLF, Tang MO, et al. (2000) Efficacy and tolerability of continuous overdrive atrial pacing in atrial fibrillation. Europace 2:286-291.

[37] Carlson MD, Ip J, Messenger J, Beau S, Kalbfleisch S, Gervais P, et al. (2003) A new pacemaker algorithm for the treatment of atrial fibrillation: results of the Atrial Dynamic Overdrive Pacing Trial (ADOPT). Journal of the American College of Cardiology. 20:627-633.

[38] Murgatroyd FD, Nitzsche R, Slade AK, Limousin M, Rosset N, Camm AJ, et al. (1994) A new pacing algorithm for overdrive suppression of atrial fibrillation. Pacing and Clinical Electrophysiology. 17:1966-1973.

[39] Lee MA, Weachter R, Pollak S, Kremers MS, Naik AM, Silverman R, et al. (2003) The effect of atrial pacing therapies on atrial tachyarrhythmia burden and frequency. Journal of the American College of Cardiology. 41:1926-1932.

[40] Adler S, Ziegler P, Koehler J, Holbrook R, Hettrick DA. (2001) Post mode switch overdrive pacing algorithm reduces atrial tachyarrhythmia recurrence in patients with bradycardia and atrial tachyarrhythmias. Circulation. 104:II-624.

[41] Platonov PG. (2007) Interatrial conduction in the mechanisms of atrial fibrillation: from anatomy to cardiac signals and new treatment modalities. Europace 9:vi10-vi16.

[42] Bailin SJ, Adler S, Giudici M. (2001) Prevention of chronic atrial fibrillation by pacing in the region of Bachmann's bundle: results of a multicenter randomized trial. Journal of Cardiovascular Electrophysiology 12:912-917. 
[43] Padeletti L, Pieragnoli P, Ciapetti C, Colella A, Musilli N, Porciani MC, et al. (2001) Randomized crossover comparison of right atrial appendage pacing versus interatrial septum pacing for prevention of paroxysmal atrial fibrillation in patients with sinus bradycardia. American Heart Journal, 142:1047-1055.

[44] Saksena S, Prakash A, Ziegler P, Hummel JD, Friedman P, Plumb VJ, et al. (2002) Improved suppression of recurrent atrial fibrillation with dual-site right atrial pacing and antiarrhythmic drug therapy. Journal of the American College of Cardiology. 40:1140-1150.

[45] Adler SW, Wolpert C, Warman EN, Musley SK, Koehler JL, Euler DE. (2001) Efficacy of pacing therapies for treating atrial tachyarrhythmias in patients with ventricular arrhythmias receiving a dual-chamber implantable cardioverter defibrillator. Circulation. 104:887-892.

[46] O Souza, S Gursoy, F Simonis, G Steurer, E Andries, P Brugada (1992) Right-sided versus left-sided radio frequency ablation of the His bundle. Pacing and Clinical Electrophysiologhy. 15:1454-1459.

[47] Kalbfleisch SJ, Williamson B, Man KC, Vorperian V, Hummel JD, Calkins H, et al. (1993) A randomized comparison of the right- and left-sided approaches to ablation of the atrioventricular junction American Journal of Cardiology. 72:1406-1410.

[48] Brignole M. (2000) Ablate and pace: palliating the symptoms? American Journal of Cardiology. 86:4K-8K.

[49] Brignole M, Gianfranchi L, Menozzi C, Alboni P, Musso G, Bongiorni MG, et al. (1997) Assessment of atrioventricular junction ablation and DDDR mode-switching pacemaker versus pharmacological treatment in patients with severely symptomatic paroxysmal atrial fibrillation: a randomized controlled study. Circulation. 96:2617-2624.

[50] Kay GN, Ellenbogen KA, Giudici M, Redfield MM, Jenkins LS, Mianulli M, Wilkoff B. (1998) The Ablate and Pace Trial: a prospective study of catheter ablation of the AV conduction system and permanent pacemaker implantation for treatment of atrial fibrillation. Journal of Interventional Cardiac Electrophysiology. 2:121-135.

[51] Gasparini M, Auricchio A, Metra M, Regoli F, Fantoni C, Lamp B, et al. (2008) Longterm survival in patients undergoing cardiac resynchronization therapy: the importance of performing atrio-ventricular junction ablation in patients with permanent atrial fibrillation. European Heart Journal. 29:1644-1652.

[52] Leon AR, Greenberg JM, Kanuru N, Baker CM, Mera FV, Smith AL, et al. (2002) Cardiac resynchronization in patients with congestive heart failure and chronic atrial fibrillation: effect of upgrading to biventricular pacing after chronic right ventricular pacing. Journal of the American College of Cardiology. 39:1258-1263.

[53] Brignole M, Botto GL, Mont L, Oddone D, Iacopino S, De Marchi G, et al. (2012) Predictors of clinical efficacy of 'Ablate and Pace' therapy in patients with permanent atrial fibrillation. Heart. 98:297-302.

[54] Nowinski K, Gadler F, Jensen-Urstad M, Bergfeldt L. (2002) Transient proarrhythmic state following atrioventricular junction radiofrequency ablation: pathophysiologic mechanisms and recommendations for management. American Journal of Medicine. 113:596-602.

[55] Evans GT, Scheinman MM, Bardy G, Borggrefe M, Brugada P, Fisher J, et al. (1991) Predictors of in-hospital mortality after DC catheter ablation of atrioventricular junction. Results of a prospective, international, multicenter study. Circulation. 84:1924-1937. 
[56] Ozcan C, Jahangir A, Friedman PA, Patel PJ, Munger TM, Rea RF, et al. (2001) Longterm survival after ablation of the atrioventricular node and implantation of a permanent pacemaker in patients with atrial fibrillation. New England Journal of Medicine. 344:1043-1051.

[57] Epstein AE, DiMarco JP, Ellenbogen KA, Estes NA, Freedman RA, Gettes LS, et al. (2008) ACC/AHA/HRS 2008 Guidelines for Device-Based Therapy of Cardiac Rhythm Abnormalities: a report of the American College of Cardiology/American Heart Association Task Force on Practice Guidelines. Journal of American College of Cardiology. 51:e1-62.

[58] Linde C, Leclercq C, Rex S, Garrigue S, Lavergne T, Cazeau Set al. (2002). Long-term benefits of biventricular pacing in congestive heart failure: results from the MUltisite STimulation in cardiomyopathy (MUSTIC) study. Journal of the American College of Cardiology. 40:111-118.

[59] Doshi RN, Daoud EG, Fellows C, Turk K, Duran A, Hamdan MH, Pires LA. (2005) Left ventricular-based cardiac stimulation post AV nodal ablation evaluation (the PAVE study). Journal of Cardiovascular Electrophysiology. 16:1160-1165.

[60] Brignole M, Gammage M, Puggioni E, Alboni P, Raviele A, Sutton R, et al. (2005) Comparative assessment of right, left, and biventricular pacing in patients with permanent atrial fibrillation. European Heart Journal. 26:712-722.

[61] Leclercq C, Mabo P. (2008) Cardiac resynchronization therapy and atrial fibrillation. Do we have a final answer? European Heart Journal. 29:1597-1599.

[62] Bardy GH, Lee KL, Mark DB, Poole JE, Packer DL, Boineau R, et al. (2005) Amiodarone or an implantable cardioverterdefibrillator for congestive heart failure. New England Journal of Medicine. 352:225-237.

[63] Padeletti L, Muto C, Maounis T, Schuchert A, Bongiorni MG, Frank R, et al. (2008) Atrial fibrillation in recipients of cardiac resynchronization therapy device: 1-year results of the randomized MASCOT trial. American Heart Journal. 156:520-526.

[64] Azpitarte J, Baún O, Moreno E, García-Orta R, Sánchez-Ramos J, Tercedor L. (2001) In patients with chronic atrial fibrillation and left ventricular systolic dysfunction, restoration of sinus rhythm confers substantial benefit. Chest. 120:132-8.

[65] Butter C, Winbeck G, Schlegl M et al.(2004) Management of atrial fibrillation in cardiac resynchronization therapy clinical practice of CRT: How to improve the success rate. European Heart Journal. 6: D106-D111.

[66] Chen MS, Marrouche NF, Khaykin Y, Gillinov AM, Wazni O, Martin DO, et al.(2004) Pulmonary vein isolation for the treatment of atrial fibrillation in patients with impaired systolic function. Journal of the American College of Cardiology. 43:1004-1009.

[67] Hsu LF, Jaïs P, Sanders P, Garrigue S, Hocini M, Sacher F, et al. (2004) Catheter ablation for atrial fibrillation in congestive heart failure. New England Journal of Medicine. 351:2373-2383.

[68] Gentlesk PJ, Sauer WH, Gerstenfeld EP, Lin D, Dixit S, Zado E, et al. (2007) Reversal of left ventricular dysfunction following ablation of atrial fibrillation. Journal of Cardiovascular Electrophysiology. 18: 9-14. 
Section 3

\section{Management of Complications}





\title{
Complications of Pacemaker Implantation
}

\author{
Jeffrey L. Williams and Robert T. Stevenson \\ Additional information is available at the end of the chapter
}

http://dx.doi.org/10.5772/48682

\section{Introduction}

Approximately 180000 patients undergo pacemaker implantation in the U.S each year [1]. In addition, the extreme elderly are the most rapidly growing segment of the U.S. [2,3] and pacemakers are commonly implanted in this population. There are reports of pacemaker implant complications (generally clinical trials reporting outcomes and incident complication rates) and fewer reports of complication rates in the extreme elderly (with a persistent exclusion of elderly patients from ongoing clinical trials [4]). A comprehensive review of pacemaker implant complications can help improve informed consent in preoperative patients. Major and minor complications are defined based upon prior reports of device-related complications. [5,6,7,8] Major complications have been defined as death, cardiac arrest, cardiac perforation, cardiac valve injury, coronary venous dissection, hemothorax, pneumothorax, transient ischemic attack, stroke, myocardial infarction, pericardial tamponade, and arterial-venous fistula. Minor complications have been defined as drug reaction, conduction block, hematoma or lead dislodgement requiring reoperation, peripheral embolus, phlebitis, peripheral nerve injury, and device-related infection. This chapter will include discussion of common and uncommon complications of pacemaker implantation including associated incidence as well as the associated radiographs and common clinical signs of these complications.

\section{Demographics of pacemaker implantation}

From 1993 to 2006, 2.4 million patients received a primary pacemaker and 69,000 pacemaker generator changes; women comprised $49 \%$ of pacemakers. [1] A review of studies involving pacemaker implantation in adults reveals an average age range of 69-86 years with approximately $30-40 \%$ of patients aged $>80$ years $[9,10]$. There is a tendency for higher percentage of female patients as age increases; a prior study from our Heart Rhythm Center [11] examined pacemaker implant outcomes of extremely elderly patients (>80years) with an average implant age of 86 and revealed $61 \%$ of implants were performed in females. 


\section{Preprocedural issues}

\subsection{Preoperative risk assessment}

As with all surgical interventions requiring anesthesia, recognizing and managing comorbid conditions preoperatively helps to mitigate the risks during and immediately after pacemaker implantation. The association of heart failure and other structural heart disease with cardiac conduction system disease as well as the expanding role of biventricular pacemakers specifically indicated for patients with symptomatic congestive heart failure means that there is an inherently high risk population of patients frequently served in the EP lab. Indeed, CHF increases the risk of all surgery. A decreased LVEF has been found to be a predictor of perioperative mortality and morbidity, with the highest risk group being those with an $\mathrm{EF}<35 \%$; the very patients brought to the lab for resynchronization therapy. Pre-procedural management of $\mathrm{CHF}$ is not only integral to our practice but vitally important to the safety of the procedure. Patients certainly should not be in a state of decompensated heart failure.

\subsection{Infection}

Patients who present with systemic infection and positive blood cultures carry the highest risk of infection. Infection of implantable devices is one of the most feared complications due to the dismal prognosis of untreated infections and risk of device removal. Often we are asked to evaluate patients for bradyarrhythmias when they happen to be identified at the time of hospitalization for infectious etiologies such as pneumonia and urosepsis as well as post cardiac surgery. Pre-implant evaluation for potential sources of infection is critical. The estimated rate of infection of permanent endocardial pacing leads is between 1 and $2 \%$, however the range is from under 1 percent to greater than $10 \%$. In the PEOPLE study [12], device infection requiring removal was correlated to fever within 24 hours of device implant, temporary pacing prior to implant, and early reintervention for lead revision or hematoma evacuation. The likelihood of infection was nearly doubled by the presence of a temporary system. The association with temporary intravenous pacing wires certainly implies an association with any indwelling lines including central lines and PICC lines. The duration of hospitalization prior to implant was not correlated to higher risk of infection. Infections were negatively correlated with de-novo implantation and perioperative antibiotic prophylaxis. The latter intervention is considered controversial. Of 28,860 Danish patients [13], 3.6\% had a lead related complication by 3 months. Risk factors for lead related complications included operator inexperience ( $<25$ implants).

\subsection{Procedural management of iodinated contrast agents}

\subsubsection{Contrast Induced Nephropathy (CIN)}

Contrast-induced nephropathy is a surprisingly common complication of radio-contrast procedures occurring in $15 \%$ of cases. It is defined as an absolute increase in creatinine of $0.5 \mathrm{mg} / \mathrm{dl}$ (in patients starting under 2 ) or an increase of $25 \%$ of baseline and typically peaks 
at 48-72 hours after exposure. Creatinine may remain above baseline for 7-14 days. Naturally, the best way avoid this complication is to abstain from its use. Single and dual lead pacing systems can safely be implanted without the use of contrast at all; Prior data from our Heart Rhythm Center [11] revealed that contrast was used in 55 of 92 (59.8\%) of the pacemaker implantations with a mean intravenous contrast usage of 9.6cc. We often use contrast to ensure that the subclavian /axillary venous system is patent and to help guide venous access. No contrast was used for generator changes and there were no contrast reactions. CRT implantation may require contrast agents to define the coronary (CS) anatomy. The vast majority of patients undergoing CRT implants are patients with heart failure and its associated comorbidities which very frequently include diabetes and chronic kidney disease, therefore a working knowledge and respect for these agents is a necessity. We typically use 5cc of iso-osmolar contrast in a 1:1 ratio with NSS and limit CS venography to 1 cine if possible. For patients with GFR 30-60 $\mathrm{ml} / \mathrm{min}$, hydration can usually be achieved with 4-6 glasses of water the evening before the procedure. ACE inhibitors, ARBs, and NSAIDS can be held the day prior and day of exposure and be resumed 24 hours after exposure. For patients with a GFR $<30 \mathrm{ml} / \mathrm{min}$, the above recommendations should be followed with consideration of one or both of the following: 1.) IV hydration using $1 \mathrm{~L}$ of $\mathrm{NaCl} 0.45 \%$ with $50 \mathrm{mEq} \mathrm{NaHCO} 3$ (1/2 normal saline with 1 amp of sodium bicarbonate) run at $1 \mathrm{ml} / \mathrm{kg} / \mathrm{hr}$ for 12 hours. For same day procedures, this can be administered at $3 \mathrm{ml} / \mathrm{kg} / \mathrm{hr}$ for 1 hour. 2.) N-acetylcysteine 600mg PO BID the day prior and day of the procedure, totaling 4 doses. It must be noted that besides preprocedural hydration, the evidence is not conclusive that bicarbonate and acetylcysteine offer additional benefit. Treatment of CIN after evident is largely supportive and infrequently requires short-term dialysis.

CS lead placement can be performed successfully without the use of contrast in patients at risk of CIN. [8] CS lead placement at our Heart Rhythm Center without the use of contrast begins with CS access by engagement of the CS with a 5French octapolar deflectable electrophysiology (EP) catheter or hydrophilic coated $0.035^{\prime \prime}$ guidewire that the CS sheath is advanced over. Next, a 0.014 " guidewire is advanced out distally and the entire CS is probed for LV branch vessels in 360 degree fashion; we then work proximally and if no braches are found (including very proximal posterolateral "bailout" vessels) then we repeat the process. We have demonstrated a $97.3 \%$ success rate in LV lead placement with these techniques. [8]

\subsubsection{Contrast allergies}

Immediate anaphylactic reactions including angioedema, bronchospasm, arterial hypotension, and shock can occur within minutes of and up to 60 minutes after injection. [14] The reported incidence of severe immediate reactions to ionic contrast material (CM) is 0.1$0.4 \%$ and with newer non-ionic, low osmolar or iso-osmolar contrast is $0.02-0.04 \%$ but death rates from the two materials do not differ. [15] Although incompletely understood, direct release of histamine from circulating basophils and eosinophils is probably the primary mechanism with IgE mediated mast cell activation (i.e. true allergy) being a much less frequent secondary mechanism. A skin testing study showed that only $4 \%$ of patients with anaphylaxis symptoms had an IgE-mediated mechanism [16]. Delayed reactions from 1 hour 
to 7 days after injection of CM are T-cell mediated and most typically are skin reactions. [15] Patients with even mild anaphylactoid (immediate) reactions should be considered high risk in future $\mathrm{CM}$ administration. Indeed, a distinction between immediate anaphylactoid and non-immediate anaphylactoid reactions may be more clinically relevant than a history of mild, moderate, and severe reactions. [17] For purposes of reporting, CM reactions are graded 1-3. Grade 1 reactions include one episode of nausea/vomiting or sneezing, grade 2 reactions being fever/chills, hives, and more severe nausea/vomiting, and grade 3 reactions are potentially life-threatening and include angioedema, bronchospasm, laryngospasm, pulmonary edema, hypotension, and shock.[18] It is common practice to pre-medicate with corticosteroids with or without H1 blockers in patients with a history of moderate or severe immediate reactions, despite the fact that randomized trials comparing pretreatment strategies are severely lacking. No trials of pre-treatment have tested a steroid-antihistamine combination which is the most commonly utilized. [18] In a consensus document published by Marcos et al in 2001, 91\% of survey respondents administered corticosteroids at least 11 hours prior to $\mathrm{CM}$ administration (though dose frequency varied from 1-3x) and 55\% used an H1 blocker (diphenhydramine 25-50mg) typically administered once. [14] H2 blockers are used, but rarely. In a meta-analysis including 10,011 patients with no history of contrast allergy, routine pre-treatment with steroids alone reduced respiratory symptoms after contrast injection (frequently ionic, high osmolar) from $1.4 \%$ to $0.4 \%$ and Grade III symptoms from $0.9 \%$ to $0.2 \%$. Only a "double dose" steroid regimen reduced Grade III symptoms. [18] In this same cohort, there were no "disastrous" consequences. Cutaneous manifestations were more often prevented by $\mathrm{H} 1$ antagonists and respiratory symptoms show improvement with steroid pretreatment. [18] Essential information to be sought from the patient prior to administration of contrast is history of previous CM reaction, asthma, renal insufficiency, diabetes, and metformin therapy. [19]

Routine pre-medication of all patients to receive CM is probably not warranted given the overall low incidence of a reaction; in fact some have advocated abandoning this procedure all together. [18] Patients with a history of severe CM allergy who will likely need IV CM injection should probably receive pre-exposure prophylaxis with corticosteroids as well as $\mathrm{H} 1$ antagonists although strong evidence of benefit is lacking. [16] The likely mechanism for the benefit of corticosteroids is a reduction in circulating basophils and eosinophils available for direct activation. If CM administration cannot be delayed for 4-6 hours after steroid injection, some would omit use and administer only H1 blockers. [19] One can also consider the addition of $\mathrm{H} 2$ antagonists such as ranitidine but evidence is also lacking. Low or isoosmolar contrast such as ioxaglate, iohexol, or ioversol should be used due to the lower overall incidence of reactions in patients with a history of asthma or a CM allergy. The specific $\mathrm{CM}$ causing the prior reaction should be sought and avoided if possible. Cutaneous allergic testing can be performed on patients with a history of anaphylactic reactions and a "skin test negative" CM should be used but it should be noted that only a fraction of those patients will have a positive skin test.[15,18] The ESUR guidelines on prevention of CM reactions recommend Prednisone 50mg $\mathrm{PO}$ at 13, 7, and 1 hours prior or methylprednisolone $32 \mathrm{mg}$ orally 12 and 2 hours prior to exposure to $\mathrm{CM}$ in addition to 
diphenhydramine 50mg IV, IM, or PO 1 hour prior to exposure. Despite this, reactions are reported in those with prior reactions with corticosteroid pretreatment.

\subsection{Thyroid}

Hypothyroidism has been found in $0.5-0.8 \%$ of the population demonstrated by elevated serum levels of thyroid-stimulating hormone (TSH) or decreased serum thyroxine levels. [20] Undiagnosed (and hence untreated) hypothyroidism can lead to major perioperative complications including severe hypotension or cardiac arrest following induction of anesthesia, extreme sensitivity to narcotics and anesthetics with prolonged unconsciousness and hypothyroid coma following anesthesia and surgery. [20] Ideally, hypothyroidism is caught early and thyroxine administered until the patient is euthyroid (generally, 4-6weeks).

Hyperthyroidism affects approximately $0.2 \%$ of men and $2 \%$ of women and may cause atrial fibrillation, congestive heart failure and thrombocytopenia. [21] In addition, anesthetic drugs may be affected by the hypermetabolic state of hyperthyroidism. When total intravenous anesthesia is used (often at our center when high frequency jet ventilation is used to minimize respiratory motion), propofol infusion rates should be increased to reach anesthetic blood concentrations because the clearance of propofol is increased in hyperthyroid patients. [21] Supportive management of thyroid crisis includes hydration, cooling, inotropes and steroids. Beta-blockade and antithyroid drugs are used as the first line of treatment.

Generally, more thyroid related perioperative complications stem from hypothyroidism as opposed to hyperthyroidism however, recognition of either prior to implantation is important. Our Heart Rhythm Center generally obtains TSH prior to all device implantation and allows 4-6weeks for the patient to become euthyroid prior to proceeding with surgery. Emergent cases with thyroid abnormalities require close coordination with anesthesiology and will generally be undertaken with general anesthesia.

\section{In-hospital complications}

\subsection{Sedation / airway:}

Less than $20 \%$ of electrophysiology (EP) programs in the United States exclusively use anesthesia professionals for procedural sedation. [22] Minor complications (e.g., atelectasis, fever, vascular congestion) may simply be reflective of common postoperative pulmonary complications (PPC's) seen after general anesthesia. Atelectasis can be seen on CT scan in up to $90 \%$ of patients who are anesthetized [23] and PPC's have been found to occur in $9.6 \%$ of patients. [24]. There are data to suggest that patients undergoing invasive EP procedures may require deep conscious sedation that often is converted to general anesthesia;[25] thus, the use of general anesthesia (including HFJV) during EP procedures may enhance patient safety.[26,27] The advantage of general anesthesia was mostly studied in patients undergoing complex procedures, such as AF ablation, where precise electroanatomical mapping is required. There is not much data supporting the use of general anesthesia 
(which may increase costs) versus conscious sedation in routine PM implantations. In our center, we often use general anesthesia with a laryngeal mask airway to minimize patient movement during LV lead implantation.

\subsection{Pneumothorax}

Pneumothorax may occur in as many as 3-4\% $[28,29]$ and as few as $0-1 \%[8,10,11,30,31,32]$ but generally ranges from $1-3 \%(5,9,33,34,35,36,37]$ of patients undergoing pacemaker implantation. Routine chest radiographs are often performed immediately after pacemaker implantation though clinical signs of pneumothorax include hypoxia, shortness of breath, pleuritic pain, and hypotension. Figure 1 depicts the radiographic appearance of small, medium, and large pneumothoraces. Figure 2 depicts a pseudopneumothorax that resulted from external artifact; close review of radiographs by radiologists can limit false-positive pneumothorax interpretations. Emergent treatment of pneumothorax includes decompression of the pressure tension by thoracentesis or chest tube. Oftentimes, high concentrations of inspired oxygen can lead to a resolution of a pneumothorax that comprises less than $30 \%$ lung volume. (38) This conservative treatment of pneumothorax can reduce morbidity and duration of hospitalization and avoid invasive drainage procedures. The traditional treatment of patients with traumatic hemo- or pneumo-thoraces has been an insertion of a chest tube (CT). CT have larger caliber than pigtail catheters and can cause significant trauma during insertion, cause pain, prevent full lung expansion, and worsen pulmonary outcomes. [39] Pigtail catheters, smaller and less invasive than chest tubes, have been used successfully in patients with nontraumatic pneumothorax. Pigtail catheters have demonstrated a non-significant increase $(11 \%$ vs $4 \%$ for CT) in the tube failure rate (defined by a requirement for an additional tube or by recurrence requiring intervention) [39].

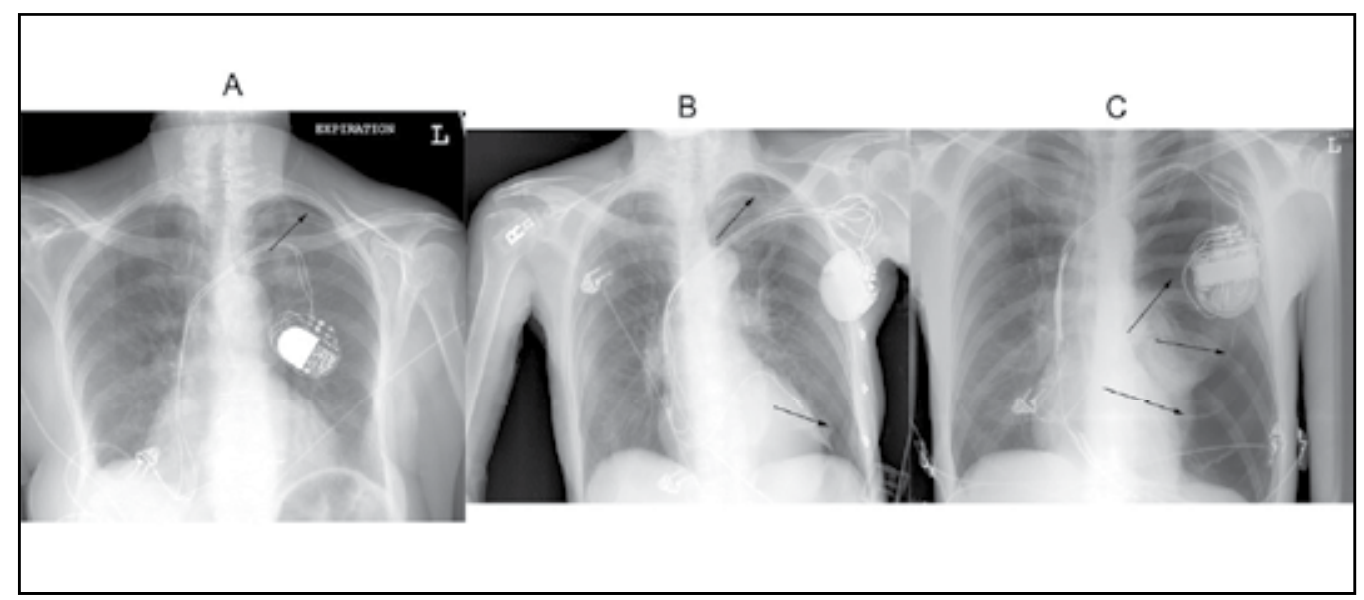

Figure 1. Examples of Pneumothoraces, Small (A), Medium (B), and Large (C). The edge of the pneumothorax is indicated by the arrows. A small left apical pneumothorax is shown in A. A moderatesized apical and basilar pneumothorax is shown in B. An almost complete collapse of the left lung is shown in C. Please note that examples shown in B and C are from defibrillator implantations. 


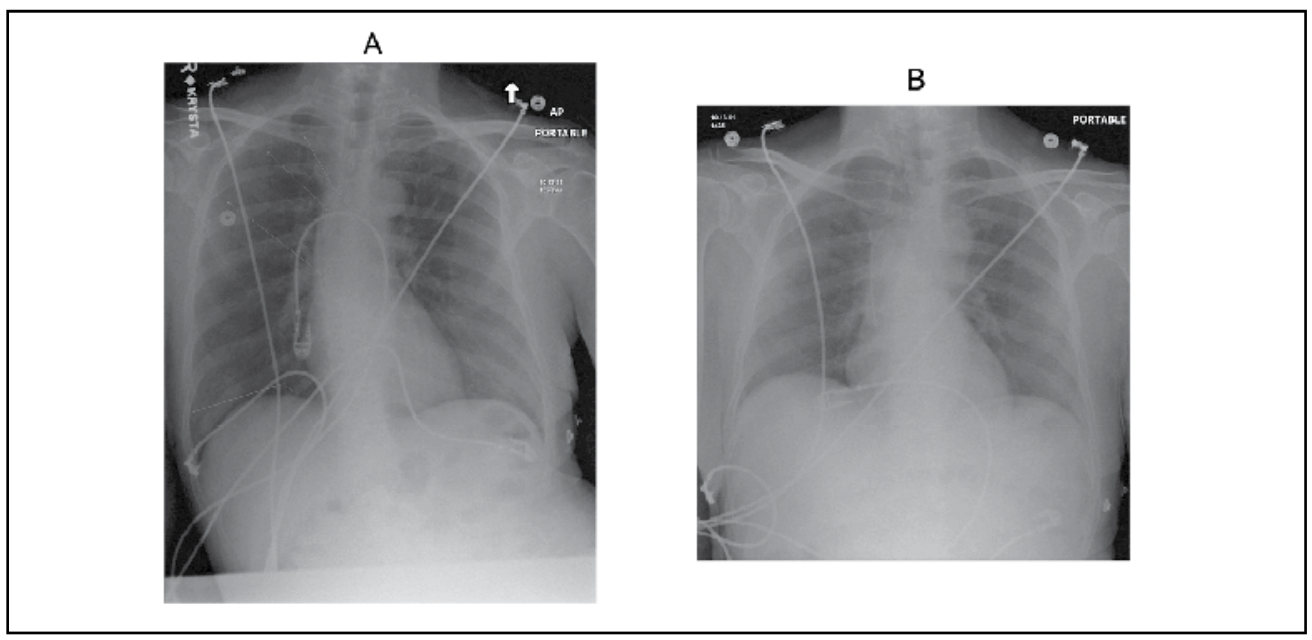

Figure 2. Pseudopneumothorax after removal of temporary internal jugular vein temporary pacemaker. Figure A shows an outline (arrows) that appears to indicate a pneumothorax in an asymptomatic patient. Repeat CXR indicates that initial findings have resolved (with no intervention) and was likely external artifact from gown or skin.

\subsection{Vascular access and hemothorax}

The axillary venous approach has been associated with less frequent pneumothorax and subclavian crush syndrome. [40,41] The axillary vein is the continuation of the basilica vein that terminates immediately beneath the clavicle at the outer border of the first rib, at which point it becomes the subclavian vein. [42] Direct subclavian venous punctures are associated with increased rate of pneumothorax [5] while cephalic vein cutdown has been associated with the lowest rate of pneumothorax and lead damage. [33,31] Fluoroscopicguided, first rib approach to axillary vein access is the most effective means to access the vessel while minimizing the risk of pneumothorax. [42] A prior study did examine pacemaker implantation complication rates of 632 consecutive implants at a single noncommunity institution.[33] They found a $0.6 \%$ rate of hemothorax with a substantially large incidence of complications experienced by low-volume $(<12$ implants per year) implanters.

Hemothorax can be caused by pacemaker lead placement (more frequently atrial lead perforation) as well as vascular access damage to the subclavian and axillary veins as well as the vena cava. Figure 3 depicts a post-implant CXR of a hemothorax from damage to the superior vena cava during upgrade of dual chamber pacemaker to biventricular defibrillator. This patient experienced pain during passage of the introducer placed over a guidewire seen in inferior vena cava with subsequent development of right pleural effusion. The injury was believed to have occurred during passage of CS guiding catheter introducer over the wire. Recognition of new effusions should be treated as possible procedural related hemothorax and surgical consultation is warranted. 


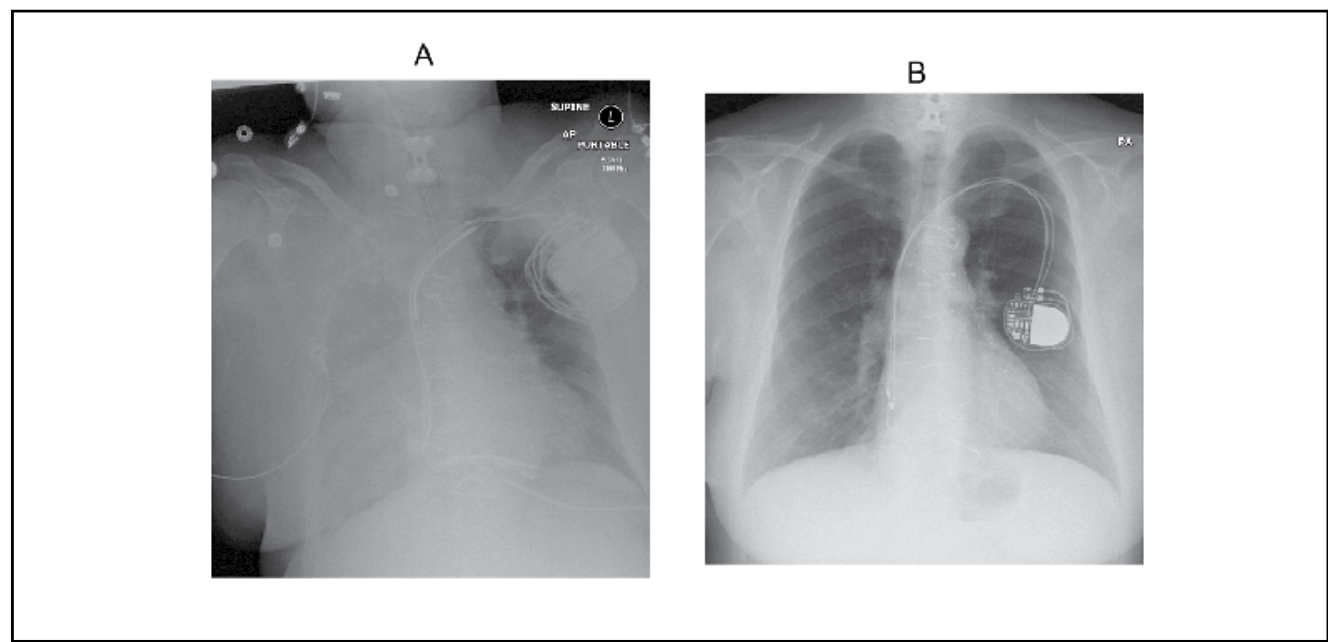

Figure 3. Hemothorax after Device Implantation (A) From Wire/Sheath Trauma to the Superior Vena Cava. The right hemithorax has a layered effusion with blunting of the right costophrenic angle. Image $\mathrm{B}$ depicts the preoperative radiograph prior to upgrade of pacemaker to defibrillator.

\subsection{Perforation / tamponade}

Perforation (both acute and subacute) has been reported to occur in up to $1 \%$ of pacemaker implantations. [5,8,10,31,32,35,36,37] In addition, asymptomatic subclinical perforation may occur in $15 \%$ of patients after device implantation. [43] Symptoms of perforation include pleuritic chest pain from pericarditis, diaphragmatic or intercostal muscle stimulation and, in the presence of pericardial effusion, patients may develop shortness of breath and hypotension as tamponade develops. [44] Others signs/symptoms of perforation include right bundle-oid paced QRS morphology (though we have seen RBBB configuration and diaphragmatic stimulation in RV apical lead position) or friction rub after implant. If perforation is suspected, urgent evaluation of the patient and device function is warranted though lead parameters are often within normal limits. [44] Figure 4 shows examples of coronary sinus damage that can occur during LV lead implantation. Figure 5 depicts right ventricular lead perforations. Cardiac surgery is typically not required for a majority of patients diagnosed with cardiac perforation from a pacemaker implantation. Rather, most cases can be managed with pericardiocentesis for symptomatic effusions and repositioning of the lead in the EP laboratory with close cardiothoracic surgical collaboration. [45,46,44] Figure 6 shows a large cardiac silhouette developing after pacemaker implantation that was due to large pericardial effusion. The effusion was treated with pericardiocentesis (with no evidence of reaccumulation) and did not require lead repositioning. Though perforation and subsequent tamponade are infrequent complication of pacemaker implantation, they can be responsible for significant patient morbidity and mortality. The risks of perforation cannot be underestimated; death from tamponade with subsequent cardiac arrest was responsible for $21.8 \%$ of the mortality in a worldwide study of perforation after ablation for atrial fibrillation. [47] 


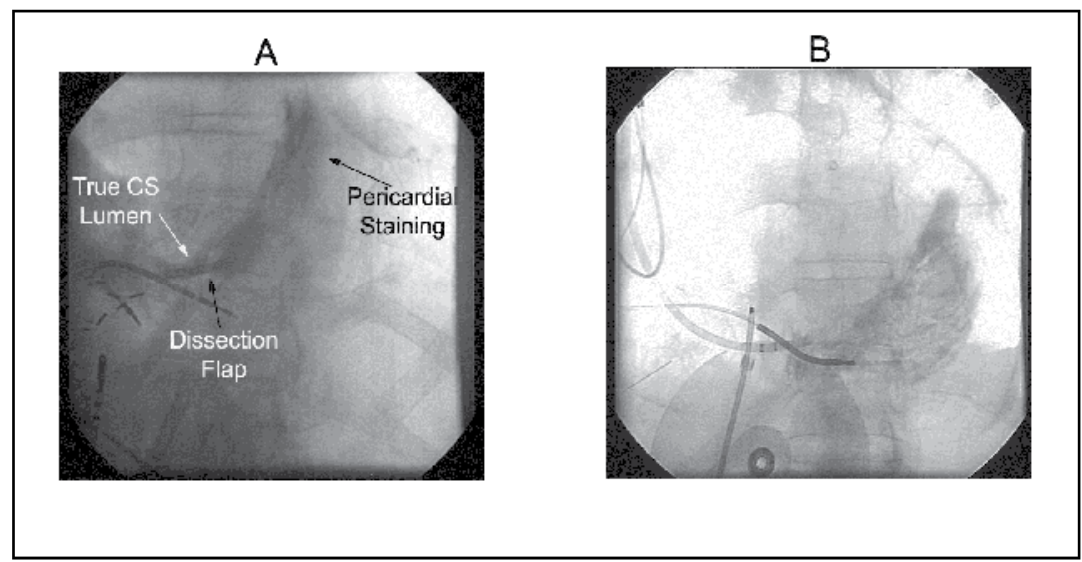

Figure 4. Damage to the coronary sinus during left ventricular lead implantation. Image A depicts a dissection/perforation flap and the resulting pericardial staining from engaging the coronary sinus with a deflectable electrophysiology recording catheter. Image B shows a similar instance of pericardial staining with no focal dissection flap or perforation. Both patients underwent successful LV lead implantation at the time.

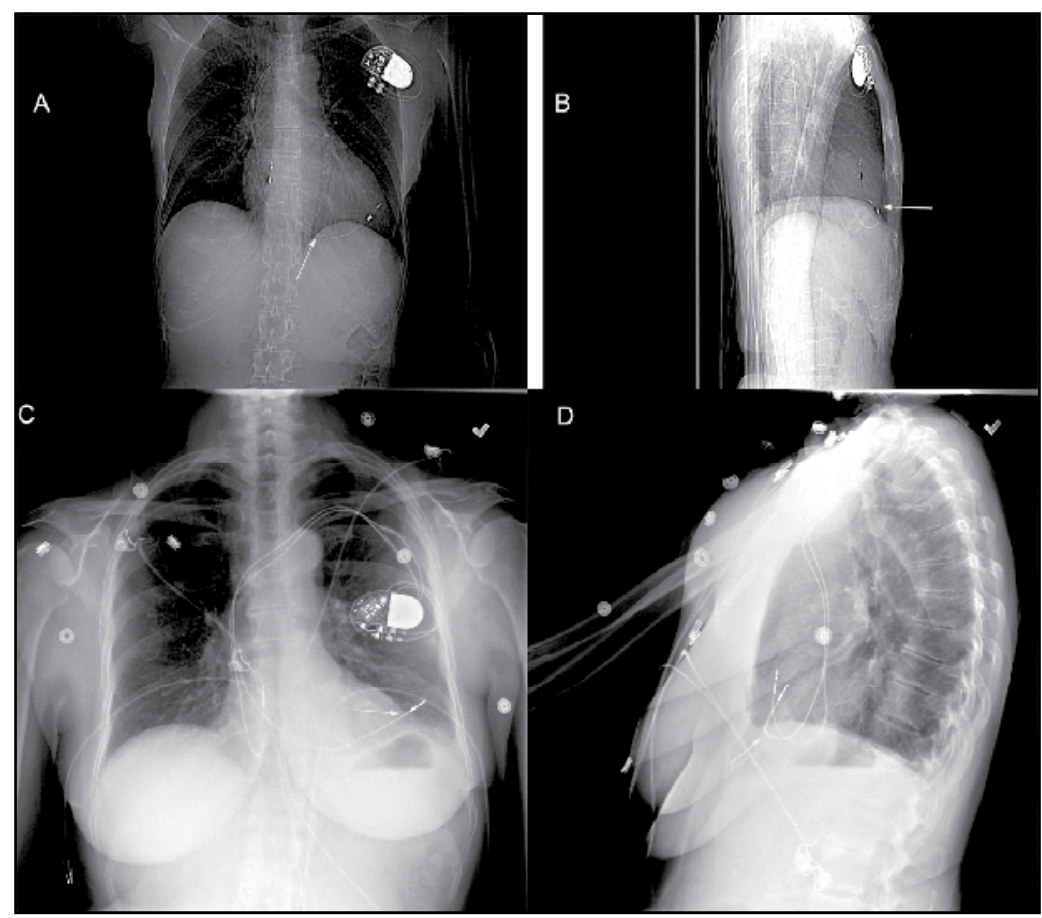

Figure 5. Examples of Right Ventricular Pacemaker Lead perforation. Images A and B depict an RV lead perforation that exits the right ventricular base in $\mathrm{A}$ (arrow) and reenters near the right ventricular apex in $B$ (arrow). Images $C$ and $D$ depict an right ventricular apical perforation. The lead is seen exiting the cardiac silhouette in C (arrow); the lateral view (D) depicts an abrupt change in lead course (arrow) that is often seen in right ventricular apical perforations as the lead courses posteriorly in the pericardial space. 


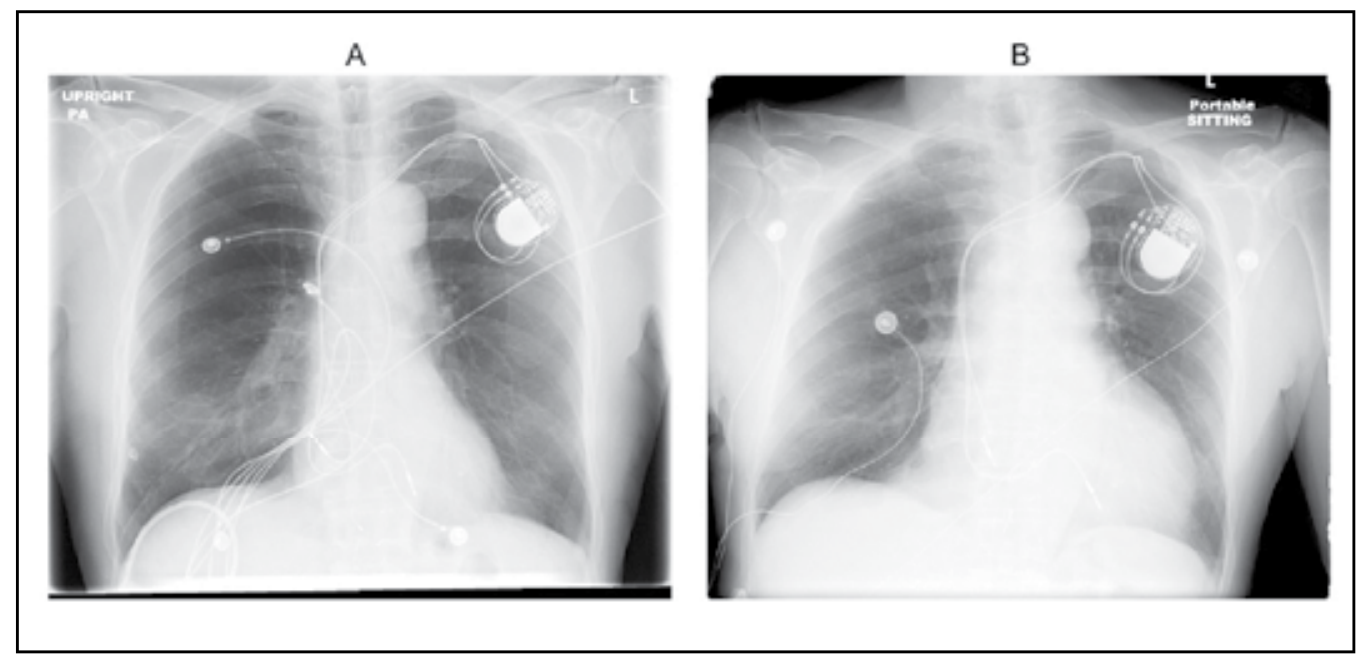

Figure 6. Chest radiograph appearance of large pericardial effusion after RV lead perforation. Immediate post-implant CXR (A) shows normal appearance of the cardiac silhouette. Two weeks postoperative CXR performed because patient reported symptoms of chest pressure (B) shows enlarged cardiac silhouette. Patient responded to pericardiocentesis with no lead repositioning.

\subsection{Complications of Left Ventricular (LV) lead placement via the coronary sinus}

The emergence of resynchronization therapy has led to an increase in attempts at left ventricular lead placement via the coronary sinus. The MIRACLE study program [48] reported a 91.6\% success rate for LV lead placement, while COMPANION [49] revealed an $89 \%$ success rate for LV lead placement. Another report indicated a similar $92 \%$ success rate with LV lead placement. [50] Though we counsel our patients on a LV lead placement success rate at $88-92 \%$, our center demonstrated a $97 \%$ success rate (64 of 66 patients) with $\mathrm{LV}$ lead placement within the range from 2:30 to 5:30 o'clock in the left anterior oblique (LAO) view. [8]

Complications of biventricular pacing, specifically LV lead placement, include cardiac perforation, coronary sinus dissection, electrical trauma (damage to the native conduction system), failure to place the lead, dislodgement of the lead, and diaphragmatic stimulation. [51] CS dissections or perforations, cardiac perforations, or cardiac vein dissection or perforation was reported in 45 of $2078(2 \%)$ in the MIRACLE study program. [48] Figure 4 depicts damage done to the coronary sinus during LV lead implantation. Loss of LV capture and diaphragmatic stimulation leading to interruption of resynchronization therapy has been found to occur in $10 \%$ and $2 \%$ of patients, respectively. [52] The development of new LV leads with up to 4 electrodes offer the possibility of numerous pacing vectors that can minimize loss of capture and diaphragmatic stimulation. 


\subsection{Arrhythmias (SVT, VT, VF)}

The incidence of sustained atrial, pacemaker-mediated and ventricular rhythm disturbances after pacemaker implantation is low. [53]. In patients without prior atrial arrhythmias, Jordaens et al found early atrial fibrillation (during the first week) in 2 of 112 patients and late atrial fibrillation was seen in seven patients, flutter in one, yielding a total incidence of $8.9 \%$ for 22 months. There were no significant differences with respect to age, etiology, electrocardiographic diagnosis, pacing history, or the measured intracardiac $\mathrm{P}$ wave between the group with and the group without atrial fibrillation. Ventricular fibrillation has been reported to occur in $0.1 \%$ of all patients undergoing pacemaker implantation and up to $0.6 \%$ of patients aged $>90$ years undergoing pacemaker implantation. [10]

It has been reported that ventricular tachyarrhythmias may be present in $12-31 \%$ of patients months to years after pacemaker implantation [54] but this may reflect underlying substrate issues. However, there are several situations where pacemaker implantation may cause the ventricular tachyarrhythmias. These include pacemaker lead irritation of the right ventricular inflow [55] and outflow tracts [56], pacemaker stimulus on T wave [57], reentrant circuit around endocardial pacemaker lead [58] and bradycardia-dependent VT facilitated by long pause caused by myopotential inhibition of a VVI pacemaker. [59]

\subsection{Death}

In-hospital death generally occurs in less than $1 \%$ of pacemaker implantations $[5,8,10,11,37]$ however there is a concern that death is underreported as some studies do not specifically mention perioperative death. $[9,30,31,32,33]$ The most common causes of confirmed device related in-hospital deaths are perforations (subclavian artery, brachiocephalic trunk, right atrium, and right ventricle). The most common cause of non-device related in-hospital deaths is myocardial infarction as well as less commonly pulmonary embolism, stroke, heart failure, and sepsis. [60]

\subsection{Pocket hematoma}

The incidence of pocket hematoma has been reported at $4.9 \%$ and leading to prolonged hospitalization in $2.0 \%$ of all patients. [61] Reoperation for pocket hematoma was required in $1.0 \%$ of patients. High-dose heparinization, combined acetylsalicylic acid (ASA)/thienopyridine treatment after coronary stenting, and low operator experience were independently predictive of hematoma development. [61] In addition, development of postoperative hematoma places the patient at elevated risk of device infection. [62] There is data to suggest that warfarin causes fewer pocket complications than heparin products. Specifically, temporarily interrupting anticoagulation is associated with increased thromboembolic events, whereas cessation of warfarin with bridging anticoagulation is associated with a higher rate of pocket hematoma and a longer hospital stay. [63] 


\subsection{Hospital lengths of stay}

There is little data available on average length of stays post pacemaker implantation. An estimate of 2-3days as an average length of stay post implant can be made from the available studies. $[5,8,11,37]$ There is evidence that complications cause a substantial increase in the length of stay up to 16days. [64] The mean complication costs are $\$ 4345 \pm \$ 1540$ for pacemaker lead revision, $\$ 24,459 \pm \$ 14,585$ for pacemaker infection, and $\$ 6187 \pm \$ 2631$ for hematoma evacuation. [64]

\section{Subacute post-implant complications (<30days)}

\subsection{Pacemaker and lead function / failures}

Electrocardiographic signs of pacemaker malfunction can be grouped into four categories: failure to capture, failure to output, undersensing, and inappropriate pacemaker rate. Sensing abnormalities may occur in $3 \%$ of patients, failure to capture in $1 \%$, and failure to capture and inappropriate pacemaker rate in another $1 \%$ of patients. [65]

\subsubsection{Failure to capture}

Loss of capture after pacemaker implantation has many causes: Dislodgement, Elevated Thresholds, Inappropriate Lead Placement, Fracture, Insulation Failure, Loose set screw, Exit Block ( $>4$ weeks), Perforation, Battery/circuit Failure, Air in Pocket, and Metabolic/Drugs (Flecainide). Lead dislodgement, the most common cause of failure to capture, [65] has been reported to occur in up to $4-6 \%$ of pacemaker implantations $[28,35]$ but is generally reported with a $1-3 \%$ incidence $[5,10,11,30,32,33,34,36,37]$. Figure $7 \mathrm{~A}, \mathrm{~B}$, and $\mathrm{C}$ depicts examples of right atrial, right ventricular, and left ventricular lead dislodgements that may result in loss of capture. Lead dislodgements are treated by repositioning in the EP laboratory.

\subsubsection{Failure to output}

Failure to pace with no obvious pacemaker output may be caused by battery or circuit failure, lead fracture, internal insulation failure, oversensing, loose set screw, or crosstalk. Random component failure is rare however, total battery depletion can occur if routine pacemaker followup is inadequate. [65] Once initial end-of-life indicators appear, there is usually a period of months before the battery reaches a critically low voltage and pacing fails. [65] The incidence of pacemaker lead fracture has been reported at $0.1 \%$ to $4.2 \%$ per patient-year and usually occurs adjacent to the generator or near the site of venous access. [66] Figure 8 shows a lead fracture discovered at reoperation when patient presented in complete heart block with no ventricular capture almost one year from pacemaker implant. Figure 9 depicts chest radiograph findings of lead fracture (failure to pace) versus a pseudofracture (normal pacing function). Finally, air in header may cause noise oversensing (as air released out of header) as shown in Figure 10. Other electrical signals that may cause 


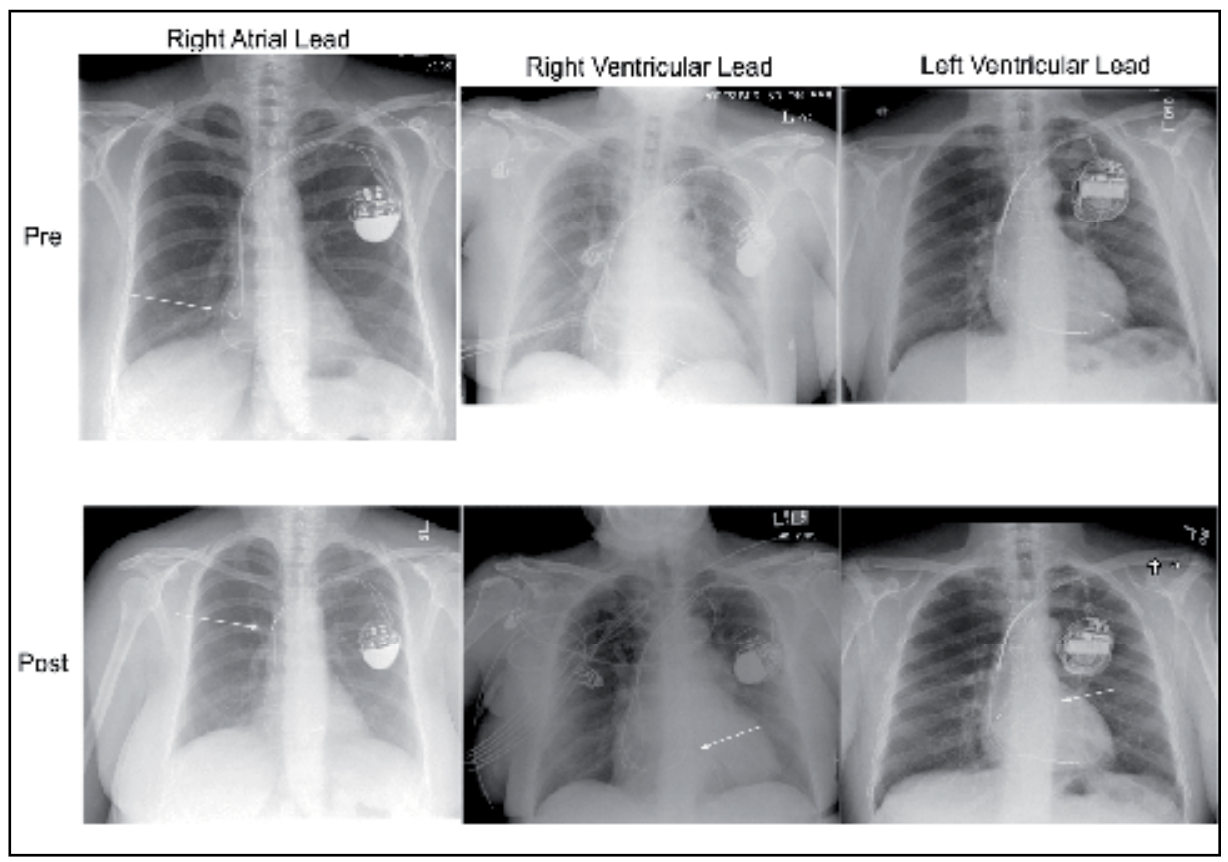

Figure 7. Right Atrial (A), Right Ventricular (B), and Left Ventricular (C) Leads Before (Pre) and After (Post) Dislodgements. Right atrial lead became dislodged after patient twiddled with device. Right ventricular lead dislodged by moving more basilar in position (arrow) one day after implant. Left ventricular lead dislodged and reseated itself in the body of coronary sinus 3 months after initial placement (arrow).

oversensing include diaphragmatic myopotentials (especially with extending bipolar sensing), T waves, $\mathrm{P}$ waves, and environmental noise.

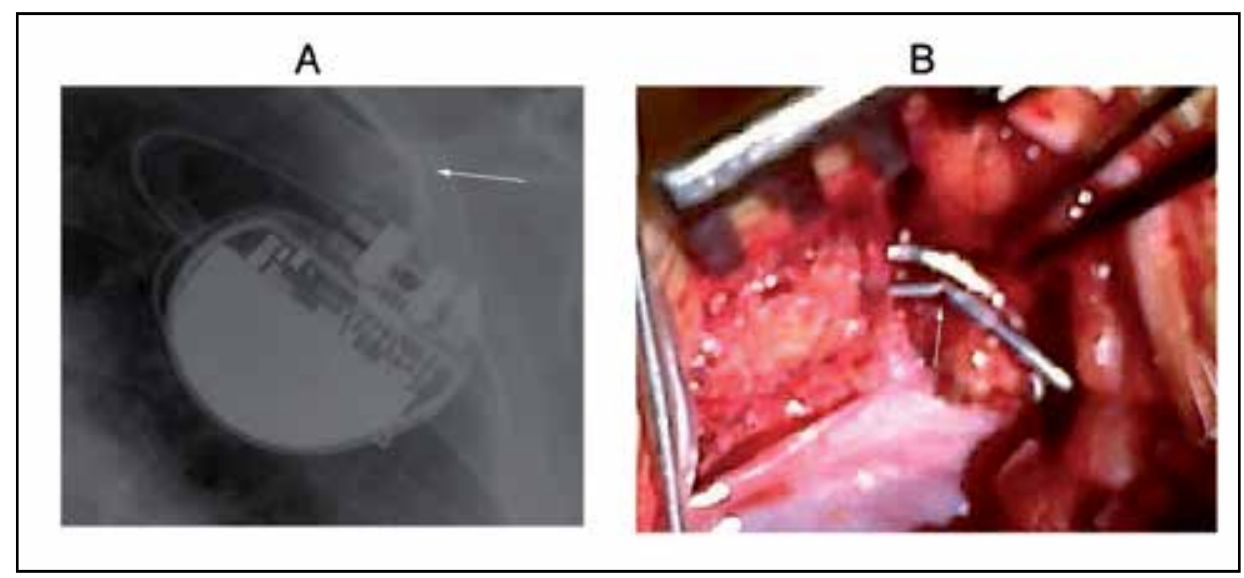

Figure 8. Right Ventricular Lead Fracture. Ventricular lead that was fractured one year after implantation resulting in failure to pace. Figure 8A shows appearance of right ventricular lead on CXR that was suspicious for fracture location (arrow). Figure 8B depicts the intraoperative appearance of lead that was likely site of fracture (arrow). 


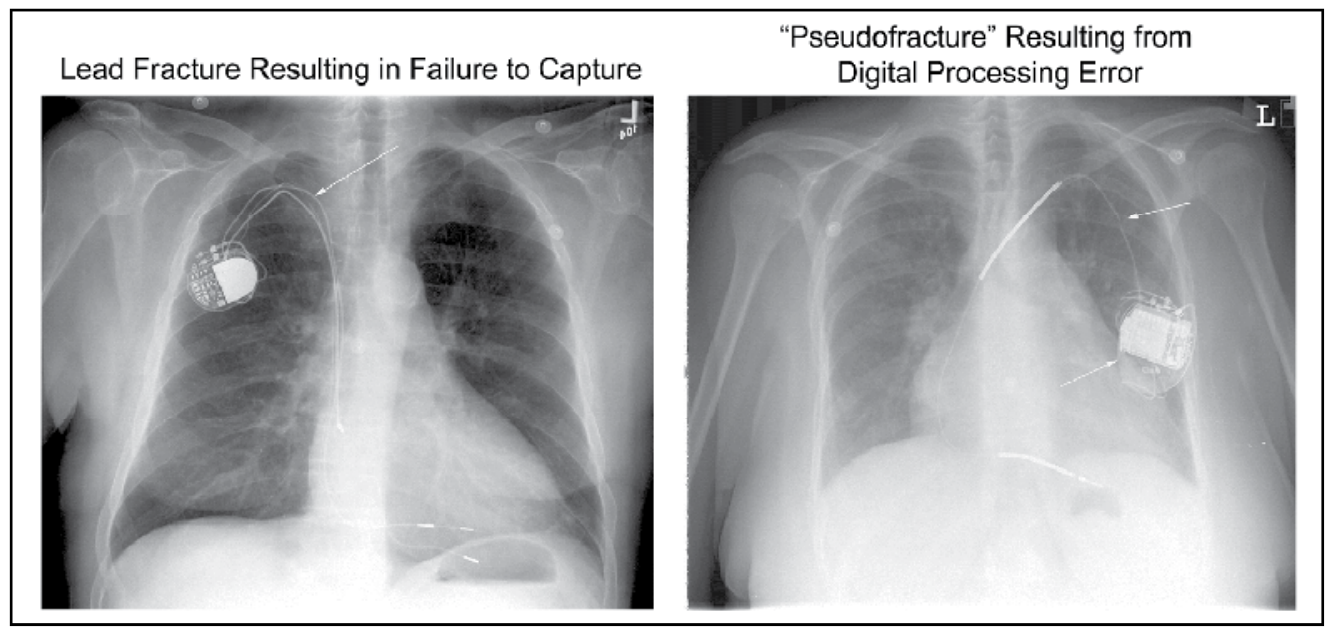

Figure 9. The radiograph in A shows a lead fracture that resulted in no capture. The radiograph in B depicts a "pseudofracture" where digital frame shift causes artifact to simulate a lead fracture in a properly functioning lead.

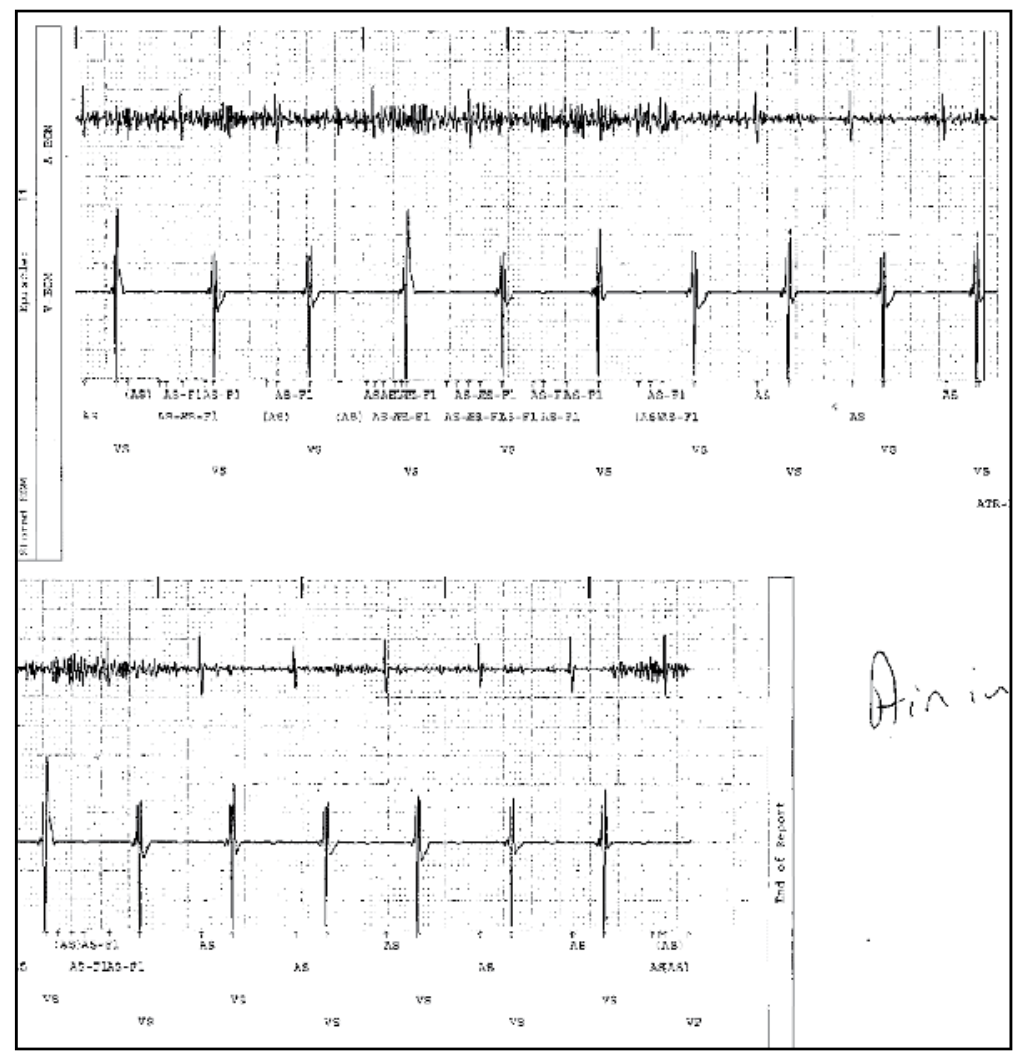

Figure 10. Noise caused by air in header. Atrial electrogram during device interrogation revealed high-frequency noise during sinus rhythm. This patient was not pacemaker dependent so there was no failure to pace. 


\subsubsection{Undersensing}

Undersensing of intrinsic cardiac activity results in inappropriate pacing output that competes with intrinsic activity. Undersensing is most likely caused by lead dislodgement, poor lead position at time of implantation, or an interruption in the insulation of the pacing lead. [65]

\subsection{Hospital readmission}

The average rate of hospital readmission within 30 days of pacemaker implant is $4-6 \%$. $[8,11,28,35]$ We examined possible factors influencing readmission rates in extreme elderly undergoing pacemaker implantation. [11] Overall, increased age and Device Type (e.g., single-chamber, dual-chamber, biventricular, generator change) demonstrated a nonsignificant trend toward increased readmission rate. The order of decreasing significance in a multivariate analysis of readmissions was: Device Type $>$ Age $>$ Creatinine $>$ Urgent/Emergent $>$ EF $>$ Sex $>$ Weight.

\subsection{Death}

Early all-cause mortality 30-days after pacemaker implantation has been reported in 0.1$0.7 \%$ of patients. $[30,5,36]$ Death rates may be increased $(2 \%)$ in the extreme elderly aged $>80$ years due to increased age-related mortality in this group. [11]

\section{Late complications (>30days)}

\subsection{Lead function / failures}

\subsubsection{Twiddling}

Originally described in 1968 [67], twiddling refers to patient manipulation of pacemaker can or leads that may lead to malfunction. It has a reported incidence of $0.07 \%$ in a series of 17000 patients. [68] Figure 11 depicts lead orientation before and after patient twiddling resulted in lead dislodgement.

\subsubsection{Exit block}

Transient disruptions should be excluded first: metabolic and electrolyte abnormalities, drug effects, extreme hypothyroidism (myxedema), and cardiac ischemia. There is an expected rise in capture threshold in the 2-6 week period after lead placement attributed to local inflammation or foreign body reaction at the tip-tissue interface. In the era of epicardial and early endocardial leads, this was a much greater concern. Passive fixation endocardial leads have, on average, lower stimulation thresholds than active fixation leads due to lack of trauma at tissue interface. The degree that the capture threshold increases is markedly blunted with steroid eluting endocardial leads which have thus become generally preferred for their more favorable delivery characteristics having overcome the problem of 
A. Initial Post-Implant CXR

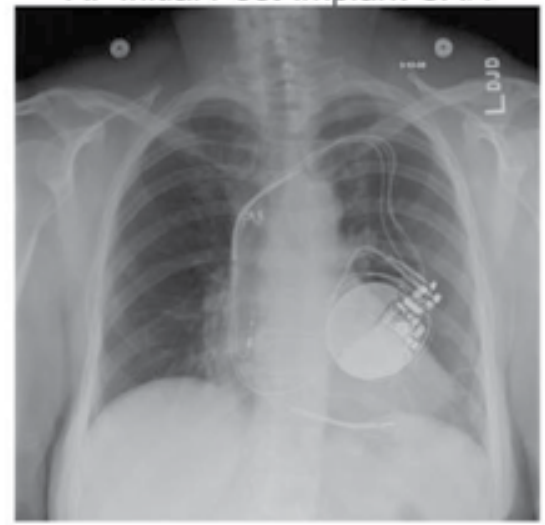

B. CXR After "Twiddling"

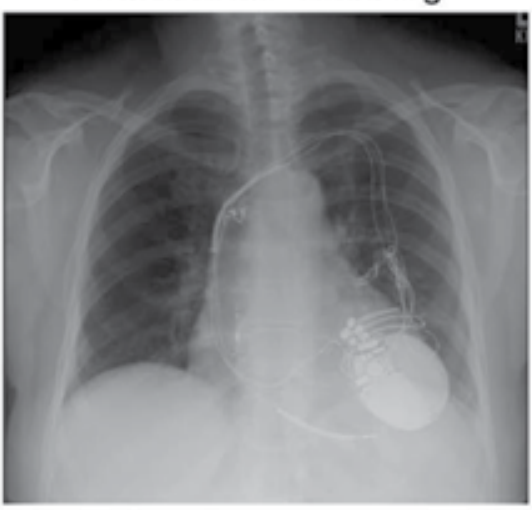

Figure 11. A and B. Lead orientation before and after patient twiddling resulted in lead dislodgement. Image A depicts the post-implant radiograph baseline lead positioning after biventricular defibrillator implant. Image B shows retracted right and left ventricular leads and leads tangled in the pocket superior to device can that is rotated.

higher stimulation thresholds. [69,70] The first randomized trial to compare a standard active fixation lead to a similar designed lead with a steroid eluting reservoir was reported in 1995. [71] Prior to that time, passive fixation leads were generally preferred because the capture threshold was relatively lower than standard active fixation leads. The disadvantage to passive fixation leads was an inability to perform atrial mapping, unreliable lateral wall stability, and requirement for placement in the atrial appendage which may be difficult in patient who have undergone bypass. The most dramatic difference in stimulation threshold between steroid eluting leads and standard endocardial leads was in the magnitude of increase in the acute phase as well as the duration of peak before returning to the chronic threshold. [71] The steroid lead returned to chronic capture threshold by week 2 whereas the non-steroid eluting lead remained above chronic threshold for 12 weeks. A non-significant increase in atrial lead dislodgements occurred with the steroid lead $(0 \%$ vs $2 \%, p=0.58)$. Lower capture thresholds allow for lower programmed output to maintain $2 x$ safety margin, ultimately improving generator longevity. A rise in capture threshold may occur beyond 6 weeks after implantation (chronic phase of lead maturation). As the threshold steadily rises, it may exceed the maximum output of the pulse generator, known as exit block. Exit block is recognized by high pacing thresholds without radiographic evidence of dislodgement. It may be related to inflammation or fibrosis at the electrode-myocardium interface and generally presents $>4$ weeks after implantation. [65] Some patients and particularly pediatric patients are particularly prone to this phenomena and may lead to multiple lead revisions. 


\subsection{Device/lead advisories}

A large multicenter Canadian observational study showed that the complication rate from device replacement for an advisory indication was an astounding 9.1\%. [72] Of these, 5.9\% required reoperation and there were 2 deaths. Naturally, the risk of an adverse outcome during replacement must be balanced by the risk of death due to device malfunction. Pacemakers and defibrillators have saved thousands of lives but as is true of all man-made devices, malfunctions have and will continue to occur. In response to a marked increase in device advisories in 2005, and to balance alarmism with protection of patients with a high risk situation, the Heart Rhythm Society (HRS), utilizing the HRS Task Force on Device Performance Policies and Guidelines published guidelines in 2006. [73] Recognizing that physicians and patients need timely and accurate information regarding device performance, arguably the most important outcome was a call for greater transparency of post market analysis and reporting of failures. Device performance is defined as the percentage of devices that are in service and functioning appropriately in living individuals over time and depends not only on the characteristics of the device but the skill of the implanting physician and caregivers following the device. [73] Data compiled from 19902002 from FDA annual reports showed that confirmed device malfunctions leading to device explantation were about $0.1-0.9 \%$ for pacemakers and $0.7-3.9 \%$ for ICDs. [73] Although failure rates are low, there is a negative psychological impact on patients who have a device which is under advisory, particularly if pacer dependent or if placed for a secondary indication.

To assist in communication from industry to physicians and patients it is proposed that terminology be standardized. The term "recall" was changed to "Class I Advisory" which is just short of a directive for device replacement because of a reasonable probability that malfunction could result in death or significant harm. Class II and class III recalls are subsequently referred to as advisory notices (non-life threatening malfunctions) and safety alerts (potential malfunctions). This information is disseminated from industry via standardized Physician Device Advisory Notifications and Patient Device Advisory Notifications which are also available on the manufacturer's website. Prior experience tells us the advisory information should be disseminated to physicians just before patients. Advisories should include general information about the malfunction and potential clinical implications but should acknowledge that treatment decisions should ultimately be determined by patients in consultations with physicians. The situations where device replacement is recommended are 1) When mechanism of malfunction is known and likely to be recurrent or lead to patient death, 2) The patient is pacer dependant, 3) The device was placed for a secondary prevention indication or have received appropriate therapy, 4) The device is approaching EOL. Conservative management (enhanced non-invasive and remote monitoring) should be considered when 1) The rate of malfunction is very low in non-pacer dependant patients or primary prevention without history of appropriate therapy, 2) The patient has significant comorbidities or high operative risk even when the risk of device malfunction is substantial. 3) Remote monitoring and software reprogramming can minimize risk (i.e., non physiologic noise). 


\subsection{Infection}

Up to $60 \%$ of patients present with localized infection involving the device pocket whereas the remaining patients may present with endovascular infection but no evidence of inflammation of the device pocket. [74] Approximately $10 \%$ of patients may have intracardiac vegetations identified by transesophageal echocardiogram, though can still undergo percutaneous lead extraction safely [75]. See Figure 12 for echocardiographic image of vegetation adherent to device lead. Generally, the most common pathogen isolated is aerobic gram-positive organisms, of which $90 \%$ are Staphylococcus species, with a high rate of methicillin resistance $(\sim 50 \%)$. [74] The risk of pacemaker infection is lower than that of implantable defibrillators. The presence of epicardial leads and postoperative complications at the generator pocket are significant risk factors for early-onset ICD infection, whereas longer duration of hospitalization at the time of implantation and chronic obstructive pulmonary disease were associated with late-onset ICD infections. [62] In one of the largest studies of pacemaker infections [76], repeated operative procedures after the first pacemaker implantation were associated with a substantial incremental risk of infection. Female sex, older age, and preoperative antibiotics given at the initial implant were associated with a lower risk of later infection. The pacing mode, indication for pacing, and complexity of the procedure were not independently associated with the risk of later infection. Sixty percent of infections have been found to occur within 90days of implant [77] though a large number of infections occur during the late follow-up ( $>1$ year post-implant) (76). Generator changes and cardiac resynchronization therapy/dual-chamber devices have also been implicated as independent predictors of infection. [77]

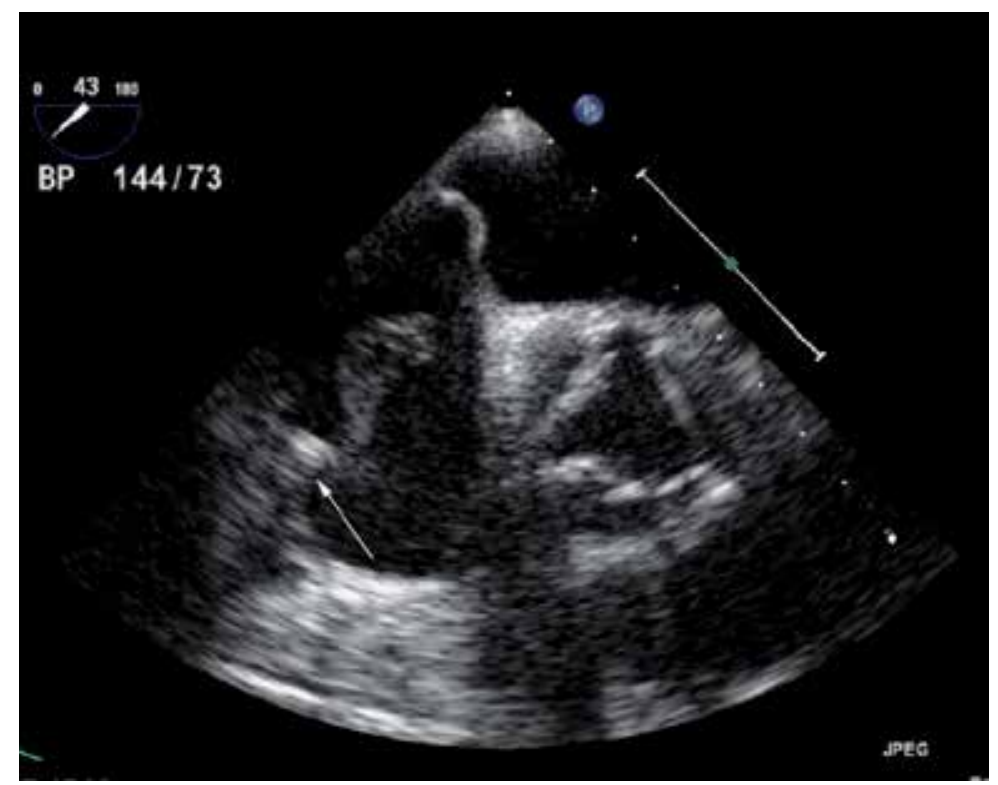

Figure 12. Transesophageal echocardiographic image of vegetation adherent to pacemaker lead. This is a short axis view showing a large, mobile vegetation (encircled) on a right ventricular lead (arrow) in a patient with persistent bacteremia. 
The generally accepted means of device infection treatment is removal of the generator and all implanted leads. [78] In a large series of device extractions including 1838 leads [75], post-operative 30 -day mortality was $10 \%$ though no deaths were related directly to the extraction procedure. Another series of device extractions reported a $0.5 \%$ rate of intraprocedural mortality, $4.6 \%$ rate of in-hospital mortality, and $2.6 \%$ rate of relapsing infections within 1 year of reimplantation. [74]

\subsection{Pacemaker syndrome}

Occurs most commonly with single chamber ventricular pacemakers (e.g., VVI or VVIR modes) and symptoms are due to loss of atrioventricular synchrony. It must be noted that pacemaker syndrome can occur with any pacing mode if AV synchrony is lost. Symptoms include malaise, weakness, cannon A waves, $\mathrm{CP}$, cough, confusion, or syncope.

\subsection{Venous thrombosis}

Upper extremity deep venous thrombosis (or stenosis) is uncommon in the general population but venous stenosis has been seen in up to 33-64\% of patients after implantation of pacing leads. $[79,80]$ Statistically significant factors that have been associated with an increased risk include previous transvenous temporary leads [80], left ventricular ejection fraction $<40 \%$ [80], systemic infection [81], absence of anticoagulation, use of hormone treatment, personal history of venous thrombosis, and presence of multiple leads. [82] Symptoms may include shoulder or neck discomfort, ipsilateral arm edema with cyanosis, dilated collateral cutaneous veins around the shoulder, or jugular vein distension. [83] Venography is considered the gold standard for diagnosis but compressive ultrasonography is an effective and economical means of confirming the clinical diagnosis. [83] Treatment may include anticoagulation (warfarin and/or heparin), extraction of old nonfunctioning lead to create a new venous channel, or venoplasty to reduce venous stenosis or allow the implantation of subsequent leads. [83,84]

\section{Predicting risks for procedural complications}

There is some evidence that elderly patients are at increased risk of complications following pacemaker implantation. [85] Armaganijan et al [85] found that any early complication occurred in $5.1 \%$ of patients $\geq 75$ years of age compared to $3.4 \%$ of patients aged $<75$ years. The concomitant use of temporary transvenous pacemakers or steroid use within 7 days of implant have been shown to increase rates of post implant pericardial effusion. [45] Weaker predictors of post implant effusions were the use of helical screw ventricular leads, body mass index $<20$, older age, and longer fluoroscopy times. [45] Pneumothorax has been found to more common in older, lighter females. [5] A prior study examining predictors of complications in extremely elderly patients undergoing pacemaker implantation [11] found overall rates of implant complications comparable to data from younger patient populations while experiencing a higher 30day all-cause mortality (that may have been attributable to elevated all-cause mortality rates in this age-group). Multivariate analysis revealed that 
female sex, device type, and urgent/emergent placement demonstrated a non-significant trend toward increased rates of complication; increased age and device type demonstrated a non-significant trend toward increased readmission rate.

Higher ( $>12$ implants/year) versus lower volume operators $(<12$ implants/year) have also demonstrated diverging rates of complication. [33] Finally, more complex devices (dualchamber vs single ventricular chamber pacemakers) have been associated with higher rates of complications. [10,30,31,32,35,37] however, there is data that does not demonstrate increased rates of complications in dual-chamber devices. [9,11,34] Finally, it has been suggested that physician training (specifically, board-certification or board-eligibility in clinical cardiac electrophysiology) may result in lower rates of lead dislodgement. $[7,86]$

\section{Conclusion}

Major and minor complications occur in approximately $4-7 \%$ of patients within $30 \mathrm{~d}$ of pacemaker implantation. [5,10,11,32,36,35] Permanent pacemakers are commonly implanted in patients over the age of 65 and this is the most rapidly growing segment of the U.S. population. That being said, pacemaker therapy is also associated with an $11-40 \%$ risk of complications in the pediatric population; the most common complications in this segment are pneumothorax, hematoma, and infection. [87] Figure 13 depicts the incidence of the most common complications after pacemaker implantation. Prompt recognition and treatment of complications after pacemaker implantation is essential for all implanting physicians regardless of background.

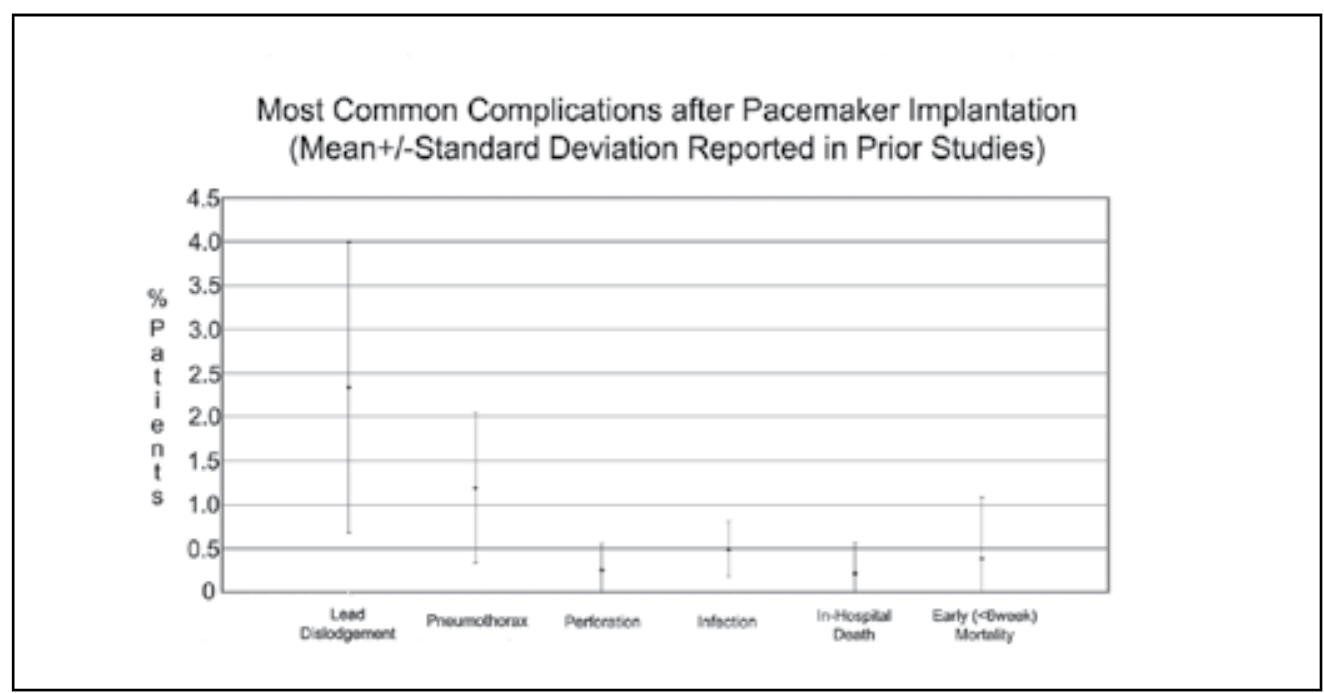

Figure 13. Most Common Complications Seen After Pacemaker Implantation. This figure depicts the most common complications seen after pacemaker implantation from available prior. The data are for complications seen within 30-42days depending upon the study parameters as referenced. 


\section{Author details}

Jeffrey L. Williams and Robert T. Stevenson

The Good Samaritan Hospital, Heart Rhythm Center, Lebanon Cardiology Associates, Lebanon Pennsylvania, U.S

\section{References}

[1] Kurtz SM, Ochoa JA, Lau E, Shkolnikov Y, Pavri BB, Frisch D, Greenspon AJ, "Implantation trends and patient profiles for pacemakers and implantable cardioverter defibrillators in the United States: 1993-2006," Pacing Clin Electrophysiol, V. 33, No. 6 (June 1, 2010), pp. 705-11.

[2] Perls T, "Health and disease in people over 85," BMJ, V. 339 (22 December 2009), b4715.

[3] Hobbs FB, "Current Population Reports, Series P23-190, Sixty-Five Plus in the U.S.," http://www.census.gov/population/www/pop-profile/elderpop.html. Accessed 10 March 2011.

[4] Cherubini A, Oristrell J, Pla X, Ruggiero C, Ferretti R, Diestre G, Clarfield M, Crome P, Hertogh C, Lesauskaite V, Prada G-I, Szczerbinska K, Topinkova E, Sinclair-Cohen J, Edbrooke D, Mills G, “The Persistent Exclusion of Older Patients From Ongoing Clinical Trials Regarding Heart Failure," Arch Intern Med, V. 171, No. 6 (March 28, 2011), pp. 550-556.

[5] Link MS, Estes NAM, Griffin JJ, Wang PJ, Maloney JD, Kirchhoffer JB, Mitchell GF, Orav J, Goldman L, Lamas GA, "Complications of Dual Chamber Pacemaker Implantation in the Elderly," J Intervent Cardiac Electrophys, V. 2 (1998), pp. 175-179.

[6] Alter P, Waldhans S, Plachta E, Moosdorf R, Grimm W, “Complications of Implantable Cardioverter Defibrillator Therapy in 440 Consecutive Patients," PACE, V. 28 (September 2005), pp. 926-932.

[7] Curtis JP, Luebbert JJ, Wang Y, Rathore SS, Chen J, Heidenreich PA, Hammill SC, Lampert RI, Krumholz HM, "Association of Physician Certification and Outcomes Among Patients Receiving an Implantable Cardioverter-Defibrillator," JAMA, V. 301, No. 16 (April 22/29, 2009), pp. 1661-1670.

[8] Williams JL, Lugg D, Gray R, Hollis D, Stoner M, Stevenson R, “Patient Demographics, Complications, and Hospital Utilization in 250 Consecutive Device Implants of a New Community Hospital Electrophysiology Program," American Heart Hospital Journal, V. 8, No. 1 (Summer, 2010), pp. 33-39.

[9] Rosenheck S, Geist M, Weiss A, Hasin Y, Weiss TA, Gotsman MS, "Permanent Cardiac Pacing in Octogenarians," Amer J Ger Card, V. 4, No. 6 (1995), pp. $42-47$.

[10] Nowak B and Misselwitz B, "Effects of increasing age onto procedural parameters in pacemaker implantation: results of an obligatory external quality control program," Europace, V. 11 (November 2008), pp. 75-79. 
[11] Stevenson R, Lugg D, Gray R, Hollis D, Stoner M, Williams JL, "Pacemaker Implantation in the Extreme Elderly," Journal of Interventional Cardiac Electrophysiology, V. 33, No. 1 (January 2012), pp. 51-58.

[12] Klug D, Balde M, Pavin D, Hidden-Lucet F, Clementy J, Sadoul N, Luc Rey J, Lande G, Lazarus A, Victor J, Barnay C, Grandbastien B, Kacet S, "Risk Factors Related to Infections of Implanted Pacemakers and Cardioverter-Defibrillators," Circulation, Vol. 116, (August 2007), pp.1349-1355.

[13] Kirkfeldt RE, Johansen JB, Nohr EA, Moller M, Nielsen JC, “Risk Factors for Lead Complications in Cardiac Pacing: A Population-based Cohort of 28,860 Danish Patients," Heart Rhythm, Vol.8, No.10, (October 2011), pp.1622-1628.

[14] Marcos, SK, Thomsen, HS. "Prevention of general reactions to contrast media: A consensus report and guidelines," Eur Radiol, Vol.11, N.9 (2001), pp.1720-8.

[15] Brockow K, Christiansen C, Kanny G, Clément O, Barbaud A, Bircher A, DeWachter P, Guéant JL, Rodriguez Guéant RM, Mouton-Faivre C, Ring J, Romano A, SainteLaudy J, Demoly P, Pichler WJ, ENDA and the EAACI interest group on drug hypersensitivity (2005), "Management of hypersensitivity reactions to iodinated contrast media," Allergy, Vol.60, pp.150-158.

[16] Trcka J, Schmidt C, Seitz CS, Brocker EB, Gross GE, Trautman A, “Anaphylaxis to iodinated contrast materials: nonallergic hypersensitivity or IgE-mediated allergy? AJR, V. 190, No. 3 (2008), pp. 666-670

[17] Guillaume B, Kellner F, Barnig C, "Management of Patients with History of Adverse Effects to Contrast Media When Pulmonary Artery CT Angiography is Required," Radiology, V. 245 (December 2007), pp. 919-921.

[18] Tramer MR, vonElm E, Loubeyre P, Hauser C, "Pharmacologic prevention of Serious Anaphylactic Reactions Due to Iodinated Contrast Material: Systematic Review," BMJ, V.333, (September 2006), pp. 675-678.

[19] The Royal College of Radiologists, "Standards for Intravascular Contrast Administration To Adult Patients: Second Edition," London, The Royal College of Radiologists, February 2010.

[20] Murkin JM, "Anesthesia and hypothyroidism: a review of thyroxine physiology, pharmacology, and anesthetic implications," Anesth Analg, V. 61, No. 4 (April 1982), pp. 371-83.

[21] Farling PA, “Thyroid Disease,” Br J Anaesth, V. 85, No. 1 (2000), pp. 15-28.

[22] Gaitan BD, Trentman TL, Fassett SL, Mueller JT, Altemose GT, "Sedation and analgesia in the cardiac electrophysiology laboratory: a national survey of electrophysiologists investigating the who, how, and why?" J Cardiothorac Vasc Anesth 2011; 25:647-659.

[23] Magnusson L and Spahn DR, "New concepts of atelectasis during general anesthesia," British J Anaesthesia, V. 91, No. 1 (2003), pp. 61-72. 
[24] Lawrence VA, Hilsenbeck SG, Mulrow CD, Dhanda R, Sapp J, Page CP, "Incidence and hospital stay for cardiac and pulmonary complications after abdominal surgery," J Gen Intern Med, V. 10 (1995), pp. 671-678.

[25] Trentman TL, Fassett SL, Mueller JT, Altemose GT, "Airway interventions in the cardiac electrophysiology laboratory: a retrospective review," J Cardiothorac Vasc Anesth 2009; 23:841-5.

[26] DiBiase L, Conti S, Mohanty P, et al. General anesthesia reduces the prevalence of pulmonary vein reconnection during repeat ablation when compared with conscious sedation: Results from a randomized study. Heart Rhythm 2011; 8:368-372.

[27] Williams JL, Valencia V, Lugg D, Gray R, Hollis D, Toth JW, Benson R, DeFrancescoLoukas MA, Stevenson R, Teiken PJ, “High Frequency Jet Ventilation During Ablation of Supraventricular and Ventricular Arrhythmias: Efficacy, Patient Tolerance and Safety," The Journal of Innovations in Cardiac Rhythm Management, 2 (2011), 1-7]

[28] Hargreaves MR, Doulalas A, Ormerod OJM, "Early Complications Following Dual Chamber Pacemaker Implantation: 10-Year Experience of a Regional Pacing Centre," Eur JCPE, V. 5, No. 3 (1995), pp. 133-138.

[29] Noseworthy PA, Lashevsky I, Dorian P, Greene M, Cvitkovic S, Newman D, "Feasibility of Implantable Cardioverter Defibrillator Use in Elderly Patients: A Case Series of Octogenarians," PACE, V. 27 (March 2004), pp. 373-378.

[30] Chauhan A, Grace AA, Newell SA, Stone DL, Shapiro LM, Schofield PM, Petch MC, "Early Complications After Dual Chamber Versus Single Chamber Pacemaker Implantation," PACE, V. 17, Part II, (November 1994), pp. 2012-2015.

[31] Wiegand UKH, Bode F, Bonnemeier H, Eberhard F, Schlei M, Peters W, "Long-Term Complication Rates in Ventricular, Single Lead VDD, and Dual Chamber Pacing," PACE, V. 26 (October 2003), pp. 1961-1969.

[32] Eberhart F, Bode F, Bonnemeier H, Boguschewski F, Schlei M, Peters W, Wiegand $\mathrm{UKH}$, “Long term complications in single and dual chamber pacing are influenced by surgical experience and patient morbidity," Heart, V. 91 (2005), pp. 500-506.

[33] Parsonnet V, Bernstein AD, Lindsay B, "Pacemaker-Implantation Complication Rates: An Analysis of Some Contributing Factors," JACC, V. 13, No. 4 (March 15, 1989), pp. 917-921.

[34] Aggarwal RK, Connelly DT, Ray SG, Ball J, Charles RG, "Early complications of permanent pacemaker implantation: no difference between dual and single chamber systems," Br Heart J, V. 73 (1995), pp.571-575.

[35] Kiviniemi MS, Pirnes MA, Eranen HJK, Kettunen RVJ, Hartikainen JEK, “Complications Related to Permanent Pacemaker Therapy,” PACE, V. 22 (May 1999), pp. 711-720. 
[36] Ellenbogen KA, Hellkamp AS, Wilkoff BL, Camunas JL, Love JC, Hadjis TA, Lee KL, Lamas GA, "Complications Arising After Implantation of DDD Pacemakers: The MOST Experience," Amer J Card, V. 92 (September 15, 2003), pp. 740-741.

[37] van Eck JWM, van Hemel NM, Zuithof P, van Asseldonk JPM, Voskuil TLHM, Grobbee DE, Moons KGM, "Incidence and predictors of in-hospital events after first implantation of pacemakers," Europace, V. 9 (June 2007), pp. 884-889.

[38] Chadha TS, Cohn MA, "Noninvasive treatment of pneumothorax with oxygen inhalation," Respiration, V. 44, No. 2, (1983), pp. 147-52.

[39] Kulvatunyou N, Vijayasekaran A, Hansen A, Wynne JL, O'Keeffe T, Friese RS, Joseph $\mathrm{B}$, Tang A, Rhee $\mathrm{P}$, “Two-year experience of using pigtail catheters to treat traumatic pneumothorax: a changing trend," J Trauma, V. 71, No. 5 (Nov 2011), pp. 1104-7.

[40] Fyke FE III, "Infraclavicular lead failure: tarnish on a golden route," Pacing Clin Electrophysiol, V. 16 (1993), pp. 373-376.

[41] Magney JE, Flynn DM, Parsons JA, Staplin DH, Chin-Purcell MV, Milstein S, Hunter DW, "Anatomical mechanisms explaining damage to pacemaker leads, defibrillator leads, and failure of central venous catheters adjacent to the sternoclavicular joint," Pacing Clinical Electrophysiol, V. 16 (1993), pp. 445-447.

[42] Belott P, "How to access the axillary vein," Heart Rhythm, V. 3, No. 3 (March 2006), pp. 366-369.

[43] Hirschl DA, Jain VR, Spindola-Franco H, Gross JN, Haramati LB, "Prevalence and Characterization of Asymptomatic Pacemaker and ICD Lead Perforation on CT," PACE, V. 30 (January 2007), pp. 28-32.

[44] Wang NC, Williams JL, Jain SK, Shalaby A, "Post-Pacemaker Pulsations," Amer J Med, V 122, No. 4 (April 2009), pp. 345-347.

[45] Mahapatra S, Bybee KA, Bunch TJ, et al, "Incidence and predictors of cardiac perforation after permanent pacemaker implantation," Heart Rhythm, V. 2 (2005), pp. 907-911.

[46] Geyfman V, Storm RH, Lico SC, Oren IV JW, "Cardiac tamponade as complication of active-fixation atrial lead perforations: proposed mechanism and management algorithm," Pacing Clin Electrophysiol, V. 30 (2007), pp. 498-501.

[47] Cappato R, Calkins H, Chen S-A, Davies W, Iesaka Y, Kalman J, Kim Y-H, Klein G, Natale A, Packer D, Skanes A, "Prevalence and Causes of Fatal Outcome in Catheter Ablation of Atrial Fibrillation," JACC, Vol. 53, No. 19 (May 12, 2009), 1798-803.

[48] Leon AR, Abraham WT, Curtis AB, et al.; for the MIRACLE Study Program, "Safety of Transvenous Cardiac Resynchronization System Implantation in Patients with Chronic Heart Failure: Combined Results of Over 2000 Patients from a Multicenter Study Program," J Am Coll Cardiol, 2005;46(12):2348-56.

[49] Bristow MR, Saxon LA, Boehmer J, et al., "Cardiac-Resynchronization Therapy with or without an Implantable Defibrillator in Advanced Chronic Heart Failure for the 
Comparison of Medical Therapy, Pacing, and Defibrillation in Heart Failure (COMPANION) Investigators," N Engl J Med, 2004;350(21):2140-50.

[50] D'Ivernois C, Lesage J, Blanc P, “Where are left ventricular leads really implanted? A study of 90 consecutive patients," Pacing Clin Electrophysiol, 2008;31(5):554-9.

[51] Ellery SM and Paul VE, “Complications of biventricular pacing," European Heart Journal Supplements, V. 6, Supp. D (2004), pp. D117-D121.

[52] Knight BP, Desai A, Coman J, Faddis M, Yong P, “Long-term retention of cardiac resynchronization therapy," JACC, V. 44, No. 1 (July 7, 2004), pp. 72-77.

[53] Jordaens L, Robbens E, Van Wassenhove E, Clement DL, "Incidence of arrhythmias after atrial or dual-chamber pacemaker implantation," Eur Heart J, V. 10, No. 2 (Feb 1989), pp. 102-107.

[54] Faber TS, Gradinger R, Treusch S Morkel C, Brachmann J, Bode C, Zehender M, "Incidence of ventricular tachyarrhythmias during permanent pacemaker therapy in low-risk patients results from the German multicentre EVENTS study," European Heart Journal, V. 28, No. 18 (2007), pp. 2238-2242.

[55] Datta G, Sarkar A, Haque A, "An Uncommon Ventricular Tachycardia due to Inactive PPM Lead," ISRN Cardiology, V. 2011 (2011), Article ID 232648, 3 pages.

[56] Bohm A, Pinter A, Preda I, "Ventricular tachycardia induced by a pacemaker lead," Acta Cardiologica, V. 57, No. 1 (2002), pp. 23-24.

[57] Freedman A, Rothman MT, Mason JW, “Recurrent ventricular tachycardia induced by an atrial synchronous ventricular-inhibited pacemaker," Pacing and Clinical Electrophysiology, V. 5, No. 4 (1982), pp. 490-494.

[58] Li W, Sarubbi B, Somerville J, "Iatrogenic ventricular tachycardia from endocardial pacemaker late after repair of tetralogy of Fallot," Pacing and Clinical Electrophysiology, V. 23, No. 12 (2000), pp. 2131-2134.

[59] Iesaka Y, Pinakatt T, Gosselin AJ, Lister JW, "Bradycardia dependent ventricular tachycardia facilitated by myopotential inhibition of a VVI pacemaker," Pacing and Clinical Electrophysiology, V. 5, No. 1 (1982), pp. 23-29.

[60] Schulza N, Puschelb K, Turkc EE, “Fatal complications of pacemaker and implantable cardioverter defibrillator implantation: medical malpractice?" Interactive CardioVascular and Thoracic Surgery, V. 8 (2009), pp. 444-448.

[61] Wiegand UKH, LeJeune D, Boguschewski F, Bonnemeier H, Eberhardt F, Schunkert H, Bode F, “Pocket Hematoma After Pacemaker or Implantable Cardioverter Defibrillator Surgery: Influence of Patient Morbidity, Operation Strategy, and Perioperative Antiplatelet/Anticoagulation Therapy," CHEST, V. 126, No. 4 (October 2004), pp. 1177-1186.

[62] Sohail MR, Hussain S, Le KY, Dib C, Lohse CM, Friedman PA, Hayes DL, Uslan DZ, Wilson WR, Steckelberg JM, Baddour LM; Mayo Cardiovascular Infections Study Group, "Risk factors associated with early- versus late-onset implantable 
cardioverter-defibrillator infections," J Interv Card Electrophysiol. V. 31, No. 2 (Aug 2011), pp. 171-83.

[63] Ahmed I, Gertner E, Nelson WB, House CM, Dahiya R, Anderson CP, Benditt DG, Zhu DWX, "Continuing warfarin therapy is superior to interrupting warfarin with or without bridging anticoagulation therapy in patients undergoing pacemaker and defibrillator implantation," Heart Rhythm, V. 7, No. 6 (June 2010), pp. 745-749.

[64] Ferguson TB Jr, Ferguson CL, Crites K, Crimmins-Reda P, “The additional hospital costs generated in the management of complications of pacemaker and defibrillator implantations," J Thorac Cardiovasc Surg, V. 111 (1996), pp. 742-752.

[65] Hayes DL and Vlietstra RE, "Pacemaker Malfunction," Annals of Internal Medicine, V. 119, No. 8 (October 15, 1993), pp. 828-835.

[66] Alt E, Volker R, Blomer H, "Lead fracture in pacemaker patients," Thoracic Cardiovasc Surg, V. 35 (1987), pp. 101-104.

[67] Bayliss CE, Beanlands DS, Baird RJ, "The pacemaker-twiddler's syndrome: a new complication of implantable transvenous pacemakers," Can Med Assoc J, V. 99 (1968), pp. 371-3.

[68] T. Fahraeus and C. J. Hoijer, "Early pacemaker twiddler syndrome," Europace, Vol. 5 (July 2003), pp.

[69] Ellenbogen KA, Wood MA, Gilligan DM, Zmijewski M, Mans D and The CAPSURE Z Investigators (1999), "Steroid Eluting High Impedance Pacing Leads Decrease Short and Long-Term Current Drain: Results from a Multicenter Clinical Trial," Pacing and Clinical Electrophysiology, V.22 No.1 (January 1999), pp. 39-48.

[70] Fortescue EB, Berul Cl, Cecchin F, Walsh EP, Triedman JK, Alexander ME, "Comparison of Modern Steroid-Eluting Epicardial and Thin Transvenous Pacemaker Leads in Pediatric and Congenital Heart Disease Patients," J Interv Card Electrophysio, V.14, No.1 (October 2005), pp.27-36.

[71] Crossley GH, Brinker JA, Reynolds D, Spencer W, Johnson B, Hurd H, Touder L, ZmijewskinM, "Steroid Elution Improves the Stimulation Threshold in an ActiveFixation Atrial Permanent Pacing Lead," Circ V. 92 (1995), pp.2935-2939.

[72] Gould, PA. Outcome of Advisory ICD replacement: One year follow-up. Heart Rhythm Vol5, No12, December 2008.

[73] Carlson MD, Wilkoff, BL. Recommendation from the HRS task force on device performance policies and guidelines. Heart Rhythm 2006;3:1250-1273.

[74] Tarakji KG, Chan EJ, Cantillon DJ, Doonan AL, Hu T, Schmitt S, Fraser TG, Kim A, Gordon SM, Wilkoff BL et al, "Cardiac implantable electronic device infections: Presentation, management, and patient outcomes," Heart Rhythm V. 7, No. 8 (August 2010 ), pp. 1043-1047.

[75] Grammes JA, Schulze CM, Al-Bataineh M, Yesenosky GA, Saari CS, Vrabel MJ, Horrow J, Chowdhury M, Fontaine JM, Kutalek SP, “Percutaneous pacemaker and implantable cardioverter-defibrillator lead extraction in 100 patients with intracardiac 
vegetations defined by transesophageal echocardiogram," J Am Coll Cardiol, V. 55, No. 9 (March 2010), pp. 886-94.

[76] Johansen JB, Jørgensen OD, Møller M, Arnsbo P, Mortensen PT, Nielsen JC, “Infection after pacemaker implantation: infection rates and risk factors associated with infection in a population-based cohort study of 46299 consecutive patients ," Eur Heart J, V. 32, No. 8 (April 2011) pp. 991-998.

[77] Nery PB, Fernandes R, Nair GM, Sumner GL, Ribas CS, Menon SM, Wang X, Krahn AD, Morillo CA, Connolly SJ, Healey JS, “Device-related infection among patients with pacemakers and implantable defibrillators: incidence, risk factors, and consequences," J Cardiovasc Electrophysiol, V. 21, No. 7 (July 2010), pp. 786-90.

[78] Wilkoff BL, "How to treat and identify device infections," Heart Rhythm, V. 4 (2007), pp. 1467-1470.

[79] Oginosawa $Y$, Abe $H$, Nakashima $Y$, "The incidence and risk factors for venous obstruction after implantation of transvenous pacing leads," Pacing Clin Electrophysiol, V. 25 (2002), pp. 1605-1611.

[80] DaCosta SS, Scalabrini NA, Costa A, Caldas JG, Martinelli FM, "Incidence and risk factors of upper extremity deep vein lesions after permanent transvenous pacemaker implant: A 6-month follow-up prospective study," Pacing Clin Electrophysiol, V. 25 (2002), pp. 1301-1306.

[81] Bracke F, Meijer A, Van Gelder B, "Venous occlusion of the access vein in patients referred for lead extraction: Influence of patient and lead characteristics," Pacing Clin Electrophysiol, V. 26 (2003), pp. 1649-1652.

[82] van Rooden CJ, Molhoek SG, Rosendaal FR. Schalij MJ, Meinders AE, Huisman MV, "Incidence and risk factors of early venous thrombosis associated with permanent pacemaker leads," J Cardiovasc Electrophysiol, V. 15 (2004), pp. 1258-1262.

[83] Rozmus G, Daubert JP, Huang DT, Rosero S, Hall B, Francis C, “Venous Thrombosis and Stenosis After Implantation of Pacemakers and Defibrillators," J Interv Cardiac Electrophysiol, V. 13 (2005), pp. 9-19.

[84] Worley SJ, Gohn DC, Pulliam RW, Raifsnider MA, Ebersole B, Tuzi J, "Subclavian venoplasty by the implanting physicians in 373 patients over 11 years," Heart Rhythm, V. 8, No. 4 (April 2011), pp. 526-533.

[85] Armaganijan LV, Toff WD, Nielsen JC, Andersen HR, Connolly SJ, Ellenbogen KA, Healey JS, "Are Elderly Patients at Increased Risk of Complications Following Pacemaker Implantation? A Meta-Analysis of Randomized Trials," Pacing Clin Electrophysiol, Epub ahead of Print, (2011 Oct 31).

[86] Cheng A, Wang Y, Curtis JP, Varosy PD, “Acute Lead Dislodgements and In-Hospital Mortality in Patients Enrolled in the National Cardiovascular Data Registry Implantable Cardioverter Defibrillator Registry," J Am Coll Cardiol, V. 56 (2010), pp. 1651-1656. 
[87] Czosek RJ, Meganathan K, Anderson JB, Knilans TK, Marino BS, Heaton PC, "Cardiac rhythm devices in the pediatric population: Utilization and complications," Heart Rhythm, V. 9, No. 2 (Feb 2012), pp. 199-208. 


\title{
Device-Related Endocarditis and Infected Leads Extraction: The Dark Side of The Moon
}

\author{
Michele Rossi, Giuseppe Musolino, Giuseppe Filiberto Serraino \\ and Attilio Renzulli
}

Additional information is available at the end of the chapter

http://dx.doi.org/10.5772/48463

\section{Introduction}

Cardiac rhythm management devices are being increasingly implanted worldwide not only for symptomatic bradycardia, but also for the management of arrhythmia and heart failure (Adabag et al. 2011; COMPANION Investigators 2004; Multicenter Automatic Defibrillator Implantation Trial Investigators 1996; Sudden Cardiac Death in Heart Failure Trial Investigators 2005). The benefit of cardiac resynchronization therapy (CRT) with permanent pacemakers (PPM) as first invasive step to treat the failing heart (Adabag et al. 2011; COMPANION Investigators 2004) and the survival advantage of internal cardiac defibrillator (ICD) in patients with end stage heart failure compared with medical therapy alone (Multicenter Automatic Defibrillator Implantation Trial Investigators 1996; Sudden Cardiac Death in Heart Failure Trial Investigators 2005), have supported a more liberal implantation policy of these devices. Moreover the widespread use of the trans-catheter aortic valve implantation (TAVI) for the percutaneous treatment of severe aortic stenosis, in high risk patients that would, otherwise, deemed inoperable by conventional surgery, has carried along a high post procedural implantation rate of PPMs in this elderly subgroup of patients (Bates et al. 2011; D'Ancona et al. 2011). It seems logical to expect an increased rate of device related infections to follow the boom of PPMs and ICDs implantation in the last two decades (Voigt et al. 2006). Of the 400,000 -500,000 permanent pacemaker leads implanted worldwide each year, around $10 \%$ may eventually fail or become infected, becoming potential candidates for removal (Byrd et al. 1999). Device infections can be local, involving the insertion site of PPM or ICD box, or systemic because of the spreading along the PPM leads and, in worst case scenario, lead to septic shock and device-related endocarditis. Device-related endocarditis has been reported in $23 \%$ of infected PPMs, the remainder being pocket infections (Sohail et al. 2007). The infection can spread over the cardiac structures and typically involves the tricuspid valve. Right-sided endocarditis accounts for only 5-10\% of cases of infective 
endocarditis (Chan et al. 1989) and occurs predominantly in selected patient's subgroup, such as: intravenous drug users, patients with pacemakers, ICD or central venous lines and with congenital heart diseases (Robbins et al. 1986). The tricuspid valve can show massive vegetations with or without valve regurgitation (figure 1).

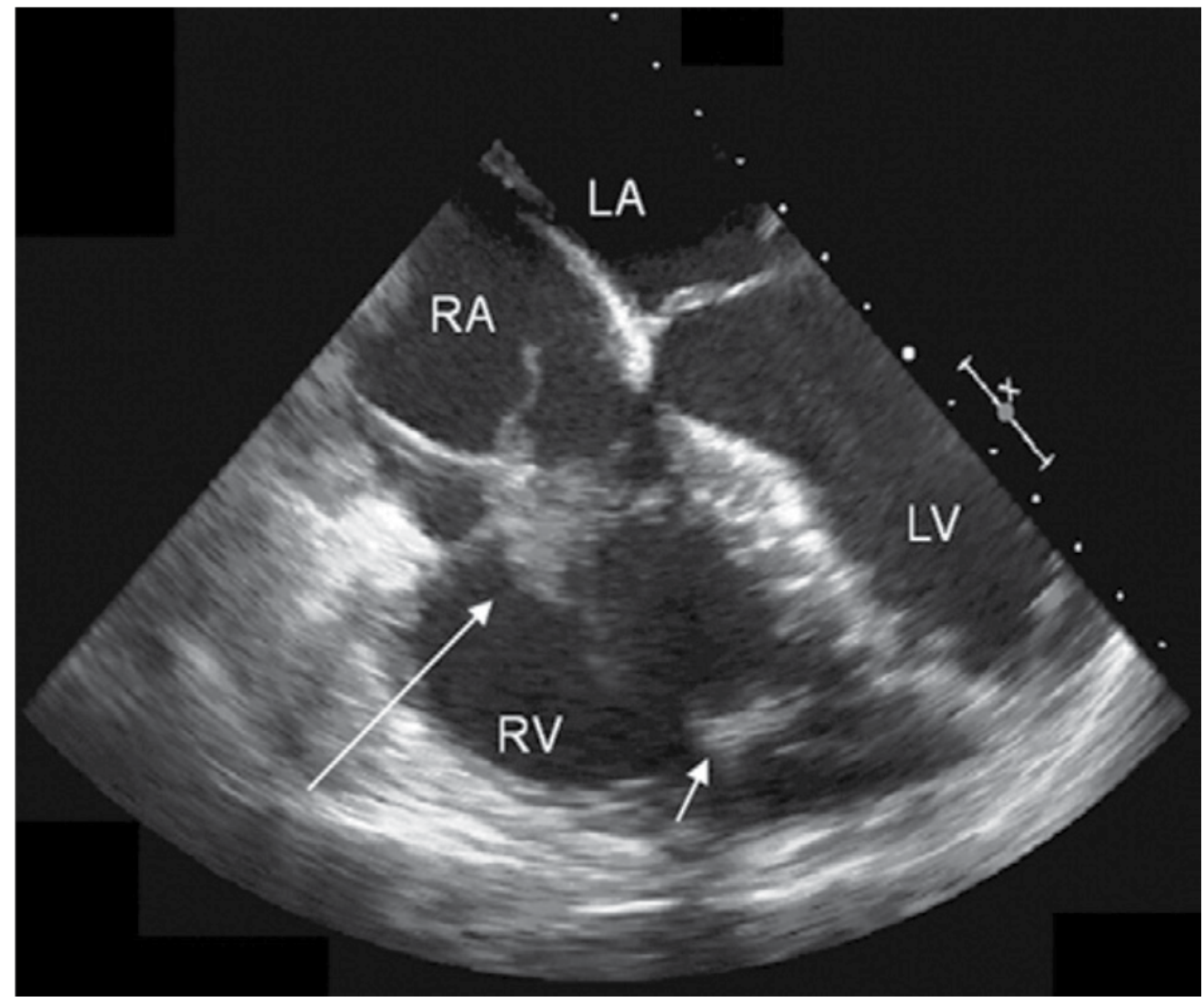

LA: left atrium; LV: left ventricle; RA: right atrium; RV: right ventricle.

Figure 1. ${ }^{T}$ Transesophageal echocardiography -four chamber view - shows multiple vegetations on the tricuspid valve (long arrow) and pacing leads (short arrow).

This chapter will focus on the presentation, diagnosis and management of device-related endocarditis and explore different extraction techniques - both percutaneous and surgical.

\section{Device-related endocarditis}

Mortality rates for infected PPM devices range from 31\% to $66 \%$ when the device is not explanted (Cacoub et al. 1998). Better outcomes, with mortality rates of $18 \%$ or less, have been reported when a combined management with device removal and antimicrobial therapy is adopted (Klug et al. 1997). Pacemaker related sepsis or endocarditis is a class I

${ }^{1}$ From Rossi et al. with permission. 
indication for lead extraction, according to the recently updated device infection guidelines (Baddour et al. 2010). Standard treatment includes removal of infected PPM device combined with six weeks of antimicrobial therapy. Management of device-related endocarditis is challenging and requires collaborative efforts between cardiologists, surgeons, and infective disease specialists.

\subsection{Clinical presentation}

The presentation can be acute, with onset of symptoms in the first 6 weeks after the last procedure on the implant site, or chronic, with $>6$ weeks from the last procedure on the implant site to the onset of symptoms. In the acute form, the short time elapsed between PM implantation and the occurrence of infection facilitates the diagnosis. The vast majority of patients will have systemic symptoms from septic shock to fever, pneumonia, pulmonary embolism, associated with local signs of infection at the PM site. In the chronic form, the delay between the onset of symptoms and the implant time makes it difficult to diagnose PM-lead infection. Often delays in diagnosis of chronic device-related endocarditis are related to the fact that PM-lead infection was not considered in the differential diagnosis or because, possible clues were ignored: for example, blood cultures positive for S. epidermidis were erroneously considered contamination of the specimens (Klug et al. 1997). The most common chronic presentation would be fever or chills with asthenia, and wasting, sometimes associated with symptoms and signs of low tract respiratory infection (cough, expectoration, bronchitis, pneumonia, pulmonary abscess, pleural effusion). History of pulmonary embolism, arthralgia, spondylitis or signs of local infection at the PM site could be present. The diagnosis of systemic infection related to PM-lead infection must be systematically considered in the presence of chronic fever, recurrent bronchitis, or pulmonary infection or in case of recurrent or persistent evidence of infection at the implant site (Klug et al. 1997). More over endocarditis of the right heart should be specifically excluded, regardless the presence or absence of tricuspid regurgitation (Love et al. 2000).

\subsection{Diagnosis}

Patients will have elevated markers of inflammation. Erythrocyte sedimentation rate (ESR) and CRP will be elevated, often along with high withe cell count (WCC) due to an increase in polymorphonuclear cells. Positive blood cultures will confirm the diagnosis. Blood cultures should be taken on 3 consecutive days and integrated with cultures at the site of battery pocket if appropriate (wounds, local infection, or PM exteriorization). The Duke criteria are useful to define systemic infection related to PM-lead infection (tab.1), but as suggested by Klug et al (1997), the importance of some clinical criteria, should, probably, be highlighted, such as, local symptoms of infection at the PM site and pulmonary infections, to facilitate the diagnosis (tab.2).

Chest roentgenogram will often show signs of pulmonary infection or pleural effusion. Echocardiography is essential to confirm the diagnosis and to clarify whether treatment will require removal of the infected pacing system. However, a single negative transthoracic 
echocardiogram (TTE) is not enough to exclude the diagnosis of lead-related endocarditis. Although TTE has about $80 \%$ sensitivity in the detection of vegetations in the right heart (Love et al. 2000), often patients present with PM-lead-related endocarditis with intact tricuspid leaflets (Klug et al. 1997). This is the reason why a transoesophageal echocardiogram (TOE) should always be performed in the diagnostic algorithm when device-related endocarditis is suspected. Ventilation perfusion pulmonary scintigraphy can corroborate the diagnosis showing multiple septic lung embolisms. It is wise to perform also a wider range of investigations to exclude other sources of infection. These will normally include a dental pantomogram, sinus radiographs and abdominal ultrasound.

\section{Definite infective endocarditis}

Pathological criteria

Microorganisms: demonstrated by culture or histology in vegetation, in a vegetation that has embolized, or in intracardiac abscess, or

Microorganisms demonstrated by culture of the lead

Clinical criteria(as listed in Table 2)

Two major criteria, or

One major and three minor criteria, or

Five minor criteria

\section{Possible infective endocarditis}

Findings consistent with infective endocarditis that fall short of "definite" but not "rejected"

\section{Rejected}

Firm alternate diagnosis explaining evidence of infective endocarditis, or

Resolution of infective endocarditis syndrome, with antibiotic therapy for $\leq 4$ days, or

No pathological evidence of infective endocarditis at surgery or autopsy, with antibiotic therapy for $\leq 4$ days

Table 1. ${ }^{2}$ Modified Duke Criteria for Diagnosis of Infective Endocarditis on PM Leads

${ }^{2}$ From Klug et al. with permission 


\section{Major criteria}

$\underline{\text { Positive blood culture for infective endocarditis }}$

Typical microorganisms for infective endocarditis from two separate blood cultures

Streptococcus viridans, Streptococcus bovis, HACEK group, or

Community-acquired Staphylococcus aureus or enterococci, in the absence of a primary focus, or

Persistently positive blood culture, defined as microorganism consistent with infective endocarditis from Blood cultures drawn $>12$ hours apart, or

All of three or a majority of four or more separate blood cultures, with first and last drawn at least 1 hour apart

Evidence of endocardial involvement:

Positive echocardiogram for infective endocarditis:

Oscillating intracardiac mass on PM leads or on the endocardial structure in contact with PM leads

Abscess in contact with PM leads

\section{Minor criteria}

Fever $>38^{\circ} \mathrm{C}$

Vascular phenomena: arterial embolism, septic pulmonary infarcts, mycotic aneurysm, intracranial hemorrhage, Janeway lesions

Immunologic phenomena: glomerulonephritis, Osler nodes, Roth spots

Echocardiogram: consistent with infective endocarditis but not meeting major criterion as noted previously (sleevelike appearance)

Microbiological evidence: positive blood culture but not meeting major criterion as noted previously.

Table 2. ${ }^{3}$ Definition of Terms Used in the Proposed Modified Diagnostic Criteria

\subsection{Pathophysiology}

It is commonly accepted that the most common portal of entry to develop device-related endocarditis is the subcutaneous site of insertion of the pacing system. Extension along the lead into the vascular system is the usual explanation for the localization of the infection to the lead. Bacterial colonization of the lead during the course of bacteremia whose origin is not related to the pacing system might be possible but has been less well documented. Staphylococci are responsible for the vast majority of these infections, especially S. epidermidis in the chronic group and S. aureus in the acute group. A fungal infection is rare and more subtle to indentify. Fungal endocarditis is associated with high mortality and usually presents with scant growth of the microorganism in blood cultures (Figure 2). A

${ }^{3}$ From Klug et al. with permission 
high index of suspicion for fungal endocarditis should be maintained in individuals with implantable pacemakers and fever of an uncertain source, especially in the context of negative blood cultures(Leong 2006). However, there is a $20-30 \%$ of device-related endocarditis with negative BC (Klug et al. 1997).
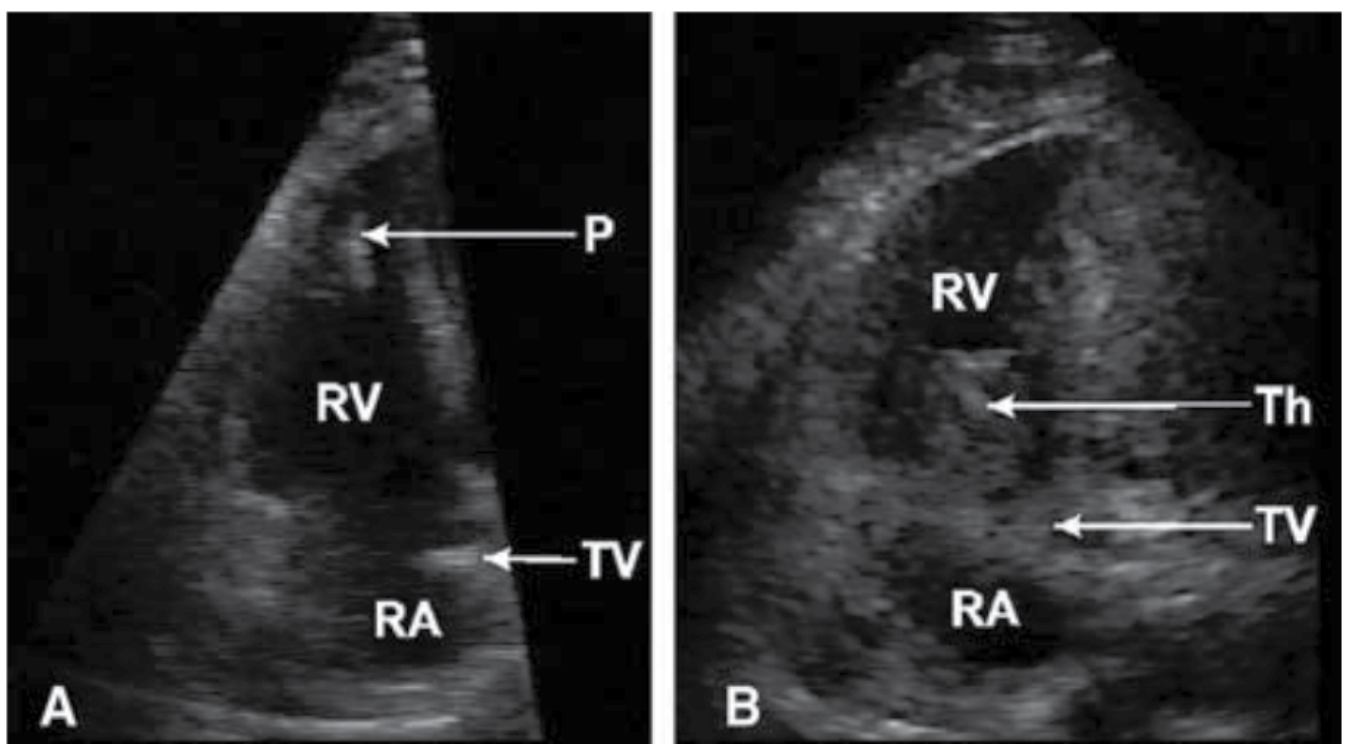

Figure 2. ${ }^{4} \mathrm{~A}$ : Transthoracic echocardiogram of the right atrium (RA), tricuspid valve (TV) and right ventricle $(R V)$ demonstrating a pacemaker lead $(\mathrm{P})$ and no valvular vegetations. $\mathrm{B}$ : Second transthoracic echocardiogram of the right heart, seven weeks after the initial echocardiogram, demonstrating a right ventricular pacemaker lead thrombus (Th)

\subsection{Management}

The removal of the entire pacing system should be performed immediately rather than attempting prolonged antibiotic therapy alone. As in the study by Camus et al, the high rate of uncontrolled infection or relapse among patients with septicemia in relation to PMmaterial infection confirms the need for (Camus et al. 1993). Moreover immediate removal of the entire pacing system should be performed in all cases both for systemic infection related to PM-lead contamination and for infection of the PM pocket or the subcutaneous part of the lead (Panidis et al. 1984). Cultures of the leads and of the PM should be done after the extraction.Complete PPM or ICD removal should be performed when patients undergo valve replacement or repair for infective endocarditis, because the pacing system could serve as a nidus for relapsing infection and subsequent seeding of the surgically treated heart valve. Hardware removal is not required for superficial or incisionalinfection at the pocket site if there is no involvement of the device, 7-10 days of antibiotic therapy with an oral agent with activity against staphylococci is reasonable (Baddour 2010).

${ }^{4}$ From Leong et al. with permission. 


\section{Technique of extraction: Surgical or percutaneous?}

After implantation, transvenous device leads usually undergo fibrotic encapsulation by activation of different cellular and humoral mechanisms (Esposito et al. 2002). The ensuing fibrotic lead adhesions tend to increase over time. Young patients, however, usually develop fibrotic adhesions earlier than elderly. On the contrary, systemic lead infection seems to counteract or dissolve fibrotic adherences. Current literature suggests that, the best outcome is achieved with percutaneous removal of infected devices by applying external traction on the leads (Sohail et al. 2007; Ruttmann et al. 2006). However, while simple traction is often successful in newly placed leads, it can be problematic with chronic leads and cause catastrophic complications, ranging from septic embolic phenomena to tricuspid valve injury, subclavian vein laceration, hemothorax, pocket hematoma, massive hemorrhage, and lead fracture requiring urgent surgical intervention (Sohail et al. 2007; Ruttmann et al. 2006; Panidis et al. 1984). Damage to the left sided cardiac structures is rare but may be a complication of an infected lead extraction, manifesting as iatrogenic ventricular septum disruption with consequent aortic valve leaflet prolapse and massive acute aortic regurgitation (fig.3)(Rossi et al. 2011).

Chronically implanted leads are fixed to the myocardium by fibrous tissue. Fibrous scar tissue may also encase the lead along its course. Furthermore, fragility of the lead and its tendency to break when extraction force is applied to overcome resistance imparted by the scar tissue add to the challenge of lead extraction. Thus, selecting the appropriate extraction procedure for chronically implanted leads is an important issue.

\subsection{Percutaneous extraction}

The removal of the entire pacing system should be performed in one session. Newpercutaneous PM and ICD lead extraction techniques have been developed to overcome the problem of a difficult extraction with the aim to reduce damage to the cardiac structures produced by the simple counter traction. Telescoping mechanical sheaths and locking stylets were introduced during the late 1980s and early 1990s. Special tools for femoral lead extraction soon followed. They can be used though a superior approach (jugular or subclavian vein) using locking stylet ; or via a femoral vein approach using double lasso catheters (Needle's eye snare) (Byrd et al. 1999; Bracke et al. 2001; Fearnot et al. 1990). In the superior vena cava approach, a locking stylet is introduced into the lead and locked close to the distal electrode in order to apply traction directly to the tip. If gentle traction is not successful, telescoping sheaths can be advanced over the lead to disrupt fibrous binding of the lead to veins or myocardium. When necessary, the tip of the lead is freed by countertraction, the sheath being positioned against the myocardium to prevent inversion during traction on the lead. In the transfemoral approach, the pacing lead is grabbed with a deflecting guide wire or retriever through a long sheath inserted from the femoral vein. The proximal end of the lead is pulled down from the subclavian vein. Then the outer sheath is advanced over the lead to disrupt the scar tissue, as with the superior approach. When the myocardium is reached countertraction is applied. 


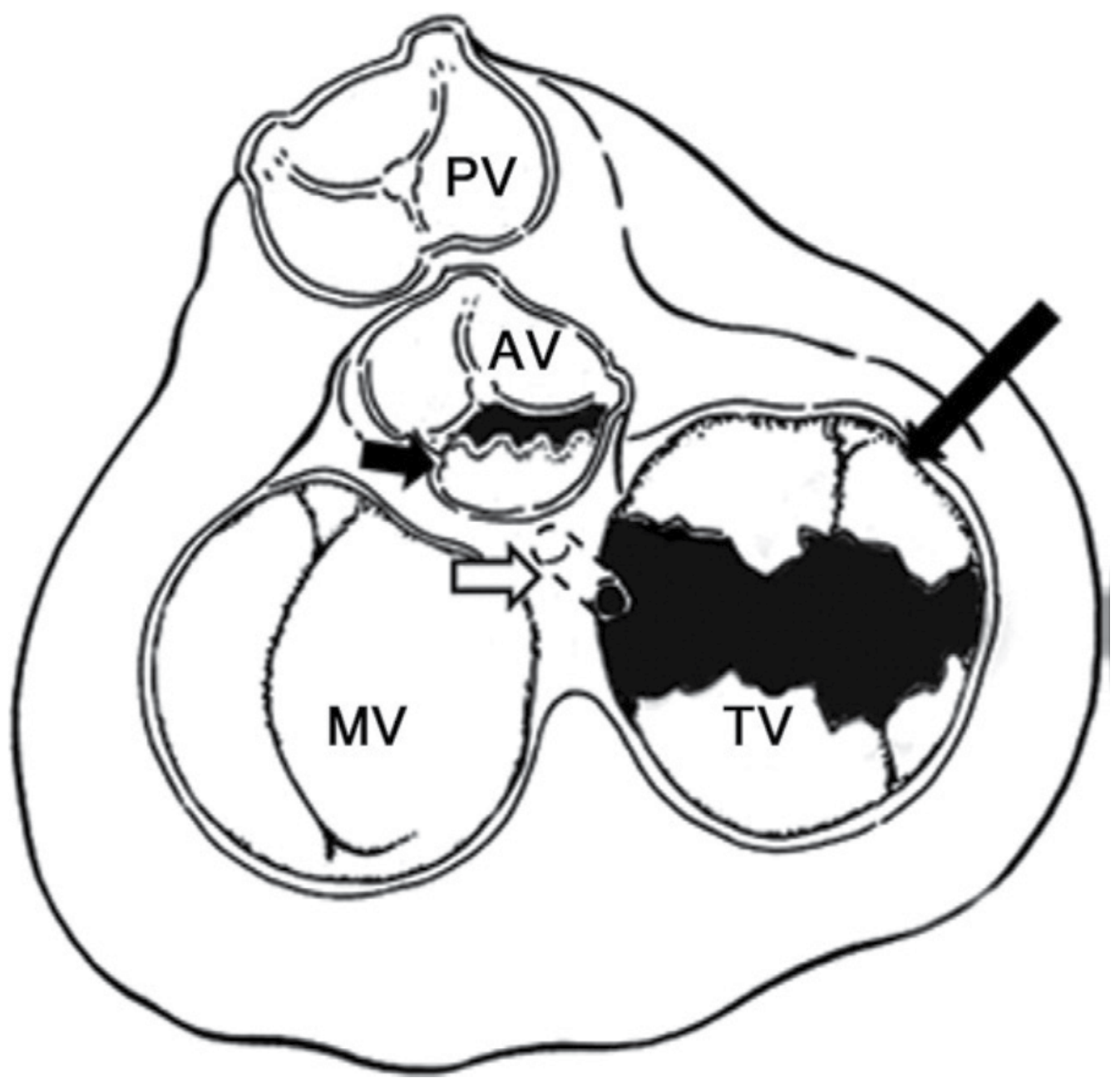

AV: aortic valve; MV: mitral valve; PV: pulmonary valve; TV: tricuspid valve.

Figure 3. ${ }^{5}$ Four-valve view of the fibrous skeleton of the heart showing disruption of the tricuspid valve (long black arrow), ventricular septal defect (white arrow), and prolapse of the noncoronary cusp of the aortic valve (short black arrow).

\subsubsection{Laser extraction}

Progress has also been made in developing other systems for lead extraction powered with laser energy. The first laser-assisted lead extraction performed in 1994 was a major breakthrough. The laser extraction sheath offers a method for removal by "cutting" through scar tissue, without excessive use of force (such as with purely mechanical systems). It appears to be an efficient tool for removal of chronic implanted infected leads but its use is

\section{${ }^{5}$ From Rossi et al. with permission.}

Intraoperative cultures of the aortic valve and ventricular septal defect edges did not show any significant growth, supporting the hypothesis that the prolapse of the noncoronary cusp was due to lack of support on the valve structure. 
associated with a high number of bleeding complications often requiring surgical revision (see section 3.3).

\subsection{Surgical extraction}

Surgical removal has a higher complication rate and worse outcome compared with percutaneous techniques (Klug et al. 1997). Although, in concept, the surgical approach is the cleanest way to extract an infected lead together with its vegetation without risks of pulmonary or systemic septic embolism, it is generally accepted both by surgeon and cardiologist to prefer the percutaneous extraction as first attempt. However we should not forget that the surgical population is highly selected, i.e. made of cases not suitable for percutaneous extraction or with heart damage after percutaneous attempt or with severe tricuspid valve involvement. Moreover, the presence of intracardiac vegetations alone, identifies a subset of patients at increased risk for complications and early mortality from systemic infection regardless the technique of extraction (percutaneous or surgical) and appropriate antimicrobial therapy (Grammes et al. 2010). From a review of the current literature, it appears that the indication for surgical removal is mainly limited as rescue intervention to fix complicated or failed percutaneous extraction. Surgical removal generally requires median sternotomy or thoracotomy and sometimes cardiopulmonary bypass (CBP). Nevertheless there are still patients that will benefit from a surgical removal as a first attempt rather than later, especially those with large vegetations that might obstruct the main pulmonary artery or those who need the implantation of an epicardial pacing system.

A primary surgical approach to lead removal in patients with PPM/ICD infection is recommended by the current guidelines for implantable electronic device infections and management in patients who have significant retained hardware after attempts at percutaneous removal or in patients with lead vegetations $>2 \mathrm{~cm}$ in diameter, because of concerns about the risk of pulmonary embolism with percutaneous lead extraction (Baddour et al. 2010). In fact, it is useful to reconsider the indication for pacing after successful extraction of the infected pacing system.Discontinuation of pacemaker treatment after lead extraction has been reported in $13-52 \%$ of patients (Bracke et al. 2001). However when permanent pacing is a must, an epicardial system is the recommended choiceespecially after valve surgery with initial hardware removal(Baddour et al. 2010). With the surgical approach, the epicardial permanent system can be easily placed at the same time of the extraction and offers the advantage of eliminating the contact between leads and systemic circulation taking the chances of infection of the new system to the ground.

\subsection{Evidence-based discussion}

Wilkoff et al, in a randomized control trial of 465 chronically implanted leads, achieved $94 \%$ complete removal with a laser sheath against $64 \%$ with conventional sheaths, but with an higher rate of potentially life-threatening complications (Wilkoff et al. 1999). In a multicenter study over 2338 patients, Byrd et al, reported an increased risk of failed or partial extraction with increasing implant duration, doubling every three years (Byrd et al. 1999). Kennergren 
et al, in a retrospective analysis of their activity on 647 lead extractions, surprisingly showed that the implantation time was not associated with extraction failure neither there was an association between implantation time and the incidence of serious complications. Actually they showed that leads can often be extracted by a superior transvenous approach with simple traction; Laser-assisted lead extraction appeared to be a useful technique to extract leads that could not be removed by manual traction but at the cost of a higher rate of bleeding complications requiring open-chest surgical revision (Kennergren et al. 2009). Alt et al, achieved total removal of $81 \%$ of 150 leads, without major complications with the use of only locking stylets (Alt et al. 1996). Tokunaga et al, performed a surgical removal without CBP after a failed extraction using the Excimer Laser Sheath Extraction System (Tokunaga et al. 2011). The authors highlight the potential risk of perforation and lethal bleeding complications using this tool and suggest a close backup by a cardiovascular surgeon. Neuzi et al, in a randomized control trial (RCT) compared the safety and efficacy of transvenous pacemaker and implantable cardioverter-defibrillator (ICD) lead extraction, with an electrosurgical dissection sheath (EDS) system using radiofrequency (RF) current or standard countertraction lead removal in 120 consecutive patients (Neuzi et al. 2007). Although the EDS extraction system appeared quicker and more effective in complete removal of leads, they could not demonstrate a significant superiority versus the standard counter-traction method. Buongiorni et al, in a retrospective analysis of 1330 leads extraction, concluded that transvenous lead extraction is an effective and safe procedure. They showed how the use of the jugular approach, in the presence of free-floating or difficult exposed leads, increases both safety and success rate (Buongiorni et al. 2005). Kratz et al, in a retrospective analysis of 365 patients who underwent PPM or ICD lead removal, showed that performing a lead extraction in a protected environment, such as an operating room, allowed rapid and effective treatment of potential procedure-related complications. Actually, the use of several extraction tools, arterial line monitoring, transesophageal echocardiography, general anesthesia, and an experienced team, yielded complete extraction in more than $90 \%$ of patients, with a low complication rate and no procedurally related deaths (Kratz et al. 2010). Grammes et al, reported their experience using percutaneous leads extraction by simple traction of 1,838 infected leads with echocardiographic evidence of intracardiac vegetations. Post-operative 30-day mortality was 10\%; no deaths were related directly to the extraction procedure (Grammes et al. 2010). The common message that comes from the literature is that extraction of infected PM-leads is not just a "pull and go" procedure and should be performed by expert physicians, in tertiary centres, with a cardiac surgery back up to best manage their complications.

\subsubsection{Septic embolism}

There is also a concern in pacemaker related endocarditis over embolisation of vegetations adhering to the lead when endovascular extraction is attempted. Klug et al, in their series of 52 patients with device-related endocarditis, suggested to chose the technique of removal (surgical versus percutaneous) on the size of the vegetations: percutaneous when vegetation size was $\leq 10 \mathrm{~mm}$ and surgical if $>10 \mathrm{~mm}$ at transesophageal echocardiogram (Klug et al. 
1997). This policy was based on previous observations by Mugge et al and by Robbins et al, who found that embolism was more frequent with vegetation size was $>10 \mathrm{~mm}$ in endocarditis related to valve infection (Mugge et al. 1989; Robbins et al. 1986). More recently Ruttmann et al, showed that transvenous lead removal is a safe and effective even in patients with large vegetations. Embolism to the lung happens but tends to proceed mainly without complications. However there are still cases where surgical approach is preferred, such as, in presence of large vegetations that might occlude the main pulmonary artery(Ruttmann et al. 2006) or with vegetations $>2 \mathrm{~cm}$ in diameter (Baddour et al. 2010).

\section{Conclusion}

The diagnosis of endocarditis related to PM-lead infection should be systematically considered in patients with fever, history of local complications, or pulmonary pathology after PM insertion. There are two different clinical presentations: the acute form, that presents early with sepsis, often in conjunction with local signs of infection, and a chronic form, beginning several months later. The presentation may be atypical and the symptoms may occur late after the last intervention at the PM site. CRP, ESR, scintigraphy or chest CT angiogram may be of diagnostic value. TTE and TOE must be performed in search of vegetations. Immediate removal of the entire pacing system is paramount, in addition to prolonged antimicrobial therapy. We believe that multidisciplinary approach is the key to manage device-related endocarditis and good professional relationships are essential between cardiologists, surgeons, and infective disease specialists to make the appropriate decisions to best treat these complex patients. Our recommendation, in the patients' best interest, is not to embark on extracting infected leads without doing a serious ongoing individual risk-benefit analysis. Finally a word of wisdom to the future generations: even if we are moving faster towards new innovative ways to pace the heart, we will always be dealing with their complications, therefore, is worth to create a network of professionals to address them in the best possible way.

\section{Author details}

Michele Rossi, Giuseppe Musolino, Giuseppe Filiberto Serraino and Attilio Renzulli Department of Cardiac Surgery, Magna Graecia University, Catanzaro, Italy

\section{References}

Adabag S, Roukoz H, Anand IS \& Moss AJ. (2011). Cardiac resynchronization therapy in patients with minimal heart failure a systematic review and meta-analysis. J Am Coll Cardiol, Vol. 58, No. 9 (Aug 2011), pp. 935-41. ISSN 1558-3597

Alt E, Neuzner J, Binner L, Göhl K, Res JC, Knabe UH, Zehender M \& Reinhardt J. (1996). Three-year experience with a stylet for lead extraction: a multicenter study. Pacing Clin Electrophysiol. Vol 19, No. 1, (Jan 1996), pp. 18-25. ISSN 0147-8389 
Baddour LM, Epstein AE, Erickson CC, Knight BP, Levison ME, Lockhart PB, Masoudi FA, Okum EJ, Wilson WR, Beerman LB, Bolger AF, Estes NA 3rd, Gewitz M, Newburger JW, Schron EB \& Taubert KA. (2010). American Heart Association Rheumatic Fever, Endocarditis, and Kawasaki Disease Committee; Council on Cardiovascular Disease in Young; Council on Cardiovascular Surgery and Anesthesia; Council on Cardiovascular Nursing; Council on Clinical Cardiology; Interdisciplinary Council on Quality of Care; American Heart Association. Update on cardiovascular implantable electronic device infections and their management: a scientific statement from the American Heart Association. Circulation. Vol. 121, No. 3, (Jan 2010), pp. 458-77. ISSN 1524-4539

Bardy GH, Lee KL, Mark DB, Poole JE, Packer DL, Boineau R, Domanski M, Troutman C, Anderson J, Johnson G, McNulty SE, Clapp-Channing N, Davidson-Ray LD, Fraulo ES, Fishbein DP, Luceri RM \& Ip JH; Sudden Cardiac Death in Heart Failure Trial (SCDHeFT) Investigators. (2005) Amiodarone or an implantable cardioverter-defibrillator for congestive heart failure. N Engl J Med, Vol. 352, No.3 (Jan 2005), pp. 225-37. Erratum in: N Engl J Med. Vol. 352, No. 20 (May 2005), pp. 2146. ISSN1533-4406

Bates MG, Matthews IG, Fazal IA \& Turley AJ. (2011). Postoperative permanent pacemaker implantation in patients undergoing trans-catheter aortic valve implantation: what is the incidence and are there any predicting factors? Interact Cardiovasc Thorac Surg. Vol 12, No.2, (Feb 2011), pp. 243-53. ISSN 1569-9285

Bongiorni MG, Giannola G, Arena G, Soldati E, Bartoli C, Lapira F, Zucchelli G \& Di Cori A. (2005). Pacing and implantable cardioverter-defibrillator transvenous lead extraction. Ital Heart J. Vol. 6, No. 3, (Mar 2005), pp. 261-6. ISSN 1129-471X

Bracke FA, Meijer A \& van Gelder LM. (2001). Pacemaker lead complications: when is extraction appropriate and what can we learn from published data? Heart. Vol. 85, No.3, (Mar 2001), pp. 254-9. ISSN 1468-201X

Bristow MR, Saxon LA, Boehmer J, Krueger S, Kass DA, De Marco T, Carson P, DiCarlo L, DeMets D, White BG, DeVries DW \& Feldman AM; Comparison of Medical Therapy, Pacing, and Defibrillation in Heart Failure (COMPANION) Investigators. (2004). Cardiac-resynchronization therapy with or without an implantable defibrillator in advanced chronic heart failure. N Engl J Med, Vol. 350, No. 21 (May 2004), pp. 2140-50. ISSN 1533-4406

Byrd CL, Wilkoff BL, Love CJ, Sellers TD, Turk KT, Reeves R, Young R, Crevey B, Kutalek SP, Freedman R, Friedman R, Trantham J, Watts M, Schutzman J, Oren J, Wilson J, Gold F, Fearnot NE \& Van Zandt HJ. (1999). Intravascular extraction of problematic or infected permanent pacemaker leads: 1994-1996. U.S. Extraction Database, MED Institute. Pacing Clin Electrophysiol. Vol. 22, No.9, (Sep 1999), pp. 1348-57. ISSN 01478389

Cacoub P, Leprince P, Nataf P, Hausfater P, Dorent R, Wechsler B, Bors V, Pavie A, Piette JC \& Gandjbakhch I. (1998). Pacemaker infective endocarditis. Am J Cardiol. Vol. 82, No.4, (Aug 1998), pp. 480-4. ISSN 0002-9149

Camus C, Leport C, Raffi F, Michelet C, Cartier F \& Vilde JL. (1993). Sustained bacteremia in 26 patients with a permanent endocardial pacemaker: assessment of wire removal. Clin Infect Dis. Vol. 17, No. 1, (Jul 1993), pp. 46-55. ISSN 1058-4838 
Chan P, Ogilby JD \& Segal B. (1989). Tricuspid valve endocarditis. Am Heart J. Vol. 117, No.5, (May 1989), pp. 1140-6. ISSN 0002-8703

D'Ancona G, Pasic M, Unbehaun A \& Hetzer R. (2011). Permanent pacemaker implantation after transapical transcatheter aortic valve implantation. Interact Cardiovasc Thorac Surg. Vol. 13, No. 4 (Oct 2011), pp. 373-6. ISSN 1569-9285

Esposito M, Kennergren C, Holmström N, Nilsson S, Eckerdal J \& Thomsen P. (2002). Morphologic and immunohistochemical observations of tissues surrounding retrieved transvenous pacemaker leads. J Biomed Mater Res. Vol. 63, No.5, (Sept 2002), pp. 548-58. ISSN 0021-9304

Fearnot NE, Smith HJ, Goode LB, Byrd CL, Wilkoff BL \& Sellers TD. (1990). Intravascular lead extraction using locking stylets, sheaths, and other techniques. Pacing Clin Electrophysiol. Vol. 13, No. 12 Pt 2, (Dec 1990), pp. 1864-70. ISSN 0147-8389

Grammes JA, Schulze CM, Al-Bataineh M, Yesenosky GA, Saari CS, Vrabel MJ, Horrow J, Chowdhury M, Fontaine JM \& Kutalek SP. (2010). Percutaneous pacemaker and implantable cardioverter-defibrillator lead extraction in 100 patients with intracardiac vegetations defined by transesophageal echocardiogram. J Am Coll Cardiol. Vol. 55, No. 9, (Mar 2010), pp. 886-94. ISSN 1558-3597

Kennergren C, Bjurman C, Wiklund R \& Gäbel J.(2009) A single-centre experience of over one thousand lead extractions. Europace. Vol. 11, No.5, (May 2009), pp. 612-7. ISSN 15322092

Klug D, Lacroix D, Savoye C, Goullard L, Grandmougin D, Hennequin JL, Kacet S \& Lekieffre J. (1997). Systemic infection related to endocarditis on pacemaker leads: clinical presentation and management. Circulation. Vol. 95, No.8, (Apr 1997), pp. 2098107. ISSN 0009-7322

Kratz JM, Toole JM. (2010). Pacemaker and internal cardioverter defibrillator lead extraction: a safe and effective surgical approach. Ann Thorac Surg. Vol. 90, No. 5, (Nov 2010), pp. 1411-7. ISSN 1552-6259

Leong R, Gannon BR, Childs TJ, Isotalo PA \& Abdollah H. (2006). Aspergillus fumigates pacemaker lead endocarditis: a case report and review of the literature. Can J Cardiol. Vol. 22, No. 4, (Mar 2006), pp. 337-40. ISSN 0828-282X

Love CJ, Wilkoff BL, Byrd CL, Belott PH, Brinker JA, Fearnot NE, Friedman RA, Furman S, Goode LB, Hayes DL, Kawanishi DT, Parsonnet V, Reiser C \& Van Zandt HJ. (2000). Recommendations for extraction of chronically implanted transvenous pacing and defibrillator leads: indications, facilities, training. North American Society of Pacing and Electrophysiology Lead Extraction Conference Faculty. Pacing Clin Electrophysiol. Vol. 23, No. 4 Pt 1, (Apr 2000), pp. 544-51. ISSN 0147-8389

Moss AJ, Hall WJ, Cannom DS, Daubert JP, Higgins SL, Klein H, Levine JH, Saksena S, Waldo AL, Wilber D, Brown MW \& Heo M. (1996). Improved survival with an implanted defibrillator in patients with coronary disease at high risk for ventricular arrhythmia. Multicenter Automatic Defibrillator Implantation Trial Investigators. $N$ Engl J Med. Vol. 335, No. 26, (Dec 1996), pp. 1933-40. ISSN 0028-4793

Mügge A, Daniel WG, Frank G \& Lichtlen PR. (1989). Echocardiography in infective endocarditis: reassessment of prognostic implications of vegetation size determined by 
the transthoracic and the transesophageal approach. J Am Coll Cardiol. Vol. 14, No.3, (Sep 1989), pp. 631-8. ISSN 0735-1097

Neuzil P, Taborsky M, Rezek Z, Vopalka R, Sediva L, Niederle P \& Reddy V. (2007). Pacemaker and ICD lead extraction with electrosurgical dissection sheaths and standard transvenous extraction systems: results of a randomized trial. Europace. Vol. 9, No.2, (Feb 2007), pp. 98-104. ISSN 1099-5129

Panidis IP, Kotler MN, Mintz GS, Segal BL \& Ross JJ. (1984). Right heart endocarditis: clinical and echocardiographic features. Am Heart J. Vol. 107, No.4, (Apr 1984), pp. 75964. ISSN 0002-8703

Robbins MJ, Frater RW, Soeiro R, Frishman WH \& Strom JA. (1986). Influence of vegetation size on clinical outcome of right-sided infective endocarditis. Am J Med. Vol. 80, No. 2, (Feb 1986), pp. 165-71. ISSN 0002-9343

Robbins MJ, Soeiro R, Frishman WH \& Strom JA. (1986). Right-sided valvular endocarditis: etiology, diagnosis, and an approach to therapy. Am Heart J. Vol. 111, No. 1, (Jan 1986), pp. 128-35. ISSN 0002-8703

Rossi M, Pirone F, Nair S \& Trivedi U. (2011). Massive acute aortic regurgitation after infected pacemaker lead removal: a word of caution. Ann Thorac Surg. Vol. 92, No.2, (Aug 2011), pp. e29-31. ISSN 1552-6259

Ruttmann E, Hangler HB, Kilo J, Höfer D, Müller LC, Hintringer F, Müller S, Laufer G \& Antretter H. (2006). Transvenous pacemaker lead removal is safe and effective even in large vegetations: an analysis of 53 cases of pacemaker lead endocarditis. Pacing Clin Electrophysiol. Vol. 29, No.3, (Mar 2006), pp. 231-6. ISSN 0147-8389

Sohail MR, Uslan DZ, Khan AH, Friedman PA, Hayes DL, Wilson WR, Steckelberg JM, Stoner S \& Baddour LM. (2007). Management and outcome of permanent pacemaker and implantable cardioverter-defibrillator infections. J Am Coll Cardiol. Vol. 49, No.18, (May 2007), pp. 1851-9. ISSN 1558-3597

Tokunaga C, Enomoto Y, Sato F, Kanemoto S, Matsushita S, Hiramatsu Y, Aonuma K \& Sakakibara Y. (2012). Surgical removal of infected pacemaker leads without cardiopulmonary bypass after failed extraction using the Excimer Laser Sheath Extraction System. J Artif Organs. Vol. 15, No. 1, (Mar 2012), pp. 94-8. ISSN 1619-0904

Voigt A, Shalaby A \& Saba S. (2006). Rising rates of cardiac rhythm management device infections in the United States: 1996 through 2003. J Am Coll Cardiol. Vol. 48, No.3, (Aug 2006), pp. 590-1. ISSN 1558-3597

Wilkoff BL, Byrd CL, Love CJ, Hayes DL, Sellers TD, Schaerf R, Parsonnet V, Epstein LM, Sorrentino RA \& Reiser C. (1999). Pacemaker lead extraction with the laser sheath: results of the pacing lead extraction with the excimer sheath (PLEXES) trial. J Am Coll Cardiol. Vol. 33, No. 6, (May 1999), pp. 1671-6. ISSN 0735-1097 


\title{
Mechanism and Management of Pacing Lead Related Cardiac Perforation
}

\author{
Sanjay Kumar, Haidar Yassin, Opesanmi Esan, \\ Adam S. Budzikowski and John T. Kassotis \\ Additional information is available at the end of the chapter
}

http://dx.doi.org/10.5772/48630

\section{Introduction}

\subsection{Incidence and time course for cardiac perforation}

Cardiac perforation due to a pacemaker or defibrillator lead occurs at a rate of $0.4-2.0 \%$. Since first described [1], the overall incidence has decreased significantly. The reported incidence of this complication has been as low as $<1 \%$ to as high as $15 \%$ [2-4]. Of 4,280 permanent pacemaker (PPM) implants only $50(1.2 \%)$ patients developed a significant effusion and symptoms consistent with a perforation [5]. The incidence of this complication from the time of surgery decreases over time. By convention, a perforation detected within 24 hours, is classified as acute. If detected within 30 days of implantation (usually 5 days- 4 weeks), it is referred to as early (sub-acute); and, those detected after 30 days referred to as late, delayed or chronic perforation. The majority of perforations manifest within a year but rarely cases have been reported as late as five years following implantation. [6]

\subsection{RV perforation}

\subsubsection{Modeling of $R V$ perforation}

In order to develop perforation resistant leads, several models of myocardium - lead interaction have been developed. Lead related factors that affect propensity for perforation include lead thickness as well as stiffness. Refinement of the current lead technology produce leads with both a reduced thickness and stiffness. In experimental model [7] bipolar leads with silicone rubber insulation had lower stiffness as compared with those coated with polyurethane. While a lower stiffness is a desirable property, it results in a 
thinner lead with a higher penetration pressure (force per unit area). In a porcine perforation model [8] studying the effect of cylindrical punches, of variable diameter, on RV wall tissue the penetration pressure (force/cross sectional area) decreases as a function of an increasing punch diameter. The penetration pressure decreased by approximately $25 \%$ as the punch diameter was doubled. (Figure 1)
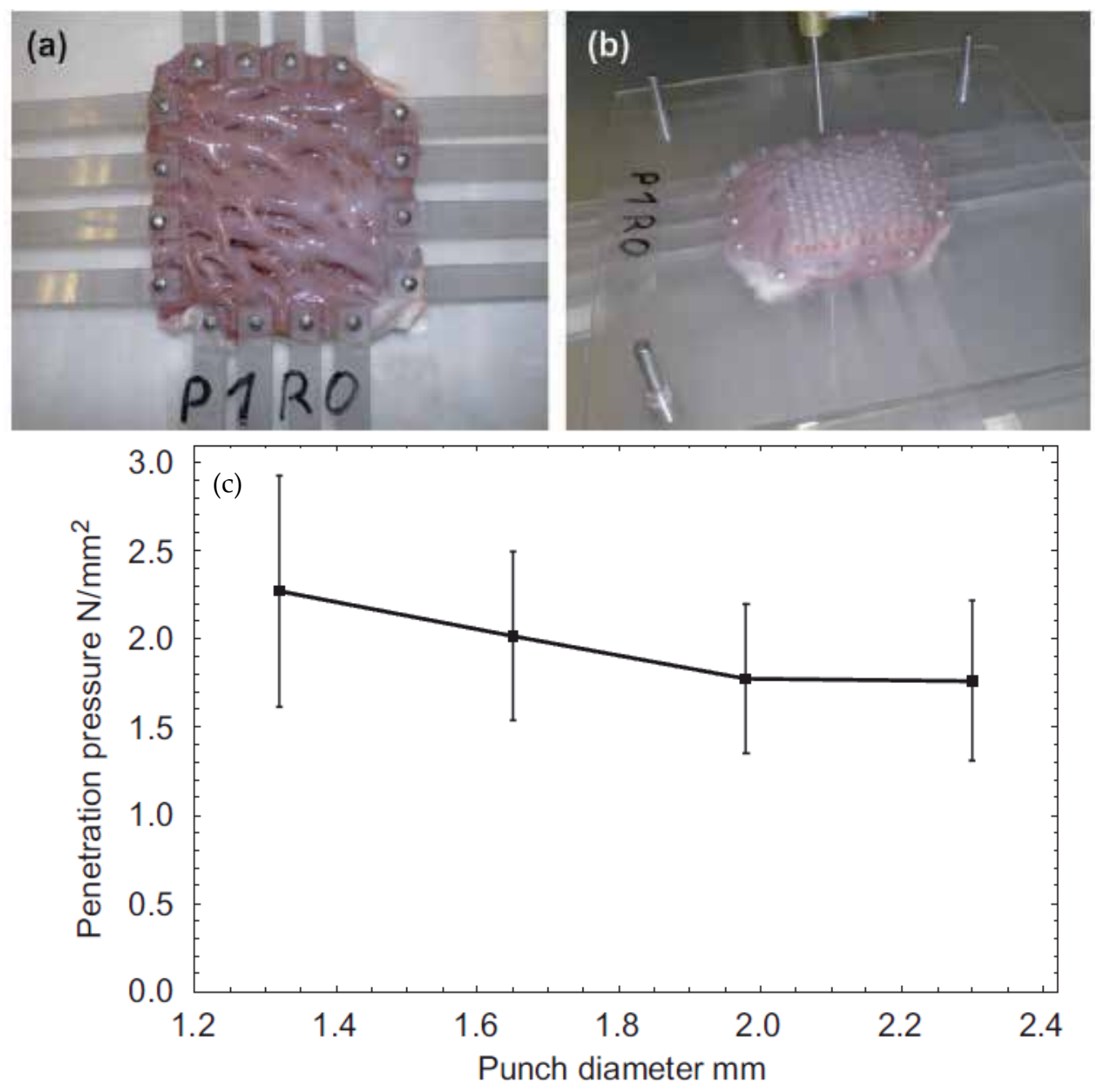

Figure 1. Figures show the porcine specimen of RV free wall fixed to the grips (a) and specimen biaxially stretched to its testing conditions (b). Figure (c) shows penetration characteristics of the right ventricular myocardium. Penetration pressure (penetration force divided by punch cross section) with respect to the punch diameter. "(With permission from Gasser TC: Journal of Biomechanics. 42:629-633, 2009.) 
Myocardium related factors which contribute to the perforation risk include the wall thickness and the viscoelastic properties of the tissue. Experimental data suggest that the myocardium is anisotropic (with the highest stiffness in the direction of the fiber's longitudinal orientation), non-linear, nearly elastic and exhibiting heterogeneity throughout a particular chamber (e.g. RV) [9].

The interaction between the lead tip and myocardium is complex, varies with the cardiac cycle, and changes over time. The lead tip penetrates the tissue, creating a fissure between local fibers. The lead wedges into this "crack", expanding the opening over time [7]. This is illustrated by the detection of a "crack" (fissure) both by light and electron microscopy, see Figures 2, 3. However, it should be noted that when a "crack" splits the plane of fibers, compared to a punch, there is higher likelihood of closure due to the opposing forces of the myocardium, which act to seal the fissure. This may explain why many perforations are subclinical. Despite advancements in the knowledge of the lead-tissue interaction and advances in lead technology, this complication has still not been eliminated.

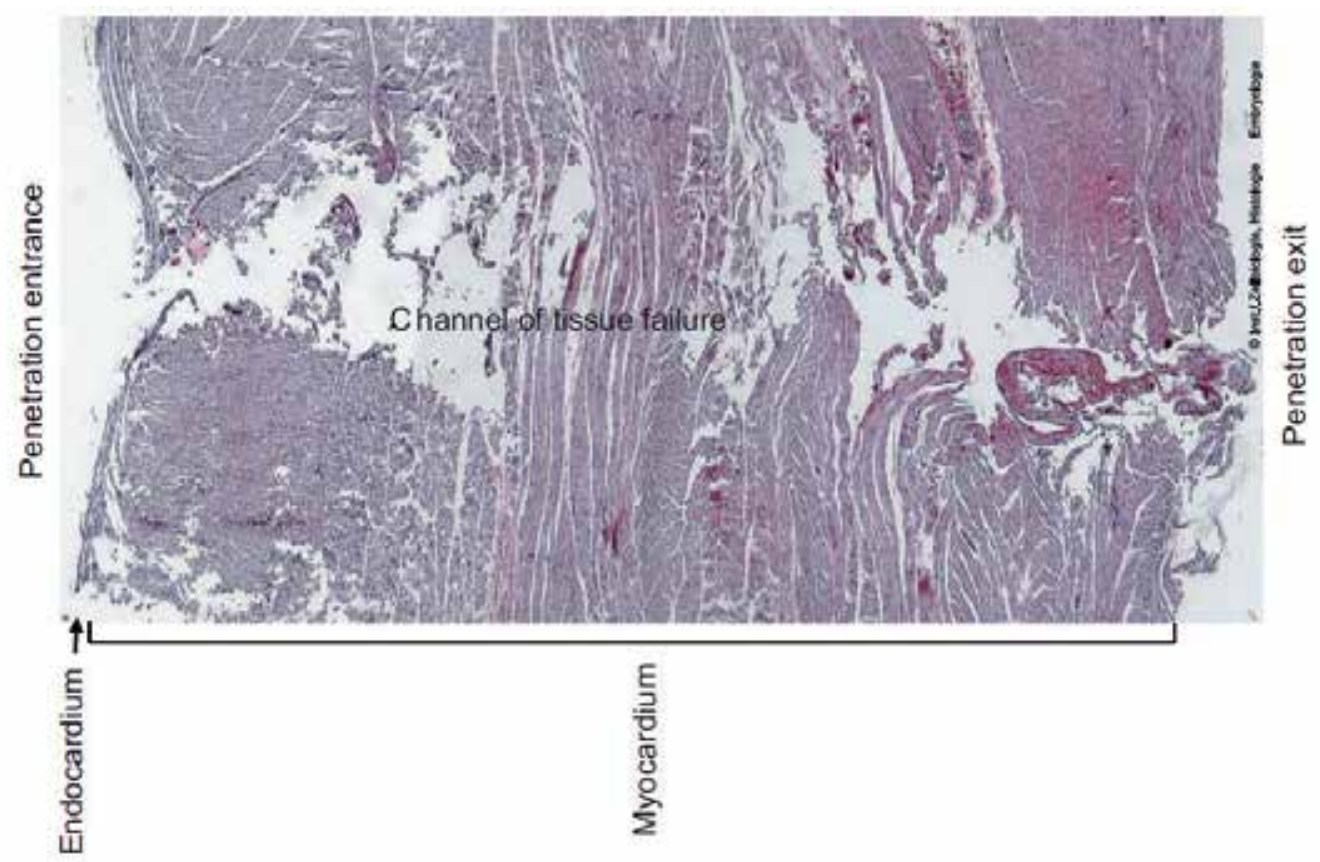

Figure 2. Microscopy of endocardium and myocardium of RV of a porcine specimen with a punch of $2.3 \mathrm{~mm}$ diameter. Picrosirius red stain illustrates tissue failure through the different layers of the ventricular tissue. “(With permission from Gasser TC: Journal of Biomechanics. 42:629-633, 2009.) 
a

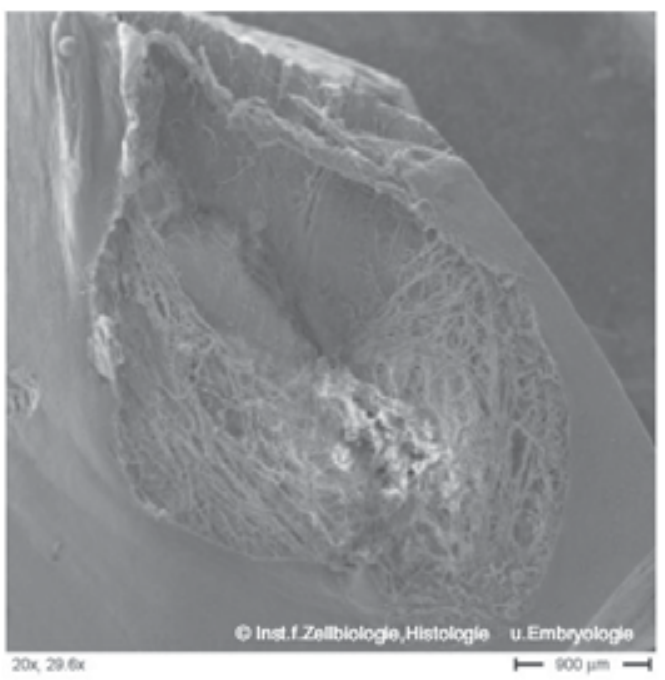

b

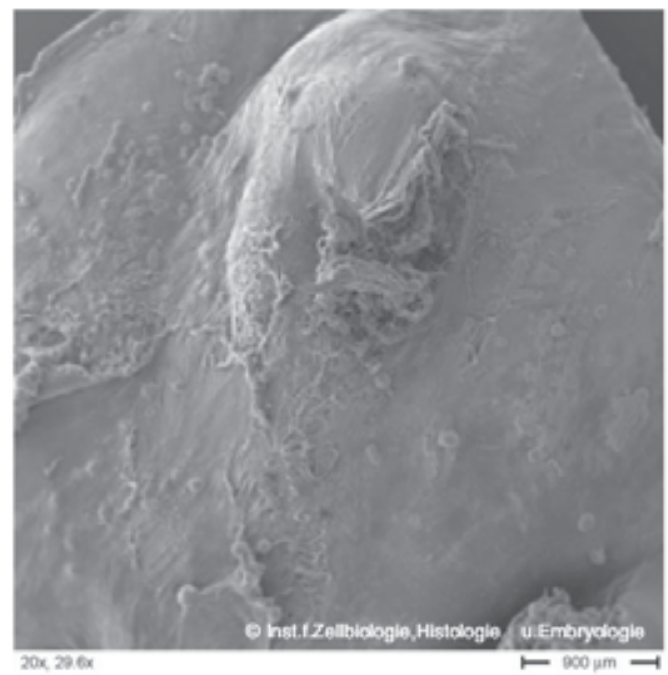

Figure 3. Electron microscopy images taken from the (a) entrance and (b) exit of the punch illustrating a splitting mode failure and the presence of remaining deformations respectively. "(With permission from Gasser TC: Journal of Biomechanics. 42:629-633, 2009.)

\subsubsection{Free wall perforation}

Hirschl et al studied the incidence of an asymptomatic lead perforation, by computed tomography and correlated with electrophysiologic data. Out of 100 consecutive PPM and intra-cardiac defibrillator (ICD) implantations, right ventricular (RV) perforation was less common ( $6 \%$ vs. $15 \%)$ than right atrial (RA) perforation, respectively [4]. However, in a recent study of 2,385 patients undergoing an electrophysiology study as well as pacemaker lead insertion, $7(0.3 \%)$ patients experienced an RV perforation compared to only $1(0.04 \%)$ RA perforation [10]. The predisposition to develop an RV perforation was a function of RV location; explained in part by the heterogeneous nature of the RV. The RV resembles a pyramidal structure with the interventricular septum (IVS) located posteriorly and the free wall located anteriorly. The RV free wall is thinner $(<0.5 \mathrm{~cm})$ than the septum (ranging from 0.6 to $0.9 \mathrm{~cm}$ ) [11], accounting for the higher risk of perforation. Positioning leads in the RV IVS may not only reduce the risk of perforation but reduce the degree of QRS widening, possibly minimizing asynchrony. In the majority of cases the perforating lead is contained within the pericardium, however, in rare cases the lead can migrate along the pericardium traversing the diaphragm into the abdominal cavity. Figure 4 and 5 (chest $x$-ray and CT scan) illustrate an example of a RV free wall perforation. 

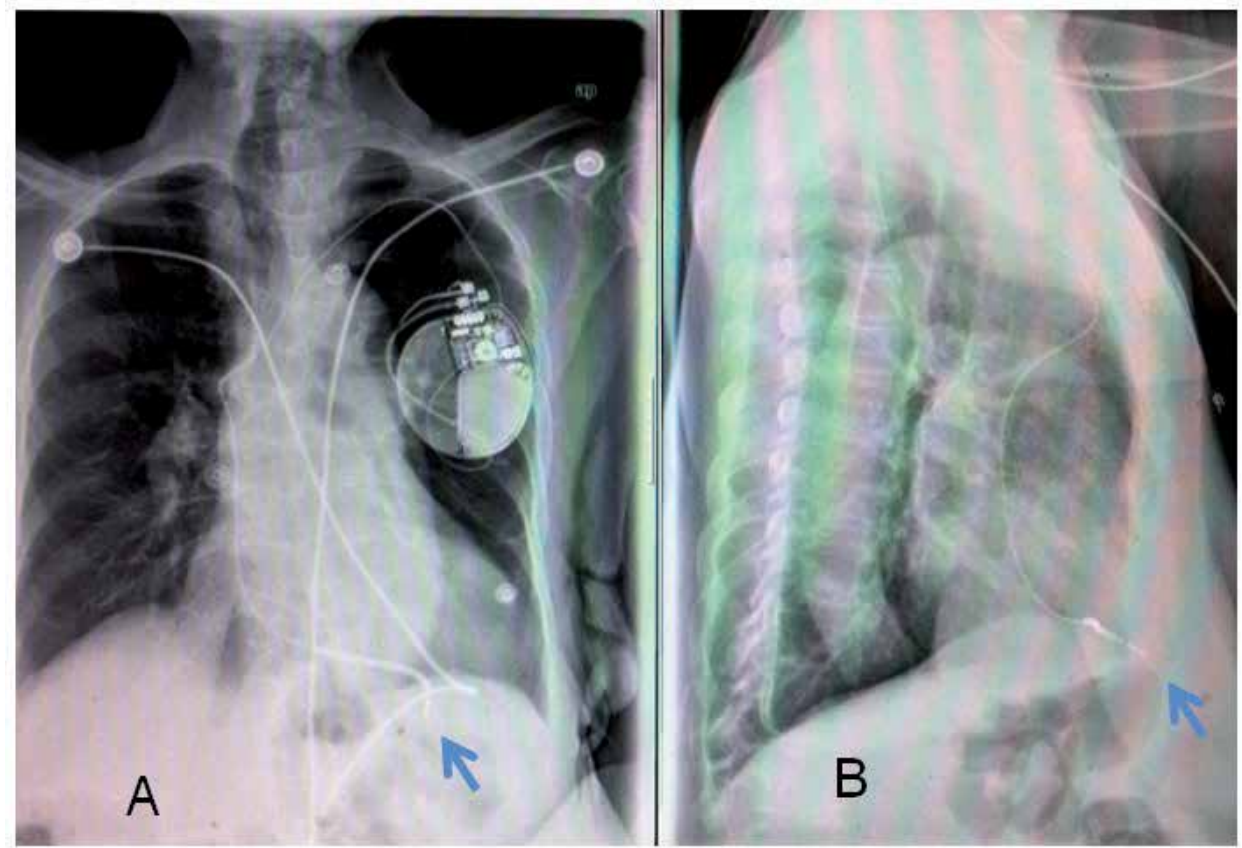

Figure 4. RV lead (arrows) perforating through the RV and sharply pointing below the diaphragm in PA view (A) and the lead is excessively close to the sternum and gastric air under the left hemidiaphragm in lateral view (B).

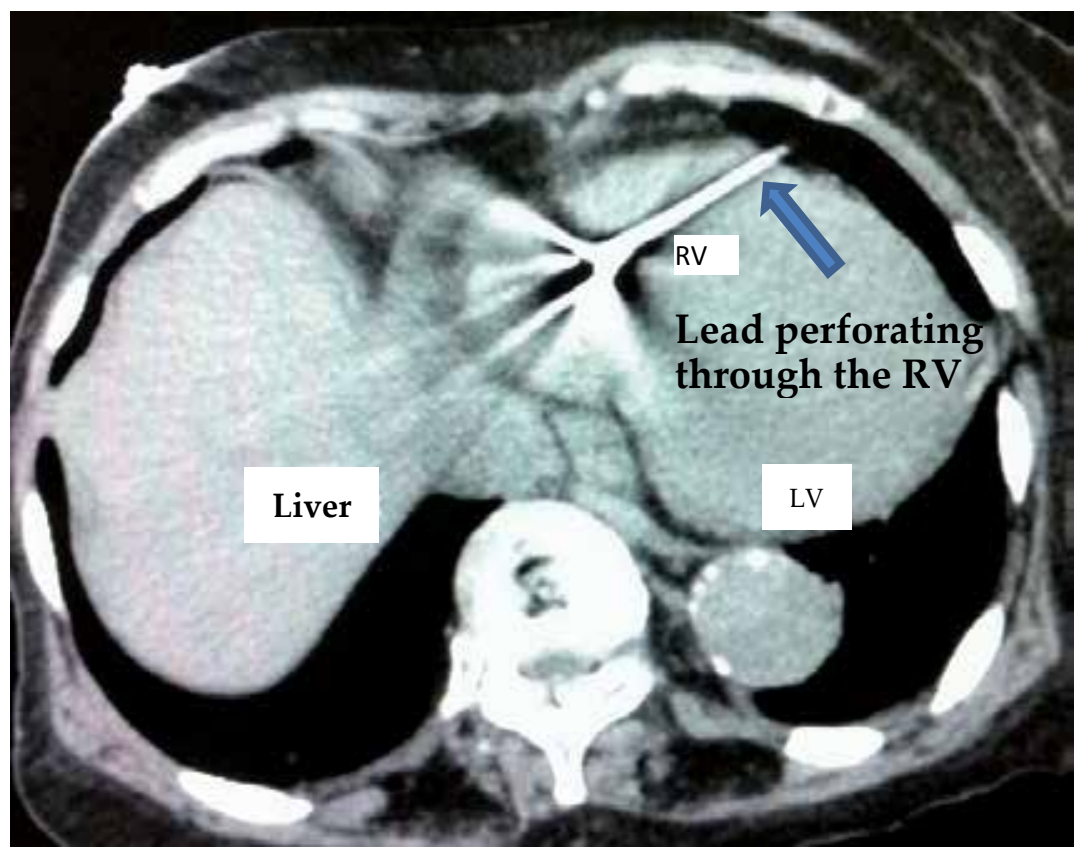

Figure 5. Axial view of CT scan of chest of the same patient in figure 4, showing the RV lead perforation through the RV apex. $R V=$ right ventricle, $L V=l$ eft ventricle 


\subsubsection{Migration to the LV cavity}

As a consequence of the thicker IVS, direct perforation through the septum and migration of lead tip into the left ventricle (LV) is rare [12]. However, the RV PPM lead may end up in LV due to mal-positioning during implantation. A common route for the lead to access the LV is a patent foramen ovale [13] or atrial septal defect [14]. These patients may remain asymptomatic for a long time, and often lead malposition is detected incidentally. It can be detected by fluoroscopy or chest radiography, presence of right bundle branch block (RBBB) with pacing on electrocardiogram (ECG) or when the patient presents with symptoms of a peripheral embolism (e.g. cerebrovascular accident) [14]. LV epicardial pacing may result when the electrode perforates through the RV apex migrating and remaining along the LV epicardial surface [15].

\subsection{RA perforation}

The RA is more susceptible to perforation as compared to the RV, attributable to the thinner wall of the atrium [4]. Thicker electrodes and active fixation leads are more likely to cause this form of perforation. Most commonly, one observes RA appendageal perforation. Age, gender, device type and anticoagulation status appear to increase the risk for perforation [16]. The lead may traverse through the pericardium into the lung field. Although, surgical intervention may be needed most cases are treated with percutaneous drainage or simple lead repositioning.

\subsection{Coronary Sinus (CS) dissection/perforation}

CS perforation is a relatively rare complication during pacemaker implantation. The rate of CS perforation has seen an upturn with increased CS cannulation during LV lead insertion for cardiac resynchronization therapy (CRT). The incidence of CS dissection and CS perforation have been reported to vary from $2 \%-4 \%, 0.3 \%-2 \%$, respectively [17]. Cardiac tamponade and pericarditis have been reported with a CS perforation [18].

In the early experience with PPM implantation the CS was selected for the purpose of RA pacing, affording a more stable catheter position. With a high perforation risk, tamponade and thrombosis, complicating lead insertion with CS manipulation, the RA became the preferred site. Enhanced stability with the RA appendage as the preferred site occurred once active fixation leads became mainstream. Figure 6 shows a CS lead perforating through the body of coronary sinus. During CRT, LV pacing is achieved by placing an electrode via the os of the CS to one of its tributaries. This procedure may result in a CS dissection. The overwhelming majority of CS dissections are without incident, with the dissection flap sealed by blood flow within the CS. Even if contrast extravasates out of the CS, the low perfusion pressure within the CS prevents any significant bleeding. In the majority of cases the true lumen of the CS can be re-accessed. The lead serves to tamponade the dissection or perforation and the procedure can proceed with successful lead placement. Serial echocardiograms are needed to assure any significant pericardial blood accumulation. If 
significant blood accumulation occurs within the pericardium this can be relieved via a percutaneous approach, very rarely patients require surgical drainage.

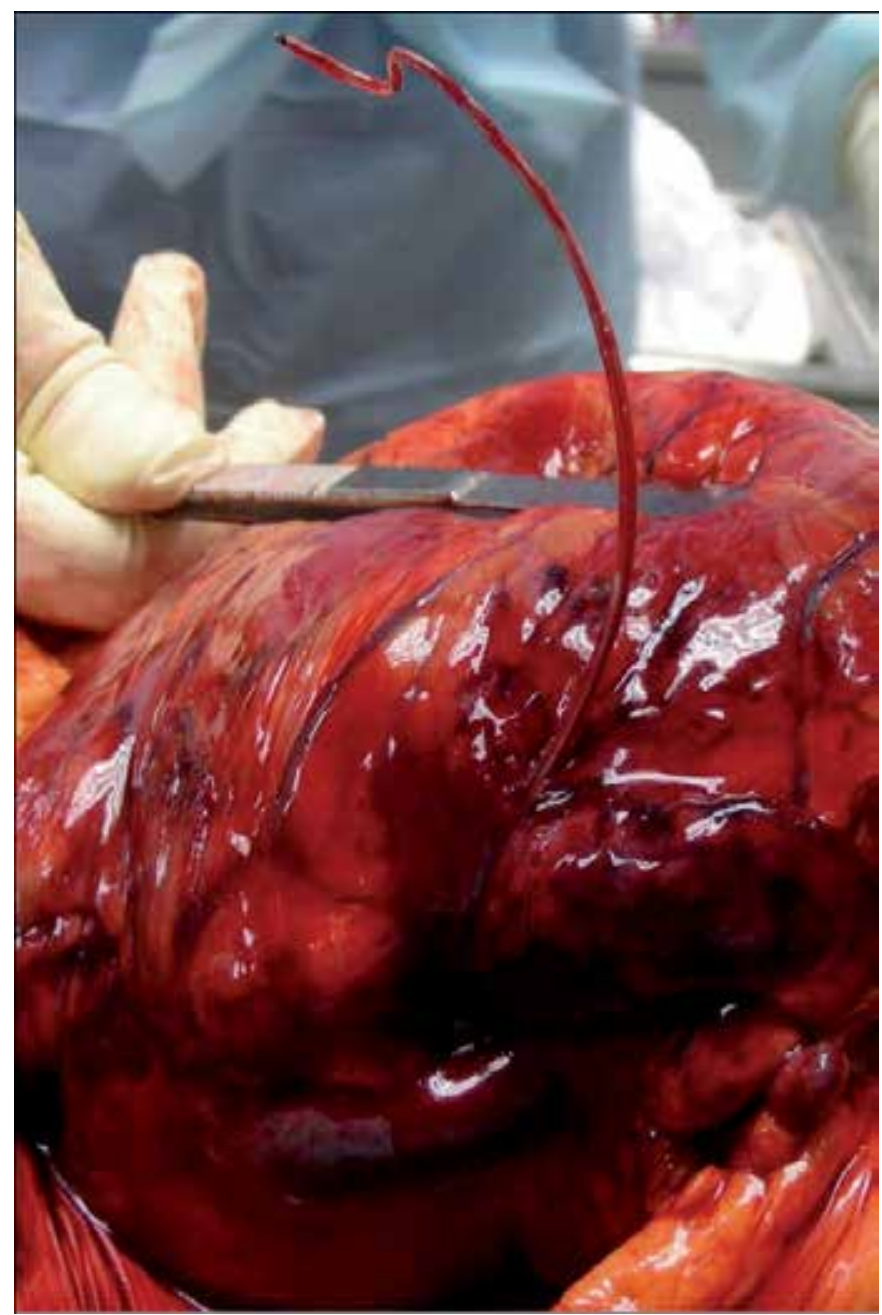

Figure 6. CS lead is perforating through coronary sinus as seen during autopsy. Patient was asymptomatic and died for unrelated reason. Courtesy of Jenny Libien, MD. Department of Pathology, SUNY Downstate. Brooklyn, NY.

\subsection{Great vessel injury/dissection}

Transvenous leads can be placed through various venous access sites, including, axillary, subclavian or cephalic veins. Innominate or internal jugular veins have been used as well. Lead placement via persistent left sided superior vena cava has been reported [19] and in such cases implantation could be challenging. These electrodes are designed to traverse the circulation from the point of vessel entry proximal to the generator to the targeted cardiac chamber. As one can imagine, injury can theoretical occur anywhere through this entire route. 
Commonly, venous injury, hematoma or even inadvertent arterial puncture can occur during the time of implantation and usually respond to compression. Perforation of the innominate vein has been reported and is attributed to its curvaceous course. This tortuosity increases with age, with a higher predisposition in the elderly [20]. The use of the internal jugular vein was associated with complications in 3 out of 92 implantations, including one patient who suffered permanent recurrent laryngeal nerve injury and two with thrombophlebitis. [21]

\section{Diagnostic approach}

\subsection{Presentation and symptoms}

Manifestations of a cardiac perforation depend largely on the site, presence and rate of accumulation of pericardial fluid. Approximately $1 \%-7 \%$ of patients present acutely; $1 \%$ present early and $0.1 \%$ present as a late perforation. [2] Acute perforations often present with chest pain, dyspnea and signs of pericardial effusion or tamponade. Asymptomatic perforations are not infrequent and as a result the diagnosis is often delayed. The most common presentation of a delayed perforation is a hemopericardium (with or without cardiac tamponade), pericarditis, diaphragm or chest wall muscle stimulation, loss of capture and pneumothorax [22]. Penetration to the superior mediastinum may result in largyngeal nerve injury. Rarely patient's may present with a mediastinal bleed.

The presentation of patients with cardiac perforation can be variable; therefore, a high index of suspicion needs to be maintained for a rapid diagnosis. Patients present with chest pain, shortness of breath, presyncope or syncope. The physical examination may reveal signs of cardiac tamponade (hypotension, tachycardia, elevated jugular venous pressure, distant heart sounds, pulsus paradoxus etc.), friction rub, ascites or leg edema. In addition, signs of pneumothorax or pleural effusions may be noted. On ECG, a new RBBB may be noted, suggesting insertion or migration of the lead into the LV cavity, occasional penetration into the epicardium.

\subsection{Analysis of interrogation data}

Upon suspicion of a perforation the device should be interrogated immediately. Routine device checks are performed to ensure normal pacing and or sensing function. A thorough device evaluation can be helpful in increasing one's suspicion of lead migration. In many instances one will detect an alteration in pacing function. New onset diaphragmatic, pectoralis or intercostal muscle stimulation with intermittent or constant failure to sense and capture should increase ones suspicion of a lead migration and possible perforation. However, one should keep in mind that a normal interrogation does not exclude the presence of a cardiac perforation.

\subsection{Use of remote monitoring for the early detection of a perforation}

Most of the current defibrillators and some PPMs have the capacity for home monitoring. They routinely transmit data regarding rhythm and system integrity at set time intervals. In 
the correct setting, abnormalities in the transmitted data should increase one's level of supposition for a perforation. A perforation often leads to a change in impedance and/or pacing threshold with a loss of capture. Several published reports have shown that early detection of a perforation was made via home monitoring. The perforations were detected 12 days and 4 weeks, respectively, following the insertion of the device [20,21]. With growing support for the use of home monitoring the number of cases diagnosed via this modality is expected to grow.

\subsection{Imaging studies}

Multi-detector computed tomography (MDCT) is the imaging modality of choice in diagnosing a lead perforation, identifying the pericardial effusion while, assisting in the therapeutic approach. However, a simple chest x-ray (preferably postero-anterior and lateral view) should be the starting point in the diagnosis of a cardiac perforation. An enlarged cardiac silhouette, pneumothorax, pleural effusion can also be supportive in making the diagnosis. Usually the lead tip should be within $3 \mathrm{~mm}$ of the cardiac border; if the tip extends outside the cardiac border, a perforation should be suspected. Physicians should familiarize themselves with the expected position of the RA or RV leads. In this regard, a baseline $x$-ray is useful. If a lead deviates significantly from its original or expected position or has developed an unusual contour, one should suspect perforation. Figure 7 shows the normal cardiac anatomy with respect to pacing leads. On a frontal radiograph, the tip of the right ventricular lead should be seen to the left of the spine. On the lateral view, it should traverse anteriorly towards the RV [23]. However, in some centers leads are preferentially positioned in RV outflow tract (free wall or septal aspect). In these cases a left lateral view (45 degree) x-ray would display the RV lead to be pointing to the left in the case of free wall and to the right in case of septal positioning of the lead. Similarly, a left lateral x-ray would show the RV lead projecting anteriorly in case of free wall or posteriorly in case of septal positioning of the lead. [24]

Fluoroscopy plays an important role during positioning of the leads. In this regard the implanter needs to have a thorough understanding of flouroscopic anatomy, in order to confirm proper positioning and detect a mal-positioned lead. In the case of a perforation, the lead electrodes can extend outside of the cardiac silhouette and not move with the contraction of the heart [22]. Further clues to the early detection of a cardiac perforation include: an unexplained decrease in blood pressure; a decreased pulsatility of the cardiac silhouette; an increase in the size of the cardiac silhouette; and, an abnormal position of lead relative to pericardial outline [23].

Echocardiography is widely available and easily performed to detect a cardiac perforation. Echocardiography can detect pericardial fluid, cardiac tamponade, malposition as well as extension through the myocardium but rarely is able to identify the extra-cardiac presence of the lead. [25] On occasion the perforation may not cause any detectable pericardial effusion or there may be poor data acquisition, hence the absence of an effusion does not rule out a cardiac perforation. 


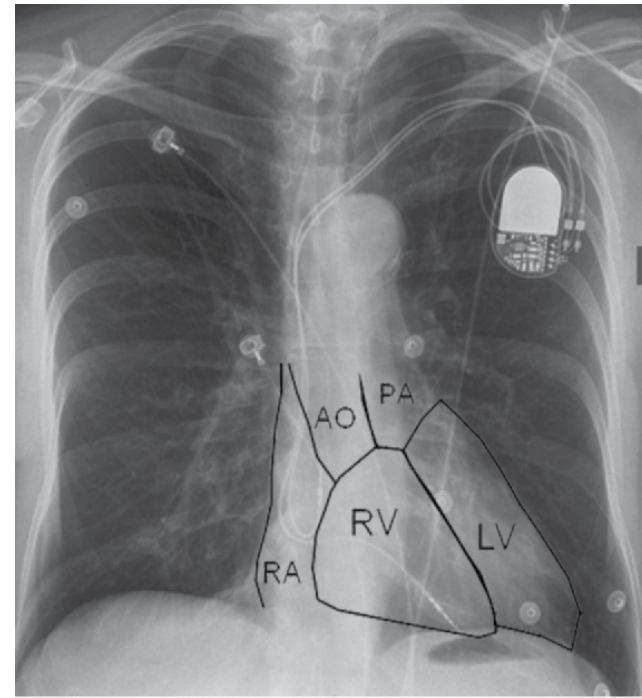

a.

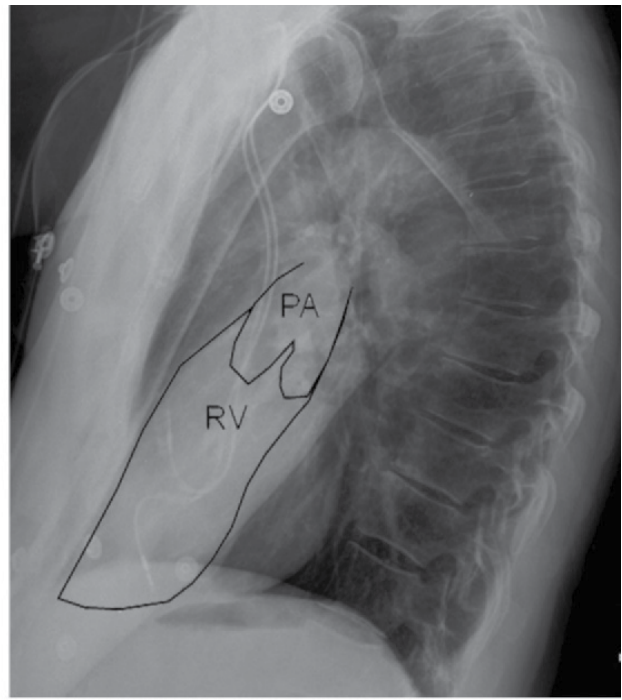

b.

Figure 7. Normal cardiac anatomy. Frontal (a) and lateral (b) chest radiographs show the aorta (AO in a), left ventricle (LV in a), main pulmonary artery $(P A)$, right atrium $(R A$ in a), and right ventricle $(R V)$. “(With permission from Aguilera AL: Radiographics. 31:1669-1682,2011.)

\section{Lead design and propensity for perforation}

\subsection{Active vs. passive fixation leads}

The type of lead fixation mechanism contributes to the risk of perforation. Studies have shown that active fixation (screw-in) leads are associated with a higher risk of perforation, related to lead thickness and over-torquing. It has been speculated that the newer, thinner leads, exert a higher force per unit area on the endocardium, and exhibit a higher incidence of delayed perforations [25]. In addition, the tip force and the myocardial counterforce are variable, changing over time and with each cardiac cycle. When the lead tip force exceeds the counterforce a perforation can occur. On the other hand, passive fixation lead placed in vulnerable region of the RV (apex) may exhibit an even higher risk of perforation compared to the active fixation leads. It is speculated that successful insertion of a passive fixation lead uses the RV apex, whose trabeculations are used to anchor the lead; however, this represents the most vulnerable zone within the chamber. The higher incidence of perforation associated with active fixation leads may be attributed to the mere fact that we are actively "screwing" the lead into the RV wall, making sure we have adequate tissue contact by monitoring the current of injury.

\subsection{Defibrillation lead size and risk of perforation}

Recent studies have reported a surprisingly high incidence of cardiac perforation with small body (7F) active fixation defibrillator leads (Riata) as compared to Sprint Fidelis leads (3.8\% vs. $0 \%$ ) [23]. The reasons for higher perforation rate are largely unknown but may include 
lead design and breeches in structural integrity [26]. Another important property in assessing the propensity of a lead to perforate is "thickness". Older manufactured leads were thicker than the newer models. Thicker leads are stiffer and exert a higher pressure on the cardiac tissue thus associated with higher cardiac perforations.

\subsection{Preformed J shape lead design and risk for retained wire perforation}

One of the first and most serious lead recalls involved the Accufix atrial pacing lead which was recalled due to a high risk of J retention wire fracture. The J wire fracture protruded with time through the insulation resulting in a high rate of atrial perforation [27], (Figure 8). Many of these leads needed to be extracted and were associated with a significant morbidity and mortality, due to the friability of the lead. Temporary atrial and ventricular epicardial pacing wires are routinely placed after cardiac surgery. These wires are routinely removed from patients prior to discharge. They are pulled and cut at skin level and allowed to retract into the pericardial sac. These wires can migrate to the RV extend into the pulmonary artery [28] or migrate to the left atrium and subsequently to the carotid arteries [29].

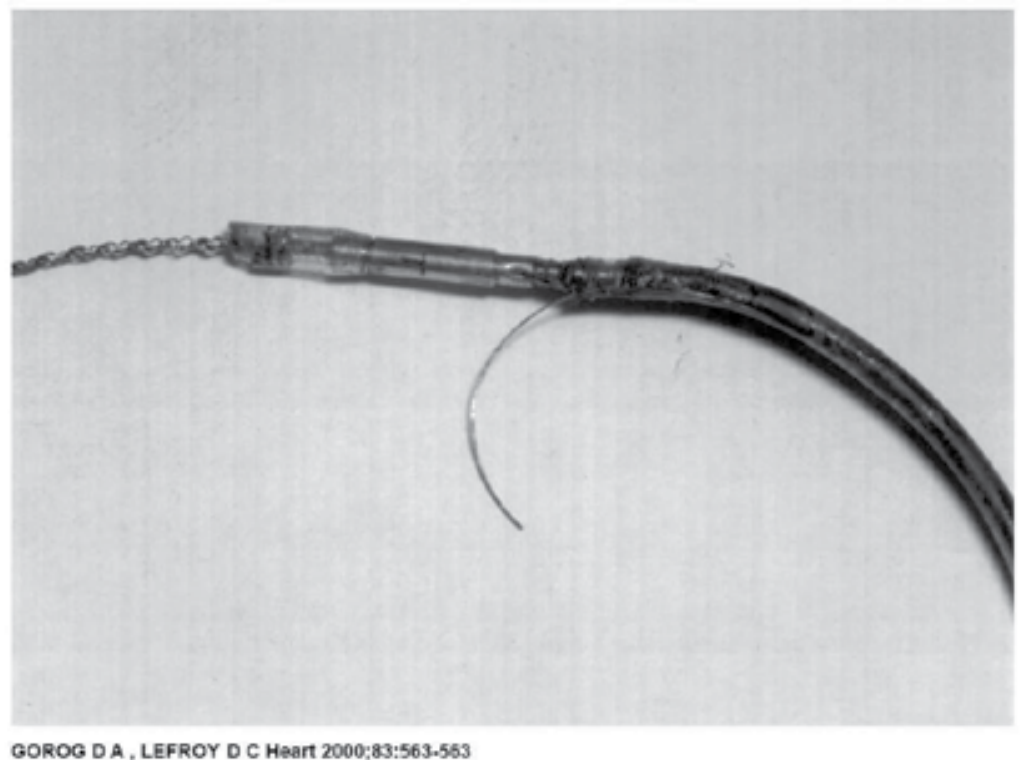

Figure 8. J-retention wire protruding through the insulation. (With Permission from Gorog DA: Heart. 83:563-563, 2000. )

\section{Patient risk factors and risk for perforation}

Cardiac perforation may be associated with intrinsic myocardial, as well as, patient-specific factors rendering this, rather uncommon complication more predictable. Mahapatra et al [5] have investigated the risk factors for the development of symptomatic pericardial effusion consistent with cardiac perforation in more than 4,000 patients. The multivariate analysis identified variables associated with perforation included: steroid use (HR 3.2); temporary 
pacemaker wire insertion (HR 2.7); active-fixation leads (HR 2.5); low body-mass index (HR 2.5); advancing age (HR 1.4); and, longer fluoroscopy times (HR 1.3). Predictors with a lower perforation potential included, a pulmonary artery pressure $>35 \mathrm{~mm} \mathrm{Hg}$ (probable protective effect of hypertrophied RV) and BMI $>30$ (univariate predictor only). Interestingly, steroid use is associated with cardiac atrophy, mediated by a muscle specific protein called muscle ring finger-1 [30].

The specific lead type, site of implantation, and the occurrence of traumatizing factors may result in a higher incidence of RV perforation. Although factors, such as defibrillator leads, excessive slack during lead implantation, smaller diameter leads with higher resistance, chest wall trauma especially soon after implantation and multiple shocks delivered around the time of implantation are yet to be validated, they remain important considerations when one considers the predisposition to perforation. It has previously been suggested that RV free wall and/or apical insertions, especially in the elderly are associated with higher perforation rate attributed to the thinner wall in that region, which atrophies with age. In addition, the use of anticoagulants may increase the risk of perforation.

\section{Implantation techniques to minimize the risk of perforation}

Certain steps should be taken to reduce the incidence of lead related cardiac perforation. It is imperative that in obtaining an informed consent, patients should be made aware of the symptoms to watch for following a surgery for a more rapid recognition of complications. Only experienced implanters with highly trained personnel, in dedicated electrophysiology laboratories, should perform these procedures.

Careful patient selection and avoidance of temporary pacing wires would be helpful in reducing this complication. In addition, selection of more compliant (less stiff) leads, wider lead tip area and placement in the RV septum can further reduce this risk. It has also been suggested that excessive slack on the lead after placement can increase the tension on the free wall resulting in a late perforation. Post-procedure, a high index of suspicion needs to be maintained; while the clinical examination, routine chest x-ray, ECG and pacemaker interrogation including remote monitoring can help expedite the diagnosis.

\section{Management of lead perforation}

The management of cardiac perforation depends on the timing of this complication. Acutely, the pacing lead can be repositioned and the patient is monitored with serial echocardiography. The risk of bleeding during removal of a lead increases rapidly in non-acute perforations. Subacute or delayed perforation is dealt with on a case by case basis. In some cases, when the cardiac perforation is asymptomatic or not associated with pacing/sensing malfunction or mediastinal bleeding or if the risk of lead removal outweighs the non-removal, the lead can be left in place. Patients can be observed and watched for lead migration and at that point lead extraction could be performed. [16, 30,31] Simple lead positioning can be utilized in some cases especially in acute or early stages when minimal fibrotic adhesions facilitate repositioning. [32] If there is bleeding within or outside of the mediastinum, accompanied by 
lung or other vascular damage, especially if the lead was recently implanted, the lead should be explanted surgically to insure adequate surgical correction of the problem.

\subsection{Non-thoracotomy approach}

Active Fixation leads can be removed transvenously under direct fluoroscopic visualization. However, an Expert Consensus Statement published by the Heart Rhythm Society [33] recently classifies following conditions as class III indication for transvenous extraction of nonfunctional leads. They cited the following in their decision: lead removal is not indicated in patients with a known anomalous placement of leads through structures other than normal venous and cardiac structures, (e.g. subclavian artery, aorta, pleura, atrial or ventricular wall or mediastinum) or through a systemic venous atrium or systemic ventricle. Additional techniques including surgical backup should be deployed in the appropriate clinical scenario. Simple perforations can be managed with a percutaneous approach. In one series of 11 patients with an RV perforation, in 10 patients, the leads were removed by simple traction under fluoroscopic guidance in the operating room with surgical backup support. In one patient, surgical intervention was required after a pericardiocentesis, underscoring the need for close monitoring [34]. In the analysis of 33 consecutive patients who developed cardiac perforation with associated with tamponade, within 24 hours of procedure (electrophysiology or device implant), nineteen (58\%) patients were managed conservatively with intravenous fluid and pressor support. Fourteen $(42 \%)$ required a pericardiocentesis. The authors suggested that in the setting of acute tamponade associated with a large pericardial effusion (> $2 \mathrm{~cm}$ ), associated with RV diastolic collapse, pericardiocentesis would likely be needed. As a less invasive measure, video assisted thoracoscopy surgery (VATS) with adjuvant anterior minimally invasive anterior thoracotomy has also been used [35].

\subsection{Thoracotomy approach}

A surgical approach is recommended when cardiac tamponade is the presenting feature, complicating a lead extraction or an anomalous approach to lead implantation is present. Passive fixation leads are bulky and have tines potentially worsening damage if the leads are "pulled" transvenously.

During the procedure the lead tip is freed up from the tissue, and then the body of the lead is removed transvenously. Surgeons can also place epicardial leads as an alternative approach. It is recommended that pericardium be cleansed after extracting the contents of the hemopericardium to reduce the risk of future constrictive pericarditis [36). A surgical approach results in an increased hospital stay and its concomitant risks. In this regard the use of VATS with adjuvant anterior minimally invasive anterior thoracotomy appears promising in reducing the hospital stay and its associated morbidities.

\section{Summary}

The overall incidence of a cardiac perforation as a result of a transvenous lead insertion is low. Perforations can exhibit a range of manifestations, from asymptomatic to life-threatening. In 
order to minimize complications, temporary pacing lead placement should be avoided unless absolutely necessary. Extreme caution should be used when implanting a device in patients who are on steroids or anticoagulants. Once perforation is detected the management depends on the duration of implant (acute vs. chronic), accompanying findings (tamponade or pneumothorax) and the type of lead. In the case of an acute perforation, leads can be repositioned under fluoroscopy. Hemopericardium with tamponade can be managed via percutaneous pericardiocentesis and on occasion may require surgical drainage.

\section{Abbreviations}

$\mathrm{BMI}=$ body mass index

$\mathrm{CRT}=$ cardiac resynchronization therapy

$\mathrm{CS}=$ coronary sinus

$\mathrm{CT}=$ computerized tomography

$\mathrm{ECG}=$ electrocardiogram

FDA $=$ federal drug administration

IVS= interventricular septum

$\mathrm{HR}=$ hazard risk

$\mathrm{LV}=$ left ventricle

PPM or ppms= pacemaker or pacemakers

$\mathrm{RA}=$ right atrium

$\mathrm{RBBB}=$ right bundle branch block

$\mathrm{RV}=$ right ventricle

VATS= video assisted thoracoscopy surgery

\section{Author details}

Sanjay Kumar, Haidar Yassin, Opesanmi Esan, Adam S. Budzikowski and John T. Kassotis* Division of Cardiovascular Medicine, Clinical Cardiac Electrophysiology Section, SUNY Downstate, Brooklyn, NY, USA

\section{References}

[1] Barold SS, Center S. Electrographic diagnosis of perforation of the heart by pacing catheter electrode. Am J Cardiol. 1969 Aug;24 (2):274-8.

[2] Ellenbogen KA, Wood MA, Shepard RK. Delayed complications following pacemaker implantation. Pacing Clin Electrophysiol. 2002 Aug;25 (8):1155-8.

[3] Sivakumaran S, Irwin ME, Gulamhusein SS, Senaratne MP. Postpacemaker implant pericarditis: incidence and outcomes with active-fixation leads. Pacing Clin Electrophysiol. 2002 May;25 (5):833-7.

[4] Hirschl DA, Jain VR, Spindola-Franco H, Gross JN, Haramati LB. Prevalence and characterization of asymptomatic pacemaker and ICD lead perforation on CT. Pacing Clin Electrophysiol. 2007 Jan;30 (1):28-32.

" Corresponding Author 
[5] Mahapatra S, Bybee KA, Bunch TJ, Espinosa RE, Sinak LJ, McGoon MD, et al. Incidence and predictors of cardiac perforation after permanent pacemaker placement. Heart Rhythm. 2005 Sep;2 (9):907-11.

[6] Alla VM, Reddy YM, Abide W, Hee T, Hunter C. Delayed lead perforation: can we ever let the guard down? Cardiol Res Pract. 2010;2010.

[7] Cameron J, Mond H, Ciddor G, Harper K, McKie J. Stiffness of the distal tip of bipolar pacing leads. Pacing Clin Electrophysiol. 1990 Dec;13 (12 Pt 2):1915-20.

[8] Gasser TC, Gudmundson P, Dohr G. Failure mechanisms of ventricular tissue due to deep penetration. J Biomech. 2009 Mar 26;42 (5):626-33.

[9] Forsell C, Gasser TC. Numerical simulation of the failure of ventricular tissue due to deep penetration: the impact of constitutive properties. J Biomech. 2011 Jan 4;44 (1):45-51.

[10] Aliyev F, Celiker C, Turkoglu C, Karadag B, Yildiz A. Perforations of right heart chambers associated with electrophysiology catheters and temporary transvenous pacing leads. Turk Kardiyol Dern Ars. 2011 Jan;39 (1):16-22.

[11] Lang RM, Bierig M, Devereux RB, Flachskampf FA, Foster E, Pellikka PA, et al. Recommendations for chamber quantification: a report from the American Society of Echocardiography's Guidelines and Standards Committee and the Chamber Quantification Writing Group, developed in conjunction with the European Association of Echocardiography, a branch of the European Society of Cardiology. J Am Soc Echocardiogr. 2005 Dec;18 (12):1440-63.

[12] James $M$, Townsend $M$, Aldington S. An unusual complication of transvenous temporary pacing. Heart. 2003 Apr;89 (4):448.

[13] Zaher MF, Azab BN, Bogin MB, Bekheit SG. Inadvertent malposition of a permanent pacemaker ventricular lead into the left ventricle which was initially missed and diagnosed two years later: a case report. J Med Case Reports. 2011;5 (1):54.

[14] Raghavan C, Cashion WR, Jr., Spencer WH, 3rd. Malposition of transvenous pacing lead in the left ventricle. Clin Cardiol. 1996 Apr;19 (4):335-8.

[15] Meyer JA, Millar K. Perforation of the right ventricle by electrode catheters: a review and report of nine cases. Ann Surg. 1968 Dec;168 (6):1048-60.

[16] Polin GM, Zado E, Nayak H, Cooper JM, Russo AM, Dixit S, et al. Proper management of pericardial tamponade as a late complication of implantable cardiac device placement. Am J Cardiol. 2006 Jul 15;98 (2):223-5.

[17] Azizi M, Castel MA, Behrens S, Rodiger W, Nagele H. Experience with coronary sinus lead implantations for cardiac resynchronization therapy in 244 patients. Herzschrittmacherther Elektrophysiol. 2006 Mar;17 (1):13-8.

[18] Kitamura K, Jorgensen CR, From AH. Transvenous atrial and ventricular pacing from the coronary sinus complicated by perforation and cardiac tamponade. Chest. 1971 Jul;60 (1):95-8.

[19] Innasimuthu AL, Rao GK, Wong P. Persistent left-sided superior vena cava--a pacing challenge. Acute Card Care. 2007;9 (4):252.

[20] Igawa O, Adachi M, Yano A, Miake J, Inoue Y, Ogura K, et al. Brachiocephalic vein perforation on three-dimensional computed tomography. Europace. 2007 Jan;9 (1):74-5.

[21] Brodman R, Furman S. Pacemaker implantation through the internal jugular vein. Ann Thorac Surg. 1980 Jan;29 (1):63-5. 
[22] Krivan L, Kozak M, Vlasinova J, Sepsi M. Right ventricular perforation with an ICD defibrillation lead managed by surgical revision and epicardial leads--case reports. Pacing Clin Electrophysiol. 2008 Jan;31 (1):3-6.

[23] Aguilera AL, Volokhina YV, Fisher KL. Radiography of cardiac conduction devices: a comprehensive review. Radiographics. 2011 Oct;31 (6):1669-82.

[24] McGavigan AD, Roberts-Thomson KC, Hillock RJ, Stevenson IH, Mond HG. Right ventricular outflow tract pacing: radiographic and electrocardiographic correlates of lead position. Pacing Clin Electrophysiol. 2006 Oct;29 (10):1063-8.

[25] Villanueva FS, Heinsimer JA, Burkman MH, Fananapazir L, Halvorsen RA, Jr., Chen JT. Echocardiographic detection of perforation of the cardiac ventricular septum by a permanent pacemaker lead. Am J Cardiol. 1987 Feb 1;59 (4):370-1.

[26] Hauser RG, McGriff D, Retel LK. Riata implantable cardioverter-defibrillator lead failure: Analysis of explanted leads with a unique insulation defect. Heart Rhythm. 2011 Dec 28.

[27] Kay GN, Brinker JA, Kawanishi DT, Love CJ, Lloyd MA, Reeves RC, et al. Risks of spontaneous injury and extraction of an active fixation pacemaker lead: report of the Accufix Multicenter Clinical Study and Worldwide Registry. Circulation. 1999 Dec 7;100 (23):2344-52.

[28] Worth PJ, Conklin P, Prince E, Singh AK. Migration of retained right ventricular epicardial pacing wire into the pulmonary artery: a rare complication after heart surgery. J Thorac Cardiovasc Surg. 2011 Sep;142 (3):e136-8.

[29] Juchem G, Golczyk K, Kopf C, Reichart B, Lamm P. Bizarre case of migration of a retained epicardial pacing wire. Europace. 2008 Nov;10 (11):1348-9.

[30] Henrikson CA, Leng CT, Yuh DD, Brinker JA. Computed tomography to assess possible cardiac lead perforation. Pacing Clin Electrophysiol. 2006 May;29 (5):509-11.

[31] Sadamatsu K. Complication of Pacemaker Implantation: An Atrial Lead Perforation. www.intechopen.com/download/pdf/pdfs_id/13788

[32] Refaat MM, Hashash JG, Shalaby AA. Late perforation by cardiac implantable electronic device leads: clinical presentation, diagnostic clues, and management. Clin Cardiol. 2010 Aug;33 (8):466-75.

[33] Wilkoff BL, Love CJ, Byrd CL, Bongiorni MG, Carrillo RG, Crossley GH, 3rd, et al. Transvenous lead extraction: Heart Rhythm Society expert consensus on facilities, training, indications, and patient management: this document was endorsed by the American Heart Association (AHA). Heart Rhythm. 2009 Jul;6 (7):1085-104.

[34] Laborderie J, Barandon L, Ploux S, Deplagne A, Mokrani B, Reuter S, et al. Management of subacute and delayed right ventricular perforation with a pacing or an implantable cardioverter-defibrillator lead. Am J Cardiol. 2008 Nov 15;102 (10):1352-5.

[35] Welch AR, A.R., Yadav, P., Lingle, K. Subacute Right Ventricular Pacemaker Lead Perforation: Often talked about in consent forms but very rarely seen. The Journal of Innovations in Cardiac Rhythm Management. 2011;2:442-5.

[36] Saksena S. Defibrillation thresholds and perioperative mortality associated with endocardial and epicardial defibrillation lead systems. The PCD investigators and participating institutions. Pacing Clin Electrophysiol. 1993 Jan;16 (1 Pt 2):202-7. 


\title{
Intravascular Lead Extractions: Tips and Tricks
}

\author{
Spyridon Koulouris and Sofia Metaxa
}

Additional information is available at the end of the chapter

http://dx.doi.org/10.5772/48496

\section{Introduction}

The transvenous insertion of implantable pacemaker (PM) and implantable cardioverter defibrillator (ICD) leads was a major milestone in antiarrhythmic therapy with the use of cardiac devices. Indeed, based on data published over the last decade the indications for ICD therapy have further expanded [1,2] while cardiac resynchronization therapy (CRT) through bi-ventricular pacing has significantly improved mortality and quality of life in patients with heart failure and ventricular dyssynchrony [3,4,5]. Unfortunately, this exponential increase in the implantation rate of cardiac devices has been accompanied by a parallel increase in the need for explanting some of those [6]. This has been mainly attributed to the so called "increased total lead exposure time" resulting from the expanding indications for device treatments, the implantation of more leads per patient and the longer average life expectancy of device-recipients [7]. Lead removal has been performed only in limited centers from physicians with some expertise in this subject. The volume of procedures in these centers has also been increasing in a continuous manner and the techniques applied have become more and more sophisticated and effective. Indeed, the options for lead extraction were initially very limited and dedicated tools were not available. Life threatening situations such as infection with sepsis were the only reason to attempt a lead removal with these highly morbid and often ineffective techniques [8]. As a necessity to overcome these limitations a significant evolution in lead extraction technology occurred over the past 30 years. More simple, safe and efficacious techniques are nowadays widely used in clinical practice [9].

Dealing with a possible lead extraction, the main technical problems that have to be taken into consideration are: the endovascular reaction surrounding the intravenous lead, the physical characteristics of the lead affecting its removability and the lack of direct visualization along the intravascular route. Fibrotic scar tissue develops at areas of endothelial contact and engulfs the leads. This process begins with thrombus development along the lead at the time of implantation. Fibrosis of the thrombus occurs next resulting in 
almost complete encapsulation of the lead with a fibrin sheath within 4-5 days post implant [10,11]. Calcification of the fibrous tissue may even occur over time especially in young patients [12]. The most common adhesion sites include the venous entry site, the superior vena cava and the electrode-endocardial interface [13]. (Figure 1). In the majority of patients multiple areas of scar tissue are found. This scar resists against lead explantation and specific manipulations are needed to overcome this particular obstacle. In addition, lead to lead interaction and binding in the case of multiple leads as well as along each of the shocking coils of the ICD leads may happen, which may pose further limitations in the extraction procedure (Figure 2).

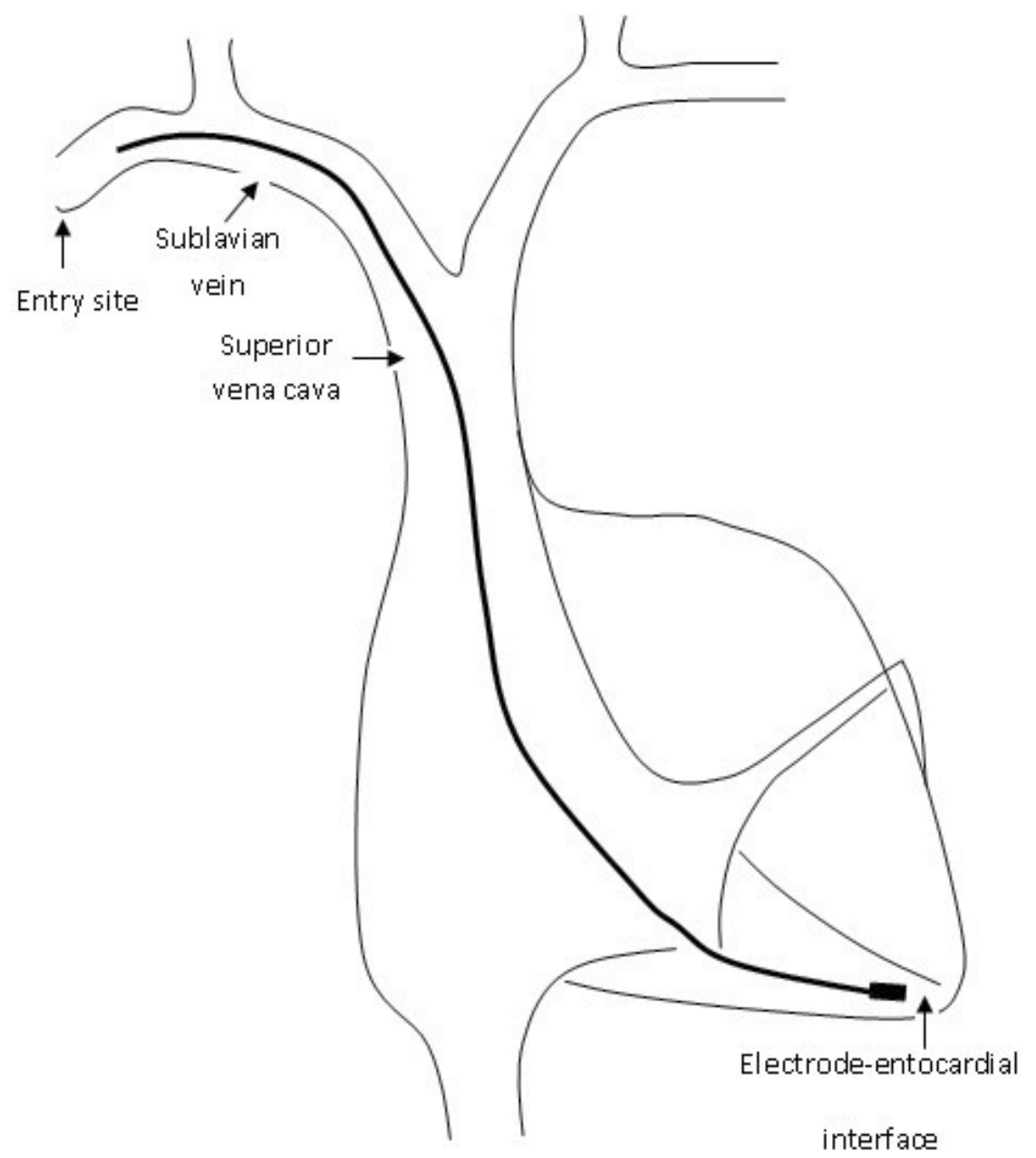

Figure 1. Location of areas of lead adherence.

On the other hand, the material and construction of the lead may promote or resist the development of scar tissue and may also largely affect the lead removability through its specific tensile strength characteristics. To combat the formation of fibrous connections, 
manufacturers have recently attempted to produce ICD coils coated with expanded polytetrafluoroethylene (ePTFE) or back-filled with medical adhesive (MABF). Both have been shown to be easier to extract due to decreased incidence of fibrosis on and around the filters of the coils [14]. Finally, the indirect control on the procedure from the operator due to the lack of direct visualization urges the outmost care and experience in order to avoid any major or even life-threatening consequences. Because lead extraction is not frequently performed, few high-volume centers can provide the best patient care along with opportunities for adequate physician training in this field. Both European and American Societies of Electrophysiology have set standards for training and accreditation in order to overcome these limitations. Generally, a minimum of 40 lead extractions as the primary operator is required to be considered fully trained, and 20 leads per year is needed to maintain competency $[6,15]$.

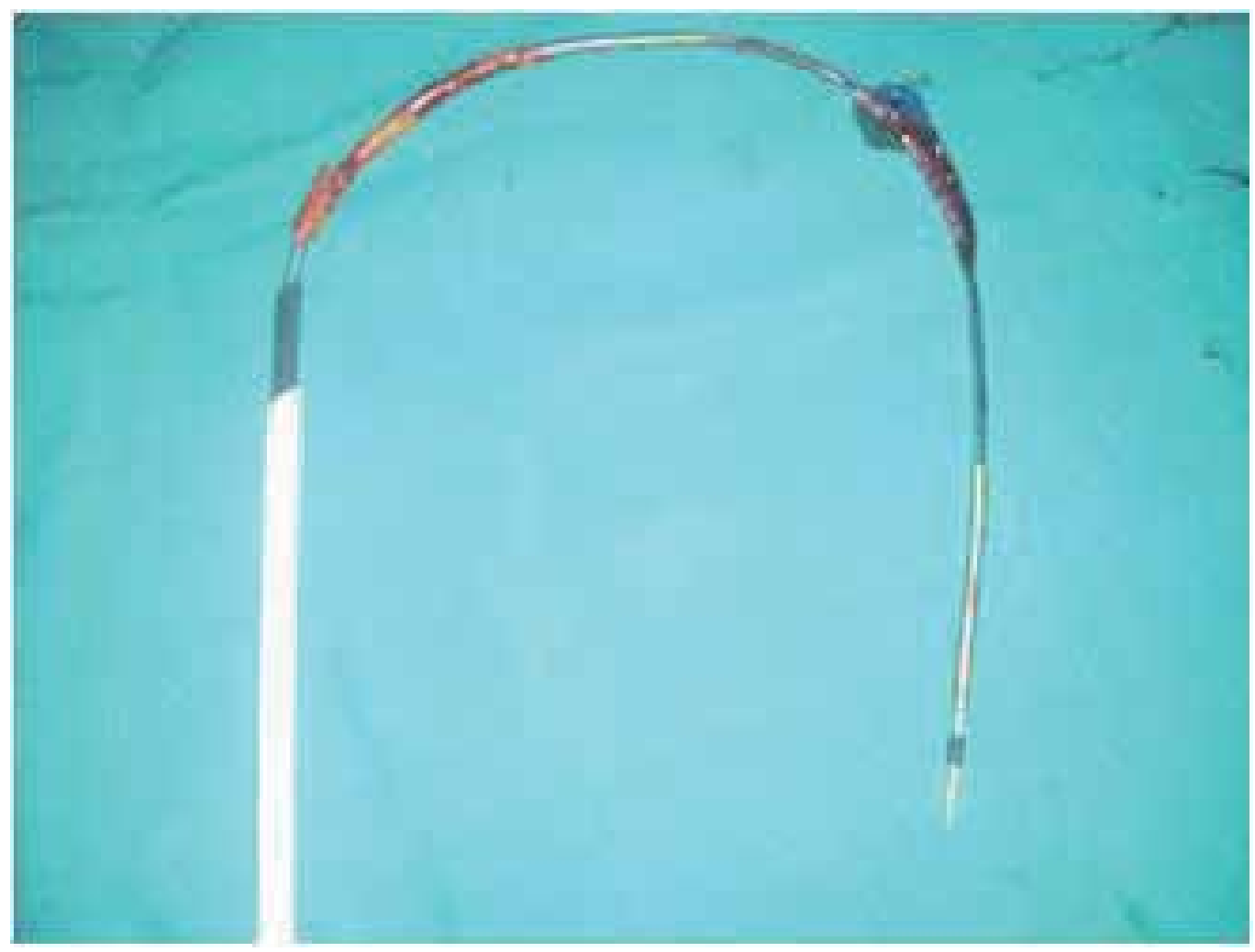

Figure 2. Extensive scarring over an extracted ICD lead.

\section{Indications for lead extraction}

Indications for device removal can be divided in two categories: infectious and non-infectious. Non-infectious indications include malfunctioning leads or leads which through their presence can cause harm to the patient (for example thrombosis of the superior vena), as well as leads that have to be removed in order to upgrade a device. In all published reports, 
infection seems to be the most common indication for lead extraction $(54-60 \%$ of all extraction procedures)[16,17].

Previous reports have indicated an overall rise in the rate of device infection which might have been attributed to the wider implantation of ICDs for primary prevention of sudden cardiac death in a population whose health status is by definition relatively poor (patients post myocardial infarction with low ejection fraction and clinical signs of heart failure). Another reason could have been the subsequent generator changes whose rate has been following the increasing rate of initial implantation. Both ICD implantation and generator replacement have been clearly associated with a higher rate of device infections $[18,19]$. On the other hand, others have more recently reported that referrals for extraction for infection and upgrade of the device have remained relatively stable in contrast to the incidence of lead failure which seems to have decreased over the last decade (Figure 3) [20].

\section{Changing relative indications for lead extractions over 7 years at a tertiary referral center}

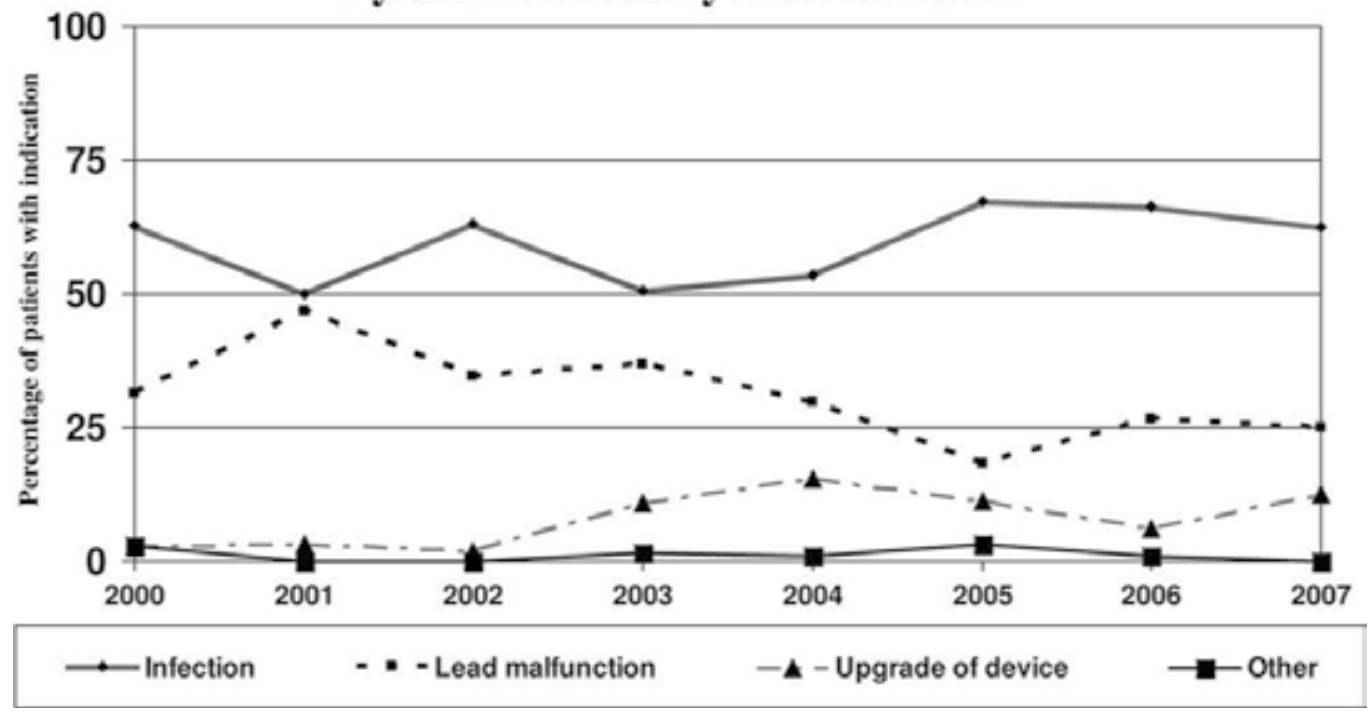

Figure 3. Indications for lead extraction in a cohort of 498 patients. There is a clear trend in the decreasing indication for lead removal due to malfunction. Referrals for extraction for infection and upgrade of the device have remained relatively stable over time (From: Jones SO, et al. Large, single-center, single-operator experience with transvenous lead extraction: Outcomes and changing indications. Heart Rhythm 2008:5;520-525, with permission) 
The indication to remove a lead in all the above circumstances is largely dependent on patient's age, general condition, the potential of future problems, the risk to extract and the potential harmful circumstances associated with the lead presence such as subclavian or superior vena cava thrombosis. However, infection of the device regardless of its presentation makes the removal of the whole system unavoidable. This has been shown to be the only effective way to totally resolve this potentially life-threatening health problem. Staphylococcal infections dominate the responsible flora. In a recent survey Methicillinsensitive S. aureus was found in 25\% Methicillin-resistant S. aureus was found in $34 \%$ and Coagulase-negative S. species were found in $14 \%$ of the cases of pacemaker endocarditis [21]. It should be noted that even if the infection is by clinical examination found to be confined to the pocket of the device, complete removal of the system including the leads has to be performed in order to avoid future relapse of the disease in the form of endocarditis. Studies have shown that leads not thought to be infected may in fact be heavily colonized by bacteria entering the systemic circulation at the pocket site [22,23]. Indeed, the majority of extraction procedures are currently performed for infections localized to the device pocket. Nevertheless, a more widespread infection is not uncommon. In a recent review of 189 patient admitted in a single tertiary center with device infection, pocket infection was present in $52 \%$ of them while $17 \%$ had evidence of pocket infection with blood stream infection and $23 \%$ had developed device-related endocarditis [24] Finally, even in patients presenting with an erosion of the pocket, as a consequence of infection or mechanical pressure or both, the system should be considered contaminated and has to be completely removed. Of note, adherence of the generator or leads to the skin often proceeds erosion and is an indication for extraction too (Figure 4) $[6,15]$. Nevertheless, a few authors may still advocate a conservative approach with debridement and chronic antibiotic administration in elderly, infirmary patients with a limited life expectancy [25].

Controversy continues to exist regarding the other indications for lead removal. The risk posed by abandoned leads is relatively low. Thus many physicians would recommend simply abandoning malfunctioning leads. The opposite is however true as well. In most of the series the attempts to remove abandoned leads has been associated with a relatively low risk of complications. Accordingly, in the hands of more experienced operators nonfunctioning leads may become a challenge for an extraction attempt [26]. Moreover, the risk of venous obstruction seems to increase proportionally to the number of leads and this has raised some additional concerns regarding the safety of leaving "orphan" leads in place. Accordingly, the decision of explanting a non-infected lead needs to be individualized in most of the cases. The cause of malfunction is insulation defect in the majority of pacemaker lead failures [27]. In the case of ICDs, high-voltage coil failures and disruption of the polyurethane inner insulators represent the most common reason that may lead to a lead replacement. Of note, ICD lead failure rate is not at all negligible. In a recent survey failure rate reached $20 \%$ in 10-year-old leads [28].

A comprehensive list of lead removal indications has been recently published in Heart Rhythm Society expert Consensus Statement (Table 1). Nevertheless, it is important to 
remember that selection of the patients for lead extraction should be done on an individual case-by-case basis taking always into account the patient's clinical picture and general health status, the lead characteristics and the operator's experience along with the availability of the specific facilities and tools. Only in the case that the risk of extraction is lower in comparison to the risk of lead abandonment a procedure should be attempted.

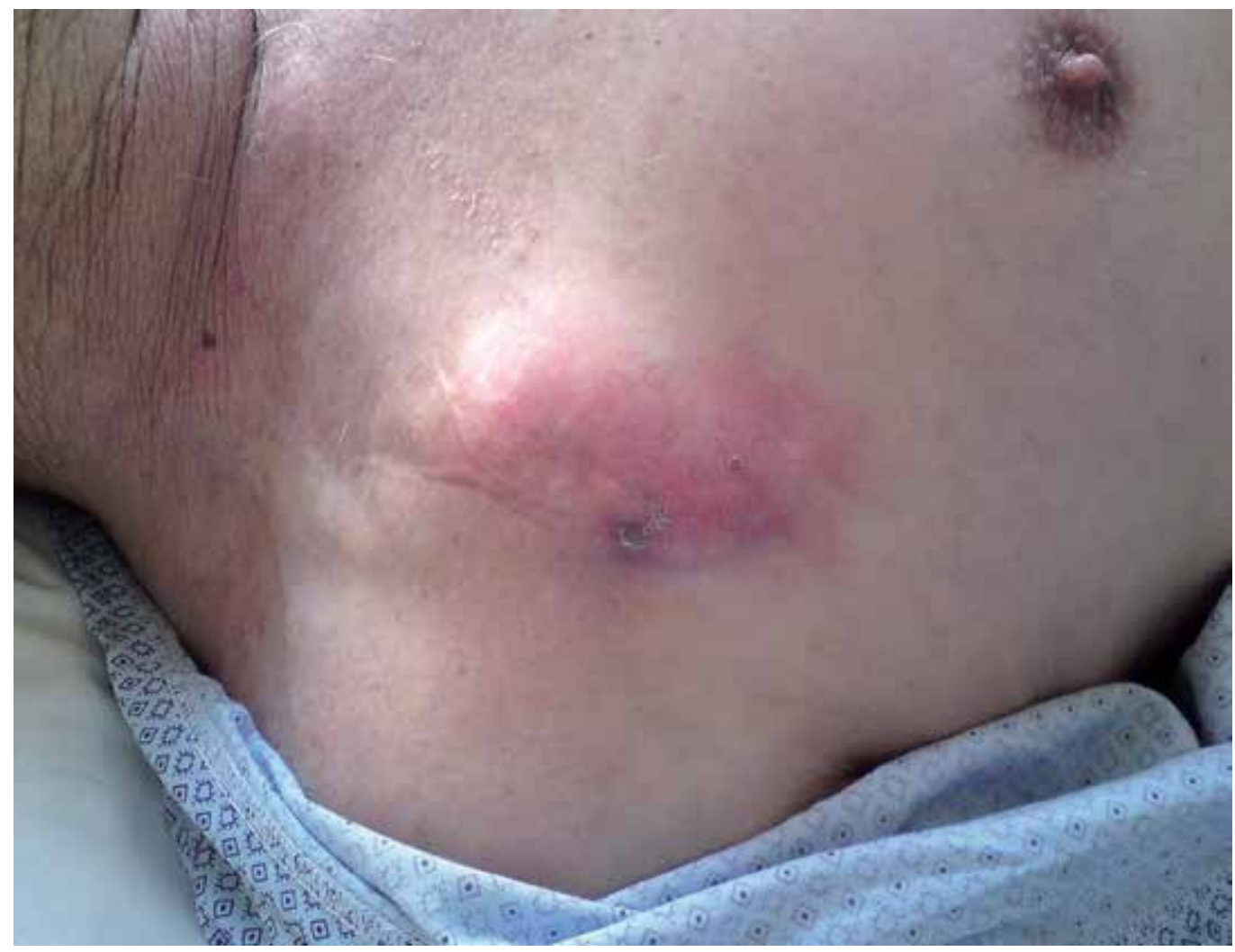

Figure 4. Device adherence to the overlying skin with initiation of erosion

\section{The extraction procedure}

The goal of extraction techniques of chronic pacemaker and defibrillator leads is to present an approach that is successful in extracting all leads and minimizes or eliminates complications. Separating the lead from the encapsulating inflammatory tissue is the most crucial step in this process. However, regardless of the technique that will be used, clinicians must be prepared to deal with the fact that this procedure may vary from a simple to an extremely complicated one. Thus, careful planning of the procedure along with meticulous patient preparation seems mandatory. 


\begin{tabular}{|c|c|c|c|c|}
\hline Indication & $\begin{array}{l}\text { Class I } \\
\text { Procedure should be } \\
\text { performed }\end{array}$ & $\begin{array}{l}\text { Class IIa Reasonable } \\
\text { to perform } \\
\text { procedure }\end{array}$ & $\begin{array}{l}\text { Class IIb } \\
\text { Procedure may be } \\
\text { considered }\end{array}$ & $\begin{array}{l}\text { Class III Procedure } \\
\text { should not be } \\
\text { performed }\end{array}$ \\
\hline Infection & $\begin{array}{l}\text { 1. Definite infection of } \\
\text { CIED e.g.device } \\
\text { endocarditis or sepsis } \\
\text { (LOE: B) } \\
\text { 2. CIED pocket } \\
\text { infection e.g. abscess, } \\
\text { erosion or chronic } \\
\text { draining sinus } \\
\text { (LOE: B) } \\
\text { 3. Valvular } \\
\text { endocarditis w/o } \\
\text { definite lead and/or } \\
\text { device involvement } \\
\text { (LOE: B) } \\
\text { 4. Occult gram- } \\
\text { positive bacteraemia } \\
\text { (LOE: B) }\end{array}$ & $\begin{array}{l}\text { Persistent occult } \\
\text { gram-negative } \\
\text { bacteraemia (LOE:B) }\end{array}$ & & $\begin{array}{l}\text { 1. Superficial or } \\
\text { incisional infection } \\
\text { w/o involvement } \\
\text { of device/leads } \\
\text { (LOE: C) } \\
\text { 2. Chronic } \\
\text { bacteraemia due to } \\
\text { a source other than } \\
\text { CIED when long } \\
\text { term suppressive } \\
\text { antibiotics are } \\
\text { required (LOE: C) }\end{array}$ \\
\hline $\begin{array}{l}\text { Thrombosis or } \\
\text { venous stenosis }\end{array}$ & $\begin{array}{l}\text { 1. Clinically } \\
\text { significant TE events } \\
\text { associated } \\
\text { with thrombus on } \\
\text { lead or fragment } \\
\text { (LOE: C) } \\
\text { 2. Bilateral SCV or } \\
\text { SVC occlusion } \\
\text { precluding } \\
\text { implant of needed TV } \\
\text { lead (LOE: C) } \\
\text { 3. Planned stent } \\
\text { deployment in vein } \\
\text { with TV lead already } \\
\text { to avoid entrapment } \\
\text { (LOE: C) } \\
\text { 4. Symptomatic SVC } \\
\text { stenosis/occlusion } \\
\text { (LOE: C) } \\
\text { 5. Ipsilateral venous } \\
\text { occlusion precluding } \\
\text { implant of additional } \\
\text { lead when } \\
\text { contralateral implant } \\
\text { contraindicated (AVF, } \\
\text { shunt or vascular } \\
\text { access port, } \\
\text { mastectomy) } \\
\text { (LOE: C) }\end{array}$ & $\begin{array}{l}\text { Ipsilateral venous } \\
\text { occlusion precluding } \\
\text { ipsilateral implant of } \\
\text { additional lead w/o } \\
\text { contraindication to } \\
\text { contralateral implant } \\
\text { (LOE: C) }\end{array}$ & & \\
\hline
\end{tabular}




\begin{tabular}{|c|c|c|c|c|}
\hline Indication & $\begin{array}{l}\text { Class I } \\
\text { Procedure should be } \\
\text { performed }\end{array}$ & $\begin{array}{l}\text { Class IIa Reasonable } \\
\text { to perform } \\
\text { procedure }\end{array}$ & $\begin{array}{l}\text { Class IIb } \\
\text { Procedure may be } \\
\text { considered }\end{array}$ & $\begin{array}{l}\text { Class III Procedure } \\
\text { should not be } \\
\text { performed }\end{array}$ \\
\hline Functional leads & $\begin{array}{l}\text { 1. Life threatening } \\
\text { arrhythmias due to } \\
\text { retained leads } \\
\text { (LOE: B) } \\
\text { 2. Leads, due to } \\
\text { design or failure, may } \\
\text { pose immediate threat } \\
\text { if left in place } \\
\text { (LOE: B) } \\
\text { 3. Leads that interfere } \\
\text { with CIED function } \\
\text { (LOE: B) } \\
\text { 4. Leads that interfere } \\
\text { with treatment of } \\
\text { malignancy } \\
\text { (radiation, surgery) } \\
\text { (LOE: C) }\end{array}$ & & $\begin{array}{l}\text { 1. Leads w/potential } \\
\text { interference with } \\
\text { CIED function } \\
\text { (LOE: C) } \\
\text { 2. Leads, due to } \\
\text { design or failure, } \\
\text { with potential threat } \\
\text { if left in place (LOE: } \\
\text { C) } \\
\text { 3. Abandoned leads } \\
\text { (LOE: C) } \\
\text { 4. Need for MRI } \\
\text { imaging w/o } \\
\text { alternative (LOE: C) } \\
\text { 5. Need for MRI } \\
\text { conditional CIED } \\
\text { system (LOE: C) }\end{array}$ & $\begin{array}{l}\text { 1. Redundant leads } \\
\text { with <1 year life } \\
\text { expectancy } \\
\text { (LOE: C) } \\
\text { 2. Known } \\
\text { anomalous lead } \\
\text { placement (SCA, } \\
\text { Ao, pleura, etc) or } \\
\text { through a systemic } \\
\text { atrium or } \\
\text { ventricle* (LOE: C) } \\
\text { 3. }{ }^{*} \text { Can be } \\
\text { considered } \\
\text { w/surgical backup }\end{array}$ \\
\hline $\begin{array}{c}\text { Non-functional } \\
\text { leads }\end{array}$ & & $\begin{array}{l}\text { 1. Leads, due to } \\
\text { design or failure, } \\
\text { with potential threat } \\
\text { if left in place (LOE: } \\
\text { C) } \\
\text { 2. CIED implant } \\
\text { would yield }>4 \text { leads } \\
\text { on one } \\
\text { side or }>5 \text { leads } \\
\text { through SVC } \\
\text { (LOE: C) } \\
\text { 3. Need for MRI } \\
\text { imaging w/o } \\
\text { alternative (LOE: C) }\end{array}$ & $\begin{array}{l}\text { 1. At time of } \\
\text { indicated CIED } \\
\text { procedure w/o } \\
\text { contraindication to } \\
\text { TLE (LOE: C) } \\
\text { 2. Need for MRI } \\
\text { conditional CIED } \\
\text { system (LOE: C) }\end{array}$ & $\begin{array}{l}\text { 1. Redundant leads } \\
\text { with }<1 \text { year life } \\
\text { expectancy } \\
\text { (LOE: C) } \\
\text { 2. Known } \\
\text { anomalous lead } \\
\text { placement (SCA, } \\
\text { Ao, } \\
\text { pleura, etc) or } \\
\text { through a systemic } \\
\text { atrium or } \\
\text { ventricle* (LOE: C) } \\
\text { 3. }{ }^{*} \text { Can be } \\
\text { considered } \\
\text { w/surgical backup }\end{array}$ \\
\hline Chronic pain & & $\begin{array}{l}\text { Severe chronic pain } \\
\text { at device or lead } \\
\text { insertion site with } \\
\text { significant } \\
\text { discomfort not } \\
\text { manageable } \\
\text { by medical or } \\
\text { surgical techniques } \\
\text { and w/o } \\
\text { acceptable alternative } \\
\text { (LOE: C) }\end{array}$ & & \\
\hline
\end{tabular}

Table 1. Indications for transvenous lead extraction Ao, aorta; CIED, cardiovascular implantable electronic device; DRE, device related endocarditis; LOE, level of evidence; SCA, subclavian artery; SCV, subclavian vein; SVC, superior vena cava; TE, thromboembolic; TLE, transvenous lead extraction; TV, transvenous; w/, with; w/o, without. 


\subsection{Pre-procedural and patient preparation}

Extractions can be performed either in the electrophysiology / catheterization laboratory on in the operating room. The site varies according to the preference and the availability of each center [29]. In any case, a cardiothoracic surgical back up should be always immediately available to intervene in case of life threatening complications. In the presence of such a team, safety is comparable in both settings [30]. In addition to the stand-by surgeon, the required personnel include the physician performing the procedure, a "scrubbed" and a "non-scrubbed" assistant, a third "outside the door" assistant to provide equipment and assist in an emergency, anesthesia support and an x-ray technician or other personnel to operate the fluoroscopy. Regarding the instrumentation, a full range of extraction tools should be available. Additional emergency equipment that should be present in the room or immediately available includes sets for pericardiocentesis, chest drainage, vascular repair, thoracotomy, sternotomy and cardio-pulmonary bypass. In addition, equipments for transthoracic and transesophageal echocardiography, temporary pacing and general anesthesia as well as vasopressors and other emergency medications should also be available [15].

A detailed patient history should be obtained and a complete physical examination should be performed before the patient arrives to the interventional suite. Co-morbidites (anticoagulation therapy, renal impairment, allergies and antibiotic resistance) should be carefully taken into account when planning the procedure. Details about prior implantations and about the hardware in place are also mandatory. Technical characteristics of the lead should be known in advance. The vascular route has to be also explored in advance. Chest fluoroscopy can define the number, type and location of leads. Extravascular coursing can be detected through chest computed tomography [31]. Venography may be useful in case that a vascular access problem is anticipated. A transesophageal echocardiogram should be performed in all infected patients to check for vegetations. The size, shape and friability of vegetations may preclude transvenous extraction and support the decision to take the patient to the operating room. Although a clear cut-off point for the vegetation size has not been defined, many physicians would advocate surgical removal of leads of infected leads with large vegetations $(>1-1,5 \mathrm{~cm})$ [32],[33]. More recent evidence, however, suggests that even larger vegetations can be safely removed percutaneously [21]. Laboratory examinations should include: blood typing and crossmatch, a full blood count, coagulation profile, electrolytes, renal and liver function tests, virology screen (Hep B, C and HIV), Creactive protein and erythrocyte sedimentation rate. A pregnancy test for young females should not be omitted [15] Finally, the patient preparation concludes with the obtaining of written informed consent.

The day of the procedure 4 packs of red blood cells should be immediately available. The patient is prepared with chlorexidine or povidone iodine and wrapped in a way to allow access ipsilaterally and contralaterally to the site of implantation, as well as to permit emergent pericardiocentesis, thoracentesis, thoracotomy, sternotomy or cardiopulmonary 
bypass. Large bore iv. cannulae in peripheral veins are placed bilaterally to allow for rapid fluid infusion. A percutaneous arterial line is placed for direct blood pressure monitoring. Non-invasive automated blood pressure measurements, electrocardiographic monitoring and pulse oximetry are also available throughout the procedure. Femoral venous access is obtained for possible rapid fluid administration, for potential upgrade to a transfemoral approach or to facilitate the placement of a temporary pacing electrode. For patients who are pacemaker dependent, a temporary pacemaker lead inserted via the internal jugular or the femoral vein is adequate if the system is not infected and will be immediately replaced. In the case of infected leads several strategies of inserting a longer lasting temporary lead have been described (for example placing an active fixation permanent lead through the internal jugular vein) in order to permit for an adequate lead-free interval of antibiotic administration before the permanent system will be re-implanted. The externalized pulse generator after it has been cleaned and sterilized has been successfully used for temporary pacing in some centers [34]. In patients with ICDs tachycardia therapies should be switched off in order to prevent inappropriate shocks.

\subsection{Techniques and tools}

Generally speaking, leads can be removed with one of the following techniques:

a. Manual traction without tools

b. Traction mediated by some sort of weight or by application of a clamp to the stretched lead

c. Mechanical sheaths, with or without the use of a locking stylet

d. Laser-assisted lead extraction, with or without the use of a locking stylet

e. Open chest extraction, with or without transvenous extraction tools

f. Thransthoracic extraction using a paraternal, subxyphoid or intercostals approach [7].

If a decision has been made to proceed via the transvenous route after the device has been opened, the pulse generator is removed and the leads to the vascular entrance are freed through careful dissection usually with the aid of electroacautery. The incision is usually performed at the site of the initial one, although some physicians make a second incision over the venous entry site of the leads. Infected pockets should undergo thorough revision and microbial cultures of the tissue should be obtained. Irrigation with hydrogen peroxide or chlorexidine and meticulous removal of all infected tissue must follow. It is not clear if a complete capsulectomy needs to be done, although this is dictated by common sense in the case an ipsilateral implantation is planned. After the leads have been dissected all the way down to the venous entry site, the anchor sleeves are removed along with any suture remnants. It is essential that the leads be completely freed and remain intact. Damaged leads can be hard to extract. When using cautery it should be kept instead that polyurethane insulation is more heat sensitive than silicone [7]. Back bleeding issues may arise when dissecting at the venous entry site. A 2-0 suture placed as a snare in the surrounding tissues may help to solve this problem. 
At this stage trasvenous extraction can be successfully performed in one of the following ways applied commonly in a steward fashion:

Simple traction after the insertion of a regular stylet can be sufficient for recently implanted leads. Some experts advocate the gain of ipsilateral venous access through the introduction of a thin (i.e. 5 French) dilator and a guide wire prior to the traction attempt [3]. We do not routinely follow this practice. We simply place moderate traction on the free part of the lead trying to avoid stretching of the insulation or the induction of ectopy in the electrocardiogram. Unfortunately, there is not a priori certainty which lead will be successfully removed through this simple procedure. With traction, fibrous encapsulation often provides sufficient friction to prevent the force applied from being transmitted to the tip of the lead. When more force is needed, the tensile strength of the insulation or the conductor can be exceeded resulting in stretching or rupture. The lead may become irreversibly damaged complicating the extraction process and even leaving part of it indwelling in the venous circulation. Thus, it is of paramount importance for the lead to be removed in on piece. This can happen only if the operator has control of the body lead throughout the procedure binding the elements of the lead together. In that case, the exposed part of the lead can be used as a handle to remove its endovascular segments which will not be the case if the lead becomes distorted or elongated. Although it may be possible to snare fragments of the lead that have remained intravascularly after the main body of the lead has been removed, this may become increasingly hard at times.

Regarding the type of the lead which can be extracted by simple traction, there is always a better chance for recently implanted and active fixation than passive fixation leads, especially if they are isodiametric and can be unscrewed before extraction. Sometimes this is not feasible because the mechanism is damaged or tissue is plugging the helix. In that case manual counterclockwise rotation of the lead body may unscrew the lead. However, to achieve this, the lead body must be free of adhesions in its entire course. In the case of atrial leads, where the helix has often extended through the thin atrial wall, failure to retract the helix makes traction particularly dangerous in removing a plug of atrial tissue with subsequent tamponade. As a rule, traction should not be placed on a lead not fully unscrewed unless a cardiac surgeon is present and the operating room is ready to accept the patient.

In general, invagination of the myocardium may complicate any case of unopposed traction. Arrhythmias and hypotension can be the result of myocardial rupture, avulsion of a tricuspid valve leaflet or rupture of the superior vena cava or the subclavian veins. To avoid these complications, prolonged graded traction has been introduced. Historically speaking Bilgutay et al [35] created a graded weight and pulley system to deliver gentle traction on the externalized portion of the lead. This system required prolonged hospitalization with bed rest, increased the risk of infection and was proven frequently unsuccessful. This technique has been totally replaced nowadays from the use of locking stylets. 
Traction via a locking stylet is directly applied at the tip, bypassing the conductor and the insulation. If manual traction is unsuccessful, the inner lumen is reamed with a conventional stylet to remove debris and the lead is cut approximately $5 \mathrm{~cm}$ from the vascular entry with a sharp scissor to maintain the shape of the spiral conductors. Care must be taken not to damage the distal lead, which should be firmly held by the assistant with his fingers or with a soft clamp if available. The central lumen of the lead is then identified and a locking stylet is inserted through it. To avoid pulling out the core and leaving the outer insulation in place, a ligature is used to tie down the insulation with the rest of the lead components and with the locking stylet. To choose the locking stylet of the appropriate size, the inner lumen diameter hat to initially be measured with the insertion of a series of gauze pins. A locking stylet of a size corresponding to the largest pin was chosen. This is not longer necessary since most of the contemporary stylets are designed to accommodate a wide range of conductor coil diameters. Locking stylets consist of a straight non-expandable wire that can be locked into the coil close to the tip of the lead. This specific design permits to focus the force of traction as close to the lead tip as possible. As a consequence, the risk of lead disruption is reduced and the likelihood of complete removal of the lead is increased [36],[37]. Several types of stylets with different locking exist. The most commonly used are: the Liberator (Cook Medical, Bloomington, Indiana, USA), the Lead Locking Device (LLD) EZ (Spectranetics, Colorado Springs, Colorado, USA) and the Extor Set (VascoMed, Binzen, Germany) (Figure 5).

The locking mechanisms of the Liberator and the Extor Set are at the distal tip of the stylet providing focal traction at the tip of the lead, whereas the LLD EZ stylet grabs the lead in multiple areas and delivers stable traction along the entire lead length. An additional advantage of both the Extor and the LLD is that they provide the ability to unlock and reposition after initial deployment. This can facilitate the advancement of the locking stylet to the lead tip in cases the later is very tortuous or has sharp bends. In that case, the clinician can advance the stylet to the obstruction, lock the stylet, free the lead at that point, unlock the stylet and advance it to the next obstruction point, repeat the maneuver and manage to reach the lead tip at the end. The Bulldog Lead Extender (Cook Medical) is a tool that can be useful if a lead cannot receive a locking stylet due to extensive damage or a solid core design. It consists of a wire with a threadable handle through which the lead is passed and secured, thereby locking the insulation and conductor to the extender. This way the exposed part of the lead is securely grasped and extended to a workable length allowing a potentially more effective direct traction.

There are still limitations to the use of a locking stylet. If the conductor is broken or distorted, e.g. with subclavian crush syndrome, it is not possible to introduce the stylet. It can also lack grip and dislocate during traction or too much force can damage the delicate locking mechanism. Further, as traction is still exerted via the distal conductor coil, this can unwind or disconnect from the electrode. As with direct traction, there is risk of invagination of the myocardium. 

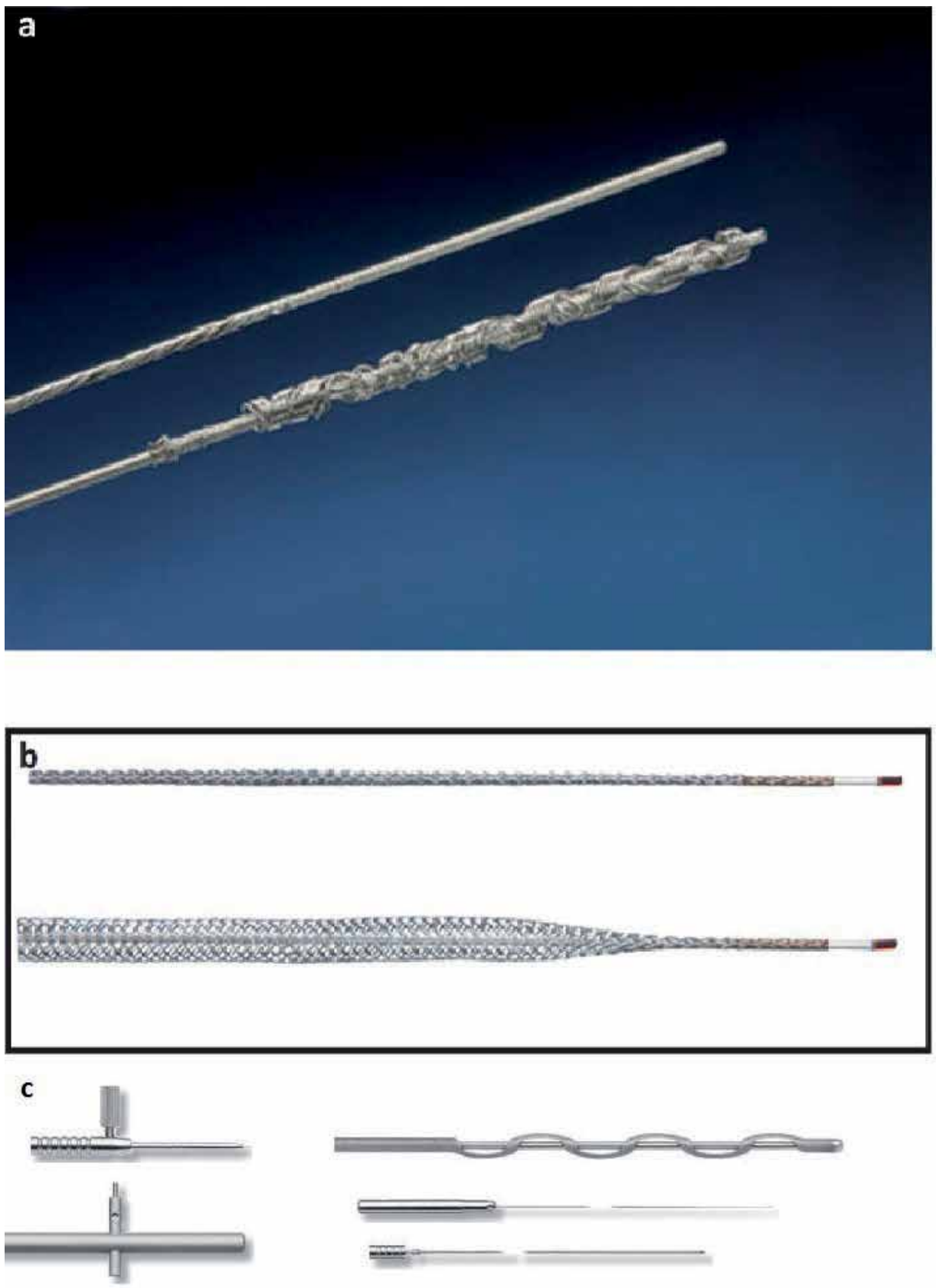

Figure 5. Various types of locking stylets. (A) The Liberator Locking Stylet (Cook Medical, Bloomington, Indiana, USA). (B) The Lead Locking Device (LLD) EZ (Spectranetics, Colorado Springs, Colorado, USA). In contrast to the Liberator locking stylet, the LLD locking stylet has a braided mesh over the entire length of a solid lead that expands when deployed. (C) The Extor Set (VascoMed, Binzen, Germany) (from Maytine et al. The challenges of transvenous lead extraction. Heart 2011;97:425-434, modified with permission) 
Counter-pressure and Counter-traction: to overcome the limitations of a locking stylet, telescoping sheaths can be advanced over the lead with alternating counterclockwise and clockwise motions with moderate pressure. Fibrous bindings can be mechanically disrupted (counter-pressure). The outer sheath also functions as a guiding catheter facilitating the movement of inner sheath and alignment of the inner sheath and the lead. It is of paramount importance to use a locking stylet at the same time, as the leads are often too fragile to withstand the traction necessary to counter the forces applied to advance the sheath. Once the distal electrode is reached, the outer sheath can be positioned against the myocardium to prevent inversion (counter-traction). By pulling on the locking stylet, for several minutes if necessary, the tip of the lead is pulled inside the outer sheath. The force is thus concentrated at a small area of the scar tissue and the myocardium without gross displacement of the myocardium (Figure 6).

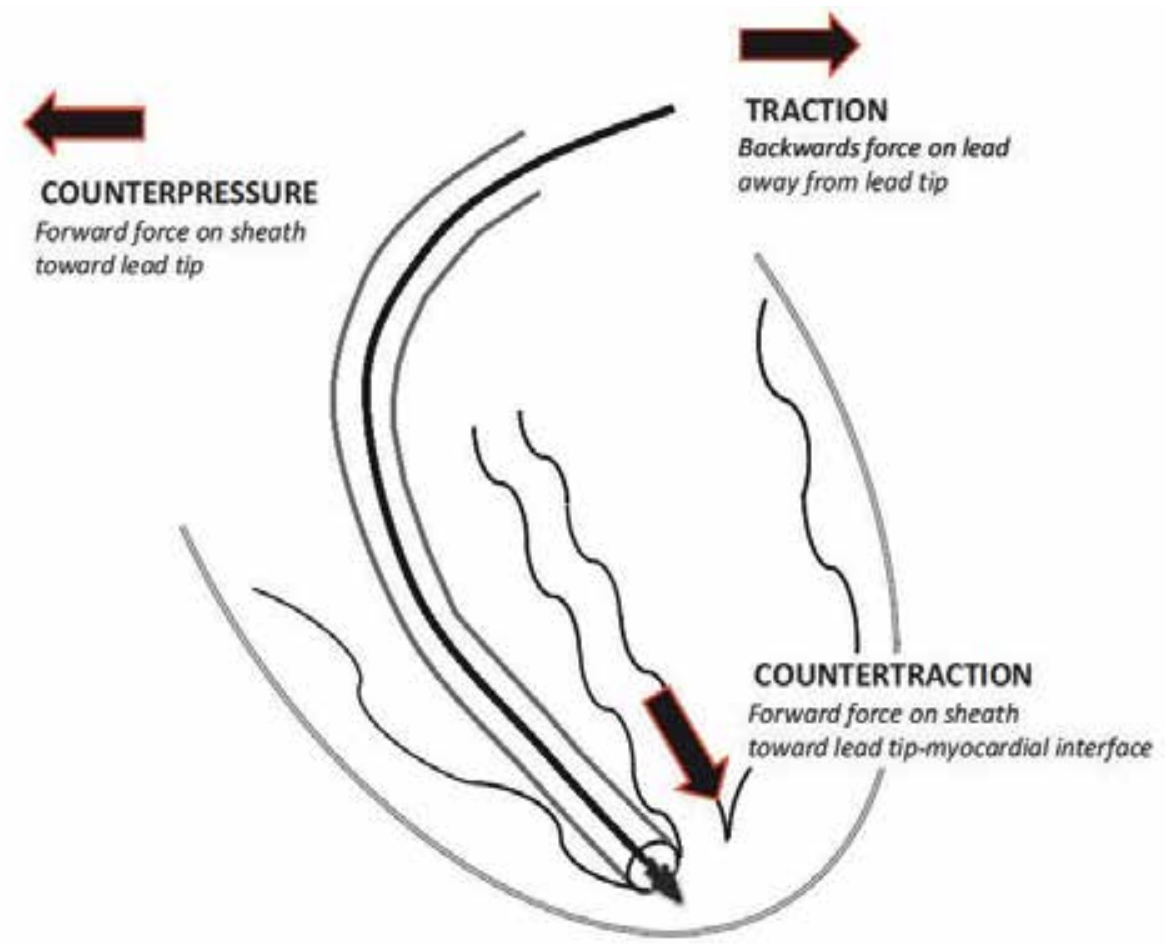

Figure 6. Schematic representation of the forces of counterpressure, traction, and countertraction. (from Maytine et al. The challenges of transvenous lead extraction. Heart 2011;97:425-434, with permission)

Telescopic sheaths are made of different materials (stainless steel, Teflon, and polypropylene) and are available in various sizes (7-16 French) (Figure 7). Sheath selection is determined by the clinical situation and the operator's preference and experience. Teflon is soft and flexible but is unable to cut through dense scar tissue, while polypropylene is stiffer and better at disrupting encapsulating scar but must be used with caution so as to avoid vascular injury. Stainless steel sheaths are employed only to deal with dense and calcified fibrosis. If despite the use of a stainless steel sheath, tight adhesions prevent further 
advancement to reach the tip, changing to a power sheath and/or upsizing to a larger sheath may solve the problem.
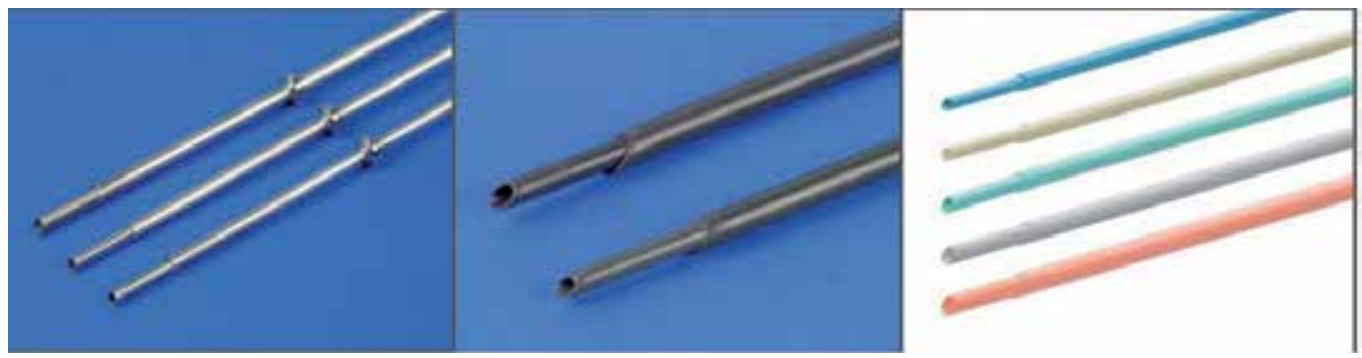

Figure 7. Various types of telescopic sheaths (from Maytine et al. The challenges of transvenous lead extraction. Heart 2011;97:425-434, with permission)

Even the removal of large vegetations (up to $4 \mathrm{~cm}$ ) has been successfully attempted by very experienced operators with the use of 16 French sheaths [7]. Although counter-traction prevents invagination of the myocardium and diminishes the chance of rupture, perforation of the myocardium is still possible, especially in the thin-walled atrium.

Powered sheath assisted extraction: If lead removal still proves unsuccessful, a powered sheath can be used as an alternative. Powered sheaths use a source of energy to make the dissection of encapsulating fibrous tissue easier and more efficient, thus enabling the advancement of the sheath along the lead with reduced countertraction and counterpressure forces. One such powered sheath is the Excimer Laser System (Spectranetics). It consists of optic fibers spirally warped between the inner and outer tubing of the sheath. At the tip of the device the fibers are arranged in a ring. Pulsed laser light is emitted from the fibers to ablate the tissue. The device is connected to a $308 \mathrm{~nm} \mathrm{XeCl} \mathrm{excimer} \mathrm{laser} \mathrm{(Spectranetics} \mathrm{CVX-300),}$ which delivers pulsed light. As the penetration depth of $308 \mathrm{~nm}$ light in vascular tissue is $\sim 100 \mu$, it is completely absorbed in the tissue immediately in front of the tip. This results in an ablation depth, depending on the applied force, between 2 and $15 \mu$ per pulse in the experimental setting. The influence of force is explained by increasing the mechanical effect of the micro-bubbles entrapped beneath the tip of the device in creating microscopic tears [38]. The sheath is advanced under fluoroscopic guidance over the lead body utilising the standard techniques of counterpressure and countertraction, and laser energy is delivered when encapsulating fibrous tissue halts sheath advancement. Tissue in direct contact with the sheath tip is ablated to a depth of $50 \mathrm{~mm}$ until the distal electrode is reached. It should be kept in mind that countertraction is still necessary to dislocate the lead tip. Great care must be paid to proper traction/countertraction techniques to prevent complications. Loss of coaxial orientation of the sheath and the lead can result in vascular injury. The leading edge of the bevel of the laser sheath should be oriented away from the vessel wall and the laser energy should be stopped before the lead tip is reached. Care must also be taken to avoid 
damage of the tricuspid valve. The ablation results in a shearing of the fibrous bindings, often leaving a rim of scar tissue around the lead. Compared with mechanical telescoping sheaths, laser assisted extraction results in more frequent complete lead removal and shortened extraction times without an increase in procedural risk [39]. [40].

The Perfecta Electrosurgical Dissection Sheath (Cook Medical) represents another type of powered sheath. The electrosurgical dissection sheath consists of an inner polytetrafluoroethylene (PTFE) sheath with bipolar tungsten electrodes exposed at the distal tip and an outer sheath for counterpressure and countertraction. Radiofrequency energy is delivered between the bipoles to dissect through fibrous binding sites, much like a surgical cautery tool, although the lead tip must be liberated with countertraction. In contrast to the Excimer Laser Sheath, the Electrosurgical Dissection Sheath permits a localised application of radiofrequency energy with linear rather than circumferential dissection of the encapsulating fibrous tissue. The focused and steerable dissection plane offers the potential advantages of improved precision.

However, the sheath may have to be repositioned repeatedly as a result. The Electrosurgical Dissection Sheath offers a cost effective alternative to the Excimer Laser System without compromising safety or efficacy [41].

Finally, the Evolution and Evolution Shortie Mechanical Dilator Sheaths (Cook Medical) are 'hand powered' mechanical sheaths that consist of a flexible, braided stainless steel sheath with a stainless steel spiral cut dissection tip. The sheath is attached to a trigger activation handle that rotates the sheath and allows the threaded metal end to bore through calcified and dense adhesions [42].

An inferior vena cava or transfemoral approach also named as "the inferior approach" has been developed as the alternative mode to be used often only after the approach via the implant vein ("superior approach") has failed. It is also the procedure of choice for removal of broken or cut lead with free-floating ends. This technique is an old one [43] and over the years the following tools have been developed to assist in the extraction of leads by the femoral vein:

1. The Byrd Femoral Work Station (Cook Vascular Inc, Leechburg, PA)

2. The Dotter retriever Snare

3. The Curry Loop Snare

4. The Amplatz Snares $25 \mathrm{~mm}, 25 \mathrm{~mm}$ (Microvena Corp)

5. The Needls Eye Snares (Cook Vascular Corp) [44] (Figure 8)

Virtually all the femoral extraction techniques use some form of snaring. Two fundamental techniques have evolved. The first uses the combination of a wire loop and catheter to snare free ends or free-floating leads. The other creates a loop around the lead to be removed when there is no free end available for simple snaring. In any case, a long sheath is introduced via the femoral vein and positioned close to the lead. Then, a retriever is inserted through the sheath to grab and secure the lead close to the tip. The isodiametric proximal part of the lead (with the connector cut off) is pulled down through 
the fibrous scar tissue. The outer sheath is then advanced over the doubled up lead to disrupt the scar tissue, while the lead is kept under tension by the retriever. When the sheath reaches the tip, counter-traction is applied. In comparison with the superior approach, only a short distance of scar tissue needs to be disrupted as the proximal isodiametric part of the lead can be simply pulled down. Therefore, even if no locking stylet has been used to reinforce the lead, the shorter distance to cover decreases the chance of elongating the lead. "Reversibility" is one of the most important principles to be followed with snaring techniques since the process of grasping a lead must be totally reversed and the lead freed again if necessary. If this situation cannot be reversed thoracotomy remains the only solution.

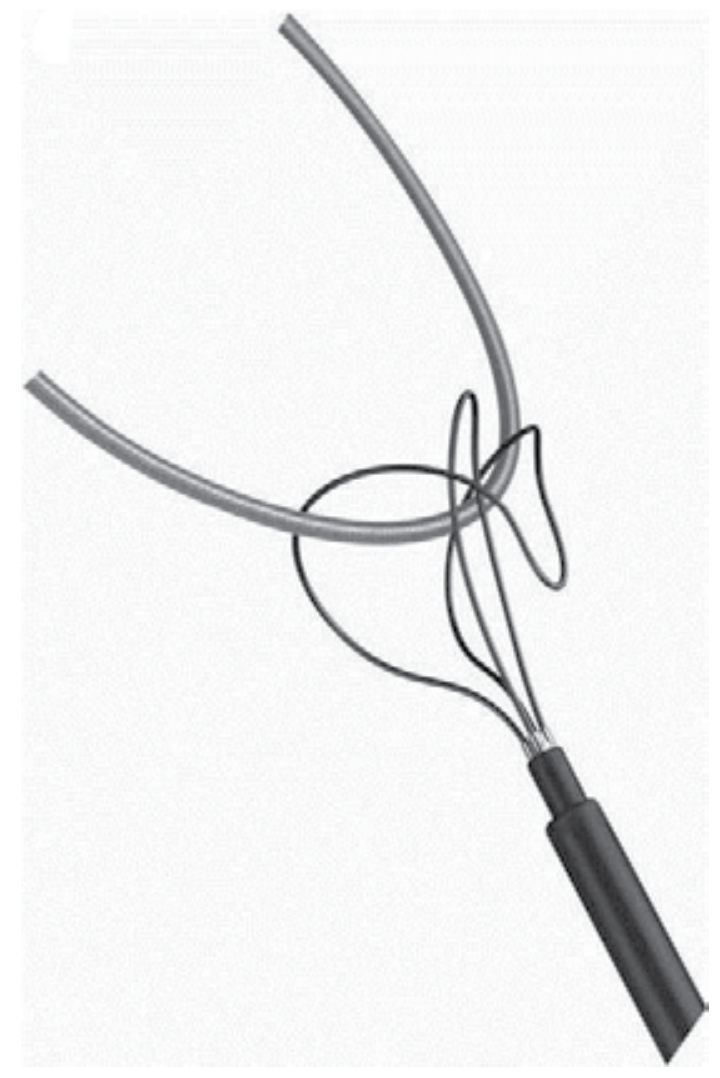

Figure 8. The Needle's Eye snaring tool

Beyond that, the main challenge of femoral retrieval remains manipulating the tools and snaring the lead in three dimensions under the guidance of two dimensional fluoroscopic imaging. The recent description of a novel technology to facilitate extraction and the maintenance of vascular access proposed a hybrid superior and inferior approach, with femoral snaring of the lead to stabilise the lead while countertraction and counterpressure are used through the right jugular vein to free the lead, reiterating the clinical importance of femoral retrieval [45],[46]. 


\section{Complications}

The major risks of transvenous lead extraction include: cardiac perforation (1-4\%), emergency cardiac surgery (1-2\%) and death (0.4-0.8\%). ${ }^{34}$ (Table 2). However, the risk of an individual varies according to the presence or absence of the following factors:

- $\quad$ Age of the patient (risk increase with advanced age)

- $\quad$ Gender of the patient (risk higher in female patients)

- Comorbidities

- Presence of calcifications on the leads

- Presence and size of vegetations

- Duration of implant

- Physical characteristics of the lead (fragility, condition)

- Presence of multiple leads

- Presence of ICD leads, especially with a superior vena cava coil

\section{Major complications}

Death

Cardiac avulsion requiring intervention

(percutaneous or surgical)

Vascular injury requiring intervention

(percutaneous or surgical)

Pulmonary embolism requiring surgical intervention

Respiratory arrest/anesthesia related complication prolonging hospitalization

Stroke

Cardiovascular implantable electronic device infection at previously non-infected site

\section{Minor complications}

Pericardial effusion not requiring intervention

Haemothorax not requiring intervention

Pocket haematoma requiring reoperation

Upper extremity thrombosis resulting in medical treatment

Vascular repair near implant site or venous entry site

Hemodynamically significant air embolism

Migrated lead fragment without sequelae

Blood transfusion as a result of intraoperative blood loss

Pneumothorax requiring a chest tube

Pulmonary embolism not requiring

surgical intervention

Table 2. Potential complications of transvenous lead extraction.

Complications result primarily from forces applied to separate leads from fibrous connections within the large vessels and the heart. Disruption of the superior vena cava or brachiocephalic vein is the most devastating complication of lead extraction, as it results in swift exsanguination in the thoracic cavity and is very difficult for the surgeon to control or repair. The superior vena cava has a wall thickness of sometimes $<1 \mathrm{~mm}$ and is 
vulnerable for damage by the sheaths. Of note, the pathway of least resistance is the vessel wall rather than the scar. Of course, damage to the superior vena cava and its branches veins may be minimized with a femoral approach. Some have suggested the use of a large balloon to tamponade bleed from the superior vena cava until the surgeon arrives. Death can occur or emergency surgery may be needed due the above complication but also due to cardiac tamponade from cardiac rupture or due pulmonary embolism from the dislodgment of large lead vegetations. Infection with re-implantation is another potential problem to consider. Proper care of the infected pocket is essential to prevent recurrent infection. Some operators elect to leave the wound open to heal by secondary intention while others prefer tight suturing of tissues to eliminate any residual cavity. As a rule, a new device should never be placed in a previous infected pocket. However, the most important principle in preventing lead extraction complications is to avoid lead extraction by meticulous operative technique at the time of the initial implantation and by early recognition of potential problems in the immediate postoperative period [47].

\section{Lead extraction success rate}

The Expert Consensus of the Hearth Rhythm Society has defined the success of the lead extraction procedure in two different ways [6]: Complete Procedural Success has been considered the removal of all targeted leads and all lead material from the vascular space, with the absence of any permanently disabling complication or procedure related death. On the other hand as Clinical Success has been defined the removal of all targeted leads and lead material from the vascular space, or retention of a small portion of the lead that does not negatively impact the outcome goals of the procedure. This may be the tip of the lead or a small part of the lead (conductor coil, insulation, or the latter two combined) when the residual part does not increase the risk of perforation, embolic events, perpetuation of infection or cause any undesired outcome. Finally, the Committee defined as Failure the inability to achieve either complete procedural or clinical success, or the development of any permanently disabling complication or procedure related death.

Results of transvenous extraction have been repeatedly reported as associated with a high success rate of complete $(>90 \%)$ or partial $(>95 \%)$ removal with a concomitant low rate of complications in experienced centers [48],[49],[50]. The use of extraction sheaths ranged from $60-80 \%$ in these studies. Nevertheless, the success rate of manual only traction alone or with the use of locking stylets is not negligible ranging form $15-30 \%$ in recent studies. More recently, de Bie et al [51] reported a substantially higher clinical success rate of $\sim 85 \%$ in $>250$ removal procedures with the use of manual traction without the assistance of extraction sheaths. This finding was particularly true for leads implanted $>2.6$ years. Since it is not clearly defined when an attempt should be considered unsuccessful, the efficacy of these simpler techniques seem to be largely dependent of the availability of the more complex 
ones (i.e. telescopic or laser sheaths). That means that if an operator has a wide range of tools available, he will give up more easily on a resistant to traction lead, moving to more sophisticated techniques and strategies [51].

\section{Removal of left ventricular pacing leads}

Nowadays CRT is considered a standard therapy used to improve symptoms and prognosis in heart failure. Increasing evidence confirming the benefits of CRT has led to widespread implantation of CRT devices with technically challenging procedures, followed by frequent dislodgement of the coronary sinus (CS) lead or infections and requirement for extraction and re-implantation of the device. During the last decade, evolutionary changes have emerged in CS lead technology and techniques to optimize CRT function during implantation and permit the removal of left ventricular (LV) pacing leads with minor complications.

Coronary sinus lead implantation is a complex procedure with several limitations and hazards. According to several studies, the procedure is usually time consuming with long fluoroscopy times and the implantation success rate is reduced compared to conventional procedures (estimated between $90 \%$ to $97 \%$ ) [52-56]. With the introduction of special delivery sheaths and the so-called 'over the wire technology' the breakthrough of LV pacing became reality in the early 2000s, yet lead stability remains problematic mainly due to different coronary sinus anatomies [57-58]. Because no muscular trabeculae is found in the CS for anchoring of the lead, the tip has to be pushed as distal as possible in a wedge position in a lateral or a postero-lateral CS branch and is typically non-actively fixated. Such an ideal position of the LV lead cannot be reached in several cases owing to small and tortuous venous anatomy, phrenic nerve stimulation or sub-optimal hemodynamics due to close proximity to the right ventricular lead. As a result, detectable CS lead dislodgement has been observed in about $4-8.6 \%$ of patients during follow-up, accompanied by loss of capture and need for repositioning $[52,59,60]$. To overcome these limitations, pre-shaped leads (curved in one or more dimensions) have been developed to offer stability even in proximal positions and in larger veins. Coronary sinus side branch stenting has also been performed in several occasions [61-62]. Finally, the development of an active fixation CS lead (Attain StarFix 4195, Medtronic) has also been achieved [63]. This lead body has a 55D polyurethane coating that expands into pleated loops near the electrode tip to increase its diameter and promote fixation (practically reaching stability of $100 \%$ ), along with a small amount of steroid at the electrode tip to reduce inflammation in the surrounding tissue [64] (Figure 9).

In parallel to the rapid growing experience with the implantation of CS leads, a new and interesting field relating to LV pacing lead extraction and subsequent re-implantation has been developed. It should be noted, however, that in comparison to the extensive data available on conventional pacing and ICD lead extraction, the experience with LV leads is still limited. Similarly to conventional devices, infection seems to be the primary 
indication for removal in the case of CRT devices too. On the other hand, it might be expected that removing leads from fragile and tortuous CS vein tributaries, especially with the use of larger-bore sheaths (mechanical or laser) which may not fit into the distal branches, would lend itself to a higher risk of complications. Indeed, the high rate of hemopericardium and dense scar tissue in-growth or vein occlusion in animal models $[65,66]$ were early findings suggesting the need for extreme care in the removal of CS leads and for detailed preoperative knowledge of the CS anatomy. Nevertheless, published data so far suggest that CS leads can be safely and successfully removed percutaneously and that CS lead extraction is not more hazardous than conventional PM/ICD lead extraction (Table 3), although post-extraction complete occlusion of the branch vein previously implanted with an LV lead followed by re-implantation complications may become a particular problem occasionally [67]. It appears that CS leads implanted for $<2$ years are amenable to manual traction [68] and even extraction of active fixation CS leads has been reported due to prolonged manual traction alone or careful use of a laser sheath within the CS. [69],[70],[71].

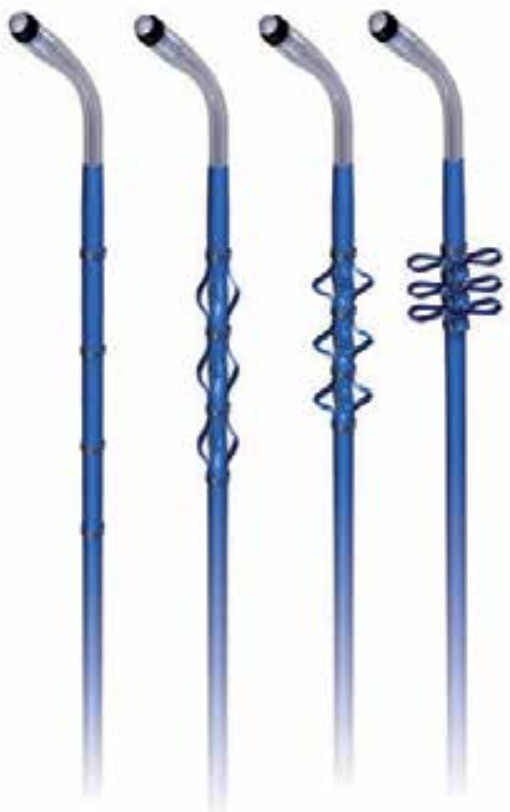

Figure 9. Medtronic Attain StarFix 4195 Coronary Sinus Lead at four stages of deployement.

All the previously mentioned techniques have been successfully used for the removal of CS infected or malfunctioning leads. Tyers et al [72] reported a series of 14 left ventricular lead extractions, all successfully removed with the use of locking stylets and powered sheaths. Bongiorni et al [73] have reported one of the earlier and larger single centre experiences on extraction of LV pacing leads: manual traction using a standard stylet only was effective in the majority of cases (73\%) and mechanical dilation with polypropylene sheaths was necessary $(27 \%)$ when tight adherence was found along the catheter course. 
In particular, adherence in CS lead extraction was located more commonly in the systemic veins (subclavian vein $60 \%$, innominate vein $30 \%$, superior vena cava $20 \%$, right atrium $20 \%)$ rather than inside the CS (10\%). When areas of adherence were found inside the CS (never in its branches), dissection through a transfemoral approach was chosen and no major complications were seen. Safety and efficacy of transvenous CS lead removal was also confirmed by Di Cori et al [74] in a large, single-center experience involving extraction of 147 CS pacing leads. Nearly one-third of procedures were resistant to manual traction and thus required mechanical dilation or transfemoral approach. Complications were rare, there was no predictable pattern among manual traction or mechanical dilation removal techniques and fibrous adherence sites were also found mainly in non-CS locations (subclavian vein $66 \%$, innominate vein $48 \%$, superior vena cava $32 \%$, right atrium $20 \%$ and CS $14 \%$ ). Most recently, Williams et al [75] confirmed their high procedural success of 10-year experience regarding percutaneous removal of CS leads. Among 71 patients they explanted 60 CS leads and 143 non-CS leads: CS extraction had $0 \%$ operative mortality $-2.8 \%$ postprocedural mortality (in hospital $<30$ days) - minor complication rates $5.6 \%$ and major complication rates $1.4 \%$. The majority of CS leads were extracted using manual traction and laser sheath dissection was required in $10 \%$ (laser was used within the CS only in two cases).

In conclusion, extensive data are available on conventional pacing and ICD lead extraction but only limited experience with LV leads exists. The LV pacing leads may be removed easily by manual traction in a large number of cases, but coronary sinus (CS) adherences may complicate extraction requiring mechanical dilation or ablative extraction techniques. In addition to CS remnant adherences, post-extraction venous occlusion might complicate the eventual re-implantation. Nevertheless, CS lead extraction seems to be not more hazardous than conventional pacemaker and defibrillator lead extraction. The evolving use of CRT in current clinical practice, is expected to improve the techniques and provide us with more data regarding the feasibility and safety of LV lead removal.

\begin{tabular}{|c|c|c|c|c|c|c|c|c|c|}
\hline Author & $\begin{array}{l}\text { CS leads } \\
\text { extracted }\end{array}$ & infection & sepsis & malfunction & $\begin{array}{l}\text { time from } \\
\text { implant } \\
\text { (months) }\end{array}$ & $\begin{array}{l}\text { manual } \\
\text { traction }\end{array}$ & $\begin{array}{c}\text { mechanical } \\
\text { dilation }\end{array}$ & $\begin{array}{c}\text { major } \\
\text { compli- } \\
\text { cations }\end{array}$ & $\begin{array}{c}\text { success } \\
\text { rate }\end{array}$ \\
\hline $\begin{array}{c}\text { Bongiorni } \\
\text { et } \mathrm{al}^{73}\end{array}$ & 37 & $43.3 \%$ & $29.7 \%$ & $27 \%$ & $19.5 \pm 16.5$ & $73 \%$ & $27 \%$ & $0 \%$ & $100 \%$ \\
\hline $\begin{array}{l}\text { Williams } \\
\text { et } \mathrm{al}^{75}\end{array}$ & 60 & $31 \%$ & $31 \%$ & $38 \%$ & 35.8 & $90 \%$ & $10 \%$ (laser) & $1.4 \%$ & $98 \%$ \\
\hline Di Cori et $\mathrm{al}^{74}$ & 147 & $56 \%$ & $24 \%$ & $20 \%$ & $29 \pm 25$ & $70 \%$ & $30 \%$ & $0.7 \%$ & $99 \%$ \\
\hline $\begin{array}{c}\text { De Martino } \\
\text { et al }{ }^{77}\end{array}$ & 12 & $58 \%$ & & $42 \%$ & $13.9 \pm 11.7$ & $100 \%$ & & $0 \%$ & $100 \%$ \\
\hline Hamid et al ${ }^{68}$ & 32 & 56.2 & & $43.8 \%$ & $26.5 \pm 28.7$ & $87.5 \%$ & $\begin{array}{l}12.5 \% \\
\text { (laser) } \\
\end{array}$ & $0 \%$ & $100 \%$ \\
\hline Tyers et $\mathrm{al}^{72}$ & 14 & & & & not available & & & $7 \%$ & $100 \%$ \\
\hline Kasravi et al ${ }^{76}$ & 14 & & & & $17.4 \pm 12.2$ & & & $0 \%$ & $100 \%$ \\
\hline
\end{tabular}

Table 3. Coronary sinus lead extraction: differences and similarities between reports 


\section{Author details}

Spyridon Koulouris and Sofia Metaxa

$1^{\text {st }}$ Cardiology Department, Evangelismos Hospital, Athens, Greece

\section{References}

[1] Bardy GH, Lee KL, Mark DB, et al. Amiodarone or implantable crdioverter-defirbrillator for congestive heart failure. N Engl J Med 2005;352:225-237

[2] Moss AJ. Zareba W, Hall WJ, et al. Prophylactic implantation of a defibrillator in patients with myocardial infarction and reduced ejection fraction. N Engl J Med 2002;346:877883

[3] Abraham WT, Fischer WG, Smith AL, et al. Cardiac reynchronization therapy in chronic heart failure. N Engl J Med 2002;346:1845-1853

[4] Bristow MR, Saxon LA, Boehmer J, et al. Cardiac-resynchronization therapy with or without an implantable defibrillator in advanced chronic heart fialure. N Engl J Med 2004;350:2140-2150

[5] Tang AS, Wells GA, Talajic M, et al, the Resynchronization-Defibrillation for Ambulatory Heart Failure Trial (RAFT) Investigators. Cardiac-resynchronization therapy for mild-to-moderate heart failure. N Engl J Med 2010;363:2385-2399

[6] Wilkoff BL, Love CI, Byrd BL, et al. Transvenous lead extraction: Heart Rhythm Society expert consensus on facilites, training, indications and patient management: this document was endorsed by the American Heart Association (AHA). Heart Rhythm 2009;6:1085-1104

[7] Kennergren C. A European prespective on lead extraction: Part I. Heart Rhythm 2008;5:160-162

[8] Madigan NP, Curtis JJ, Sanfelippo JF, et al. Difficulty of extraction of chronically implanted tined ventricular endocardial leads. J Am Coll Cardiol 1984;3:724-731

[9] Farooqi FM, Talsania S, Hamid S, et al. Extraction of cardiac rhythm devices: indications, techniques and outcomes for the removal of pacemaker and defibrillator leads. Int J Clin Pract 2010;64:1140-1147

[10] Robboy SJ, Harthorne JW, Leinbach RC, et al. Autopsy findings with permanent pervenous pacemakers. Circulation 1969;39:495-501

[11] Huang TY, Baba N. Cardiac pathology of transvenous pacemakers. Am Heart J 1971;83:469-474

[12] Cooper JM, Stephenson EA, Berul CI, et al. Implantable cardioverter defibrillator lead complications and laser extraction in children and youg adults with congenital heart disease: implications for implantation and management. J Cardiovasc Electrophysiol 2003;14:344-349

[13] Smith HJ, Fearnot NE, Byrd CL, et al. Five-years experience with intravascular lead extraction. Pacing Clin Electrophysiol 1994;17:2016-2020 
[14] Hackler JW, Sun Z, Lindsay BD, et al. Effectiveness of implantable cardioverterdefibrillator lead coil treatments in facilitating ease of extraction. Heart Rhythm 2010;7:890-897

[15] Deharo JC, Bongiorni MG, Rozkovec A, et al. Pathways for training and accreditation for transvenous lead extraction: a European Heart Rhythm Association position paper. Europace 2012;14:124-134

[16] Smith HJ, Fearnot NE, Byrd CL, et al. Five-years experience with intravascular lead extraction. U.S. Lead Extraction Database. Pacing Clin Electrophysiol 1994:17:2016-2020

[17] Roux JF, Page P, Dubuc M, et al. Laser lead extraction: predictors of success and complications. Pacing Clin Electrophysiol 2007;30:214-20

[18] Cabell CH, Heidehreich PA, Chu VH, et al. Increasing rates of cardiac device infections among Medicare beneficiaries: 1990-1999. Am Heart J 2004;147:582-586

[19] Voigt A, Shalaby A, Saha S. Rising rates of cardiac rhythm management device infection in the United States:1996 through 2003. J Am Coll Cardiol 2006;48:590-591

[20] Jones SO, Eckart RE, Albert CM, Epstein L. Large, single-center, single-operator experience with transvenous lead extraction: Outcomes and changing indications. Heart Rhythm 2008:5;520-525

[21] Grammes JA, Schulze CM, Al-Bataineh M, et al. Paercutaneous pacemaker and implantable cardioverter-defibrillator lead extraction in 100 patients with intracardiac vegetations defined by transesophageal echocardiography. J Am Coll Cardiol 2010; 55:886-894

[22] Klug D, Wallet F, Lacroix D, et al. Local symptoms at the site of pacemaker implantation indicate latent systemic infection. Heart 2004;90:882-886

[23] Baddour LM, Epstein AE, Erickson CC, et al. Update on cardiovascular electronic device infections and their management: a scientific statement from the American Heart Association. Circulation 2010;121:458-477

[24] Sohail MR, Uslan DZ, Khan AH, et al. Management and outcome of permanent pacemaker and implantable cardioverter-defibrillator infections. J Am Coll Cardiol 2007;49:1851-1859

[25] Field M, Jones S, Epstein L. How to select patients for lead extraction. Heart Rhythm 2007;4:978-985

[26] Bracke FA, Meijer A, Van Gelder B. Pacemaker lead complications: when is extraction appropriate and what can we learn from published data? Heart 2001:85:254-259

[27] Hauser RG, Hayes DL, Kallinen LM, et al. Clinical experience with pacemaker pulse generators and transvenous leads: an 8-year prospective multicenter study. Hear Rhythm 2007;4:154-160

[28] Kleemann T, Becker T, Doenges K, et al. Annual rate of transvenous defibrillation lead defects in implantable cardioverter- defibrillators over a period of $>10$ years. Circulation 2007;115:2474-2490

[29] Henrickson CA, Zhang K, Brinker JA. A survey of the practice of lead extraction in the United States. Pacing Clin Electrophysiol 2010;33:721-726 
[30] Franceschi F, Dubuc M, Deharo JC, et al. Extraction of transvenous leads in the operating room versus electrophysiology laboratory: a comparative study. Heart Rhythm 2011;8:1001-1005

[31] Hirschl DA, Jain VR, Spindola-Franco H, et al. Prevalence and characterization of asymptomatic pacemaker and ICD lead perforation on CT. Pacing Clin Electrophysiol 2007;30:28-32

[32] Chiu WS, Nguyen D. Pacemaker lead extraction in pacemaker endocarditis with lead vegetation: usefulness of transesophageal echocardiography. Can J Cardiol 1998;14:87-9

[33] Sohail MR, Uslan DZ, Khan AH, et al. Infective endocarditis complicating permanent pacemaker and implantable cardioverterdefibrillator infection. Mayo Clin Proc 2008;83:46-53

[34] Henrikson CA, Brikner JA. How to prevent, recognize and manage complications of lead extraction. Part III: Procedural factors. Heart Rhythm 2008;5:1352-1354

[35] Bilgutay AM, Jensen NK, Schmidt WR, et al., Incarceration of transvenous pacemaker electrode. Removal by traction, Am Heart J1969;77:377-9

[36] Fearnot NE, Smith HJ, Goode LB, et al. Intravascular lead extraction using locking stylets, sheaths, and other techniques. Pacing Clin Electrophysiol 1990;13:1864-1870

[37] Goode LB, Byrd CL, Wilkoff BL, et al. Development of a new technique for explantation of chronic transvenous pacemaker leads: five initial case studies. Biomed Instrum Technol 1991;25:50-53

[38] Gijsbers GH, van den Broecke DG, Sprangers RL, et al. Effect of force on ablation depth for a $\mathrm{XeCl}$ excimer laser beam delivered by an optical fiber in contact with arterial tissue under saline. Lasers Surg Med 1992;12:576-584

[39] Wilkoff BL, Byrd CL, Love CJ, et al. Pacemaker lead extraction with the laser sheath: results of the pacing lead extraction with the excimer sheath (PLEXES) trial. J Am Coll Cardiol 1999;33:1671-1676

[40] Byrd CL, Wilkoff BL, Love CJ, et al. Clinical study of the laser sheath for lead extraction: the total experience in the United States. Pacing Clin Electrophysiol 2002;25:804-808

[41] Neuzil P, Taborsky M, Rezek Z, et al. Pacemaker and ICD lead extraction with electrosurgical dissection sheaths and standard transvenous extraction systems: results of a randomized trial. Europace 2007;9:98-104

[42] Dello Russo A, Biddau R, Pelargonio G, et al. Lead extraction: a new effective tool to overcome fibrous binding sites. J Interv Card Electrophysiol 2009;24:147-150

[43] Massumi RA, Ross AN. Atraumatic nonsurgical technique for removal of broken catheters from the cardiac acvities. New Engl J Med 1967;227:195

[44] Belott PH. Lead extraction using the femoral vein. Heart Rhythm 2007;4:1102-1107

[45] Fischer A, Love B, Hansalia R, et al. Transfemoral snaring and stabilization of pacemaker and defibrillator leads to maintain vascular access during lead extraction. Pacing Clin Electrophysiol 2009;32:336-339 
[46] Bongiorni MG, Soldati E, Arena G, et al. Transvenous removal of diffcult pacing and ICD leads: a new technique through the internal jugular vein. Pacing Clinc Electrophysiol 2000:23:696

[47] Henrikson CA, Brikner JA. How to prevent, recognize and manage complications of lead extraction. Part I: Avoiding lead extraction - Infectious issues. Heart Rhythm 2008;5:1083-1087

[48] Kennergren C, Bjurman C, Wiklund R, Gabel J. A single-centre experience of over one thousand lead extractions. Europace 2009;11:612-617

[49] Bongiorni MG, Soldati E, Zucchelli G, et al. Transvenous removal of pacing and implantable cardiac defibrillating leads using single sheath mechanical dilatation and multiple venous approaches: high success rate and safety in more than 2000 leads. Eur Heart J 2008;29:2886-2893

[50] Wazni O, Epstein LM, Carrillo RG, et al. Lead extraction in the contemporary setting: the LexICon Study. An observational retrospective study of consecutive laser lead extractions. J Am Coll Cardiol 2010;55:579-586

[51] de Bie M, Fouad D, Borleffs JW, et al. Trans-venous lead removal without the use of extraction sheaths, results of $>250$ removal procedures. Europace 2012; 14: 112-116

[52] Abraham WT, Fisher WG, Smith AL et al for the MIRACLE Study Group. Cardiac resynchronization in chronic heart failure. N Engl J Med 2002; 346: 1845-1853.

[53] Bristow MR, Saxon LA, Boehmer J et al. Comparison of Medical Therapy, Pacing and Defibrillation in Heart Failure (COMPANION) Investigators. Cardiac resynchronization therapy with or without an implantable defibrillator in advanced chronic heart failure. N Engl J Med 2004; 350: 2140-2150.

[54] Cazeau S, Leclercq C, Lavergne $T$ et al. Multisite Stimulation In Cardiomyopathies (MUSTIC) Study Investigators. Effects of multisite biventricular pacing in patients with heart failure and intraventricular conduction delay. N Engl J Med 2001; 344: 873-880.

[55] Clelang JG, Daubert JC, Erdmann E et al. Cardiac Resynchronization - Heart Failure (CARE-HF) Study Investigators. The effect of cardiac resynchronization on morbidity and mortality in heart failure. N Engl J Med 2005; 352: 1539-1549.

[56] Azizi M, Castel MA, Behrens S, et al. Experience with coronary sinus lead implantations for cardiac resynchronization therapy in 244 patients. Herzschr Elektrophys 2006; 17: 13-18.

[57] Purerfellner H, Nesser HJ, Winter S, et al. EASYTRAK Clinical Investigation Study Group; European EASYTRAK Registry. Transvenous left ventricular lead implantation with the EASYTRAK lead system: the European experience. Am J Cardiol 2000; 86: 157K-164K.

[58] Sack S, Heinzel F, Dagres N et al. Stimulation of the left ventricle through the coronary sinus with a newly developed 'over the wire' lead system - early experiences with lead handling and positioning. Europace 2001; 3: 317-323.

[59] Leon AR, Abraham WT, Curtis AB et al. MIRACLE Study Program. Safety of transvenous cardiac resynchronization system implantation in patients with chronic 
heart failure: combined results over 2000 patients from a multicenter study program. J Am Coll Cardiol 2005; 46: 2348-2356.

[60] Bulava A, Lukl J. Single-centre experience with coronary sinus lead stability and longterm pacing parameters. Europace 2007; 9: 523-527.

[61] Szilagyi S, Merkely B, Zima E et al. Minimal invasive coronary sinus lead reposition technique for the treatment of phrenic nerve stimulation. Europace 2008: 10: 1157-1160.

[62] Szilagyi S, Merkely B, Roka A et al. Stabilization of the coronary sinus electrode position with coronary stent implantation to prevent and treat dislocation. J Cardiovasc Electrophysiol 2007; 18: 303-307.

[63] Nagele H, Azizi M, Hashagen S, et al. First experience with a new active fixation coronary sinus lead. Europace 2007; 9: 437-441.

[64] Baranowski B, Yerkey M, Dresing T, et al. Fibrotic tissue growth into the extendable lobes of an active fixation coronary sinus lead can complicate extraction. PACE 2011; 34: e64-e65.

[65] Tacker WA, Vanvleet JF, Shoenlein WE, et al. Post mortem changes after lead extraction from the ovine coronary sinus and great cardiac vein. PACE 1998; 21: 296-298.

[66] Wilkoff BL, Belott P, Scheiner A, et al. Extractibility of coronary sinus defibrillation leads improves with ePTFE and medical adhesive coatings. PACE 2001; 24: 560

[67] Burke MC, Morton J, Lin AC, et al. Implications and outcome of permanent coronary sinus lead extraction and reimplantation. J Cardiovasc Electrophysiol 2005; 16: 830-837.

[68] Hamid S, Arujna A, Khan S et al. Extraction of chronic pacemaker and defibrillator leads from the coronary sinus: laser infrequently used but required. Europace 2009; 11: 213-215.

[69] Baranowski B, Yerkey M, Dresing T, et al. Fibrotic tissue growth into the extendable lobes of an active fixation coronary sinus lead can complicate extraction. Pacing Clin Electrophysiol 2011; 34: e64-e65.

[70] Hamid S, Arujuna A, Rinaldi CA. A shocking lead in the coronary sinus. Europace 2009; 11: 833-834.

[71] Curnis A, Bontempi L, Coppola G et al. Active-fixation coronary sinus pacing lead extraction: a hybrid approach. Int J Cardiol 2011; Sep 8 \{Epub ahead of print\}

[72] Tyers GF, Clark J, Wang Y, Mills P, Bashir J. Coronary sinus lead extraction. Pacing Clin Electrophysiol 2003; 26: 524-526.

[73] Bongiorni MG, Zucchelli G, Soldati E et al. Usefulness of mechanical transvenous dilation and location of areas of adherence in patients undergoing coronary sinus lead extraction. Europace 2007; 9: 69-73.

[74] Di Cori A, Bongiorni MG, Zucchelli G et al. Large, single-center experience in transvenous coronary sinus lead extraction: procedural outcomes and predictors for mechanical dilatation. Pacing Clin Electrophysiol 2012; 35: 215-222.

[75] Williams SE, Arujuna A, Whitaker J et al. Percutaneous lead and system extraction in patients with cardiac resynchronization therapy (CRT) devices and coronary sinus leads. Pacing Clin Electrophysiol 2011; 34: 1209-1216. 
[76] Kasravi B, Tobias S, Barnes MJ, et al. Coronary sinus lead extraction in the era of cardiac resynchronization therapy: single center experience. Pacing Clin Electrophysiol 2005; 28: 51-53.

[77] De Martino G, Orazi S, Bisignani G et al. Safety and feasibility of coronary sinus left ventricular leads extraction: a preliminary report. J Interv Card Electrophysiol 2005; 13 : 35-38. 


\title{
Emergencies of Implantable Cardioverter Defibrillator
}

\author{
Baris Bugan \\ Additional information is available at the end of the chapter
}

http://dx.doi.org/10.5772/47440

\section{Introduction}

Since the first implant in 1980, the implantable cardioverter defibrillator (ICD) has become the first line therapy for sudden cardiac death and it's technology has developed from a non-programmable device into a sophisticated multi-programmable, multi-functional device with extensive diagnostic and therapeutic options [1,2]. Programmable options of the new generation ICDs include arrhythmia detection, tachyarrhythmia therapy, pacing function, and stored intracardiac data.

An ICD consists of pulse generator, endocardial electrode, and defibrillation coils. ICD senses the rhythm and detects ventricular tachycardia (VT) and/or ventricular fibrillation $(\mathrm{VF})$ and may deliver the following antitachycardia therapies [2,3]:

1. Anti-tachycardia pacing (ATP),

2. Low energy cardioversion,

3. High energy defibrillation.

All ICDs are programmed several heart rate zones to detect tachyarrhythmia. The VF zone is programmed to a rapid heart rate, often with a heart rate of $\geq 180 \mathrm{bpm}$. Monomorphic and stable VT may allow to program other therapy zones such as ATP. ATP may allow termination of a tachyarrhythmia via pacing impulses that are faster than the underlying VT rate and prevent defibrillator shocks (Figure 1-2) [2,4].

Randomized clinical secondary prevention trials have demonstrated the effectiveness of ICD therapy for arrhythmic death and total mortality in survivors of cardiac arrest [5]. Syncope of undetermined origin with inducible ventricular tachyarrhythmias is also indication for ICD implantation. Low incidence of sudden cardiac death and high incidence of appropriate ICD therapy has been shown in patients with syncope of undetermined origin and inducible ventricular tachyarrhythmias at follow-up [6]. Prophylactic therapy in patients at risk for 
sudden cardiac death (primary prevention) was first based on the results of MADIT, MUSTT, and MADIT 11 , then later expanded upon results from SCD-HeFT [7-9]. Multiple randomized ICD trials reported that ICD benefit increases dramatically with time [10]. Indications for ICDs have expanded considerably since approval of the first ICD in 1985 [11]. Table 1 and 2 summarizes the Class I and Class II indications for ICD implantation in adults $[11,12]$. In this section we discuss ICD-releated problems including implantation of device detection and therapy of VT/VF.

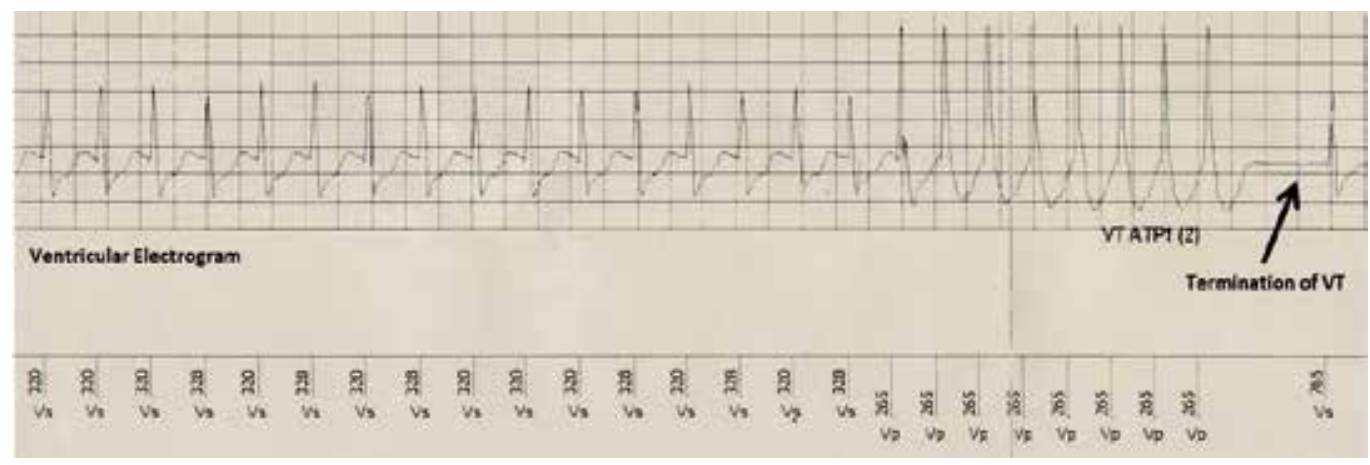

Figure 1. Stored ventricular electrogram showing a successful anti-tachycardia pacing (ATP) therapy, converting a rapid ventricular tachycardia to sinus rhythm.

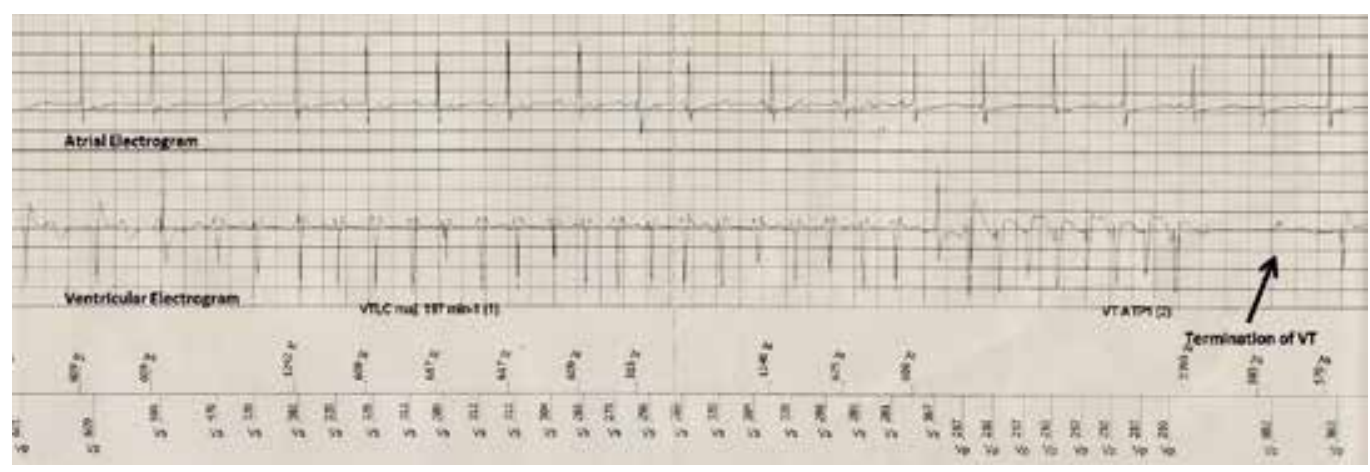

Figure 2. Stored atrial and ventricular electrogram showing a successful anti-tachycardia pacing (ATP) therapy, converting a rapid ventricular tachycardia to sinus rhythm.

\section{Class I}

1. Survivors of cardiac arrest due to VT or VF not due to a reversible cause.

2. Spontaneous sustained VT in association with structural heart disease.

3. Syncope of undetermined origin with clinically relevant, hemodynamically significant sustained VT or VF induced at EPS. 


\section{Class I}

4. Patients with LVEF less than or equal to $35 \%$ due to prior MI who are at least 40 days post-MI and are in NYHA functional Class II or III.

5. Patients with nonischemic DCM who have an LVEF less than or equal to $35 \%$ and who are in NYHA functional Class II or III.

6. Patients with LV dysfunction due to prior MI who are at least 40 days post-MI, have an LVEF less than or equal to $30 \%$, and are in NYHA functional Class I.

7. Nonsustained VT due to prior MI, LVEF less than or equal to $40 \%$, and inducible VF or sustained VT at EPS.

Abbreviations: DCM, Dilated Cardiomyopathy; EPS, Electrophysiologic Study; LVEF, Left Ventricular Ejection

Fraction; MI, Myocardial Infarction; NYHA, New York Heart Association; VF, Ventricular Fibrillation; VT, Ventricular Tachycardia.

Reproduced from Epstein AE, Dimarco JP, Ellenbogen KA et al: ACC/AHA/HRS 2008 Guidelines for device-based therapy of cardiac rhythm abnormalities. a report of the American College of Cardiology/American Heart Association task force on practice guidelines (Writing Committee to Revise the ACC/AHA/NASPE 2002 Guideline Update for Implantation of Cardiac Pacemakers and Antiarrhythmia Devices) JACC 2008;51: e1-e62.

Table 1. Indications of ICD Implantation in Adults

\section{Class IIa}

1. Unexplained syncope, significant LV dysfunction, and nonischemic DCM.

2. Sustained VT and normal or near-normal ventricular function.

3. ARVD/C who have 1 or more risk factors* for SCD.

4. HCM who have 1 or more major risk factorst for SCD.

5. Brugada syndrome who have had syncope.

6. Brugada syndrome who have documented VT that has not resulted in cardiac arrest.

7. Catecholaminergic polymorphic VT who have syncope and/or documented sustained VT while receiving beta blockers.

8. Long-QT syndrome who are experiencing syncope and/or VT while receiving beta blockers.

9. Non hospitalized patients awaiting transplantation.

10. Cardiac sarcoidosis, giant cell myocarditis, or chagas disease.

\section{Class IIb}

1. Nonischemic heart disease who have an LVEF of less than or equal to $35 \%$ and who are in NYHA functional Class I.

2. Familial cardiomyopathy associated with sudden death.

3. Long-QT syndrome and risk factors for SCD. 


\section{Class IIb}

\section{LV noncompaction.}

5. Syncope and advanced structural heart disease in whom thorough invasive and noninvasive investigations have failed to define a cause.

Abbreviations: ARVD/C, Arrhythmogenic Right Ventricular Dysplasia/Cardiomyopathy; CABG, Coronary Artery Bypass Grafting; DCM, Dilated Cardiomyopathy; EPS, Electrophysiologic Study; HCM, Hypertrophic Cardiomyopathy; LVEF, Left Ventricular Ejection Fraction; NYHA, New York Heart Association; SCD, Sudden Cardiac Death; VF, Ventricular Fibrillation; VT, Ventricular Tachycardia.

* The risk factors include induction of VT during electrophysiological testing, detection of nonsustained VT on noninvasive monitoring, male gender, severe RV dilation, and extensive RV involvement.

† The major risk factors include prior cardiac arrest, spontaneous sustained VT, spontaneous nonsustained VT, family history of SCD, syncope, LV thickness greater than or equal to $30 \mathrm{~mm}$, and an abnormal blood pressure response to exercise. Reproduced from Epstein AE, Dimarco JP, Ellenbogen KA et al: ACC/AHA/HRS 2008 Guidelines for device-based therapy of cardiac rhythm abnormalities. a report of the American College of Cardiology/American Heart Association task force on practice guidelines (Writing Committee to Revise the ACC/AHA/NASPE 2002 Guideline Update for Implantation of Cardiac Pacemakers and Antiarrhythmia Devices) JACC 2008;51: e1-e62.

Table 2. Indications of ICD Implantation in Adults

\subsection{Implantable cardioverter defibrillator related problems}

In the early years, ICD's generator was implanted in the abdomen and epicardial leads and patches were placed via thoracotomy associated with significant morbidity and mortality [2]. With the new generation of endocardial leads and significant reduction in size of the devices, the procedure can be performed under local anaesthesia. Moreover, ICD generator is implanted in the pectoral region and endocardial lead is positioned at the right ventricular apex $[2,3]$.

The ICD related problems can be classified as mechanical complications and pacing system malfunction including detection and therapy of VT/VF (Table 3). Mechanical complications are related to implantation procedure such as pneumothorax, hemothorax, subclavian artery puncture, thrombosis, pocket hematoma, infection, lead dislodgement, and myocardial perforation $[3,13,14]$.

Venous access can be administered via the cephalic, subclavian, or axillary vein for ICD implantation and pneumothorax is mostly associated with the blind puncture approach of the subclavian vein. Review of randomized clinical trials showed that the incidence of pneumothorax was low and observed approximately 0,9\% [13].

\begin{tabular}{|c|l|}
\hline Mechanical Complications & \multicolumn{1}{c|}{ Pacing system malfunction } \\
\hline Pneumothorax & $\begin{array}{l}\text { Shocks delivered by the device } \\
\text { Inappropriate ICD Shock } \\
\text { Electrical Storm }\end{array}$ \\
\cline { 1 - 1 } Hemothorax & \\
\hline Subclavian artery puncture & \\
\hline Thrombosis & Ineffective Therapy \\
\hline Pocket hematoma &
\end{tabular}




\begin{tabular}{|c|c|}
\hline Mechanical Complications & Pacing system malfunction \\
\hline Infection & Drug Effects on ICD \\
\hline $\begin{array}{c}\text { Lead dislodgement, and myocardial } \\
\text { perforation }\end{array}$ & ICD-Pacemaker Interactions \\
\hline
\end{tabular}

Table 3. Implantable cardioverter defibrillator related problems

Pocket hematoma is not directly life threatening, but it may increase the risk of infection. Pocket hematoma was observed approximately $2,2 \%$ in clinical trials $[13,15]$. Infection is a rare complication of pacemaker and ICD implantation, with the incidence ranging from $0,8 \%$ to $5,7 \%$. Clinical manifestations include skin infection, pocket infection, and endocarditis. Staphylococcus aureus and S. epidermidis are the most common organisms [3]. Endocarditis has a fearful mortality rate as high as $27 \%$ [16]. Randomized clinical trials demonstrated that infection rates decreased with routine use of antibiotic prophylaxis at the time of implantation or generator change and hence routine prophylaxis with antistaphylococcal antibiotics is recommended [3,17].

Venous thrombosis in the subclavian vein and superior vena cava was observed in $14 \%$ of pacemaker and ICD implantations in a prospective trial. Clinical manifestations vary from subclinical to superior vena cava obstruction syndrome [18]. Intracardiac thrombosis generally occurs in the right atrium around the lead. The size and location of the thrombus determine the clinical manifestation. Intravascular and intracardiac thrombosis may cause acute or recurrent pulmonary embolism. Treatment is usually initiated with intravenous heparinization and followed by oral anticoagulation [3].

Lead dislodgement with myocardial perforation is a rare complication with an incidence of $<1 \%$ and it is defined as perforation of a device lead through the myocardium. Clinical manifestations vary from asymptomatic to sudden cardiac death [14].

Pacing system malfunction can be grouped into following categories [2,3,19].

1. Shocks delivered by the device

a. Inappropriate ICD Shock

b. Electrical Storm

2. Ineffective Therapy

3. Drug Effects on ICD

4. ICD-Pacemaker Interactions

\section{Shocks delivered by the device}

Although randomized clinical trials have demonstrated the effectiveness of ICD therapy, both appropriate and inappropriate ICD shocks are painful, physically and mentally disturbing, and potentially arrhythmogenic. In addition, inappropriate shocks are associated with a higher risk of all-cause mortality [20,21]. Even appropriate ICD shocks increase mortality, mostly due to progressive heart failure [22]. Most single shocks are appropriately 
delivered to therapy of VT/VF, but multiple shocks are more often classified as inappropriate [2]. When a patient presents with an ICD shock, history, physical examination, and device interrogation should be performed. Stepwise approach for patients with ICD shock is essential and the first step is to differentiate a real tachycardia from a device recorded tachycardia originating from another source (Figure 3) [2,19].

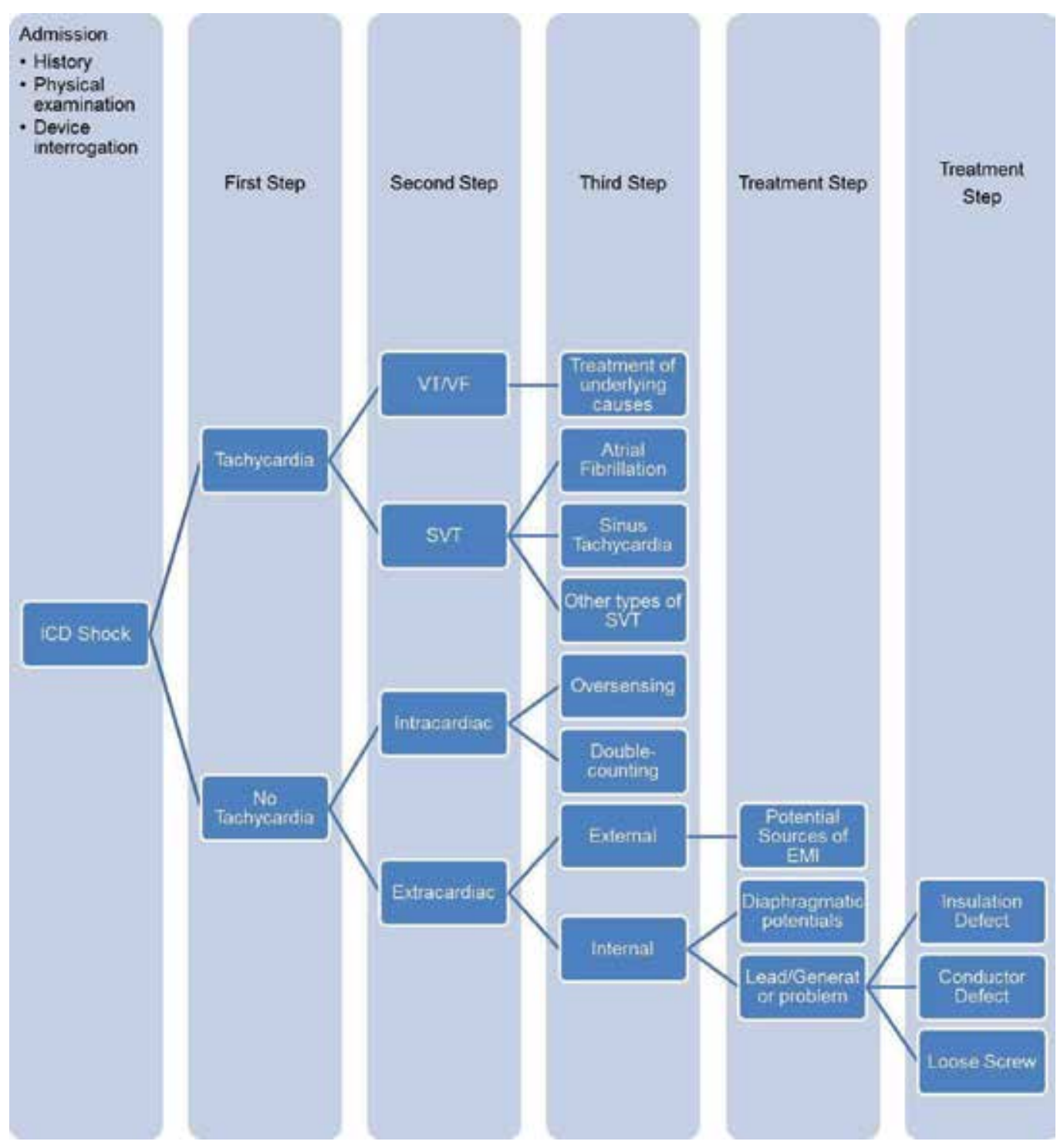

Figure 3. Stepwise Algoritm for Patients with ICD Shock. Adapted from van Erven L, Schalij MJ. Arrhythmias: Troubleshooting implantable cardioverter-defibrillator related problems. Heart 2008;94(5):649-60. Abbreviations: EMI, Electromagnetic interference; ICD, implantable cardioverter defibrillator; SVT, supraventricular tachycardia; VF, ventricular fibrillation; VT, ventricular tachycardia. 


\subsection{Inappropriate ICD shock}

Inappropriate shock is the most common adverse effect observed among ICD patients and the incidence varies from $13 \%$ to $22 \%$ [21-24]. Inappropriate shock may be caused by misdiagnosis of supraventricular tachycardia (SVT) as VT/VF or inappropriate sensing originating from internal and external sources (Table 4) [2,21]. These sensed events must occur at a rate higher than the programmed cut-off rate [2]. Atrial fibrillation (AF) is the most common cause of inappropriate shock, followed by sinus tachycardia, atrial flutter, atrial tachycardia and less commonly other types of SVT, like atrioventricular nodal reentry tachycardia [19,25,26]. History of AF, younger age, no statin use, interim appropriate shocks, a maximal heart rate during exercise close to the detection interval, and a low cut-off rate for VT-detection have been found to be independent risk factors for inappropriate shock $[21,26]$.

Various algorithms, including tachyarrhythmia stability, sudden onset, and morphology of rhythm have been developed to discriminate between SVT and ventricular arrhythmias. Sinus tachycardia is differentiated from VT based on sudden onset criterion. While sinus tachycardia increases gradually, VT has a sudden increase in ventricular rate. But this criterion might not differentiate slow VT from the sinus tachycardia due to minimal cycle length difference $[27,28]$. Stability algorithm based on regularity of VT is an effective discriminator to differantiate AF from VT; however, AF with faster ventricular rate can be regular and VT can show some irregularity. Ventricular arrhythmias have different origin and wave form, resulting in a morphology district from supraventricular rhythm. These criteria help to discriminate between SVT and ventricular arrhythmias [2]. It is important to remember that these discriminators don't work in the VF zone and hence adjusting detection intervals and therapy zones more carefully may reduce recurrent shocks. In addition, the use of medications and/or appropriate ablation procedure may prevent recurrences of SVT $[19,29]$.

\begin{tabular}{|l|l|}
\hline \multicolumn{1}{|c|}{ Tachycardia Related Causes } & \multicolumn{1}{c|}{ Non-Tachycardia Related Causes } \\
\hline Supraventricular Tachycardia & Oversensing of Intracardiac Signals \\
- Atrial Fibrillation (most common) & - Oversensingof P or T Wave \\
- Sinus Tachycardia & - Double Counting of R Wave \\
- Atrial Flutter & - Sensing of Pacemaker Artifact \\
- Atrial Tachycardia & Oversensing of Extracardiac Signals \\
- Other Types of Arrhythmia (like & - Conductor or Connector Defect \\
AVNRT) & - Myopotentials \\
& - Electromagnetic Interference \\
\hline
\end{tabular}

Abbreviations: AVNRT, Atrioventricular Nodal Reentry Tachycardia; ICD, implantable cardioverter defibrillator.

Table 4. Causes of Inappropriate ICD Shocks 
When the device interrogation doesn't reveal a real tachycardia, the device recorded signals originate from intracardiac or extracardiac sources (Table 4) $[2,30]$. Oversensing of P or T wave and double counting of $\mathrm{R}$ wave are easily diagnosed from intracardiac electrocardiograms. T-wave oversensing occurs more frequently during exercise. A high $\mathrm{T}$ wave amplitude, a low $\mathrm{R}$ wave amplitude and younger age may contribute to $\mathrm{T}$ wave oversensing as a cause of inappropriate shocks. T wave oversensing and double counting of $\mathrm{R}$ wave can be managed by reprogramming the sensitivity level of ICD $[2,30]$. Devicerelated causes of inappropriate shocks include inappropriate sensing due to lead/device malfunction or dislodgement. Conductor defect or connector problems are associated with postural changes. Impedance may be within normal limits. On the other hand, if lead impedance is high, conductor fracture should be suspected, while low lead impedance is associated with insulation defect. Insulation defect may lead to oversensing of signals and inappropriate shocks. Upper extremity isometric exercises, deep breathing may lead to diaphragmatic oversensing and cause inappropriate shocks [2,19]. Electromagnetic interference (EMI) is recognised as high frequency, low amplitude signals that refers to noise on the ventricular channel from environmental sources. Potential sources of EMI are listed in Table 5. It is believed that using household appliances, such as televisions, radios, microwaves, toasters, and electric blankets do not effect functions of ICDs. A detailed history is useful in the diagnosis of this problem [2,19,31 ].

\begin{tabular}{|l|l|}
\hline \multicolumn{1}{|c|}{ Non-Medical Sources } & \multicolumn{1}{c|}{ Medical Sources } \\
\hline - Electronic Article Surveillance Devices & - Transthoracic Cardioversion \\
- Cellular Phones & - Magnetic Resonance Imaging \\
- Metal Detector Gates & - Radiation Therapy \\
- Arc Welding & - Electrocautery \\
& - Lithotripsy \\
\hline
\end{tabular}

Table 5. Sources of Electromagnetic Interference

\subsection{Electrical storm}

Electrical storm refers to the occurrence of three or more episodes of VT or VF in a 24-hour period and it is associated with episodes of ATP (Figure 4) and/or multiple shocks (Figure 5) [3,32]. A patient with electrical storm should be immediately hospitalized and monitorized to determine the appropriateness of the shocks. Intravenous amiodarone is the first line therapy and the first step is to evaluate reversible causes of VT/VF such as electrolyte abnormality and ischemia. All clinicians should keep in mind that proarrhythmic effects of antiarrhythmic drugs may also lead to electrical storm. Intravenous $\beta$-blockers to suppress adrenergic stimulation, intubation and sedation, anti-ischemic therapy, intra-aortic balloon pump and ventricular assist devices for hemodynamic support have been used to suppress the ventricular arrhythmias. The ICD can be interrogated to ascertain programmed parameters to attempt pace termination of VT. Catheter ablation of VT/VF may be used as a last treatment way in selected patients $[3,32,33]$. 


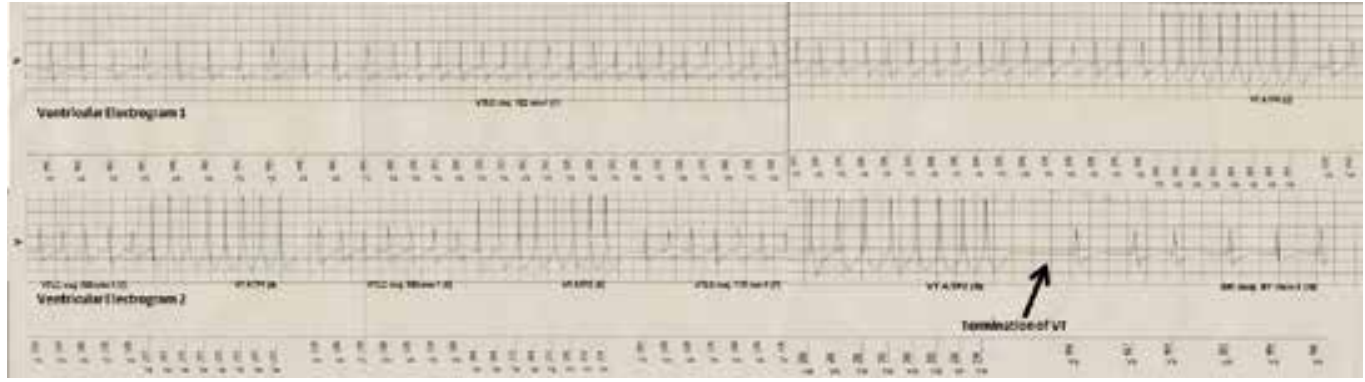

Figure 4. Continuous intracardiac electrogram recordings from interrogation of an ICD in a 56-year-old man with sustained VT. Electrical storm terminates with four episodes of ATP therapy.

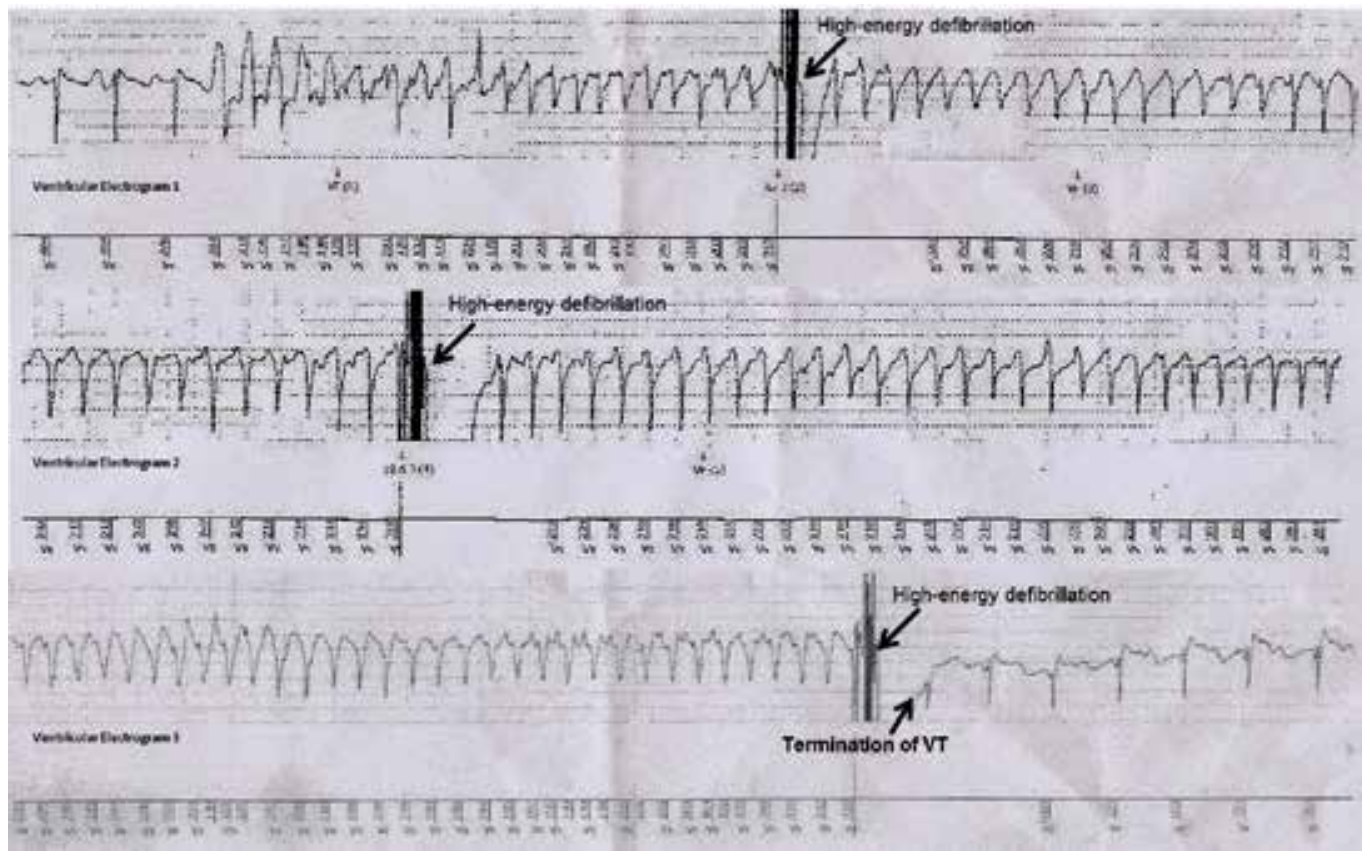

Figure 5. Continuous intracardiac electrogram recordings from interrogation of an ICD in a 62-year-old man with sustained VT. Electrical storm terminates with three episodes of high-energy defibrillation. 


\section{Ineffective therapy}

Ineffective therapy refers to delayed or absent ICD therapy delivery during VT/VF and it can be lethal $[2,3]$. Undersensing and lack of detection of VT/VF, and device related problems are the main causes of ineffective ICD therapy. Lead malfunction or displacement, generator malfunction, exposure to EMI, use of antiarrhythmic drug, and pacemaker-ICD interaction may cause undersensing of VT/VF. High cut-off rate for detection, battery depletion with prolonged charge time, exposure to EMI, and slower VT may obstruct the detection of VT/VF [3]. Consequently, device failure have resulted in unnecessary deaths. Although these deaths may be infrequent, patients with an ICD should be evaluated to assess the battery status, charge time, lead integrity and function, and underlying rhythm every three months and after each exposure to EMI [3,34]. Alternatively, if remote monitoring is used, the office follow-up may be spaced out according to the recent HRS/EHRA guideline [35].

\section{Drug effects on ICD}

The primary indications to initiate antiarrhythmic agents in patients with ICD are suppression of the burden of the arrhythmias. Antiarrhythmic drug use has been reported to range from $49 \%$ to $69 \%$ in various trials [36]. Adding concomitant therapy with antiarrhythmic drugs to ICD patients results in following beneficial effects [3,36,37]:

1. Reduction in frequency of ICD therapy by decreasing the episodes of VT/VF or making them nonsustained,

2. Reduction of VT rate to allow ATP therapy,

3. Reduction in the frequency of recurrence of SVT to prevent inappropriate shocks,

4. Reduction in the defibrillation threshold (DFT),

5. Improvement in quality of life.

Although evidence supports above beneficial effects, the potential deleterious effects of antiarrhythmic drugs on ICD function include [3,36,37]:

1. Increased ICD discharge due to proarrhythmias or making them sustained

2. Slowing of the VT rate below cut-off detection rate for VT

3. Changing the QRS morphology that causes sensing alteration

4. Elevation of the DFT.

5. Elevation of the pacing threshold

6. Heart failure exacerbation

Drug effects on the pacing threshold vary with the class of drug and there is little or no effect on pacing threshold except class IC agents, especially flecainide [38]. The most potentially dangerous effect of drugs on ICD function is an increase in DFT that can lead to ineffective ICD therapy $[3,36]$. Generally, the drugs that block fast inward sodium channel and shorten the action potential duration can cause an increase in DFT, while potassium channel blocking drugs that prolong the action potential duration (e.g., sotalol) tend to decrease DFTs and these drugs are favorable choices in patients with high DFTs [3]. Class IA 
drugs do not seem to have significant effects on the DFT whereas class IB drugs that block fast inward sodium channel (lidocaine and mexiletine) have consistently shown elevation in DFT $[3,36,39]$. The effect of class IC agents on the DFT is less clear. As opposed to the class I agents, class III agents tend no effect or to lower the DFT except amiodarone. Intravenous amiodarone had no effect on DFT, while oral amiodarone was associated with an increase in DFT in animal and human studies and hence DFT should be re-evaluated whenever antiarrhythmic agents especially amiodarone is administered [3,36].

\section{ICD-pacemaker interactions}

ICD-Pacemaker interactions involve only patients with separate PM and ICD systems implanted, which is a very rare scenario nowadays. The potential adverse interaction between ICD and pacemaker include [3,40]:

1. ICD effect on pacemaker function,

2. Pacemaker effect on ICD function.

Defibrillation shocks can lead to transient failure of pacemaker sensing and pacing because of exposure of the myocardium to high current density [40]. After an ICD shock, pacemaker function needs to be evaluated as reprogramming or damage to the PM system can ocur. Bipolar pacing systems may be less sensitive to this issue. Another potential adverse effect is pacemaker reprogramming during ICD interrogation; this can be prevented by keeping adequate spatial separation between the pacemaker and ICD generators. In patients with ICDs and separate pacemakers, the pacing stimulation can cause ICD oversensing or undersensing and hence result in inappropriate therapy or failure to deliver therapy during VT/VF [3,40]. In addition, the electrical artifact between the pacemaker and the ICD lead can be oversensed by the ICD and cause inappropriate shocks [3].

\section{Conclusion}

Evolution of ICD technology allows accurate detection and therapy of arrhythmias, while also reducing unnecessary shocks and ineffective therapy. However, patients with ICDrelated problems are increasingly encountered worldwide due the growing number of implantations. Regular outpatient follow-up is crucial to find out these problems before occurence of clinical manifestations.

Consequently, clinicians, who may not be electrophysiologists, should use stepwise approach (Figure 3), gathered from the patient's history, physical examination, and device interrogation as most problems can be solved by simple ICD reprogramming and/or a change in medical therapy.

\section{Author details}

Baris Bugan

Malatya Military Hospital, Cardiology Service, Malatya, Turkey 


\section{References}

[1] Mirowski M, Reid PR, Mower MM et al. (1980) Termination of malignant ventricular arrhythmias with an implanted automatic defibrillator in human beings. The New England journal of medicine. 303:322-4.

[2] van Erven L, Schalij MJ. (2008) Troubleshooting implantable cardioverter-defibrillator related problems. Heart. 94:649-60.

[3] Banker R, Mitchell R, Badhwar N, Goldschlager N. (2010) Pacemaker and Implantable Cardioverter-Defibrillator Emergencies. In: Jeremias A, Brown DL, editors. Cardiac Intensive Care. 2 ed, Saunders: Elsevier. pp 310-338.

[4] Wathen M. (2007) Implantable cardioverter defibrillator shock reduction using new antitachycardia pacing therapies. American heart journal. 153:44-52.

[5] Connolly SJ, Hallstrom AP, Cappato R et al. (2000) Meta-analysis of the implantable cardioverter defibrillator secondary prevention trials. AVID, CASH and CIDS studies. Antiarrhythmics vs Implantable Defibrillator study. Cardiac Arrest Study Hamburg . Canadian Implantable Defibrillator Study. European heart journal. 21:2071-8.

[6] Link MS, Costeas XF, Griffith JL, Colburn CD, Estes NA, Wang PJ. (1997) High incidence of appropriate implantable cardioverter-defibrillator therapy in patients with syncope of unknown etiology and inducible ventricular arrhythmias. Journal of the American College of Cardiology. 29:370-5.

[7] Moss AJ, Hall WJ, Cannom DS et al. (1996) Improved survival with an implanted defibrillator in patients with coronary disease at high risk for ventricular arrhythmia. Multicenter Automatic Defibrillator Implantation Trial Investigators. The New England journal of medicine. 335:1933-40.

[8] Buxton AE, Lee KL, Fisher JD, Josephson ME, Prystowsky EN, Hafley G. (1999) A randomized study of the prevention of sudden death in patients with coronary artery disease. Multicenter Unsustained Tachycardia Trial Investigators. The New England journal of medicine. 341:1882-90.

[9] Moss AJ, Zareba W, Hall WJ et al (2002). Prophylactic implantation of a defibrillator in patients with myocardial infarction and reduced ejection fraction. The New England journal of medicine. 346:877-83.

[10] Gasparini M, Nisam S. (2012) Implantable cardioverter defibrillator harm? Europace : European pacing, arrhythmias, and cardiac electrophysiology : journal of the working groups on cardiac pacing, arrhythmias, and cardiac cellular electrophysiology of the European Society of Cardiology. Epub ahead of print.

[11] Gregoratos G, Abrams J, Epstein AE et al. (2002) ACC/AHA/NASPE 2002 guideline update for implantation of cardiac pacemakers and antiarrhythmia devices: summary article: a report of the American College of Cardiology/American Heart Association Task Force on Practice Guidelines (ACC/AHA/NASPE Committee to Update the 1998 Pacemaker Guidelines). Circulation. 106:2145-61.

[12] Epstein AE, Dimarco JP, Ellenbogen KA et al. (2008) ACC/AHA/HRS 2008 Guidelines for device-based therapy of cardiac rhythm abnormalities. a report of the American College of Cardiology/American Heart Association task force on practice guidelines (Writing Committee to Revise the ACC/AHA/NASPE 2002 Guideline Update for 
Implantation of Cardiac Pacemakers and Antiarrhythmia Devices) Journal of the American College of Cardiology. 51: e1-e62.

[13] van Rees JB, de Bie MK, Thijssen J, Borleffs CJ, Schalij MJ, van Erven L. (2011) Implantation-related complications of implantable cardioverter-defibrillators and cardiac resynchronization therapy devices: a systematic review of randomized clinical trials. Journal of the American College of Cardiology. 58:995-1000.

[14] Celik T, Kose S, Bugan B, Iyisoy A, Akgun V, Cingoz F. (2009) Hiccup as a result of late lead perforation: report of two cases and review of the literature. Europace : European pacing, arrhythmias, and cardiac electrophysiology : journal of the working groups on cardiac pacing, arrhythmias, and cardiac cellular electrophysiology of the European Society of Cardiology. 11:963-5.

[15] Klug D, Balde M, Pavin D et al. (2007) Risk factors related to infections of implanted pacemakers and cardioverter-defibrillators: results of a large prospective study. Circulation. 116:1349-55.

[16] Klug D, Lacroix D, Savoye C et al. (1997) Systemic infection related to endocarditis on pacemaker leads: clinical presentation and management. Circulation. 95:2098-107.

[17] Da Costa A, Kirkorian G, Cucherat M et al. (1998) Antibiotic prophylaxis for permanent pacemaker implantation: a meta-analysis. Circulation. 97:1796-801.

[18] Spittell PC, Vlietstra RE, Hayes DL, Higano ST. (1990) Venous obstruction due to permanent transvenous pacemaker electrodes: treatment with percutaneous transluminal balloon venoplasty. Pacing and clinical electrophysiology : PACE. 13:271-4.

[19] Saeed M. (2011) Troubleshooting implantable cardioverter-defibrillators: an overview for physicians who are not electrophysiologists. Texas Heart Institute journal / from the Texas Heart Institute of St Luke's Episcopal Hospital, Texas Children's Hospital. 38:355-7.

[20] Irvine J, Dorian P, Baker B et al. (2002) Quality of life in the Canadian Implantable Defibrillator Study (CIDS). American heart journal. 144:282-9.

[21] van Rees JB, Borleffs CJ, de Bie MK et al. (2011) Inappropriate implantable cardioverterdefibrillator shocks: incidence, predictors, and impact on mortality. Journal of the American College of Cardiology. 57:556-62.

[22] Mishkin JD, Saxonhouse SJ, Woo GW et al. (2009) Appropriate evaluation and treatment of heart failure patients after implantable cardioverter-defibrillator discharge: time to go beyond the initial shock. Journal of the American College of Cardiology. 54(22):1993-2000.

[23] Grimm W, Flores BT, Marchlinski FE. (1993) Shock occurrence and survival in 241 patients with implantable cardioverter-defibrillator therapy. Circulation. 87:1880-8.

[24] Nunain SO, Roelke M, Trouton T et al. (1995) Limitations and late complications of third-generation automatic cardioverter-defibrillators. Circulation. 91:2204-13.

[25] Jodko L, Kornacewicz-Jach Z, Kazmierczak J et al. (2009) Inappropriate cardioverterdefibrillator discharge continues to be a major problem in clinical practice. Cardiology journal. 16:432-9.

[26] Weber M, Block M, Brunn J et al. (1996) [Inadequate therapies with implantable cardioverter-defibrillators--incidence, etiology, predictive factors and preventive strategies]. Zeitschrift fur Kardiologie. 85:809-19. 
[27] Klein GJ, Gillberg JM, Tang A et al. (2006) Improving SVT discrimination in singlechamber ICDs: a new electrogram morphology-based algorithm. Journal of cardiovascular electrophysiology. 17:1310-9.

[28] Schaumann A, von zur Muhlen F, Gonska BD, Kreuzer H. (1996) Enhanced detection criteria in implantable cardioverter-defibrillators to avoid inappropriate therapy. The American journal of cardiology. 78:42-50.

[29] Ferreira-Gonzalez I, Dos-Subira L, Guyatt GH. (2007) Adjunctive antiarrhythmic drug therapy in patients with implantable cardioverter defibrillators: a systematic review. European heart journal. 28:469-77.

[30] Rauwolf T, Guenther M, Hass N et al. (2007) Ventricular oversensing in 518 patients with implanted cardiac defibrillators: incidence, complications, and solutions. Europace: European pacing, arrhythmias, and cardiac electrophysiology : journal of the working groups on cardiac pacing, arrhythmias, and cardiac cellular electrophysiology of the European Society of Cardiology. 9:1041-7.

[31] Kolb C, Zrenner B, Schmitt C. (2001) Incidence of electromagnetic interference in implantable cardioverter defibrillators. Pacing and clinical electrophysiology : PACE. 24:465-8.

[32] Eifling M, Razavi M, Massumi A. (2011) The evaluation and management of electrical storm. Texas Heart Institute journal / from the Texas Heart Institute of St Luke's Episcopal Hospital, Texas Children's Hospital. 38:111-21.

[33] Nademanee K, Taylor R, Bailey WE, Rieders DE, Kosar EM. (2000) Treating electrical storm : sympathetic blockade versus advanced cardiac life support-guided therapy. Circulation. 102:742-7.

[34] Hauser RG, Kallinen L. (2004) Deaths associated with implantable cardioverter defibrillator failure and deactivation reported in the United States Food and Drug Administration Manufacturer and User Facility Device Experience Database. Heart rhythm : the official journal of the Heart Rhythm Society. 1:399-405.

[35] Wilkoff BL, Auricchio A, Brugada J et al. (2008) HRS/EHRA expert consensus on the monitoring of cardiovascular implantable electronic devices (CIEDs): description of techniques, indications, personnel, frequency and ethical considerations. Heart Rhythm. 5(6):907-25.

[36] Page RL. (2000) Effects of antiarrhythmic medication on implantable cardioverterdefibrillator function. The American journal of cardiology. 85:1481-5.

[37] Goldschlager N, Epstein A, Friedman P, Gang E, Krol R, Olshansky B. (2001) Environmental and drug effects on patients with pacemakers and implantable cardioverter/defibrillators: a practical guide to patient treatment. Archives of internal medicine. 161:649-55.

[38] Greene HL. (1996) Interactions between pharmacologic and nonpharmacologic antiarrhythmic therapy. The American journal of cardiology. 78:61-6.

[39] Echt DS, Gremillion ST, Lee JT et al. (1994) Effects of procainamide and lidocaine on defibrillation energy requirements in patients receiving implantable cardioverter defibrillator devices. Journal of cardiovascular electrophysiology. 5:752-60.

[40] Brode SE, Schwartzman D, Callans DJ, Gottlieb CD, Marchlinski FE. (1997) ICDantiarrhythmic drug and ICD-pacemaker interactions. Journal of cardiovascular electrophysiology. 8:830-42. 


\section{Frontiers of Pacemaker Technology}





\title{
Nonlinear Cardiac Dynamics
}

\author{
Charles L. Webber, Jr. \\ Additional information is available at the end of the chapter
}

http://dx.doi.org/10.5772/48753

\section{Introduction}

\subsection{The pulse as window into the heart}

The beating heart has attracted attention since antiquity and probably before recorded history [1]. Some cultures have centered the human soul within the heart because of its vivacious and incessant actions. Careful reading of the Hebraic Psalms, for example, attributes thinking, feeling and soulish behaviors to this remarkable organ. Consider King David's anguish: "I am benumbed and badly crushed; / I groan because of the agitation of my heart. / Lord, all my desire is before You;/ And my sighing is not hidden from You. / My heart throbs, my strength fails me; / And the light of my eyes, even that has gone from me" [2]. Since time immemorial the cardiac pulse has been viewed as a window into the heart [1].

To the modern observer, the vigorous motions of the heart are a strong reminder that living physiological systems are dynamical in nature. Over the last century the dynamics of the heart have been refined and redefined by analogy to naturally rhythmical systems of nature extending to mathematical modeling and simulations. Indeed, the whole concept of homeostasis, a foundational so-called law of physiology, is even under revision. If homeostasis implies that a system is static with no motion, maybe the prime exemplar of homeostasis is the cadaver state! Possibly a simplistic but meaningful redefinition of physiology is "If it wiggles, it's physiology; if it stops wiggling it's anatomy" [3]. In this sense and beyond homeostasis, homeodynamics is being promoted as a better descriptor of living systems [4]. In terms of the heart, there are a variety of pressures fluctuations and heart rates that are permitted and even necessitated by the extant dynamical state (sleeping, sitting, running, etc.).

\section{The sinusoidal cardiac pulse}

The ubiquitous sine wave holds a unique position in the history of linear motions from seawave patterns to planetary revolutions around the solar mass. As shown in Figure 1, the sine wave may be considered a good first approximation to the waxing and waning of 
ventricular and aortic pressure traces, both of which are attributed to alternations between ventricular muscle contractions (active phase) and relaxations (quiescent phase). The heart beats through the lifespan of the individual, but this does not infer that the heart gets no rest. It is good to remember that the "wake/sleep" cycle of this automatic organ is measured in terms of milliseconds, not hours or days. Rest in this context means that the myocardium is electrically silent, but the heart still continues to consume energy throughout both systolic and diastolic phases.

On closer inspection, however, the mechanical motions of the heart are highly complex and ventricular pressure waves and arterial pressure pulses are not well-fit by the simple sine wave. A single sine wave has a characteristic amplitude and period that repeats forever (infinite series), and knowing the details of one wave allows one to accurately extrapolate into the future with mathematical confidence. But the heart is not like this. Each beat and each period of the heart is different, unique to the moment and suited for the current environmental challenge. Besides this, the heart is a noisy system (noisy dynamic) simply because it resides within a noisy world. There is dynamical noise arising from within the organ itself, the heart is jostled by breathing and stepping motions, neuronal reflexes are constantly modulating cardiac function, and external noise from the environment shape and

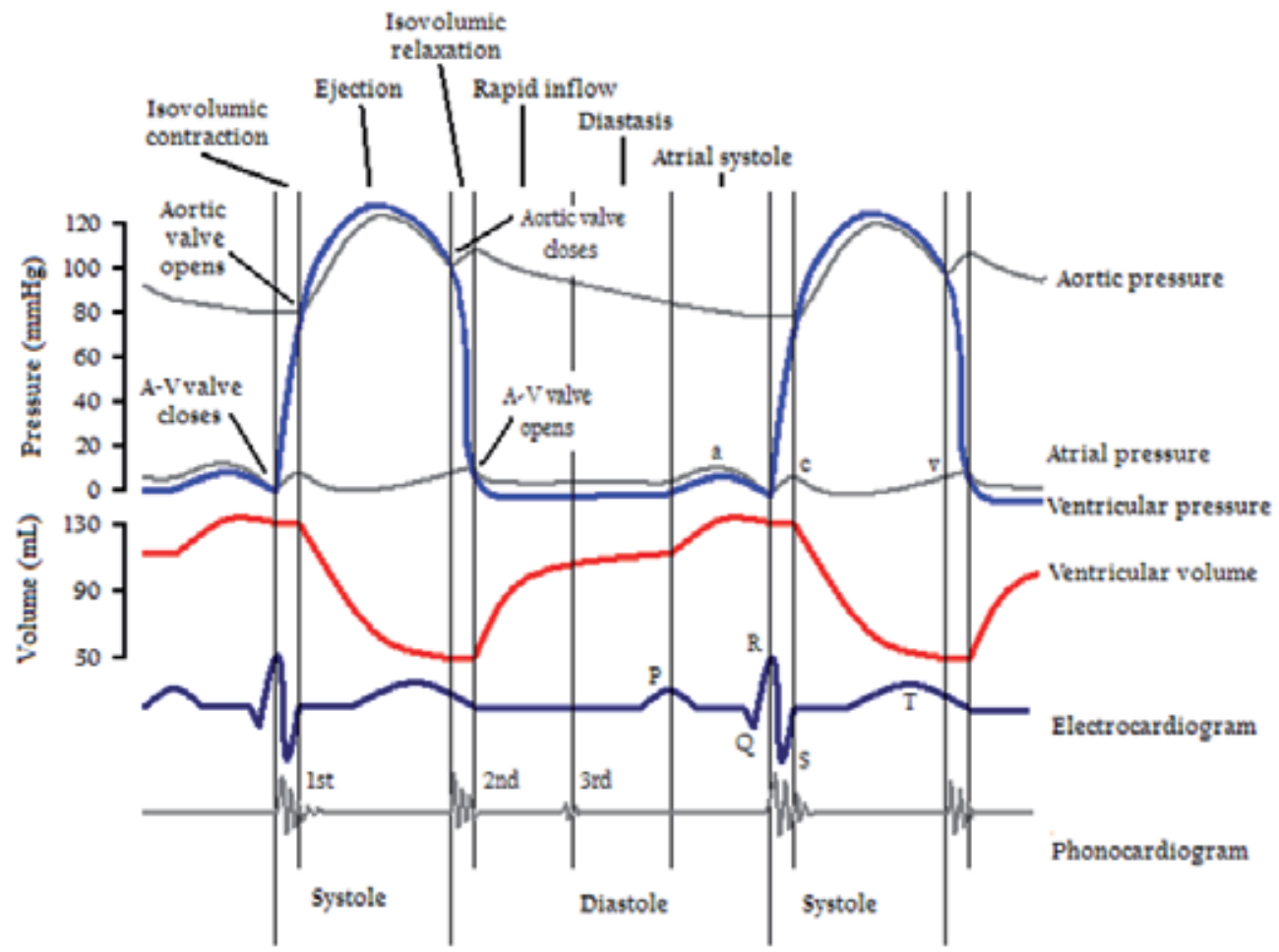

Figure 1. The famous Wiggers diagram displaying the time variations in cardiac electrical and mechanical functions as recorded by a polygraph. Note that the shape of the ventricular and aortic pressure traces can, on first pass, be mimicked by a simple sine wave. (from Wikepedia: http://en.wikipedia.org/wiki/Wiggers_diagram) 
mold the activity of the heart. Thus, not only is the heart dynamic in nature, it is also very flexible, perfectly suited to the world in which we live.

\section{Electrical underpinnings of the cardiac pulse}

Cardiac dynamics go way beyond mechanical pressure generation (which can be sensed by arterial vessel palpitation), but also depends upon electrical activities inherent within the myocardium (which are insensible, save by electronic amplification and display). The heart remains mechanically silent until it is electrically activated. But activation depends upon electro-mechanical coupling which is calcium dependent in cardiac myocytes. The physiological principle (cause $\rightarrow$ effect) as shown in Figure 2 is this: electrical activity (phase $0) \rightarrow$ calcium induced calcium release (CICR of phase 2) $\rightarrow$ mechanical contraction of atrial and ventricular muscle cells [5]. In one study, the median left ventricular electromechanical delay (EMD) of 103 control subjects was $17 \mathrm{~ms}$, with calcium release preceding mechanical force generation [6]. The summation of electrical depolarizations of ventricular action potentials (phase 0 in Fig. 2) are registered as the QRS waves in the ECG (electrocardiogram in Fig 1). Likewise, the summation of electrical repolarizations of ventricular action (Phase 3 in Fig. 2) are registered as $T$ waves in the ECG (electrocardiogram in Fig 1). Note that because of the long phase 2 plateau of the ventricular action potential, there is an isopotential phase between the $\mathrm{Q}$ and $\mathrm{T}$ waves, the QT interval.

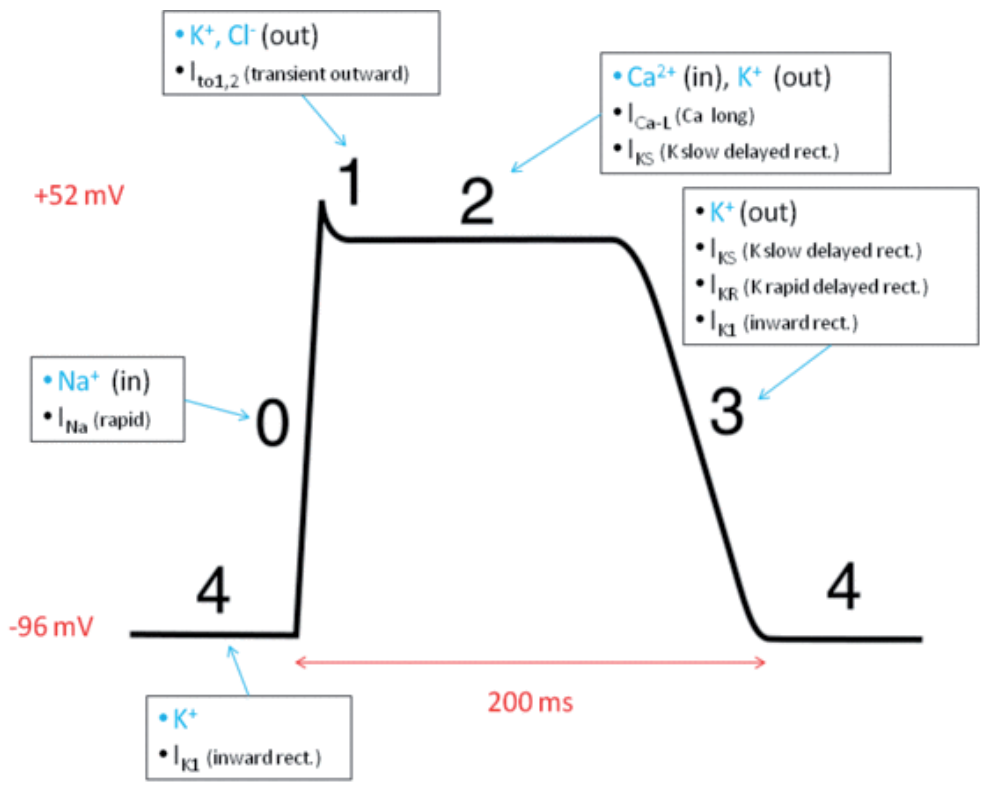

Figure 2. The five phases of the ventricular muscle action potential. (from Wikepedia: http://en.wikipedia.org/wiki/Cardiac_action_potential)

What all this means is that there are different levels or different dimensions of cardiac rhythmicity. One could study cardiac mechanics and blood pressures, one could examine electrocardiological signals of the whole organ or isolated cardiac myocytes, or one might 
delve into the dynamics of calcium fluxes within single heart cells. Dynamical details abound for the clever investigator to mine, interpret and hopefully apply to cardiac patients with abnormal dynamics for a myriad of different, sometimes subtle, reasons. But before one can discuss abnormalities in cardiac functioning, normal rhythms, beatings and cyclings of the heart must first be comprehended as best as possible. What is normal in this respect must cover a wide territory or legal (healthy) dynamical possibilities (homeodynamics). Because so many demands are placed on the heart, the normal heart must be flexible enough to survive environmental challenges and heavy workloads. It is.

\section{The rate of the heart}

Starting off most simply, cardiac dynamics can be captured in the variable heart rate. As an aside, some reports in the literature erroneously label as adjustable fixed parameters those observables which are in reality fluctuating physiological variables [7]. Heart rate can be detected manually by palpitation of the radial systolic pulse or automatically measured from an intravascular catheter (beats per minute). Heart rate of a different flavor can be recorded from surface electrocardiograms or cardiac muscle electrograms (electrical pulses per minute). These two heart rates are similar but not identical. Notably, in some diseased hearts it is possible for the developed ventricular pressure to be so weak that cardiac ejections occur only on every other contraction cycle. In this case the radial pulse rate would be half that of the ECG rate. Since artificial pacemakers take their cues not from hemodynamic feedbacks, but from non-physiological indicators, their clinical effectiveness can be compromised in certain situations.

However measured, the fundamental problem with heart rate is that it represents an average over several beats in which case beat-to-beat periods (instantaneous rates) are not identical. Here the variability of the dynamic can be captured in the standard deviation. Still, this is not a fool-proof definition of variability since these deviations from the mean may not be normally distributed (non-Gaussian). To the extent that these physiology-math mismatches are real, attempting to capture natural variability in terms of homestatic and Gaussian statistics is a huge error both conceptually and practically [8]. Means and standard deviations are linear descriptors fully appropriate for linear systems. But what if, in fact, cardiac dynamics are actually non-linear? The truth is that it is now widely recognized that the functions and fluctuations of the heart are highly nonlinear [9]. Might it be concluded that as the sine wave is to the arterial pressure pulse, so are the mean and standard deviation to cardiac dynamics?

As second point to grasp is that taking the mean filters out and minimizes (morphs, contorts or even destroys) dynamical details. Worse yet, taking means create fictive realities that do not exist in nature. For example, it is well known that the heart accelerates as one starts exercising. And even when the heart rate levels off at some plateau correlated to the work load of the exercise regime, it is proper to stay the system is in some kind of "steady-state?" What does steady-state really mean anyway? Again, in the spirit of homeodynamics [4], maybe such states are better comprehended as quasi-steady states or simply dynamic states 
within a corralled boarder consistent with the status of the individual. And over time, the dynamic state can move in time and space either by following deterministic rules within a narrow regime or by allowing for some stochastic expansion of the perimeter border in which multiple motions are permitted and encouraged.

\section{The cardiac period}

If the cardiomyocyte is the functional unit of the myocardium, the cardiac period is the fundamental time signature of the heart cycle. This cardiac period can be measured in different ways and yielding different values, yet time intervals are superior to rates since no ratios are encountered. The absolute interval (neither relative nor ratio) could span the time from one systolic beat to another between aortic pressure pulses, or it could be the time lapse from one R wave to the next in the ECG (see Fig. 1). RR intervals must be understood as discrete events weather or not they form random point processes or are connected entities with deterministic structures. Quantitative analyses can distinguish the two possibilities, but the discrete RR interval remains the fundamental marker of cardiac timings.

The second marker of cardiac timing represents the smooth flow of events occurring between beats. In this case (see Fig. 1) the variable could be the continuous ventricular or aortic pressure trace, the simple ECG presented as a time series, or even a cardiac electrogram measured with electrodes embedded in the heart. In either case, mechanical or electrical, the variable of interest is a single continuous function, one that is not discretized into intervals (e.g. analogous to a sine wave). Many models of cardiac function, mechanical or electrical, can take the form of continuous differential equations that are parameterized to captures the contours of the wave being mimicked. High digitization rates are, of course, required to faithfully capture accurate waveforms.

Sequences of discrete cardiac intervals and continuous heart variables can be studied in the time domain (functions of time) as displayed on polygraph recordings. However, another perspective on discrete and continuous events is to transpose the signals into the frequency domain. For example, if one computed the frequency spectra of the ECG, the resultant spectrum would consist of the power of the signal at different frequency bands (not shown). One of the most useful techniques, however, has been computing the frequency spectra of sequenced cardiac periods using the Fast Fourier transform [10]. Three typical peaks emerge from such an approach as shown in Figure 3. High frequency oscillations (HFO. 0.15-0.40 $\mathrm{Hz}$ ) are typically attributed to ventilation effects on the cardiac cycle due to the waxing (inspiration) and waning (expiration) of vagal efferent inputs to the sinoatrial node. This is known as the classic respiratory sinus arrhythmia (SA) [12]. The HFO peak (hatched area) near $0.3 \mathrm{~Hz}$ represents a breathing frequency of 18 breaths per minute. Low frequency oscillations (LFO, 0.04-0.15 Hz) are associated with sympathetic activity with some parasympathetic contamination as it were. Nevertheless, the LFO/HFO ratio is commonly computed as a measure of autonomic imbalance with high ratios favoring sympathetic tone and low ratios favoring parasympathetic tone. Lastly, the origination of very low frequency oscillations (VLFO, 0.01-0.04 Hz) is very controversial but may be related to heart period 
changes driven by the very slow oscillation in the renin-angiotensin-aldosterone system or thermoregulation [13]. One major clinical investigation has produced standards of RR spectral measurements, physiological interpretation, and clinical utilization [14].

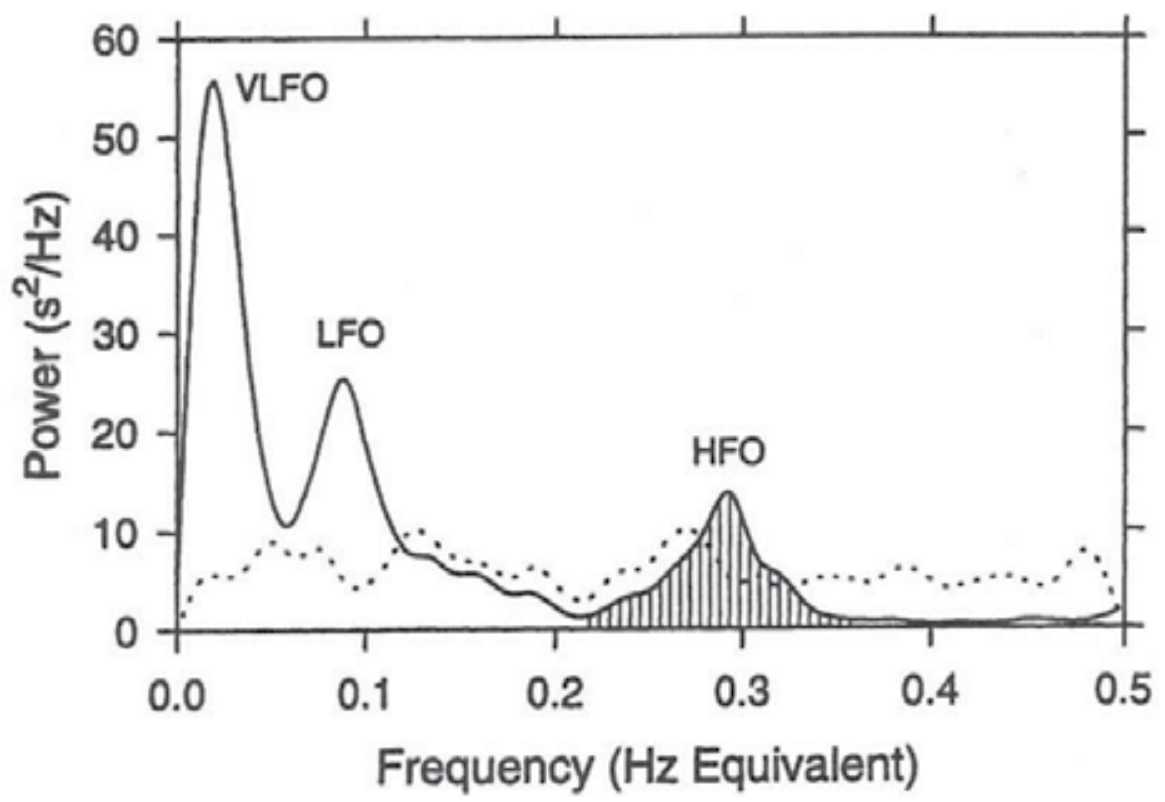

Figure 3. Power spectrum of human PP intervals before (solid line) and after random shuffling (dotted line) of intervals. Reproduced from reference [11] with permission.

The spectral analysis of RR intervals is commonly known as heart rate variability (HRV). The name is kind of a misnomer, because the spectral analyses are performed on heart periods not heart rates (a ratio). Nevertheless, all such computations are classified as linear and one dimensional. That is, the Fourier spectrum consists of the linear sums of sine and cosine waves which reconstruct the time domain signals (even square waves can be approximated with an infinite number of summations of wide ranging frequencies). With the growing appreciation that physiological signals are, among other things, highly nonlinear, another popular methodology has come to the forefront, approximate entropy (ApEn) [15]. This technique uses RR intervals as the input and treats then as chains of information. Low values of ApEn indicate that the cardiac signals have a deterministic structuring, whereas high ApEn values signal that the signals are more random or stochastic. There are technical problems associate with ApEn measures of complexity [3], but the utility of the approach cannot be denied.

\section{The cardiac period in recurrence space}

Physiological systems, including the cardiovascular system, are not only nonlinear, but they can be nonstationary, high-dimensional, and noisy. Such "miss-behaved" dynamics are characteristic of real-world systems. To tame the signals rendering them suitable for 
classical analyses often requires filtering, smoothing, clipping of outliers, detrending, interval replacements, etcetera, all of which contort the original signals. For this reason the first introduction of recurrence plots in the physics literature [16] became highly attractive to physiologists $[17,18]$. Recurrence plot can be generated from any time-varying (or spacevarying) signals consisting of either discrete intervals or continuous flows. The attraction of recurrence plots is that they are distribution-free, model-free, and have utility in dissecting out meaningful information from signals living on a dynamical transient and buffeted by noise. Higher dimensions are captured in surrogate variables by employing the principles of the embedding theorem of Takens [19]. What this means is that the windowed signal is divided up into short vectors of length EMBED and compared with each other exhaustively. If two vectors "match" by falling beneath a threshold radius value, then the leading points of the paired vectors are said to be recurrent. To render the data visible, a point is placed at the intersection of each and every vector $i$ and vector $j$ (e.g. for $i=1$ to 500 and $j=1$ to 500 ) falling within the allowed radius.
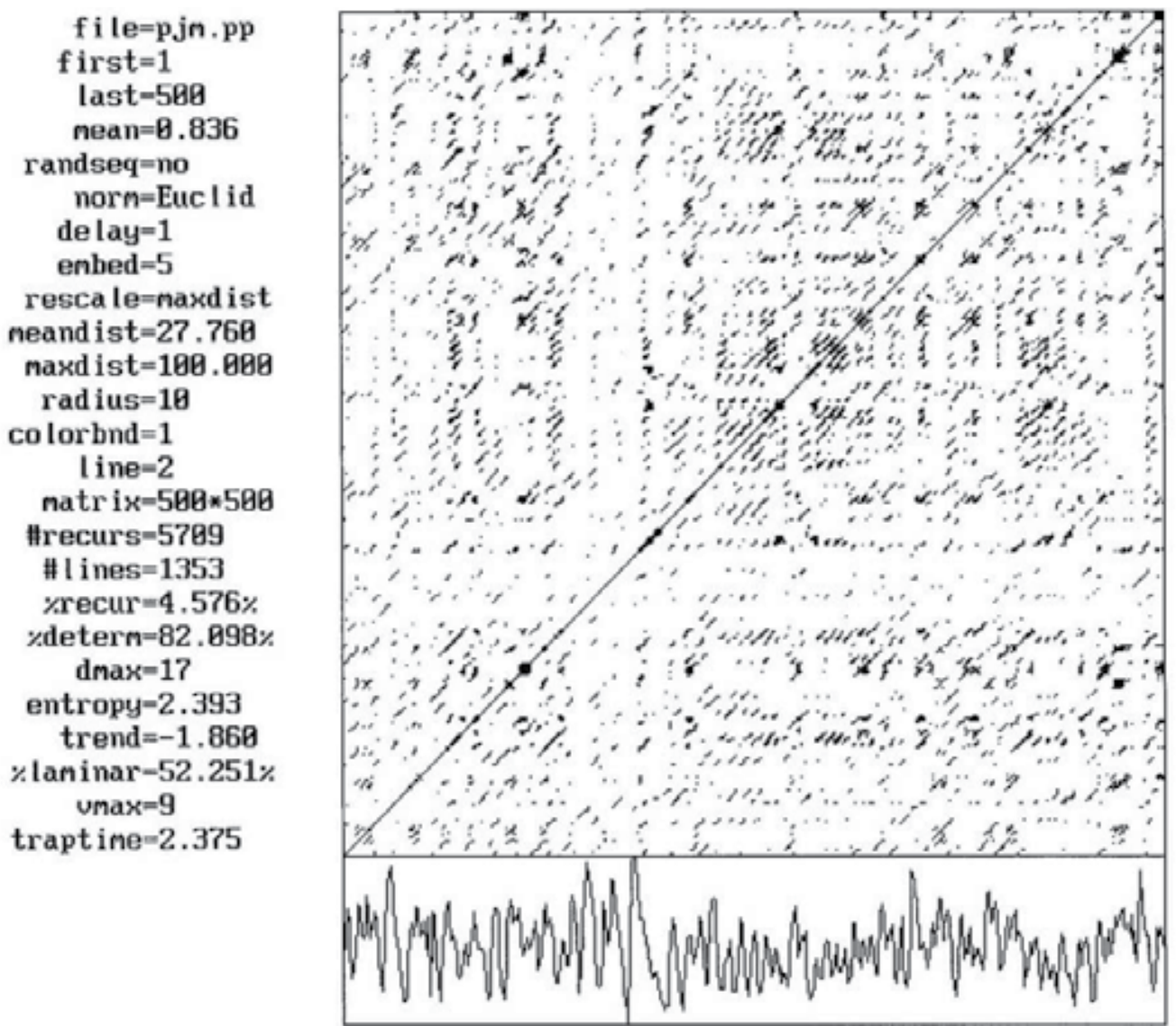

Figure 4. PP time series (bottom) and RR recurrence plot (top) along with recurrence parameters and quantifications (left). The plot is symmetrical on either side of the line-of-identity. The PP time series has 500 points displayed (417.9s) with HRV fluctuations centered on mean of 71.8 beats per min. 
The recurrence plot of a 500 sequential PP intervals from a disease-free human is shown in Figure 4. In this case the embedding dimension was set to 5 such that all possible vectors of 5 points were compared with each other. The plot presents with lacelike delicacy revealing hidden patterns that are not seen in the one-dimensional time series beneath the plot. For quantitation, however, eight recurrence variables are extracted from the plot (\%recur, \%determ, dmax, entropy, trend, \%laminar, vmax, traptime). Each variable has a critical, non-biased definition [20,21] which report eight unique perspectives on the data derived from the time series. In the example shown, the density of points in the plot (\%recur) is $4.576 \%$ and the probability of diagonal lines in the plot (\%determ) is $82.098 \%$. However, after randomly shuffling the PP intervals into a "nonsense" sequence (destroying the dynamical structure), \%recur fell to $0.162 \%$ and \%determ fell to $46.535 \%$ (not shown). These shuffling changes indicate that the original time series had deterministic structures attributed to physiological laws of the heart.

Proper implementation and interpretation of recurrence strategies requires careful selection of recurrence parameters for which a tutorial primer has been written [22] and freeware developed [23]. Recurrence windows can also move through very long time series, converting the eight unique recurrence variables into separate time series for further analysis. Little to no changes in these variables after random shuffling indicates rule-free signals. But such pure stochasticities are very rare in the world of structured physiological systems.

\section{Partitioning the cardiac period as a terminal dynamic}

Most modern quantifications of the cardiac dynamics, either linear or nonlinear, revert back to the fundamental RR or PP interval. Electrically speaking, during this time period two specific and alternating dynamics are occurring, electrical depolarization/repolarization during the beat and electrical isopotential between beats. Clean separation of these two phases can be realized by simply dividing the PP interval into two parts: the PT interval (the dynamic trajectory) and the TP interval (the stochastic pause) as shown in Figure 5A. Most interesting is Figure 5B in which the PP interval, the PT interval and the PT interval are all plotted as functions of time (or cardiac cycle number). The PP interval shows the typical respiratory sinus arrhythmia as expected. However, most of the variability is carried by the TP intervals when the heart is at isopotential rest, not the PT intervals when the heart is selfexcited, actively contracting, and repolarizing. This arrangement forms what is called a terminal dynamic [24].

What is a terminal dynamic? Simply stated, a terminal dynamic is a dynamic the reaches its terminus [25]. It comes to its end and simply stops. The incoming plane (active) is not asymptotic to the runway, but it actually lands and comes to a standstill at the gate (resting). The ant that runs (active) makes intermittent pauses along its path (resting). In the same way the heart follows an almost stereotypic trajectory while being electrically depolarized and repolarized (active) before it come to its rest during the inter-beat isopotential of the ECG (resting). The key here is that while the dynamic is "living" on the transient trajectory, 
it is robust against noise. On the other hand, once the dynamic has ended and the dynamic is paused, it is at this point (technically, its singularity) when it becomes most vulnerable to external noise. This is why cardiac pacemakers can only pace the myocardium (e.g. select the next active trajectory) between beats during quiescent phases of the electrical cycle. The complex interplay of ion channel activation and inactivation during the active phase generates a refractory period for external stimuli.
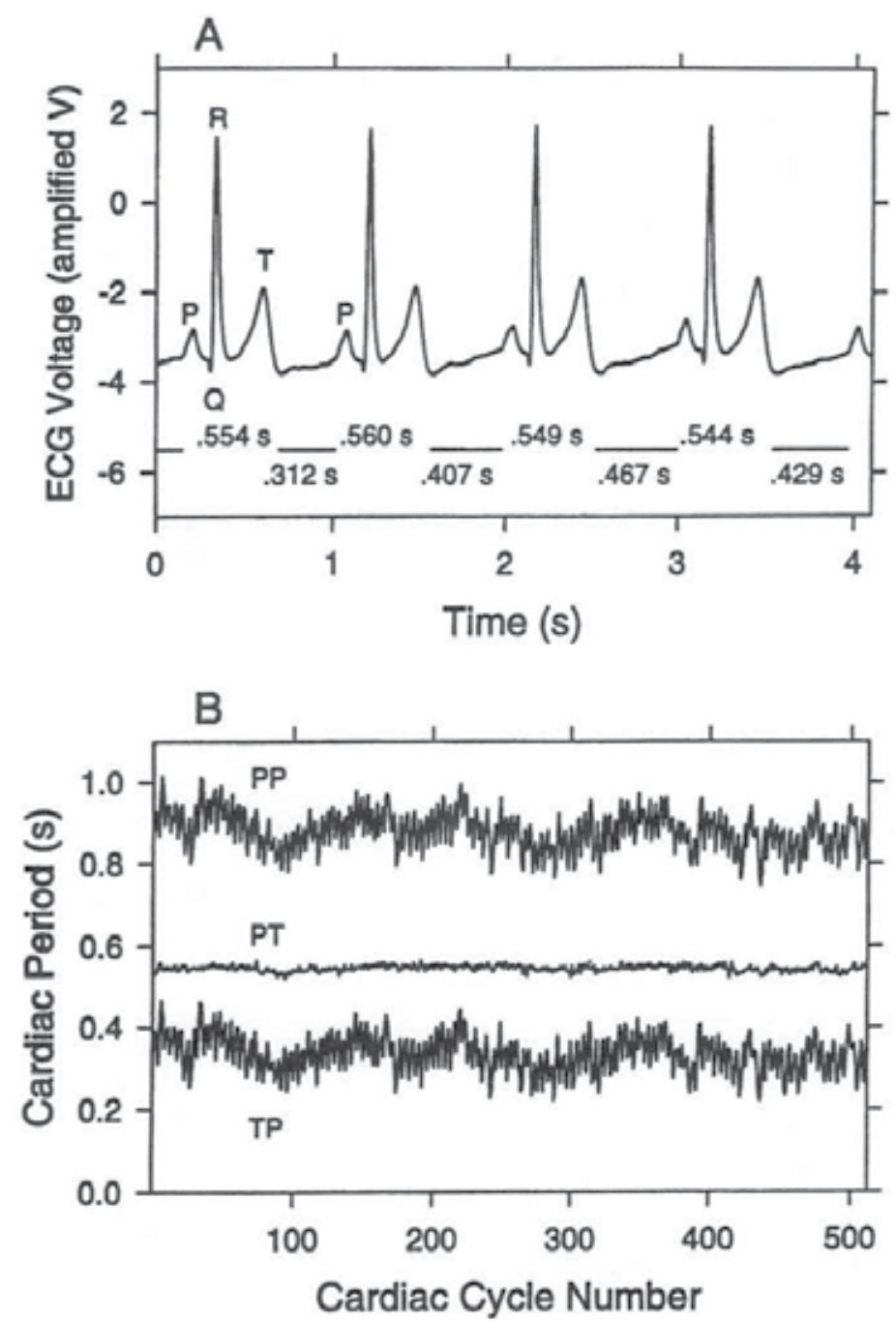

Figure 5. (A) Partitioning of PP intervals into PT and TP intervals for 512 beats. (B) HRV in PP intervals is carried almost exclusively in the TP intervals, not the PT intervals. Reproduced from reference [11] with permission.

How can a terminal dynamic be modeled? Well, instead of devising some fancy differential equation depicting beat after ECG beat, a better approach would be to write a single equation for the active phase of the heart which starts and comes to its end [26]. Then a 
pause or varying duration can be interposed before initiating the next beat. To impart more reality to the picture, the trajectory selection could come randomly, say, from a dozen slightly different differential equations (imparting a realistic wobbling in the active trajectory path). Likewise, the pause period could be randomly selected from a collection of actual cardiac isopotential times.

Thus the cardiac dynamic recorded in the ECG is really an alternation between a deterministic trajectory (which can be modeled by a differential equation) and a stochastic pause (which can only be randomly sampled from a collection of measure realtime pauses). This pause is termed the cardiac singularity, from which one of many trajectories can be selected for the next beat. The dynamic is also called a terminal dynamic, because the active heart actually stops (rests) at a terminus each and every beat. When one thinks about it, this type of modeling constitutes a piecewise deterministic system. The trajectory is the deterministic piece and the pause is the stochastic piece. Thus during the PP interval the heart alternates between determinism (PT interval) and stochasticity (TP interval).

\section{Atrial fibrillation}

One of the most common arrhythmias in human medicine is atrial fibrillation due to ectopic foci with possible spiral wave reentries [27]. Modeling of these three-dimensional patterns is very difficult, and control of atrial fibrillation is not fool-proof [28]. The thesis of a recent report is that atrial fibrillation may arise from unstable cardiac singularities [21]. As hinted at in the previous section, a singularity can be understood as the disruption or termination of an otherwise continuous event. For example, a sine wave has two singularities, one at its peak and another at its nadir. Under the influence of gravity, a ball tossed up into the air reaches its first unstable singularity at its peak. Then falling back to earth (the trajectory) again, the ball experiences a few bouncing cycles (unstable singularity-trajectory alternations) before finally coming to rest on the ground (stable singularity). Balancing a pencil on it stub end is easy (stable singularity), but balancing the same pencil on its point is very difficult (unstable singularity).

Beyond sine waves, bouncing balls and balancing pencils, how can singularities be identified in the cardiac electrogram and what might they have to do with the detection of electrical patterns that may precipitate out as full atrial fibrillation? In the bottom panels of Figure 6, two atrial electrograms are shown as recorded from roving bipolar electrodes in human patients [21]. Each window consists of 2048 points spanning 2.10 seconds in time (digitization frequency $977 \mathrm{~Hz}$ ). The first recording shows a spike pattern at 254 pulses per minute $(4.2 \mathrm{~Hz}$, Fig. $6 \mathrm{~A})$ and the second recording shows a spike pattern at 881 pulses per $\min (14.7 \mathrm{~Hz}$, Fig. 6B). Although these rates are much too fast for each depolarization to be conducted through the atrioventricular node, the can still wreak havoc with arrhythmias in the main pumping chambers of the heart. 
Recurrence plots of these two atrial signals clearly display singularities as square blocks stair-casing upward along the central diagonal line (line of identity, Fig. 6A and Fig. 6B). These singularities are coincident with the quasi-isopotentials periods in the electrograms. Off-central recurrences form rectangular boxes due to variability in isopotential durations among the windowed beats (analogous to the TP variability discussed above). Clearly, the faster the arrhythmia, the smaller the singularity that is inscribed. The postulate is that as atrial singularities become vanishingly small, then unrestrained atrial fibrillation is unleashed. Somewhere along the continuum to oblivion, the singularities become unstable, triggering fibrillation. The clinical application of this methodology may evolve such that mapped regions in the atria lacking singularities may be candidate sites for ablation in halting the dysrhythmia expressed within the larger tissue [29].
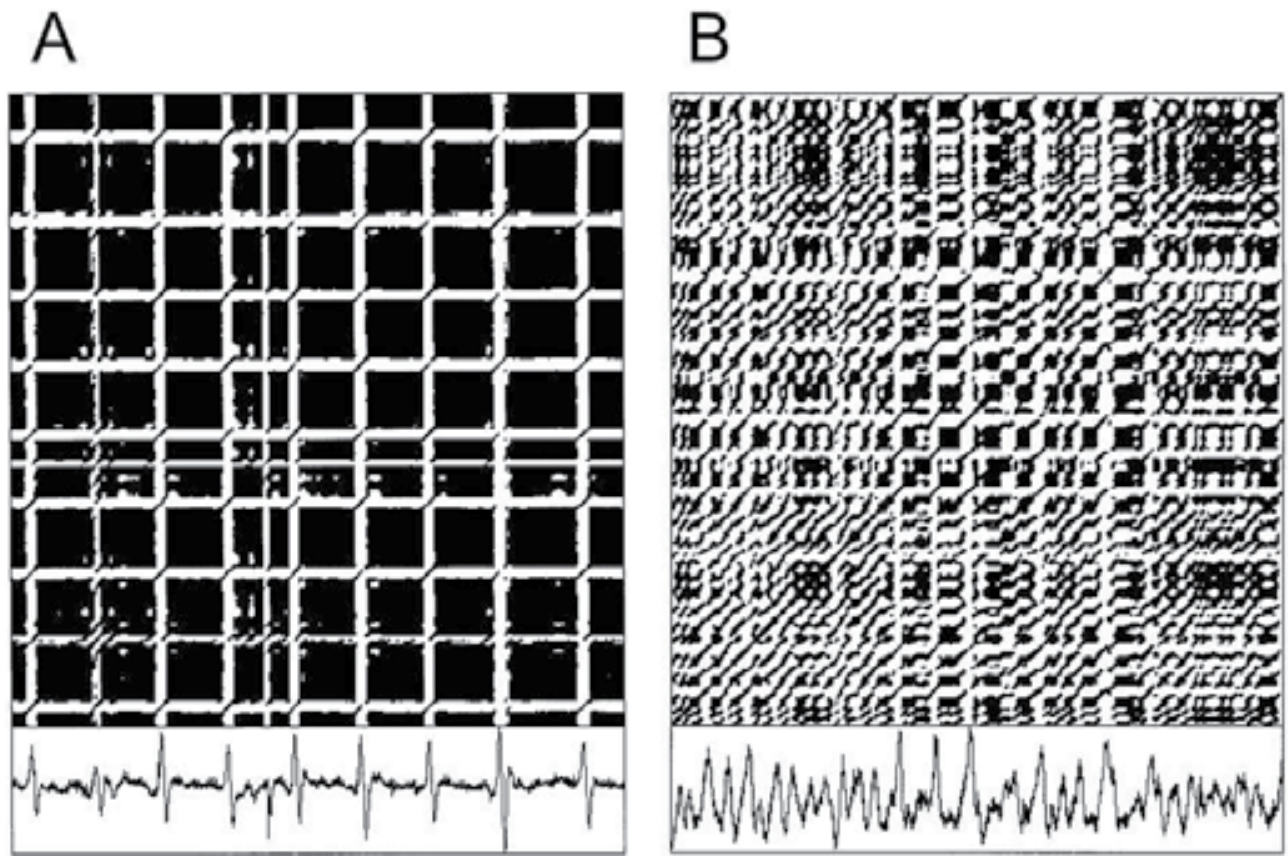

Figure 6. Singularities in atrial electrograms. (A) Fast atrial waves $(4.2 \mathrm{~Hz})$ with relatively long singularities. (B) Very fast atrial waves $(14.7 \mathrm{~Hz})$ with relatively short singularities. Recurrence parameters: delay $=1$, embedding dimension $=15$, norm $=$ Euclidean, radius $=15 \%$ of maximum distance, line $=2$. Data from reference [21].

\section{Conclusions}

The heart is a homeodynamic organ with automatic rhythms expressed in both mechanical electrical domains. Mechanical activities presume electrical underpinnings, but the reverse is not necessarily true except for possible stretch-activated electrical channels [30]. To get a window into cardiac dynamics, this chapter has majored on linear and nonlinear analyses that tease apart the cardiac cycle as defined as the R-R (or P-P) interval. For clinical medicine, the quest has been to define cardiovascular dynamics that may forecast unstable 
patterns (arrhythmias) or fatal events (asystole). Can it be that dis-homeodynamics is a kind of dynamical disease [31]?

Numerous studies have examined heart rate variability (HRV) and concluded that high HRV is good (how high?) and low HRV is bad (how low?). There are methodological problems associated with the measurement of HRV restricted to linear the perspective (spectral analysis) and low-dimensionality perspective (approximate entropy). One thing is for sure - autonomic imbalance and heart rate variability are important risk factors for cardiovascular disease [32]. Importantly, the reciprocity or co-activation of the sympathetic and parasympathetic branches of the autonomic nervous system is decidedly non-linear [33] and non-Gaussian [34]. This being true, it becomes apparent and necessary to apply the proper nonlinear tools to assess the signals. One proven too is recurrence quantification analysis (RQA) which has a demonstrated utility in diagnosing nonstationary cardiac dynamics [35].

As can be seen, the normal cardiac dynamic (mechanical or electrical) has a wealth of descriptors. With these in hand, it becomes easier to detect and possibly predict heart dysfunction and failure. Returning to the concept of homeodynamics, as people age or become ill, the dynamic range of cardiac functionality becomes limited. The loss of complexity and high-dimensional dynamics become markers for dysrhythmias, fibrillations and possibly even death [36].

Although current implanted artificial pacemakers are limited in their computational capacity, advances in nonlinear dynamics are being investigated to augment signal filtering and facilitate event detection [37-38].

\section{Author details}

Charles L. Webber, Jr.

Department of Cell \& Molecular Physiology, Loyola University Chicago, Stritch School of Medicine, Maywood, IL, USA

\section{References}

[1] Hajar R (1999) The Pulse in Antiquity. Heart Views 1: 89-94.

[2] King David (1995) Psalm 38:8-10. New American Standard Bible. Zondervan Publishing pp. 808-809.

[3] Webber CL Jr (2005) The Meaning and Measurement of Physiologic Variability? Crit. Care Med 33: 677-678.

[4] Rose S (1997) - Lifelines: Biology, Freedom, Determinism. Oxford University Press, 334 pp.

[5] Bers DM (2002) - Excitation-Contraction Coupling and Cardiac Contractile Force. Kluwer Academic Publishers, 452 pp. 
[6] Badano LP, Gaddi O, Peraldo C, Lupi G, Sitges M, Parthenakis F, Molteni S, Pagliuca MR, Sassone B, Di Stefano P, De Santo T, Menozzi C, Brignole M. (2007) Left Ventricular Electromechanical Delay in Patients with Heart Failure and Normal QRS Duration and in Patients with Right and Left Bundle Branch Block. Europace 9: 41-47.

[7] Ferrer R, Artigas A (2011) Physiologic Parameters as Biomarkers: What Can we Learn from Physiologic Variables and Variation? Crit Care Clin 27: 229-240.

[8] West, B.J. (2010). Homeostasis and Gauss statistics: Barriers to Understanding natural Variability. J Eval Clin Pract 16: 403-408.

[9] González, J.J., Cordero, J.J., Feria, M., Pereda, E. (2000). Detection and Sources of Nonlinearity in the variability of Cardiac R-R Intervals and Blood Pressure in Rats. Am. J. Physiol. Heart Circ. Physiol. 279: H3040-H3046.

[10] Grafakos L (2003) - Classical and Modern Fourier Analysis. Prentice Hall, 931 pp.

[11] Webber CL Jr, Zbilut JP (1998) Recurrent Structuring of Dynamical and Spatial Systems. In: Complexity in the Living: A Modelistic Approach. Colosimo A, ed, Proc Int Meet, Feb. 1997, University of Rome “La Sapienza," pp. 101-133.

[12] Mazzeo, A.T., La Monaca, E., Dileo, R., Vita, G., Santamaria, L.B. (2011). Heart Rate Variability: A Diagnostic and Prognostic Tool in Anesthesia and Intensive Care. Acta Anaesthesiol Scand 55: 797-811.

[13] Taylor JA, Carr DL, Myers CW, Eckberg DL (1998) Mechanisms Underlying Very-LowFrequency RR-Interval Oscillations in Humans. Circ 98: 547-555.

[14] MalikM (1996) Heart Rate Variability: Standards of Measurement, Physiological Interpretation, and Clinical Use. Circ 93: 1043-1065.

[15] Pincus, S.M. (1991). Approximate Entropy as a Measure of System Complexity. Proc. Natl Acad Sci 88: 2297-2301.

[16] Eckmann, J.-P., Kamphorst, S. O., \& Ruelle, D. (1987). Recurrence Plots of Dynamical Systems. Europhys Lett, 4, 973-977.

[17] Zbilut, J.P., Webber, C.L., Jr. (1992). Embeddings and Delays as Derived from Quantification of Recurrence Plots. Phys Let A 171: 199-203.

[18] Webber, C.L., Jr., Zbilut, J.P. (1994) Dynamical Assessment of Physiological Systems and States using Recurrence Plot Strategies. J Appl Physiol 76: 965-973.

[19] Takens F (1981) Detecting Strange Attractors in Turbulence. In: Rand D \& Young L-S, Lecture Notes in Mathematics, Vol. 898, Dynamical Systems and Turbulence, Warwick 1980. Springer-Verlag, pp. 366-381.

[20] Webber, CL Jr, Zbilut, JP (2007) Recurrence Quantifications: Feature Extractions from Recurrence Plots. Int J Bifurcation Chaos 17: 3467-3475.

[21] Webber CL Jr, Hu Z, Akar J (2011) Unstable Cardiac Singularities May Lead to Atrial Fibrillation. Int J Bifurcation Chaos 21: 1141-1151.

[22] Webber, C.L., Jr., Zbilut, J.P. (2005) Recurrence Quantification Analysis of Nonlinear Dynamical systems. In: Tutorials in Contemporary Nonlinear Methods for the Behavioral Sciences, (Chapter 2, pp. 26-94), Riley MA, Van Orden G, eds. Retrieved December 1, 2004 http://www.nsf.gov/sbe/bcs/pac/nmbs/nmbs.jsp

[23] Webber, C.L., Jr. (2012) Introduction to Recurrence Quantification Analysis. RQA Version 14.1 README.PDF: http://homepages.luc.edu/ cwebber/ 
[24] Zbilut JP, Webber CL Jr, Zak M (1998) Quantification of Heart Rate Variability using Methods Derived from nonlinear Dynamics. In: Analysis and Assessment of Cardiovascular Function. Drzewiecki G and Li JK-J, eds. Springer Verlag, New York, Chapter 19, pp. 324-334.

[25] Giuliani A, Giudice PL, Mancini AM, Quatrini G., Pacifici L., Webber CL Jr, Zak M, Zbilut JP (1996) A Markovian Formalization of Heart Rate Dynamics Evinces a Quantum-like Hypothesis. Biol Cyber 74: 181-187.

[26] Zbilut JP, Hübler A, Webber CL Jr. (1996) Physiological Singularities Modeled by Nondeterministic Equations of Motion and the Effect of Noise. In: Fluctuations and Order: The New Synthesis. Millonas MM, ed, Springer-Verlag, Chapter 24, pp. 397-417.

[27] Nattel S (2002) New Ideas about AtrilaFibrillation 50 Years On. Nature 414: 219-226.

[28] Garfinkel A, Chen PS, Walter DO, Karagueuzian HS, Kogan B, Evans SJ, Karpoukhin M, Hwang C, Uchida T, Gotoh M, Nwasokwa O, Sager P, Weiss JN (1997) Quasiperiodicity and Chaos in Cardiac Fibrillation. J Clin Inv 99: 305-314.

[29] Wilber DJ, Pappone C, Neuzil P, De Paola A, Marchlinski F, Natale A, Macle L, Daoud EG, Calkins H, Hall B, Reddy V, Augello G, Reynolds MR, Vinekar C, Liu CY, MPH; Berry SM, Berry DA (2010) Comparison of Antiarrhythmic Drug Therapy and Radiofrequency Catheter Ablation in Patients With Paroxysmal Atrial Fibrillation - A Randomized Controlled Trial. J Am Med Assoc 303: 333-340.

[30] Kohl P, Sachs F, Franz MR (2011) Cardiac Mechano-Electric Coupling and Arrhythmias. Oxford Univesity Press 477 pp.

[31] Beliar J, Glass L, Heiden UAD, Milton J (1995) Dynamical Disease: Mathematical Analysis of Human Illness. Am Inst Physics 220 pp.

[32] Thayer JF, Yamamoto SS, Brosschot JF (2010) The Relationship of Autonomic Imbalance, Heart Rate Variability and Cardiovascular Disease Risk Factors. Int J Cardiol 141: 122-131.

[33] Mourot L, Bouhaddi M, Gandelin E, Cappelle S, Nguyen NU, Wolf J-P, Rouillon JD, Hughson R, Regnard J. (2007) Conditions of Autonomic Reciprocal Interplay versus Autonomic Co-activation: Effects on Non-linear Heart Rate Dynamics. Autonomic Neuroscience: Basic and Clinical 137: 27-36.

[34] Kiyono K, Hayano J, Watanabe E, Struzik ZR, Yamamoto Y (2008) Non-Gaussian Heart Rate as an Independent Predictor of Mortality in Patients with Chronic Heart Failure. Heart Rhythm 5:261-268.

[35] Zbilut JP, Thomasson N, Webber CL Jr (2002) Recurrence Quantification Analysis as a Tool for Nonlinear Exploration of Nonstationary Cardiac Signals. Med Engin Physics 24: 53-60.

[36] Beckers F, Verheyden B, Aubert AE (2006) Aging and Nonlinear Heart Rate Control in a Healthy Population. Am J Physiol - Heart 290: H2560-H2570.

[37] Polpetta A, Banelli P )2008) Fully digital pacemaker detection in ECG signals using a non-linear filtering approach. Conf Proc IEEE Eng Med Biol Soc. 2008:5406-5410.

[38] Aström M, Olmos S, Sörnmo L (2006) Wavelet-based event detection in implantable cardiac rhythm management devices. IEEE Trans Biomed Eng. 53: 478-484. 
Chapter 12

\title{
Mobile and Wireless Technologies on Sphygmomanometers and Pulsimeters for Patients with Pacemakers and Those with Other Cardiovascular Diseases
}

\author{
Ching-Sung Wang and Teng-Wei Wang \\ Additional information is available at the end of the chapter
}

http://dx.doi.org/10.5772/48565

\section{Introduction}

The population with pacemaker implants varies by age, sex or race. Over 100,000 pacemakers are implanted every year in the United States with approximately 500,000 Americans having an implanted permanent pacemaker device [1]. Pacemakers can be affected by electromagnetic interference in several different ways, including temporary inhibition of the pacemaker, temporary function at the fixed noise rate, temporary function at the fixed magnet rate, permanent inhibition or malfunction and random reprogramming. For any of these results to occur, the E field strength must be greater than $200 \mathrm{~V} / \mathrm{m}$ or the magnetic field strength must be greater than 10 Gauss [2].

Mobile tele-medicine is a future development trend. There is much research on it, but most of them discuss GSM, GPRS, 3G, WiMax or LTE. Another key point of mobile tele-medicine is variable electronic medical instrumentation. Our research is expected to integrate embedded system design, wireless communication and medical know-how to implement remote medical care. Long-term blood pressure management is very important for patients who are at risk of stroke or heart attack, therefore, monitoring blood pressure and the ability to immediately call for help are both important. This research will use a portable manometer combined a Bluetooth and GSM cell phone. It can monitor BP and transmit emergency information from wherever people live and wherever they go.

Cellular telephones can interfere with the function of implanted cardiac pacemakers $[3,4,5]$. However, when telephones are placed over the ear, the normal position, this interference does not pose a health risk [5]. Barbaro V, et al.'s research indicated influence between a 
GSM mobile phone and an implanted pacemaker. Electromagnetic interference effects were detected at a maximum distance of $10 \mathrm{~cm}$ with the pacemaker programmed at its minimum sensing threshold. When the phone antenna was in direct contact with the patient's skin over the implant, electromagnetic interference effects occurred at the maximum ventricular and atrial sensing thresholds of $4 \mathrm{mV}$ and $2.5 \mathrm{mV}$, respectively [6]. Therefore, decreasing wireless emission power and increasing pacemaker emission distance are the most useful methods of lowering the magnetic interference to the pacemaker. In order not to affect users' habits of using mobiles and to avoid the electromagnetic interference effects to a pacemaker, we have designed a wristable sphygmomanometer and pulsimeter which uses Bluetooth power class II $[7,8]$ as the device for short-distance transmission. Furthermore, by combing with a cellular phone, we can do prolonged, distant observation and treatment immediately for patients who require adjustments to pacemaker settings or those with other cardiovascular diseases.

\section{System architecture}

This research aims at designing a wristable sphygmomanometer and pulsimeter. We designed a non-invasive sphygmomanometer [9] to remind the user to measure blood pressure every hour. A pulsimeter is an electronic device which is based on a pressure sensor and can observe pulse continuously. In order to avoid the influence of electromagnetic waves on the pacemaker, this research uses the Bluetooth power class II equipment for short-distance wireless data transmission from the sphygmomanometer to the client-end cellular phone, then uses the GPRS (General Packet Radio Service) $[10,11]$ to do the long distance data transmission. This can transmit blood pressure and pulse rate to a Remote Medical Server and then provide them to specialist doctors for reference data.

\subsection{Client-side hardware architecture}

Because of the connecting method of GPRS is a packet switch [10,11], it does not connect with the Remote Medical Server continuously. Therefore, this research utilized a GSM (Global System for Mobile Communications) circuit connection (voice connection) [10,11,12] for the Remote Medical Server to inform the wristable sphygmomanometer and pulsimeter, and to drive the sphygmomanometer to measure blood pressure immediately. Then, the sphygmomanometer will send the results of blood pressure measurements to the Remote Medical Server by GPRS [10,11]. Figure 1 details the block diagram of the client-side hardware architecture.

\subsection{Server-side hardware architecture}

Figure 2 details the block diagram of the server-side hardware architecture [13]. Server-side includes a PSTN (Public Switched Telephone Network) phone controller and a monitoring terminal. Furthermore, the PSTN phone controller is constructed by an ATA (Analogue Telephone Adapter), microcontroller and the accessories, which takes the incoming call from the client. W681388 [14] is used as the baseband IC of ATA for ring detection and coding of voice signals. PIC24FJ64 [15] is used as a microcontroller, which receives the 
command of the monitoring terminal through the UART interface and controls the W681388 Hook ON/OFF and voice output through the SPI (Serial Peripheral Interface) interface. W681388 is made by Winbond. PIC24FJ64 is made by Microchip.

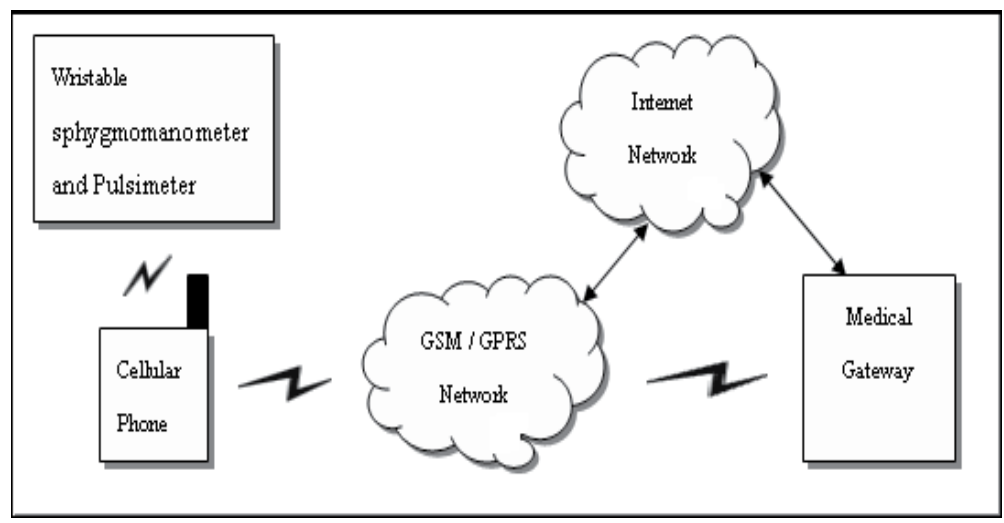

Figure 1. Client-side hardware architecture

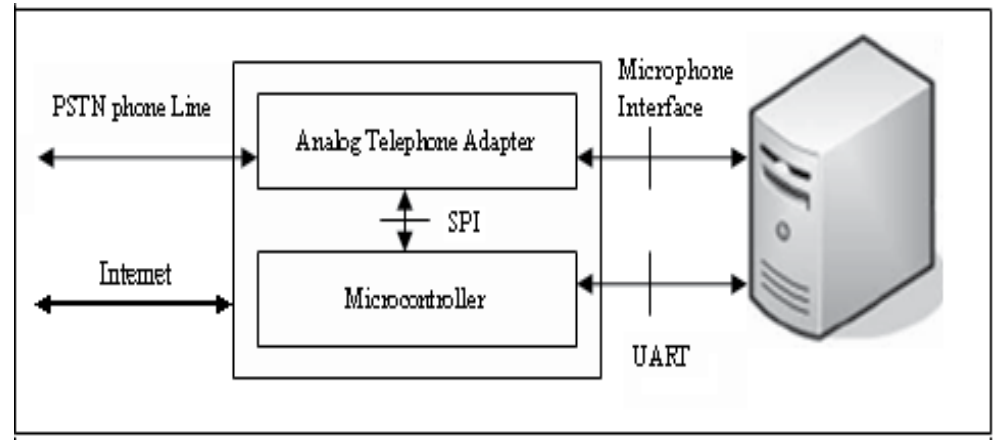

Figure 2. Server-side hardware architecture

\subsection{Client-end cellular phone hardware architecture}

Figure 3 details the block diagram of client-end cellular phone hardware architecture, which includes two main parts, Bluetooth and GSM/GPRS.

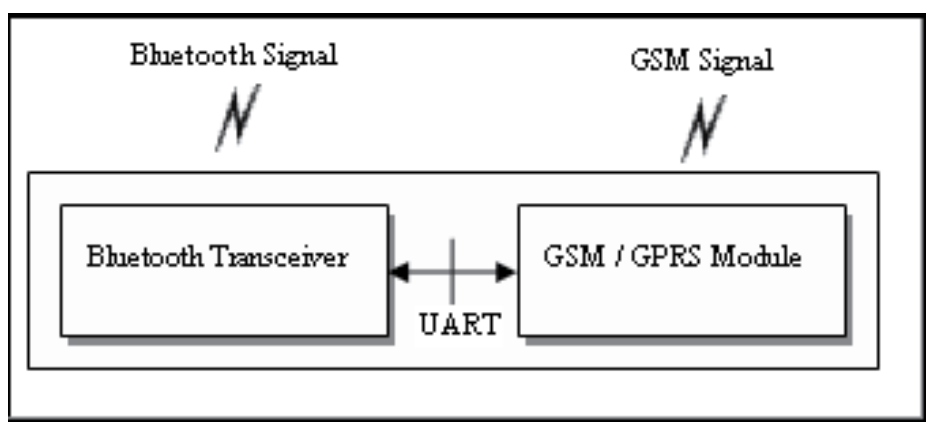

Figure 3. Client-end Cellular Phone Hardware Architecture 
1. Bluetooth transceiver $[16,17,18]$ : the hardware framework is similar to the Bluetooth transceiver of wristable sphygmomanometer and pulsimeter. It communicates with GSM/GPRS module by UART interface.

2. GSM Module [18]: includes Baseband part and RF part. The main components are Baseband IC (including DSP), TFT-LCD, NOR Flash, SRAM, Power Management IC and RF IC. We use MT6219 made by MediaTek as the platform for the GSM/GPRS system.

\subsection{Tele-care system architecture}

Figure 4 details the block diagram of the tele-care system architecture. The data is acquired from the wristable sphygmomanometer which transmits to the client-end cellular phone by the Bluetooth device. IMEI (International Mobile Equipment Identity) [19] measure the time and blood pressure data which were transmitted to the server-end cellular phone and all the data are classified, saved then applied.

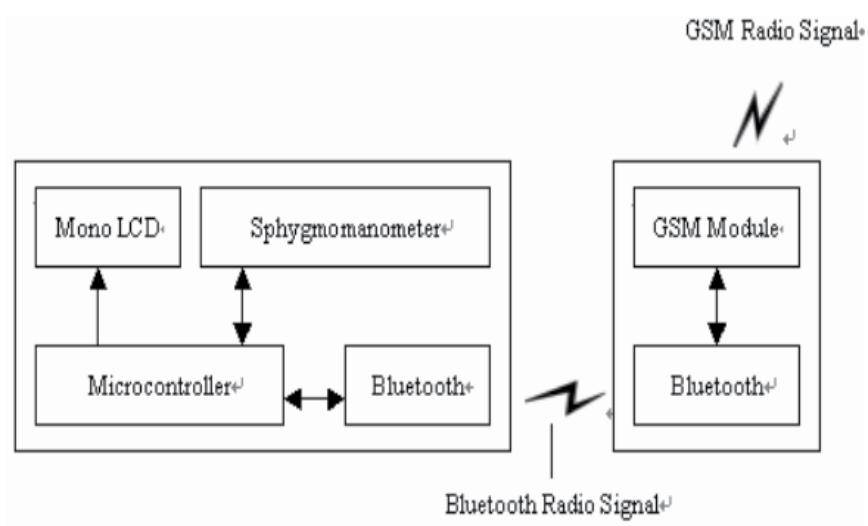

Figure 4. Tele-care system architecture

\subsection{Tele-emergency announcement system architecture}

Figure 5 details the block diagram of the tele-emergency announcement system architecture. When the data exported by the motion sensor is fixed for a period of time, the microcontroller goes into emergency mode. Then the client-end cellular phone establishes a voice connection with the server-end cellular phone. After the connection is established, it transmits IMEI [20], location, time, and blood pressure in DTMF (Dual-tone multifrequency) format.

\section{Hardware architecture}

The hardware system of this research is divided into two parts, one is a wristable sphygmomanometer and pulsimeter; the other one is a client-end cellular phone. Details are discussed below. 


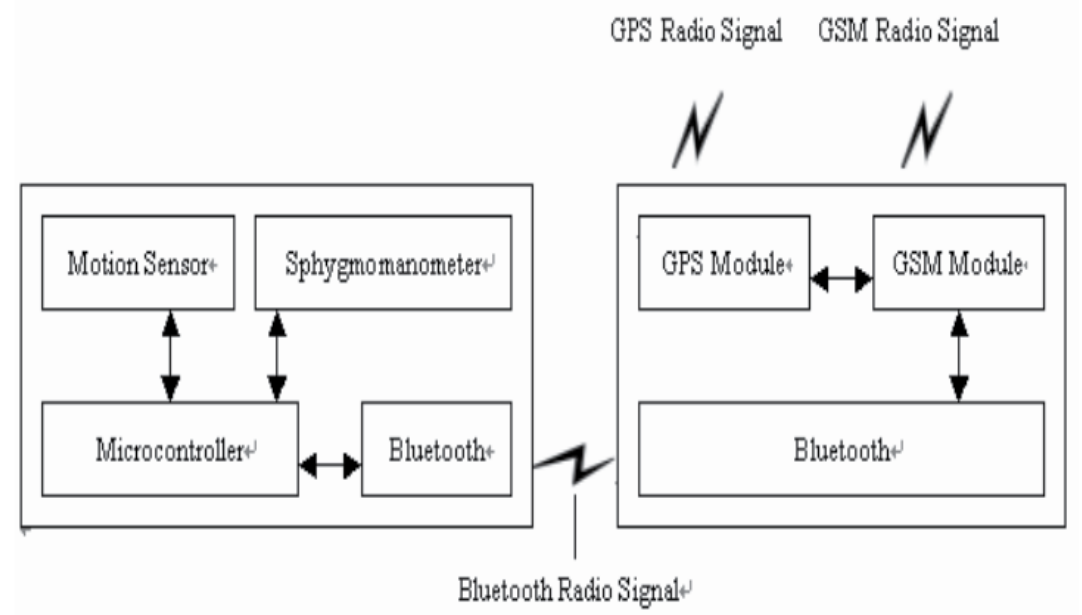

Figure 5. Tele-emergency announcement system architecture

\subsection{Wristable sphygmomanometer and pulsimeter}

Figure 6 details the block diagram of the wristable sphygmomanometer and pulsimeter hardware architecture. The Controller unit of the wristable sphygmomanometer and pulsimeter is the microcontroller [16], which controls and reads the peripheral units. Here is the description of each unit.

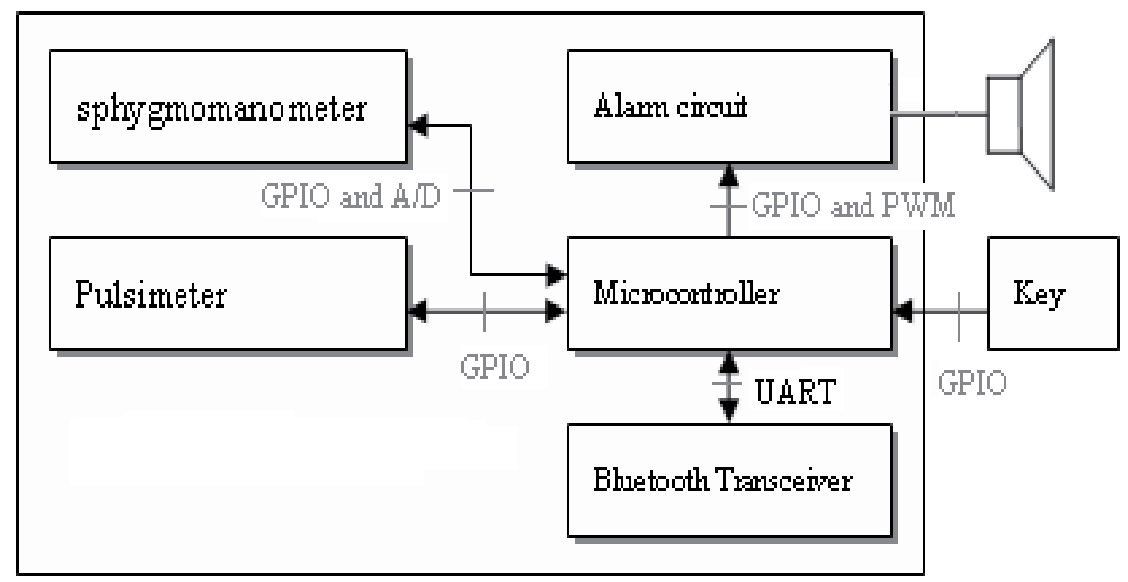

Figure 6. Wristable sphygmomanometer and pulsimeter hardware architecture

1. Sphygmomanometer $[9,16,17,18]$ : Figure 7 details the block diagram of the wristable sphygmomanometer hardware architecture. The wristable sphygmomanometer consists of a 16 bit RISC microcontroller, Bluetooth transceiver, mono LCD, motion sensor and accessories. The microcontroller controls the other components. This is an electronic medical instrument; the microcontroller communicates with the device by the 
UART interface of 8051 . Bluetooth module $[16,18]$ : the project uses BC3 made by CSR as the Bluetooth transceiver. The microcontroller communicates with the Bluetooth by UART interface and controls all the process by software. Mono LCD[16,21]: mono LCD displays all information in the system. The mono LCD was driven by the microcontroller and communicated to by the parallel LCD interface. Motion sensor [16,22]: we use the MXC6202 chip made by MEMSIC as the motion sensor. It detects the movements of the object and gives all kinds of information for the microcontroller to classify every situation. It uses $\mathrm{I} 2 \mathrm{C}$ as the interface.

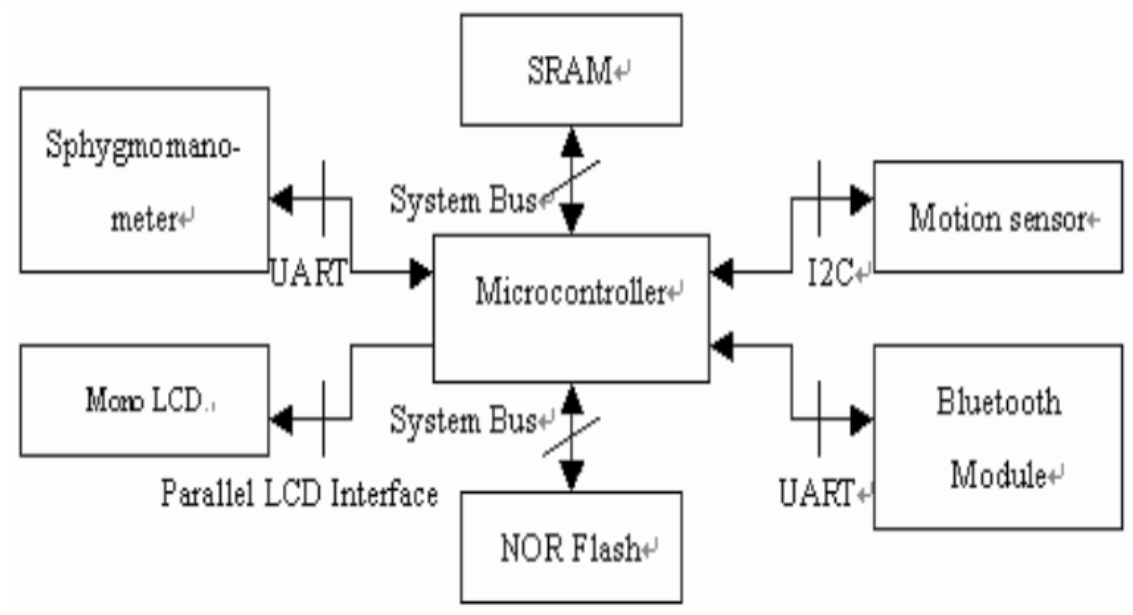

Figure 7. Wristable sphygmomanometer hardware architecture

Details of the sphygmomanometer include the LDO (Low Dropout Regulators) as power controller, pressure sensor, filter, amplifier circuit and accessories. The project is using the pressure sensor of the SCC series which is manufactured by HoneyWell's company. The pressure sensor will transmit different messages according to the changing pressure. The messages pass through the procedures of magnifying (magnify the micro-message form sensor), and filtering (noise removal), and then all the analogue messages are sent to the microcontroller via A/D (Analogue/Digital) interface. Figure 8 shows the implementation of the sphygmomanometer device architecture.

2. Pulsimeter $[9,16,17,18]$ : the pulsimeter includes LDO, pressure sensor, filter, amplifier circuit and accessories. The circuit is using the SCC series made by HoneyWell, but it has a different sensitivity pressure sensor. Putting the sensor pad over the radial pulse, the pressure sensor will convert the normal pulse to a larger voltage output by magnifying, filtering and then converting (transforming to digital signal), and finally inputs it into the microcontroller. Figure 9 shows the block diagram of the pulsimeter.

3. Bluetooth transceiver [16, 23]: the project uses BC3 made by CSR as the Bluetooth transceiver. The microcontroller communicates with the Bluetooth by UART (Universal asynchronous receiver/transmitter) interface and controls all the processes using the software. 


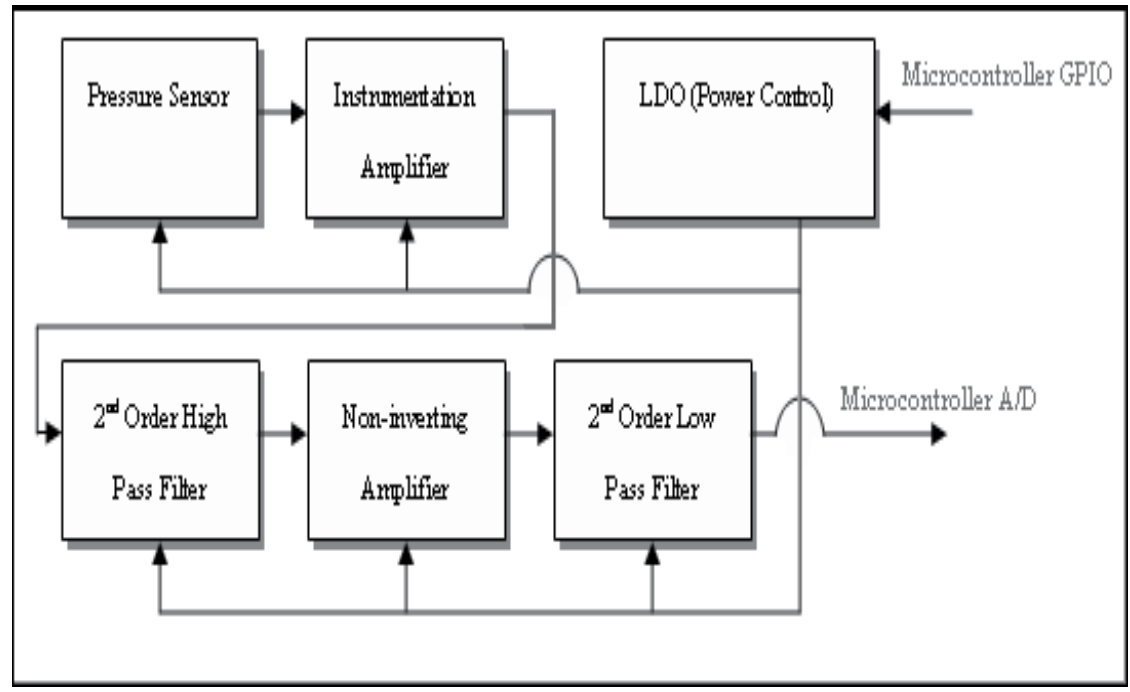

Figure 8. Sphygmomanometer device

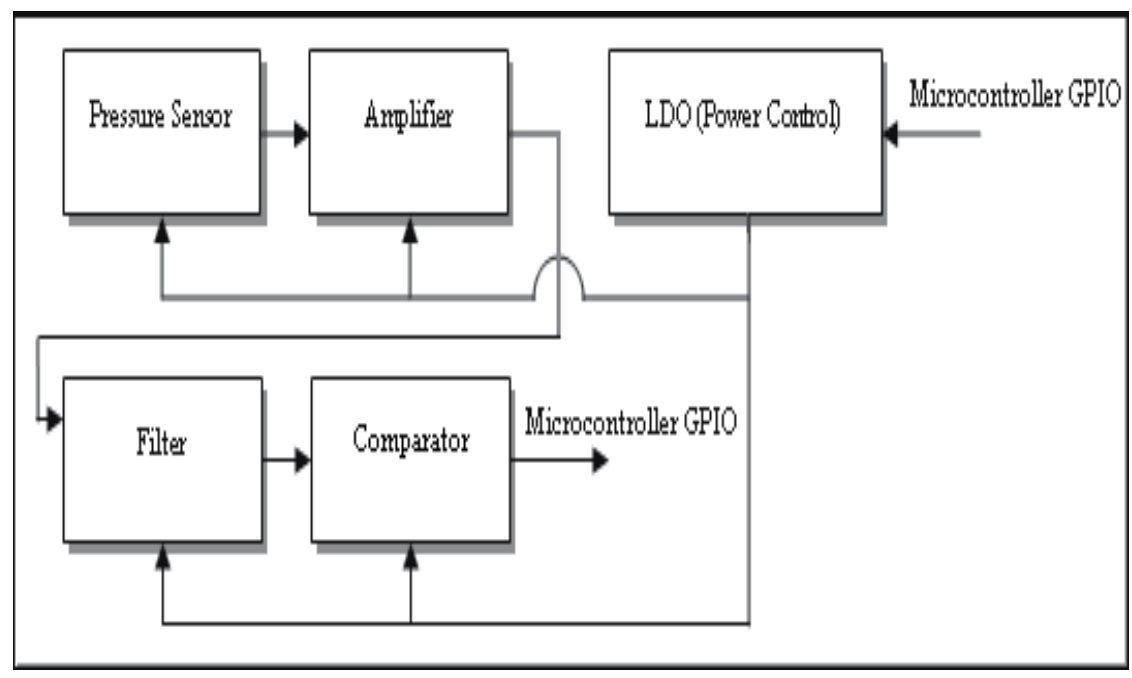

Figure 9. Pulsimeter device

4. Alarm circuit: the main purpose of this part is to send a notice to the users and then to perform blood pressure measurement. The microcontroller can activate the alarm circuit every hour. At the other end, the Remote Medical Server can also order a blood pressure measurement, so that the users can measure their blood pressure when required.

\subsection{Wireless personal area network base on Bluetooth}

Bluetooth provides point to point and multipoint wireless connection according to the Internet concept. Within any active communication scope, all devices are treated the same. The first one requesting communication is called master and the passive one accepting the 
signal is called slave. A master and one or more salves construct the Pico-net of Bluetooth [24], [25]. Because not all mobile phones with Bluetooth function in the current market support Serial Port Profile, the GPS device used in this research cannot connect with all the mobile phones with Bluetooth via the Bluetooth function. In order to cover most mobile phones with Bluetooth in the market in this research and make an active Pico-net, we used the Bluetooth device of BAD (Body Activity Detector) as master, and the Bluetooth devices of the mobile phone and GPS as slave. The Bluetooth of BAD connects with the Bluetooth of the mobile phone and GPS, and forms a PAN (Personal Area Network) upon Bluetooth. Figure 10 indicates the Bluetooth Pico-net in this system.

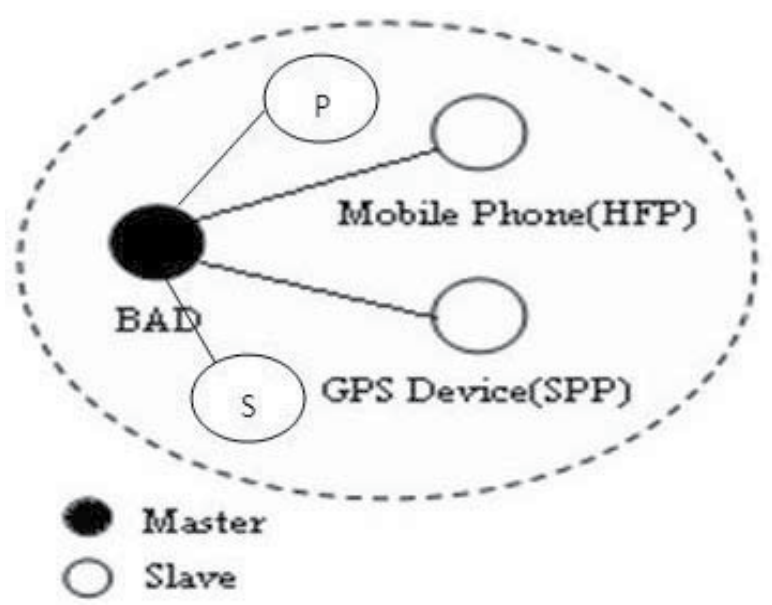

Figure 10. Bluetooth Pico-net

\section{Software algorithm}

\subsection{Continuous pulse and blood pressure measurement, and the monitoring procedure}

Pulse monitor procedure [16, 17, 18, 21]: the microcontroller reads the pulsimeter every minute, then transmits the results to the client-end cellular phone by the Bluetooth SPP (Serial Port Profile). Blood pressure measure procedures [9, 16, 17, 18, 21]: the microcontroller enables an alarm system to remind the user every hour to measure blood pressure. Users need to put their hand in the proper position so that the sphygmomanometer can better detect the pulse. When the users put their hand in the proper position and push the trigger button, the microcontroller controls the pulsimeter's LDO to stop the action and start measuring the blood pressure. After that, the microcontroller transmits the blood pressure to the cellular phone by Bluetooth SPP, and activates the pulsimeter. If the user doesn't perform the blood pressure measurement, the microcontroller would re-activate the pulsimeter after a while. The software algorithm of continuous pulse and blood measurement, and the monitoring procedure is shown in Figure 11 and Figure 12. 


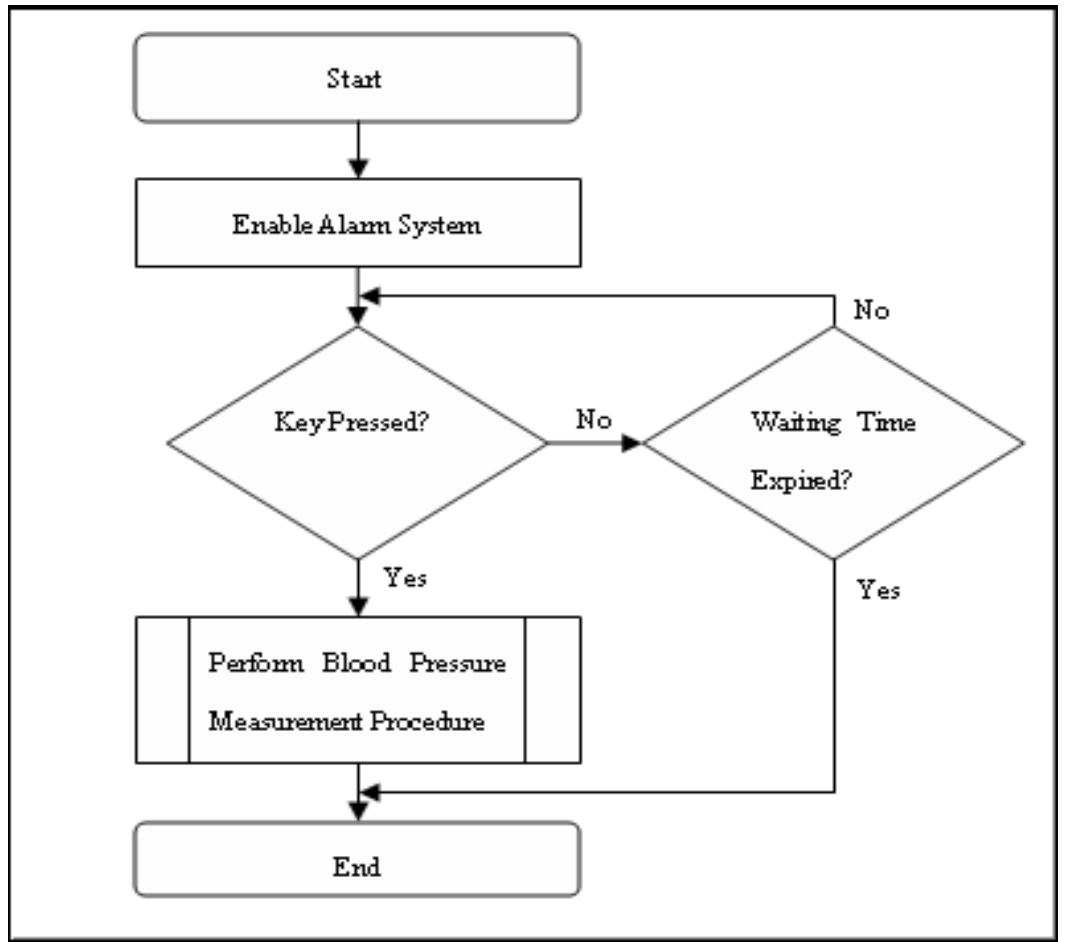

Figure 11. The software algorithm of continuous pulse and blood measuring, and the monitoring procedure

\subsection{Client-end cellular phone data handling procedure}

When the Bluetooth of the mobile receives the data from the Bluetooth of the wristable sphygmomanometer and pulsimeter, it analyses blood pressure or pulse data and stores (including the measurement time) locally first. Finally, it establishes a GPRS connection. After successful connection, the mobile scans the stored blood pressure and pulse data, and then transmits them to the Remote Medical Server if the data has not yet been sent. The software algorithm of the mobile data handling procedure is shown on Figure 13 [13, 21, 22,25].

\subsection{Remote Medical Server claim for measuring blood pressure}

If the physician considers that it is necessary to get the patient's current blood pressure immediately, he/she can call the client-end cellular phone using the GSM module on the Remote Medical Server. While the client-end cellular phone receives the incoming call from the server-end, the Bluetooth SPP transmits the command of measuring blood pressure to the wristable sphygmomanometer and pulsimeter. While the wristable sphygmomanometer and pulsimeter receive the command from the Remote Medical Server, they activate blood pressure measurements and transmit procedures. The software algorithm of the Remote Medical Gateway claims for measuring blood pressure are shown in Figure 14 [13, 21, 22, 25]. 


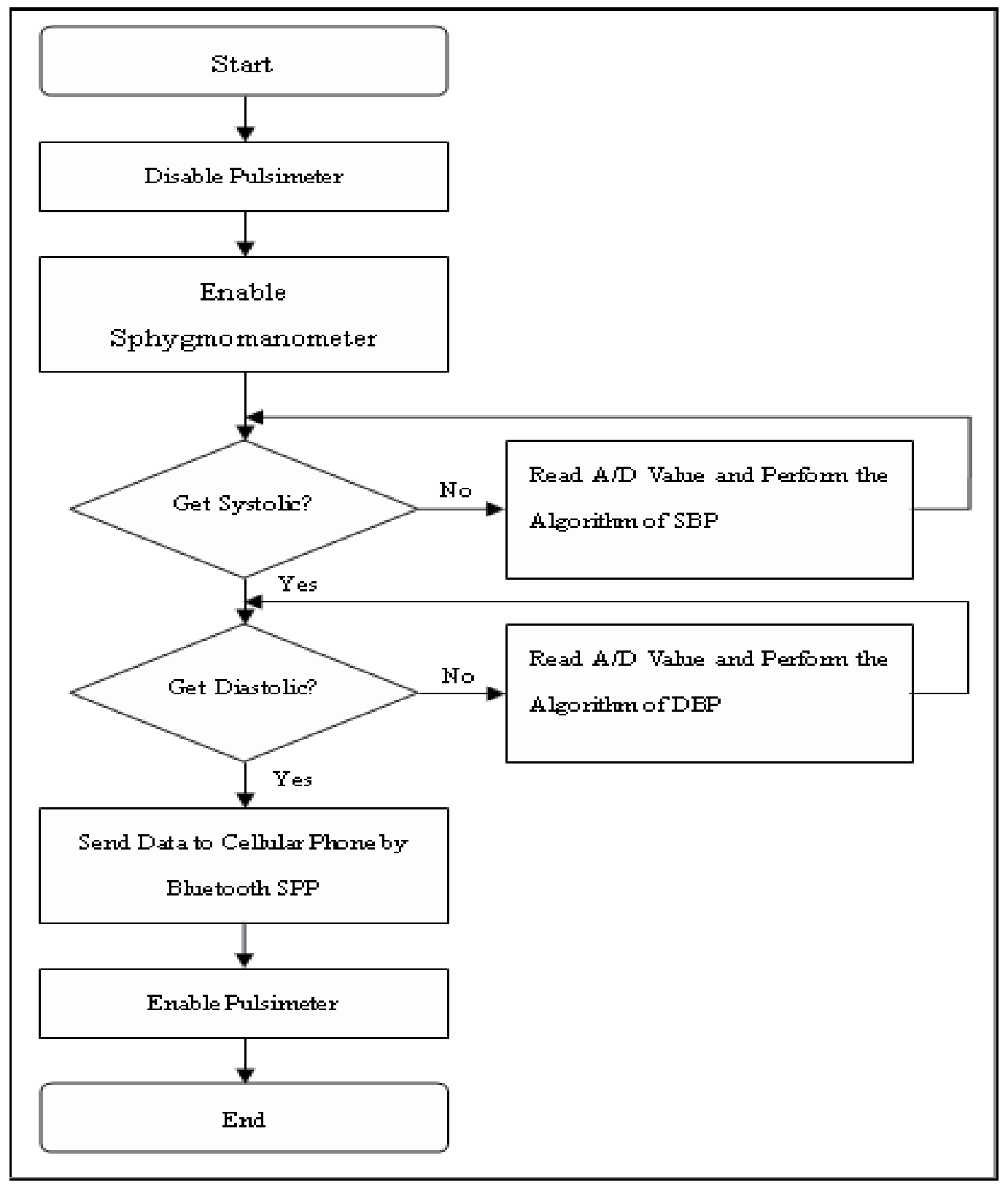

Figure 12. The software algorithm of the blood pressure measurement procedure 


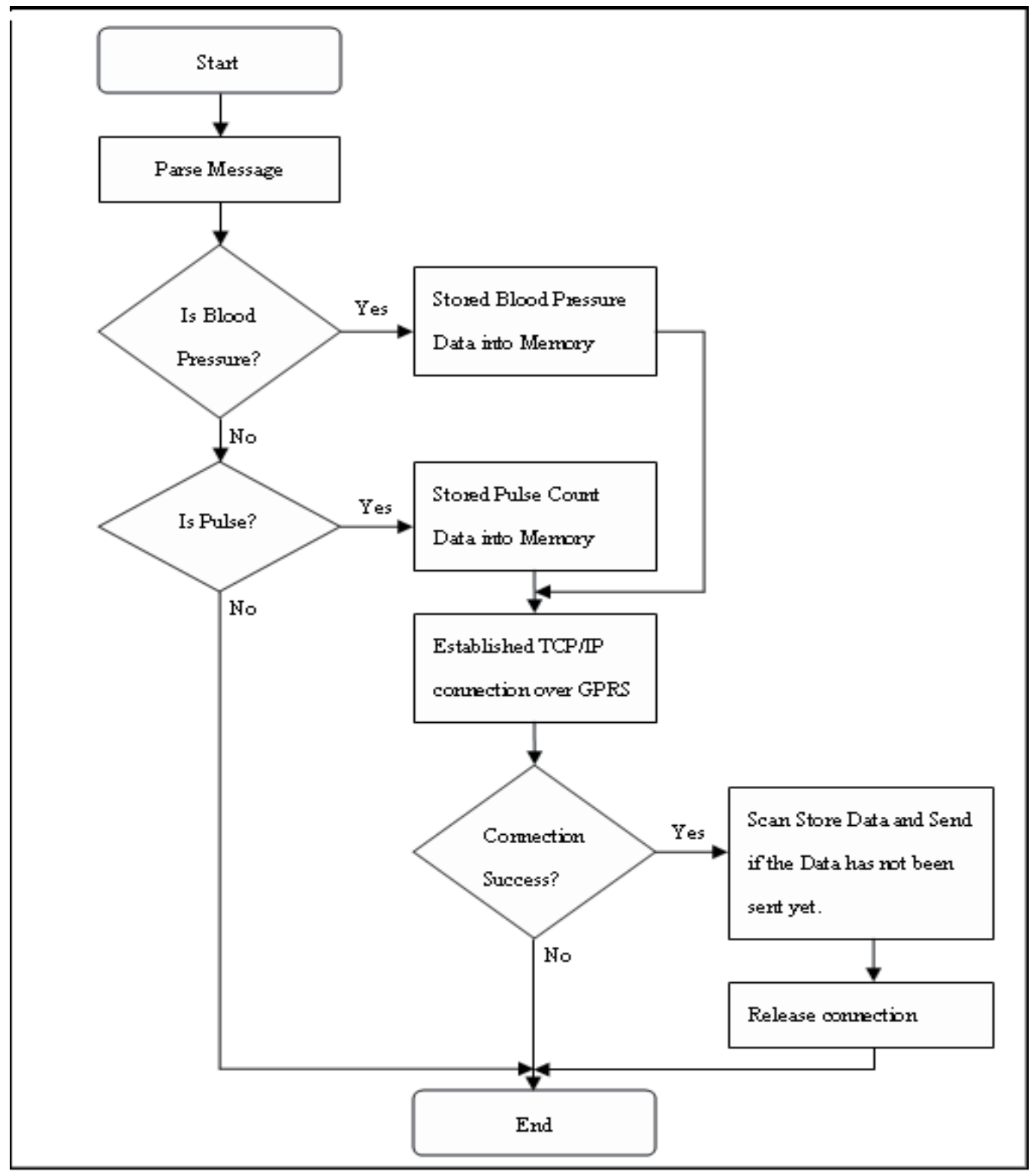

Figure 13. The software algorithm of client-end cellular phone data handling procedure 


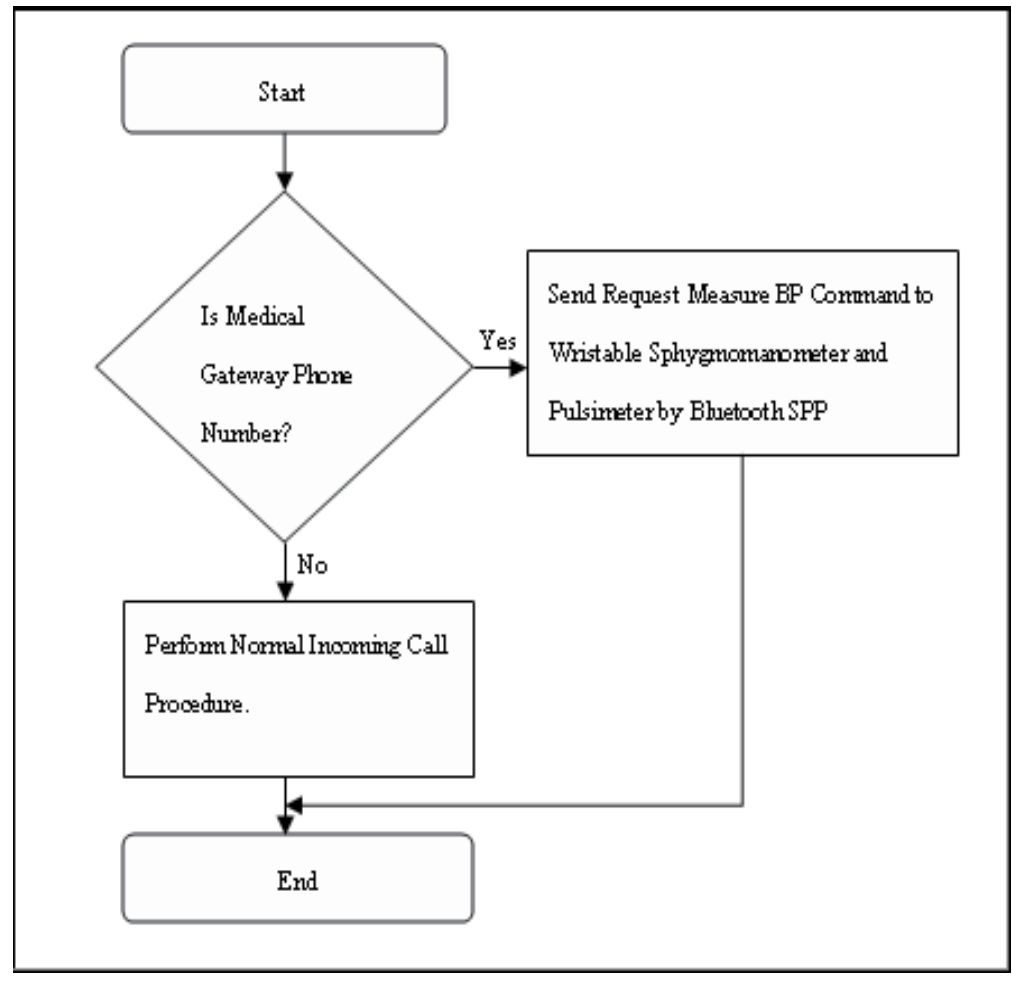

Figure 14. The software algorithm of remote medical server claim for measuring blood pressure

\subsection{The whole system operation procedure}

1. The procedure of measuring blood pressure by the wristable sphygmomanometer of the tele-care system is shown in Figure 15 [16,21,27,28,29]. The measurement was triggered by the user. When the microcontroller receives the trigger, it starts the procedure. First, the sphygmomanometer measures the pressure and sends back the data. The data are shown on the mono LCD and transmitted to the client-end cellular phone by Bluetooth SPP (Serial Port Profile).

2. The procedure of measuring blood pressure using the client-end cellular phone of telecare system is shown in Figure 16 [20,28,29,30,31,32]. When the Bluetooth of the clientend cellular phone receives signals, it checks the format and the Checksum of the data. The data is saved and sent to the sever-end cellular phone by Send Message over SD (Circuit Switched Data) if it is correct.

3. The procedure of measuring blood pressure using the server-end cellular phone of telecare system is shown in Figure 17 [19,20,29,30,31]. When the server-end cellular phone receives a short message from the client-end cellular phone, it checks the format and the Checksum of the data. If it is correct, the short message is decoded and saved according to IMEI. Then the message is erased.

4. The emergency procedure of the wristable sphygmomanometer of the tele-emergency announcement system is shown in Figure 18 [16,27,28,32]. The microcontroller reads the 
data which was acquired from the motion sensor every second. If the data repeats 30 times, it is called the Emergency Task and Alarm. If the user pushes the button, the Emergency Task will stop. Otherwise it will trigger sphygmomanometer measuring. The microcontroller sends an emergency message to the client-end cellular phone by Bluetooth SPP, once the number which is received from the sphygmomanometer measurement is defined as dangerous.

5. The procedure of emergency of the client-end cellular phone of tele-emergency announcement system is shown in Figure 19 [19,20,28,29,30,31,32]. When the Bluetooth of the client-end receives signals, it checks the format and the Checksum of the data. If it shows an emergency message, it is called the Emergency Task. In order to transmit a clear DTMF tone, the phone closes the microphone and forces the system to send out a Caller ID. Then the client-end cellular phone establishes voice connection with the server-end cellular phone. After the connection is established, it transmits IMEI, location, time and blood pressure in DTMF format.

6. The procedure of emergency of the server-end cellular phone of tele-emergency announcement system is shown in Figure 20 [19,20,29,30,31]. When the server-end mobile receives the incoming call from the client-end mobile phone, it will be forced to connect, and turn off the microphone, then switch audio path to the DTMF decoder [33]. After 2 seconds, if the controller receives the correct format and Checksum from the DTMF decoder, the phone will make a specific tone at the loudest setting, start the vibrator, then an emergency message will show on the display. It won't stop the tone and vibrator, even if it is disconnected.

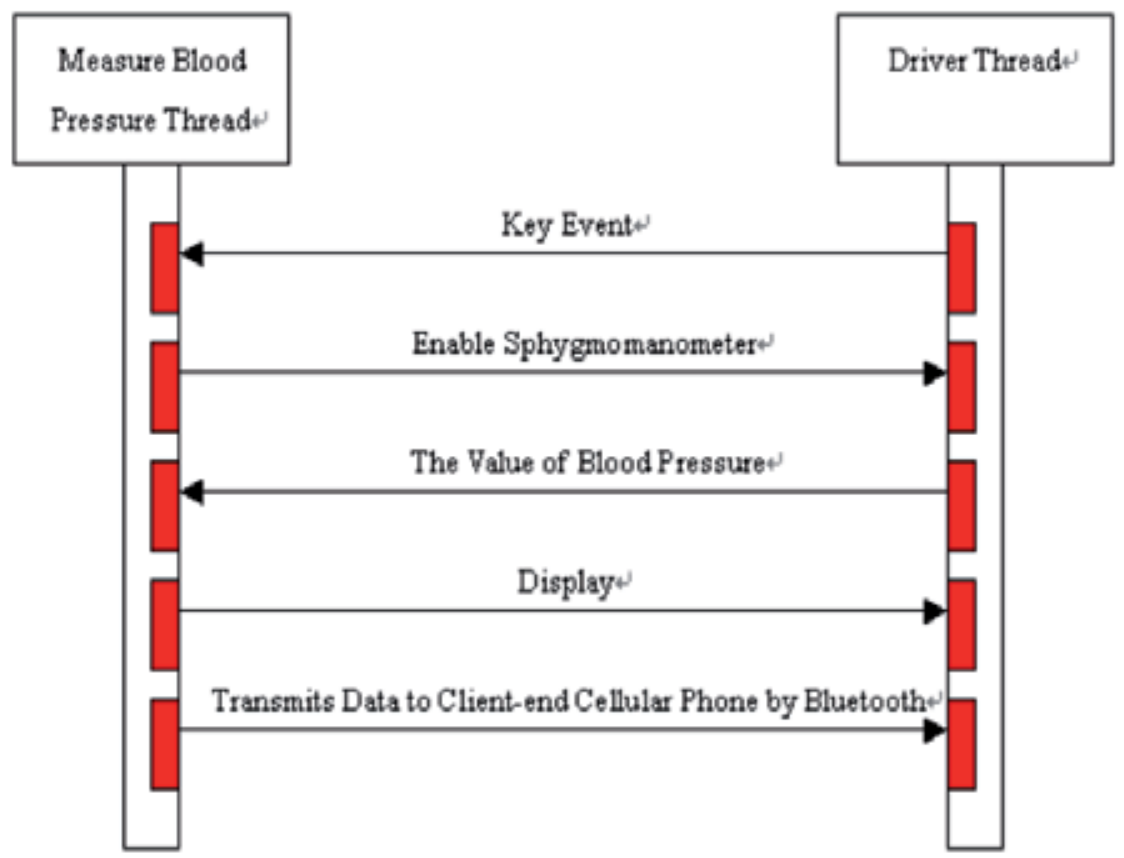

Figure 15. Procedure of measuring blood pressure by the wristable sphygmomanometer of the tele-care system 


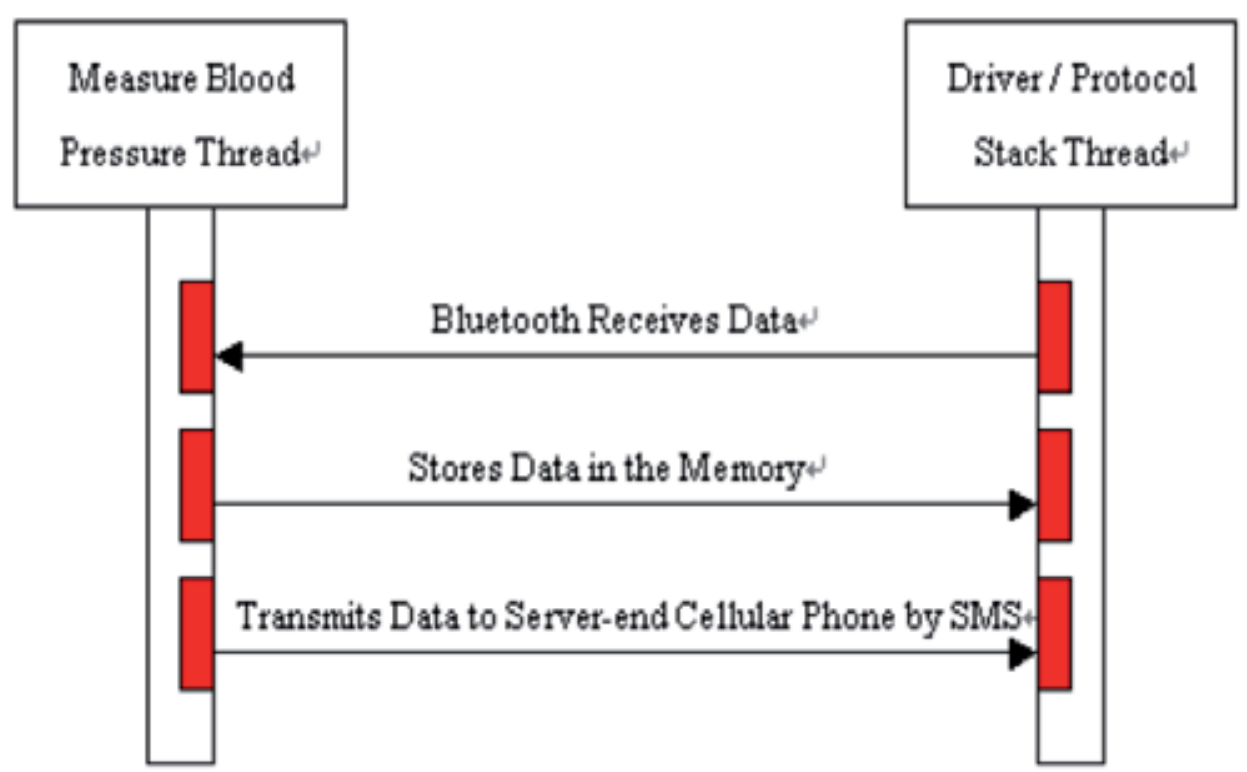

Figure 16. Procedure of measuring blood pressure using the client-end cellular phone of the tele-care system

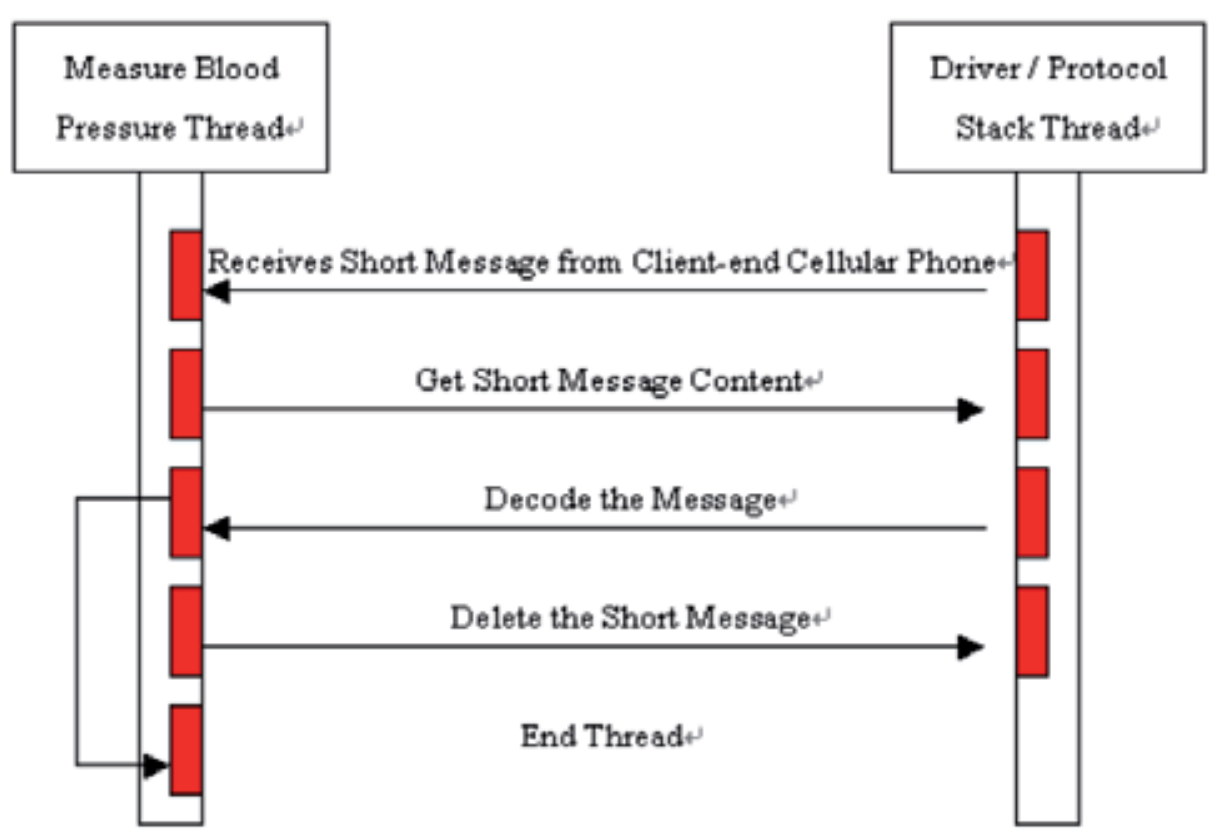

Figure 17. Procedure of measuring blood pressure of the server-end cellular phone of tele-care system 


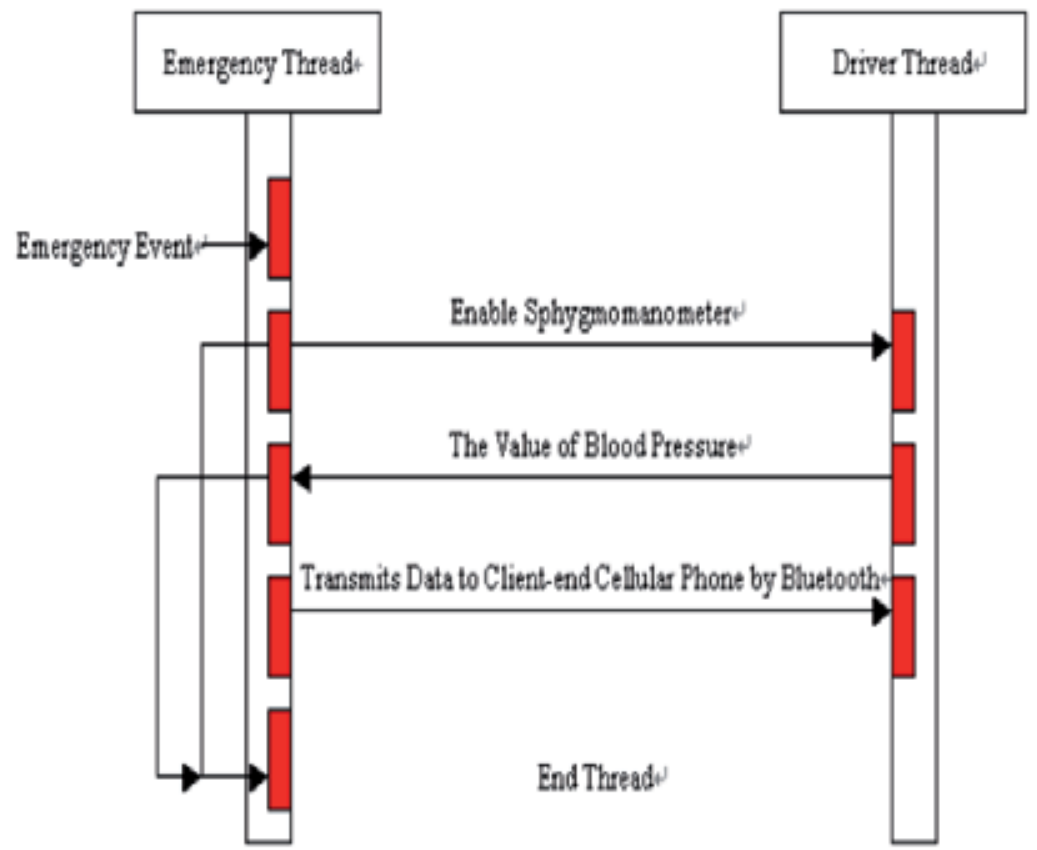

Figure 18. Emergency procedure of the wristable sphygmomanometer of the tele-emergency announcement

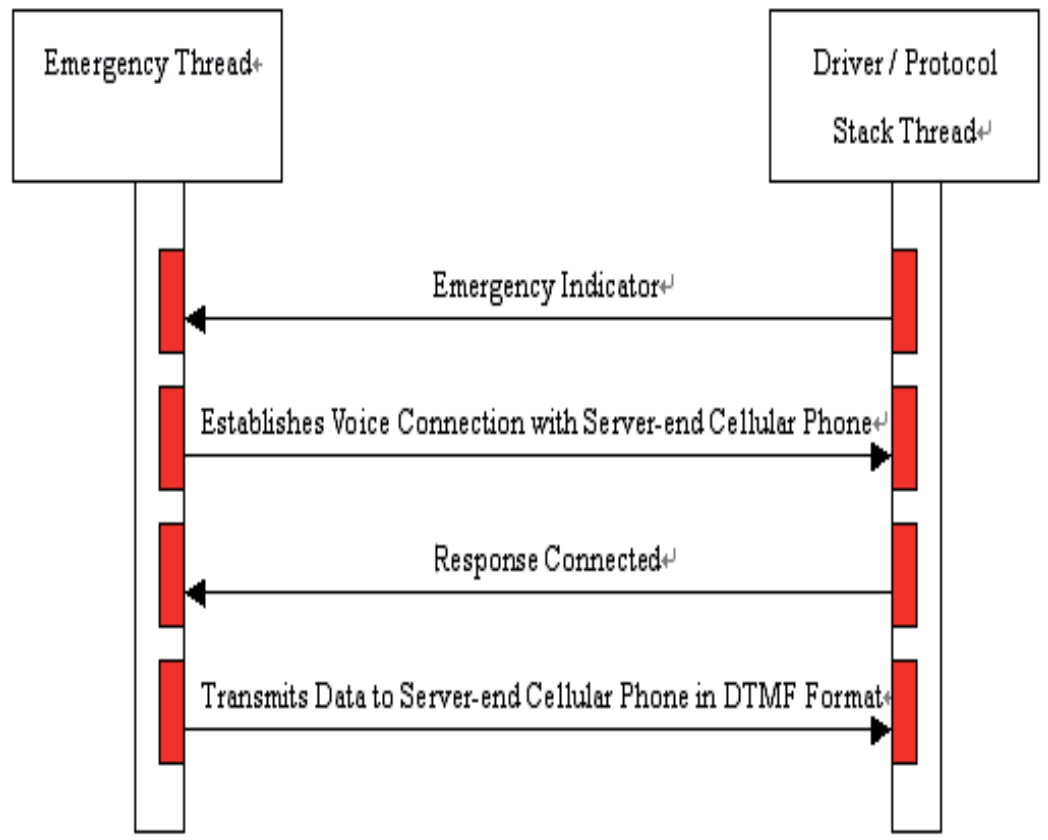

Figure 19. Emergency procedure of the wristable sphygmomanometer of the tele-emergency announcement system 


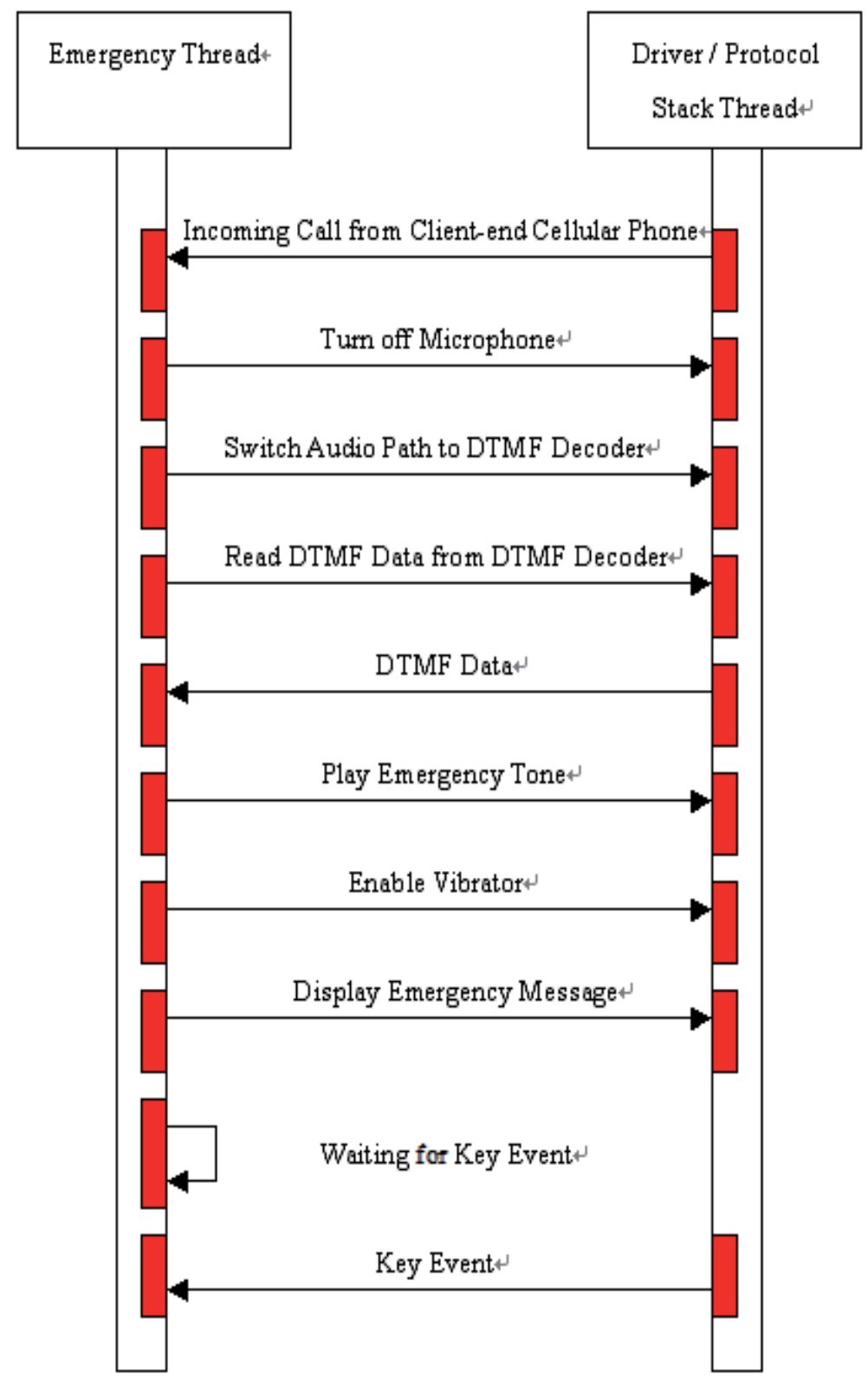

Figure 20. Emergency procedure of the server-end cellular phone of tele-emergency announcement system

\section{Remote monitoring measurement example}

We conducted an experiment using SpO2 and ECG using the GPS device and the 3G system of an Android smart phone to form a remote monitoring system, which can be used in the daily care of patients with implanted pacemakers. 
This system is categorized into three main structures, client, server and first aid unit. The client includes $\mathrm{BAD}$, a biomedical signal monitoring device (including ECG and $\mathrm{SpO}_{2}$ monitoring devices) and an Android mobile phone with Bluetooth communication ability. When BAD detects an abnormal health response from the user, it will give the mobile a report by means of the Bluetooth. In the meantime, the operating biomedical device, with sending signals back, allows GPS coordination to take place. These messages would be sent back to the server through the mobile phone's Internet system. The server-end is predominantly used to save the data, to analyse it and to communicate with others as shown in Figure 21.

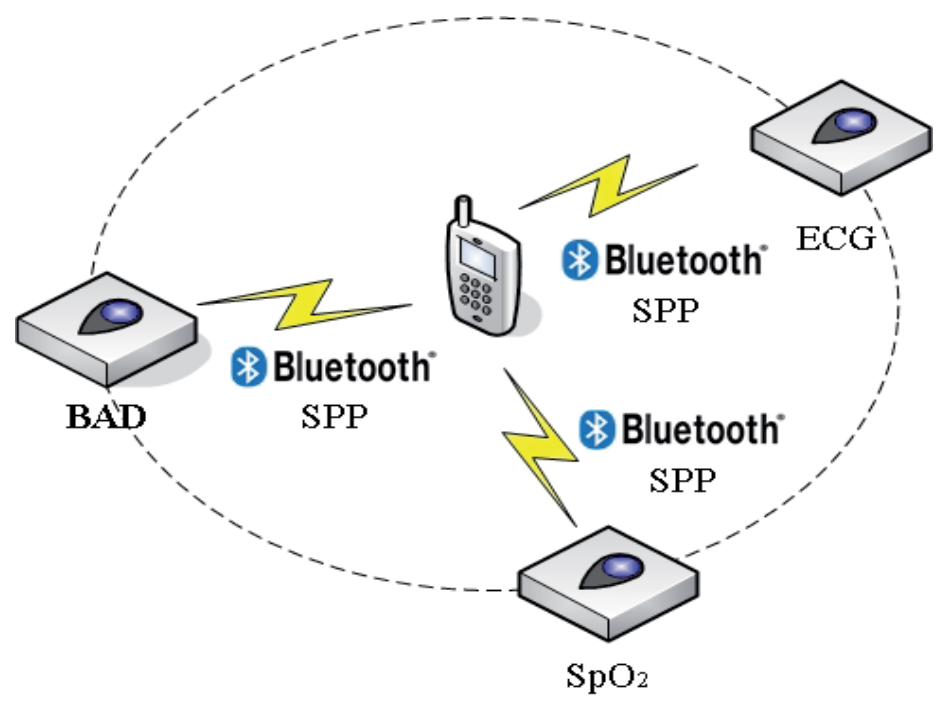

Figure 21. Bluetooth Pico-net

\subsection{System communication}

Figure 22 is the system communication plot. BAD, ECG and SpO2 will shut down the system into their power save mode after Bluetooth and cell phone pairing. When the BAD detects an emergency, it will resume normal power and contact the cell phone to call out ECG and $\mathrm{SpO}_{2}$ processing.

\subsection{Emergency procedure}

After receiving a BAD emergency signal, the system will enter the emergency procedure mode as shown in Figure 23. First it will check the signal again. If it is still an emergency signal, it will start the GPS to find out where the user is and identify whether the target moves. If the target stays put, it will start ECG and $\mathrm{SpO}_{2}$ Bluetooth devices. After receiving the data, it ativates the cell phone monitoring and displaying, and alerts nearby people for further assistance. At the same time, the data is sent back to the server and informs First Aid Units of the need for further help. Figure 24 shows the biomedical signals of ECG and $\mathrm{SpO}_{2}$ on the Android smart phone. 


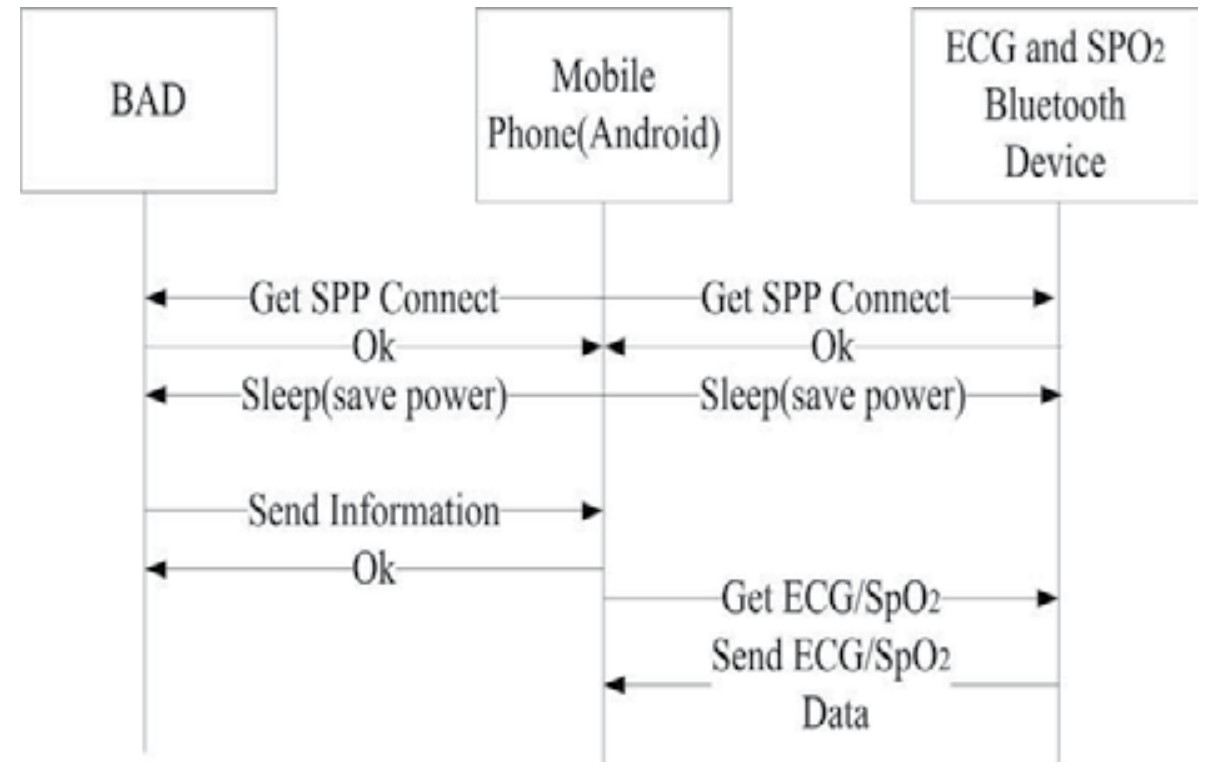

Figure 22. System communication

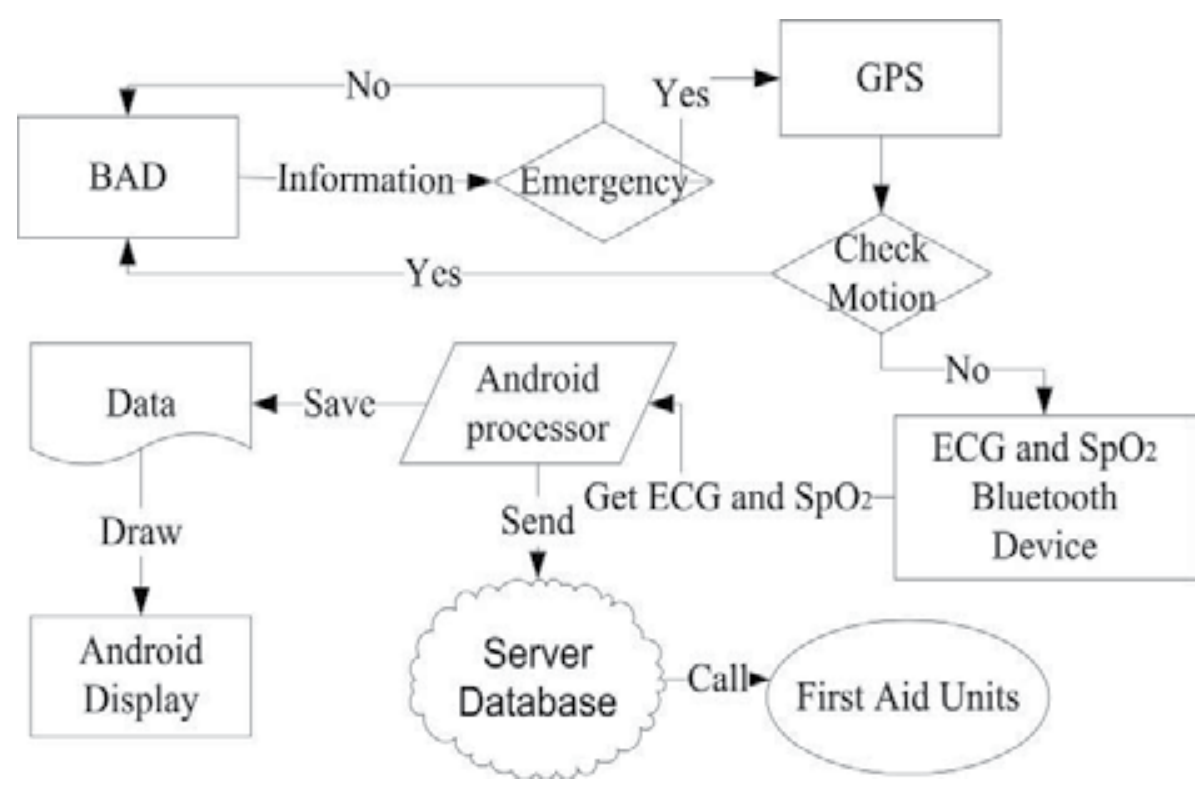

Figure 23. Emergency procedure

\subsection{Web-end}

The remote monitoring personnel confirm the biomedical information through the WEB interface, such as the ECG shown in Figure 25, and receive the signal when the patient needs help. At the same time, it uses GPS to confirm the client position to assist the ambulance to arrive at the right place (see Figure 26). 


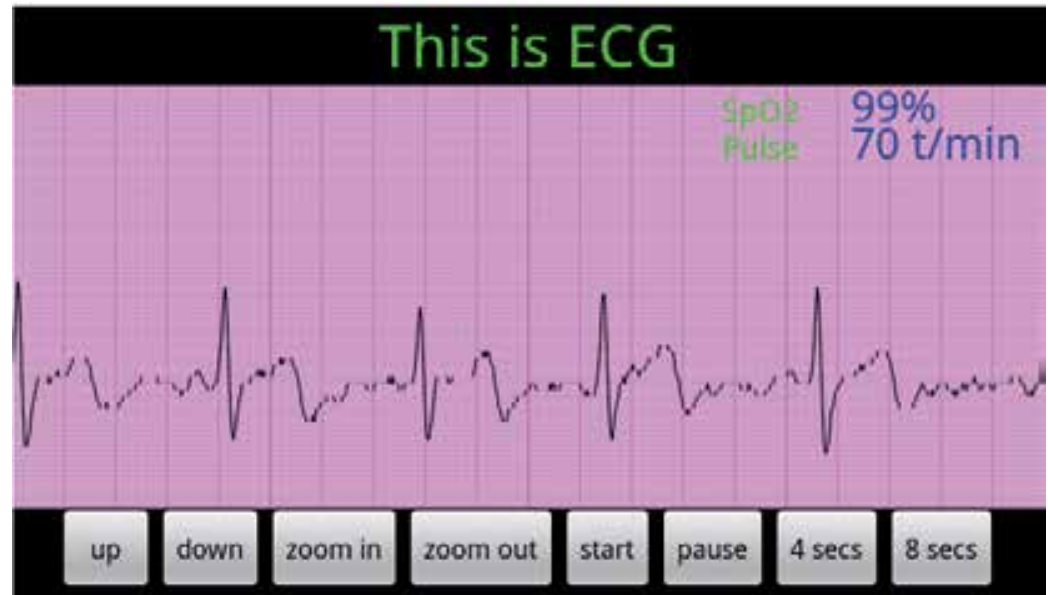

Figure 24. The biomedical signals ECG and $\mathrm{SpO}_{2}$ on the Android smart phone

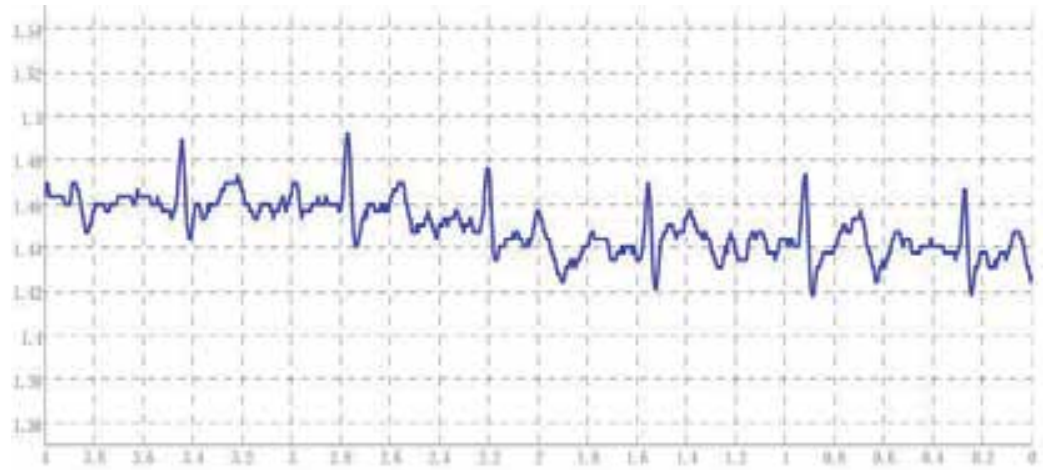

Figure 25. Server Web-end

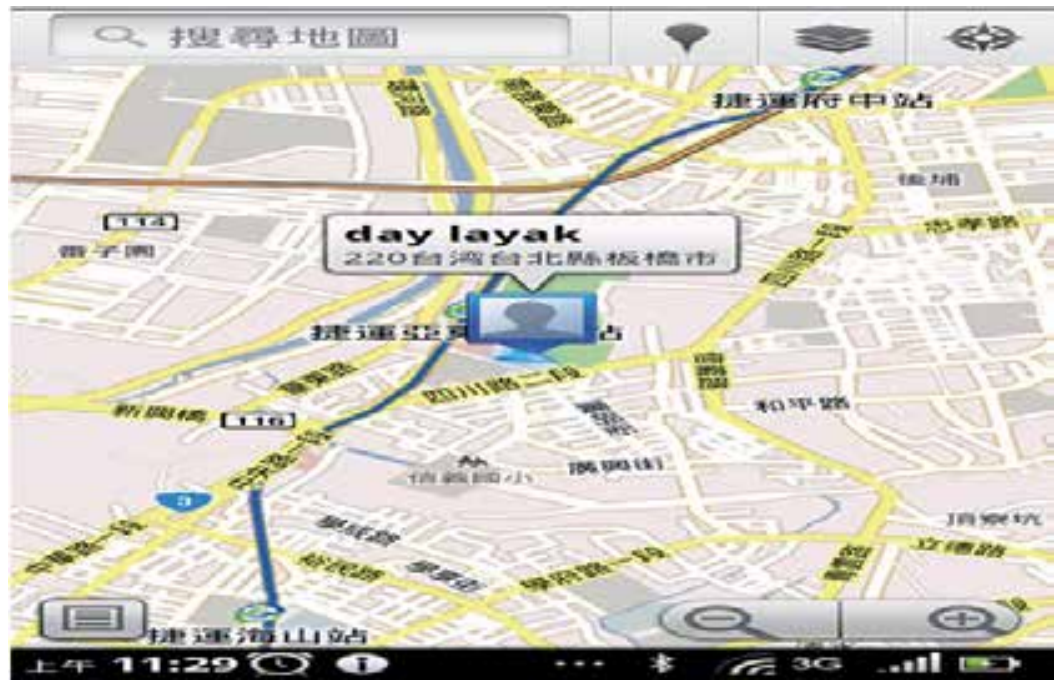

Figure 26. GPS location 


\section{Conclusion}

It may be dangerous to have continuously operating mobile connection close to the heart for patients with artificial pacemakers as it may consider the electromagnetic wave from the mobile to be a cardiac signal, leading to oversensing. However, frequent pulse and blood pressure measurement may be necessary for certain patient groups, such as those with high arrhythmia risk, hypertension, coronary artery disease or heart failure. As Bluetooth technology is safe to use in proximity of pacemakers, we have designed a portable medium based on the Bluetooth power class II RF test specification. We expect that this system will benefit these patients without causing inconvenience to their daily activities or risking adverse interaction with the pacemaker.

\section{Author details}

Ching-Sung Wang

Department of Electronic Engineering, Oriental Institute of Technology, Taipei, Taiwan

Teng-Wei Wang

The Third Department of Clinical Research Institute, Peking University, Peking, China

\section{References}

[1] http://www.surgeryencyclopedia.com/La-Pa/Pacemakers.html

[2] Tacey S, Ronald A, "The Effects of Electromagnetic Fields on Cardiac Pacemakers" IEEE Transactions on Broadcasting, Vol.38,No.2,June 1992

[3] Barbaro V, Bartolini P, Donato A, et al. "Do European GSM mobile cellular phones pose a potential risk to pacemaker patients?" PACE 1995; 18: 1218-24

[4] Okan Erdogan, MD, "Electromagnetic Interference on Pacemakers", Indian Pacing Electrophysiol J. 2002 Jul-Sep; 2(3): 74-78

[5] Hayes DL, Wang PJ, Reynolds DW, et al. "Interference with cardiac pacemakers by cellular telephones". N Eng J Med 1997; 336: 1473-9

[6] Bluetooth, Specification of the Bluetooth System Core, Version 2.0, 2004

[7] Bluetooth, Radio Frequency Test Suite Structure (TSS) and Test Purposes (TP) Specification 1.2, Revision 1.2.3, 2004-08-24

[8] Nissila, S, Sorvisto, M, Sorvoja, H, Vieri-Gashi, E, Myllyla, R, “Non-invasive blood pressure measurement based on the electronic palpation method", Engineering in Medicine and Biology Society, 1998. Proceedings of the 20th Annual International Conference of the IEEE, Volume 4, 29 Oct.-1 Nov. 1998 Page(s):1723 - 1726 vol.4

[9] Yi-Bing Lin, Imrich Chlamtac, "Wireless and Mobile Network Architectures", Wiley Computer Publishing, 1 edition (October 2, 2000)

[10] Mouly, M, Pautet, M-B, “The GSM System for Mobile Communications”, Telecom Publishing (June 1992) 
[11] Ching-Sung Wang, Jung-Hunag Lee, Yiu-Tong Chu, "Mobile Telemedicine Application and Technologies on GSM", Bioinformatics and Biomedical Engineering, 2007. ICBBE 2007. The 1st International Conference on 6-8 July 2007 Page(s):1125 - 1128

[12] MediaTek "AT Command Set", Revision: 0.03, Sep. 1. 2004

[13] Ching-Sung Wang, Chien-Wei Liu, Teng-Wei Wang, “Tele-care for emergency announcements" Journal of Biomedical Science and Engineering, pp.822-827, Aug.,2010

[14] Winbond Inc., "W681388 Single Programmable Extended CODEC/SLIC", ver1.0, 2006.

[15] Microchip Inc., "PIC24FJ64GA004 Family Data Sheet", 2007.

[16] National Semiconductor "SC14440/431/432, SC14435, SC14436/437/438. Baseband Processor for PP/FP DECT and WDCT", V1.1, June 16, 2005

[17] Champion "CM2838, 300mA Low Esr CMOS LDO With Enable", Rev 1.1, 2004/0501

[18] HoneyWell , “SSC Series Pressure Sensors 0 - 5 psi Through 0 - 300 psi” , 2002

[19] ETSI “Digital cellular telecommunications system (Phase 2+); International Mobile station Equipment Identities (IMEI); (GSM 02.16 version 6.2.0 Release 1997)", 200007

[20] ETSI “Digital cellular telecommunications system (Phase 2+); Technical realization of the Short Message Service (SMS); (GSM 03.40 version 7.4.0 Release 1998)", 199912

[21] ELAN “EM62100 65 COM/ 132 SEG STN LCD Driver Product Specification”, version 1.5, September 2006

[22] MEMSIC “MXC6202xJ/K Data Sheet”, Rev A, 2006

[23] CSR, "BlueCore3-ROM CSP Product Data Book", Nov 2006

[24] Specification of the Bluetooth System Core, Version 2.0, 2004

[25] Pravin Bhagwat Reefedge Inc., "Bluetooth: technology for short-range wireless apps", IEEE Internet Computing, Volume 5, Issue 3, May-June 2001 Page(s): 96 103.

[26] MediaTek “MT6219 GSM/GPRS Baseband Processor Data Sheet”, Revision 1.00, Feb 02, 2004.

[27] ATMEL "AT89C2051 8-bit Microcontroller with 2K Bytes Flash Data Sheet", 6/05.

[28] Bluetooth SIG, "Specification of the Bluetooth System Profile, Part K:5 Serial Port Profile", version 1.1, 2001

[29] MediaTek “Customer Device Driver Document", Revision: 0.1, Nov 03, 2003

[30] PixTel “Writing Applications Using PixTel MMI Platform”, version: 1.3, July 10, 2004

[31] ETSI “Digital cellular telecommunications system (Phase 2+); AT command set for GSM Mobile Equipment(ME);(GSM 07.07 version5.9.1 Release 1996)", December 1999 
[32] Bluetooth SIG, Specification of the Bluetooth System Core, version 2.0, 2004

[33] MITEL “MT8870D/MT8870D-1 Data Sheet”, May 1995 


\title{
Phrenic Nerve Pacing: Current Concepts
}

\author{
Jorge F. Velazco, Shekhar Ghamande and Salim Surani
}

Additional information is available at the end of the chapter

http://dx.doi.org/10.5772/48808

\section{Introduction}

Anatomy: The diaphragm is the main inspiratory muscle in mammals, it is a highly active muscle with a duty cycle of $30-40 \%$, and therefore, the diaphragm may be particularly susceptible to inactivity or disuse (1). It is innervated by the phrenic nerves that arise from the nerve roots $\mathrm{C} 3$ through $\mathrm{C} 5$, and is primarily composed of fatigue-resistant slow-twitch type I and fast-twitch IIa myofibers. As the diaphragm contracts, the abdominal contents are displaced caudally, abdominal pressure increases and the lower ribcage expands (2). The phrenic nerve contains sensory, motor and sympathetic nerve fibers, and it supplies motor supply to the diaphragm and sensory supply to the central tendon (3). Both phrenic nerves run along the anterior scalene muscle deep to the carotid sheath. The right phrenic nerve passes over the brachiocephalic artery and posterior to the subclavian vein and over the right atrium. It enters the diaphragm at the level of T8 vertebrae. The left phrenic nerve also runs posterior to the left subclavian vein and passes over the pericardium of the left ventricle and penetrates the left hemidiaphragm separately (3). See Figures 1 and 2.

Pathophysiology: Diaphragmatic dysfunction can manifest as weakness or paralysis, and is related to different entities. Patients with bilateral diaphragmatic paralysis are more likely to have symptoms like dyspnea or recurrent respiratory failure; other complications include subsegmental atelectasis and infections of lower respiratory tract (2). See Figure 3.

Of the 11,000 new cases of spinal cord injury (SCI), approximately one half $(56.4 \%)$ are sustained at the cervical level (4). Currently, the average age of injury is 37.6 , and about $80 \%$ are male (5). Cervical SCI often leads to an interruption of the descending bulbospinal respiratory pathways, resulting in respiratory muscle paresis and/or paralysis. The more rostral the level of injury the greater the likelihood of a major respiratory compromise, however the most caudal segment of the spinal cord with normal motor function defines the motor level of injury. The injuries above the level of the phrenic motoneurons (C3, C4, C5) 


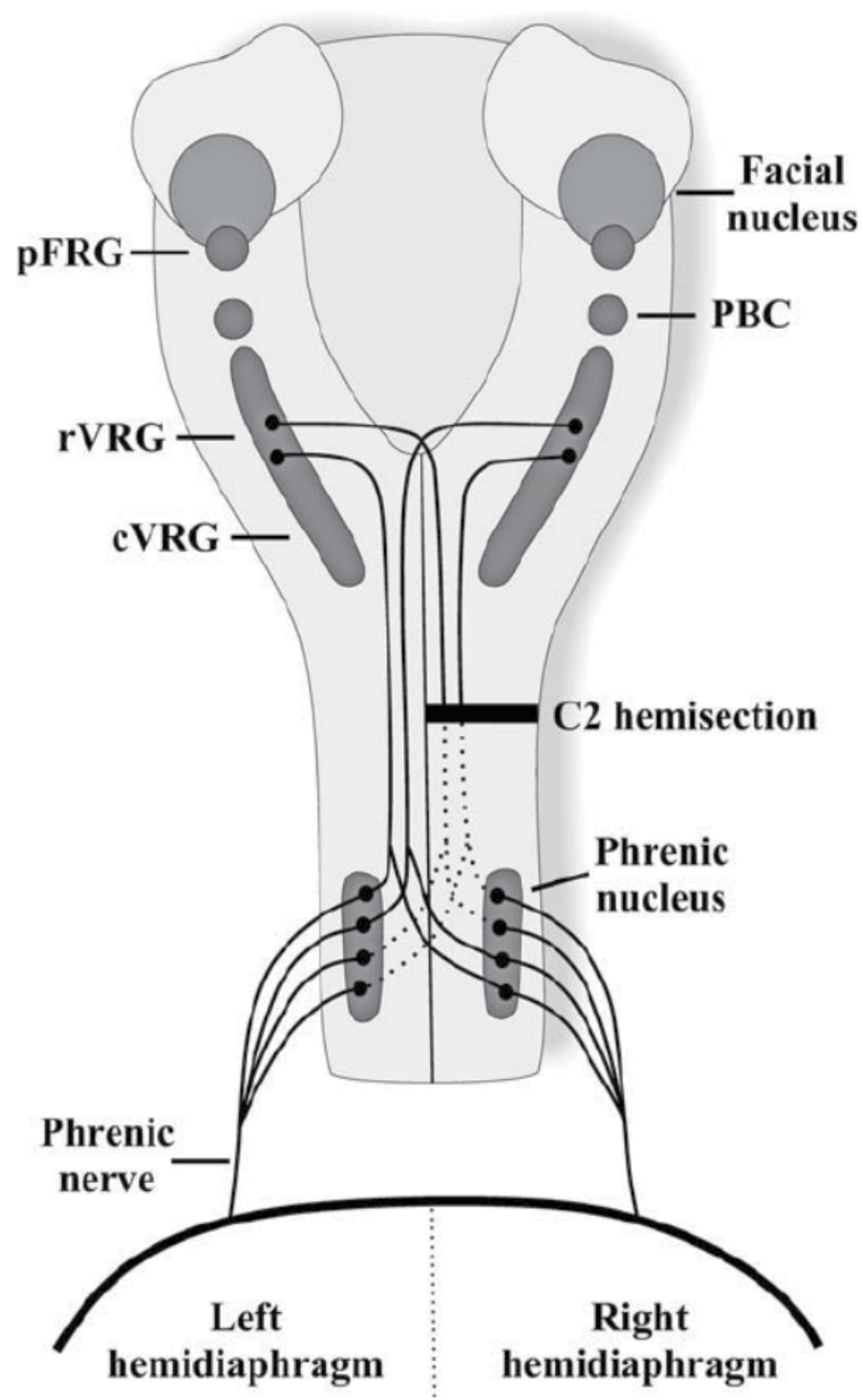

Legend: pFRG: parafacial respiratory group; rVRG: rostral ventral respiratory group; cVRG: caudal ventral respiratory group; PBC: preBotzinger complex.

Reproduced with permission. Effect of Spinal Cord Injury on the Respiratory System: Basic Research and Current Clinical Treatment Options. Beth Zimmer et al. J Spinal Cord Med. 2007; 30:319-330

Figure 1. Schematic drawing of the major respiratory neural centers and pathways in the rat. Respiratory rhythm generation is formed by neurons located within the pFRG and the PBC. Impulses are transmitted to premotor neurons located within the VRG with inspira-tory neurons located predominantly in rVRG and expira-tory neurons localized to cVRG. Premotor neurons project either unilaterally or bilaterally down to phrenic motor neurons located on both sides of the spinal cord (C3-C6). Crossed respiratory axons (arising from VRG neurons bilaterally) have been localized the level of the phrenic nucleus and contribute to the expression of the crossed phrenic phenomenon. Phrenic axons from each side of the spinal cord form the phrenic nerve, which projects to each half of the diaphragm. 


\section{A Normal}
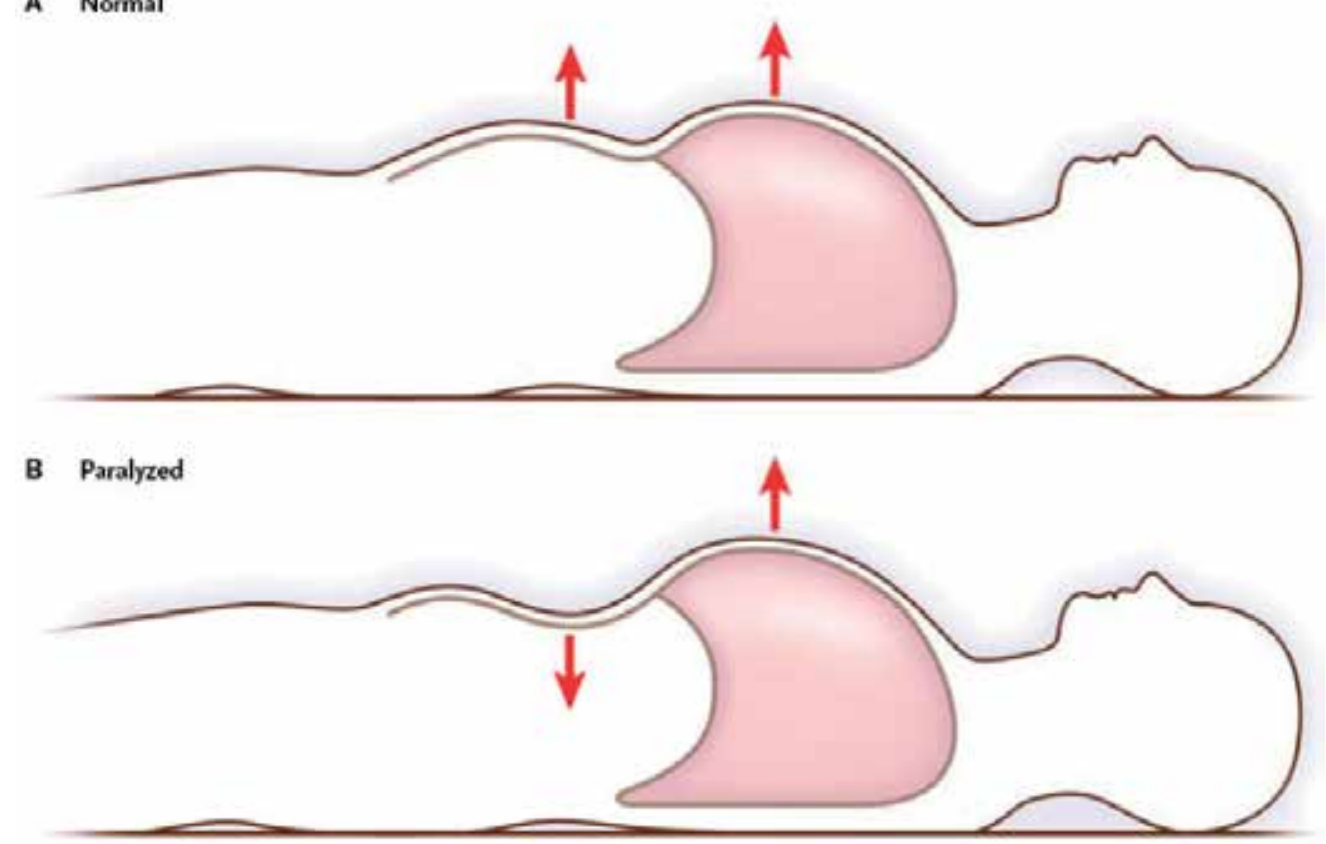

Reproduced with permission. Dysfunction of the Diaphragm. McCool, MD et al. N Engl J Med 2012; 366:932-42

Figure 2. Comparison of Rib-Cage and Abdominal Motion in Normal and Paralyzed Diaphragms. Panel A shows how normal diaphragmatic contraction results in an outward motion of the abdomen and rib cage (arrows). Panel B shows how diaphragmatic paralysis results in a paradoxical inward motion of the abdomen (down-ward-pointing arrow) during inspiration. The accessory inspiratory muscles contract, lifting the rib cage (upward-pointing arrow) and lowering the intrathoracic pressure. This change causes the flaccid diaphragm to move in a cephalad direction and the anterior abdominal wall to move inward.

cause virtually complete paralysis of both the inspiratory and expiratory muscles with mechanical ventilator dependency $(5,6)$. It may be possible to maintain neuromuscular transmission and functional properties of diaphragm muscle fibers after spinal cord injury when motoneurons are not directly injured (1). More than $80 \%$ of injuries are traumatic in nature, and up to $1.5 \%$ of such injuries are sustained in children. Many of these patients cannot be eventually weaned off mechanical ventilation, and require chronic ventilator support in as much as $4 \%$ of the cases $(4,6,7,8)$. In the United States more than 100,000 tracheostomies are performed for patients who are unable to be weaned from ventilator $(8,9)$. In most of these cases the lungs, chest wall, and respiratory muscles are usually physiologically normal (4). Acute SCI is complicated by respiratory complications in $67 \%$ of cases with pneumonia being the most common cause of death $(6,9,10)$. The C1-C4 injury group had pneumonia in $63 \%$ of cases at a mean interval of 30 days post-injury. Patients with high level lesions associated with bilateral diaphragm paralysis cannot achieve and sustain a vital capacity of $5 \mathrm{~mL} / \mathrm{Kg}$ (10). Controlled mechanical ventilation leads to rapid atrophy of the diaphragm. Within 18 hours of mechanical ventilation there is a decrease in type I muscle fibers with conversion to less functional fast-twitch type IIb muscle fibers (8). 


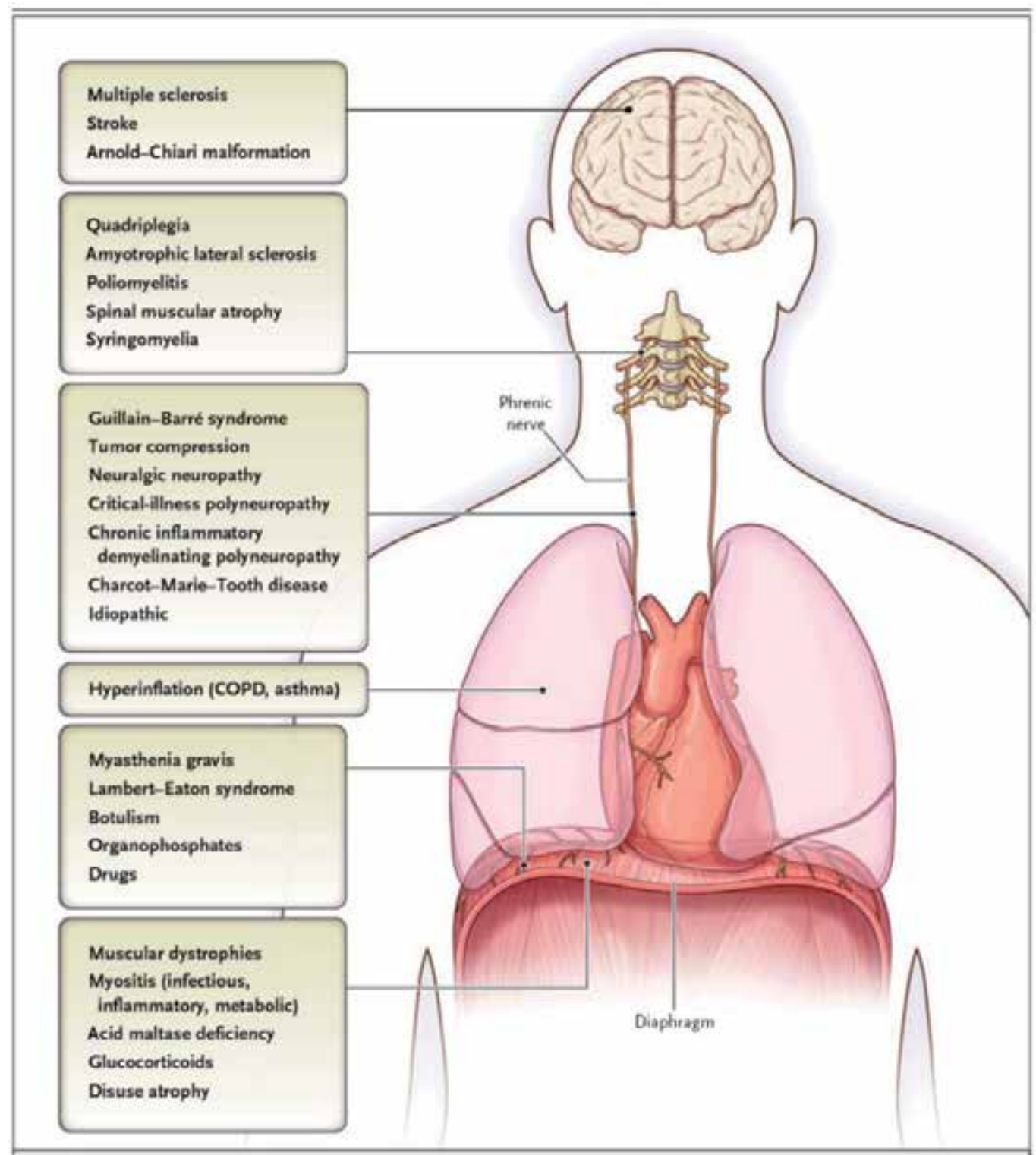

Reproduced with permission. Dysfunction of the Diaphragm. McCool, MD et al. N Engl J Med 2012; 366:932-42

Figure 3. Causes of Diaphragmatic Dysfunction, According to Level of Impairment. Disorders that occur at various regions in the body can lead to diaphragmatic dysfunction. COPD denotes chronic obstructive pulmonary disease.

Beyond one year the chances of recovery of fully independent breathing is probably less than $5 \%$ of patients with complete high tetraplegia (10). Loss of diaphragm function reduces survival. It is estimated that a 15 year-old who sustains a high SCI and survives the first 24 hours, the need of mechanical ventilation decreases life expectancy by 21 years (7); in the case of a 40 year-old patient with tetraplegia and ventilator dependence who survives the 
first 24 hours life expectancy is 7 years. It is dramatically reduced to 1.8 years in a 60 yearold with SCI $(8,9)$.

Mechanical ventilation in tracheostomy patients has several complications such as speech difficulties, reduced mobility, difficulty with transfers, social limitations, loss of sense of smell, equipment malfunction, increased respiratory infections, and increased cost of care approaching $\$ 200,000$ a year $(7,8,10,11)$.

Central Alveolar Hypoventilation Syndrome (CAHS) or "Ondine's curse" is extremely rare condition that shares the ventilatory failure pattern along with preserved lung functions, normal chest wall mechanics, and physiologically normal respiratory muscles. It is characterized by failure of the autonomic control of breathing during sleep (12). The term originates from a German myth about Ondine, an oceanic nymph who punished her unfaithful lover with the loss of all movements that did not involve his conscious will. Thus, he was condemned to remain awake forever to be able to breathe $(12,13)$. Blunted response of the respiratory center to carbon dioxide leads to hypoventilation and central apneas resulting in hypercarbia and hypoxemia; typically exacerbated during periods of sleep. Pulmonary hypertension and cor pulmonale lead to death (12).

Besides trauma, other causes of phrenic nerve involvement leading to chronic diaphragm dysfunction include vasculitis, neuralgic amyotrophy (14).

Amyotrophic lateral sclerosis is a relentlessly progressive neuromuscular disease of unknown origin that affects both upper and lower motor neurons causing weakness, hyperreflexia and atrophy (15). The incidence is 0.2 to 2.4 cases per 100,000 populations; it is most commonly diagnosed in middle age, affects more men than women. Life expectancy of ALS is 3 to 5 years after diagnosis (15). The major cause of mortality in patients with ALS is respiratory failure because of the progressive decline of 3-5\% per month of Forced Vital Capacity (FVC) due to progressive loss of motor neurons leading to respiratory muscle weakness. Phrenic nerve pacing is a novel method being investigated to treat the diaphragm involvement in ALS (11).

Diaphragm pacing is a technique of artificial ventilation using electrical stimulation of the phrenic nerves to produce diaphragm contraction, providing many ventilator-dependent tetraplegic patients with freedom from mechanical ventilation $(16,17,18,19)$. Compared with mechanical ventilation, phrenic pacing provides more natural breathing dynamics, reduces the occurrence of respiratory infection, and improves quality of life. Additionally earlier home discharge and lower infections drive down health care costs (20). Diaphragm muscle pacing, phrenic nerve pacing, and combined intercostal and unilateral diaphragm pacing techniques are currently been used to wean patients from ventilators and reduce the incidence of infection, atelectasis and respiratory failure (6).

Diaphragm pacing is conducted with low frequency electrical stimulation at a slow repetition rate to condition the diaphragm muscle against fatigue (21). 


\section{History}

The use of electricity for therapeutic purposes dates back to $15 \mathrm{AD}$, when Scribonious Largus, a court physician to the Roman emperor Claudius began using electric shocks from the torpedo ray fish to treat gout pain and headaches (22).

Sophisticated techniques for electrical stimulation of excitable tissue in neuromuscular disorders have been developed over the past three decades. Caldani in 1786 was probably the first to note movement of the diaphragm upon electrical stimulation of the phrenic nerve. In 1818 Ure applied electricity to the phrenic nerve of a recently hung criminal producing "strong and laborious respirations". In 1873, Hufeland proposed stimulating the phrenic nerve to treat asphyxia in neonates. During $19^{\text {th }}$ century attempts were made to apply direct electrical stimulation to the diaphragm or to phrenic nerves in the neck $(21,23,24)$.

By 1878, phrenic nerve stimulation was accepted as a rational procedure. Ziemssen used placement of large well-moistened sponges firmly over phrenic nerves at the outer borders of sternocleidomastoid muscles at the lower end of the scalene muscles to electrically stimulate the nerves (21).

The advent of mechanical ventilation led to abandonment of efforts at diaphragm stimulation as a means of supporting ventilation except for a few case reports. Sarnoff in 1948 that provided adequate ventilation with transcutaneous phrenic nerve stimulation for up to 52 hours in paralyzed patient with cerebral aneurysm. He also demonstrated this in bulbar poliomyelitis $(3,4,16,21,25)$. Sarnoff et al experimented extensively with electrical stimulation of the phrenic nerve and demonstrated that submaximal electrical stimulation of only one phrenic nerve could affect normal oxygen and carbon dioxide exchange; they called this method electrophrenic respiration (EPR) (26). In 1966 Glenn and coworkers first treated a patient with chronic ventilatory failure, using a radiofrequency system with an implantable receiver and electrode but an external power source. This technique was adopted as the gold standard of clinical application for more than 20 years. The surgery involved placement of electrodes on the phrenic nerve in the neck and thorax through a thoracotomy $(9,16,22,25,27,28)$. The initial device consisted of an external battery-powered transmitter with an antenna placed on the skin and a radio receiver placed subcutaneously beneath the antenna (3). See Figure 4.

Successful use of implantable pacemakers for the heart has prompted further interest in development of electrical stimulation of excitable tissues including the diaphragm. Improvements in pacemaker equipment design and intensive-care technology have increased the chances of using diaphragm pacing for the treatment of respiratory failure due to diaphragm paralysis (29). By 2004 approximately 1600 patients worldwide had been implanted with one of these pacing systems during last 35 years $(9,22)$.

In multicenter trials of SCI patients, $98 \%$ of patients were liberated from their ventilators with the diaphragm pacing system, which led to US Food and Drug Administration (FDA) approval for this indication in 2008. 


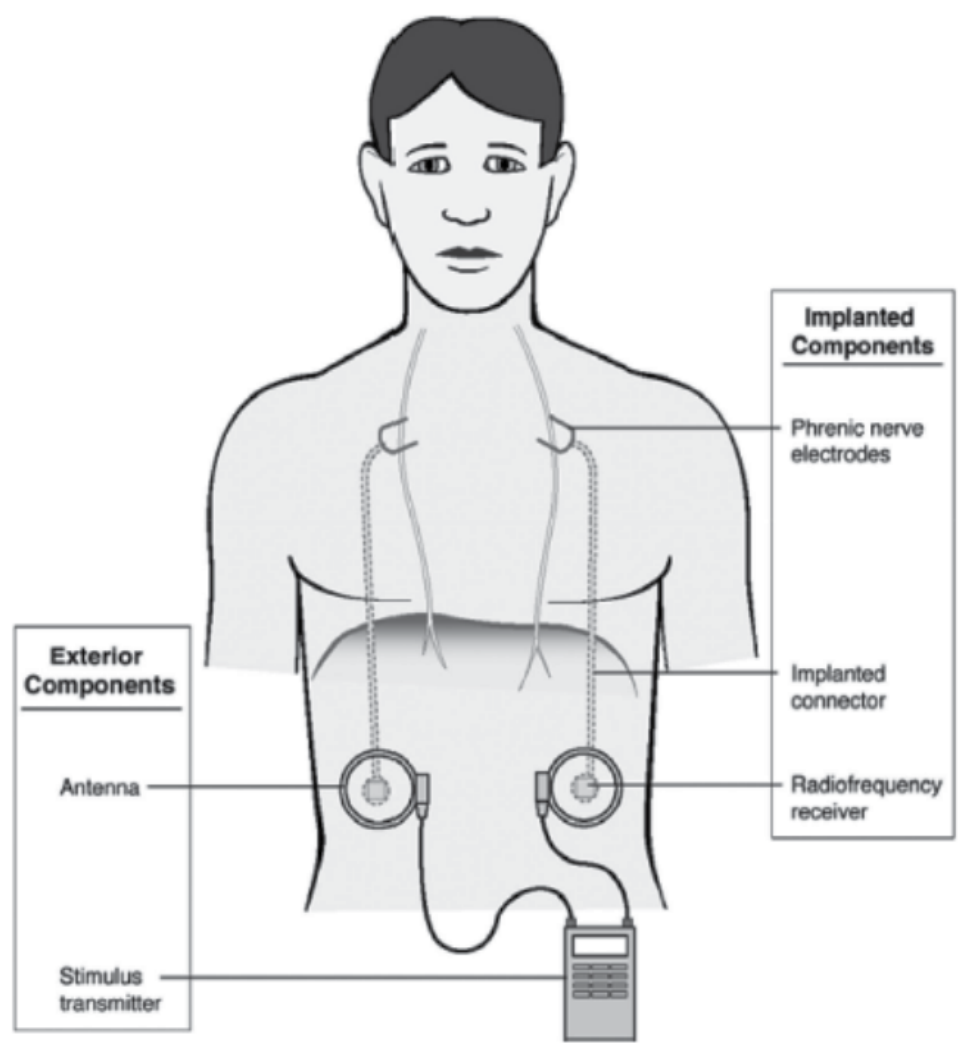

Reproduced with permission by Functional Electrical Stimulation Therapies after Spinal Cord Injury. David Gater et al. NeuroRehabilitation 28 (2011)231-248

Figure 4. Functional electrical stimulation of the diaphragm for respiration

\section{Indications}

The accepted indications for phrenic nerve pacing are respiratory paralysis after Cervical $\mathrm{SCI}$ above the origin of the phrenic nerve neurons (C1-C2) that are ventilator-dependent and the chronic Central Hypoventilation Syndrome associated with central apneas $(2,13,23,26,30,31)$. Diaphragm pacing has also been tried in chronic obstructive pulmonary disease (COPD), ALS $(15,32,33)$ and chronic hiccup $(34,35)$. Glenn et al elaborated on indications for the use of these pacemakers.

A diaphragmatic pacemaker may be considered for:

1. Chronic, respiratory failure requiring invasive mechanical ventilation via permanent tracheostomy on either a temporary, intermittent or continuous basis.

2. A central neurological cause for the respiratory failure such as

a. Alveolar hypoventilation, either primary or secondary to a brainstem disorder.

b. Interruption of neuronal conduction at the upper cervical level, above the C3 level

3. Integrity of the intrathoracic section of the phrenic nerve 
4. Acceptable pulmonary function

5. Normal level of consciousness (36).

Acquired CAHS has been described with medullary tumors, infection (particularly poliomyelitis), upper cervical trauma, some mitochondrial diseases, degenerative diseases (eg multiple sclerosis), and non-specific ischemic insults (12).

In the case of ALS patients, the goal is to initiate phrenic pacing before tracheostomy is required in order to maintain diaphragm strength. This will help convert diaphragm muscle fibers from the fast-twitch type IIb to slow-twitch type I and perhaps have a trophic effect that allows the phrenic motor neurons to live longer (32).

Diaphragm pacing has also been tried for Arnold Chiari malformation, meningomyelocele, neurofibromatosis, incomplete $\mathrm{C} 3-\mathrm{C} 4$ fractures, $\mathrm{C} 4-\mathrm{C} 5$ fractures with ascending paralysis to C2-C3 level, radiation induced phrenic nerve injury (3).

\section{Evaluation}

Diaphragm pacing is a costly undertaking, and depending on the specific technique of implantation, variable amounts of risks are associated with the surgical procedure. Therefore, potential candidates should be carefully screened and meet specific eligibility criteria. Initial evaluation should thoroughly assess psychological factors $(4,37,38)$. Some centers will even wait a year after injury to consider diaphragmatic pacemaker implantation (9). Patients with lesions of lower motor neurons, of the phrenic nerve itself or its roots, or with diaphragm weakness not due to disuse atrophy should not be consider for pacing $(28,39)$.

The ideal candidates for phrenic nerve pacing are those with normal phrenic nerves, diaphragms, and lungs $(5,21)$. Pacing may not be effective in patients with lesions involving the segment of the cord C 3 and C5 as some or all of the phrenic nerve cell bodies or lower motor neurons may be destroyed (21). Glenn et al reported that of 77 cases selected, 48 patients had defects in respiratory control, and 29 had lesions of the upper motor neurons of the phrenic nerve (21). For patients who do not have intact phrenic nerves, conventional or intramuscular diaphragmatic pacing is not an option (5).

Once suspected, diaphragmatic dysfunction can be confirmed by a number of tests. Chest radiographs may reveal elevated diaphragms and basal subsegmental atelectasis. Sniff tests are not useful to diagnose bilateral diaphragmatic paralysis $(2,38)$. Pulmonary function tests, especially measurements of upright and supine vital capacity, are non invasive and may support or refute the diagnosis(2,14,38). Arterial blood gas values during mechanical ventilation $(23,36,38)$ and a sleep study when possible, add diagnostic information (23).

Maximal static inspiratory pressure and sniff nasal inspiratory pressure have limitations due to patient effort. Both tests are markedly reduced to less than $30 \%$ of the predicted value, in those with bilateral diaphragm paralysis, as well as tetraplegia patients $(2,6)$. 
Other tests to assess diaphragmatic function could be invasive like transdiaphragmatic pressure (Pdi) or noninvasive like ultrasonography (2). Measurement of Pdi provides the difference between gastric and esophageal pressures during tidal breathing, maximal sniff maneuvers (sniff Pdi), maximal inspiratory efforts against a closed glottis (Pdi max), or transcutaneous electrical or magnetic stimulation of the phrenic nerve (twitch Pdi) $(2,25,38)$.

A twitch Pdi greater than $10 \mathrm{cmH}_{2} \mathrm{O}$ with unilateral phrenic-nerve stimulation or greater than $20 \mathrm{cmH}_{2} \mathrm{O}$ with bilateral phrenic-nerve stimulation also rules out clinically significant weakness. Ultrasonography of the diaphragm at its zone of apposition with the rib cage shows thickening of the diaphragm reflecting shortening during inspiration. Loss of thickening is diagnostic of diaphragmatic paralysis (2). Ultrasonography is ideal in children because it is noninvasive, painless, safe and portable. Image quality is superior in children compared with adults because of the small body size and lower body fat (40).

Electromyography (EMG) of the diaphragm during tidal breathing or after phrenic nerve stimulation is limited by technical issues of electrode placement, "crosstalk" from adjacent muscle groups, and variable distance of electrodes to muscle related to subcutaneous thickness when electrodes are placed at standardized places like seventh and ninth intercostal spaces $(4,18)$.

Phrenic nerve integrity must be demonstrated by EMG twitch of diaphragm contraction in response to electrical stimulation prior to considering diaphragmatic pacemaker insertion. This is tested by cervical magnetic stimulation and/or electrical stimulation of the phrenic nerve in the neck $(20,21,36,38,39)$. However a recent French series which looked at long term outcomes, found benefit in phrenic nerve pacing even in patients who had injuries dating back several years. $(20,39)$. EMG response of the diaphragm is considered normal if the latency was within normal range between 5.5 and $6.5 \mathrm{~ms}$ for cervical magnetic stimulation, and between 6.5 and $8 \mathrm{~ms}$ for electrical stimulation. In patients on prolonged mechanical ventilation, due to diaphragm atrophy, conduction times may be prolonged $(4,5,20)$. Fluoroscopic evaluation of diaphragm movement during phrenic nerve stimulation, is useful to exclude both false-positive and false-negative results of phrenic nerve stimulation. The diaphragm should descend at least 3 to $4 \mathrm{~cm}$ during stimulation as visualized fluoroscopically $(4,23)$. Phrenic nerve function can also be assessed by measurements of transdiaphragmatic pressure Pdi (4,39). See Figure 5.

\section{Surgical implantation techniques}

The goal of any diaphragmatic pacing system is to provide safe and effective activation of the diaphragm, sufficient to provide adequate ventilation to meet metabolic requirements (4).

In the past the pacemaker electrodes were placed at the cervical area (25); but is presently discouraged for several reasons. Cervical phrenic nerve stimulation may result in incomplete diaphragm activation in the presence of an accessory branch from a lower segment of the cervical spinal cord that joins the main trunk of the phrenic nerve in the lower neck region or thorax.(4). 


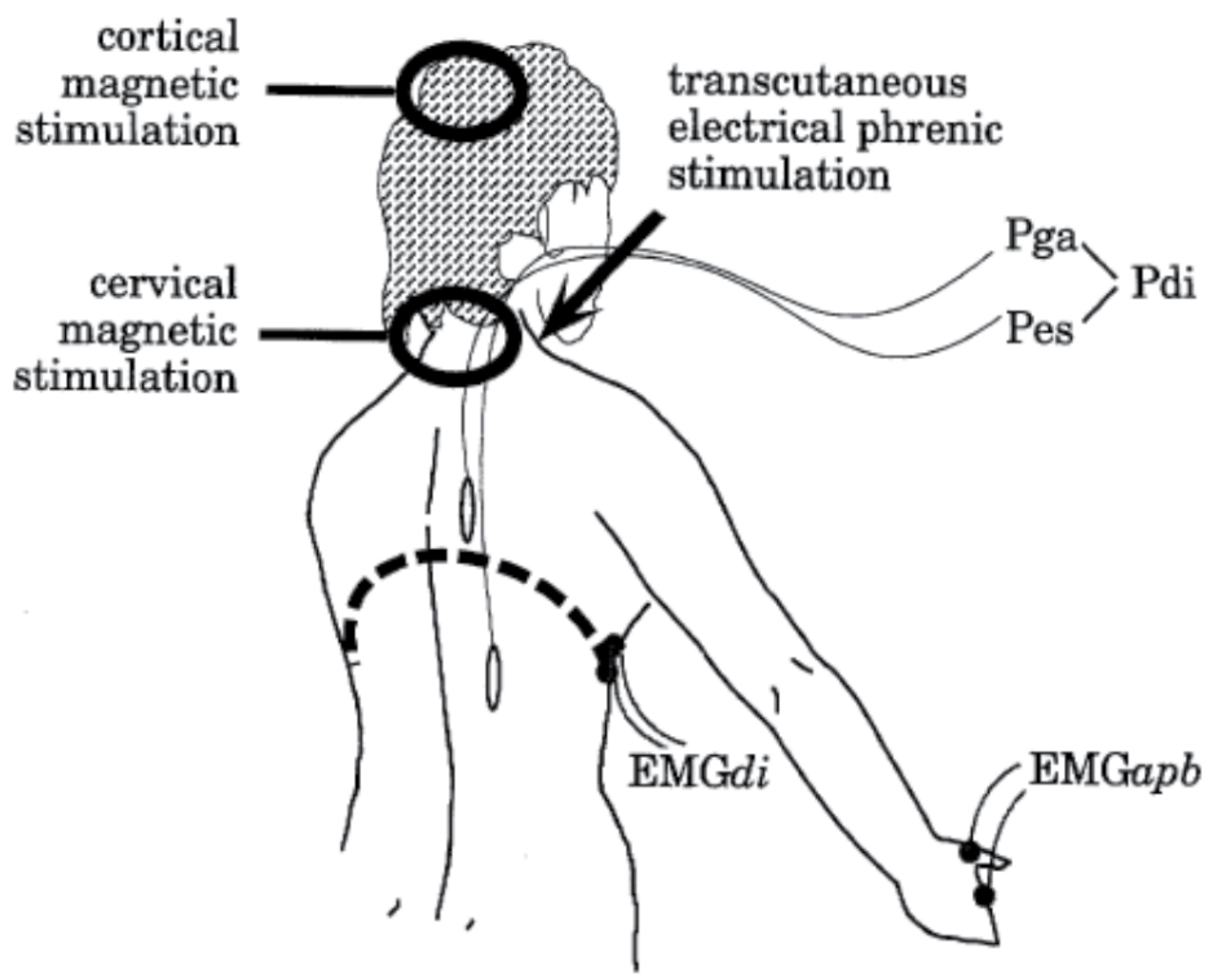

Reproduced with permission. Assessment of the Motor Pathway to the Diaphragm using Cortical and Cervical Magnetic Stimulation in the Decision-making process of phrenic Pacing. Thomas Similowski et al. Chest 1996; 110:1551-57

Figure 5. Schematic representation of the experimental setup used to study the patients. Diaphragmatic EMG (EMGdi), esophageal pressure (Pes), and gastric pressure (Pga) were measured in response to cortical and cervical magnetic stimulation, and to transcutaneous electrical stimulation of the phrenic nerve in the neck. Pdi was derived from Pes and Pga. An EMG ofthe APB (EMGapb) was used as control in some patients.

In Glenn's series, bilaterally thoracotomy procedures were performed 10 to 14 days apart. Once phrenic nerves were exposed surgically the platinum electrodes were placed directly in contact with the perineurium of the nerve in a tunnel behind the nerve that was large enough to allow free movement of the electrode within it. Then, the electrodes were connected to the receiver in the subcutaneous pocket were it was tested prior to skin closure (21).

Le Pimpec-Barthes et al successfully used Video-assisted thoracic surgery (VATS) for the implantation. The endoscopic camera was inserted in the pleural space through a second intercostal space incision. Careful dissection of the phrenic nerve was carried out in front of the vena cava on the right and in the pulmonary hilum on the left. Then quadripolar electrodes were implanted in both sides, 2 above and 2 below the nerve. Each Teflon patch 
was sutured to pleura or pericardium to prevent dislodgement. Then wires were brought out through a mini-thoracotomy, and tunneled under the pectoralis major muscle to a subcutaneous pocket. The wires were then connected to the subcutaneous radiofrequency receivers on each side. Intraoperative testing confirmed correct placement. No nerve injury or other significant intraoperative complications were observed (20). Robotic surgical placement has also been tested successfully including in patients with tetraplegia (4).

Another minimally invasive approach advocated intramuscular placement of the stimulating electrodes in the diaphragm laparoscopically $(5,9)$. In this method, identification of motor points of the phrenic nerves was done by a mapping procedure which involved electrical stimulation each hemidiaphragm with a probe at several locations. Two electrodes were placed in each diaphragm to stimulate all branches of the phrenic nerve maximally at the point where stimulation evoked the best motor response of the diaphragm $(5,9,11)$. After testing all 4 wires are brought out through the epigastric port, and then tunneled to the chest where they were connected to the transcutaneous exiting wires. Onders emphasized that the accurate identification of motor points is crucial for successfully pacing the diaphragm (9). DiMarco et al described further improvement of the technique with coarse mapping and direct visualization of diaphragmatic contraction $(4,15)$. A significant difference was noted in patients with ALS whose diaphragms had weaker contractions with the typical mapping stimulation.-Nevertheless, the laparoscopic procedure can be safely performed at multiple independent hospital sites for ventilator-dependent tetraplegia and ALS (11). Laparoscopic approach has cost and simplicity advantages and can be performed on an outpatient basis. In addition there is no manipulation of the phrenic nerve by itself (17).

Assouad et al have tried placing the electrodes through a cervical incision thoracic endoscopic surgery (CITES). Here a mediastinoscopy is followed by bilateral pleurotomies, and then a gastroscope is used in the pleural space to locate the phrenic nerve penetration points at the dome of the diaphragm. Finally needle and electrode hooks were placed close to the entry point of the phrenic nerves (19).

There are three commercially available diaphragmatic pacing systems depicted in table 1: a) Avery Biomedical Devices, b) Atrotech OY, and c) Medimplant systems. Each system is designed for lifetime use (4)

\section{Settings}

After surgical implantation, a period of 2 weeks should elapse before initiation of diaphragm pacing $(20,28,35)$. The diaphragm is weakened from prolonged mechanical ventilation, therefore, at the beginning a process of conditioning is carried through gradual lengthening of periods of stimulation $(28,38)$. Thus, a strategy of minimizing diaphragm fatigue during pacing should involve the gradual conditioning with low-frequency stimulation and a slow respiratory rate (29). Diaphragmatic conditioning is a process by which the fast-contracting fibers (anaerobic, highly glycolytic fibers), which are highly susceptible to get fatigued, are converted to slow-contracting fatigue-resistant (aerobic oxidative fibers), which enable permanent ventilatory support using diaphragmatic pacing $(4,36)$. 


\begin{tabular}{|c|c|c|c|c|}
\hline Device & $\begin{array}{l}\text { AVERY I-107A } \\
\text { (superceded) }\end{array}$ & AVERY I-110A & ATROTECH & MEDIMPLANT \\
\hline Receiver size (mm) & 46 (diam) $\times 16$ & 30 (diam) $\times 8$ & $49($ diam $) \times 8.5$ & $56 \times 53 \times 14$ \\
\hline Battery life (hr) & 160 & 100 & $\begin{array}{l}160-320(12 \mathrm{~V}) \\
8(9 \mathrm{~V})\end{array}$ & 24 \\
\hline Controller + battery weight $(\mathrm{kg})$ & 3.6 & 0.54 & $\begin{array}{l}0.45+0.6(12 \mathrm{~V}) \\
0.45+0.045(9 \mathrm{~V})\end{array}$ & $0.8+0.62$ \\
\hline Controller size (mm) & $179 \times 114 \times 97$ & $146 \times 140 \times 25$ & $185 \times 88 \times 28$ & $170 \times 130 \times 51$ \\
\hline Breaths/min & $10-50$ & $6-24$ & $8-35$ & $5-60$ \\
\hline Pulse interval (ms) & $35-170$ & $40-130$ & $10-100$ & $25-170$ \\
\hline Pulse width (microsec) & 150 & 150 & 200 & $100-1000$ \\
\hline Sigh possible & yes & yes & yes & yes \\
\hline Electrodes & Monopolar; bipolar & Monopolar; bipolar & Quadripolar & Quadripolar \\
\hline $\begin{array}{l}\text { No. of receivers to stimulate both } \\
\text { hemidiaphragms }\end{array}$ & 2 & 2 & 2 & 1 \\
\hline
\end{tabular}

Reproduced with permission. Electrical Stimulation to Restore Respiration. Journal of Rehabilitation Research and Development. Vol 33. N 2. April 1996: 123-132

Table 1. Technical features of phrenic nerve stimulators

While on mechanical ventilation, many patients are generally maintained with large tidal volumes. Diaphragmatic pacing is likely to generate smaller tidal volumes leading to respiratory acidosis therefore ventilator adjustments are needed initially (4).

Glenn initiated 5 minutes per hour of stimulation with low frequency stimulation $(11 \mathrm{~Hz})$ at slow rate ( 8 to 10 per minute). Tidal volumes at the beginning and end of each stimulation period were measured. Once tidal volumes at the end of stimulation period were at least $75 \%$ of the beginning volumes, the length of pacing was progressively increased by one to two minutes per hour until the diaphragm was paced 30 minutes per hour. After that, periods of pacing were increased by 5 minute per hour daily. Mechanical ventilation was discontinued during pacing once minute volume was adequate $(>5 \mathrm{~L} / \mathrm{min}$ in adults). Diaphragm conditioning is performed during daytime. Each hour of pacing with good minute volume is followed by an hour of rest on the ventilator. Pacing duration is incrementally increased by 15 minutes per session until 6 hours are reached. These periods are progressively extended to 8, 10 and 12 hours. When pacing on each side has been extended to 12 hours, while producing satisfactory minute volumes and normal arterial blood gases, the patient can be completely removed from mechanical ventilation. This weaning process usually takes 2 to 3 months, which is the time frame required for diaphragmatic fiber recovery $(21,28,36,38,41)$. A considerably longer period of conditioning is required in children because of immaturity of respiratory components (21). Every few weeks the pulse frequency and respiratory rate are decreased until the lowest values were reached at which ventilation was still adequate (41). Once the maximal stimulation time has been established, several schedules for maintenance can be followed. For patients who respond normally to stimulation of either side of the diaphragm, they recommended pacing each side alternately, 12 hours at the time (28). The voltage required to effect minimal (threshold) and maximal diaphragm motion during pacing was confirmed or adjusted about once a month (41). Alternatively uninterrupted simultaneous pacing of both hemidiaphragms, using a low-frequency stimulation $(7-8 \mathrm{~Hz})$ 
with a respiratory rate of 5 to 9 per minute may be appropriate for adult patients (41). In general the transition from mechanical ventilation to chronic full-time pacing must be individualized for each patient (4). Occasionally this weaning process can be performed with a home-based ventilator weaning and conditioning program $(22,42)$.

Until full-time pacing is achieved, a tracheostomy needs to remain in place, even after discontinuation of positive pressure ventilation to facilitate intermittent suctioning. This also serves as a back up to resume mechanical ventilation in case of pacemaker failure or other issues like the lack of coordination between the paced diaphragm, the upper airway muscles, and the accessory respiratory muscles in tetraplegics $(28,34)$.

In patients with CAHS, upper airway resistance is augmented by diaphragmatic contraction or diminished upper airway muscle activity and may be induced by diaphragm pacing during sleep $(34,43)$. In this population without muscle paralysis, pacing can be more aggressively initiated, 8 to 10 hours nightly by the third week postoperatively. Moreover, CAHS patients should have baseline sleep study with and without pacing to assure adequacy of pacing and identify any upper airway obstruction (21).

ALS patients begin conditioning programs by five 30-min pacing sessions a day. If ALS patients develop hypoventilation, the amount of time paced can be increased and then used at night. Continuous positive airway pressure (CPAP) or noninvasive positive-pressure ventilation may still be needed to maintain a patent upper airway (11).

Pacemaker function should be monitored on a routine basis and emergently in situations in which patients complain of difficulty breathing. Tidal volumes can be easily measured by spirometry; both electrodes stimulating each diaphragm can be independently evaluated. Pulse oxymetry as well end-tidal $\mathrm{PCO}_{2}$ should also help to assess ventilation (4).

\section{Benefits}

Ventilation by phrenic nerve pacing appears to provide both health and lifestyle advantages to the user. Freedom from positive-pressure ventilator reduces the risks of ventilatorassociated problems like tracheomalacia, infections, bleeding, barotraumas, and tracheoesophageal fistulas $(4,22,23)$.

Other ventilator-related disasters are also prevented like sudden death from accidental disconnection from ventilator, or mechanical ventilation device failure (23).

Olfaction was altered because nasal fossae were bypassed during positive-pressure ventilation on tracheostomy patients. Thus, improved speech and sense of smell recovery contribute to a better perception of quality of life in patients with successful diaphragmatic pacing $(8,23,44)$.

Phrenic nerve pacing allows negative-pressure ventilation, leading to a decrease in respiratory infections, in addition to improved patient comfort (8).

Liberation from ventilation also leads to reduced weight of their wheelchair and simplified transfers as well as elimination of social embarrassment associated with ventilator dependency $(4,7)$. 
Additionally, patients with pacing systems felt an overall improvement in their well being by reducing fatigue, depressed mood state and sleep disruptions that result from prolonged mechanical ventilation(7). A case report has demonstrated clinical evidence of improvement in right heart failure from pacing, and a fall in red cell volume and pulmonary artery pressures (33). Better ventilation of the posterior lobes minimizes atelectasis and improves cardiac function by increasing venous return (7).

Interestingly, in patients with ALS, diaphragmatic pacing significantly reduced the rate of decline of FVC from $2.4 \%$ preimplant to $0.9 \%$ per month postimplantion extrapolating to a 24-month improvement in survival (32).

\section{Complications}

A major complication is the iatrogenic injury to the phrenic nerve during operative implantation of phrenic nerve electrode and subsequent pacemaker failure $(4,30)$. In patients with thoracoscopic pacemaker implantation the most feared complication is phrenic nerve entrapment (36).

In addition to complications common to all surgical procedures, laparoscopy may be associated to pneumothorax, capnothorax $\left(\mathrm{CO}_{2}\right.$ tracking from the pressurized abdomen to the pleural space) and subcutaneous emphysema. Right shoulder pain may result from stimulation of phrenic nerve afferents $(11,27,28,45)$. All surgical procedures involving implantation of a foreign body carry some risk of infection, with rates of approximately $3 \%$ in case of phrenic nerve pacing (4).

Phrenic pacing can worsen or induce upper airway obstruction with central alveolar hypoventilation; therefore a patent tracheostomy is necessary during sleep $(4,29)$. Similarly in ALS patients that have severe bulbar weakness the upper airway can collapse during inspiration, thus needing a tracheostomy or CPAP treatment (15).

DiMarco reported one subject developed intermittent aspiration of food during meals, due to large negative airway pressure generated during diaphragmatic contraction (27).

Potential long-term complications related to pacing include electrode dislodgement and breakage that will result in device malfunction $(22,27)$.

Pacemaker failure due to technical problems is always a possibility. Strong magnetic fields, such as magnetic resonance imaging can override the electronic system of diaphragmatic pacemakers (4).

\section{Outcomes}

Diaphragmatic pacing can allow patients the benefits of negative pressure breathing. They have been used safely up to 19 years post injury in individuals with tetraplegia (7). All of these patients were able to tolerate outpatient surgery without perioperative morbidity.

Retrospective assessments have indicated a correlation between excess mortality and neurological level of injury, older age at injury, and injury at earlier calendar years (5). 
The problems associated with bilateral phrenic nerve pacing in infants and children are related more to cor pulmonale, as result of hypoventilation, than the mechanics of pacing itself, which can lead to uncertain outcomes (13).

It has been shown to improve quality of life and to extend survival in patients with advanced respiratory muscle weakness (12).

Carefully selected patients should undergo phrenic nerve pacemaker placement by experienced surgeons. They need to have individualized monitored pacing regimens with close follow up for best outcomes $(25,37)$.

The cost savings could be significant. The average cost of ventilator-dependent patient is three times that without a ventilator. During 2004 available phrenic nerve pacing devices with the hospitalization cost more than $\$ 120,000$. One of the patients in that series who was nursing homebound was able to save $\$ 13,000$ a month to Medicaid, because he no longer needed to be in a ventilator unit (9). Another series demonstrated that the first year investment for diaphragmatic pacing is paid off after three years. When costs of a respiratory infection are incorporated in the cost analysis, the diaphragm pacing becomes more cost effective in 1 year. (18).

Overall, patients with SCI treated with phrenic nerve pacing instead of standard mechanical ventilation experienced less airway infections, had reduced costs for single use airway equipment, had improved the quality of speech, had improved quality of life, and probably derived a mortality benefit (18).

Although the number of patients who may benefit from this technology is small, the potential improvement in quality of life is substantial $(46,47)$.

Garrido-Garcia and colleague found the mortality to be $22.7 \%$ (36). The problems associated with bilateral phrenic nerve pacing in infants and children are related more to cor pulmonale, as result of hypoventilation, than the mechanics of pacing itself (13).

Diaphragmatic pacemakers are safe to use in patients with cardiac pacemakers.

Patient satisfaction in recipients of diaphragm pacing was very high particularly in tetraplegic patients on ventilators (20).

\section{Conclusion}

Over the last fifty years, significant progress has been made in diaphragmatic pacing. Improvement in surgical techniques have reduced procedural complication during implantation Research in this field has relatively lagged behind other pacemakers such as cardiac devices presumably due to less financial motivation. Ideally a diaphragmatic pacemaker should synchronize with electrical signals from the central nervous system, and adapt to changing metabolic demands. Further development of such devices promises hope for patients with chronic respiratory failure for improved quality of life and possible mortality benefit at a reasonable cost. 
In conclusion this technique is efficient in liberating selected ventilator-dependent patients with respiratory paralysis and treating alveolar hypoventilation syndromes. Future clinical trials will increase acceptance of this method as an alternative form of treatment for respiratory failure in selected patients.

\section{Author details}

Jorge F. Velazco ${ }^{1,2}$, Shekhar Ghamande ${ }^{1,2}$ and Salim Surani ${ }^{1, *}$

Texas AEM University, Texas, USA

Scott $\mathcal{E}$ White Hospital, Temple, Texas, USA

\section{References}

[1] Neuromuscular adaptations to respiratory muscle inactivity. Carlos B. Mantilla; et al. Respir Physiol Neurobiol. 2009 Nov 30; 169(2): 133-140.

[2] Dysfunction of the Diaphragm. F. Dennis McCool, M.D; et al. N Engl J Med 2012; 366:932-942.

[3] Diaphragmatic Pacemaker. Salim Surani, MD. Modern Pacemakers - Present and Future. In-Tech. 2011.453-470.

[4] Inspiratory Muscle Pacing in Spinal Cord Injury: Case Report and Clinical Commentary. Anthony F. DiMarco,MD; et al. J Spinal Cord Med 2006; 29:95-108.

[5] Respiratory Dysfunction and Management in Spinal Cord Injury. Robert Brown, MD; et al. Respir Care. 2006 Aug; 51(8): 853-870.

[6] Effect of Spinal Cord Injury on the Respiratory System: Basic Research and Current Clinical Treatment Options. M. Beth Zimmer, PhD; et al. J Spinal Cord Med. 2007; 30:319-330.

[7] Diaphragm Pacing Stimulation System for Tetraplegia in Individuals Injured During Childhood or Adolescence. Raymond P. Onders, MD; et al. J Spinal Cord Med. 2007; 30:S25-S29.

[8] Multicenter analysis of diaphragm pacing in tetraplegics with cardiac pacemakers: Positive implications for ventilator weaning in intensive care units. Raymond P. Onders, MD; et al. Surgery 2010; 148:893-898.

[9] Mapping the phrenic nerve motor point: The key to a successful laparoscopic diaphragm pacing system in the first human series. Raymond P. Onders, MD; at al. Surgery 2004; 136:819-826.

[10] Delayed diaphragm recovery in 12 patients after high cervical spinal cord injury. A retrospective review of the diaphragm status of 107 patients ventilated after acute spinal cord injury. T Oo; et al. Spinal Cord (1999) 37:117-122.

[11] Complete worldwide operative experience in laparoscopic diaphragm pacing: results and differences in spinal cord injured patients and amyotrophic lateral sclerosis patients. Raymond P. Onders; et al. Surg Endosc (2009) 23:1433-1440.

" Corresponding Author 
[12] Ondine's curse: anesthesia for laparoscopic implantation of a diaphragm pacing stimulation system. Ahtsham U. Niazi, MBBS; et al. Can J Anesth/J Can Anesth (2011); 58:1034-1038.

[13] Congenital central hypoventilation syndrome: A report of successful experience with bilateral diaphragmatic pacing. Michael Coleman; et al. Arch Dis Child. 1980 Nov; 55(11): 901-903.

[14] Diaphragm plication in adult patients with diaphragm paralysis leads to long-term improvement of pulmonary function and level of dyspnea. Michel I.M. Versteegh; et al. European Journal of Cardio-thoracic Surgery 32 (2007):449-456.

[15] Laparoscopic diaphragmatic pacer placement - a potential new treatment for ALS patients: a brief description of the device and anesthetic issues. Clifford A. Schmiesing MD; et al. Journal of Clinical Anesthesia (2010) 22, 549-552.

[16] Radio-Frequency Electrophrenic Respiration Long-Term Application to a Patient With Primary Hypoventilation. John P. Judson, MD; et al. JAMA Mar 18, 1968;203(12):129134.

[17] Phrenic Nerve Pacing in a Tetraplegic Patient via Intramuscular Diaphragm Electrodes. Anthony F. DiMarco; et al. Am J Respir Crit Care Med 2002; 166:1604-1606.

[18] Mechanical ventilation or phrenic nerve stimulation for treatment of spinal cord injuryinduced respiratory insufficiency. S Hirschfeld; et al. Spinal Cord (2008); 46:738-742.

[19] Minimally invasive trans-mediastinal endoscopic approach to insert phrenic stimulation electrodes in the human diaphragm: a preliminary description in cadavers. Jalal Assouad; et al. European Journal of Cardio-thoracic Surgery 40 (2011) e142-e145.

[20] Intrathoracic phrenic pacing: A 10-year experience in France. Francoise Le PimpecBarthes, MD; et al. J Thorac Cardiovasc Surg 2011;142:378-383.

[21] Diaphragm Pacing by Electrical Stimulation of the Phrenic Nerve. William W.L. Glenn, MD; et al. Neurosurgery 17:974-984, 1985.

[22] Functional electrical stimulation therapies after spinal cord injury. David R. Gater, Jr; et al. NeuroRehabilitation 28 (2011) 231-248.

[23] Electrical stimulation to restore respiration. Creasey, Graham, MD; et al. Journal of Rehabilitation Research and Development; Apr 1996; 33:123-132.

[24] Lung function in diaphragm pacing S.W. Epstein, MD; et al. CMA Journal Jun 9,1979; 120:1360-1367.

[25] Diaphragm pacing. John Moxham; et al. Editorial. Thorax 1988; 43:161-162.

[26] Central Hypoventilation; Long-term Ventilatory Assistance by Radiofrequency Electrophrenic Respiration. William W. L. Glenn, M.D et al. Annals of Surgery, Oct 1970; 172(4): 755-773.

[27] Phrenic Nerve Pacing Via Intramuscular Diaphragm Electrodes in Tetraplegic Subjects. Anthony F. DiMarco, MD; et al. Chest 2005; 127:671-678.

[28] Long-Term Ventilatory Support by Diaphragm Pacing in Quadriplegia. William W.L. Glenn, MD; et al. Ann Surg 1976; 183(5): 566-577.

[29] Problems Associated with Diaphragm Pacing. Takashi Nishino. Respiration 2002; 69:1213. 
[30] Twenty Years of Experience in Phrenic Nerve Stimulation to Pace the Diaphragm. William, W. Glenn, MD; et al. PACE (1980) Vol 9. pp 780-784.

[31] Eight-Year Follow-Up Study of a Patient with Central Alveolar Hypoventilation Treated with Diaphragm Pacing. Fumihiko Yasuma et al. Respiration 1998; 65:313-316.

[32] Amyotrophic lateral sclerosis: the Midwestern surgical experience with the diaphragm pacing stimulation system shows that general anesthesia can be safely performed. Raymond P. Onders, M.D; et al. The American Journal of Surgery (2009) 197, 386-390.

[33] Diaphragm pacing in ventilatory failure. S Lozewicz; et al. BMJ 1981, Oct 17; 283:10151016.

[34] The Swedish Experience in Phrenic Nerve Stimulation. Harald Fodstad. PACE, 10, January-February, Part II, 1987:246-251.

[35] Transesophageal Diaphragmatic Pacing for Treatment of Persistent Hiccups. David W. Andres, M.D; et al. Letter to Editor. Anesthesiology 2005; 102:483.

[36] Treatment of chronic ventilatory failure using a diaphragmatic pacemaker. H GarridoGarcia; et al. Spinal Cord (1998) 36:310-314.

[37] Long-Term Follow-Up of bilateral Pacing of the Diaphragm in Quadriplegia. John F. Elefteriades, MD; et al. Letter to the Editor. N Engl J Med 1992; 326:1433-1434.

[38] Transdiaphragmatic pressure in quadriplegic individuals ventilated by diaphragmatic pacemaker. Honesto Garrido-Garcia; et al. Thorax 1996; 51:420-423.

[39] Assessment of the Motor Pathway to the Diaphragm Using Cortical and Cervical Magnetic Stimulation in the Decision-making Process of Phrenic Pacing. Thomas Similowski, MD; et al. Chest 1996; 110:1551-1557.

[40] Ultrasound evaluation of piglet diaphragm function before and after fatigue. Keith C. Kocis; et al. J Appl Physiol 83:1654-1659, 1997.

[41] Ventilatory Support by Pacing of the Conditioned Diaphragm in Quadriplegia. William W.L. Glenn, MD; et al. N Eng J Med 1984; 310:1150-1155.

[42] Lo stimolatore frenico, valido supporto ventilatorio per l'assistenza domiciliare dei pazienti tetraplegici. Caso clinico. A. M. Giglio; et al. Minerva Anestesiol 2002; 68:567571.

[43] Conditioning of the diaphragm by phrenic nerve pacing in primary alveolar hypoventilation. Pearce G Wilcox; et al. Thorax 1988; 43:1017-1018.

[44] Diaphragm pacing restores olfaction in tetraplegia. D. Adler; et al. Eur Respir J 2009; 34: 365-370.

[45] Chest Pain and Diaphragmatic Pacing after Pacemaker Implantation. John L. Jefferies, MD; et al. Texas Heart Institute Journal. Volume 32, Number 1, 2005:106-107.

[46] Diapham pacing. Gerhard A Baer; et al. Correspondence. Thorax 1988; 43:743-744.

[47] Efficacy of Combined Inspiratory Intercostal and Expiratory Muscle Pacing to Maintain Artificial Ventilation. Anthony F. DiMarco; et al. Am J Respir Crit Care Med 1997; 156:122-126. 



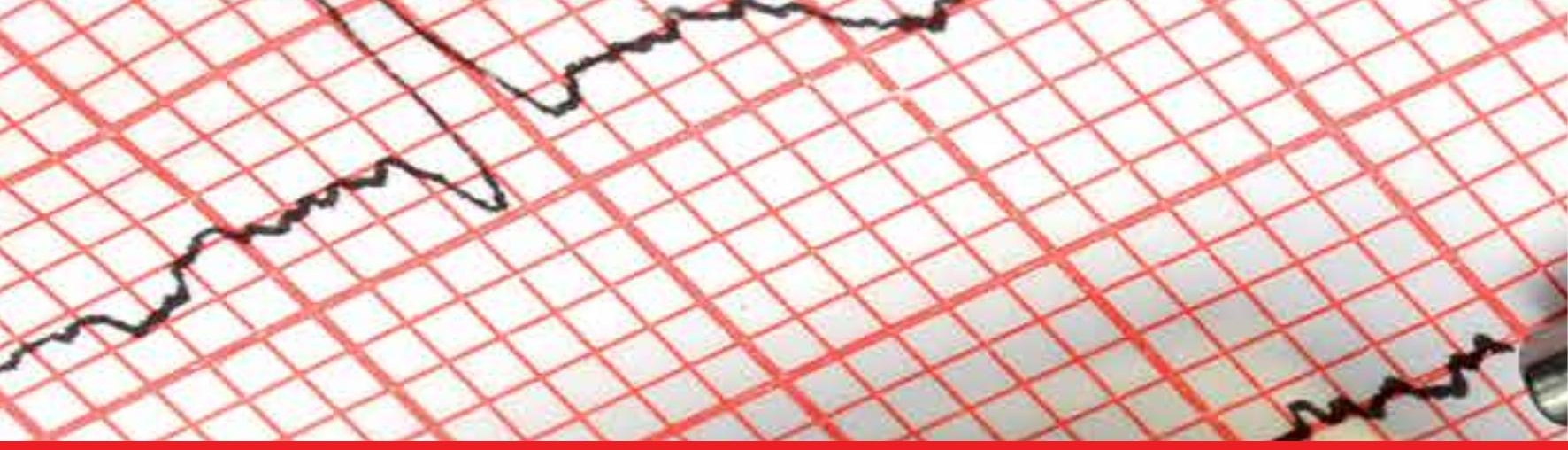

\section{Edited by Attila Roka}

Patients with implanted pacemakers or defibrillators are frequently encountered in various healthcare settings. As these devices may be responsible for, or contribute to a variety of clinically significant issues, familiarity with their function and potential complications facilitates patient management. This book reviews several clinically relevant issues and recent advances of pacemaker therapy: implantation, device followup and management of complications. Innovations and research on the frontiers of this technology are also discussed as they may have wider utilization in the future. The book should provide useful information for clinicians involved in the management of patients with implanted antiarrhythmia devices and researchers working in the field of cardiac implants. 